\title{
Resonant Second Generation Slepton Production at the Tevatron
}

\author{
Von der Fakultät für \\ Mathematik, Informatik und Naturwissenschaften der \\ Rheinisch-Westfälischen Technischen Hochschule Aachen zur \\ Erlangung des akademischen Grades eines Doktors der \\ Naturwissenschaften genehmigte Dissertation
}

vorgelegt von

\author{
Diplom-Physiker \\ Christian Tobias Autermann \\ aus Ibbenbüren, Münsterland
}

Berichter: Univ.-Prof. Dr. T. Hebbeker

Univ.-Prof. Dr. L. Feld

Tag der mündlichen Prüfung: 21.12.2006

Diese Dissertation ist auf den Internetseiten der Hochschulbibliothek online verfügbar. 



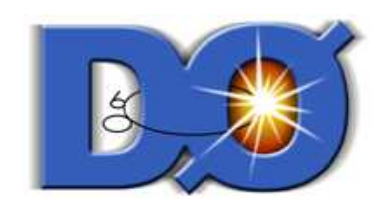

\section{Resonant Second Generation Slepton Production at the Tevatron}

\section{Christian Autermann}

III. Physikalisches Institut A, RWTH Aachen, Germany

A search for R-parity violating supersymmetry with the D $\varnothing$ detector at the Fermilab Tevatron $p \bar{p}$-collider is presented. Assuming a non-zero $L Q \bar{d}$ coupling $\lambda_{2 j k}^{\prime}$ leads to final states with two muons and jets. A total integrated luminosity of $375 \mathrm{pb}^{-1}$ collected between April 2002 and August 2004 is utilized. The observed number of events is in agreement with the Standard Model expectation, and limits on $\not R_{p}$ supersymmetry are derived.

For a non-zero $\lambda_{211}^{\prime}$ coupling resonant slepton production is possible. Three independent channels $\tilde{\mu} \rightarrow \tilde{\chi}_{1}^{0} \mu, \tilde{\mu} \rightarrow \tilde{\chi}_{2}^{0} \mu$ and $\tilde{\nu}_{\mu} \rightarrow \tilde{\chi}_{1}^{ \pm} \mu$ are combined within the mSUGRA framework, and worldbest limits as a function of the coupling parameter $\lambda_{211}^{\prime}$ and other SUSY parameters are derived. In case of any non-zero $L Q \bar{d}$ coupling $\lambda_{2 j k}^{\prime}$ with $j=1,2$ and $k=1,2,3$ neutralino masses below $41.5 \mathrm{GeV}$ can be excluded at $95 \%$ CL, significantly extending results previously obtained by $\mathrm{D} \emptyset$.

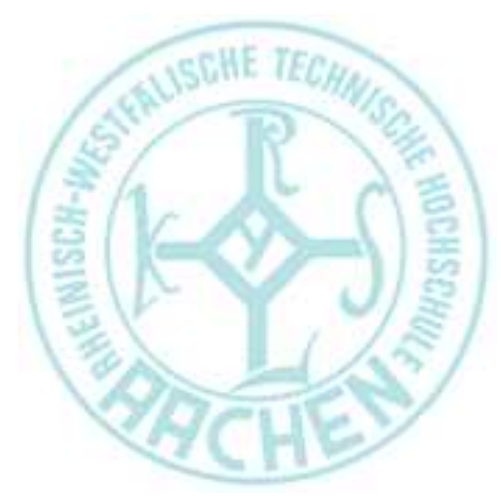


Man hört nur die Fragen, auf welche man im Stande ist, eine Antwort zu finden

Friedrich Nietzsche, Die fröhliche Wissenschaft 


\section{Abstract}

In this thesis a search for $R$-parity violating supersymmetry is presented. Two different approaches, determined by the event topologies, are chosen to search for resonant slepton production and for the pair and associated production of gauginos.

To the resonant slepton production three different signal channels contribute; $\tilde{\mu} \rightarrow \tilde{\chi}_{1}^{0} \mu$, $\tilde{\mu} \rightarrow \tilde{\chi}_{2,(3,4)}^{0} \mu$ and $\tilde{\nu}_{\mu} \rightarrow \tilde{\chi}_{1,(2)}^{ \pm} \mu$. These three channels are analyzed separately. The slepton-mass - gaugino-mass plane ist scanned systematically for an excess in the data. Effective 2-dimensional cuts have been developed, to separate signal-like events from background. The analysis profits from the ability to reconstruct the neutralino as well as the slepton mass. The 2D cuts must be very flexible, to account for the different event topologies in the three channels, while scanning the slepton- and gaugino-masses from a few $\mathrm{GeV}$ to several hundred $\mathrm{GeV}$.

The pair and associated production of gauginos and their decay via any $L Q \bar{d}$ coupling $\lambda_{2 i j}^{\prime}$ with $j=1,2$ and $k=1,2,3$ does not comprise a resonance. Therefore the search is not able to benefit from a mass reconstruction. The two muon charges are not correlated, so that the selection of only like-sign di-muon final states is the chosen method to suppress Standard Model background processes.

No indication of $\not R_{p}$ supersymmetry production or any disagreement between data and Standard Model expectation have been found. Therefore exclusion limits with $95 \%$ confidence level (CL) have been calculated. Model independent limits on cross section times branching ratio are given. These limits only depend on the masses of the contributing particles of the process. The predicted cross section of any given model can be compared to these cross section limits to determine the exclusion contour in that model.

The three resonant slepton production channels $\tilde{\mu} \rightarrow \tilde{\chi}_{1}^{0} \mu, \tilde{\mu} \rightarrow \tilde{\chi}_{2,(3,4)}^{0} \mu$ and $\tilde{\nu}_{\mu} \rightarrow \tilde{\chi}_{1,(2)}^{ \pm} \mu$ are combined within the minimal super gravity (mSUGRA) model to the world's best limit on the relevant coupling $\lambda_{211}^{\prime}$ in dependence of the neutralino and the slepton masses. A lower bound on the slepton mass depending only on $\lambda_{211}^{\prime}$ is derived.

If any $L Q \bar{d}$ coupling $\lambda_{2 i j}^{\prime}$ with $j=1,2$ and $k=1,2,3$ is larger than 0.01 , then neutralino masses below $41.5 \mathrm{GeV}$ and gluino masses below $285 \mathrm{GeV}$ can be excluded within mSUGRA. This result is a significant extension of the D $\varnothing$ Run I bounds. 



\section{Zusammenfassung}

In dieser Arbeit wird eine Suche nach $R$-Parität verletzender Supersymmetrie vorgestellt. Auf Grund der verschiedenen Ereignistopologien wurden zwei verschiedene Herangehensweisen gewählt um zum einen nach Resonanter sLeptonproduktion und zum anderen nach Paar- bzw. assozierter Produktion von Gauginos zu suchen.

Im Falle der resonanten Sleptonproduktion tragen drei verschiedene Signalkanäle bei; $\tilde{\mu} \rightarrow \tilde{\chi}_{1}^{0} \mu, \tilde{\mu} \rightarrow \tilde{\chi}_{2,(3,4)}^{0} \mu$ und $\tilde{\nu}_{\mu} \rightarrow \tilde{\chi}_{1,(2)}^{ \pm} \mu$. Diese drei Kanäle wurden seperat analysiert. Es wurden effektive 2-dimensionale Schnitte entwickelt, die signalartige Ereignisse vom Untergrund trennen. Diese Schnitte müssen flexibel sein, um den sehr unterschiedlichen Ereignistopologien in allen drei Kanälen und allen möglichen sLepton- und GauginoMassen, von einigen wenigen $\mathrm{GeV}$ bis mehreren hundert $\mathrm{GeV}$, Rechnung zu tragen.

Im Fall der Paar- bzw. assozierten Produktion von Gauginos gibt es keine Resonanz und somit nicht die Möglichkeit, von einer Massen-Rekonstruktion zu profitieren. Da aber die Ladung beider Muonen nicht korreliert ist, konnte durch eine Selektion von Ereignissen mit gleich-geladenen Muonen eine hinreichende Untergrund-Unterdrückung erreicht werden.

Es wurde kein Hinweis auf R-Parität verletzende Supersymmetrie oder eine signifikante Abweichung vom Standard Modell in den Daten gefunden. Deshalb wurden Ausschlussgrenzen mit 95\% Vertrauensniveau (CL) berechnet. Modellunabhängige Limits auf das Produkt aus Produktionswirkungsquerschnitt und Verzeigungsverhältnis werden präsentiert. Der vorhergesagte Wirkungsquerschnitt eines beliebigen Modells kann mit diesen Limits verglichen werden, um Ausschlussgrenzen für dieses Modell zu gewinnen.

Die drei Kanäle der resonanten sLepton Produktion, $\tilde{\mu} \rightarrow \tilde{\chi}_{1}^{0} \mu, \tilde{\mu} \rightarrow \tilde{\chi}_{2,(3,4)}^{0} \mu$ und $\tilde{\nu}_{\mu} \rightarrow \tilde{\chi}_{1,(2)}^{ \pm} \mu$ sind im Rahmen des minimalen Supergravitations-Modells (mSUGRA) zu dem weltweit besten Limit auf die relevante Kopplungsstärke $\lambda_{211}^{\prime}$, in Abhängigkeit von der Neutralino- und der sLeptonmasse, kombiniert worden. Für eine bestimmte Kopplungsstärke $\lambda_{211}^{\prime}$ können erstmalig Grenzen auf sLeptonmassen, unabhängig von anderen Parametern, angegeben werden.

Im Falle einer $L Q \bar{d}$ Kopplung $\lambda_{2 j k}^{\prime}$ mit $j=1,2$ und $k=1,2,3$, die größer ist als 0.01, können Neutralinomassen unterhalb von $41.5 \mathrm{GeV}$ und Gluinomassen unterhalb von $285 \mathrm{GeV}$ im Rahmen von mSUGRA ausgeschlossen werden. Dieses Ergebnis ist eine signifikante Verbesserung der Ausschlussgrenzen von DØ Run I. 



\section{Contents}

1 Introduction 1

2 Introduction to R-Parity Violating Supersymmetry 3

2.1 The Standard Model and its Limitations . . . . . . . . . . . . . . . . . 3

2.1.1 Local Gauge Symmetries . . . . . . . . . . . . . . . 4

2.1 .2 Higgs Mechanism . . . . . . . . . . . . . . 9

2.1.3 The Lagrangian of the Standard Model . . . . . . . . . . . . . 11

2.1.4 Neutrino Masses . . . . . . . . . . . . . . . . 11

2.1.5 The Hierarchy Problem of the Standard Model . . . . . . . . . . 12

2.1.6 Solutions of the Hierarchy Problem . . . . . . . . . . . . . . 14

2.2 Supersymmetry . . . . . . . . . . . . . . . . . 16

2.2.1 Explicit Supersymmetry Breaking . . . . . . . . . . . 19

2.2.2 The Wess-Zumino Model . . . . . . . . . . . . . 20

2.2.3 The Minimal Supersymmetric Standard Model . . . . . . . . . 22

2.2.4 Spontaneous Supersymmetry Breaking . . . . . . . . . . . 25

2.2 .5 Minimal Supergravity . . . . . . . . . . . . . . 27

2.2 .6 The Mass Spectrum . . . . . . . . . . . . . . 29

2.3 R-Parity . . . . . . . . . . . . . . . . 35

2.3.1 Resonant Slepton Production at the Tevatron . . . . . . . . . . 37

2.3.2 Pair and Associated Gaugino Production . . . . . . . . . . . 41

2.4 Existing bounds on R-Parity violating SUSY . . . . . . . . . . . . 44

2.4 .1 Indirect bounds . . . . . . . . . . . . . . . . . . . 44

2.4.2 Searches at $e^{+} e^{-}$Colliders. . . . . . . . . . . . . 46

2.4.3 Searches at Lepton - Hadron Colliders . . . . . . . . . . . . . 51

2.4.4 Searches at Hadron — Hadron Colliders . . . . . . . . . . . . . 54 


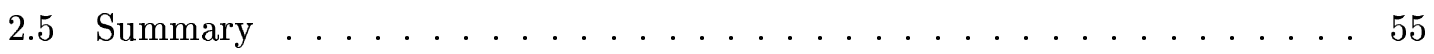

3 The Experimental Setup $\quad 57$

3.1 General Aspects of Collider Physics . . . . . . . . . . . . . . . 57

3.1.1 Interactions of Particles with Matter . . . . . . . . . . 58

3.1.2 Hadronization . . . . . . . . . . . . . . . 60

3.2 The Tevatron Accelerator . . . . . . . . . . . . . 61

3.3 The $\mathrm{D} \emptyset$ Detector . . . . . . . . . . . . . . 65

3.3.1 Coordinate System . . . . . . . . . . . . . 65

3.3.2 The Luminosity System . . . . . . . . . . . . . 67

3.3.3 The Central Tracking Systems . . . . . . . . . . . 71

3.3.4 The Calorimeter System . . . . . . . . . . . . . 76

3.3.5 The Muon System ..................... 80

3.4 Trigger, Data Acquisition and Processing . . . . . . . . . 86

3.4 .1 Level $1 \ldots \ldots \ldots \ldots \ldots$

3.4 .2 Level $2 \ldots \ldots \ldots \ldots \ldots \ldots$

$3.4 .3 \quad$ Level $3 \quad \ldots \ldots \ldots \ldots \ldots$

3.4.4 Computing and Software .................... 93

4 Data Samples $\quad 99$

4.1 Recorded Data . . . . . . . . . . . . . . . . 99

4.2 Multijet QCD Extraction from Data . . . . . . . . . . . . 101

4.2.1 QCD Sample without requiring a $b$-tag . . . . . . . . . . 102

4.2.2 QCD Sample with $b$-tag . . . . . . . . . . . . . 102

4.2.3 QCD Removal Using a Momentum Dependent Muon Isolation . . . 105

4.3 Monte Carlo Simulation . . . . . . . . . . . . . . . . . 105

4.3.1 Standard Model Background . . . . . . . . . . . . 105

4.3.2 Signal Monte Carlo . . . . . . . . . . . . . . . . 108

4.3.3 Higher Order Corrections . . . . . . . . . . . . . . 108

4.3 .4 Multiple Interactions . . . . . . . . . . . . . . . . . . 110

4.3.5 Parton Distribution Functions . . . . . . . . . . . . . . 111 
5 Object Identification $\quad 113$

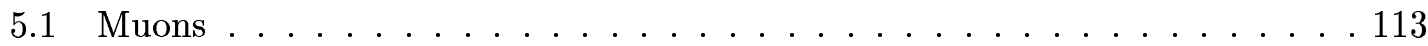

5.1.1 Di-Muon Trigger Efficiency . . . . . . . . . . . . . . 114

5.1 .2 Muon Reconstruction Efficiency . . . . . . . . . . . . 117

5.1.3 Muon Track Finding and Matching Efficiency . . . . . . . . . . 120

5.1 .4 Muon Isolation Efficiency . . . . . . . . . . . . . . . . 122

5.1.5 "Medium" Muon Efficiency . . . . . . . . . . . . . . . . . . 124

5.1.6 Muon Momentum Smearing . . . . . . . . . . . . . 127

5.1 .7 Summary Muon Efficiencies . . . . . . . . . . . . . 127

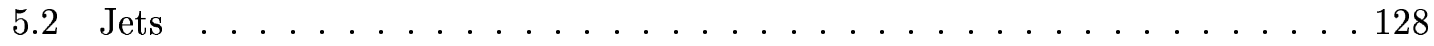

5.2 .1 Jet Algorithm . . . . . . . . . . . . . . . . . . 128

5.2 .2 Jet Identification . . . . . . . . . . . . . . . . 130

5.2 .3 Jet Reconstruction Efficiency . . . . . . . . . . . . . 131

5.2 .4 Jet Energy Scale . . . . . . . . . . . . . . . . . . . 133

5.2.5 Jet Energy Resolution . . . . . . . . . . . . . . 136

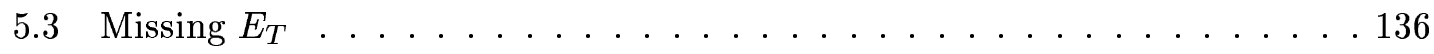

5.4 Taus . . . . . . . . . . . . . . . . . . . 138

6 Data Analysis 139

6.1 The Common Event Preselection . . . . . . . . . . . . . . 139

6.2 Search for Resonant Slepton Production . . . . . . . . . . . . . . . 144

6.2.1 Signal Monte Carlo Studies . . . . . . . . . . . . . . . . . . . 144

6.2 .2 Event Selection . . . . . . . . . . . . . . . 152

6.2 .3 Systematic Uncertainties . . . . . . . . . . . . . . . 165

6.2 .4 The Final Sample . . . . . . . . . . . . . . . 168

6.2 .5 Candidate events . . . . . . . . . . . . 170

6.3 Search for Gaugino Pair and Associated Production . . . . . . . . . 173

6.3.1 Signal Monte Carlo Studies . . . . . . . . . . . . . . . . 173

6.3.2 Event Selection . . . . . . . . . . . . . . . 174

6.3 .3 Systematic Uncertainties . . . . . . . . . . . . . . . 177

6.3 .4 The Final Sample . . . . . . . . . . . . . . . . . 177

6.3 .5 Candidate event $\ldots \ldots \ldots \ldots$. . . . . . . . . . . . . . . . . . . 
$\begin{array}{lll}7 & \text { Results } & 181\end{array}$

7.1 The $\mathrm{CL}_{s}$ Limit Calculation Method . . . . . . . . . . . . 181

7.2 Limits on Resonant Slepton Production . . . . . . . . . . . . . . 183

7.2.1 Model Independent Limits . . . . . . . . . . . . . . . . . . . 183

7.2.2 Combined Limits within mSUGRA . . . . . . . . . . . . . 183

7.3 Limits on Gaugino Pair and Associated Production . . . . . . . . . . . 190

7.3.1 Cross Section Limits . . . . . . . . . . . . . . . . 190

7.3.2 Interpretation within mSUGRA . . . . . . . . . . . 192

8 Conclusions $\quad 195$

A Feynman Diagrams of the Signal 199

A.1 Neutralino Decay Channels . . . . . . . . . . . . . . . . 199

A.2 Chargino Decay Channels . . . . . . . . . . . . . . . . 201

A.3 Neutralino 2 Decay Channels . . . . . . . . . . . . . . . . 202

$\begin{array}{ll}\text { B Susygen Modification } & 203\end{array}$

C Final Results in Detail 205

C.1 Channel $\tilde{\mu} \rightarrow \tilde{\chi}_{1}^{0} \mu$ with $\tan \beta=5$ and $\operatorname{sign}(\mu)=-1 \ldots \ldots \ldots 20 \ldots$

C.2 Channel $\tilde{\mu} \rightarrow \tilde{\chi}_{2,(3,4)}^{0} \mu$ with $\tan \beta=5$ and $\operatorname{sign}(\mu)=-1 \ldots \ldots 209$

C.3 Channel $\tilde{\nu}_{\mu} \rightarrow \tilde{\chi}_{1,(2)}^{ \pm} \mu$ with $\tan \beta=5$ and $\operatorname{sign}(\mu)=-1 \ldots \ldots \ldots \ldots \ldots \ldots$

$\begin{array}{ll}\text { References } & \mathbf{2 1 5}\end{array}$

$\begin{array}{ll}\text { Acknowledgments } & \mathbf{2 3 5}\end{array}$ 


\section{Chapter 1}

\section{Introduction}

Usually, supersymmetric models (SUSY) assume $R$-Parity conservation. The direct consequence is that the lightest supersymmetric particle, the LSP, is stable. This assumption is favored, because a neutral LSP could solve one of the big questions in current physics: The dark matter problem.

However, $R$-Parity need not be conserved; in fact, there is hardly any theoretical argument for $R$-parity to be conserved. Also the dark matter needs not to be supersymmetric, many other theories predict heavy weakly interacting particles. $R$-Parity violating (RPV or $\not R_{p}$ ) potentials can be added to any SUSY Lagrangian. This allows for single sparticle production and its decay to already known Standard Model particles, at the price of lepton and baryon number violation. With RPV it is possible to explain neutrino masses by neutrino - neutralino mass mixing. In chapter 2 the Standard Model, as well as its shortcomings leading to the introduction of supersymmetric models shall be discussed.

The search for $\not_{p}$ supersymmetry is performed with the $\mathrm{D} \emptyset$ experiment, a multi-purpose detector, located at one of two interaction points of the Tevatron accelerator, at Fermilab, near Chicago. The Tevatron is a proton-anti-proton collider which was completed in 1983 and led to the discovery of the heaviest quark, the top, in 1995 with the two detectors $\mathrm{CDF}$ and $\mathrm{D} \varnothing$. The accelerator was upgraded, together with both experiments, and started the Run II in 2001, reaching now a center of mass energy of $1.96 \mathrm{TeV}$. A total integrated luminosity of up to $8 \mathrm{fb}^{-1}$ is planned to be delivered and recorded per experiment until 2009. The total integrated luminosity utilized for this analysis coresponds to $374 \mathrm{pb}^{-1}$. The accelerator and the detector will be discussed in chapter 3 as well as the event trigger and the event data model.

Three different types of RPV couplings may exist, but this analysis concentrates on $L Q \bar{d}$ couplings, which couple Lepton $(L)$ superfields with quark $(Q)$ and down-quark like $(\bar{d})$ superfields. With three lepton and quark generations, there are $27 L Q \bar{d}$-couplings $\lambda_{i j k}^{\prime}$. In this analysis all RPV-coupling parameters are assumed to be negligibly small, except for one single coupling $\lambda_{2 j k}^{\prime}$. 
With a non vanishing $L Q \bar{d}$-coupling $\lambda_{211}^{\prime}, 2^{\text {nd }}$ generation sleptons - smuons or muon sneutrinos - can be produced resonantly at hadron colliders like the Tevatron or the Large Hadron Collider (LHC), accomplishing rather high production cross sections. In general only $R$-parity conserving pair and associated production of gauginos is possible. The analysis presented here is sensitive to all $\lambda_{2 j k}^{\prime}$ couplings with $j=1,2$ and $k=1,2,3$. The resonant production of sleptons via $\lambda_{211}^{\prime}$ is studied with special attention. All studied couplings result in final states consisting of two muons and jets.

To determine the Standard Model and the potential signal contributions in the data all relevant processes were simulated with Monte Carlo Generators, as illustrated in chapter 4. In a two-muon preselection dataset all possible signal contributions in the data are still negligibly small, compared to the Standard Model background processes. This allows a precise estimation of systematic uncertainties. Detector and reconstruction-algorithm efficiencies have been studied in great detail to minimize these uncertainties and to determine muon and jet identification efficiencies, as will be discussed in detail in chapter 5 .

Signal Monte Carlo studies and the various search strategies for $R$-parity violating supersymmetry are discussed in detail in chapter 6 . Finally the results of both searches; resonant slepton production, and pair and associated production of gauginos, are presented in chapter 7 , before this thesis is concluded in chapter 8 . 


\section{Chapter 2}

\section{Introduction to R-Parity Violating Supersymmetry}

In this chapter the Standard Model and its shortcomings, as well as possible solutions to these problems shall be discussed. The basics of a new supersymmetric theory and the concept of R-parity are introduced, which is the theoretical foundation of SUSY production at colliders. Finally, existing limits on the relevant coupling strengths are reviewed.

This theoretical introduction is based on the lectures of the SLAC Summer Institute (SSI) 2004 [1, 2], the lectures given at the Maria Laach Summer School 2002 [3], the Supersymmetry Primer [4] and other literature which is cited along the way.

\subsection{The Standard Model and its Limitations}

The Standard Model (SM) is an effective field theory that has been very successfully tested up to energy ranges of the order of the electroweak scale $\Lambda_{E W}$ at the LEP collider and of the order of $1 \mathrm{TeV}$ at the Tevatron. It describes all known particles and their interactions up to these energy scales. Three of the four fundamental forces; electromagnetism, the weak force and the strong force are unified in the theory. The gravitational force is about 40 orders of magnitude weaker than every other force, so that the impact of the SM on present experiments is not inhibited by the lack of a consistent quantum gravitation field theory. Nevertheless the Standard Model will ultimately break down at energy scales where gravity can no longer be neglected.

The Standard Model is the theory of the unification of the electromagnetic, the weak and the strong force. The dynamics are described by gauge theories, which is a very important concept in field theory. The principles of gauge symmetry leading to a consistent renormalizable quantum field theory will be discussed in the following section. The problems of the Standard Model will be discussed briefly and solutions leading to the introduction of supersymmetry will be motivated. 


\subsubsection{Local Gauge Symmetries}

In 1917 the mathematician E. Noether showed [5], that if a Lagrangian $\mathcal{L}(q, \dot{q})$ of any system, where the $q$ are the generalized coordinates, has a global symmetry, i.e. the Lagrangian is invariant under a symmetry transformation $q \Rightarrow q(s)$ parametrized by $s$ so that $\frac{d}{d s} L(q(s), \dot{q}(s))=0$, then this results in a conservation equation. The Noether Theorem predicts, that the Noether current $\mathcal{C}$ is conserved, i.e. $\frac{d}{d t} \mathcal{C}=0[6]$ :

$$
\mathcal{C}=\frac{\partial L(q(s), \dot{q}(s))}{\partial \dot{q}(s)} \cdot \frac{d q(s)}{d s}
$$

This statement can be proved by calculating the time derivative using the chain rule:

$$
\begin{aligned}
\frac{d}{d t} \mathcal{C} & =\frac{d}{d t}\left(\frac{\partial L(q, \dot{q})}{\partial \dot{q}(s)} \cdot \frac{d q(s)}{d s}\right) \\
& =\left(\frac{d}{d t} \frac{\partial L(q, \dot{q})}{\partial \dot{q}(s)}\right) \cdot \frac{d q(s)}{d s}+\frac{\partial L(q, \dot{q})}{\partial \dot{q}(s)} \cdot \frac{d \dot{q}(s)}{d s} \\
& =\frac{\partial L(q, \dot{q})}{\partial q(s)} \cdot \frac{d q(s)}{d s}+\frac{\partial L(q, \dot{q})}{\partial \dot{q}(s)} \cdot \frac{d \dot{q}(s)}{d s} \\
& =\frac{d}{d s} L(q(s), \dot{q}(s))=0 .
\end{aligned}
$$

To obtain the line (2.4) the Euler-Lagrange equation was used:

$$
\frac{d}{d t} \frac{\partial L(q, \dot{q})}{\partial \dot{q}}-\frac{\partial L(q, \dot{q})}{\partial q}=0
$$

If for example the Lagrangian $\mathcal{L}=\sum_{i} \frac{m_{i}}{2} \dot{\vec{x}}_{i}^{2}-\frac{1}{2} \sum_{i, k} V\left(\vec{x}_{i}-\vec{x}_{k}\right)$ is invariant under the translation $\vec{x}_{i} \rightarrow \vec{x}_{i}+s \cdot \vec{e}_{a}$, then following Noethers theorem the term $\mathcal{C}=m_{i} \dot{x}_{a}^{(i)}$ is conserved, which is the momentum of particle $i$ in direction $\vec{e}_{a}$.

In quantum field theories particles are described by complex fields, depending on the space-time coordinate $x$, e.g. the fermion field spinor $\Psi(x)$. The kinematics of the massless fermion field are described by the Lagrangian:

$$
\mathcal{L}_{\text {Fermion }}=i \bar{\Psi}_{i} \gamma^{\mu} \partial_{\mu} \Psi_{i}
$$

In gauge theories the interactions between fermions are introduced by requiring the invariance of the theory under $x$-depending unitary transformations $U(x)$, which is called a local symmetry:

$$
\Psi(x) \rightarrow \Psi^{\prime}(x)=U(x) \Psi(x)
$$


For each symmetry of the theory there is one gauge transformation which can be expressed as:

$$
U(x)=\exp \left(-i \sum_{j=1}^{N} \chi_{j}(x) Q_{j}\right)
$$

The set of all gauge transformation of a theory form a Lie group, where the $Q_{i}$ are the $N$ generators of this group. The $\chi_{j}(x)$ are real, space-time depending functions specifying the local transformations. The generators of a Lie algebra obey the relation

$$
\left[Q_{i}, Q_{j}\right]=i \sum_{k=1}^{N} f_{i j k} Q_{k}
$$

The totally antisymmetric structure constants $f_{i j k}$ vanish, if the gauge group is abelian.

The Lagrangian $\mathcal{L}_{\text {Fermion }}$ contains partial derivatives of the fields with respect to the space-time coordinates $\partial_{\mu} \Psi(x)$. The transformation $U(x)$ is also space-time dependent, so that additional terms enter the equations of motion when the operator $\partial_{\mu}$ acts on the transformed field spinor $U(x) \Psi(x)$. Since the equations of motion were required to be invariant under the local, i.e. space time dependent, symmetry transformation, a redefinition of the partial derivative $\partial_{\mu}$ to a covariant derivative $D_{\mu}$ is necessary:

$$
\partial_{\mu} \Rightarrow D_{\mu}=\partial_{\mu}+i g \sum_{i=1}^{N} Q_{i} A_{\mu}^{i}(x)
$$

where the $N$ gauge fields $A_{\mu}^{i}$ are spin-1 boson fields which couple to the fermion spin- $\frac{1}{2}$ fields $\Psi$. The coupling constant $g$ is not predicted.

The transformation of the gauge boson fields $A_{\mu}^{i}$ is determined in general by the overall invariance requirements of the theory.

$$
A_{\mu}^{i}(x) \rightarrow A_{\mu}^{i^{\prime}}(x)=A_{\mu}^{i}(x)-\frac{1}{g} \partial_{\mu} \chi_{i}(x)-\sum_{j, k}^{N} f_{i j k} \chi_{j}(x) A_{\mu}^{i}(x) .
$$

In non-abelian gauge theories where the commutator $\left[Q_{i}, Q_{j}\right]$ of Eq. 2.10 does not vanish (i.e. $f_{i j k} \neq 0$ ), called Yang-Mills theories [7], boson self-interaction terms $\mathcal{L}_{\mathrm{YM}}=$ $-\frac{1}{4} A_{i}^{\mu \nu} A_{\mu \nu}^{i}$ and boson-fermion interaction terms become possible. The forces between fermions are realized by the exchange of gauge bosons. The field-strength tensor $A_{\mu \nu}^{i}(x)$ of the gauge field $A_{\mu}^{i}$ is generally given by:

$$
A_{\mu \nu}^{i}(x)=\partial_{\mu} A_{\nu}^{i}(x)-\partial_{\nu} A_{\mu}^{i}(x)-g \sum_{j, k}^{n} f_{i j k} A_{\mu}^{j}(x) A_{\nu}^{k}(x) .
$$


The total Lagrangian is of the following form:

$$
\mathcal{L}=i \bar{\Psi}_{i} \gamma^{\mu} D_{\mu} \Psi_{i}-\frac{1}{4} A_{i}^{\mu \nu} A_{\mu \nu}^{i}
$$

The details of the Standard Model and its Lagrangian shall be discussed in the following. The Standard Model fermions are summarized in Tab. 2.1, the gauge bosons in Tab. 2.2. Gauge theories are renormalizable, in fact local gauge invariance is a necessary requirement for a theory to be renormalizable for massless gauge bosons [8] as well as for massive bosons [9]. Though the carriers of the forces, the gauge bosons, cannot be massive because a mass term of the form $m_{A}^{2} A^{\mu} A_{\mu}$ is not invariant under local gauge transformations, some vector bosons with non-zero masses are observed. So an independent mechanism responsible for giving mass to the bosons must exist, this "Higgs Mechanism" is discussed in Sec. 2.1.2.

\section{Quantum Chromodynamics}

The gauge theory of the strong force is quantum chromodynamics (QCD) [10] a Yang-Mills theory based on the gauge symmetry group SU(3). The strong force acts only on quarks, which carry a "color" charge. The three dimensional color space is spanned by the base colors red, green and blue. The additional color charge for quarks allows for bound states like $q q q$ and $q \bar{q}$, known as baryons and mesons. The Pauli exclusion principle for fermions is obeyed, if the quarks inside baryons have different color charges. Recently also hints for penta-quarks $q q q q \bar{q}$ were claimed [11]. The $n^{2}-1=8$ generators of the $\mathrm{SU}(3)_{\text {color }}$ Lie group correspond to eight massless spin-1 gauge bosons, called gluons $G_{\mu}^{a}, a=1 \ldots 8$. Since the $\mathrm{SU}(3)$ is a non-abelian group gluon-gluon self-interaction occurs and the gluons carry a color and anti-color charge. Because of the self-interaction the coupling constant grows as the distance between the quarks increases. This implies confinement: Single quarks or gluons cannot be free, since only quark-anti-quark pairs, quark triplets, and combinations of these can be color-neutral, i.e. white. The opposite behavior at very short distances is described as asymptotic freedom $[12,13]$. The interaction of charged particles becomes relatively weak at short scales, so that they are asymptotically free. The covariant derivate of the strong force is given by

$$
\partial_{\mu} \quad \Rightarrow \quad D_{\mu}=\partial_{\mu}+i \frac{g_{s}}{2}\left(\lambda_{i} \cdot G_{\mu}^{i}(x)\right)
$$

where the Gell-Mann matrices $\lambda_{i}$ are the irreducible representations of the $\mathrm{SU}(3)$ generators. The gauge transformation of the gluon fields $G_{\mu}^{a}$ are:

$$
G_{\mu}^{i}(x) \quad \rightarrow \quad G_{\mu}^{i^{\prime}}(x)=G_{\mu}^{i}(x)-\frac{1}{g_{s}} \partial_{\mu} \beta_{i}(x)-f_{i j k} \beta_{j}(x) G_{\mu}^{k}(x)
$$

The $\beta_{i}$ are space-time dependent phase transformation functions and the coupling $g_{s}$ is related to the QCD coupling constant $\alpha_{s}=g_{s}^{2} / 4 \pi$. 


\begin{tabular}{cccccccc}
\hline \hline & & Generation & \multicolumn{4}{c}{ Quantum number } \\
& $1^{s t}$ & $2^{n d}$ & $3^{r d}$ & $Q$ & $T$ & $T_{3}$ & $Y$ \\
\hline \multirow{2}{*}{ leptons } & $\left(\begin{array}{c}\nu_{e} \\
e\end{array}\right)_{L}$ & $\left(\begin{array}{c}\nu_{\mu} \\
\mu\end{array}\right)_{L}$ & $\left(\begin{array}{c}\nu_{\tau} \\
\tau\end{array}\right)_{L}$ & 0 & $1 / 2$ & $+1 / 2$ & -1 \\
& $e_{R}$ & $\mu_{R}$ & $\tau_{R}$ & -1 & $1 / 2$ & $-1 / 2$ & -1 \\
\hline \multirow{2}{*}{ quarks } & $\left(\begin{array}{c}u \\
d\end{array}\right)_{L}$ & $\left(\begin{array}{c}c \\
s\end{array}\right)_{L}$ & $\left(\begin{array}{c}t \\
b\end{array}\right)_{L}$ & $+2 / 3$ & $1 / 2$ & $+1 / 2$ & $+1 / 3$ \\
& $c_{R}$ & $t_{R}$ & $+2 / 3$ & 0 & 0 & $+4 / 3$ \\
& $u_{R}$ & $s_{R}$ & $b_{R}$ & $-1 / 3$ & 0 & 0 & $-2 / 3$ \\
\hline \hline
\end{tabular}

Table 2.1: The fermions of the Standard Model arranged in $\mathrm{SU}(2)_{L} \times \mathrm{U}(1)_{Y}$ multiplets and their quantum numbers; electrical charge, weak isospin and hypercharge.

\section{Electroweak interactions}

Quantum electrodynamics (QED) was united with the weak force within the GlashowSalam-Weinberg (GSW) model $[14,15,16]$, which is represented by a $\mathrm{SU}(2)_{L} \times \mathrm{U}(1)_{Y}$ gauge group. The index $L$ denotes the weak isospin and $Y$ the weak hypercharge. The Lie group $\mathrm{U}(1)_{E M}$ describing the quantum electrodynamics is a subgroup of the GSW group $\mathrm{U}(1)_{E M} \subset \mathrm{SU}(2)_{L} \times \mathrm{U}(1)_{Y}$. The associated spin-1 gauge boson is the photon $A_{\mu}$, the generator and coupling strength is the electrical charge $q$. The photon is electrically neutral and has no self-interaction, because $\mathrm{U}(1)$ is abelian. Though $\mathrm{U}(1)_{E M}$ is mathematically identical with $\mathrm{U}(1)_{Y}$ it has a different physical meaning. In the GSW-model the generator of the $\mathrm{U}(1)_{E M}$ group, the electrical charge $q$ is replaced by the $\mathrm{U}(1)_{Y}$ weak hypercharge $Y$. The electrical charge is then defined by the Gell-Mann-Nishijima equation ${ }^{1}$, involving the third component of the $\mathrm{SU}(2)_{L}$ weak isospin $T_{3}$ and the hyper-charge:

$$
q=T_{3}+\frac{Y}{2}
$$

The coupling strength of the fermions to the weak isospin $\vec{T}$ is given by $g_{1}$ and similarly the coupling strength to the weak hypercharge is given by $g_{2}$. The electro-weak mixing angle $\theta_{w}$, or Weinberg-angle, characterizes the ratio of the two electro-weak couplings $g_{1}$ and $g_{2}$ :

$$
\tan \theta_{w}=\frac{g_{1}}{g_{2}}
$$

The four gauge bosons associated to $\mathrm{SU}(2)_{L} \times \mathrm{U}(1)_{Y}$, the vector boson triplet under $\mathrm{SU}(2)_{L}, \vec{W}_{\mu}=\left\{W_{\mu}^{1}, W_{\mu}^{2}, W_{\mu}^{3}\right\}$ and the $B_{\mu}$ boson from $\mathrm{U}(1)_{Y}$, mix, to form the masseigenstates $W_{\mu}^{ \pm}, Z_{\mu}^{0}$ and $A_{\mu}$ :

\footnotetext{
${ }^{1}$ In some references also another definition of the weak hyper-charge, $q=T_{3}+Y$, is used.
} 


\begin{tabular}{llclccc}
\hline \hline Symmetry & Gauge boson & Field & Interaction & Q & Mass [GeV] & Width [GeV ] \\
\hline \multirow{3}{*}{$\mathrm{SU}(2)_{L} \times \mathrm{U}(1)_{Y}$} & Photon $\gamma$ & $A_{\mu}$ & electromag. & 0 & 0 & - \\
& $W^{ \pm}$-Boson & $Z_{\mu}$ & electroweak & 0 & 91.2 & 2.5 \\
\hline $\mathrm{SU}(3)_{C}$ & Gluon $g$ & $W_{\mu}^{ \pm}$ & weak & \pm 1 & 80.4 & 2.1 \\
\hline \hline
\end{tabular}

Table 2.2: The gauge bosons of the Standard Model and their electrical charge, mass and width [17].

$$
\begin{aligned}
W_{\mu}^{ \pm} & =\frac{1}{\sqrt{2}}\left(W_{\mu}^{1} \mp W_{\mu}^{2}\right) \\
Z_{\mu} & =-B_{\mu} \sin \theta_{w}+W_{\mu}^{3} \cos \theta_{W} \\
A_{\mu} & =B_{\mu} \cos \theta_{w}+W_{\mu}^{3} \sin \theta_{W} .
\end{aligned}
$$

The bosons $W_{\mu}^{ \pm}, Z_{\mu}^{0}$ acquire mass through the Higgs mechanism (Sec. 2.1.2) while the photon field $A_{\mu}$ remains massless.

While the photon has a pure vectorial coupling to the charged fermions, the charged weak current $W^{ \pm}$couplings have a vector minus axial-vector $(V-A)$ structure. The interaction mediated by the $W^{ \pm}$involve only left-handed fermions (and right-handed antifermions). The left-handed and right-handed parts of a generic fermion field, $\Psi_{L}=P_{L} \cdot \Psi$ and $\Psi_{R}=P_{R} \cdot \Psi$ are defined through the two chirality operators

$$
P_{L}=\frac{1-\gamma^{5}}{2} \quad \text { and } \quad P_{R}=\frac{1+\gamma^{5}}{2}
$$

For massive fermions there is always a frame of reference, in which the fermion field has a left-handed part. The chirality corresponds to definite helicity states only in the case of massless fermions.

The local gauge invariance for the $\mathrm{SU}(2)_{L} \times \mathrm{U}(1)_{Y}$ group is established through the covariant derivative

$$
\partial_{\mu} \quad \rightarrow \quad D_{\mu}=\partial_{\mu}+i g_{2} T_{i} \cdot W_{\mu}^{i}+i \frac{g_{1}}{2} Y \cdot B_{\mu}
$$

The field strength tensors of the electro-weak GSW theory are:

$$
\begin{aligned}
\vec{W}_{\mu \nu} & =\partial_{\mu} \vec{W}_{\nu}-\partial_{\nu} \vec{W}_{\mu}-g \vec{W}_{\mu} \times \vec{W}_{\nu} \quad \text { and } \\
B_{\mu \nu} & =\partial_{\mu} B_{\nu}-\partial_{\nu} B_{\mu} .
\end{aligned}
$$

The Standard Model is finally represented by the $\mathrm{SU}(3)_{C} \times \mathrm{SU}(2)_{L} \times \mathrm{U}(1)_{Y}$ symmetry group. The associated gauge bosons are summarized in Tab. 2.2.

In principle mixing between elementary fermions which share the same quantum numbers can occur, i.e. within the Majorana-type neutrinos and the supersymmetric (Majorana) neutralinos, the up-type quarks or the down-type quarks. The quark symmetry- and the 
quark mass-eigenstates are correlated by a unitary transformation in flavor space. Only the charged weak current is affected and one expression of the quark mixing matrix is given by the unitary Cabibbo-Kobayashi-Maskawa matrix $V_{C K M}[18,19]$.

$$
\left(\begin{array}{c}
d^{\prime} \\
s^{\prime} \\
b^{\prime}
\end{array}\right)=\left(\begin{array}{lll}
V_{u d} & V_{u s} & V_{u b} \\
V_{c d} & V_{c s} & V_{c b} \\
V_{t d} & V_{t s} & V_{t b}
\end{array}\right) \cdot\left(\begin{array}{c}
d \\
s \\
b
\end{array}\right)
$$

Where the $q^{\prime}$ are the quark eigenstates as given in Tab. 2.1 and the $q$ are the mass eigenstates. The $V_{i j}^{C K M}$ matrix elements are not predicted by theory but can be obtained by studying charged current processes [17].

\subsubsection{Higgs Mechanism}

The electroweak gauge bosons $W^{ \pm}$and $Z^{0}$ have non-zero masses, but mass terms of type $m^{2} W_{\mu} W^{\mu}$ are not allowed in the Lagrangian, because they would destroy the local gauge invariance. The Higgs mechanism [20] provides a solution. An additional term

$\mathcal{L}_{\text {Higgs }}$, which is invariant under the gauge transformations, is added to the Standard Model Lagrangian:

$$
\mathcal{L}_{\text {Higgs }}=\left(D^{\mu} \Phi\right)^{\dagger}\left(D_{\mu} \Phi\right)-V\left(\Phi^{\dagger} \Phi\right)
$$

where $\Phi$ is a two-component scalar field

$$
\Phi=\left(\begin{array}{c}
\phi^{+} \\
\phi^{0}
\end{array}\right)=\frac{1}{\sqrt{2}}\left(\begin{array}{c}
\phi_{1}+i \phi_{2} \\
\phi_{3}+i \phi_{4}
\end{array}\right)
$$

The potential $V(\Phi)$ must be symmetric $V(-\Phi)=-V(\Phi)$, so that its simplest form is given by

$$
V(\Phi)=\mu^{2} \Phi^{\dagger} \Phi+\lambda\left(\Phi^{\dagger} \Phi\right)^{2}
$$

The parameter $\lambda$ is positive $\lambda>0$, since the potential must increase $V(\Phi) \rightarrow \infty$ for large $\Phi \rightarrow \pm \infty$. The parameter $\mu^{2}$ is negative $\mu^{2}<0$, so that a non-trivial minimum of the Higgs potential exists, as shown in Fig. 2.1. The set of minima form a circle which is parametrized by:

$$
-\frac{\mu^{2}}{2 \lambda}=\frac{v^{2}}{2}
$$

The $\mathrm{SU}(2)_{L} \times \mathrm{U}(1)_{Y}$ symmetry of the Higgs potential is spontaneously broken. If the vacuum expectation value for the charged Higgs field $\phi^{+}=\frac{1}{\sqrt{2}}\left(\phi_{1}+i \phi_{2}\right)$ is set to 0 then the resulting state is invariant under $\mathrm{U}(1)_{E M}$ and the photon field $A_{\mu}$ remains massless. By choosing one ground state, e.g. $\phi_{1}=\phi_{2}=\phi_{4}=0$ and $\phi_{3}=v$ the vacuum expectation 
value for the neutral Higgs field becomes $\left\langle\phi^{0}\right\rangle=\frac{v}{\sqrt{2}}$. Expanding the scalar field $\Phi(x)$ around the ground state gives:

$$
\Phi(x)=\frac{1}{\sqrt{2}}\left(\begin{array}{c}
0 \\
v+h(x)
\end{array}\right),
$$

where the only remaining degree of freedom, $h(x)$, is the physical Higgs boson. The other three degrees of freedom of the SU(2) doublet field $\Phi(x)$ reappear as mass terms, leading to longitudinal polarization degrees of freedom of the three heavy electroweak gauge bosons. Using the representation of the Higgs field as given by Eq. (2.31), the boson mass terms can be identified in the Higgs Lagrangian $\mathcal{L}_{\text {Higgs }}$. The $\mathrm{SU}(2)_{L} \times \mathrm{U}(1)_{Y}$ eigenstates mix to the mass eigenstates $W_{\mu}^{ \pm}, Z_{\mu}^{0}$ and $A_{\mu}$ as discussed previously in Sec. 2.1.1:

$$
m_{W^{ \pm}}=\frac{v}{2} g_{2} \quad \text { and } \quad m_{Z^{0}}=\frac{v}{2} \sqrt{g_{1}^{2}+g_{2}^{2}} .
$$

A priori $v$, and therefore the size of the $W^{ \pm}, Z^{0}$ boson masses, is not known. However, the boson masses as well as the electroweak mixing angle $\theta_{w}$, can be measured and allow for a precise test of the Standard Model, as these values are related as given by

$$
\cos \theta_{W}=\frac{m_{W}}{m_{Z}}
$$

The above expression is derived by comparing Eq. (2.32) with Eq. (2.18).

The mass of the Higgs boson is given by $m_{H}=\sqrt{2 \lambda v}$ and is not predicted by the theory and not measured yet. The best lower bound on $m_{H}$ is $114.4 \mathrm{GeV}$ [21]. The Standard Model fermions obtain mass via Yukawa couplings to the Higgs doublet field $\Phi$. This is discussed in detail in Ref. [22]. The fermion mass terms in the SM Lagrangian are:

$$
\mathcal{L}_{\text {Yukawa }}=-\frac{v+\phi}{\sqrt{2}}\left(g_{\nu} \bar{\nu} \nu+g_{l} \bar{l} l+g_{u} \bar{u} u+g_{d} \bar{d} d\right)
$$

where the mass of the fermion $f$ is given by $m_{f}=\frac{v}{\sqrt{2}} g_{f}$.

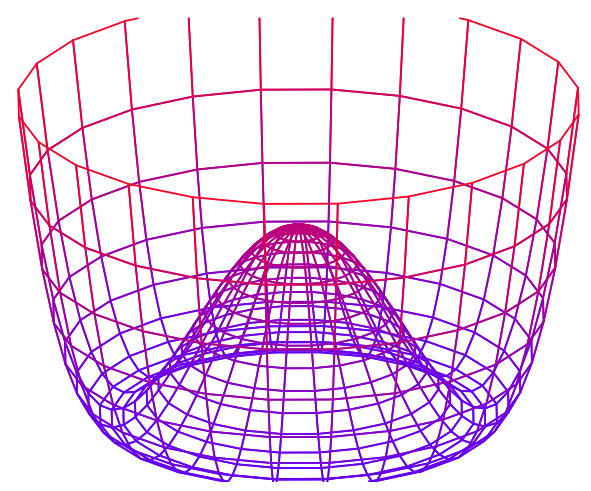

Figure 2.1: The Higgs potential. The symmetry is broken spontaneously, if $\mu^{2}<0$, otherwise the minimum is trivial and the ground state is not degenerate. 


\subsubsection{The Lagrangian of the Standard Model}

In summary, the total Standard Model Lagrangian is given by:

$$
\begin{aligned}
\mathcal{L}_{\mathrm{SM}} & =\mathcal{L}_{\text {Fermion }}+\mathcal{L}_{\text {Yang-Mills }}+\mathcal{L}_{\text {Yukawa }}+\mathcal{L}_{\text {Higgs }} \quad \text { with } \\
\mathcal{L}_{\text {Fermion }} & =\bar{\Psi}_{L} i \gamma^{\mu} D_{\mu} \Psi_{L}+\bar{\Psi}_{R} i \gamma^{\mu} D_{\mu} \Psi_{R}, \\
\mathcal{L}_{\text {Yang-Mills }} & =-\frac{1}{4} G_{a}^{\mu \nu} G_{\mu \nu}^{a}-\frac{1}{4} W_{i}^{\mu \nu} W_{\mu \nu}^{i}-\frac{1}{4} B^{\mu \nu} B_{\mu \nu}, \\
\mathcal{L}_{\text {Yukawa }} & =-g_{f} \bar{L} \Phi R-g_{f} \bar{R} \Phi^{\dagger} L, \quad \text { and } \\
\mathcal{L}_{\text {Higgs }} & =\left(D^{\mu} \Phi\right)^{\dagger}\left(D_{\mu} \Phi\right)-V\left(\Phi^{\dagger} \Phi\right),
\end{aligned}
$$

where $\mathcal{L}_{\text {Fermion }}$ are the kinematic terms of the fermion fields $\Psi$ and their interactions with the gauge boson fields using the covariant derivative $D_{\mu}$ as defined in Eq. (2.15) and (2.23). The $\mathcal{L}_{\text {Yang-Mills }}$ terms contain the kinetic-energy and self-interaction terms of the gauge bosons associated with the local symmetry groups. The Higgs Lagrangian $\mathcal{L}_{\text {Higgs }}$ gives mass to the vector bosons introduced by the spontaneous electro-weak symmetry breaking. The terms $\mathcal{L}_{\text {Yukawa }}$ provide mass terms for fermions via Yukawa couplings of the Higgs to the fermions, where the $L$ denote left-handed doublets and the $R$ right-handed singlets.

\subsubsection{Neutrino Masses}

In 1998 the Super-Kamiokande experiment found evidence for oscillation of atmospheric neutrinos, consistent with a mass difference between $\nu_{\mu}$ and $\nu_{\tau}$ of $5 \cdot 10^{-4} \leq \Delta m^{2} \leq$ $6 \cdot 10^{-3} \mathrm{eV}^{2}[23]$. This indication of non-zero neutrino masses were later confirmed by other measurements, as reviewed in [24]. The Standard Model does not foresee non-zero neutrino masses and no right-handed neutrino field $\nu_{r}$. To accommodate neutrino masses, a lepton number conserving Dirac mass term has to be added to the Model:

$$
\mathcal{L}_{\mathcal{D}}=m_{D} \bar{\nu}_{L} \nu_{R}
$$

This implicates the introduction of the $\nu_{R}$ field. Since the neutrino is electrically neutral, the occurrence of a Majorana mass term for neutrinos becomes possible, in contrast to charged leptons:

$$
\mathcal{L}_{\mathcal{M}}=m_{M} \bar{\nu}_{R}^{c} \nu_{R}
$$

The Majorana mass term causes neutrinos to be their own anti-particles. The direct consequence is, that the lepton number $L$ is not conserved. This could be validated experimentally by detecting the neutrino-less double beta-decay $(0 \nu \beta \beta)$ [25].

Both Dirac and Majorana mass terms appear automatically in the Lagrangian, if the neutrinos obtain mass via the "seesaw" mechanism [26], which gives a natural explanation 
for the small neutrino masses in comparison to other charged leptons or quarks. If the elements of the seesaw-matrix

$$
\left(\bar{\nu}_{L} \bar{\nu}_{R}^{c}\right)\left(\begin{array}{cc}
m_{M}^{L} & m_{D} \\
m_{D} & m_{M}^{R}
\end{array}\right)\left(\begin{array}{c}
\bar{\nu}_{L}^{c} \\
\bar{\nu}_{R}
\end{array}\right)
$$

underly a hierarchy, such that $m_{M}^{R} \gg m_{D} \gg m_{M}^{L}$ then the eigenvalues of the seesaw matrix are $m_{M}^{R}$ and $m_{M}^{L}=-\frac{\left(m_{D}\right)^{2}}{m_{M}^{R}}$. Some neutrinos are naturally light, while others become heavy, hence the name "seesaw" mechanism. If the Majorana mass $m_{M}^{R}$ is of the order of the GUT scale and the Dirac mass $m_{D}$ of the order of the electroweak scale, then this leads to small masses of the known (Majorana) left-handed neutrinos $m_{M}^{L}$.

If this is true, and if CP violation in the light neutrino sector is found, then this will be a hint for $\mathrm{CP}$ violation with heavy neutrinos. This could be an explanation for the leptogenesis in the early universe.

R-parity violating interaction terms are connected to this topic, as they are also able to violate the lepton number $L$.

\subsubsection{The Hierarchy Problem of the Standard Model}

As all field theories, the Standard Model is valid only for a specific energy range and has to be matched to another effective field theory [27] at some energy scale $\Lambda$. Since the Standard Model is renormalizable, this cut-off scale can be in principle at infinity. However, there is a more physical scale, at which the Standard Model breaks down. Since gravity is not included, and therefore not described by the Standard Model, the cut-off scale is the scale at which gravity becomes a strong and no longer negligible force.

Near the Planck scale, at $\Lambda_{\text {Planck }} / \sqrt{8 \pi}=2.4 \cdot 10^{18} \mathrm{GeV}$ electron-positron pair production through gravitational force is possible. This can be calculated following the experiment done by $\mathrm{H}$. Cavendish in 1798 [28], evaluating the gravitational potential at the compton wavelength $r=\hbar / m_{e} c$ of the electron and comparing it to $m_{e} c^{2}$, the rest energy of the electron.

Some parameters of the Standard Model can have sensitivity to the details of this Ultra Violet (UV) theory [1]. If both theories are well known, the energy dependence can be predicted precisely. If the ultra-violet theory is unknown, then the dependence can be estimated by letting the energy scale rise from $\Lambda$ to $2 \Lambda$ :

- Finite quantities of the Standard Model have no UV sensitivity;

- Dimensionless couplings like gauge or Yukawa couplings are proportional to $\ln \Lambda$ and have therefore no UV sensitivity;

- Dimension-full coefficients of higher dimension like the 4-fermion coupling in Fermi theory are proportional to $\Lambda^{-1}$ and are therefore UV sensitive, but suppressed; 
- Only dimension-full coefficients of lower dimension have real UV sensitivity, like the scalar Higgs mass-squared $m_{H}^{2}$ or the vacuum energy. They are proportional to the cutoff scale $\Lambda$.

The Higgs mass is a measurable quantity. The actual measureable Higgs mass does not depend on any energy scale and can be precisely calculated within a fundamental theory. In the Standard Model however, the Higgs mass-squared parameter is in 1-loop approximation directly proportional to the cut-off scale:

$$
m_{H}^{2}=m_{0}^{2}+\lambda \frac{\Lambda^{2}}{16 \pi^{2}}
$$

Until today the Higgs mass $m_{H}$ and the cut-off scale $\Lambda$ are unknown. There can be four scenarios [1]:

1. The natural case is $m_{H} \approx m_{0}$, so that the higher order corrections are small compared to $m_{H}$. With $m_{0}^{2} \approx(100 \mathrm{GeV})^{2}[21]$ for the scale $\Lambda \lesssim 4 \pi \cdot 100 \mathrm{GeV}$ follows, so that the scale dependent correction is small and under control.

2. In general a sensitive parameter $m$ to the cut-off scale can be small compared to the cut-off scale $m \ll \Lambda$ if a higher, unknown symmetry controls this parameter $m$. The symmetry, or more precisely the extent of the symmetry breaking is responsible for the value of $m$, in contrary to large, unnatural higher corrections of the order of $\Lambda$. This is as good as the natural case and is called symmetry-natural.

3. The third, supernatural possibility is the result of some tuning at the cut-off scale which fixes the parameter $m$ to another parameter $M$ of the UV theory $m=M$, due to some feature of the high energy theory.

4. Finally the last possibility is $m_{H} \ll \Lambda$ because of large radiative corrections to the low energy theory. The UV theory must be fine-tuned at the matching scale $\Lambda$. This is unnatural. "If this is true, then god doesn't like us" (J. Lykken).

Up to now, no direct evidence of physics beyond the Standard Model has been found. One explanation for this could be the fact that there is no new physics beyond the Standard Model up to the energy scale $\Lambda_{\text {Planck }} / \sqrt{8 \pi}=10^{18} \mathrm{GeV}$ where gravitation becomes a no longer negligible force. This is the worst case, because then no explanation for a small Higgs mass $\lesssim 1 \mathrm{TeV}$ exists. The higher order corrections to the Higgs mass are also proportional to $\Lambda_{U V}$. They appear to be fine tuned over 34 orders of magnitude $\left(\frac{m_{0}^{2}}{\Lambda^{2}} \approx 10^{-34}\right)$. This fine tuning is strongly disfavored by most physicists and known as the Hierarchy Problem of the Standard Model. The Problem is often characterized by the ratio of the involved scales:

$$
\frac{M_{W}}{M_{\text {Planck }}} \approx 10^{-16}
$$

This shall be discussed in more depth in Sec. 2.2.3 because the typical feature of supersymmetry, i.e. the immanent symmetry between particles and sparticles provides a (super) natural solution. 


\subsubsection{Solutions of the Hierarchy Problem}

There are several ways to solve the Hierarchy Problem of the Standard Model, because several assumptions were made to "create" this problem.

- The gravitational force becomes a strong force near the Planck scale. To calculate this energy scale, the gravitational potential was assumed to be given by

$$
V_{\text {Grav }}(r)=-G \frac{m_{1} m_{2}}{r}\left(1+\alpha e^{-\frac{r}{\lambda}}\right)
$$

with $\alpha \equiv 0$. However, this does not have to be true. Gravitation as given by Eq. 2.45 with $\alpha \neq 0$ has been studied for ranges $r$ down to the order of a micro-meter and exclusion limits on $\alpha$ and the energy scale $\lambda$ have been set, see Fig. 2.2(a).

The scale up to which Gravity is understood is smaller then $1 \mathrm{eV}$. Even the scale up to which no abnormal gravitational effects have been found is only $\sim 1 \mathrm{TeV}$. This leaves still an unknown terrain of 16 to 28 orders of magnitude to $\Lambda_{\text {Planck }}$, over which the law of gravitation is extrapolated.

Lately, another model was introduced [29], in which the gravitational and gauge interactions become united at the weak scale. The observed weakness of gravity on distances $\gtrsim 1 \mathrm{~mm}$ is due to the existence of $n \geq 2$ new compact spatial dimensions, large compared to the weak scale. The Planck scale $\Lambda_{\text {Planck }} \sim G_{N}^{-1 / 2}$ is not a fundamental scale; its enormity is a consequence of the large size of the new dimensions.

The cut-off scale is lowered by the size $R$ of the extra-dimensions:

$$
M_{\Lambda}=\left(\frac{M_{\text {Planck }}^{2}}{R}\right)^{\frac{1}{3}} .
$$

DØ has searched for Large Extra Dimensions in di-muon final states and has set the most stringent limit of $1.1 \mathrm{TeV}$ for $n=2$ on the fundamental scale in this channel $[30]$.

- The Planck scale is not necessarily the lowest possible cut-off scale for the Standard Model. Standard Model parameters can lead to a cut-off scale $\Lambda \ll \Lambda_{\text {Planck }}$. In Fig. 2.2(b) the constraints on the Higgs mass $m_{H}$ in dependence of an energy scale $\Lambda$ and the theoretical uncertainties are shown.

Since the Higgs coupling $\lambda$ is running, as given by Eq. 2.47,

$$
\lambda(\Lambda)=\frac{\lambda\left(m_{h}\right)}{1-\frac{3 \lambda\left(m_{h}\right)}{4 \pi} \ln \frac{\Lambda^{2}}{m_{h}^{2}}},
$$




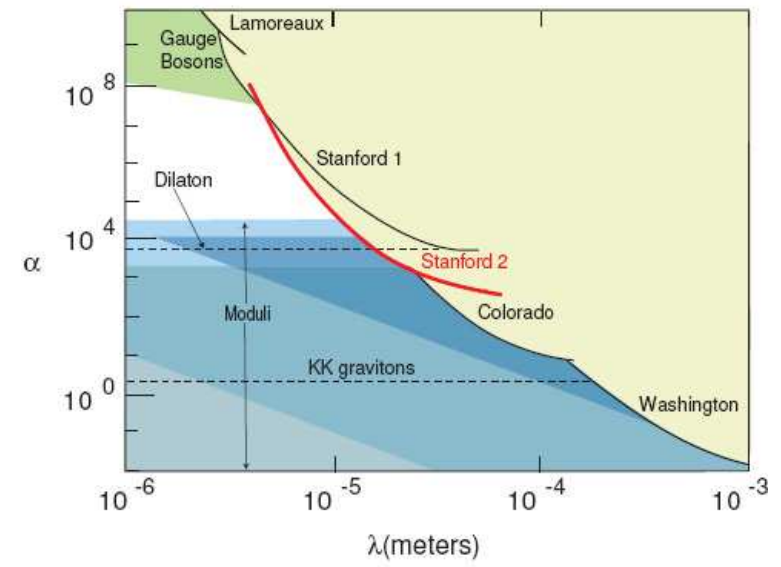

(a)

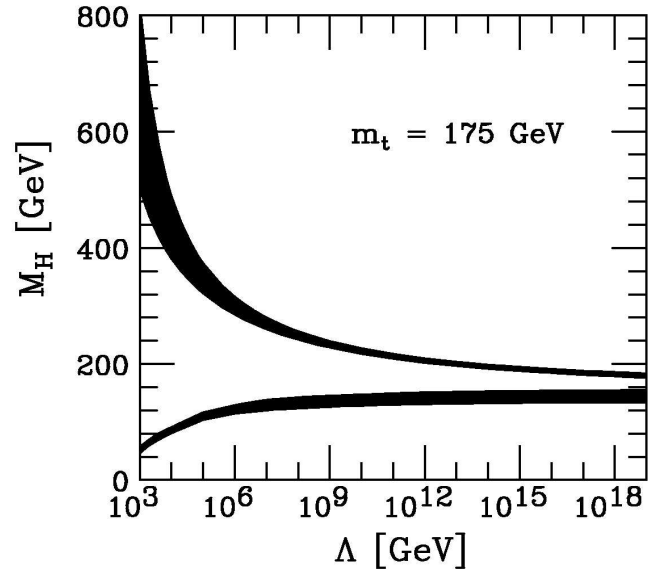

(b)

Figure 2.2: (a) Exclusion contour from [31] on Yukawa like deviations from Newtonian Gravitation in the $\alpha-\lambda$ space. (b) Lower and upper bounds on the Higgs mass $M_{H}$, for a top mass $M_{t o p}=$ $175 \mathrm{GeV}$ and $\alpha_{s}\left(M_{Z}\right)=0.118$, taken from [32].

and must remain finite and positive, an upper limit on $m_{H}$ can be derived. For small Higgs masses the contributions from gauge and Yukawa couplings to the one-loop beta-function $\beta_{\lambda}$ of the Higgs coupling are important:

$$
\beta_{\lambda}=24 \lambda^{2}+12 \lambda_{g_{t}}^{2}-6 g_{t}^{4}+\text { gauge contributions. }
$$

Because of the negative contribution of the top quark, as given by Eq. 2.48, the coupling $\lambda$ can become negative. This gives the lower limit on $m_{H}$ as displayed in Fig. 2.2(b). Only for a very small range of Higgs mass values $m_{H}$ between $160 \mathrm{GeV}$ and $170 \mathrm{GeV}$ a Standard Model cut-off at the large Planck scale $\Lambda_{P}$ is possible [32].

- Finally, there may in fact be a new theory near $1 \mathrm{TeV}$. With $\frac{M_{W}}{1 T e V}=0.1$ there is no hierarchy problem left. This, and related scenarios like supersymmetry shall be discussed in the next sections.

\section{Naturalness or the Little Hierarchy Problem}

If the Standard Model was replaced at the $\mathrm{TeV}$ scale by another theory then the Standard Model can be considered a natural theory. This easy and satisfying possibility is almost completely ruled out by electroweak precision measurements at LEP [21]. This is the Little Hierarchy Problem.

A solution to the Little Hierarchy Problem can be the introduction of a symmetry at the TeV scale, like the Little Higgs Model [33]. This allows the matching scale to be located at $\sim 10 \mathrm{TeV}$. This symmetry-natural explanation is obtained at the cost of very strict terms on the nature of the Higgs. 


\section{Supernatural UV theories}

A more favored explanation is, that $\Lambda$ is in fact larger than $1 \mathrm{TeV}$ because of some tuning, but no fine-tuning. In supersymmetry models this happens in a natural way, because there are relationships between high energy and low energy parameters, i.e. the symmetry between bosons and fermions.

It is widely expected, that the Standard Model is replaced by another effective theory, like supersymmetry, at the TeV scale. This new theory should be natural, with a cut-off scale close to the scale where gravity becomes a strong force. This can be the Planck scale, if our present knowledge of gravity is true and if there are no extra-dimensions. There is no new Hierarchy Problem, because of the naturalness of the new theory.

However, the new UV theory need not be natural. Then this theory has to be replaced again at an even higher scale and so forth, until ultimately the cut-off scale $\Lambda_{\text {Planck }}$ is reached. But it is possible, that the Planck scale, or for example the string-scale, are only a few $\mathrm{TeV}$ away. The string-theory is no field theory and has therefore no hierarchy problem. It could be the ultimate high energy model of the world.

In the following the replacement of the Standard Model by a supersymmetric model is assumed.

\subsection{Supersymmetry}

An immanent feature of supersymmetric models is the fundamental symmetry between fermions and bosons. One motivation for the introduction of such symmetries are the higher order corrections to the Higgs mass, as given by the Feynman diagrams in Fig. 2.3.

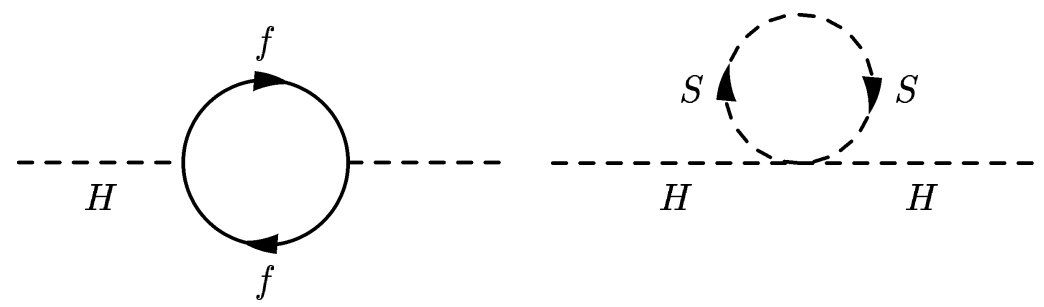

(a)

(b)

Figure 2.3: One loop corrections to the Higgs mass due to (a) fermions $f$ and (b) complex scalar particles.

If the Higgs field couplings to fermions $\Psi$ and to scalar particles $\xi$ are given by the Lagrangian terms

$$
\mathcal{L} \sim-\lambda_{f} H \bar{\xi} \xi-\lambda_{S}|H|^{2}|\Psi|^{2}
$$

then the resulting corrections to the Higgs mass-squared are: 


$$
\begin{aligned}
\delta\left(m_{H}^{2}\right)= & \frac{\left|\lambda_{f}\right|^{2}}{16 \pi^{2}}\left[-2 \Lambda_{U V}^{2}+6 m_{f}^{2} \ln \left(\frac{\Lambda_{U V}}{m_{f}}\right)+\ldots\right]+ \\
& \sum_{S=s_{l}, s_{r}} \frac{\lambda_{S}}{16 \pi^{2}}\left[\Lambda_{U V}^{2}-2 m_{S}^{2} \ln \left(\frac{\Lambda_{U V}}{m_{S}}\right)+\ldots\right]
\end{aligned}
$$

Since fermions can have two polarization states, two scalars are needed in order to keep a balanced number of degrees of freedom. The two scalar couplings are denoted $s_{l}$ and $s_{r}$. Within the Standard Model the terms would cancel each other just by chance, which results in the hierarchy problem, as discussed in Sec. 2.1.5. The natural value of the Higgs mass does not seem to be of the order of some hundred $\mathrm{GeV}$ but rather of the order of the cut-off scale $\Lambda_{U V}$, which may be as large as the Planck scale (The Hierarchy Problem).

If a supersymmetry between fermions $\Psi_{i}$ and bosons $\xi_{i}$ is introduced, then the symmetry controls the couplings and $\lambda_{S}=\left|\lambda_{f}\right|^{2}$. Each SM fermion is accompanied by two bosonic super-partners, corresponding to both chiral states of the fermion, of similar mass and vice-versa for bosons. All fermions and bosons are arranged in super-multiplets. The divergent Higgs mass correction term will cancel naturally. The small $m_{H}$ is a feature of the new theory, no hierarchy problem is left.

Similar to other symmetries like isospin invariance, a recipe to construct a SUSY invariant action can be found. Equivalent to the nucleon or pion isospin field a chiral superfield $S$ is defined:

$$
S=\left(\begin{array}{c}
\xi \\
\Psi \\
F
\end{array}\right)
$$

where $\xi$ is a scalar boson field and $\Psi$ the associated spin- $\frac{1}{2}$ fermion field. It is important that the bosonic and fermionic degrees of freedom are equal. This is accomplished by introducing the auxiliary, complex, not propagating $F$ field. It can be expressed purely algebraically in terms of the scalar fields. If a function $W\left(S_{1}, S_{2}, \ldots, S_{i}\right)$ with an arbitrary number $i$ of superfields $S_{i}$ is defined, that must depend only on the $S_{i}$ or on the $S_{i}^{\dagger}$, but not on the superfields and on their hermitecal adjoints, then the following Lagrangian guarantees an action which is invariant under supersymmetric transformations:

$$
\begin{aligned}
\mathcal{L}= & \sum_{i}\left(\partial_{\mu} \xi_{i}\right)^{*}\left(\partial_{\mu} \xi_{i}\right)+\frac{i}{2} \sum_{i} \bar{\Psi}_{i} \not \partial \Psi_{i} \\
& -\sum_{i}\left|\frac{\partial W}{\partial S_{i}}\right|_{S_{i}=\xi_{i}}^{2} \\
& -\sum_{i, j} \bar{\Psi}_{i} \frac{1-\gamma_{5}}{2}\left|\frac{\partial^{2} W}{\partial S_{i} \partial S_{j}}\right|_{S=\xi}^{2} \Psi_{j}+\text { h.c. }
\end{aligned}
$$


where the first line represents the kinetic terms for the scalar and the fermion field, the second line gives the scalar potential and the third line the fermion-scalar-interaction. The function $W$ has to be differentiated by $S_{i}$ and finally evaluated for $S_{i}=\xi_{i}$, so that the derivation depends only on the scalar component of the superfield $S_{i}$. W is called the superpotential, and must be at most a polynomial of degree three with respect to the superfields $S_{i}$. The Wess-Zumino Model, which is discussed in Sec. 2.2.2, can be derived easily from Eq. (2.52) with $\xi=\frac{A+i B}{\sqrt{2}}$ and $W(S)=\frac{1}{2} m S^{2}+\frac{1}{3} m S^{3}$.

While the above recipe works for any combination of chiral superfields, a similar recipe to construct supersymmetric gauge theories (containing gauge, spin-1 fields) can be found in $[2,4]$. The Lagrangian density for a gauge supermultiplet is then:

$$
\mathcal{L}_{\text {gauge }}=-\frac{1}{4} F_{\mu \nu}^{a} F^{\mu \nu a}-i \lambda^{\dagger a} \bar{\sigma}^{\mu} D_{\mu} \lambda^{a}+\frac{1}{2} D^{a} D^{a}
$$

where $\lambda^{a}$ is a spin- $\frac{1}{2}$ gaugino field, the superpartner of a massless gauge boson field $A^{a}$. Together $\lambda^{a}$ and $A^{a}$ form a gauge supermultiplet. The index $a$ runs over the representation of the gauge group $\left(a=1 . .8\right.$ for $\mathrm{SU}(3)_{c}$ color, $a=1,2,3$ for $\mathrm{SU}(2)_{L}$ weak isospin, $a=1$ for $\mathrm{U}(1)_{Y}$ weak hypercharge). The Yang-Mills field strength $F_{\mu \nu}^{a}$ is defined as:

$$
F_{\mu \nu}^{a}=\partial_{\mu} A_{\nu}^{a}-\partial_{\nu} A_{\mu}^{a}-g f^{a b c} A_{\mu}^{b} A_{\nu}^{c}
$$

and the covariant derivative of the gaugino field $D_{\mu} \lambda^{a}$ is:

$$
D_{\mu} \lambda^{a}=\partial_{\mu} \lambda^{a}-g f^{a b c} A_{\mu}^{b} \lambda^{c} .
$$

The auxiliary field $D^{a}$ is in analogy with the role of the auxiliary field $F_{i}=\partial f / \partial S_{i}$ in the chiral supermultiplet case. Therefore $D^{a}$ can also be expressed in terms of the scalar field:

$$
D^{a}=-g S^{*} T^{a} S
$$

where the hermitian matrices $\left(T^{a}\right)_{j}^{i}$ represent the gauge group that transforms the chiral supermultiplets $S$. The commutator is $\left[T^{a}, T^{b}\right]=i f_{a b c} T^{c}$, so if for example the gauge group is $S U(2)$, then the $T^{a}$ are $\frac{1}{2}$ times the Pauli matrices and $f_{a b c}=\epsilon_{a b c}$ is the totally antisymmetric tensor.

Finally, in a supersymmetric gauge theory the complete scalar potential can be written as:

$$
\begin{aligned}
V & =\left|F^{i}\right|^{2}+\frac{1}{2}\left|D^{a}\right|^{2} \\
& =\sum_{i}\left|\frac{\partial W}{\partial S_{i}}\right|_{S_{i}=\xi_{i}}^{2}+\frac{1}{2} \sum_{a} g_{a}^{2}\left(S^{*} t^{a} S\right)^{2} \\
& =W^{i} F_{i}-W_{i j} \xi_{i} \xi_{j}+\text { h.c. }
\end{aligned}
$$

The two terms are called $F$-term and $D$-term contributions. The $F$-terms are fixed by Yukawa couplings and fermion mass terms and the $D$-terms are fixed by the gauge in- 
teractions. $W^{i}$ is the short form of $\partial W /\left.\partial S_{i}\right|_{S_{i}=\xi_{i}}$ and similarly $W_{i j}=\partial^{2} W / \partial S_{i} S_{j}$. This potential is a subset of the total SUSY Lagrangian $\mathcal{L}_{S U S Y} \supset-V$.

\subsubsection{Explicit Supersymmetry Breaking}

If supersymmetry is realized by nature, it has to be a broken symmetry, because otherwise the SUSY particle masses would have been found at the energy scale of their SM partners. The quadratically divergent corrections to the Higgs mass will continue to cancel, independent of the mass of the partner particles, as can be seen in Eq. (2.50). However, the dimensionless couplings of the supersymmetric theory must not be changed. Terms that break supersymmetry spontaneously without introducting new $\Lambda^{2}$ divergences are called Soft SUSY Breaking terms. The Lagrangian $\mathcal{L}_{\text {soft }}$ obviously must be invariant under supersymmetric operations, but the vacuum state does not have to be, similar to the electro-weak symmetry breaking, to allow for a spontaneous breaking.

Besides the important observation, that the divergent $\Lambda^{2}$ term vanishes independently of the superpartner masses, a rough estimation of the SUSY masses can be extracted from Eq. (2.50). The corrections to $m_{H}^{2}$ are of the following form:

$$
\begin{aligned}
\delta\left(m_{H}^{2}\right) & \approx \frac{|\lambda|^{2}}{16 \pi^{2}} m_{\text {soft }}^{2} \ln \left(\Lambda / m_{\text {soft }}\right) \\
& \lesssim m_{H}^{2} \lesssim(200 \mathrm{GeV})^{2}
\end{aligned}
$$

where the coupling $\lambda$ is schematic for the various dimensionless couplings. The mass of the heaviest SM particle $m_{\text {top }}$ is negligible, compared to the heaviest SUSY mass $m_{\text {soft }}$ generated by soft SUSY breaking terms. The remaining radiative correction to the Higgs mass should be maximally of the order of the Higgs mass itself, to be under control. $200 \mathrm{GeV}$ is the upper limit to the Higgs mass, obtained from LEP and Tevatron electroweak (EW) fits [21]. In most supersymmetry breaking models the masses of the super-partner masses do not differ by more than one order of magnitude. The cut-off scale $\Lambda$ can be as large as the Planck scale and with $\lambda \approx 1$ one finds, that the mass of the lightest superpartner should be around $1 \mathrm{TeV}$. The superpartners are within reach of the Tevatron or the future Large Hadron Collider (LHC)!

The way of breaking the supersymmetry is determined by the same considerations. In order to create not too heavy SUSY masses compared to the electroweak scale, no dimensionless supersymmetry-breaking coupling shall be considered [4]. In general, the possible soft supersymmetry breaking terms in the Lagrangian are then:

$$
\begin{aligned}
\mathcal{L}_{\text {soft }}= & -\frac{1}{2}\left(M_{\lambda} \lambda^{a} \lambda^{a}+\text { c.c. }-\left(m^{2}\right)_{j}^{i} \xi^{j *} \xi_{i}\right) \\
& -\left(\frac{1}{2} b^{i j} \xi_{i} \xi_{j}+\frac{1}{6} a^{i j k} \xi_{i} \xi_{j} \xi_{k}+\text { c.c. }\right),
\end{aligned}
$$

where the spin- $\frac{1}{2}$ gaugino field $\lambda^{a}$, the superpartner of the massless gauge boson field $A^{a}$ is defined in the context of Eq. (2.53). $M_{\lambda}$ are the gaugino masses for each gauge group, $m^{2}$ 
and $b$ the scalar mass terms and $a^{i j k}$ the trilinear scalar couplings. The Lagrangian $\mathcal{L}_{\text {soft }}$ is free of quadratic divergences [34], and $\mathcal{L}_{\text {soft }}$ breaks supersymmetry, because it involves only scalars and gauginos, not their superpartners. It meets therefore all requirements ${ }^{2}$.

\subsubsection{The Wess-Zumino Model}

The supersymmetric model by J. Wess and B. Zumino $[35,36]$ is a toy model not realized in nature, but very useful to study the basic features of supersymmetry. The theory contains two spin-0 fields, $A$ and $B$ representing a boson $\xi=\frac{A+i B}{\sqrt{2}}$, and a spin- $\frac{1}{2}$ field $\Psi$. The scalar fields are real, and therefore uncharged, and have two polarization states. The uncharged fermion field is self-conjugate $\left(\Psi=\Psi^{c}\right)$ and of Majorana type and has therefore also two degrees of polarization. A Dirac type fermion field would have four degrees. The bosonic degrees of freedom match the number of fermionic degrees.

The Wess-Zumino Lagrangian is given by:

$$
\begin{aligned}
\mathcal{L}= & \mathcal{L}_{\text {kin }}+\mathcal{L}_{\text {mass }}+\mathcal{L}_{\text {interaction }} \\
\mathcal{L}_{\text {kin }}= & \frac{1}{2}\left(\partial_{\mu} A\right)^{2}+\frac{1}{2}\left(\partial_{\mu} B\right)^{2}+\frac{i}{2}(\bar{\Psi} \not \partial \Psi)^{2} \\
\mathcal{L}_{\text {mass }}= & -\frac{1}{2} m^{2}\left(A^{2}+B^{2}\right)-\frac{1}{2} m \bar{\Psi} \Psi \\
\mathcal{L}_{\text {interaction }}= & -\frac{g}{\sqrt{2}} A \bar{\Psi} \Psi+\frac{g}{\sqrt{2}} i B \bar{\Psi} \gamma_{5} \Psi \\
& -\frac{g}{\sqrt{2}} m A^{3}-\frac{g}{\sqrt{2}} m A B^{2}-\frac{1}{4} g^{2} A^{4}-\frac{1}{4} g^{2} B^{4}-\frac{1}{2} g^{2} A^{2} B^{2}
\end{aligned}
$$

The first term $\mathcal{L}_{\text {kin }}$ is the kinematic term every theory needs. The mass term $\mathcal{L}_{\text {mass }}$ defines the masses of the three fields $A, B$ and $\Psi$. All three fields have the common mass $m$. The interaction term $\mathcal{L}_{\text {interaction }}$ contains all parity conserving interactions $(2.63)$ and self-interactions (2.64). $A$ is a scalar, because $A$ couples to the scalar $\bar{\Psi} \Psi$ and $B$ is a pseudo-scalar because it couples to the pseudo-scalar $\bar{\Psi} \gamma_{5} \Psi$. The Lagrangian selfinteraction terms must be of dimension mass to the fourth, and due to the pseudo-scalar nature of the $B$ field, only the terms $B^{2}$ and $B^{4}$ can occur. In a more general theory each coupling term would have its own coupling constant, but here only one common coupling constant $g$ and one common mass $m$ is used.

There is a symmetry involved, so that all couplings have the same factor $g$ and the masses are all equal to $m$. The symmetry links the $A, B$ and $\Psi$ fields. Symmetries are known from space time invariance, gauge invariance or internal symmetries like isospin invariance. The symmetry is in general defined by the symmetry generator or "charge" $Q$ :

$$
Q \mid \text { state }\rangle=\mid \text { new state }\rangle
$$

\footnotetext{
${ }^{2}$ Another trilinear scaler coupling term $c_{i}^{j k} \xi^{i *} \xi_{j} \xi_{k}$ can be considered for the soft supersymmetry breaking Lagrangian, too. However, the $c_{i}^{j k}$ terms are usually neglected, because they can lead to quadratic divergences and complicate the spontaneous supersymmetry breaking [4].
} 
The commutator of the Hamiltonian $H$ and the generator $Q$ vanishes, so that $Q$ is conserved.

$$
[Q, H]=0
$$

A symmetry results in the degeneration of the spectrum; if $H \mid$ state $\rangle=E \mid$ state $\rangle$ then $H \mid$ new state $\rangle=E \mid$ new state $\rangle$ follows, because $H$ and $Q$ commute:

$$
H Q \mid \text { state }\rangle=Q H \mid \text { state }\rangle=E Q \mid \text { state }\rangle
$$

Both eigenstates $\mid$ state $\rangle$ and $\mid$ new state $\rangle$ have the same energy eigenvalue $E$, or in the restframe the same mass, as a consequence of the symmetry.

While all known symmetries of the Standard Model connect bosonic states with bosonic states and fermionic states with fermions, the symmetry of this model connects bosons with fermions and vice versa.

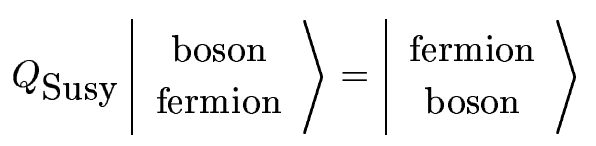

A symmetry with this feature is called a supersymmetry. The symmetry transformations of the $A, B$ and $\Psi$ fields are given by:

$$
\begin{aligned}
\delta A & =i \bar{\alpha} \gamma^{5} \Psi \\
\delta B & =-\bar{\alpha} \Psi \\
\delta \Psi & =F \alpha-i G \gamma^{5} \alpha+\left(\not \partial \gamma^{5} A\right) \alpha+i(\not \partial B) \alpha
\end{aligned}
$$

Where the spinor $\alpha$ is a constant parameter of the transformation and the auxiliary fields $F$ and $G$ are $F=m A-g\left(A^{2}-B^{2}\right)$ and $G=m B-2 g A B$. Technically, $Q$ is a fermionic charge, or in other words, $Q$ is a spin- $\frac{1}{2}$ generator. Bosonic and fermionic states are energy degenerated pairs. The fermion $\Psi$ is the superpartner of the boson $\xi=\frac{A+i B}{\sqrt{2}}$ and vice versa. The number of bosonic states is equal to the number of fermionic states and the boson mass is equal to the fermion mass. Superpartners differ in spin by $\frac{1}{2} \hbar$, and all other quantum numbers are equal.

It can be easily showed, that the transformed fields change the Lagrangian (2.60) by only a total derivate $\delta \mathcal{L}=\partial^{\mu}$ (something), so that the four-dimensional action integral is invariant under supersymmetric transformations.

$$
\delta S=\int \delta L(d x)^{4}=\int \partial^{\mu}(\text { something })(d x)^{4}=0
$$

The equations of motion and the physics are therefore invariant under the supersymmetric transformations.

As discussed previously, the quadratically divergent Higgs mass correction terms; $\lambda_{S} \Lambda^{2}$ from scalar-couplings and $\left|\lambda_{f}\right|^{2} \Lambda^{2}$ from Yukawa couplings, have to cancel, to solve the 
hierarchy problem. In the Standard Model the couplings are independent, and both terms would cancel just by chance. With some work the relevant contributions can be calculated within the Wess-Zumino model.

An important characteristic of a symmetry is the commutator of the generators, like for rotations $\left[J_{i}, J_{j}\right]=i \epsilon_{i j k} J_{k}$. To calculate the relation for $Q$ two successive transformations are applied on one field $A, B, \xi$ or $\Psi$ :

$$
\begin{aligned}
\delta_{2} \delta_{1} A & =\delta_{2}\left(i \bar{\alpha}_{1} \gamma_{5} \Psi\right) \\
& =i \bar{\alpha}_{1} \gamma_{5}\left(F \alpha_{2}-i G \gamma_{5} \alpha_{2}+\not \partial \gamma_{5} A \alpha_{2}+i \not \partial B \alpha_{2}\right) \\
\delta_{1} \delta_{2} A & =i \bar{\alpha}_{2} \gamma_{5}\left(F \alpha_{1}-i G \gamma_{5} \alpha_{1}+\not \partial \gamma_{5} A \alpha_{1}+i \not \partial B \alpha_{1}\right)
\end{aligned}
$$

If both expressions are subtracted, all terms cancel except for $i \bar{\alpha} \gamma_{5} \not \partial \gamma_{5} A \alpha^{\prime}$, since $\bar{\Psi} \gamma^{\mu} \alpha=$ $-\bar{\alpha} \gamma^{\mu} \Psi$.

$$
\delta_{2} \delta_{1} A-\delta_{1} \delta_{2} A=-2 i \bar{\alpha}_{1} \alpha_{2} \not \partial A
$$

Since in general a transformation can be written as $A \rightarrow A^{\prime}=A+\delta A=A+\bar{\alpha} Q A$ and with $\bar{\Psi} \alpha=\bar{\alpha} \Psi$ the above can be done similarly for the generators $Q$ :

$$
\begin{aligned}
\delta_{2} \delta_{1} A-\delta_{1} \delta_{2} A & =\left(\bar{\alpha}_{2} Q \bar{\alpha}_{1} Q-\bar{\alpha}_{2} Q \bar{\alpha}_{1} Q\right) A \\
& =-\bar{\alpha}_{1} \alpha_{2}(\bar{Q} Q+Q \bar{Q}) A \\
& =-\bar{\alpha}_{1} \alpha_{2}\{Q, \bar{Q}\} A
\end{aligned}
$$

Comparing Eq. (2.75) and (2.76) the anti-commutator for $Q$ can be identified:

$$
\{Q, \bar{Q}\}=2 i\left(\gamma_{\mu} \partial^{\mu}\right)
$$

where $i \partial^{\mu}$ is nothing else then the generator $P^{\mu}$ of the Poincaré group (translation, Lorentzinvariance and rotations). Supersymmetry is a space-time symmetry! The generator of a supersymmetric transformation acting on a field will not change any quantity (charge, mass, ...) of the particle, but the spin. A boson is transformed into a fermion and vice versa. The supersymmetry is connected to gravity, if the supersymmetry is a local symmetry.

\subsubsection{The Minimal Supersymmetric Standard Model}

The Minimal Supersymmetric Standard Model (MSSM) is the minimal extension of the Standard Model, in the sense that the fewest amount of new, supersymmetric particles are introduced. Each SM particle is assigned to a supersymmetric partner (a "sparticle"), which differs in spin by $\frac{1}{2}$. Both partners form a supermultiplet. The model has one supersymmetry generator $Q$, the MSSM is therefore a $N=1$ model.

The particle content of the MSSM is divided into two different types of multiplets. The chiral supermultiplets (Tab. 2.3) contain fermions that couple differently to the weak gauge 
bosons (depending on the helicity). All Standard Model fermions have this property. Their scalar partners are called scalar quarks or scalar leptons, or for simplicity the names are constructed by prepending an "s"; squarks or sleptons. A right-handed selectron $\tilde{e}_{R}$ is the SUSY partner of the SM right-handed electron $e_{R}$, but $\tilde{e}_{R}$ does not have a helicity itself, it is a spin-0 particle. Except for spin and the mass, the particles and sparticles share the same quantum numbers and couplings; for example the $\tilde{e}_{R}$ does not couple to the W boson, because its partner $e_{R}$ is a weak isospin singlet, and has therefore no weak charge, too.

The Standard Model Higgs boson is a spin-0 particle [17]. Therefore it builds together with its spin- $\frac{1}{2}$ partner, the higgsino, another chiral multiplet. The name of the supersymmetric partner of a boson is constructed by appending the syllable "ino". All chiral supermultiplets in the MSSM are listed in Tab. 2.3. The MSSM must contain at least two Higgs chiral super-multiplets, because of two reasons, related to the weak hypercharge: The cancellation of gauge anomalies requires $\operatorname{Tr}\left[Y^{3}\right]=\operatorname{Tr}\left[T_{3}^{2} Y\right]=0$ where $T_{3}$ is the third component of the weak isospin and $Y$ is the weak hypercharge as defined in Eq. 2.17. The traces run over all left-handed fermions in the theory. Within the Standard Model the condition is satisfied and the SM is anomaly-free. Since the higgsino must be a $\mathrm{SU}(2)_{L}$ doublet with a weak hypercharge $Y= \pm 1$ at least two isodoublets with $Y=+1$ and $Y=-1$ and therefore two Higgs super-multiplets are necessary. The second, independent reason is, that only a Higgs chiral super-multiplet with $Y=+1$ can have Yukawa couplings to up-type quarks $(u, c, t)$ and only a $Y=-1$ Higgs can give masses to down-type quarks $(d, s, b)$ and charged leptons. More information can be found in [4]. The scalar fields of the weak isodoublet with positive hypercharge and $T_{3}=+\frac{1}{2},-\frac{1}{2}$ are $H_{u}^{+}$and $H_{u}^{0}$, where the lower index refers to the Yukawa coupling to fermions and the upper index to the electrical charge, defined by the third component of the weak isospin. The fermionic partners are $\tilde{H}_{u}^{+}$and $\tilde{H}_{u}^{0}$; the second super-multiplet is accordingly denoted with the lower index $d$.

Before the electro-weak symmetry breaking, the Standard Model contains massless spin-1 vector bosons, which each have two helicity states and therefore two bosonic degrees of

\begin{tabular}{|c|c|c|c|c|c|}
\hline S & particle & spin & $\mathrm{SU}(3)_{C}, \mathrm{SU}(2)_{L}, \mathrm{U}(1)_{Y}$ & sparticle & spin \\
\hline $\bar{Q}$ & Quark $\quad q_{L}^{u}, q_{L}^{d}$ & $\frac{1}{2}$ & $\left(3,2,+\frac{1}{3}\right)$ & $\begin{array}{ll}\text { squark } & \tilde{q}_{L}^{u}, \tilde{q}_{L}^{u}\end{array}$ & 0 \\
\hline $\bar{u}$ & up-like $\quad u_{R}^{\dagger}$ & $\frac{1}{2}$ & $\left(\mathbf{3}, \mathbf{1},+\frac{4}{3}\right)$ & $\tilde{u}_{R}^{*}$ & 0 \\
\hline $\bar{d}$ & down-like $d_{R}^{\dagger}$ & $\frac{1}{2}$ & $\left(\mathbf{3}, \mathbf{1},-\frac{2}{3}\right)$ & $\tilde{d}_{R}^{*}$ & 0 \\
\hline $\bar{L}$ & $\begin{array}{l}\text { Lepton } \quad \nu, l_{L} \\
\end{array}$ & $\frac{1}{2}$ & $(1,2,-1)$ & slepton $\tilde{\nu}, \hat{l}_{L}$ & 0 \\
\hline $\bar{e}$ & up-like & $\frac{1}{2}$ & $(\mathbf{1}, \mathbf{1},-2)$ & $\tilde{l}_{R}^{*}$ & 0 \\
\hline$H_{u}$ & $H_{u}^{0}, H_{u}^{+}$ & 0 & $(1,2,+1)$ & higgsino $\tilde{\tilde{H}}_{u}^{0}, \tilde{H}_{u}^{+}$ & $\frac{1}{2}$ \\
\hline$H_{d}$ & $H_{d}^{0}, H_{d}^{-}$ & 0 & $(\mathbf{1}, 2,-1)$ & $\tilde{H}_{d}^{0}, \tilde{H}_{d}^{-}$ & $\frac{1}{2}$ \\
\hline
\end{tabular}

Table 2.3: Chiral super-multiplets in the Minimal Supersymmetric Standard Model; following the convention of [4] the chiral super-multiplets are defined using left-handed Weyl spinors, so that only the conjugates of the right-handed fermions appear. For the $\mathrm{SU}(3)_{C}$ and the $\mathrm{SU}(2)_{L}$ group the dimension of the charge multiplet is given. The hypercharge $Y$ is defined such that the electromagnetic charge is $q=T_{3}+\frac{Y}{2}$. 


\begin{tabular}{llc|c|llc}
\hline \hline particle & & spin & $\mathrm{SU}(3)_{C}, \mathrm{SU}(2)_{L}, \mathrm{U}(1)_{Y}$ & sparticle & spin \\
\hline gluon & $g$ & 1 & $(\mathbf{8}, \mathbf{1}, 0)$ & gluino & $\tilde{g}$ & $\frac{1}{2}$ \\
W boson & $W^{0}, W^{ \pm}$ & 1 & $(\mathbf{1}, \mathbf{3}, 0)$ & wino & $\tilde{W}^{0}, \tilde{W}^{ \pm}$ & $\frac{1}{2}$ \\
B boson & $B^{0}$ & 1 & $(\mathbf{1}, \mathbf{1}, 0)$ & bino & $\tilde{B}^{0}$ & $\frac{1}{2}$ \\
\hline \hline
\end{tabular}

Table 2.4: Gauge or vector super-multiplets in the Minimal Supersymmetric Standard Model. The dimension of the charge multiplets and the hypercharge quantum number $Y$ is given.

freedom. The superpartner is a spin- $\frac{1}{2}$-fermion which has also two helicity states and thus the same number of degrees of freedom. If the superpartners had different degrees of freedom, i.e. if the vector bosons were assigned to a spin- $\frac{3}{2}$ superpartner, then the theory would not be renormalizable. The gauge or vector multiplets are listed in Tab. 2.4. As before a tilde is used to denote the superpartners and the syllable "ino" is appended.

After electro-weak symmetry breaking the neutral bosons $W^{0}$ and $B^{0}$ of the electroweak gauge symmetry $\mathrm{SU}(2)_{L} \times \mathrm{U}(1)_{Y}$ mix, to form the mass eigenstates $Z^{0}$ and $\gamma$. In the case of unbroken supersymmetry the superpartner would equally mix to $\tilde{Z}^{0}$ and $\tilde{\gamma}$ with identical masses. However, the supersymmetry is broken, as known from data, so that most general mixing must be assumed. The precise mixing depends on the form of symmetry breaking.

If the soft supersymmetry breaking, as defined in general in Sec. 2.2.1, is explicitly introduced to the MSSM then the masses and the mixing parameters become calculable [4] for the price of many new parameters like masses, phases and mixing angles. $\mathcal{L}_{M S S M}$ contains 124 free parameters in total [37], including the 19 Standard Model parameters.

The mass eigenstates of the MSSM are listed in Tab. 2.5 under the assumption, that the mixing of first- and second-generation squarks and sleptons is negligible.

\begin{tabular}{lc|c|c}
\hline \hline sparticle & spin & gauge eigenstates & mass eigenstates \\
\hline squarks & 0 & $\tilde{u}_{L}, \tilde{u}_{R}, \tilde{d}_{L}, \tilde{d}_{R}$ & no mixing \\
$2^{\text {nd }}$ generation & 0 & $\tilde{c}_{L}, \tilde{c}_{R}, \tilde{s}_{L}, \tilde{s}_{R}$ & no mixing \\
$3^{\text {rd }}$ generation & 0 & $\tilde{t}_{L}, \tilde{t}_{R}, \tilde{b}_{L}, \tilde{b}_{R}$ & $\tilde{t}_{1}, \tilde{t}_{2}, \tilde{b}_{1}, \tilde{b}_{2}$ \\
\hline sleptons & 0 & $\tilde{e}_{L}, \tilde{e}_{R}, \tilde{\nu}_{e}$ & no mixing \\
$2^{\text {nd }}$ generation & 0 & $\tilde{\mu}_{L}, \tilde{\mu}_{R}, \tilde{\nu}_{\mu}$ & no mixing \\
$3^{\text {rd generation }}$ & 0 & $\tilde{\tau}_{L}, \tilde{\tau}_{R}, \tilde{\nu}_{\tau}$ & $\tilde{\tau}_{1}, \tilde{\tau}_{2}, \tilde{\nu}_{\tau}$ \\
\hline Higgs & 0 & $H_{u}^{0}, H_{d}^{0}, H_{u}^{+}, H_{d}^{-}$ & $h^{0}, H^{0}, A^{0}, H^{ \pm}$ \\
\hline gravitino & $\frac{3}{2}$ & $\tilde{G}_{0}$ & no mixing \\
gluinos & $\frac{1}{2}$ & $\tilde{g}^{0}, \tilde{W}^{0}, \tilde{H}_{u}^{0}, \tilde{H}_{d}^{0}$ & no mixing \\
neutralinos & $\frac{1}{2}$ & $\tilde{W}^{ \pm}, \tilde{H}_{u}^{+}, \tilde{H}_{d}^{-}$ & $\tilde{\chi}_{1}^{0}, \tilde{\chi}_{2}^{0}, \tilde{\chi}_{3}^{0}, \tilde{\chi}_{4}^{0}$ \\
charginos & $\frac{1}{2}$ & $\tilde{\chi}_{1}^{ \pm}, \tilde{\chi}_{2}^{ \pm}$ \\
\hline \hline
\end{tabular}

Table 2.5: Particle mass eigenstates in the minimal supersymmetric model. The mixing of the gauge eigenstates depends on the exact nature of the supersymmetry breaking. 
The MSSM Lagrangian consists of four major parts, which have been discussed already in general in the previous sections.

$$
\mathcal{L}_{M S S M}=\mathcal{L}_{\text {kin }}+\mathcal{L}_{\text {gauge }}+\mathcal{L}_{W}+\mathcal{L}_{\text {soft }}
$$

The explicit form of the MSSM Lagrangian is discussed in great detail in reference [4]. The MSSM superpotential (see also Eq. 2.57 how to derive $\mathcal{L}_{W}$ ) is given by:

$$
\begin{aligned}
W_{M S S M}= & \lambda_{e} H_{d} L \bar{e}+\lambda_{d} H Q \bar{d}+\lambda_{u} H_{u} Q \bar{u}+\mu H_{u} H_{d} \\
& +W_{R P V}
\end{aligned}
$$

where the superfields $S=H_{u}, H_{d}, Q, L, \bar{u}, \bar{d}$ are as defined in Tab. 2.3 and the dimensionless Yukawa coupling parameters $\lambda_{e}, \lambda_{u}, \lambda_{d}$ are in general $3 \times 3$ matrices in family space. All SU $(3)_{C}$ color, $\mathrm{SU}(2)_{L}$ weak isospin and family indices are suppressed here, for clarity. $W_{R P V}$ comprises R-parity violating terms, which will be discussed in Sec. 2.3. It is important to note that the appearance of this $R_{p}$ term is neither required nor forbidden. Since the nature of supersymmetry is unknown, a superpotential obeying the requirements as discussed in the context of Eq. (2.52) must be assumed and this includes in general the $W_{R P V}$ term. The presence of R-parity violating interactions must be verified or ruled out by observation.

\subsubsection{Spontaneous Supersymmetry Breaking}

The possible supersymmetry breaking terms were discussed in general in Sec. 2.2.1. In the MSSM these breaking terms were explicitly introduced and added ad hoc to the Lagrangian. However, in a self-consistent SUSY model this supersymmetry breaking should occur spontaneously. By definition, this means that the ground state $|0\rangle$ is not invariant under supersymmetry transformations $Q|0\rangle \neq 0$. Since SUSY is a space-time symmetry, Eq. (2.77), the Hamiltonian operator is related to the anti-commutator of the supersymmetry generators $Q_{i}$ :

$$
H=P^{0} \simeq Q_{i} Q_{i}^{\dagger}+Q_{i}^{\dagger} Q_{i}
$$

Consequently, the vacuum energy is larger than zero $\langle 0|H| 0\rangle>0$. If spacetime effects and fermion condensates are neglected, then $\langle 0|H| 0\rangle=\langle 0|V| 0\rangle$ follows, where $V$ is the SUSY potential, as defined in Eq. (2.57). If there is a state $|x\rangle$ for which the $F_{i}$ and $D^{a}$ vanish, then $\langle x|V| x\rangle=\langle x|0| x\rangle=0$ follows, and $|x\rangle$ is the ground state of an unbroken supersymmetry. SUSY is therefore spontaneously broken, if the $F_{i}$ and the $D^{a}$ are never simultaneously zero for any state.

Supersymmetry breaking with non-zero $D$-terms is achieved through the Fayet-Iliopoulos mechanism [38]. The following linear term is introduced to the auxiliary field of a $U(1)$ gauge supermultiplet:

$$
\mathcal{L}_{F I}=\kappa D
$$


This term is invariant under supersymmetric transformations, because $\delta D \sim D_{\mu}$ is changed by only a total derivative ${ }^{3}$. If added to the scalar potential as given in Eq. (2.53) or by Eq. (2.57) then $D$ can be rewritten to:

$$
D=\kappa-g \sum_{i} q_{i}\left|\xi^{i}\right|^{2}
$$

The $q_{i}$ are the $U(1)$ charges of the scalar fields $\xi_{i}$. Large superpotential mass terms prevent the scalar fields $\xi_{i}$ from getting vacuum expectation values (VEV). This forces the VEV of the $D$-field to $\kappa$ and the supersymmetry is broken.

Supersymmetry breaking with non-zero $F$-terms are called O'Raifeartaigh models [39]. The superpotential $f\left(S_{1}, S_{2}, \ldots, S_{i}\right)$ is defined such, that the $F_{i}=-\partial f / \partial S_{i}$ cannot be zero simultaneously, see also Eq. (2.52) for reference. The simplest example uses three chiral supermultiplets:

$$
f=-k S_{1}+m S_{2} S_{3}+\frac{y}{2} S_{1} S_{3}^{2}
$$

Where $S_{1}$ is a gauge singlet. The parameters $k, m$ and $y$ can be chosen to be real and positive by phase rotations of the fields. The scalar potential following from Eq. (2.84) is:

$$
\begin{aligned}
V & =\sum_{i}\left|\frac{\partial f}{\partial S_{i}}\right|_{S_{i}=\xi_{i}}^{2} \\
& =\left|F_{1}\right|^{2}+\left|F_{2}\right|^{2}+\left|F_{3}\right|^{2} \\
F_{1} & =k-\frac{y}{2} \xi_{3}^{* 2} ; \quad F_{2}=-m \xi_{3}^{*} ; \quad F_{3}=-m \xi_{2}^{*}-y \xi_{1}^{*} \xi_{3}^{*}
\end{aligned}
$$

Clearly, $F_{1}$ and $F_{2}$ cannot be simultaneously zero for any state, so that the given potential breaks supersymmetry spontaneously. The breaking of any global symmetry results in a massless Goldstone particle, which is in case of supersymmetry a broken fermion charge, so that the new particle is a massless neutral Weyl fermion, the goldstino.

If the MSSM is required to break supersymmetry spontaneously, than it must be extended. The Fayet-Iliopoulos mechanism is disfavored, because a changed $D$-term for $U(1)_{Y}$ would result in non-zero VEVs for some squarks or sleptons, leading to breaking of color, electromagnetism or lepton number. A new $U(1)$ symmetry, as yet unknown because it is spontaneously broken at a high mass scale or because it doesn't couple to Standard Model particles, could be introduced to fix this problem. However, in this case it is difficult to create a mass hierarchy consistent with observation. Similarly, there is no gauge-singlet candidate, whose $F$-term could develop a VEV. The O'Raifeartaigh model would result in non-viable particle spectra.

To account for these reasons, the supersymmetry soft breaking terms are expected to arise indirectly or radiatively. Supersymmetry breaking occurs in a "hidden sector" of particles that have no or only little direct couplings to the "visible sector" chiral supermultiplets of the theory. The two sectors share some interactions which are responsible for mediating

\footnotetext{
${ }^{3}$ The superscript " $\mu$ " helps to distinguish between the field $D$ and the covariant derivative $D_{\mu}$.
} 


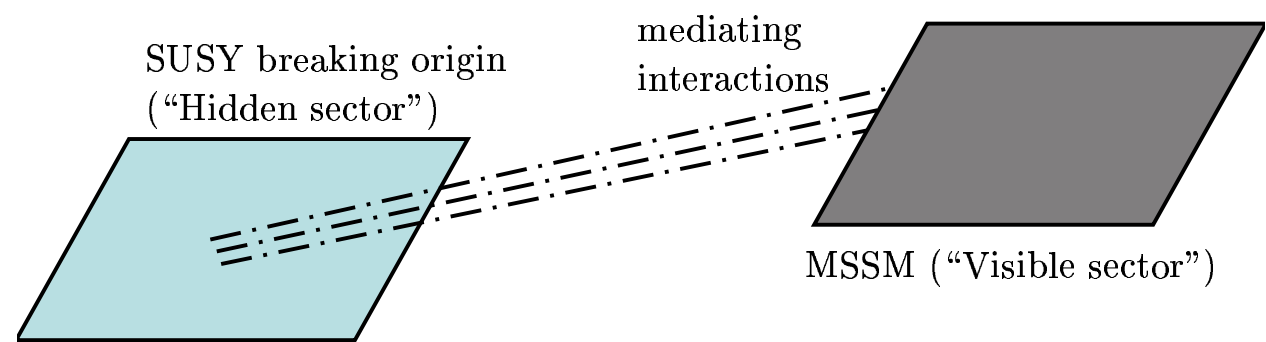

Figure 2.4: The supersymmetry breaking originates in the "hidden sector" and is mediated to the "visible sector" by gravitational or gauge interactions, depending on the chosen model.

SUSY breaking, as shown in Fig. 2.4. There are several proposals how the mediating interaction could be realized, two are summarized below:

1. Gravitational supersymmetry breaking: If SUSY is broken in the hidden sector by a vacuum expectation value $\langle F\rangle$, then the soft breaking terms in the visible sector are roughly $m_{\text {soft }}=\frac{\langle F\rangle}{M_{P}}$. As discussed previously, the $m_{\text {soft }}$ should be of the order of a few hundred $\mathrm{GeV}$, this leaves for the scale of the origin to be roughly $\sqrt{\langle F\rangle} \sim 10^{10} \mathrm{GeV}$. Another possibility is that the supersymmetry breaking is due to a gaugino condensate. The VEV for $F$ is then $\langle F\rangle \sim \Lambda^{3} / M_{P}$ with a breaking scale at approximately $\Lambda \sim 10^{13} \mathrm{GeV}$.

2. Gauge mediated supersymmetry breaking arises from loop diagrams involving messenger particles. These messengers couple to the supersymmetry violating VEV $\langle F\rangle$ and have also the common $S U(3)_{C} \times S U(2)_{L} \times U(1)_{Y}$ interactions which is the link to the visible sector. The MSSM soft terms are $m_{\text {soft }}=\frac{\alpha_{a}}{4 \pi} \frac{\langle F\rangle}{M_{\text {mess }}}$, where $M_{\text {mess }}$ is the characteristic scale of the messenger fields. If the scales are comparable $M_{\text {mess }} \sim \sqrt{\langle F\rangle}$ then the scale of the supersymmetry breaking can be very low $\sqrt{\langle F\rangle} \sim 10^{4}-10^{5} \mathrm{GeV}$, compared to the above case.

A minimal gravity mediated supersymmetry breaking scenario shall be discussed in more detail in the following section 2.2.5.

\subsubsection{Minimal Supergravity}

The minimal low energy supergravity (mSUGRA) model [40] makes use of the framework of a Grand Unified Theory (GUT) and gravity-mediated spontaneous supersymmetry breaking. Since SUSY is a space-time symmetry, the local supersymmetry invariance automatically includes gravity. In the mSUGRA model gravity occurs at some large energy scale $M_{X}$ given by the VEV of the auxiliary field $F_{X}$ responsible for the symmetry breaking, which is usually the GUT scale $\left(\left\langle F_{X}\right\rangle \sim \Lambda_{G U T} \simeq 10^{16} \mathrm{GeV}\right)$ but can theoretically be as large as the Planck scale $\left(\sim 2.4 \cdot 10^{18} \mathrm{GeV}\right)$. The supergravity Lagrangian terms which communicate between the hidden sector with spontaneous symmetry breaking and the visible sector are suppressed by powers of the Planck mass, since the gravitational coupling is proportional to $M_{P}^{-1}$. 


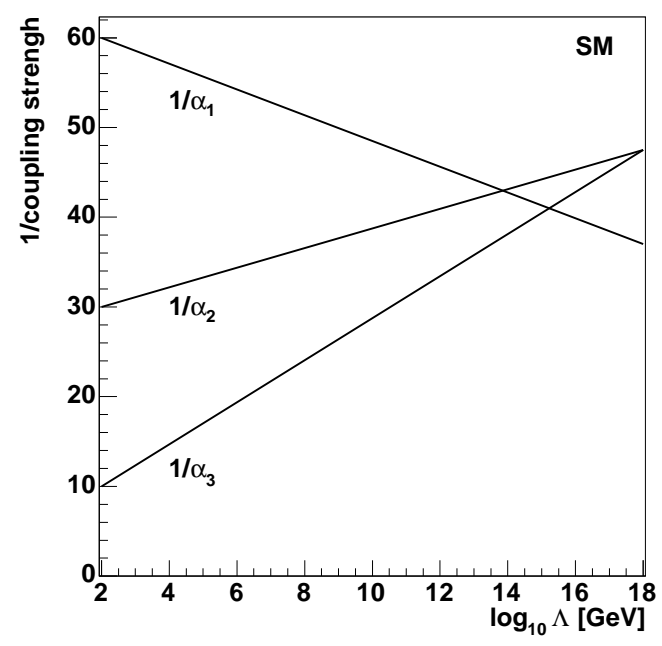

(a)

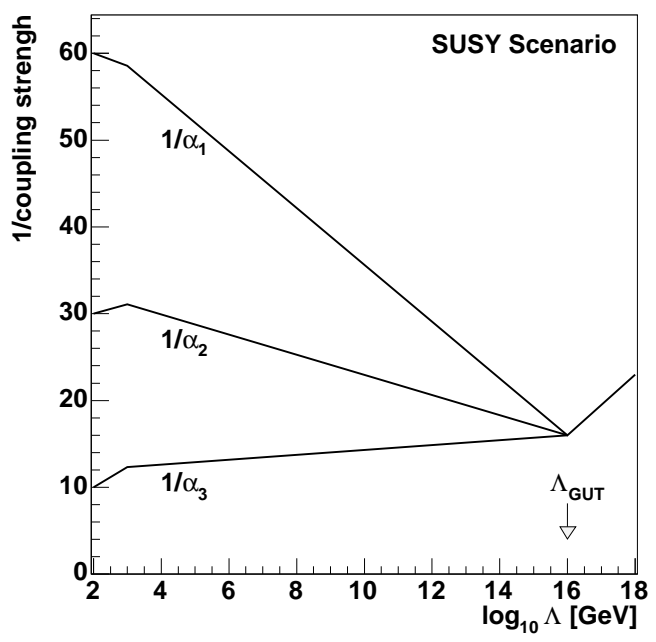

(b)

Figure 2.5: Schematic evolution of the coupling constants $\alpha_{1}$ from $\mathrm{U}(1)_{Y}, \alpha_{2}$ from $\mathrm{U}(2)_{L}$ and $\alpha_{3}$ from $\mathrm{U}(3)_{C}$ within the Standard Model (left) and in a supersymmetry scenario (right). Loop level corrections involving the superpartners change the running of the couplings so that they join in one point at $\Lambda_{G U T}$ suggesting that the known interactions arise from one grand unified force.

$$
\begin{aligned}
\mathcal{L}_{\text {gravity }}= & -\frac{F_{x}}{M_{P}} \sum_{a} \frac{1}{2} f_{a} \lambda^{a} \lambda^{a}+\text { c.c. } \\
& -\frac{F_{x} F_{x}^{*}}{M_{P}^{2}} k_{j}^{i} \xi_{i} \xi^{* j} \\
& -\frac{F_{x}}{M_{P}}\left(\frac{1}{6} y^{i j k} \xi_{i} \xi_{j} \xi_{k}+\frac{1}{2} \mu^{i j} \xi_{i} \xi_{j}\right)+\text { c.c. }
\end{aligned}
$$

Where $\xi_{i}$ and $\lambda^{a}$ are the scalar and gaugino field components of the MSSM supermultiplets. With $\left\langle F_{X}\right\rangle / M_{P}=\mathcal{O}(100 \mathrm{GeV})$ the above supersymmetry breaking Lagrangian by gravitymediation $\mathcal{L}_{\text {gravity }}$ is of the same form of the general soft-breaking Lagrangian $\mathcal{L}_{\text {soft }}$ in Eq. (2.59).

The free parameters $f_{a}, k_{j}^{i}, y^{i j k}$ and $\mu^{\prime i j}$ of Eq. (2.87) can be constrained in the GUTframework. A GUT inspired MSSM assumes, that the running couplings of the three $\mathrm{SU}(3)_{C} \times \mathrm{SU}(2)_{L} \times \mathrm{U}(1)_{Y}$ forces join at some energy scale $\Lambda_{G U T}$, see Fig. 2.5 for reference.

At this GUT scale the gaugino masses, and the scalar lepton masses are degenerate, respectively (Fig. 2.6). One common gaugino mass parameter, defined at the GUT scale, $\left(m_{1 / 2}\right)$ and one common scalar mass parameter $\left(m_{0}\right)$ are left. Together with three additional mSUGRA parameters, all other masses, mixing angles and phases of the MSSM are defined. 


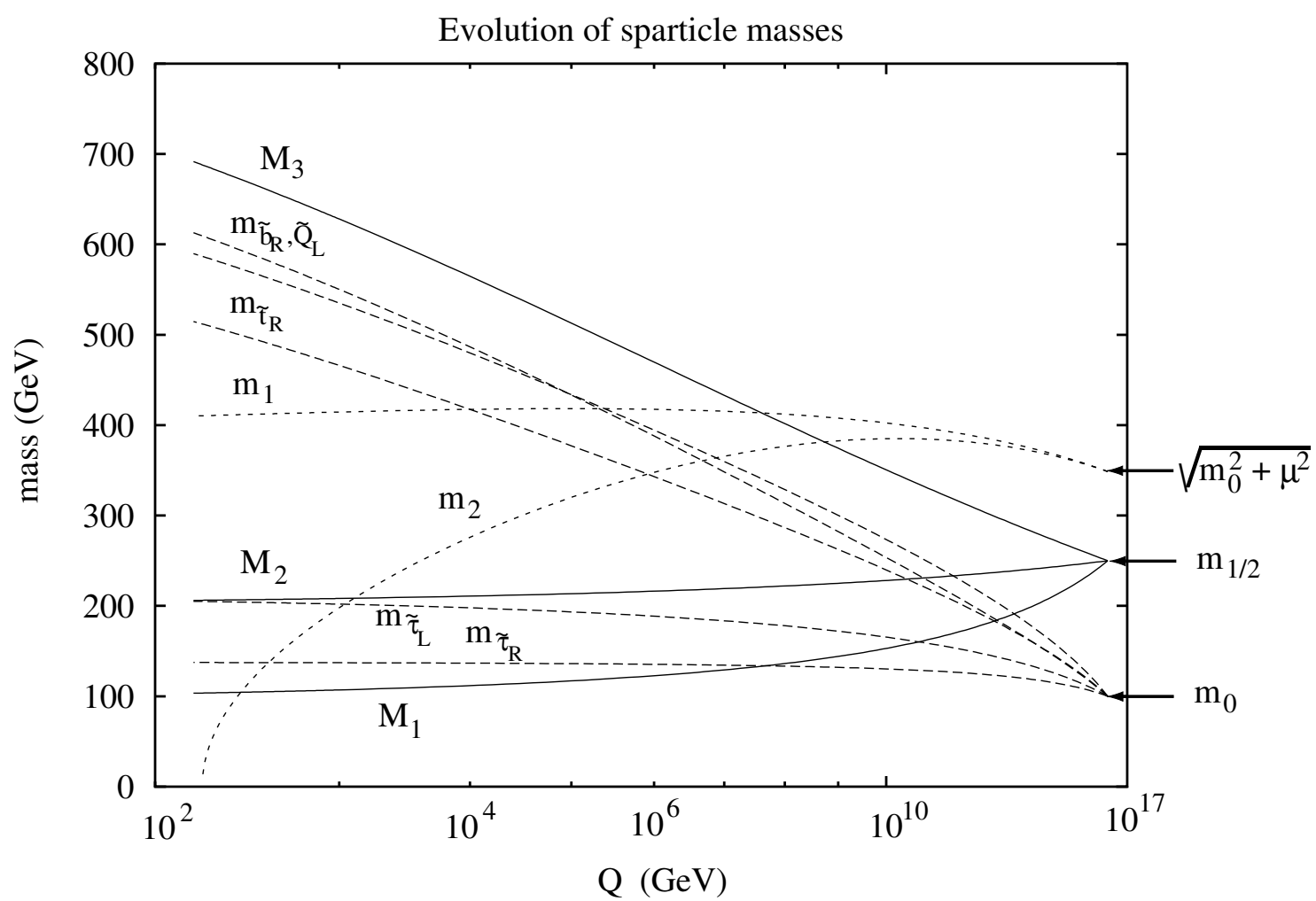

Figure 2.6: In GUT inspired supersymmetry theories like mSUGRA, the gaugino and the scalar masses are degenerated at the Grand Unification Scale. The common gaugino mass at the GUT scale is $m_{1 / 2}$ and the common scalar mass is $m_{0}$. The example [41] shown, changes with different mSUGRA parameters. The energy scale on the x-axis begins directly "before" the electro-weak symmetry breaking, where both Higgs mass parameters are still positive. The gauginos are shown in their symmetry-group eigenstates.

\subsubsection{The Mass Spectrum}

Given an underlying model for the soft breaking terms of a supersymmetry theory, as given in general by Eq. (2.59) or specified for the MSSM in [4], the masses and mixing angles of the supersymmetric, and yet undiscovered sparticles, can be computed.

In the following the mass calculations for neutralinos, charginos, sleptons and squarks within a minimal supersymmetric model with GUT assumption shall be summarized. In a GUT theory the three gauge couplings $g_{1}, g_{2}$, and $g_{3}$ are unified at all scales $\Lambda>\Lambda_{\mathrm{GUT}}$, as discussed in Sec. 2.2.5. This leads to the relationship

$$
\frac{M_{1}}{g_{1}^{2}}=\frac{M_{2}}{g_{2}^{2}}=\frac{M_{1}}{g_{3}^{2}}
$$


which is true at any renormalization group (RG) scale [4]. Within a GUT theory the ratio of the bino mass $M_{1}$, wino mass $M_{2}$, and gluino mass $M_{3}$ can be predicted at the electroweak scale:

$$
\begin{aligned}
& M_{1} \approx \frac{5}{3} \tan ^{2} \theta_{W} M_{2} \approx 0.5 M_{2} \\
& M_{3} \approx \frac{\alpha_{S}}{\alpha} \sin ^{2} \theta_{W} M_{2}=\frac{3}{5} \frac{\alpha_{S}}{\alpha} \cos ^{2} \theta_{W} M_{1}
\end{aligned}
$$

With $\alpha_{S}=0.118, \alpha=1 / 128$ and $\sin ^{2} \theta_{W}=0.23$ this leads to the following approximation at the EW scale:

$$
M_{3}: M_{2}: M_{1} \approx 7: 2: 1
$$

\section{The Higgs sector}

The electroweak symmetry breaking is slightly more complicated in supersymmetric models. The MSSM makes use of two (see Sec. 2.2.3 for the reason) Higgs doublets $\mathbf{H}_{\mathbf{u}}=$ $\left(H_{u}^{+}, H_{u}^{0}\right)$ and $\mathbf{H}_{\mathbf{d}}=\left(H_{d}^{-}, H_{d}^{0}\right)$. The ratio of the vacuum expectation values of the two neutral Higgs fields $v_{u}=\left\langle H_{u}^{0}\right\rangle$ and $v_{d}=\left\langle H_{d}^{0}\right\rangle$ is written as:

$$
\tan \beta=\frac{v_{u}}{v_{d}}
$$

The value of $\tan \beta$ is a free parameter and not fixed by present experiments.

The parameter $\mu$ characterizes the mass mixing of the Higgs doublets, defined at the EW scale. The absolute value $|\mu|$ can be calculated with knowledge of the other mSUGRA parameters. From the explicit form of the Higgs potential $V$ in the MSSM, as given for example in Ref. [4], the following relation (2.93) can be derived, from the conditions $\partial V / \partial H_{u}^{0}=0$ and $\partial V / \partial H_{d}^{0}=0$ under which the Higgs potential $V$ has a minimum.

$$
\tan (\beta)^{2}=\frac{2 \mu^{2}+2 m_{H_{u}}^{2}+m_{Z}^{2}}{2 \mu^{2}+2 m_{H_{d}}^{2}+m_{Z}^{2}}
$$

Only the sign of the higgsino mass mixing parameter $\mu$ remains as a free parameter.

The Higgs scalar fields in the MSSM have eight degrees of freedom and consist of two complex $\mathrm{SU}(2)_{L}$ doublets. When the electroweak symmetry is broken, then three degrees of freedom would become the longitudinal modes of the massive $Z$ and $W^{ \pm}$vector bosons. The remaining five scalar Higgs mass eigenstates are the CP-odd neutral scalar $A^{0}$, two charged scalars $H^{ \pm}$, and two CP-even neutral scalars $h^{0}$ and $H^{0}$. The mass eigenstates 
are given in terms of the gauge eigenstate fields [4]. The tree-level masses of the scalar Higgs fields can be found by expending the potential around the minimum:

$$
\begin{aligned}
m_{A^{0}}^{2} & =m_{H_{u}}^{2}+m_{H_{d}}^{2}+|\mu|^{2} \\
m_{h, H}^{2} & =\frac{1}{2}\left(m_{A}^{2}+m_{Z}^{2} \mp \sqrt{\left(m_{A}^{2}+m_{Z}^{2}\right)^{2}-4 m_{A}^{2} m_{Z}^{2} \cos (2 \beta)^{2}}\right) \\
m_{H^{ \pm}}^{2} & =m_{A}^{2}+m_{W}^{2}
\end{aligned}
$$

The masses of $A^{0}, H^{0}$, and $H^{ \pm}$can grow arbitrarily large, however the mass of $h^{0}$ is constrained. With Eq. 2.95 it is possible to show [4] that at tree-level the upper bound is given by:

$$
m_{h^{0}}<|\cos 2 \beta| \cdot m_{Z}
$$

Significant higher order corrections to the neutral Higgs mass $m_{h^{0}}$, e.g. from top-stop loops have to be taken into account, so that the upper bound is weaker as given by Eq. (2.97) but approximately

$$
m_{h^{0}} \lesssim 130 \mathrm{GeV}
$$

if all MSSM sparticles do not exceed $1 \mathrm{TeV}$ in mass. This bound is weakened if more supermultiplets exist and rises logarithmically with the soft masses, typically $m_{\tilde{t}}$.

\section{The Neutralinos}

The neutral higgsinos $\left(\tilde{H}_{u}^{0}\right.$ and $\left.\tilde{H}_{d}^{0}\right)$ and the neutral electroweak gauginos $\left(\tilde{B}\right.$ and $\left.\tilde{W}^{0}\right)$ mix with each other and form four neutral mass eigenstates called the neutralinos, as already shown in Tab. 2.5.

The neutralino mass terms in the Lagrangian are

$$
L_{\tilde{\chi}_{1,2,3,4}^{0}}=-\frac{1}{2}\left(\psi^{0}\right)^{T} \mathbf{M}_{\tilde{\chi}^{0}} \psi^{0}+\quad \text { c.c. },
$$

with the electrically neutral gauge-eigenstate basis $\psi^{0}=\left(\tilde{B}, \tilde{W}^{0}, \tilde{H}_{d}^{0}, \tilde{H}_{u}^{0}\right)$ and

$$
\mathbf{M}_{\tilde{\chi}^{0}}=\left(\begin{array}{cccc}
M_{1} & 0 & -c_{\beta} s_{W} m_{Z} & s_{\beta} s_{W} m_{Z} \\
0 & M_{2} & c_{\beta} s_{W} m_{Z} & -s_{\beta} s_{W} m_{Z} \\
-c_{\beta} s_{W} m_{Z} & c_{\beta} c_{W} m_{Z} & 0 & -\mu \\
s_{\beta} s_{W} m_{Z} & -s_{\beta} c_{W} m_{Z} & -\mu & 0
\end{array}\right) .
$$

The absolute values of the four eigenvalues of the matrix $\mathbf{M}_{\tilde{\chi}^{0}}$ correspond to the neutralino masses $\mathrm{m}\left(\tilde{\chi}_{1}^{0}\right), \mathrm{m}\left(\tilde{\chi}_{2}^{0}\right), \mathrm{m}\left(\tilde{\chi}_{3}^{0}\right)$, and $\mathrm{m}\left(\tilde{\chi}_{4}^{0}\right)$. 


\section{The Charginos}

Similar to the neutralinos, also the charged higgsinos $\left(\tilde{H}_{u}^{+}\right.$and $\left.\tilde{H}_{d}^{-}\right)$and the charged electroweak gauginos $\left(\tilde{W}^{+}\right.$and $\left.\tilde{W}^{-}\right)$mix. The chargino mass terms in the Lagrangian are

$$
L_{\tilde{\chi}_{1,2}^{ \pm}}=-\frac{1}{2}\left(\psi^{ \pm}\right)^{T} \mathbf{M}_{\tilde{\chi}^{ \pm}} \psi^{ \pm}+\text {c.c }
$$

with the electrically charged gauge-eigenstate basis $\psi^{ \pm}=\left(\tilde{W}^{+}, \tilde{H}_{u}^{+}, \tilde{W}^{-}, \tilde{H}_{d}^{-}\right)$and

$$
\mathbf{M}_{\tilde{\chi}^{ \pm}}=\left(\begin{array}{cccc}
0 & 0 & M_{2} & \sqrt{2} s_{\beta} m_{W} \\
0 & 0 & \sqrt{2} s_{\beta} m_{W} & \mu \\
M_{2} & \sqrt{2} s_{\beta} m_{W} & 0 & 0 \\
\sqrt{2} s_{\beta} m_{W} & \mu & 0 & 0
\end{array}\right) .
$$

The resulting chargino masses are given by:

$$
\begin{aligned}
m_{\tilde{\chi}_{1}^{ \pm}}, m_{\tilde{\chi}_{2}^{ \pm}}= & \frac{1}{2}\left(M_{2}^{2}+\mu^{2}+2 M_{W}^{2}\right. \\
& \left.\mp \sqrt{\left[M_{2}^{2}+\mu^{2}+2 M_{W}^{2}\right]^{2}-4\left[\mu M_{2}-M_{W}^{2} \sin (2 \beta)^{2}\right]^{2}}\right) .
\end{aligned}
$$

\section{The Sleptons and the Squarks}

In principle, all squarks sharing the same quantum numbers can mix, so that the squark mass eigenstates in the MSSM should be obtained by diagonalization of the $6 \times 6$ masssquared matrices for up-type quarks $\left(\tilde{u}_{L}, \tilde{c}_{L}, \tilde{t}_{L}, \tilde{u}_{R}, \tilde{c}_{R}, \tilde{t}_{R}\right)$ and for the down-type quarks $\left(\tilde{d}_{L}, \tilde{s}_{L}, \tilde{b}_{L}, \tilde{d}_{R}, \tilde{s}_{R}, \tilde{b}_{R}\right)$, respectively. The same holds for the charged sleptons $\left(\tilde{e}_{L}, \tilde{\mu}_{L}, \tilde{\tau}_{L}\right.$, $\left.\tilde{e}_{R}, \tilde{\mu}_{R}, \tilde{\tau}_{R}\right)$. The sneutrino masses should be obtained by diagonalizing the $3 \times 3$ matrix spanned by $\left(\tilde{\nu}_{e}, \tilde{\nu}_{\mu}, \tilde{\nu}_{\tau}\right)$. However, in general the mixing angles are very small, only the third generation squarks and sleptons masses differ from their first- and second-family counterparts, because of the effects of large third generation Yukawa- and soft-breaking couplings in the renormalization group equations (RGEs). For details, the reader is referred once more to reference [4].

\section{The remaining mSUGRA parameters}

To summarize, the remaining five free mSUGRA parameters are:

- The common scalar mass $m_{0}$ at the GUT scale;

- The common gaugino mass $m_{1 / 2}$ at the GUT scale. Sometimes also $M_{1}$ and $M_{2}$ the masses of the $\mathrm{SU}(2)$ gaugino eigenstates (bino and wino) and $M_{3}$ the gluino-mass, defined at the EW scale, are used;

- The sign of the mass mixing parameter $\mu$ of the Higgs doublets, which is defined at the EW scale. 
- The ratio of the vacuum expectation values of the Higgs doublets at the EW scale $\tan \beta$;

- The trilinear coupling $A_{0}$ in the Higgs sector, which controls the mixing of the third generation sfermions, taken at the GUT scale.

The masses and the mixing angles of all SUSY particles at the electro-weak scale can be computed by solving the renormalization group equations (RGE), under the assumption, that there is no new physics between the GUT and the EW scale. The RGEs are discussed in detail in [4]. The evolution of the sparticle masses is shown for an exemplary mSUGRA parameter set in Fig. 2.6. As a practical rule of thumb the relation

$$
\mathrm{m}\left(\tilde{\chi}_{1}^{ \pm}\right) \approx \mathrm{m}\left(\tilde{\chi}_{2}^{0}\right) \approx 2 \cdot \mathrm{m}\left(\tilde{\chi}_{1}^{0}\right)
$$

is valid near the EW scale. The mSUGRA parameter space for $\mu<1, \tan \beta=5$ and $A_{0}=0 \mathrm{GeV}$ is depicted in Fig. 2.7.

The mSUGRA model is a theory which is capable to replace the Standard Model and is not excluded by observations. Not only because of the spontaneous supersymmetry breaking mechanism and since there are only five free parameters, it is favored by theorists as well as experimentalists. 


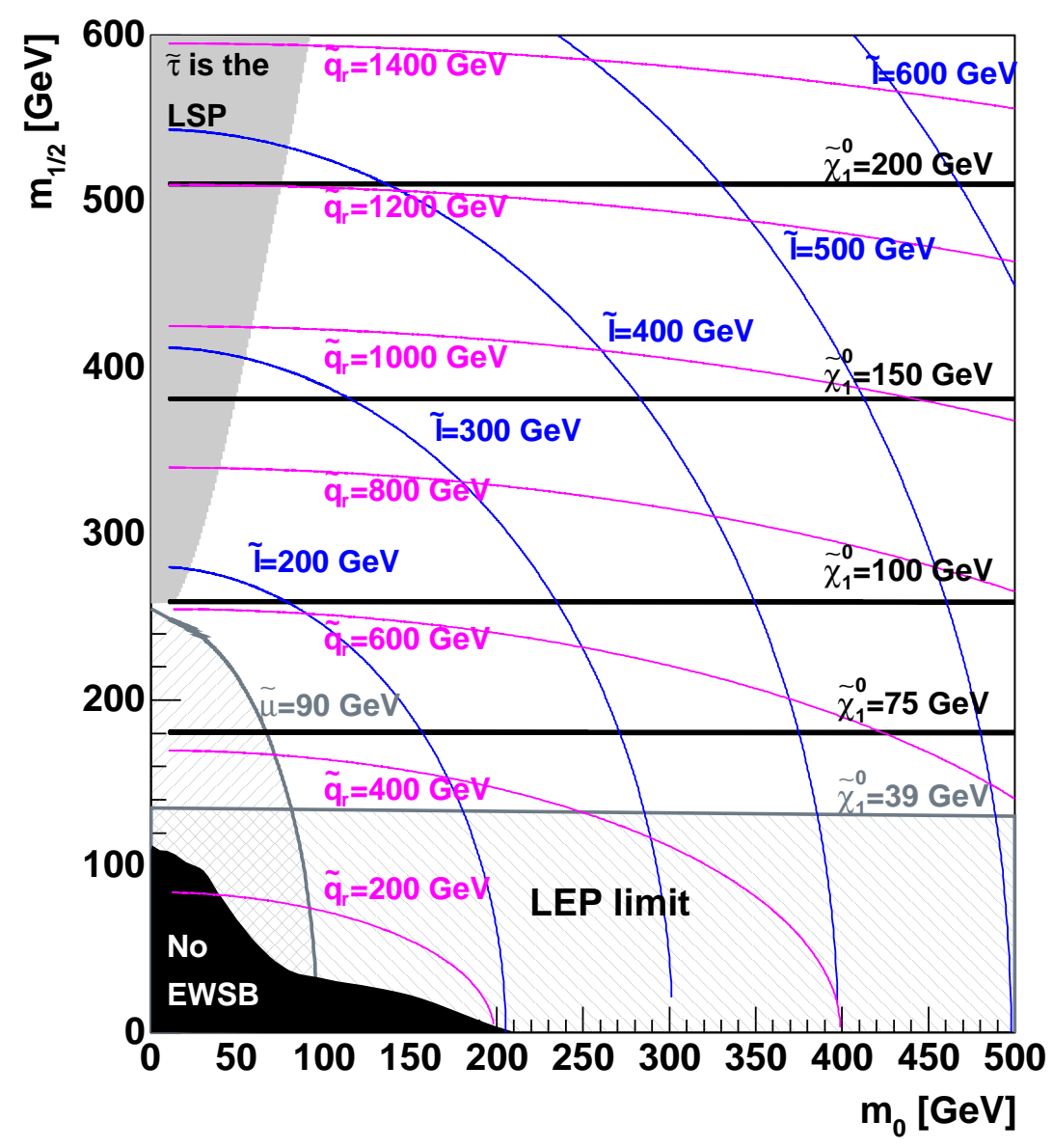

Figure 2.7: The mSUGRA parameter space for $\mu<1, \tan \beta=5$ and $A_{0}=0 \mathrm{GeV}$ in the $m_{0}-m_{1 / 2}$ parameter plane. The neutralino $\tilde{\chi}_{1}^{0}$ iso-mass lines are shown as horizontal black lines, constant slepton mass $\tilde{l}$ lines are shown in blue and constant right-handed down squark $\tilde{d}_{R}$ masses are drawn in magenta. The hatched area is excluded by LEP [42] if one R-parity violation $L Q \bar{d}$ coupling is different from zero. The stau $\tilde{\tau}$ is the LSP in the gray filled region and in the black filled region at small $m_{0}$ and $m_{1 / 2}$ no electroweak symmetry breaking occurs, because both Higgs mass parameters are positive at the EW scale. The iso-mass lines are calculated with [43] with an estimated accuracy of $\pm 5 \%$. 


\section{$2.3 \quad$ R-Parity}

In the Standard Model all fermions carry conserved lepton and baryon quantum numbers $L$ and $B$, which represent an implicit fermion number $F=L+3 B$. In a supersymmetric theory naturally all bosonic superpartners carry $L$ and $B$, while the fermionic superpartners of the Standard Model bosons $\gamma, Z, W^{ \pm}, g$ do not carry such a fermion quantum number. In particular Majorana type spin- $\frac{1}{2}$ fields, the neutralinos, are not able to carry such fermionic charges. Moreover, $L$ and $B$ are no longer necessarily conserved, since new interactions can be present in a supersymmetric theory, that can prevent lepton and baryon number conservation. One consequence is that virtual squark and slepton exchanges and loops become possible, leading for example to a possible proton decay, i.e. $p \rightarrow \pi^{0} e^{+}$, see Sec. 2.4.

In a supersymmetric theory a fermion quantum number makes no longer sense. To replace " $F$ ", a new multiplicative quantum number $R_{P}$ is introduced:

$$
R_{P}=(-1)^{L+3 B+2 S}
$$

If the lepton number $L$ or the baryon number $B$ is violated, then $R_{P}$ is violated and vice versa. The spin $S$, and therefore angular momentum conservation, cannot be violated. The discrete quantum number $R_{P}$ divides Standard Model particles from their supersymmetric partners:

$$
R_{P}= \begin{cases}+1 & \text { for ordinary SM particles } \\ -1 & \text { for their superpartners }\end{cases}
$$

$R_{P}$ originates from the continuous $R$-invariance [44,45], which is in $N=1$ SUSY models a $U(1)$ symmetry carried by the supersymmetry generator. It is abandoned in favor of its discrete version R-parity, because an unbroken R-invariance would prevent gauginos, like gluinos and gravitinos from gathering mass, once the supersymmetry is spontaneously broken. Otherwise light "R-hadrons" made of quarks, anti-quarks and massless gluinos could exist, contradicting observation [46].

If R-parity violation $\left(R_{p}\right)$ is allowed, the following trilinear and bilinear terms [47] appear ${ }^{4}$ in the MSSM superpotential, see also Eq. (2.80):

$$
W_{\not R_{p}}=\frac{1}{2} \lambda_{i j k} L_{i} L_{j} \bar{e}_{k}^{c}+\lambda_{i j k}^{\prime} L_{i} Q_{j} \bar{d}_{k}^{c}+\frac{1}{2} \lambda_{i j k}^{\prime \prime} \bar{u}_{i}^{c} \bar{d}_{j}^{c} \bar{d}_{k}^{c}+\mu_{i} L_{i} H_{1}
$$

$L$ and $Q$ are the lepton and quark $\mathrm{SU}(2)$ doublet superfields, and $\bar{e}, \bar{u}, \bar{d}$ denote the singlet superfields, as defined previously. Where the family indices are denoted as $i, j=1,2,3$.

\footnotetext{
${ }^{4}$ The superpotential $W$ is not determined by any theory, as mentioned before in the context of Eq. (2.80). Therefore, all possible terms can be considered in general. It is no coincidence, that the form of the terms in $W_{\not R_{p}}$ are similar to those appearing in the first part of Eq. (2.80) because the total superpotential $W$ must meet the requirements as defined in Sec. 2.2. To obtain the R-parity violating superpotential terms, the Higgs superfield $H_{d}$ can be replaced by the lepton superfield $L_{i}$ because they have exactly the same gauge quantum numbers, similarly for $H_{u}$ and the quark superfield singlet $\bar{u}_{i}$.
} 
The superscript $c$ refers to charge conjugation. The coupling strengths are given by the Yukawa coupling constants $\lambda, \lambda^{\prime}$ and $\lambda^{\prime \prime}$. The last term $\mu_{i} L_{i} H_{1}$ mixes the lepton and the Higgs superfields and can be rotated to zero in the superpotential [42]. The corresponding tree diagrams are given in Fig. 2.8. The notation in Eq. (2.107) is a simplification, because the summation over the gauge indices is dropped. One has for example

$$
\begin{aligned}
L_{i} L_{j} \bar{e}_{k} & \equiv\left(\epsilon_{\alpha \beta} L_{i}^{\alpha} L_{j}^{\beta}\right) \bar{e}_{k}=\left(L_{i} L_{j}^{\prime}-L_{i}^{\prime} L_{j}\right) \bar{e}_{k} \\
\bar{u}_{i}^{c} \bar{d}_{j}^{c} \bar{d}_{k}^{c} & \equiv \epsilon^{\xi \psi \zeta} \bar{u}_{i}^{\xi c} \bar{d}_{j}^{\psi c} \bar{d}_{k}^{\zeta c}
\end{aligned}
$$

where $\alpha, \beta=1,2$ are weak isospin indices, $\xi, \psi, \zeta=1,2,3$ are color indices and $\epsilon_{\alpha \beta}, \epsilon^{\xi \psi \zeta}$ are the totally antisymmetric tensors.

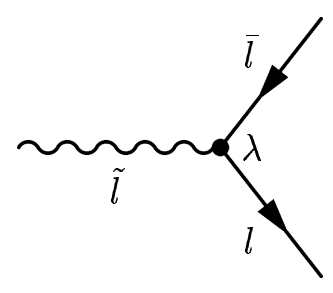

(a) $L L \bar{e}$

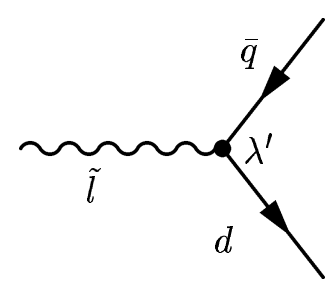

(b) $L Q \bar{d}$

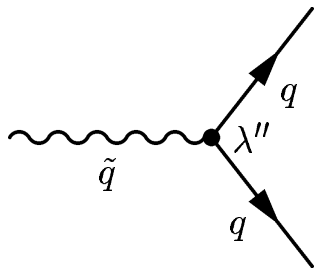

(c) $\bar{u} \bar{d} \bar{d}$

Figure 2.8: Basic interactions associated with the trilinear $\not R_{p}$ superpotential terms. The exemplary diagrams (a) and (b) violate lepton number and (c) is baryon number violating.

The constants $\lambda_{i j k}$ and similarly $\lambda_{i j k}^{\prime \prime}$ are not completely independent. Gauge invariance enforces anti-symmetry of the first two indices of the $\lambda_{i j k}$ couplings:

$$
\lambda_{i j k} L_{i} L_{j} \bar{e}_{k}^{c}=\lambda_{i j k}\left(\epsilon_{\alpha \beta} L_{i}^{\alpha} L_{j}^{\beta}\right) \bar{e}_{k}=-\lambda_{j i k}\left(\epsilon_{\beta \alpha} L_{j}^{\alpha} L_{i}^{\beta}\right) \bar{e}_{k}=-\lambda_{j i k} L_{i} L_{j} \bar{e}_{k}^{c}
$$

The above equation leads to:

$$
\lambda_{i j k}=-\lambda_{j i k}
$$

Similarly, the gauge invariance leads to the the anti-symmetry of the $\lambda_{i j k}^{\prime \prime}$ couplings with respect to the last two indices.

$$
\lambda_{i j k}^{\prime \prime}=-\lambda_{i k j}^{\prime \prime}
$$

Because of these symmetry arguments the $L L \bar{e}$ and the $\bar{u} \bar{u} \bar{d}$ terms contain a factor $\frac{1}{2}$, in contrast to the $L Q \bar{d}$ term.

Altogether, Eq. (2.107) makes use of 48 parameters; $9 L L \bar{e}$ couplings $\lambda_{i j k}, 27 L Q \bar{d}$ couplings $\lambda_{i j k}^{\prime}, 9 \bar{u} \bar{u} \bar{d}$ couplings $\lambda_{i j k}^{\prime \prime}$ and 3 dimensionful parameters $\mu_{i}$ which mix the lepton and down-type Higgs superfields. 


\subsubsection{Resonant Slepton Production at the Tevatron}

It is assumed here that only one coupling, namely $\lambda_{211}^{\prime}$ can be non-zero. This implies (muon) lepton number violation. The reason for this assumption is the existence of very strict limits on $\mathbb{R}_{p}$-supersymmetry if more than one coupling is non-zero, for example from hadron lifetimes, see Sec. 2.4.

If $\lambda_{211}^{\prime} \neq 0$, at a $p \bar{p}$ collider an initial $q \bar{q}$ pair can transform into a single smuon $\tilde{\mu}$ or into a muon sneutrino $\tilde{\nu}_{\mu}$, see Fig. 2.8(b). The slepton can then decay either into a lepton and a gaugino without violating R-parity or via the $L Q \bar{d}$-coupling $\lambda_{211}^{\prime}$ back into two quarks. Due to the smallness of $\lambda_{211}^{\prime}, R_{p}$ decay modes of sleptons (in two quarks) are suppressed in large regions of the parameter space. Only in the limit of large neutralino masses $\left(\tilde{\chi}_{1}^{0} \approx \tilde{l}\right)$ and for large $\lambda_{211}^{\prime}$, the direct $\not R_{p}$ slepton decay branching fraction can be significant. The $\tilde{l} \rightarrow q \bar{q}$ branching fraction in the slepton mass - neutralino mass plane is shown in Fig. 2.9.

The cross section for slepton production can be large if the slepton is produced resonantly (on shell). Since the momentum fractions $x_{i}$ carried by the quarks can 'adjust' to the slepton mass - if kinematically allowed at a given center of mass energy - there will always be a resonant part of the total cross section. Another advantage of this process is the relatively small kinematic suppression, since only one SUSY particle is produced.

Apart from the $s$ channel (Fig. 2.10), also $t$ and $u$ channel contributions exist, with squarks or gluons being exchanged; however, since no resonance exists, this amplitude is not enhanced and in general small compared to the $s$ channel matrix element.

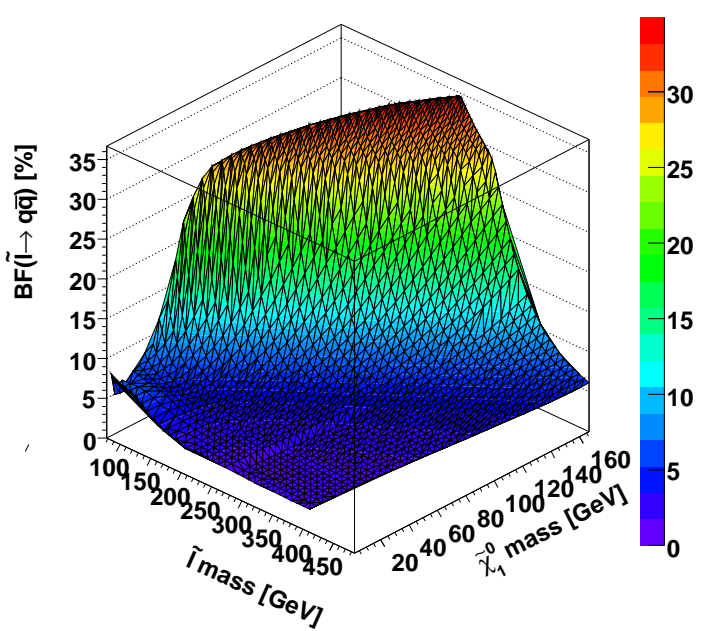

(a)

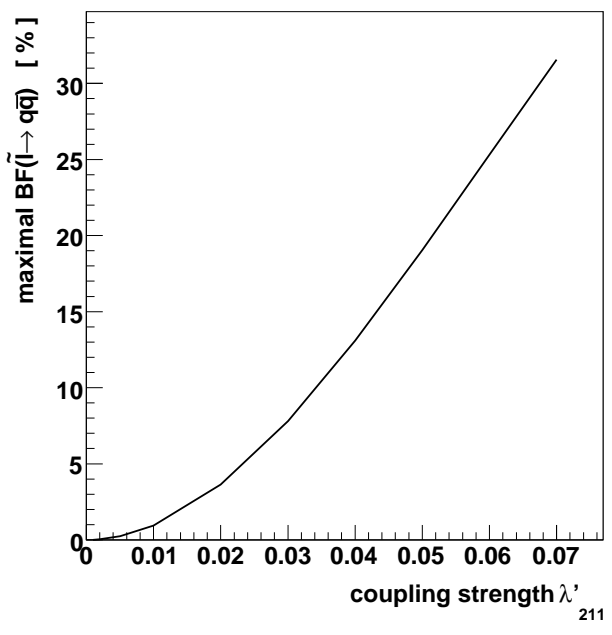

(b)

Figure 2.9: Relative slepton branching fraction to two quarks $\tilde{l} \rightarrow q \bar{q}$ in the slepton mass neutralino mass plane for $\lambda_{211}^{\prime}=0.07$ (a) and the dependence of $\operatorname{BF}(\tilde{l} \rightarrow q \bar{q})$ on the size of the coupling parameter $\lambda_{211}^{\prime}$ for a mass combination $\mathrm{m}\left(\tilde{\chi}_{1}^{0}\right)=160 \mathrm{GeV}$ and $\mathrm{m}(\tilde{l})=300 \mathrm{GeV}$. 


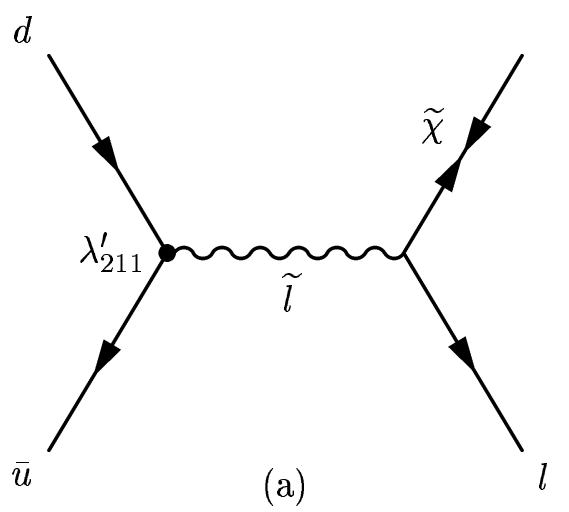

(a)

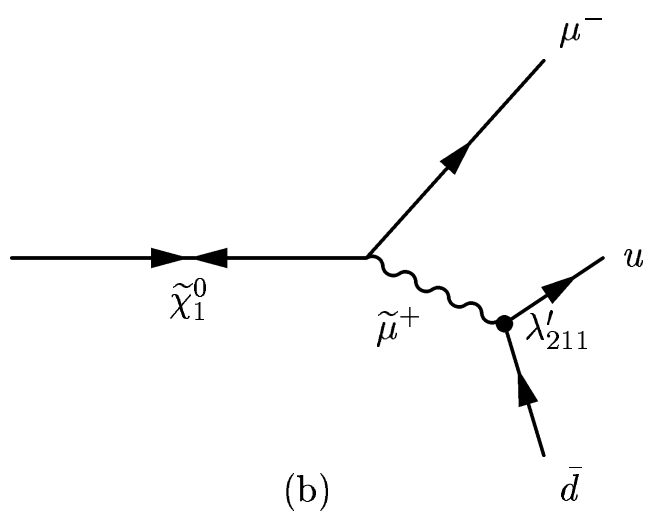

(b)

Figure 2.10: Resonant slepton production and decay into a gaugino and an accompanying lepton (a). The slepton is either a smuon $\tilde{\mu}$ or a muon sneutrino $\tilde{\nu}_{\mu}$ which both can decay into any gaugino $\tilde{\chi}_{1}^{0}, \tilde{\chi}_{2}^{0}, \tilde{\chi}_{3}^{0}, \tilde{\chi}_{4}^{0}, \tilde{\chi}_{1}^{ \pm}, \tilde{\chi}_{2}^{ \pm}$if the mass is lighter than the slepton's $\mathrm{m}(\tilde{\chi})<\mathrm{m}(\tilde{l})$, and one accompanying $\mu$ or $\nu_{\mu}$ according to electrical charge conservation. The gaugino cascade decays into the lightest neutralino, see App. A.2 and A.3 for reference. The neutralino $\tilde{\chi}_{1}^{0}$ finally decays via the R-parity violating $L Q \bar{d}$ coupling $\lambda_{211}^{\prime}$ into one lepton and two quarks (b). See App. A.1 for all possible decays.

The transition amplitude for two quarks in the initial state and one slepton in the final state (e.g. $d_{L} \bar{d}_{R} \rightarrow \tilde{\nu}_{\mu}$ ) is given by [48]:

$$
\begin{aligned}
T_{f i} & =\lambda_{211}^{\prime} \bar{d}_{R}(q) \frac{1-\gamma_{5}}{2} d_{L}(p) \\
\left|T_{f i}\right|^{2} & =\frac{1}{3 \cdot 2 \cdot 2}\left|\lambda_{211}^{\prime}\right|^{2} \cdot \operatorname{Tr}\left(\bar{d}_{R} \frac{1-\gamma_{5}}{2} d_{L} \bar{d}_{L} \frac{1+\gamma_{5}}{2} d_{R}\right)
\end{aligned}
$$

Where the factor $1 / 12$ is due to the spin and color numbers of the initial particles. With $d_{L} \bar{d}_{L}=\not p, \bar{d}_{R} d_{R}=\not d$ and $\not p \gamma_{5}=-\gamma_{5} \not p, \gamma_{5}^{2}=1$ the above can be transformed to:

$$
\begin{aligned}
\left|T_{f i}\right|^{2} & =\frac{1}{3} \frac{\left|\lambda_{211}^{\prime}\right|^{2}}{16} \cdot \operatorname{Tr}\left(\not \not\left(1-\gamma_{5}\right) \not p\left(1+\gamma_{5}\right)\right) \\
& \left.\left.=\frac{1}{3} \frac{\left|\lambda_{211}^{\prime}\right|^{2}}{16} \cdot(\operatorname{Tr}(\not \not \not p))-\operatorname{Tr}\left(\not d \gamma_{5} \not \supset \gamma_{5}\right)\right)\right) \\
& \left.=\frac{1}{3} \frac{\left|\lambda_{211}^{\prime}\right|^{2}}{16} \cdot 2 \cdot \operatorname{Tr}(\not \not \not p)\right)
\end{aligned}
$$

With $\operatorname{Tr}(\not \not \not p)=4 q_{\mu} p^{\mu}=2 \hat{s}$, where the Mandelstam variable $\hat{s}$ is the effective center of mass energy, the matrix element for this process becomes $\left|T_{f i}\right|^{2}=\frac{1}{12}\left|\lambda_{211}^{\prime}\right|^{2} \hat{s}$. The resulting cross section is then:

$$
\begin{aligned}
\hat{\sigma}\left(d \bar{d} \rightarrow \tilde{\nu}_{\mu}\right) & =\frac{\pi}{\hat{s}}\left|T_{f i}\right|^{2} \cdot \delta\left((p+q)^{2}-p_{\tilde{\nu}}^{2}\right) \\
& =\frac{\pi}{12}\left|\lambda_{211}^{\prime}\right|^{2} \delta\left(\hat{s}-m\left(\tilde{\nu}_{\mu}\right)^{2}\right)
\end{aligned}
$$




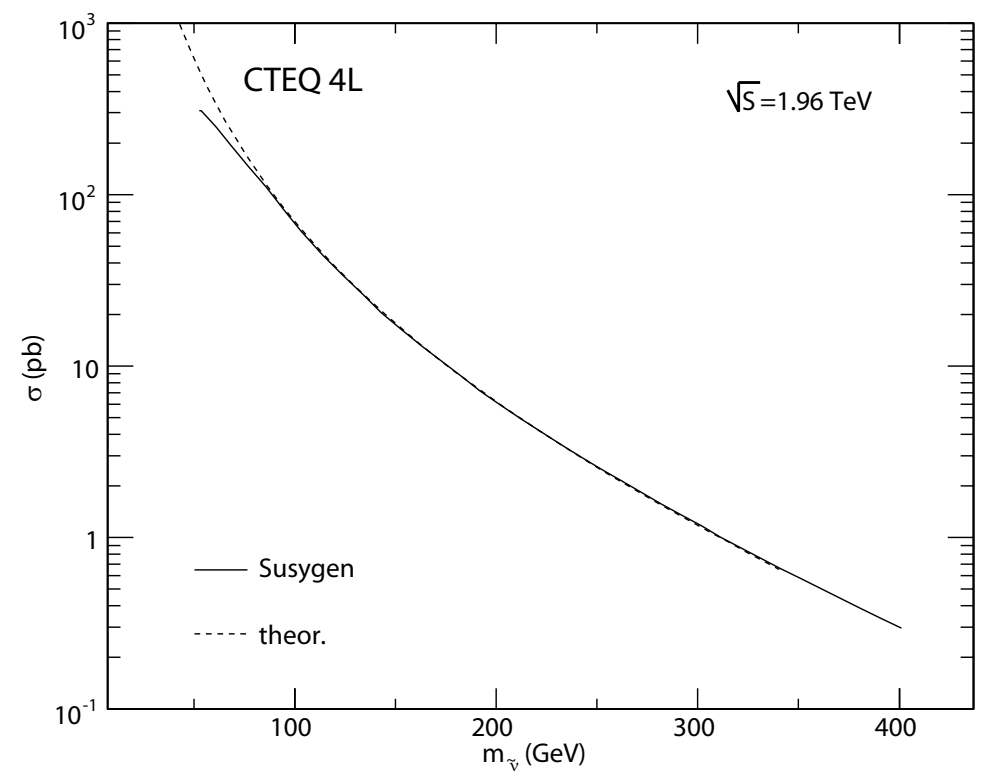

Figure 2.11: Leading order muon sneutrino cross section $p \bar{p} \rightarrow \tilde{\nu}_{\mu}$ using the parton distribution functions given by CTEQ4L. The SUSYGEN prediction (solid line) and the direct calculation [51] (dashed) using the same PDF are shown for $\lambda_{211}^{\prime}=0.07$.

Where $\hat{\sigma}$ is the parton level cross section. The parton distribution functions (PDFs) give the probability to find partons (quarks or gluons) in a hadron as a function of the fraction $x$ of the hadron's momentum carried by the parton. A parton of type $a$ (in the case of resonant sneutrino production this is either a $d$ or $\bar{d}$ ) comes from a hadron of type $A$ (this is at the Tevatron obviously a $p$ or a $\bar{p}$ ) and carries a fraction $x_{A}$ of the hadrons momentum. The probability to find it is given by $f_{a \mid A}\left(x_{A}\right) d x_{A}$. The second parton $b$ is similarly part of hadron $B$. The functions $f_{a \mid A}(x)$ are the parton distribution functions and will be discussed in more detail in Sec. 4.3.5. The proton-anti-proton cross section is then:

$$
\sigma\left(p \bar{p} \rightarrow \tilde{\nu}_{\mu}\right) \sim \sum_{a, b} \int d x f_{a \mid A}\left(x_{A}\right) \int d x f_{b \mid B}\left(x_{B}\right) \cdot \hat{\sigma}\left(d \bar{d} \rightarrow \tilde{\nu}_{\mu}\right)
$$

The leading order muon sneutrino $p \bar{p} \rightarrow \tilde{\nu}_{\mu}$ production cross section using the PDF CTEQ4L ${ }^{5}[49,50]$ as predicted by the Susygen Monte Carlo program [48], is compared to the calculation described by Eq. (2.121) using the same PDFs. Both predictions agree very well and are shown in Fig. 2.11. Resonant smuon production $u \bar{d} \rightarrow \tilde{\mu}^{+}, \bar{u} d \rightarrow \tilde{\mu}^{-}$is similarly calculable and agrees with the Susygen prediction, too.

The slepton production cross section $\sigma(p \bar{p} \rightarrow \tilde{l})$ depends on the value of $\lambda_{211}^{\prime}$ as given by Eq. (2.121). The smuon and sneutrino production cross sections are of the order of $1-10 \mathrm{pb}$ for slepton masses between $200 \mathrm{GeV}$ and $300 \mathrm{GeV}$ and $\lambda_{211}^{\prime}=0.07$. For the

\footnotetext{
${ }^{5}$ CTEQ is an abbreviation for Co-ordinated Theoretical-Experimental Project on $Q C D$
} 
resonant slepton production to be observable at Tevatron with an integrated luminosity of several $100 \mathrm{pb}^{-1}, \lambda_{211}^{\prime}$ has to be larger than $\sim 0.01$.

In addition to the two body slepton decays shown in Fig. 2.10, decays with three or more particles in the final state are possible, but these decay modes have small branching fractions for most SUSY parameter combinations. Slepton decays like $\tilde{\mu}_{L} \rightarrow W+\tilde{\nu}$ play no role, since the mass difference between the two sleptons is too small. Therefore the dominant intermediate states are:

$$
\begin{aligned}
\tilde{\mu} & \rightarrow \tilde{\chi}^{0} \mu \\
\tilde{\mu} & \rightarrow \tilde{\chi}^{ \pm} \nu_{\mu} \\
\tilde{\nu}_{\mu} & \rightarrow \tilde{\chi}^{0} \nu_{\mu} \\
\tilde{\nu}_{\mu} & \rightarrow \tilde{\chi}^{ \pm} \mu
\end{aligned}
$$

Where $\chi^{0}$ can be a neutralino of any of the four neutralino generations if the decay is kinematically allowed, similarly $\chi^{ \pm} \equiv \chi_{1}^{ \pm}, \chi_{2}^{ \pm}$. The corresponding products of cross sections times branching fraction are of the same order of magnitude. The lightest neutralino is assumed to be the lightest supersymmetric particle (LSP). The analysis presented here is insensitive to regions in the parameter space, where this is not the case. The LSP is able to decay into three fermions by violating the R-parity; an example is given in Fig. 2.10(b). All possible decay modes are given in appendix A.1. The coupling $\lambda_{211}^{\prime}$ affects the lifetime $\tau$ of the gaugino, but only for $\lambda_{211}^{\prime} \lesssim 0.01$ this results in a measurable decay length. In this analysis the value of $\lambda_{211}^{\prime}$ is always larger than $\sim 0.02$, therefore the corresponding decay length is negligible.

All slepton decay channels consist of at least two jets and in most cases of accompanying muons. This means that the search has to be done in a challenging multijet environment. The feasibility of requiring only one muon in the final state has been studied. It has been found that the lower single-muon trigger efficiency and the dramatically increasing multijet (QCD) background in this case cannot be compensated by the better signal efficiency (a factor of up to 3 ) for $\mu \mu$ final states. The rate of zero, one and two muon final states of the resonant slepton production is shown in Fig. 2.3.1. Due to these considerations, the analysis is performed on two-muon final states.

The remaining contributing channels to this analysis are therefore:

$$
\begin{array}{rlll}
\text { (i) } & \tilde{\mu} & \rightarrow & \tilde{\chi}_{1}^{0} \mu \\
\text { (ii) } & \tilde{\mu} & \rightarrow & \tilde{\chi}_{2,3,4}^{0} \mu \\
\text { (iii) } & \tilde{\nu} & \rightarrow & \tilde{\chi}_{1,2}^{ \pm} \mu
\end{array}
$$

The three channels are analyzed separately and the obtained results are interpreted within a mSUGRA scenario.

It is important to note, that the branching ratio $\tilde{\chi}_{1}^{0} \rightarrow \mu q^{\prime} \bar{q}$ is only about $50 \%$, compared to the total neutralino decay $\tilde{\chi}_{1}^{0} \rightarrow l q^{\prime} \bar{q}$ with $l \equiv \mu, \nu_{\mu}$. Since the analysis is performed on 


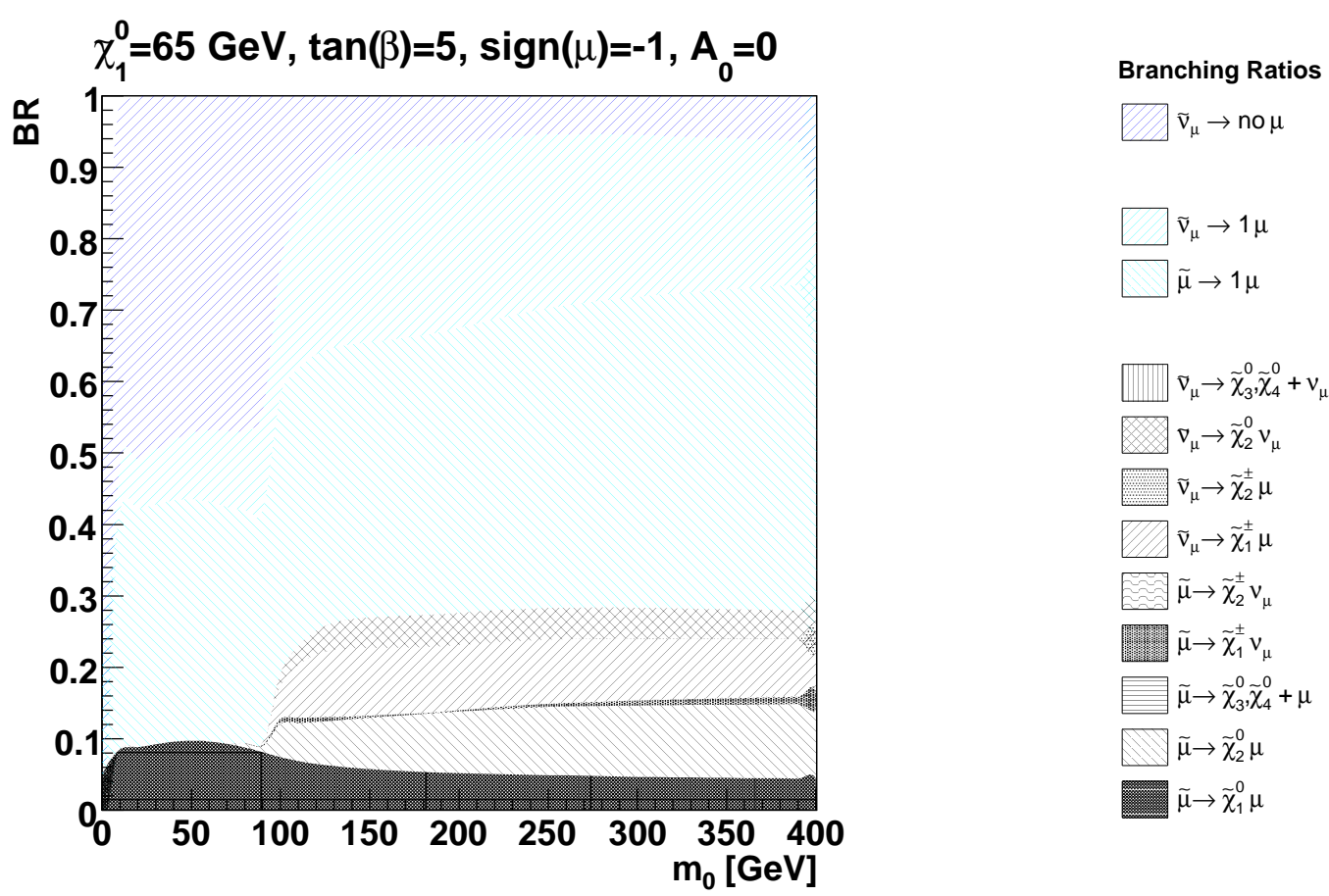

Figure 2.12: Branching ratio of the slepton decay channels into zero (top, blue), one (middle, cyan) and two (bottom, black) muons. The larger branching ratio of the one-muon final states is compensated by the smaller selection and trigger efficiency and the larger multijet background.

two-muon final states, a signal selection inefficiency of $\approx 50 \%$ is a priori inherent for all three channels.

\subsubsection{Pair and Associated Gaugino Production}

Pair- or associated production of gauginos is a R-parity conserving process, thus independent of the unknown extent of $\mathrm{R}$-parity violation. If $\mathrm{R}$-parity is violated, then this leads to the decay of the lightest neutralino $\tilde{\chi}_{1}^{0}$ via any $\mathbb{R}_{p}$ coupling, as shown in Fig. 2.10. Many different couplings can be probed simultaneously, as they lead to similar final states, deviating only in the quark or jet flavor.

In case of any non-zero $L Q \bar{d}$ coupling $\lambda_{2 j k}^{\prime}$, with ${ }^{6} j=1,2$ and $k=1,2,3$, the lightest neutralino $\tilde{\chi}_{1}^{0}$ will decay into a second generation lepton (muon or muon neutrino) and two quarks. This analysis examines two-muon final states and is sensitive to the $L Q \bar{d}$ couplings $\lambda_{2 j k}^{\prime}$.

In regions of small values of $m_{1 / 2}$ the cross section of gaugino pair- or associated production, as shown in Fig. 2.13(a, b), becomes large, as the gaugino masses depend on the mSUGRA parameter $m_{1 / 2}$. At small values of $m_{0}$, which is a region of small squark masses, also squark- and gluino pair and associated production, depicted in Fig. 2.13(c,

\footnotetext{
${ }^{6}$ The couplings $\lambda_{23 k}^{\prime}$ cannot be probed, as they lead to a neutralino decay into a top quark which is kinematically suppressed in the studied parameter space $\mathrm{m}\left(\tilde{\chi}_{1}^{0}\right)<\mathrm{m}$ (top) or $m_{1 / 2} \lesssim 500 \mathrm{GeV}$.
} 


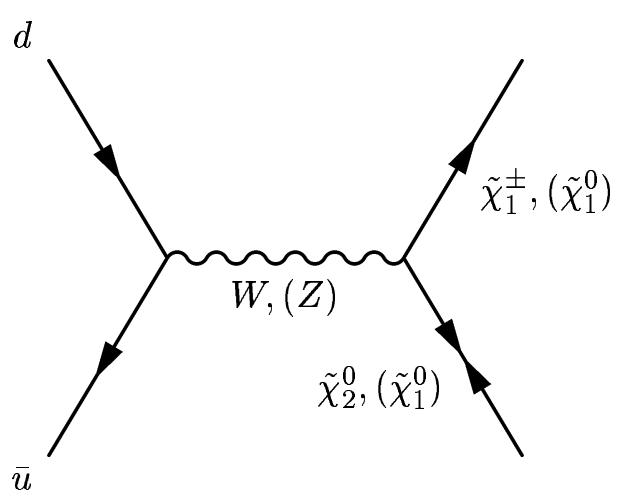

(a)

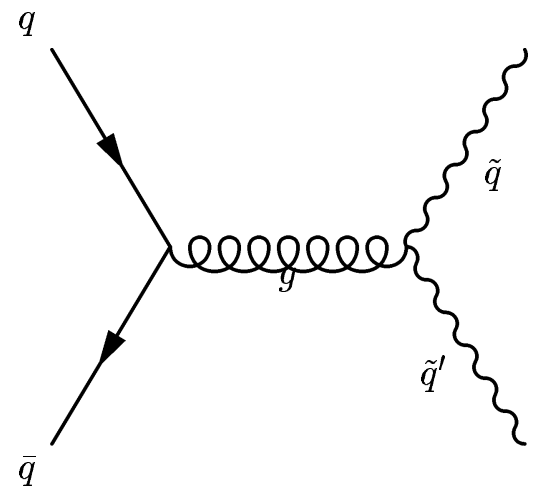

(c)

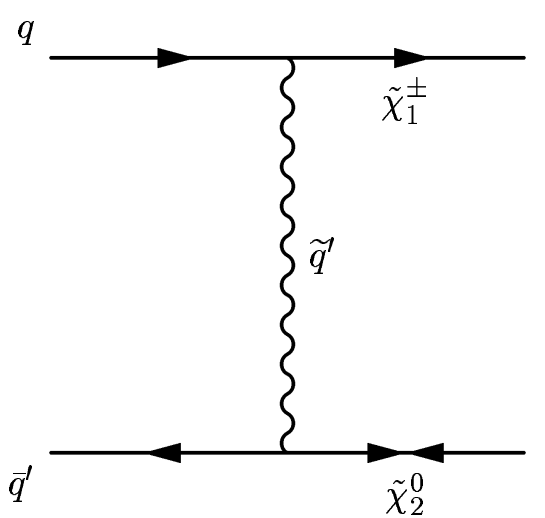

(b)

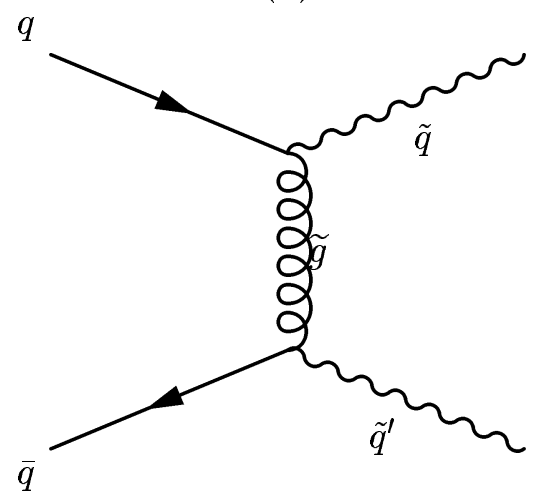

(d)

Figure 2.13: Pair- and associated gaugino production (a), (b). The gaugino cascade decays into the lightest neutralino $\tilde{\chi}_{1}^{0}$, see App. A.2 and A.3 for reference. Pair- and associated squark and gluino production via quark $q$, squark $\tilde{q}$, gluon $g$ and gluino $\tilde{g}$ exchange in the $s, t$ and $u$-channel contribute to this analysis at low values of $m_{0}(\mathrm{c}),(\mathrm{d})$.

d), is possible and contributes, because the squarks $\tilde{q}$ and gluinos $\tilde{g}$ decay predominantly R-parity conserving into a gaugino and an associated parton, $q$ or $g$.

The challenges of this analysis are the very small neutralino masses at low $m_{1 / 2}$, thus low signal selection efficiency, as the muons originate in most cases from the two neutralino decays. On the other hand $\mathrm{m}\left(\tilde{\chi_{1}^{0}}\right)$ will rise with $m_{1 / 2}$, but the gaugino pair and associated production cross section declines exponentially. The dependence of the neutralino mass and the cross section on $m_{1 / 2}$ are shown in Fig. 2.14. 


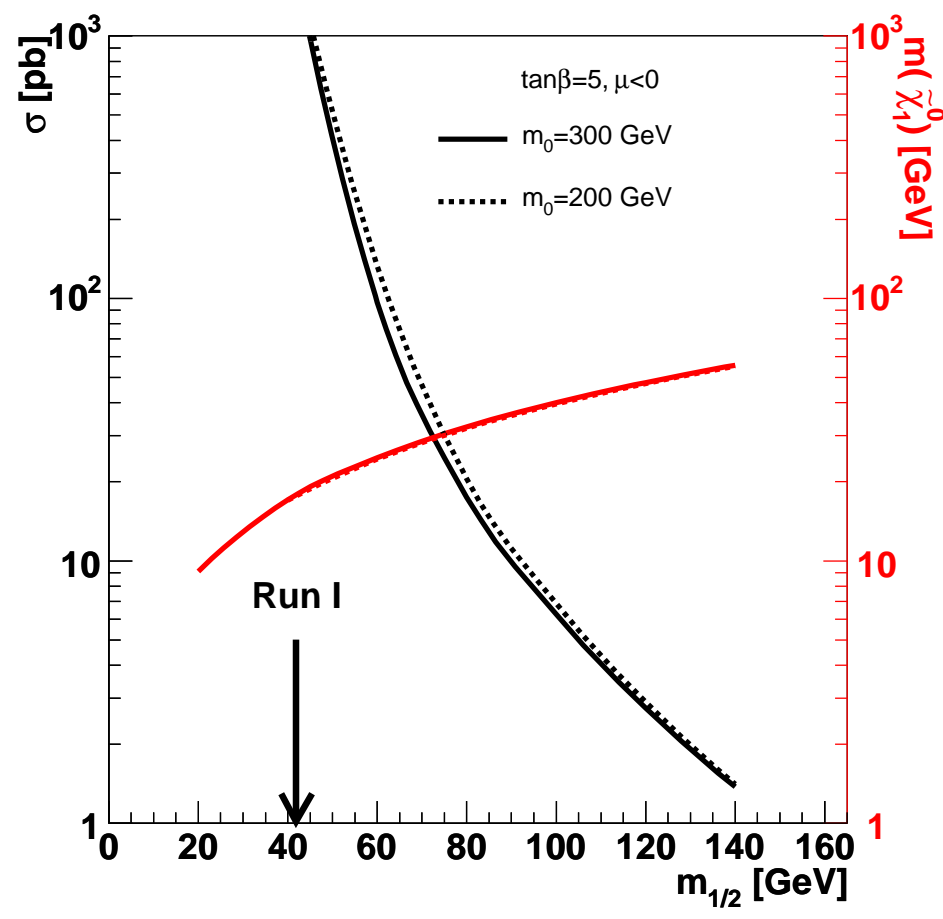

Figure 2.14: Cross section $\sigma(p \bar{p} \rightarrow \tilde{\chi} \tilde{\chi})$ and neutralino mass $\mathrm{m}\left(\tilde{\chi_{1}^{0}}\right)$ dependence on $m_{1 / 2}$ for two values of $m_{0}$. The $m_{1 / 2}$ dependence is directly opposite, while the $m_{0}$ dependence is negligible. The DØ Run I limit on $m_{1 / 2}$ for $\tan \beta=6$ is shown for reference. (NB: The intersection point is meaningless and does not represent a limit.) 


\subsection{Existing bounds on R-Parity violating SUSY}

A supersymmetric model with R-parity conservation leads to the absolute stability of the lightest supersymmetric particle. This has implications on astroparticle physics, because a neutral, uncolored LSP is a candidate for cold dark matter. Roughly $23 \%$ of the universe energy density is thought to be composed of cold dark matter. Electrical charged or colored stable LSPs would form heavy isotopes of hydrogen or bind into new heavy hadrons such as $(\tilde{t} u d)^{+}$or $(\tilde{t} d d)^{0}$. The limit on the relic number density $n_{X}$ compared to the baryon density in the universe $n_{B}$ is $n_{X} / n_{B} \leq 10^{-6} \cdot \frac{m_{x}}{\mathrm{GeV}}$ for electrical charged and uncolored particles and $n_{X} / n_{B} \leq 10^{-10}$ independent of mass $m_{X}$, for colored sparticles, such as squarks or gluinos $[52,53]$.

If in a supersymmetry model the R-parity is broken, then either the lifetime of the LSP is sufficiently large, at least of the order of the age of the universe, and the LSP remains to be a candidate for cold dark matter. This constraint leads to an upper bound of all trilinear $\not R_{p}$ couplings of $\mathcal{O}\left(10^{-20}\right)$ [42]. Or the LSP is not responsible for the dark matter and its lifetime is only a fraction of the age of the universe. This provides for a lower limit on all $\lambda_{i j k}, \lambda_{i j k}^{\prime}, \lambda_{i j k}^{\prime \prime}$ couplings of the order of $\mathcal{O}\left(10^{-12}\right)$ [42] to guarantee a sufficiently quick decay of the LSPs created in the Big Bang, because they cannot be observed today.

In summary, either the cold dark matter of the universe consists of uncharged stable supersymmetric particles, which could only be the lightest neutralino $\tilde{\chi}_{1}^{0}$, leading to extremely small values of the $\not R_{p}$ couplings and preventing the LSP decay to be observable in laboratories, or supersymmetric particles are not responsible for the dark matter and $R_{p}$ interactions could be observed at accelerators.

\subsubsection{Indirect bounds}

Up to now no evidence for a non-zero value of any R-parity violating coupling has been found. However, many bounds $[42,54]$ on $\not R_{p}$ couplings exist. In this sections the present limits on the $L Q \bar{d}$ couplings $\lambda_{2 j k}^{\prime}$ shall be reviewed.

The $\not R_{p}$ coupling constants are already constrained by several low-energy experiments. The most stringent limit, namely

$$
\lambda_{21 k}^{\prime}<0.059 \cdot \frac{\mathrm{m}\left(\tilde{d}_{k R}\right)}{100 \mathrm{GeV}}
$$

with $95 \%$ CL $[55,56,57]$ is from the lepton universality in the pion $\pi^{ \pm}$decay, Fig. 2.15. But since the down squark $\tilde{d}_{R}$ of the first family $(k=1)$ accessible at Tevatron can exceed $1 \mathrm{TeV}$ in mass, see Fig. 2.7, this limit on $\lambda_{211}^{\prime}$ is actually quite weak and can be significantly improved by the analysis presented here.

More bounds on the coupling $\lambda_{211}^{\prime}$ can be derived from neutral current interactions in fixed target experiments using $\nu_{\mu}$ and $\bar{\nu}_{\mu}$ beams and studying the neutrino interactions with 


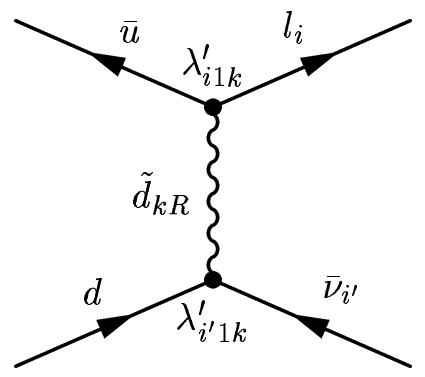

(a)

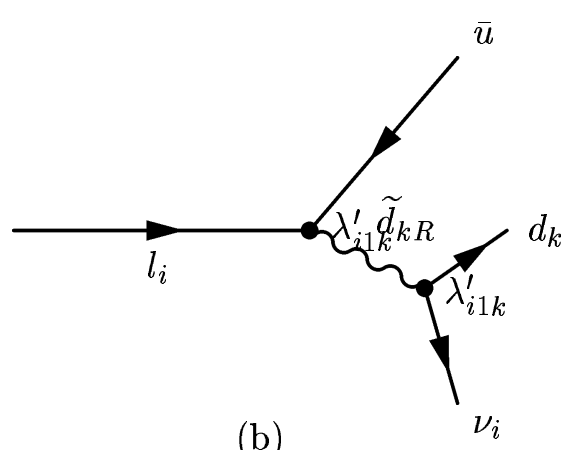

(b)

Figure 2.15: Pion decay, i.e. $\pi^{-} \rightarrow \mu^{-} \nu_{\mu}$ for $i=i^{\prime}=2$ and $k=1$ (a) and similarly the $\tau \rightarrow \pi \nu_{\tau}$ decay (b) through the $\not_{p}$ coupling $\lambda_{3 j k}^{\prime}$.

hadrons. Neutrino - Hadron scattering interactions are shown in Fig. 2.16. The resulting limits on the R-parity violating $L Q \bar{d}$ coupling constant $\lambda^{\prime}$ are [58]:

$$
\lambda_{21 k}^{\prime}<0.15 \frac{m\left(\tilde{d}_{k R}\right)}{100 \mathrm{GeV}}, \quad \lambda_{2 j 1}^{\prime}<0.18 \frac{m\left(\tilde{d}_{j L}\right)}{100 \mathrm{GeV}}
$$

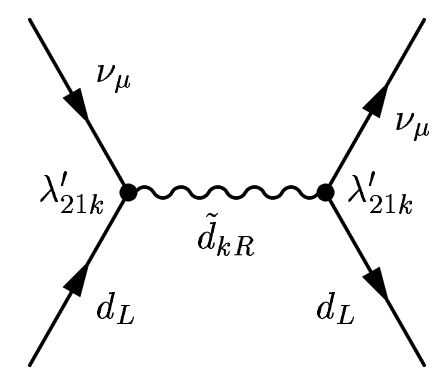

(a)

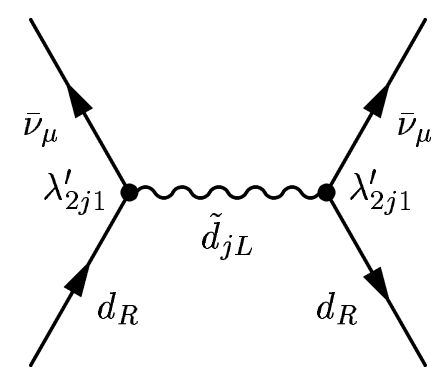

(b)

Figure 2.16: Neutrino scattering involving the R-parity violating $L Q \bar{d}$ coupling $\lambda_{2 j k}^{\prime}$. Note, that the third index references the right-handed singlet superfield.

Analysis of hadron decays up to the one-loop level can set very stringent limits on coupling constant products for all generation indices. The proton decay, Fig. 2.17, is suppressed by only $m(\tilde{q})^{-2}$, but the observed proton half-life is larger than $10^{35}$ years [17]. If the $L$ and $B$ violating couplings exist, then they must be small.

$$
\left|\lambda_{i j k}^{\prime} \lambda_{i^{\prime} j^{\prime} k^{\prime}}^{\prime \prime}\right|<\mathcal{O}\left(10^{-9}\right)
$$

And the specific bounds from the proton decay with respect to the coupling $\lambda_{211}^{\prime}$ are:

$$
\left|\lambda_{211}^{\prime} \lambda_{111}^{\prime \prime}\right|<\left(10^{-25}-10^{-27}\right) \cdot \frac{\mathrm{m}\left(\tilde{d}_{R}^{2}\right)}{\mathrm{GeV}} \quad \text { for } \quad\left[p \rightarrow \pi^{0} \mu^{+}, p \rightarrow \pi^{+} \bar{\nu}_{\mu}\right]
$$

Where $\tilde{d}_{R}$ is the right-handed down squark.

Low energy experiments like searches for single nucleon decay, the neutrinoless double beta decay, double nucleon decays, $K$ and $B$ meson mixing and rare decays and lepton number 


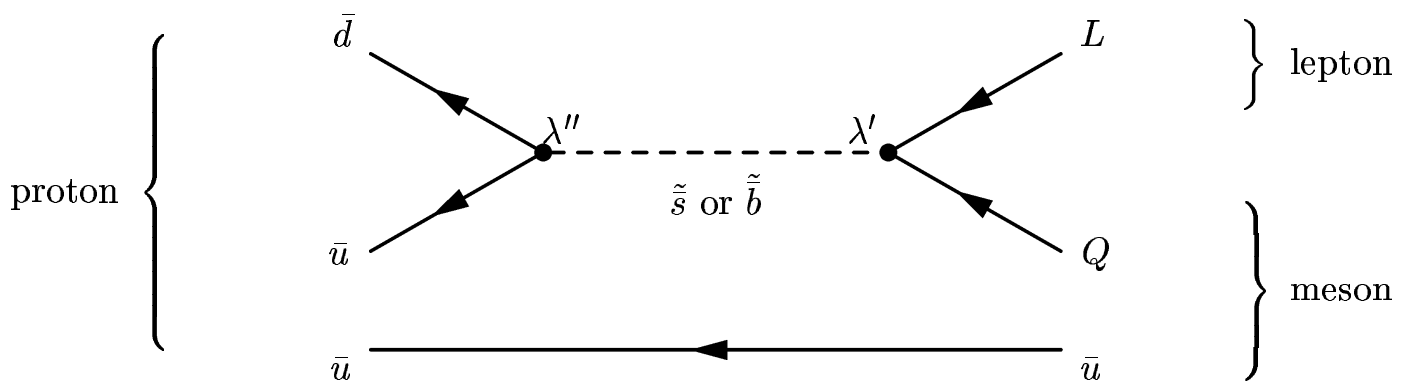

Figure 2.17: The proton decay is possible if the lepton and baryon numbers are not conserved. The squarks $\tilde{s}$ and $\tilde{b}$ carry $\mathrm{SU}(3)$ color and $B=1, L=0$, so that the baryon number is violated at the left vertex and the lepton number at the right vertex.

violating decays of leptons provide valuable informations about the R-parity violating coupling strengths. A summary of constraints on the product of two couplings of the form $\left|\lambda_{i j k}^{\prime} \cdot \lambda_{i j k}^{x}\right|$ is given in Tab. 2.6. These constraints can become very stringent, if at least one $\not_{p}$ coupling is measured to be non-zero. This supports the previous assumption, that only one coupling can be of significant size, compared to all other $R_{p}$ coupling constants.

\subsubsection{Searches at $e^{+} e^{-}$Colliders}

The four experiments; Aleph, Delphi, L3 and Opal (ADLO) at the $e^{+} e^{-}$collider LEP, operating at center-of-mass energies up to $\sqrt{s}=209 \mathrm{GeV}$, have searched primarily for Rparity conserving but also for R-parity violating supersymmetry. In particular combined LEP limits on the nine $R_{p} L L \bar{e}$ couplings $\lambda_{i j k}$ have been set [66]. For the $27 \mathrm{R}$-parity violating $L Q \bar{d}$ couplings $\lambda_{i j k}^{\prime}$ no combined LEP limits exist. However, the four experiments have searched for direct or indirect decays via $\not_{p}$ couplings of associated or pair produced sparticles, and have set limits on the involved sparticle masses and interpreted these within constrained MSSM models. These new limits extend significantly beyond previous LEP1 limits [67] from precision measurements of the $Z$-boson mass width $\Gamma_{Z}$, which is sensitive to additional decay modes, as shown in Fig. 2.18. The typical cross section limits involving $L Q \bar{d}$-couplings are of the order of $0.5 \mathrm{pb}$, leading to typical mass limits at the kinematical limit at $104 \mathrm{GeV}$. The mass limits are independent of the coupling strength $\lambda_{i j k}^{\prime}$, since no resonant $L Q \bar{d}$-channel exists at lepton-lepton colliders and the associated/pair production cross section is R-parity conserving and does not depend on $\lambda_{i j k}^{\prime}$. The coupling controls the decay width and therefore the lifetime of the sparticles. The coupling strength is assumed to be large enough, $\lambda_{i j k}^{\prime} \gtrsim 10^{-2}$, so that lifetime effects in the detector can be neglected for all searches.

The direct decays of sparticles, as shown in Fig. 2.18(a), lead in case of a non-zero $\lambda_{i j k}^{\prime}$ coupling to event topologies with two leptons and two quarks or with four quarks in the final state. In indirect decays, Fig. 2.18(b), where the sfermions decay first R-parity conserving into a fermion and a gaugino, and the gaugino cascades into the lightest neutralino, which is assumed to be the LSP, the final state consists of two leptons, four quarks and two additional fermions. Depending on the $\lambda_{i j k}^{\prime}$ under study all lepton and quark flavors can occur. 


\begin{tabular}{|c|c|c|c|}
\hline coupling & upper limit $90 \% \mathrm{CL}$ & process & diagram \\
\hline$\left|\lambda_{i 11}^{\prime} \lambda_{i 31}\right|$ & $\begin{array}{l}1.0 \cdot 10^{-2} \frac{m\left(\tilde{\nu}_{i L}\right)^{2}}{(100 \mathrm{GeV})^{2}} \\
\text { Ref. }[17,59]\end{array}$ & {$[\tau \rightarrow e \pi]$} & $q_{1}$ \\
\hline $\begin{array}{l}\left|\lambda_{i 1 k}^{\prime} \lambda_{i^{\prime} 2 k}^{\prime}\right| \\
\left|\lambda_{i j 1}^{\prime} \lambda_{i^{\prime} j 2}^{\prime}\right|\end{array}$ & $\begin{array}{l}1.9 \cdot 10^{-5} \frac{m\left(\tilde{d}_{k R}\right)^{2}}{(100 \mathrm{GeV})^{2}} \\
1.9 \cdot 10^{-5} \frac{m\left(\tilde{d}_{k R}\right)^{2}}{(100 \mathrm{GeV})^{2}} \\
\text { Ref. }[42,60]\end{array}$ & $\begin{array}{l}{[K \rightarrow \pi \nu \bar{\nu}]} \\
{[K \rightarrow \pi \nu \bar{\nu}]}\end{array}$ & $\nu_{\bar{d}}^{\nu}$ \\
\hline$\left|\lambda_{i j k}^{\prime} \lambda_{l 3 k}^{\prime}\right|$ & $\begin{array}{l}1.1 \cdot 10^{-3} \frac{m\left(d_{k R}\right)^{2}}{(100 \mathrm{GeV})^{2}} \\
\text { Ref. [61] }\end{array}$ & {$[B \rightarrow X \nu \bar{\nu}]$} & analogue to $K \rightarrow \pi \nu \bar{\nu}$ \\
\hline$\left|\lambda_{2 m k}^{\prime} \lambda_{1 m k}^{\prime}\right|$ & $\begin{array}{l}\mathcal{O}\left(5 \cdot 10^{-4}\right) \\
\text { Ref. }[62]\end{array}$ & {$[\mu \rightarrow e \gamma]$} & \\
\hline$\left|\lambda_{2 j 1}^{\prime} \lambda_{2 j 2}^{\prime}\right|$ & $\begin{array}{l}5.8 \cdot 10^{-6} \\
\text { Ref. }[63]\end{array}$ & {$\left[K_{L} \rightarrow \mu^{+} \mu^{-}\right]$} & \\
\hline $\begin{array}{l}\left|\lambda_{2 j 1}^{\prime} \lambda_{1 j 1}^{\prime}\right| \\
\left|\lambda_{21 k}^{\prime} \lambda_{11 k}^{\prime}\right|\end{array}$ & $\begin{array}{l}6.0 \cdot 10^{-8} \frac{m\left(\tilde{u}_{j L}\right)^{2}}{(100 \mathrm{GeV})^{2}} \\
1.2 \cdot 10^{-8} \frac{m\left(\tilde{d}_{k R}\right)^{2}}{(100 \mathrm{GeV})^{2}} \\
\text { Ref. }[59,64]\end{array}$ & $\begin{array}{l}{[\mu \rightarrow e(T i)]} \\
{[\mu \rightarrow e(T i)]}\end{array}$ & $\begin{array}{l}{ }^{48} \mathrm{Ti} \text { fixed target experiment, } \\
e \rightarrow \mu \text { conversion in a nucleus, } \\
t, s \text { and } u \text { channel contribution. }\end{array}$ \\
\hline $\begin{array}{l}\left|\lambda_{i j k}^{\prime} \lambda_{11 k}^{\prime \prime}\right| \\
\left|\lambda_{i j 1}^{\prime} \lambda_{1 j 1}^{\prime \prime}\right| \\
\left|\lambda_{i j k}^{\prime} \lambda_{i j k}^{\prime \prime}\right|\end{array}$ & $\begin{array}{l}10^{-25} \frac{m\left(d_{k R}\right)^{2}}{(100 \mathrm{GeV})^{2}} \\
10^{-25} \frac{m\left(\tilde{d}_{j L}\right)^{2}}{(100 \mathrm{GeV})^{2}} \\
10^{-9} \quad \text { Ref. [65] }\end{array}$ & $\begin{array}{l}{\left[p \rightarrow l^{+} X\right]} \\
{\left[p \rightarrow \nu \pi^{+}\right]} \\
{\left[p \rightarrow l^{ \pm} X\right]}\end{array}$ & see Fig. 2.17 \\
\hline
\end{tabular}

Table 2.6: Limits from low-energy experiments on the product of two $\not R_{p}$-couplings, including $\lambda_{211}^{\prime}$. The notation $\tilde{l}_{i R}$ refers to a right-handed slepton of the $i^{t h}$ generation, similarly for quarks $q$. If no chirality index is given, then both are possible. For detailed information see the references given in this table or the complete review of all $\not R_{p}$-constraints as given in [42]. 


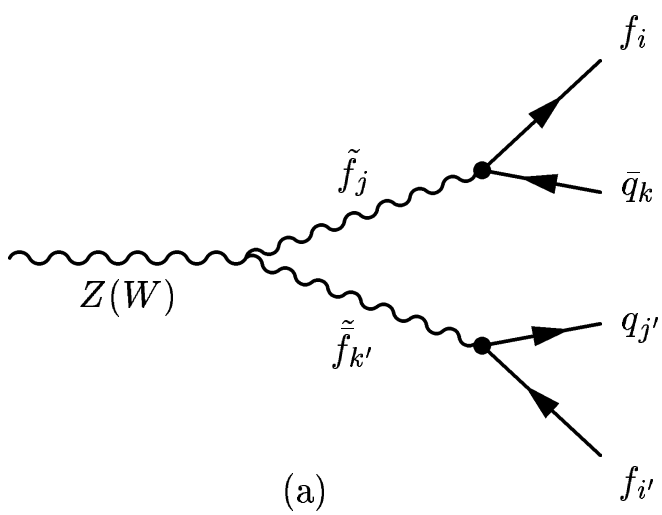

(a)

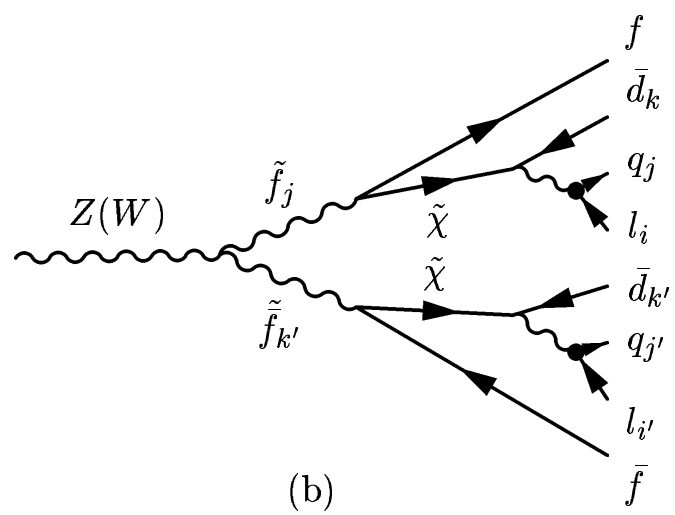

(b)

Figure 2.18: Direct decay, e.g. $e^{+} e^{-} \rightarrow Z^{0} \rightarrow \tilde{u} \tilde{u} \rightarrow \mu \bar{d}_{\mu} u$ (a) and indirect decay e.g. $e^{+} e^{-} \rightarrow W^{+} \rightarrow \tilde{\nu}_{\mu} \tilde{\mu} \rightarrow \bar{\nu}_{\mu} \tilde{\chi} \mu \tilde{\chi}$ (b) via $L Q \bar{d}$ couplings $\lambda_{i j k}^{\prime}$ at LEP. The $f_{i}$ indicate fermions, either quarks $q_{i}$ or leptons $l_{i}$ of $i^{t h}$ generation and the dot indicates the vertex with R-parity violation. The chirality indices are neglected for simplicity.

In the following a short summary of the ADLO publications with respect to limits on supersymmetry in case of non-vanishing $L Q \bar{d}$-couplings shall be given. Additional information can be found in $[42,66,68]$.

The Aleph collaboration has obtained sfermion mass limits in searches for direct decays of sparticles and mass limits on sfermions as well as gauginos in searches for indirect decays, involving non-zero $L Q \bar{d}$-couplings $[69,70]$. Aleph has set the most stringent LEP limits for any non-zero $\lambda_{i j k}^{\prime}$ coupling $[42,68]$. The exclusion countour in the $\mathrm{m}_{\tilde{l}} \mathrm{~m}_{\chi}$ plane, shown in Fig. 2.19, is covering a great part of the kinematically accessible area at LEP $\left(\mathrm{m}_{\tilde{\chi}}<\mathrm{m}_{\tilde{l}}<\frac{1}{2} \sqrt{s}\right)$. In Fig. 2.20 the Aleph limits for indirect decays via $\lambda_{211}^{\prime}$ and different mixing angles in the third family squark sector are shown.

Delphi has searched for $\not R_{p}$ non-zero $L L \bar{e}$ couplings [71] and non-zero $\bar{u} \bar{u} \bar{d}$ couplings [72], but no $L Q \bar{d}$-analysis result is published. Limits on the chargino mass were derived from searches for R-parity violation in the third lepton family [73].

The L3 experiment has published limits derived from $\tilde{\nu}$-exchange contributing to $e^{+} e^{-} \rightarrow$ $Z^{0} \rightarrow l^{+} l^{-}$via $L L \bar{e}$-couplings [74] and has analyzed chargino and neutralino decays via $L L \bar{e}$ and $\bar{u} \bar{u} \bar{d}$-couplings [75, 76]. Limits on gaugino and right handed slepton masses, as shown in Fig. 2.21, from direct decays via the $L Q \bar{d}$-couplings $\lambda_{i j k}^{\prime}$, arise from searches in events with at least four jets in the final state [77].

Opal has searched for and set cross section limits on the pair production of charged sleptons and sneutrinos [78]. These limits were interpreted as absolute mass limits on the sleptons. In case of R-parity violation through $L Q \bar{d}$-couplings limits on the left- and right-handed smuon, see Fig. 2.22, and on the left-handed sneutrino were derived.

Fig. 2.23 shows the LEP1 and LEP2 exclusion countours in the $\mu-M_{2}$ plane in case of $\not R_{p}$-SUSY through $L Q \bar{d}$, from Aleph and L3.

The LEP limits on sparticles masses in case of R-parity violating supersymmetry through any $L Q \bar{d}$-coupling $\lambda_{i j k}^{\prime} \neq 0$ are summarized in Tab. 2.7. 

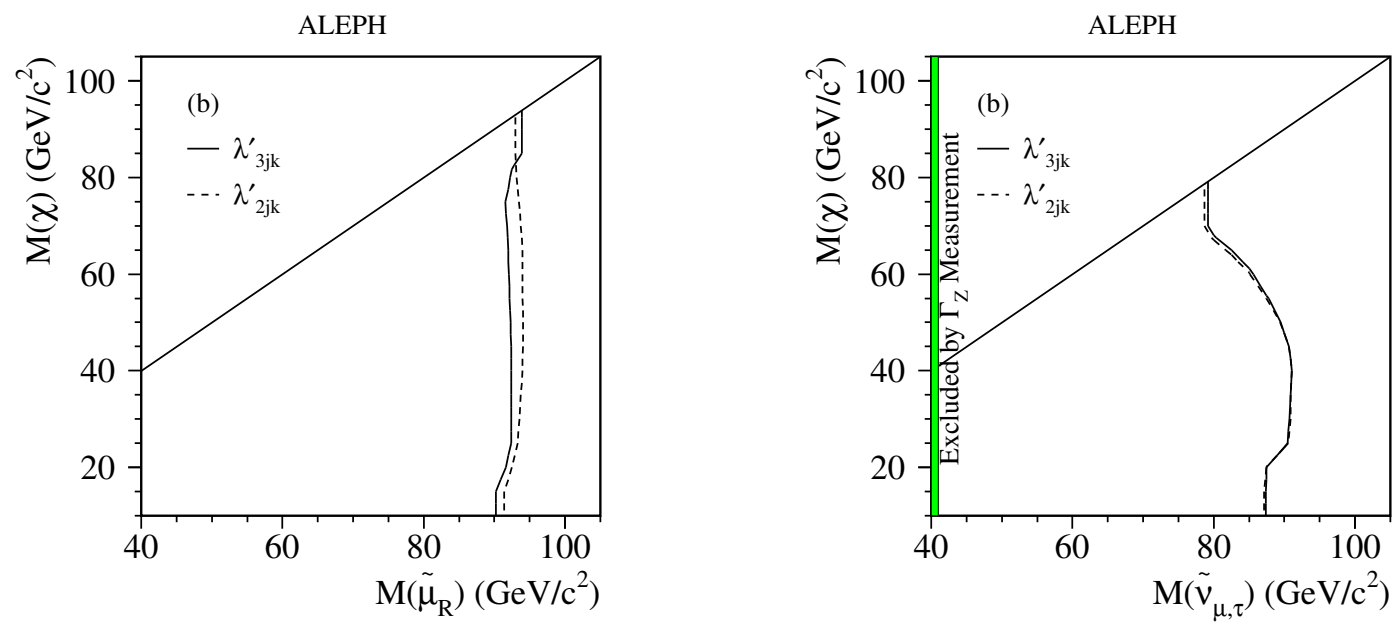

Figure 2.19: The Aleph $95 \%$ CL limits in the $\mathrm{m}_{\tilde{\mu}_{R}}-\mathrm{m}_{\chi}$ plane (left) and in the $\mathrm{m}_{\tilde{\nu}}-\mathrm{m}_{\chi}$ plane (right) for smuon or sneutrino pairs decaying indirectly via a dominant $L Q \bar{d}$-coupling [69].
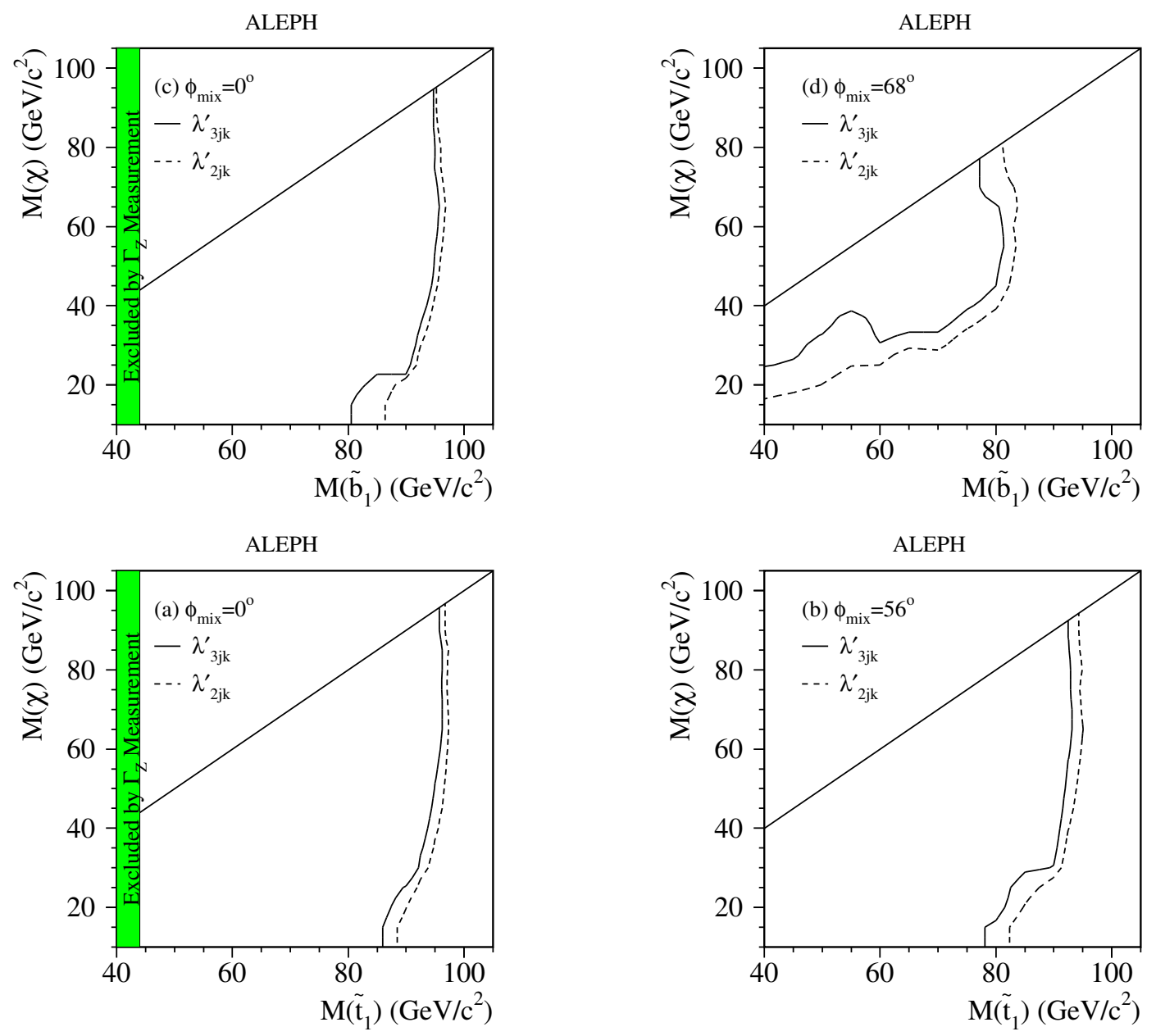

Figure 2.20: The Aleph 95\% CL limits in (a),(b) the $\mathrm{m}_{\tilde{\chi}}-\mathrm{m}_{\tilde{b}_{1}}$ plane and (c),(d) the $\mathrm{m}_{\tilde{\chi}}-\mathrm{m}_{\tilde{t}_{1}}$ plane for indirect decays via $\lambda_{211}^{\prime}$ or $\lambda_{311}^{\prime}$ for no mixing $\left(\Phi_{m i x}=0^{\circ}\right)$ and for $\Phi_{m i x}=68^{\circ}$ and $56^{\circ}$ for sbottoms and stops, respectively [69]. 

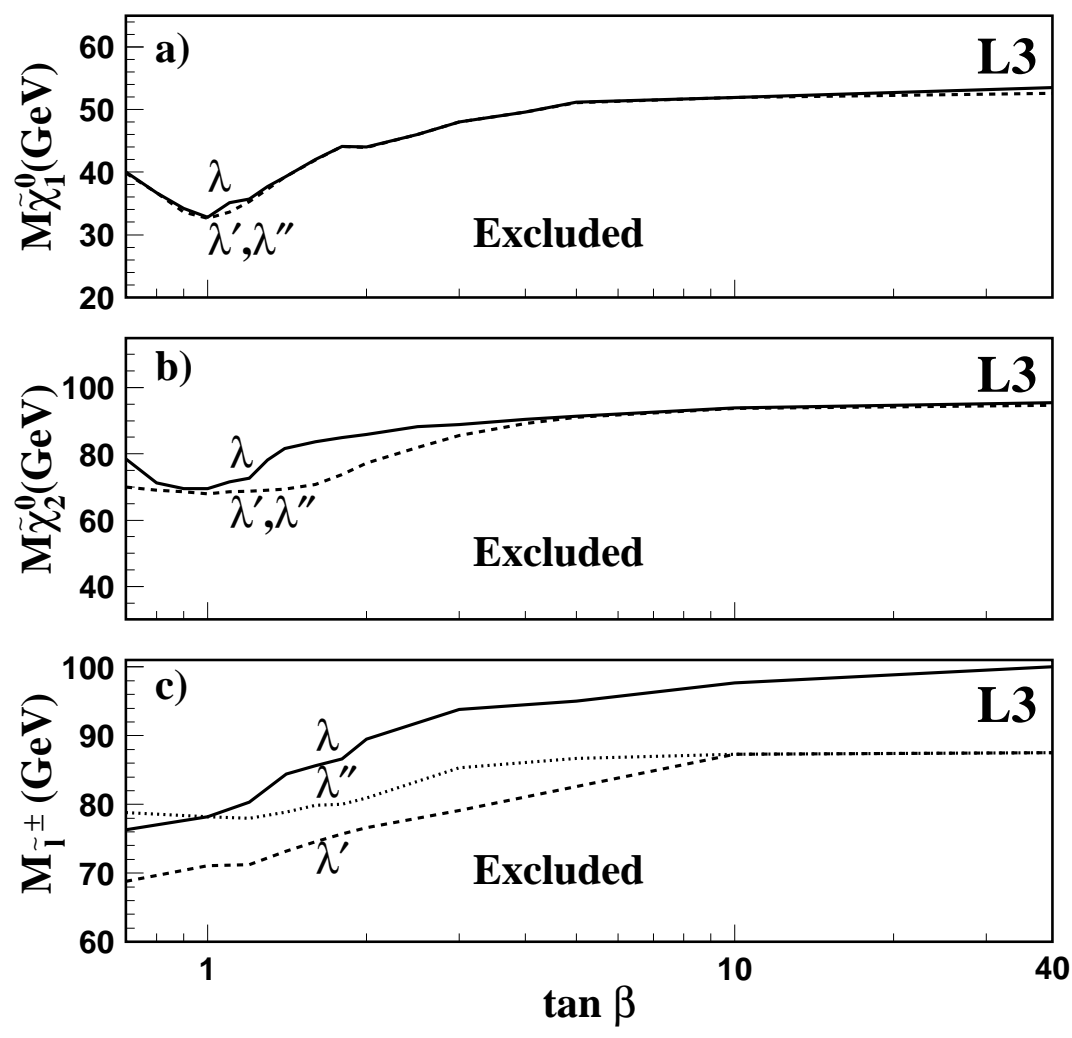

Figure 2.21: L3 95\% CL lower mass limits on the neutralino 1 (a), the neutralino 2 (b) and the right-handed slepton (c) as a function of $\tan \beta$ for $0 \leq M_{2} \leq 1000 \mathrm{GeV}$ and $-500 \mathrm{GeV} \leq \mu \leq$ $500 \mathrm{GeV}$. The $m_{0}$ parameter was chosen such, that the most conservative limit on the masses was found; $m_{0}=500 \mathrm{GeV}$ in (a) and (b) and $m_{0}=0 \mathrm{GeV}$ in (c) [77].

OPAL

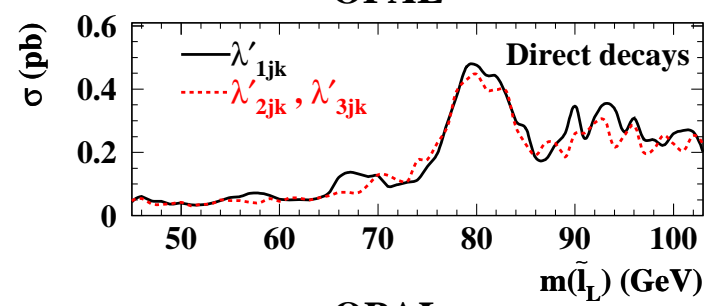

OPAL

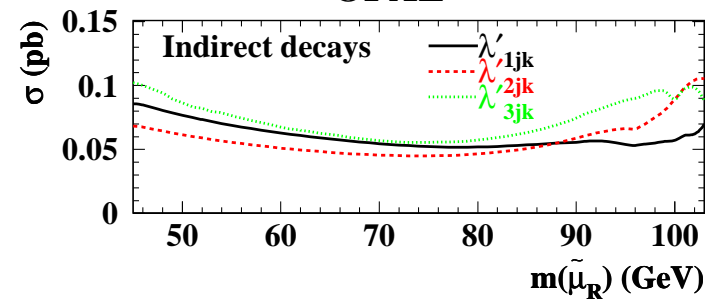

OPAL

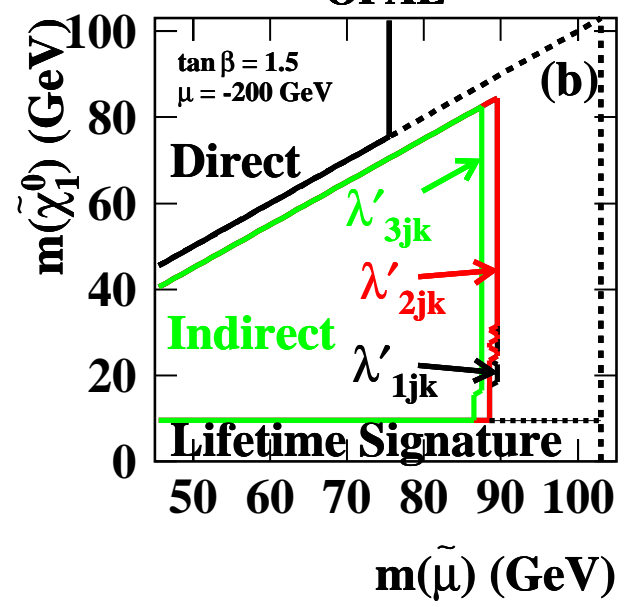

Figure 2.22: Opal 95\% CL cross section limits on direct (left, top) and indirect (left, bottom) decays of charged slepton pairs and the interpretation in the $\mathrm{m}_{\tilde{l}}-\mathrm{m}_{\tilde{\chi}}$ plane (right) [78]. 


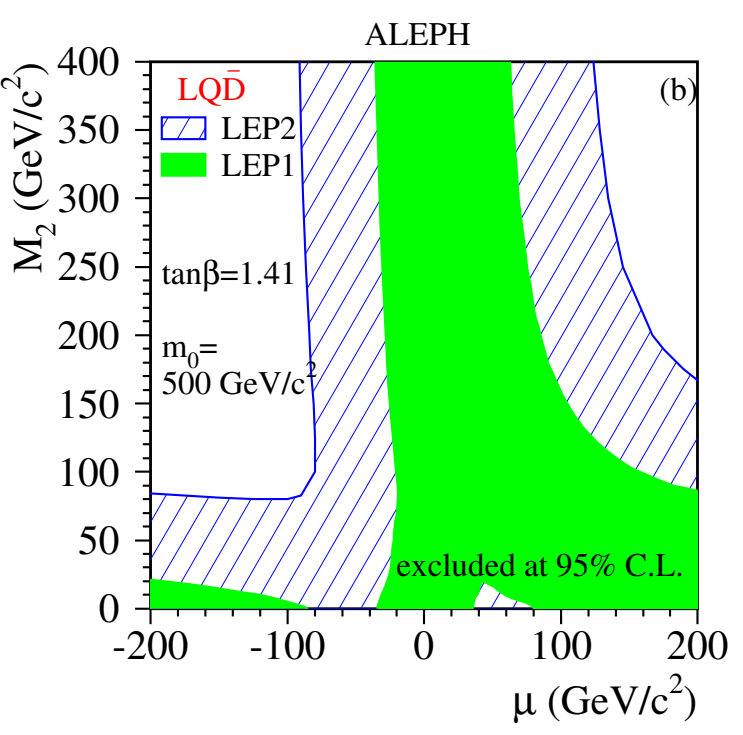

(a)

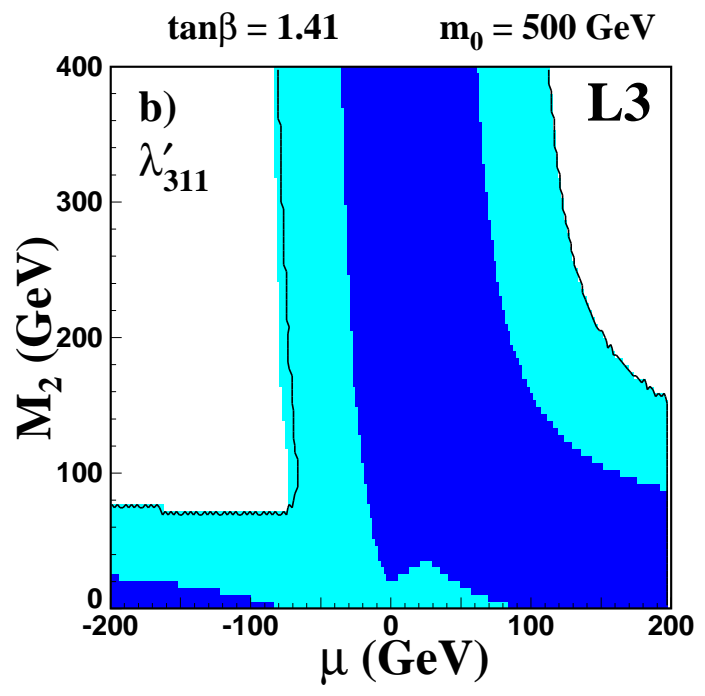

(b)

Figure 2.23: Regions in the $\mu-M_{2}$ plane excluded at $95 \% \mathrm{CL}$ at $\tan \beta=\sqrt{2}$ and $m_{0}=500 \mathrm{GeV}$ for any non-zero $L Q \bar{d}$-coupling at Aleph [69] (a) and for $\lambda_{311}^{\prime} \neq 0$ at L3 [77] (b). The darker regions were excluded by LEP1 precision measurements of $\Gamma_{Z}$. Lower limits on the chargino mass can be derived by scanning the value of $\tan \beta$.

\begin{tabular}{l|cccc|c}
\hline \hline sparticle & Aleph & Delphi & L3 & Opal & best LEP limit \\
\hline$\tilde{\mu}_{L}$ & $81 \mathrm{GeV}$ & - & - & $75 \mathrm{GeV}$ & $81 \mathrm{GeV}$ \\
$\tilde{\nu}_{\mu L}$ & $79 \mathrm{GeV}$ & - & - & $74 \mathrm{GeV}$ & $79 \mathrm{GeV}$ \\
$\tilde{\mu}_{R}$ & $90 \mathrm{GeV}$ & - & - & $87 \mathrm{GeV}$ & $90 \mathrm{GeV}$ \\
$\tilde{\nu}_{\mu R}$ & $78 \mathrm{GeV}$ & - & $77.5 \mathrm{GeV}$ & - & $78 \mathrm{GeV}$ \\
$\tilde{\chi}_{1}^{0}$ & $39 \mathrm{GeV}$ & - & $32.5 \mathrm{GeV}$ & - & $39 \mathrm{GeV}$ \\
$\tilde{\chi}_{1}^{ \pm}$ & $103 \mathrm{GeV}$ & - & $93.8 \mathrm{GeV}$ & - & $103 \mathrm{GeV}$ \\
\hline \hline
\end{tabular}

Table 2.7: Summary of LEP limits for non-zero $L Q \bar{d}$-couplings $\lambda_{i j k}^{\prime}$. The Aleph limits are the dominating constraints.

\subsubsection{Searches at Lepton - Hadron Colliders}

At the $e^{ \pm} p$-collider HERA supersymmetric particles are produced via the R-parity violating $L Q \bar{d}$ couplings $\lambda_{1 j 1}^{\prime}$ or $\lambda_{11 k}^{\prime}$ because the initial state particles. Pair production of sparticles is very difficult. The channels studied by the $\mathrm{H} 1$ and the Zeus experiment are resonant squark production and $t$-channel gaugino production. In both channels limits on the corresponding sparticles and $L Q \bar{d}$ coupling strengths have been obtained. However, no HERA experiment has set limits on any $\lambda_{2 j k}^{\prime}$ coupling.

H1 and Zeus have searched for resonant squark production $[79,80]$ in the framework of R-parity violating supersymmetry and have published limits on the couplings $\lambda_{1 j 1}^{\prime}$ and $\lambda_{11 k}^{\prime}$ (Zeus only for $j=k=3$ ). The data taken at up to $\sqrt{s}=319 \mathrm{GeV}$ correspond to 


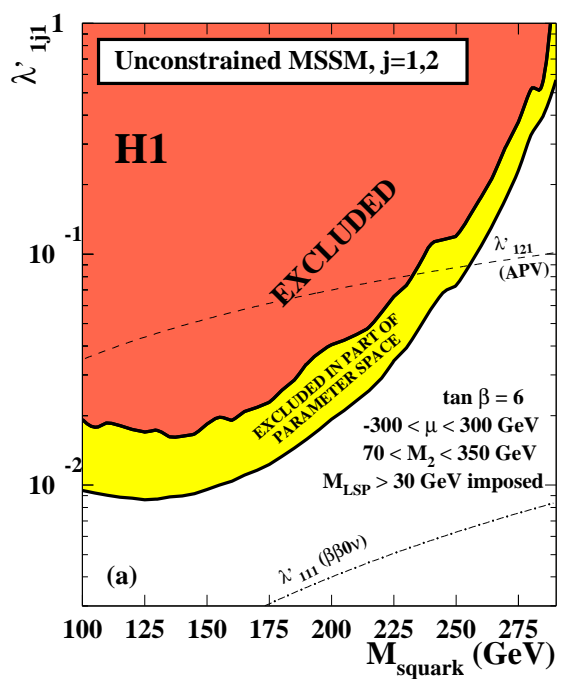

(a)

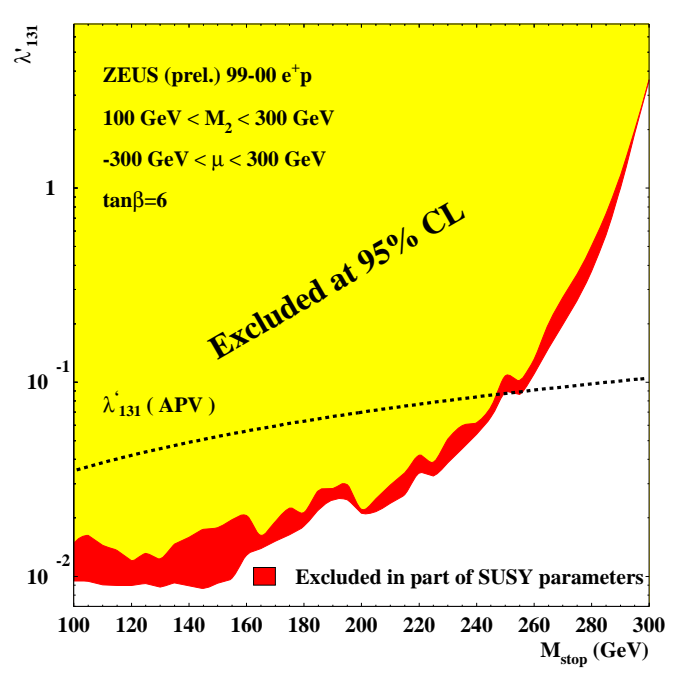

(b)

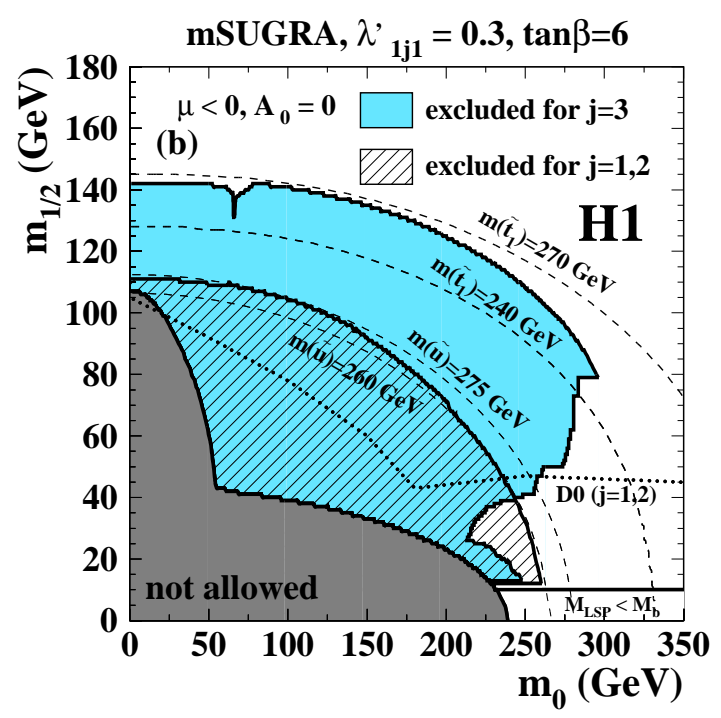

(c)

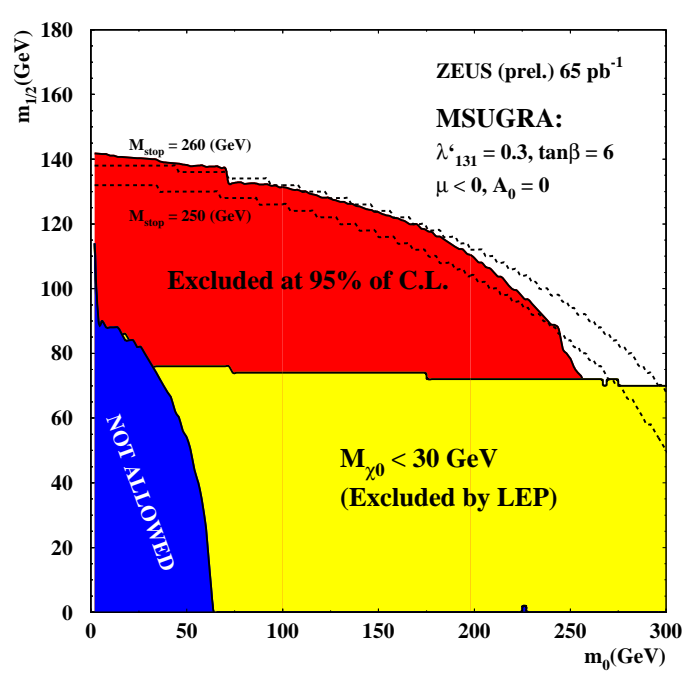

(d)

Figure 2.24: (a) H1 95\% CL exclusion contours on $\lambda_{1 j 1}^{\prime}$ for $j=1,2$ [79] and (b) Zeus limit for $j=3$ as a function of the squark mass [80]. Low-energy constraints from atomic parity violation (APV) experiments are shown as dashed lines. (c) H1 95\% CL exclusion contour in mSUGRA with $\lambda_{1 j 1}^{\prime}=0.3$ and $\tan \beta=6$ [79]. The limit from $\mathrm{D} \varnothing$ Run I on this coupling is shown as dotted line $[81,82]$. (d) Equivalent exclusion contour in the $m_{0}-m_{1 / 2}$ plane from Zeus [80]. 
an integrated luminosity of $106 \mathrm{pb}^{-1}$ (H1) and $65 \mathrm{pb}^{-1}$ (Zeus). The resonantly produced squarks can be of any flavor and will decay either directly $\tilde{u}_{j L} \rightarrow e^{+} d_{R}$, Fig. 2.25(a) or indirectly into a gauge boson and squark or into a gaugino and a quark, Fig. 2.25(b).

No evidence for squark production was found in the investigated multi-lepton and multijet final state topologies. Squark mass dependent limits on the $\lambda_{1 j 1}^{\prime}$ and $\lambda_{11 k}^{\prime}$ couplings were obtained in the framework of the MSSM. The results were interpreted as constraints on the parameters of the mSUGRA model, Fig. 2.24.

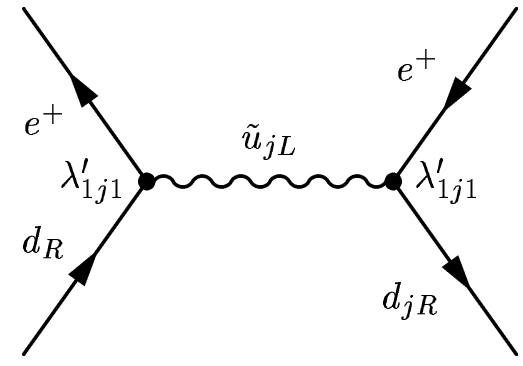

(a)

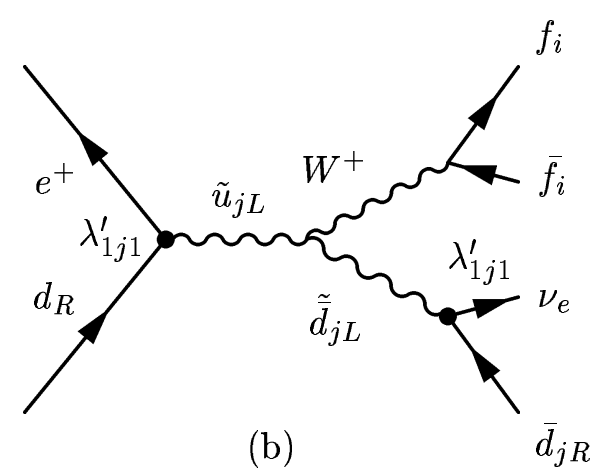

Figure 2.25: Resonant squark production at HERA via the $L Q \bar{d}$-couplings $\lambda_{1 j 1}^{\prime}$ or $\lambda_{11 k}^{\prime}$. In the latter case all chirality indices in the above diagrams must be switched.

$\mathrm{H} 1$ and Zeus have also searched for $t$-channel gaugino production, Fig. 2.26(a), and set limits in the MSSM and GMSB (gauge mediated supersymmetry breaking model) parameter planes [83]. If the gravitino is assumed to be the LSP, then gauginos can decay into a gravitino and a photon, i.e. $\tilde{\chi}_{1}^{0} \rightarrow \tilde{G} \gamma$, Fig. 2.26(b). H1 and Zeus have set limits in the neutralino - selectron mass plane in dependence of the size of the coupling strength $\lambda_{1 j 1}^{\prime}$ or $\lambda_{11 k}^{\prime}[84]$.

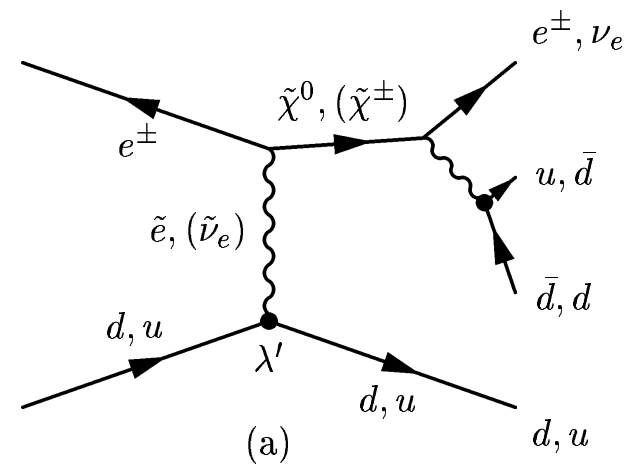

(a)

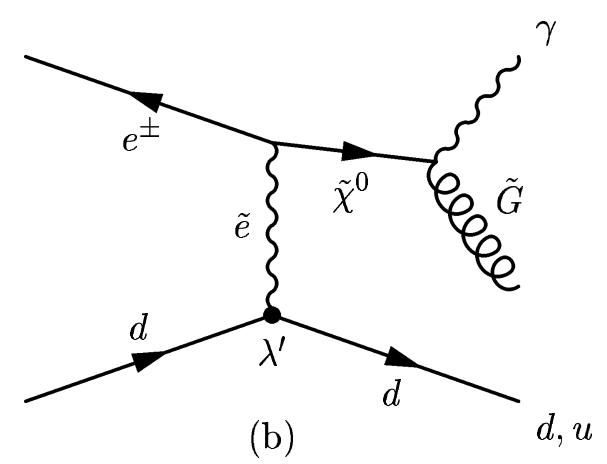

(b)

Figure 2.26: Gaugino production at HERA via the $L Q \bar{d}$-couplings $\lambda_{1 j 1}^{\prime}$ or $\lambda_{11 k}^{\prime}$. (a) Decay via the same $L Q \bar{d}$ coupling and (b) decay into a photon and a gravitino, which is assumed to be the LSP in this channel. 


\subsubsection{Searches at Hadron - Hadron Colliders}

Both experiments at the Tevatron $p \bar{p}$ collider, $\mathrm{D} \varnothing$ and CDF, have searched in Run I at center-of-mass energies of $\sqrt{s}=1.8 \mathrm{TeV}$ for $\not_{p}$ supersymmetry. The CDF experiment has searched for pair-production of squarks or gluinos and their decay via a non-zero $\lambda_{121^{-}}$ coupling in like-sign di-electron final states [85] and for pair-produced stops decaying via $\lambda_{333}^{\prime}$ into two third generation leptons and two third generation quarks [86]. No excess in the data was found and cross section limits were derived. The process diagrams are similar to those shown for the LEP searches, Fig. 2.18. CDF has updated the search for squark pair-production with $322 \mathrm{pb}^{-1}$ of Run II data and extracts from the obtained cross section limit a lower limit on the top squark mass of $\mathrm{m}(\tilde{t})>155 \mathrm{GeV}$ [87].

CDF has searched for high mass resonances decaying into oppositely charged electron/muon pairs in $344 \mathrm{pb}^{-} 1$ of Run II data [88]. The results are interpreted in terms of the R-Parity violating production and decay of the tau sneutrino $\tilde{\nu}_{\tau}$. Data is found to be consistent with the Standard Model predictions and limits on the tau sneutrino mass as a function of the product of both relevant RPV couplings $\lambda_{311}^{\prime}$ and $\lambda_{132}$ are obtained.

Beside searches for associated and pair production of sparticles, and their decay via $L L \bar{e}-$ couplings in multi-lepton final states, at D $\varnothing$ in the Run I of the Tevatron [90], and the Run II [91], the D $\varnothing$ collaboration has already searched for resonant slepton production in the Run I data $[89,92,93]$. Di-muon and di-jet final states have been analyzed within the framework of mSUGRA. About $94 \mathrm{pb}^{-1}$ integrated luminosity were utilized and exclusion contours within mSUGRA parameter space have been derived, i.e. for $\lambda_{211}^{\prime}=0.09$ as shown in Fig. 2.27.

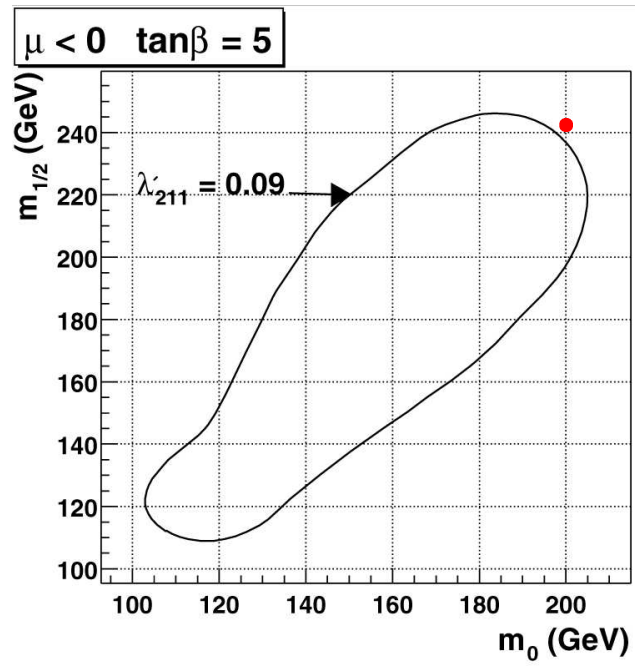

(a)

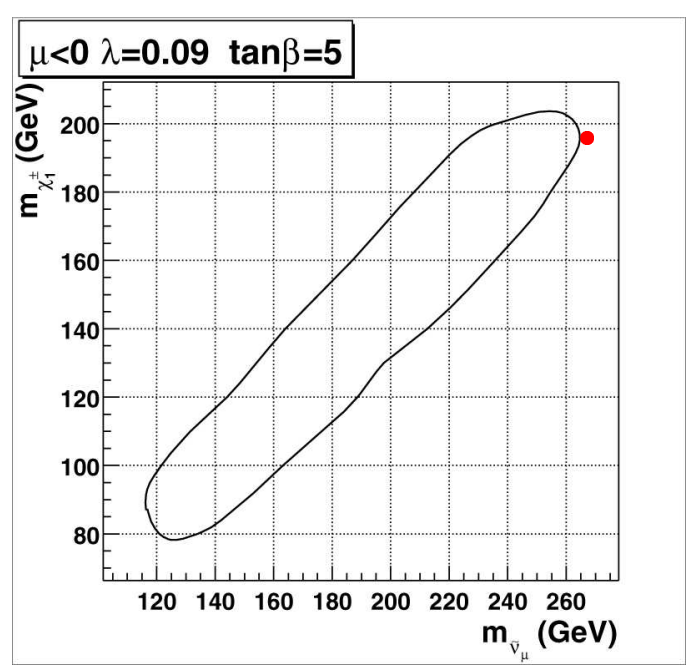

(b)

Figure 2.27: D $\emptyset$ Run I 95\% CL exclusion contours for $\lambda_{211}^{\prime}=0.09, \mu<0$ and $\tan \beta=5$ in the mSUGRA parameter $m_{0}-m_{1 / 2}$ plane (a) and in the sneutrino mass-chargino mass plane (b) [89]. The reference point as used in this analysis (discussed in Tab. 6.2) is marked with a red dot. 
$\mathrm{D} \varnothing$ Run I has searched for the pair and associated production of gauginos in the electron channel (as shown in Fig. 2.24c) and in the muon channel [82]. For $L Q \bar{d}$ couplings $\lambda_{2 j k}^{\prime}>0.01$ with $j=1,2$ and $k=1,2,3$ the obtained limits have been interpreted within mSUGRA with $\tan \beta=6, \mu<0$, and $A_{0}=0$ as shown in Fig. 2.28.

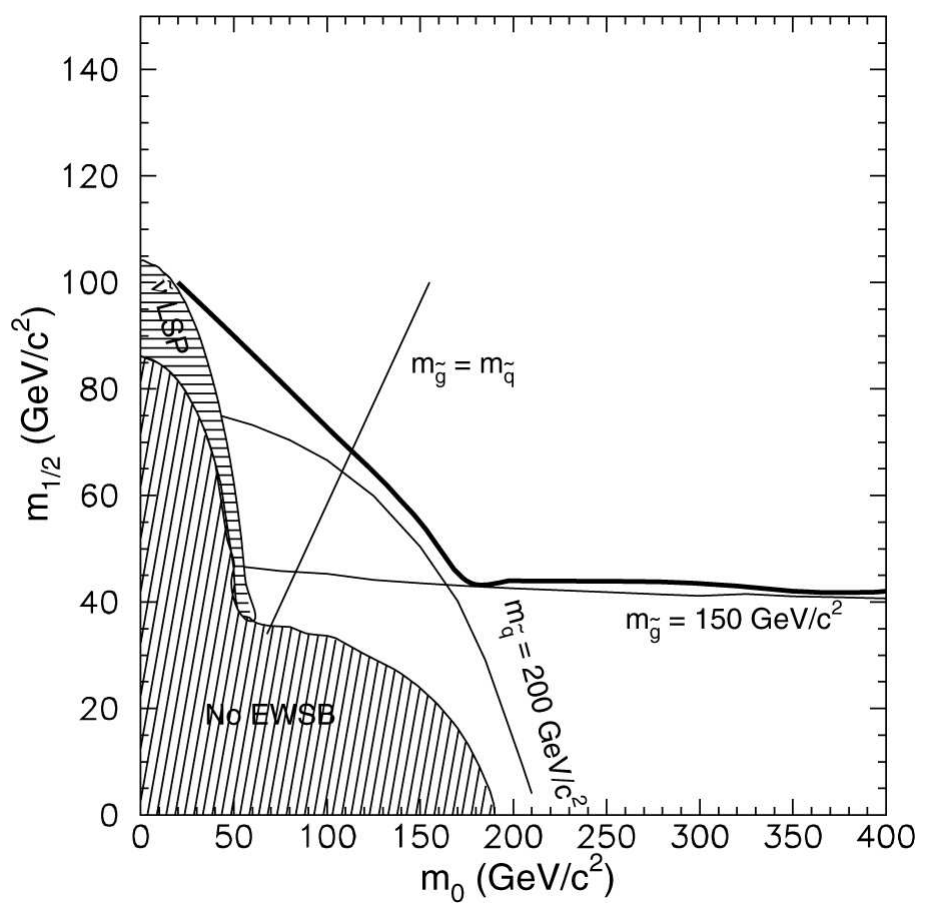

Figure 2.28: $\mathrm{D} \emptyset$ Run I exclusion contours for pair and associated gaugino production within mSUGRA with $\tan \beta=6, \mu<0$, and $A_{0}=0$ for $\lambda_{2 j k}^{\prime}>0.01$ [82].

\subsection{Summary}

In this chapter the problems of the Standard Model leading to the introduction of supersymmetry were discussed. The basic features of supersymmetric models and R-parity were given and existing limits were reviewed.

The Standard Model is a very successful high energy model for particles and their interactions. However, the quadratic divergent corrections to the Higgs mass-squared parameter make extreme fine tuning $\left(M_{W} / M_{\text {Planck }}\right)^{2}=10^{-35}$ necessary, because the cut-off scale $\Lambda$ can in principle be as large as the Planck scale and the Higgs mass vacuum expectation value is of the order of the electroweak scale. An elegant solution to this problem is the introduction of a symmetry between bosons and fermions, so that the divergent correction terms to the Higgs mass of both superpartners cancel each other, thus avoiding the hierarchy problem because no quadratic divergence is left. This symmetry automatically predicts new super-partners to every known Standard Model particle. The new sparticle masses must be heavy, as not to contradict observation, since no such sparticles has been found yet. The masses of the SUSY particles do not influence the solution of the hierarchy 
problem, because the quadratic divergent Higgs mass correction terms do not depend on the loop-particle mass, but only on the particle-Higgs coupling. However, the corrections to $m_{H}$ due to sparticle loops, should be maximally of the order of the Higgs mass vacuum expectation value to be under control, leading to expected sparticle masses $\lesssim 1 \mathrm{TeV}$. Supersymmetry is an exact symmetry, such that all parameters and couplings of both superpartners are equal, except for the mass and the spin. The superpartners differ in spin by half a unit. The supersymmetry generator transforms particles to sparticles, therefore fermions to bosons and vice versa. The anti-commutator of the symmetry generator is the translation operator. This means that supersymmetry is a space time symmetry and gravity is included naturally, if supersymmetry is a local symmetry. Since the superpartners cannot have equal masses, the supersymmetry must be broken in this respect. No new hierarchy problem will arise, if supersymmetry is broken spontaneously. The new masses and their mixing angles are free parameters, so that 110 new variables are introduced in the Minimal Supersymmetric Model. These degrees of freedom can be constrained by the assumption, that the three running coupling parameters of the $\mathrm{SU}(3)_{C} \times \mathrm{SU}(2)_{L} \times \mathrm{U}(1)_{Y}$ forces join in one point below the Planck mass. All gaugino and all sfermion masses are mass degenerate at this point. Only five SUSY model parameters are left; the common gaugino and sfermion masses at the grand unification scale, the Higgs mass mixing parameter, the ratio of the Higgs vacuum expectation values and the trilinear Higgs coupling.

The present search for resonant slepton production shall be performed as model independent as possible, depending only on the masses of the involved particles; the slepton and the gaugino masses. The result, which is either a observation or a limit, will be given with respect to the production cross section. This will finally be interpreted within the mSUGRA parameter space. The resonant slepton production cross section is directly proportional to the coupling parameter squared $\sigma \propto\left(\lambda_{211}^{\prime}\right)^{2}$, so that this analysis is also sensitive to the size of the coupling.

The analysis is extended to search for the pair and associated production of gauginos and their decay via any $L Q \bar{d}$ coupling $\lambda_{2 j k}^{\prime}$ with $j=1,2$ and $k=1,2,3$ resulting into di-muon final states.

The $L Q \bar{d}$-coupling parameters are already constraint by low energy experiments. The present most stringent limit on the $\lambda_{21 k}^{\prime}$ couplings is from the lepton universality in the pion decay. The LEP experiments have set for non-zero $L Q \bar{d}$-couplings limits on gaugino and slepton masses. The limits relevant for this analysis are summarized in Tab. 2.8. These limits will be used for comparison in the later chapters.

\begin{tabular}{lll}
\hline \hline \multicolumn{2}{l}{ parameter } & limit \\
\hline$\lambda_{21 k}^{\prime}$ & coupling & $\leq 0.059 \cdot \frac{m\left(d_{k R}\right)}{100 \mathrm{GeV}}$ \\
$\tilde{\chi}_{1}^{0}$ & neutralino mass & $\geq 39 \mathrm{GeV}$ \\
$\tilde{\chi}_{1}^{ \pm}$ & chargino mass & $\geq 103 \mathrm{GeV}$ \\
$\tilde{\mu}_{L}$ & smuon mass & $\geq 81 \mathrm{GeV}$ \\
\hline \hline
\end{tabular}

Table 2.8: Summary of all constraints with $95 \%$ CL in case of a non-zero $L Q \bar{d}$-coupling $\lambda_{211}^{\prime}$, from low energy experiments and from LEP. 


\section{Chapter 3}

\section{The Experimental Setup}

The data used for the present analysis were recorded by the $\mathrm{D} \varnothing$ experiment between April 2002 and August 2004. The DØ experiment is one of two multipurpose detectors, located at the proton-antiproton accelerator complex Tevatron at Fermilab. During the first data taking period (Run I) from 1992 - 1996, D $\varnothing$ and the second experiment CDF discovered the top-quark in $t \bar{t}$-production processes and measured the top-mass. The $W$-mass as well as gauge boson couplings were studied in great detail and greatly improved limits on supersymmetry and leptoquark particles were set. See [94] for a complete list of DØ Run I publications.

In the five years between 1996 and 2001 the accelerator complex was improved and a new pre-accelerator was built. The beam energy was increased from $900 \mathrm{GeV}$ to $980 \mathrm{GeV}$ per particle and the beam structure was changed to improve the luminosity. Both the $\mathrm{D} \varnothing$ and the CDF detectors were upgraded. The central $\mathrm{D} \varnothing$ drift-chamber was replaced with a solenoid magnet, a scintillating fiber tracker and a silicon microstrip detector. The trigger system and the detector readout were upgraded, to cope with the higher interaction frequency.

Some general aspects of collider physics shall be discussed in the following Sec. 3.1. The Tevatron accelerator and the upgraded $\mathrm{D} \varnothing$ detector shall be described briefly in Sec. 3.2 and Sec. 3.3, respectively. More detailed information can be found in [95, 96].

\subsection{General Aspects of Collider Physics}

In order to detect particles, that is to measure their trajectories and momenta, they have to interact in some kind with the detector material. The mechanisms as discussed for example in [97] will be summarized briefly in the following Sec. 3.1.1. Partons (quarks or gluons) that are created in the collision or the decay remnants of the initial (anti-) protons are not color neutral and cannot exist freely. They have to accumulate into uncolored objects, the mesons or baryons, this hadronization process is discussed in Sec. 3.1.2. 


\subsubsection{Interactions of Particles with Matter}

The particles that are created in the interaction region travel through the detector and interact with the electrons and nuclei of the detector material; this is a precondition for the particle detection. A light projectile which is colliding with a heavy nuclei will be deflected (multiple scattering), but will lose little energy unless the collision is inelastic (bremsstrahlung, pair production). A heavy particle colliding with a light target will lose energy without any significant change in direction. Multiple scattering leads to a Gaussian smearing of the particle's direction and affects theirfore the resolution of the momentum measurement. For more details on multiple scattering see [98].

Different particles interact in a different way with matter, leading to distinct signatures in the detector. Particles can be identified by measuring energy as well as energy loss. The interaction of heavy charged particles with atomic electrons or nuclei is of statistical nature, but for many interactions the average energy loss per unit path length can be calculated and measured. Ionization is the main electromagnetic contribution to the energy loss for charged particles. In Fig. 3.1 the energy loss of muons, pions and protons in different material is shown. The average energy-loss per path length for a specific particle of mass $m$ and electrical charge $q$ is predicted by the Bethe-Bloch formula:

$$
-\frac{d E}{d x}=\frac{4 \pi}{m} \cdot \frac{n \cdot q^{2}}{\beta^{2}}\left(\frac{e^{2}}{4 \pi \epsilon_{0}}\right)^{2} \cdot\left[\ln \left(\frac{2 \cdot m c^{2} \beta^{2}}{I \cdot\left(1-\beta^{2}\right)}\right)-\beta^{2}-\frac{\delta}{2}\right]
$$

Where $\beta=\frac{v}{c}$ is the ratio of the particle's velocity $v$ and the speed of light $c$, and $n$ is the electron density of the target material. $I$ is the mean excitation potential of the target, usually approximated as $I=16 \mathrm{eV} \cdot Z^{0.9}$, where $Z$ is the atomic number of the traversed material. For low velocities $(\beta \ll 1)$ the energy loss decreases with $1 / \beta^{2}$ as the velocity increases, reaching the minimum at approximately $E_{m i n} \simeq 3 \cdot m$. For strongly relativistic particles $(\beta \simeq 1)$, the energy loss increases logarithmically with the particle's momentumsquared. The momentum is given by $p=m v / \sqrt{1-\beta^{2}}$. The relativistic rise is finally leveling at a constant value, the Fermi-plateau. The $\delta$-term considers density effects, that lower the Fermi-plateau, for details see [99].

The electron $e^{ \pm}$mass is too light, so that the Bethe-Bloch equation is not applicable, here. For electron energies below $\approx 500 \mathrm{MeV} / z$ ionization dominates. More energetic electrons mainly loose energy by radiating photons in the electric field of a nucleon; this is called bremsstrahlung (deceleration radiation). Bremsstrahlung is emitted when a charged particle is decelerated. It is proportional to $1 / \mathrm{m}^{2}$, thus contributes substantially to energy loss in matter only for electrons. Other types of energy loss for electrons and positrons are Bhabha scattering $\left(e^{+} e^{-} \rightarrow e^{+} e^{-}\right)$and Møller scattering $\left(e^{-} e^{-} \rightarrow e^{-} e^{-}\right)$and in case of positrons also annihilation with atomic electrons. All types of energy loss for electrons and positrons $e^{ \pm}$in lead $(Z=82)$ are shown in Fig. 3.2.

Photons can interact with material by the photo-effect; the photon is absorbed by an atomic electron, where the nucleus ensures the momentum conservation. A photon can also be scattered by free electrons or can convert into an electron-positron pair, if the 


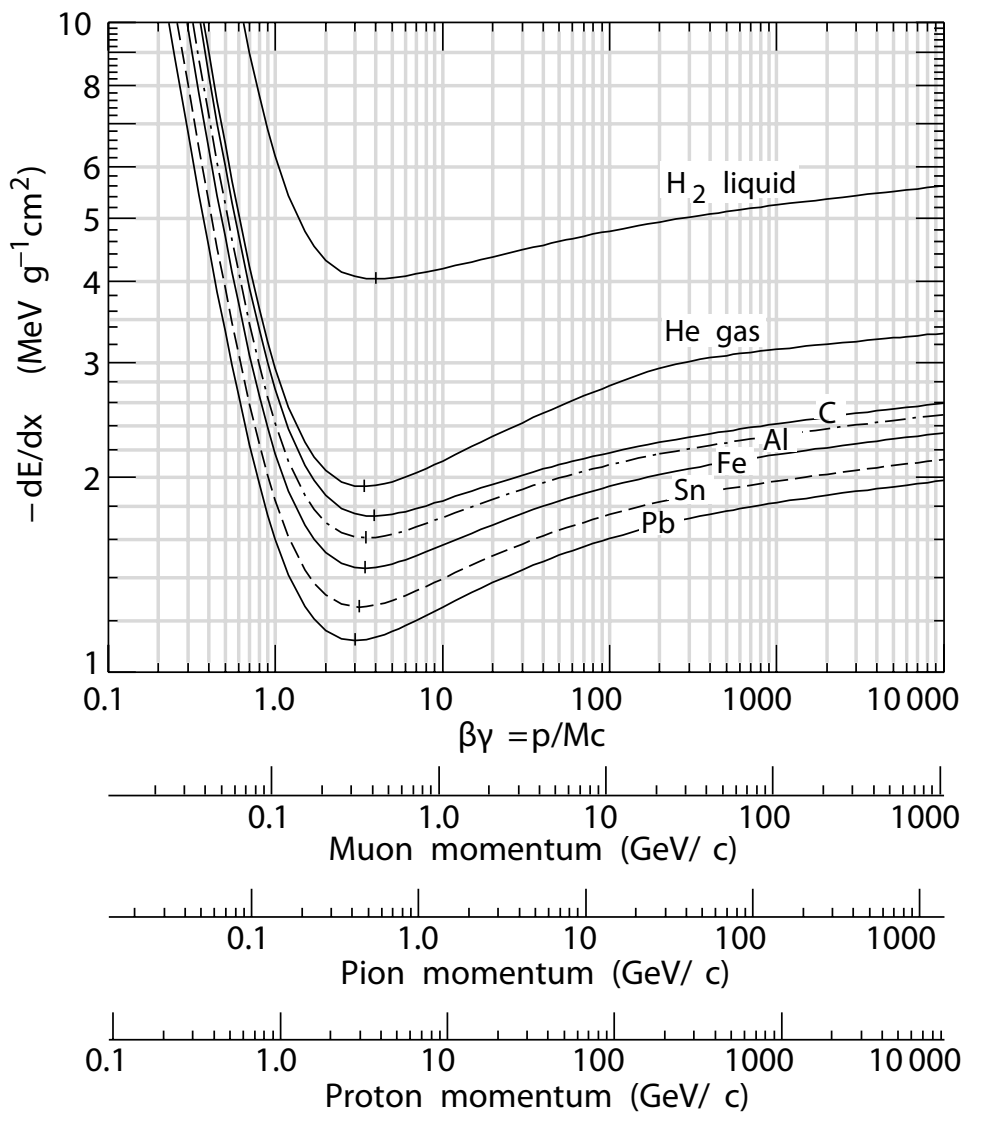

Figure 3.1: Energy loss for heavy charged particles in different materials [17] as predicted by the Bethe-Bloch equation.

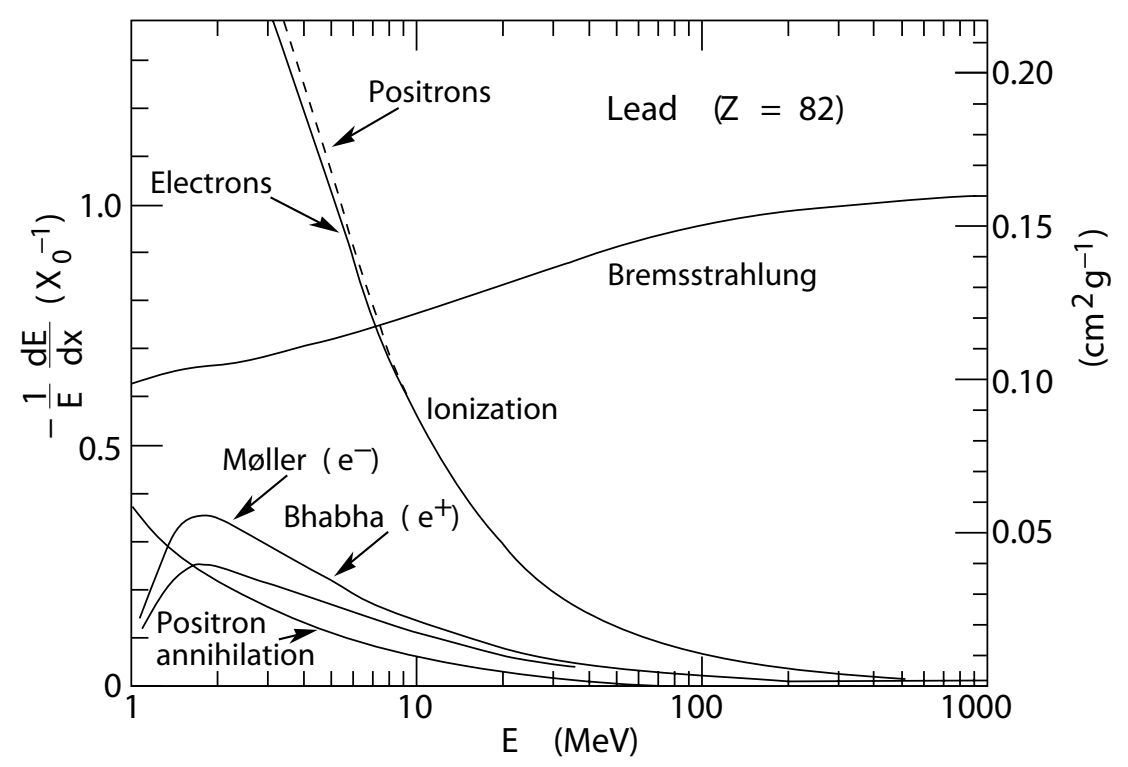

Figure 3.2: $d E / d x$ for electrons $e^{-}$and positrons $e^{+}[17]$ 
photon energy exceeds $2 m_{e}$ and a nucleus is present to ensure momentum conservation, see also Sec. 3.3.3.

Secondary particles are produced in electromagnetic processes, these particles are again mainly electrons $e^{ \pm}$and photons $\gamma$. The cascade develops through repeated similar interactions, until most of the energy is consumed for particle production. The shape of this electromagnetic shower scales longitudinally in good approximation with the radiation length $X_{0}$, and laterally with the Moliere radius [100]. The quantity radiation length $X_{0}$ refers to the distance an electron can travel before its energy is reduced to $1 / e$ due to losses. $X_{0}$ can be given without explicitly stating the material and is useful to characterize the matter density of detectors, that are usually comprised of many different materials.

Strong interacting particles hadronize, as discussed in Sec. 3.1.2. Hadrons shower in matter similar to the electromagnetic shower processes. However, the relevant processes of inelastic hadron interaction are very different. The hadronic shower is measured in terms of the nuclear interaction length $X_{\text {nuclear }}$, which in most materials is significantly larger than the electromagnetic interaction length $X_{0}$. Pions can decay purely electromagnetic $\pi^{0} \rightarrow \gamma \gamma$, so that large electromagnetic fractions can be comprised in the hadronic showers. Some amount of the initial hadron energy is converted to excitation and breakup of nuclei and cannot be detected in a calorimeter. Also particles that do not interact with the calorimeter material can be created in the cascade. Large event-to-event fluctuations have to be taken into account.

The calorimeter response to electromagnetic and hadronic particles of the same energy is different, but the calorimeter response should be as independent as possible from the shower development. In a compensating calorimeter the relative thickness of absorber and active layers are adjusted to accomplish this behavior, see [101] for details.

Neutrons are electrically uncharged hadrons, thus non-ionizing particles. They interact with nuclei through the strong force. Inelastic neutron-nucleon scattering can leave the nucleus in an excited state. The neutron can be captured by a nucleon leading to $\alpha, \beta$ or $\gamma$ emission or to fission in materials like uranium.

\subsubsection{Hadronization}

The quarks in compound objects like hadrons are asymptotically free, as described in Sec. 2.1.1, but they cannot leave the compound by themselves. The strong force acts as a guard to constrain the quarks to the compound. The repelling force increases with increasing distance. If one quark gathers enough energy in a scattering with a second particle, then it will break out of its particle (baryon or meson) by breaking it. In Fig. 2.1.1 inelastic electron-proton scattering is illustrated schematically as an example. A parton of the initial proton obtains energy in the collision, e.g. by exchanging a $W$-boson with the electron, and departs. The attached gluon tube, the carrier of the strong force, is stretched similar to an elastic band. The gluon band ultimately disrupts, corresponding to quark - antiquark production. The escaping quark can form a color-neutral meson with 


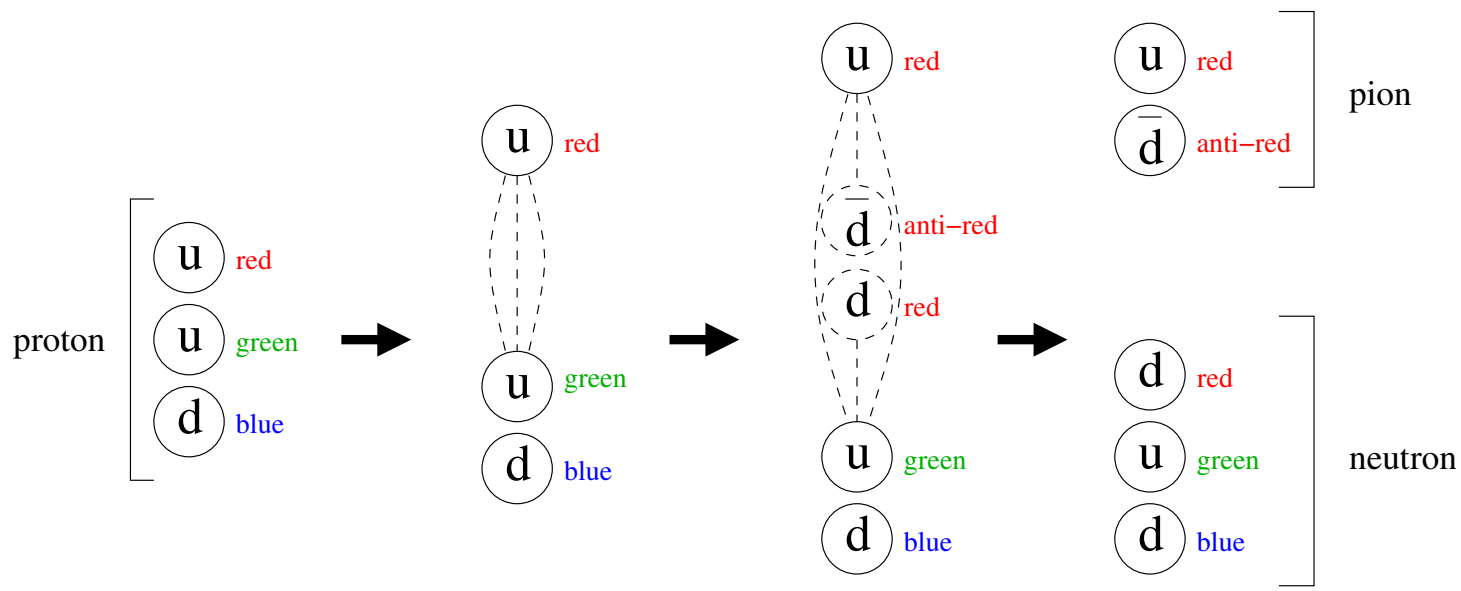

Figure 3.3: Schematic drawing of the inelastic electron-proton scattering process, i.e. $e p \rightarrow$ $e n \pi^{+}$; the electron $e$ is not shown.

the antiquark and the proton remnants and the second quark bind to a new, color-neutral baryon, in this case a neutron. This process can be written as $e p \rightarrow e n \pi^{+}$.

In Fig. 3.4 hadronization is shown schematically, for a process $q \bar{q} \rightarrow W \rightarrow q \bar{q}$. Two initial quarks form a $W$-boson which then decays back into two quarks. These high energetic quarks emit many gluons that decay into more quarks. In the gray shaded region the quarks and gluons condense to stable hadrons and leptons, the particles that are observed in the detector. This process of hadronization cannot be solved analytically up to today; because of the countless possible decays and interactions the complexity is much to large. The resulting final state hadrons (baryons and mesons) and leptons are arranged in two jets. The jet momenta correspond roughly to the momenta of the intermediate quarks, into which the $W$ decayed. Final state radiation can lead to additional particle jets. The quark momentum can be reconstructed from the detected particle jet with great precision, at $\mathrm{D} \varnothing$ with typically $10 \%$ or better, depending on energy, polar and azimuthal angle of the quark. The jet energy calibration is discussed in more detail in Sec. 5.2.4.

\subsection{The Tevatron Accelerator}

The accelerator division of FERMILAB maintains a cascade of accelerators, needed for pre-acceleration, anti-proton production, accumulation and particle storage. A sketch of the accelerator complex is shown in Fig. 3.5. The particle production and acceleration processes are very complex and difficult operations and subject to continuous research and improvements. The reader is referred to an introduction which can be found e.g. in [95], here only the basics steps shall be briefly discussed.

The acceleration process begins within the Cockroft-Walton linear accelerator. $H_{2}$ molecules are separated to ionized $H^{-}$atoms and accelerated to $750 \mathrm{keV}$ energy and passed to the LinAC linear accelerator. The LinAC boosts the particles to $400 \mathrm{MeV}$ and dumps the $H^{-}$ 


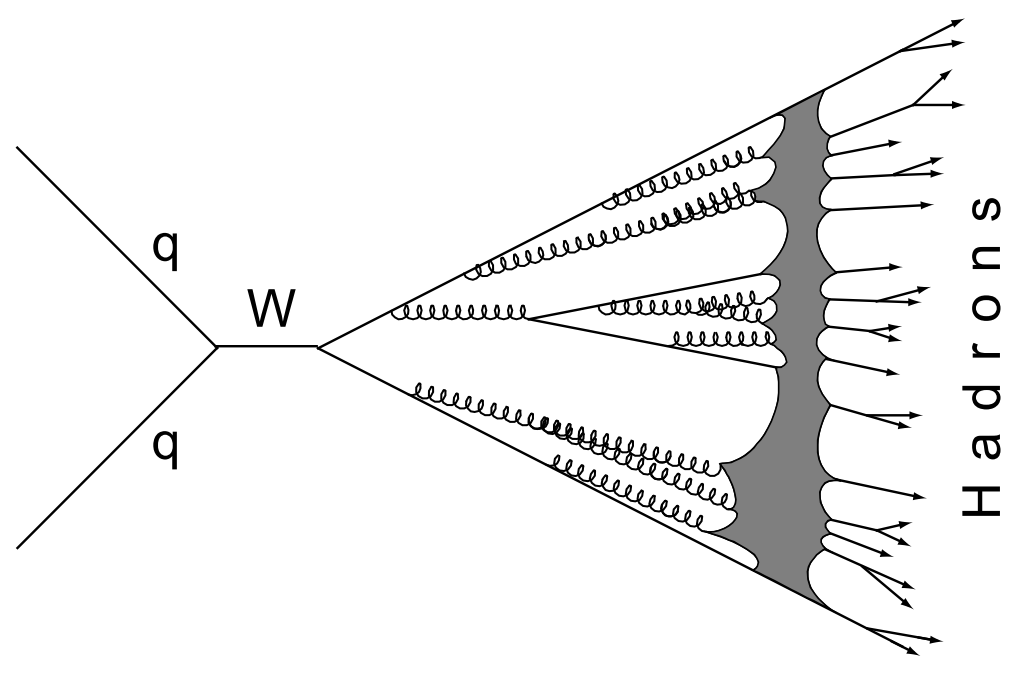

Figure 3.4: Cartoon of hadronization, from [102].

onto a graphite foil, stripping off all electrons. The $\mathrm{H}^{+}$or protons are inserted into a synchrotron (the Booster) which increases the energy to $8 \mathrm{GeV}$.

The $8 \mathrm{GeV}$ protons are transfered to the Main Injector, a storage ring that was built during the long upgrade shutdown. In the same tunnel a second storage ring was built with the intention to recycle anti-protons from the main ring. Since the recycling is too time consuming and the achieved efficiency is low, the Recycler is used only to store anti-protons, in order to unload the anti-proton accumulator. The Recycler beam pipe is equipped with permanent magnets to store $8 \mathrm{GeV}$ anti-protons, while the Injector beam line makes use of normal-conducting magnets. It accelerates either protons counterclockwise or anti-protons clockwise from 8 to $150 \mathrm{GeV}$, before they are injected into the TEVATRON main ring.

The Main Injector and the TEvatron ring were designed to allow simultaneous operation. During a physics store in the Tevatron, which lasts typically around 24 hours, the Main Injector will continuously accelerate protons from 8 to $120 \mathrm{GeV}$ which are delivered to fixed target experiments and to the anti-proton source.

The anti-proton source is a nickel-copper target with properties optimized for anti-proton production. The anti-protons are selected from the interaction products of the proton fixed target collisions. The energy spectrum of the selected anti-protons peaks at $8 \mathrm{GeV}$ and is monochromized by stochastic cooling in the Debuncher and Accumulator. Antiprotons are produced continuously and stacked in the Accumulator. The stacking efficiency depends on the amount of already stacked anti-protons. Therefore some fraction of the particles are transfered to the Recycler, which was designed to store anti-protons.

After the successful transfer of 36 proton bunches with an energy of $150 \mathrm{GeV}$ per particle from the Main Injector to the TEvatron main ring, the anti-protons are transferred. First from the Accumulator and/or the Recycler to the Main Injector and then, accelerated from 8 to $150 \mathrm{GeV}$, to the Tevatron. The reason for this sequence is to keep the anti-protons 


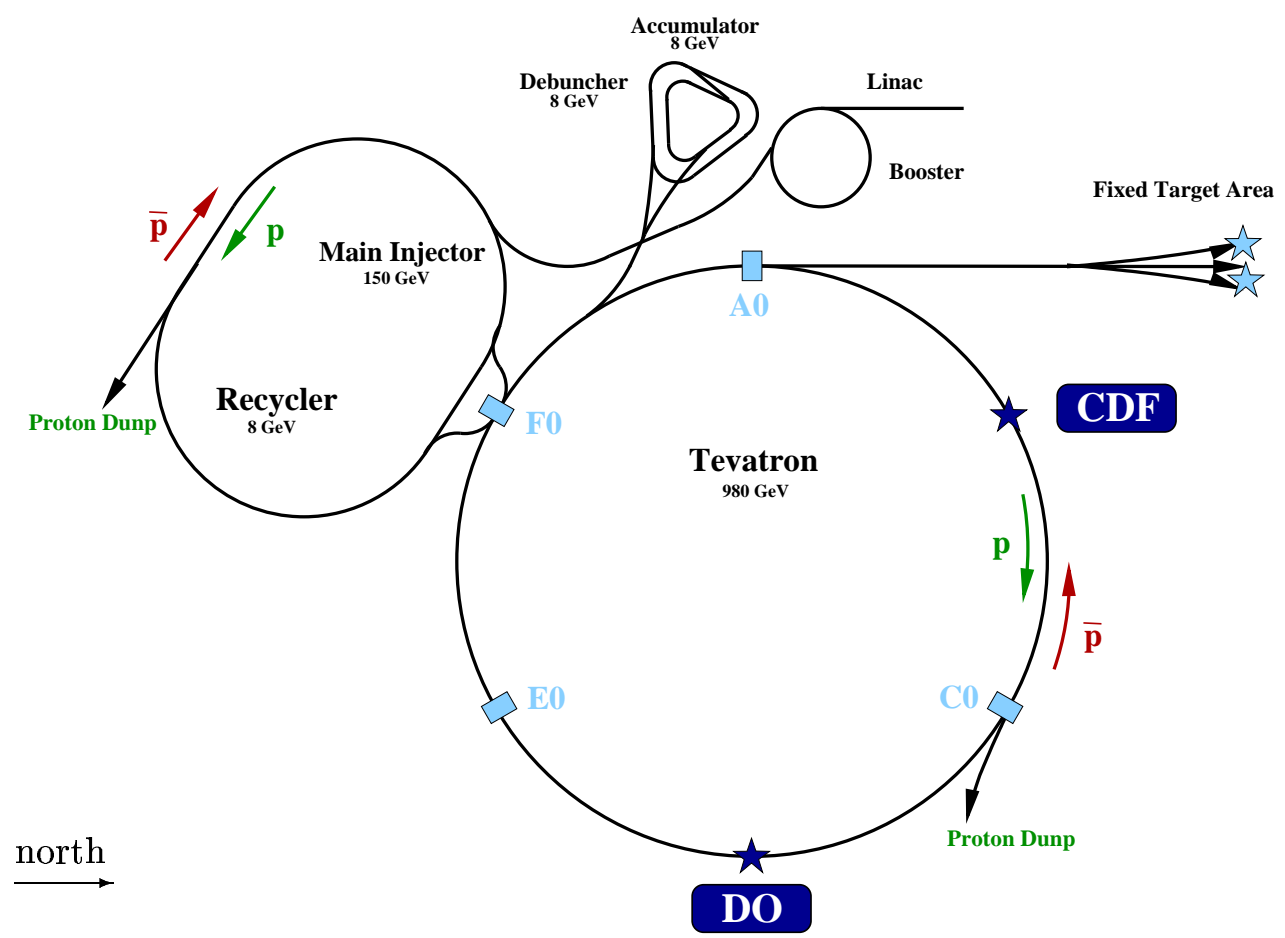

Figure 3.5: The accelerator chain of FERMILAB. The acceleration starts with the Cockroft-Walton and the LINAC linear accelerator, then the protons are passed from the BoosTER synchrotron to the Main Injector and finally to the TEVATRON main ring. The anti-protons are accumulated in the triangular shaped Accumulator as well as in the Recycler.

safe as long as possible, because of the significantly larger operating expense to produce anti-protons, compared to the comparatively simple proton acceleration. As soon as all 36 anti-proton bunches are in the Tevatron beam pipe, the energy is increased to $980 \mathrm{GeV}$ per particle. Finally, the low-beta magnets are ramped up, to focus the proton and antiproton beams into the interaction zones in the $\mathrm{D} \emptyset$ and the CDF detector.

The beam intensity and therefore the Luminosity drops exponentially, since the particle loss rate is approximately proportional to the particle flux $\dot{p} \sim p$. If the Tevatron Luminosity drops below $10 \cdot 10^{30} \mathrm{~cm}^{-2} \mathrm{~s}^{-1}$ or as soon as enough anti-protons are stacked in the Accumulator and/or in the Recycler, the old beams are dumped. The beam circulation can be stopped unintentionally if a magnet quenches. A quench is the local break-down of superconductivity in the magnet coil, i.e. triggered by a temperature fluctuation of the liquid helium coolant. This can be the result of an accidental particle loss. The magnetic field in the quenched magnet will break down, however this is in general slow compared to the circulation frequency, so that the beams can be kicked out of the beam pipe and dumped in time to avoid further damage. An unnoticed change of the beam orbit can result in the quenching of a large number of magnets.

The Tevatron is in shot setup mode, when new particles are to be inserted. In total 36 bunches of protons and 36 anti-proton bunches are filled. The bunches are grouped in 
three trains with a $7 \mu$ s separation. The bunch to bunch separation, or the interaction frequency is $396 \mathrm{~ns}$. A proton bunch contains about $2.4 \cdot 10^{11}$ protons, the anti-proton bunch about one order of magnitude less anti-protons. The longitudinal length of the bunches is $37 \mathrm{~cm}$; the number of particles per bunch and the bunch size is limited due to the repelling coulomb forces. The Tevatron main ring has a circumfence of $6283 \mathrm{~m}$, during normal operation the beams are stored with an energy of $980 \mathrm{GeV}$ per particle for up to two days. During test runs a maximal energy of $1.012 \mathrm{TeV}$ per particle has been attained. Some basic characteristics of the Tevatron are summarized in Tab. 3.1.

The Tevatron main ring is divided into six sections, named clockwise starting in the westsection from $A$ through $F$. Each section is divided into five buildings, each " $\varnothing$ " location has a long straight section with special functions; at $\mathrm{A} \emptyset$ the Tevatron is connected to the Switchyard and the beam abort is located here. The CDF detector is located at position $\mathrm{B} \varnothing$, at $\mathrm{C} \varnothing$ is the second beam abort (for protons only). The $\mathrm{D} \varnothing$ detector is named after its position in the ring. At $\mathrm{E} \varnothing$ the transfer line from the old main ring to the Tevatron was located. The Tevatron RF cavities are located at FØ, as well as the proton and anti-proton connection beam lines to the Injector and a transfer line to the anti-proton source.

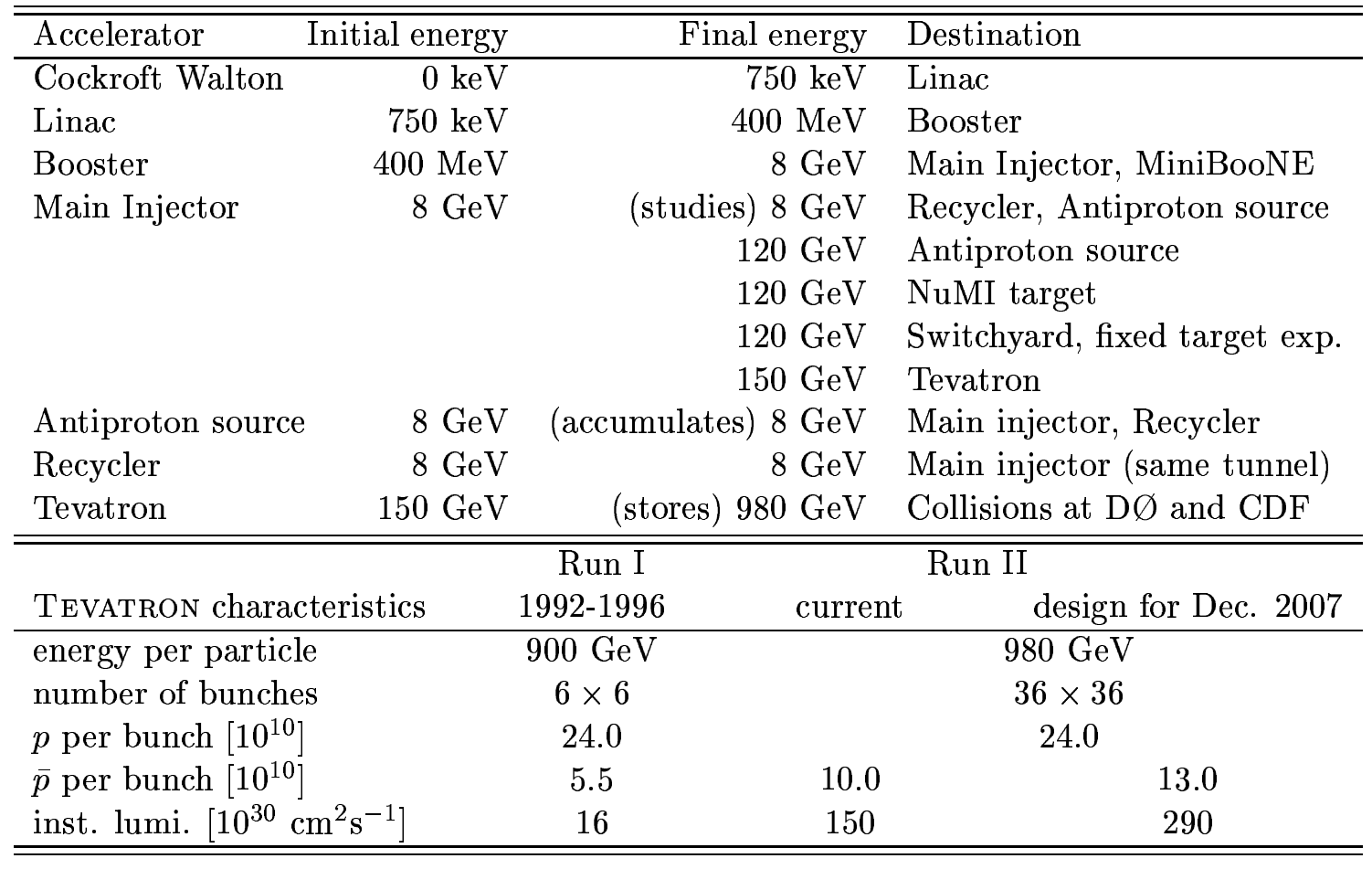

Table 3.1: Basic characteristics of the accelerator chain [95] and the TEvatron in Run I and after the upgrade in Run II. 


\subsection{The DØ Detector}

$\mathrm{D} \varnothing$ is a multipurpose detector. With its three major subsystems; the tracking system, the hermetically closed calorimeter and the muon system it is capable of detecting all kinds of physics objects, either directly or at least indirectly as for example neutrinos that leave no signal in the detector but can be reconstructed through the missing, not measured energy. An overview over the $\mathrm{D} \varnothing$ detector is given in Fig. 3.6. The major subsystems of the detector and the measurement of physics objects shall be discussed briefly in the following.

\subsubsection{Coordinate System}

In the Tevatron main ring the proton beam circulates clockwise and the anti-proton beam counter-clockwise. The protons enter the $\mathrm{D} \varnothing$ detector from the north. The proton direction defines the positive $z$-axis and the $x$-axis is pointing out of the ring, defining a right-handed cartesian coordinate system. The origin of the coordinate system is in the center of the detector. Since neither the beams, nor the beam interaction products have a preferred axis in the $x-y$ plane, often a cylindric coordinate system, symmetric to the $\mathrm{z}$-axis is used. The azimuthal angle $\phi=[0 . .2 \pi]$ is measured in the $x-y$ plane, $\phi=0,2 \pi$ is the positive $x$-axis, and $\phi=\pi / 2$ is the positive $y$-axis. The polar angle $\theta$ is replaced with the rapidity $y$.

$$
y=\frac{1}{2} \ln \left(\frac{E+p_{z}}{E-p_{z}}\right)
$$

Where $E$ is the particle's energy and $p_{z}$ the momentum in $z$-axis direction. Differences in rapidity are invariant under Lorentz boosts along the z-axis. This is especially important at hadron colliders, where the center-of-mass system of the colliding partons is not the lab rest frame. In case of high energies $E \gg m$ and therefore $E \approx|\vec{p}|$ the rapidity $y$ can be replaced with the pseudo-rapidity $\eta$ :

$$
\eta=-\ln \tan \frac{\theta}{2}
$$

The vertex position defines the origin of the coordinate system for the calculation of physics quantities. The pseudo-rapidity $\eta$ for physics objects is corrected, according to the vertex $z$-component of each event.

The interaction volume of the proton and anti-proton beams is an ellipsoidal shaped density distribution with a typical radius $r$ in $\phi$-direction of a few microns and a length $R$ in $z$-direction of the order of $50 \mathrm{~cm}$. The position of the interaction region ellipsoid is not stable, changes of the beam optic due to maintenance or upgrades cause shifts in all directions by the order of several 100 microns. The vertex distribution over the relevant runtime is shown in Fig. 3.7. The Luminosity depends on the particle density in this interaction volume, but the density is limited by the beam optics, the number of parti- 


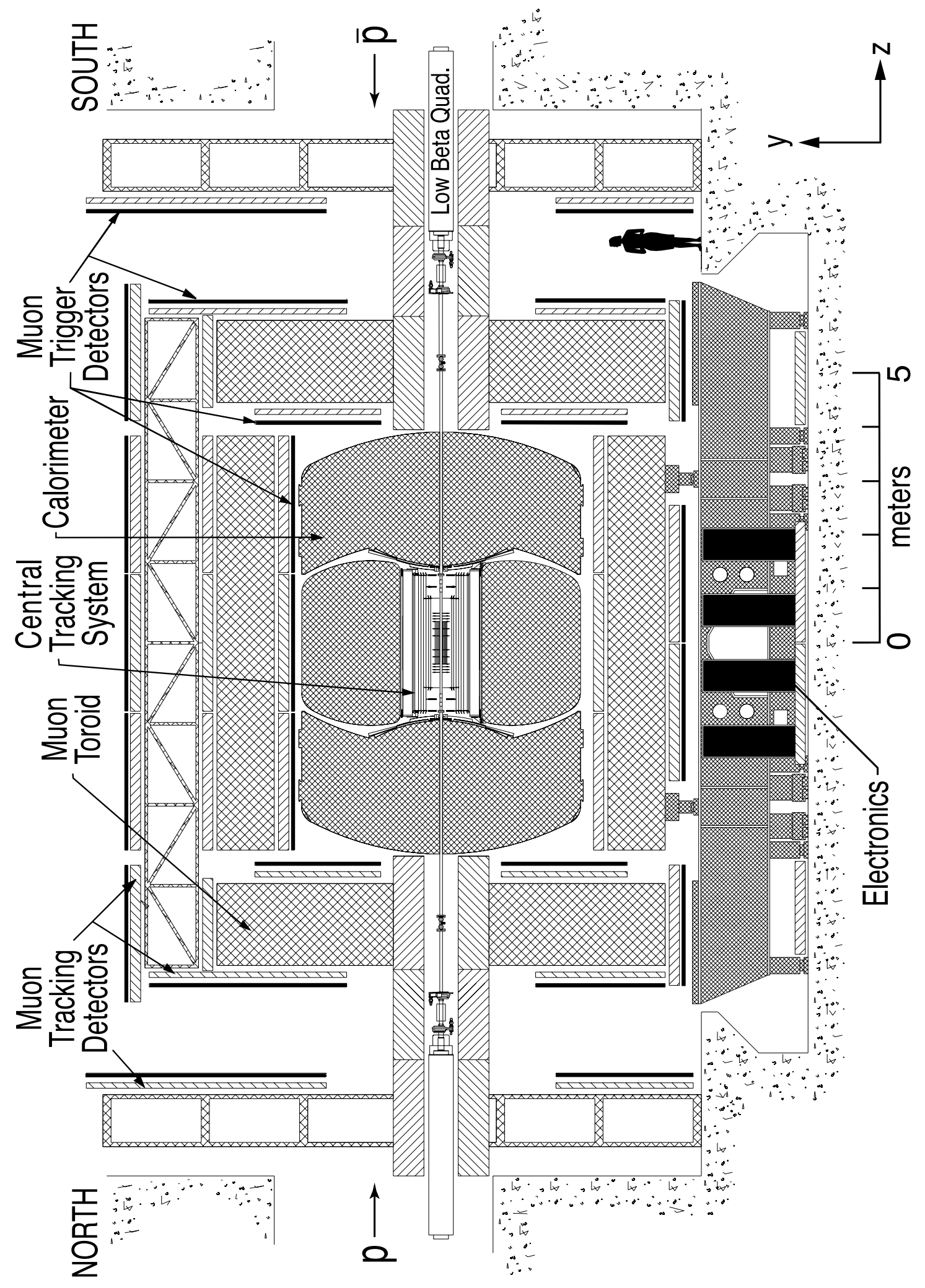

Figure 3.6: The D $\varnothing$ detector [96]. 
cles in the bunches and the repelling coulomb forces. Other constraints are due to the quantum fluctuations of synchrotron radiation or beamstrahlung and pair creation during the beambeam collision [103]. The most fundamental constraints arise from the uncertainty principle and the Fermi-Dirac statistics for fermions and are completely negligible at current accelerators [103].

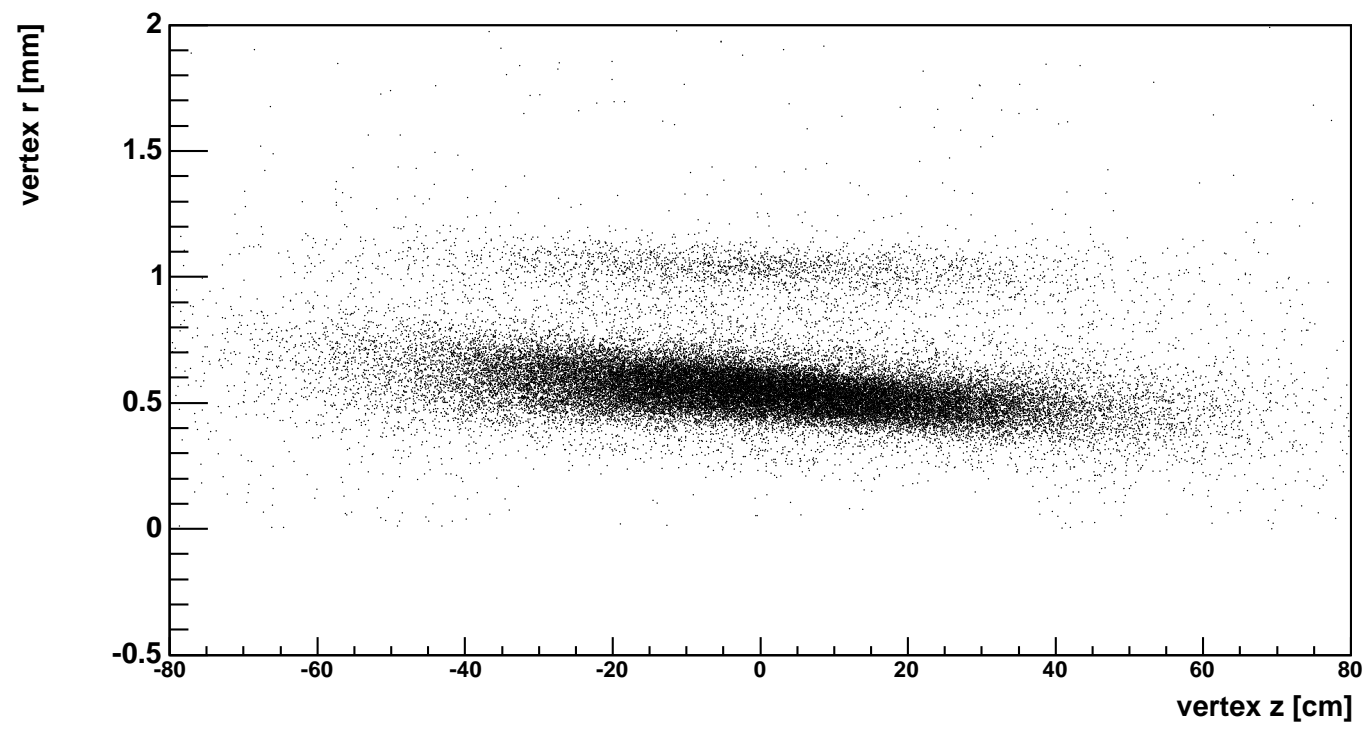

Figure 3.7: The vertex distribution at $\mathrm{D} \emptyset$, where $z$ is the distance from the detector center and $r$ the distance from the beam-pipe center. The imperfect adjustment of the low-beta focussing magnets at $\mathrm{D} \emptyset$ and the imperfect Gaussian particle density in the proton and antiproton bunches lead to shifts and the slight angle of the vertex distribution.

\subsubsection{The Luminosity System}

The luminosity is an important number to normalize the recorded data. Cross section measurements or searches for new physics depend on the precision of this number. The precise knowledge of the Luminosity is essential, because all analyses, cross section measurements and searches, depend directly on this number. The luminosity can be measured by counting events of a specific process, if the cross section of this process is known. However, to measure a cross section of a certain process one needs to know the luminosity. The Optical Theorem leads out of this quandary. The theorem connects the total cross section $\sigma_{\text {tot }}$ of a scatter process to the forward scattering amplitude $f\left(\theta_{0}\right)$, which can be measured. The Optical Theorem is known from classical electrodynamics, and can be derived [104] by calculating the forward scattering amplitude of a plane wave front $\Psi(\vec{r})=e^{i k \cdot z}$ traveling in $z$ direction, which incidents on an object in the origin of the coordinate system. Seen from a great distance the wave amplitude is then given by:

$$
\Psi(\vec{r})=e^{i k z}+f\left(\theta_{0}\right) \frac{e^{i k r}}{r}
$$


Where $f\left(\theta_{0}\right)$ is the scattering amplitude at a small angle $\theta_{0}$. With $\theta_{0} \ll 1$ and therefore $r \approx z+\frac{x^{2}+y^{2}}{2 z}$ the intensity at $\vec{r}$ can be integrated:

$$
\begin{aligned}
\oint_{A}|\Psi(\vec{r})|^{2} d r^{3} & =\int\left|e^{i k z}+\frac{f\left(\theta_{0}\right)}{r} e^{i k z} e^{i k\left(x^{2}+y^{2}\right) / 2 z}\right|^{2} d r^{3} \\
& =\int 1+2 \cdot \mathfrak{R} e \frac{f\left(\theta_{0}\right)}{z} e^{i k\left(x^{2}+y^{2}\right) / 2 z} d r^{3} \\
& =A+2 \cdot \mathfrak{R} e \frac{f\left(\theta_{0}\right)}{z} \frac{2 i \pi z}{k}
\end{aligned}
$$

Where $A$ is the area of surface over which is integrated. The expression (3.7) is the energy of an unscattered wave, diminished by the amount of the total scattered energy. Therefore the last term is the effective scattering cross section $\sigma_{t o t}$.

$$
\sigma_{t o t}=\frac{4 \pi}{k} \cdot \Im m f\left(\theta_{0}\right)
$$

This is the Optical Theorem. The process used for the luminosity calculation at TEvatron is the elastic and inelastic proton scattering, because this is the dominating process and offers therefore the opportunity to measure the instantaneous luminosity after only a few bunch crossings. A selection of these events is unnecessary, because the cross section of all other processes is negligible. The decay products of this process do leave the detector under small angles $\theta$, for this reason a rather small luminosity detector is sufficient.

The total elastic and inelastic proton scattering cross section was measured by E710 [105], E811 [106] and CDF [107] at 1.8 TeV. The measurements have been averaged and extrapolated to $1.96 \mathrm{TeV}$, as summarized in Tab. 3.2. The averaged value [108] is used for the $\mathrm{D} \varnothing$ luminosity calculation and its uncertainty is the single largest contribution to the total systematic luminosity uncertainty [109].

\begin{tabular}{lrr}
\hline \hline experiment & $\sigma_{\text {inelastic }}$ & total $\sigma_{p \bar{p}}$ \\
\hline E710 & $56.6 \pm 2.2 \mathrm{mb}$ & $72.8 \pm 3.1 \mathrm{mb}$ \\
E811 & $56.5 \pm 1.2 \mathrm{mb}$ & $71.7 \pm 2.0 \mathrm{mb}$ \\
CDF & $61.7 \pm 1.4 \mathrm{mb}$ & $80.0 \pm 2.2 \mathrm{mb}$ \\
average & $60.1 \pm 2.3 \mathrm{mb}$ & $75.9 \pm 1.0 \mathrm{mb}$ \\
\hline \hline
\end{tabular}

Table 3.2: Total and inelastic proton scattering cross section at $\sqrt{s}=1.8 \mathrm{TeV}$. All measurements are correlated with each other.

The luminosity detector consists of two arrays. Each has 24 plastic scintillation counters and photon multiplier tubes (PMT). A schematic drawing of an array is shown in Fig. 3.8 (left hand), the location of the PMT is marked by solid dots. The LM detectors are located at $z= \pm 140 \mathrm{~cm}$, Fig. 3.8 (right) between the silicon tracker and the forward calorimeter. The scintillation counters are $15 \mathrm{~cm}$ long and cover a pseudo-rapidity range of $2.7 \leq|\eta| \leq 4.4$.

To detect an event of the elastic and inelastic scattering reference process, both luminosity detectors have to be fired. Background from beam-halo can be suppressed by requiring 

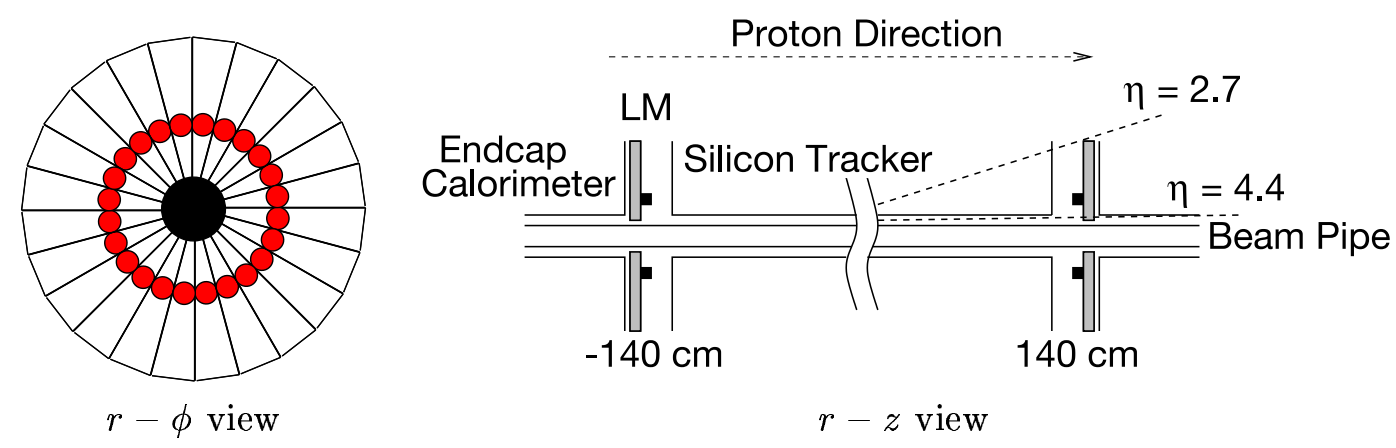

Figure 3.8: Both luminosity detectors LM (left) are located between the silicon tracking detector and the forward calorimeter (right) at $z= \pm 140 \mathrm{~cm} \mathrm{[96].}$

that the z-coordinate $z_{v}$ of the interaction vertex is within $100 \mathrm{~cm}$ of the detector center. The vertex coordinate is then $z_{v}=\frac{c}{2}\left(t_{-}-t_{+}\right)$and $t_{ \pm}$is the time-of-flight measurement at the detectors at $\pm 140 \mathrm{~cm}$.

The instantaneous luminosity $\mathrm{L}$ is averaged over one luminosity block with the length of 60 seconds. Each luminosity block is assigned to an individual, incrementing integer luminosity block number (LBN).

$$
L=\frac{f \cdot N}{\epsilon_{L M}\left(\sigma_{e}+\sigma_{i}\right)}
$$

Where $N$ is the number of detected elastic and inelastic scattering events, $\epsilon_{L M}$ the total efficiency and acceptance of the luminosity detector, $f$ the beam crossing frequency and $\left(\sigma_{e}+\sigma_{i}\right)$ the effective elastic and inelastic cross section. The efficiency and acceptance uncertainty $\delta \epsilon_{L M}$ is of similar size than the inelastic proton scattering cross section uncertainty. For the studied data taking period the total luminosity uncertainty is $6.5 \%$.

The detector is only able to distinguish if there was no event or if there was at least one event. It cannot distinguish between one and more than one event. However, with increasing luminosity more than one interaction might occur at one beam-crossing. This has to be considered in the luminosity calculation. The probability of $n$ interactions in one cross section is given by the Poisson distribution $P(n)=\frac{\mu^{n}}{n !} e^{-\mu}$, where $\mu$ is the average of interactions per crossing (see also Fig. 4.5) and can be extracted from the probability of zero interactions in a given crossing $P(0)=e^{-\mu}$. This can be measured by the luminosity detector.

Besides the detection of elastic and inelastic scattering events, the luminosity detector measures the beam-halo rates and provides a fast measurement of the z-coordinate of the interaction vertex.

In Fig. 3.9 the data taking profile of a typical store is shown, and in Fig. 3.10 the total integrated luminosity recorded by $\mathrm{D} \varnothing$ and the $\mathrm{D} \varnothing$ data taking efficiency, which is the ratio of delivered and successfully recorded luminosity, can be found. 


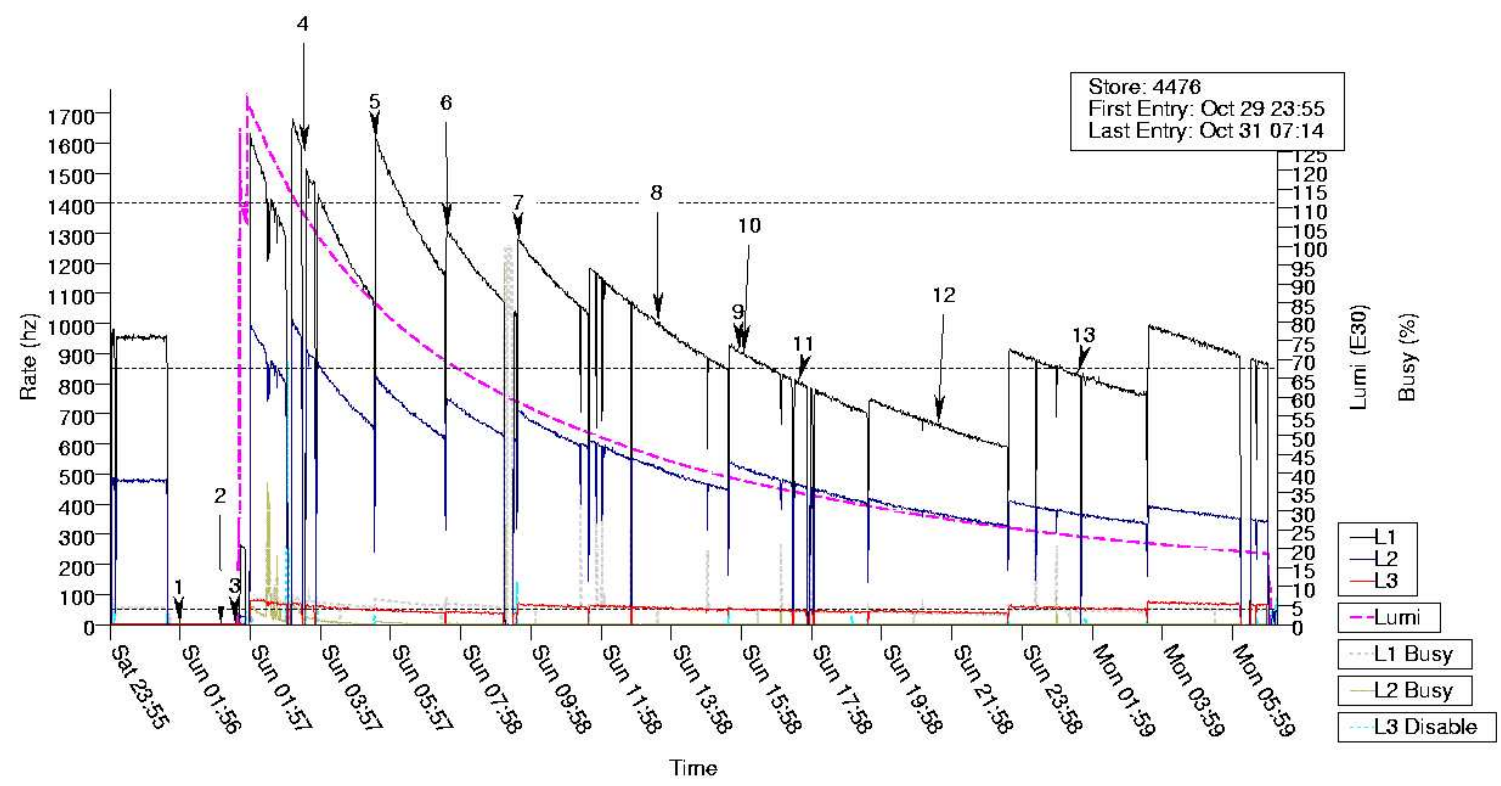

Figure 3.9: A typical physics store [110]. The magenta, dashed line refers to the instantaneous luminosity, $135 \cdot 10^{30} \mathrm{~cm}^{2} \mathrm{~s}^{-1}$ at the beginning. The solid black, blue and red lines are the Level 1 , Level 2 and the Level 3 output rates, details can be found in Sec. 3.4. Each run, referred to by numbers, is ended after approx. two hours and the set of triggers (the trigger list) is changed to account for the changed luminosity. $\mathrm{D} \emptyset$ has recorded $3.96 \mathrm{pb}^{-1}$ in this store with an absolute data taking efficiency of $89.4 \%$.
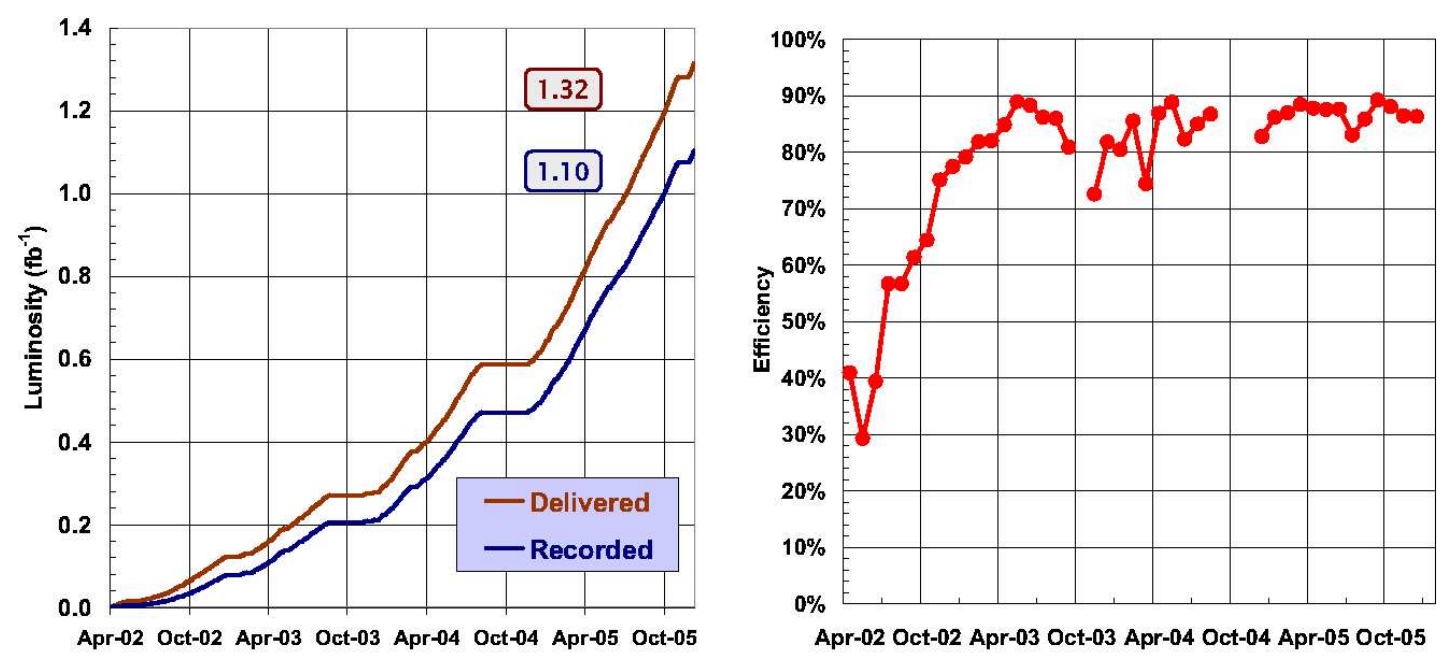

Figure 3.10: Total integrated $D \emptyset$ luminosity (left) and absolute $D \emptyset$ data taking efficiency per month (right) [110]. 


\subsubsection{The Central Tracking Systems}

Excellent knowledge about the particle trajectories is important for all studies. The tracking system at DØ in Run II consists of the silicon microstrip tracker (SMT) and the central fiber tracker (CFT) and is embedded in a superconducting solenoid magnet with radius $r=50 \mathrm{~cm}$ and length $l=2.70 \mathrm{~m}$. The magnetic field is 2 Tesla and approximately homogeneous in the inside of the magnet. Charged particles are forced onto a helix trajectory and will loose energy on their way through the tracker material. This energy deposition in form of ionization in the silicon sensors or in form of light in the fibers is detected. The five helix parameters, such as origin, direction and curvature are calculated. Momentum information can be extracted and an energy calibration of the calorimeter using $E / p$ is possible. Both tracking detectors combined locate the primary interaction vertex with a resolution of $35 \mu \mathrm{m}$. Secondary vertices, for example from b-quarks, can be detected with impact parameters better than $15 \mu \mathrm{m}$ for tracks with transverse momenta $p_{T}>10 \mathrm{GeV}$ and $|\eta|=0$. Together, the silicon microstrip and the central scintillating fiber tracker achieve a momentum resolution of $\Delta p_{T} / p_{T}^{2}=0.002 \mathrm{GeV}^{-1}$.

An overview over the tracking system is given in Fig. 3.11. The beryllium beam pipe with on outer radius of $19.05 \mathrm{~mm}$ and a wall thickness of $0.508 \mathrm{~mm}$ is surrounded by the $2.42 \mathrm{~m}$ long SMT detector. The CFT surrounds the SMT and is embedded in a 2 Tesla superconducting solenoid magnet. The magnet and therefore the complete tracking system was designed to fit into the central calorimeter. Between the magnet and the

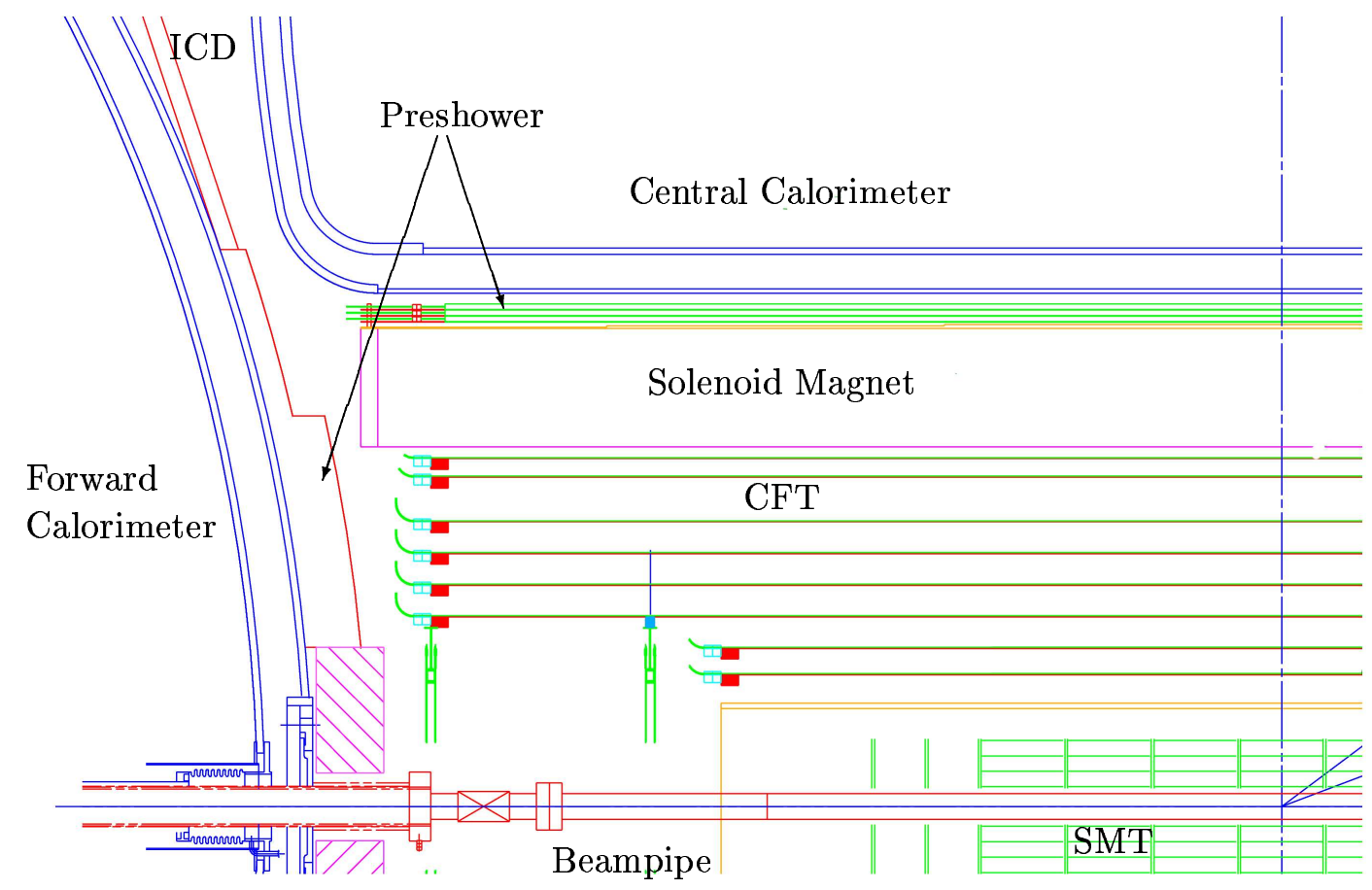

Figure 3.11: Cross section view in the rz-plane of the upper left quarter of the $D \emptyset$ tracking system [96]. 
central calorimeter and between the forward and the central calorimeter scintillating fiber preshower detectors are located.

In Fig. 3.12 a rather particular picture of the $D \emptyset$ tracking system can be found: It was taken by the tracking system itself [111]. For this picture the interaction point of reconstructed di-electron tracks of opposite charge but approximately same energy were calculated. These points are the vertices of photon conversion $\gamma \rightarrow e^{+} e^{-}$.

Photon conversion is possible if the photon energy exceeds the threshold of two electron masses $h f \geq 2 \cdot m_{e}$. At the threshold, however, the kinetic energy of the electrons is small and the momentum is negligible. Since momentum conservation must be given, the photon momentum $p_{\gamma}=h f$ must be absorbed by the nucleus of some surrounding material, as discussed in Sec. 3.1.1. Therefore photon conversion takes place in material, and a material map by vertex-reconstruction can be obtained.
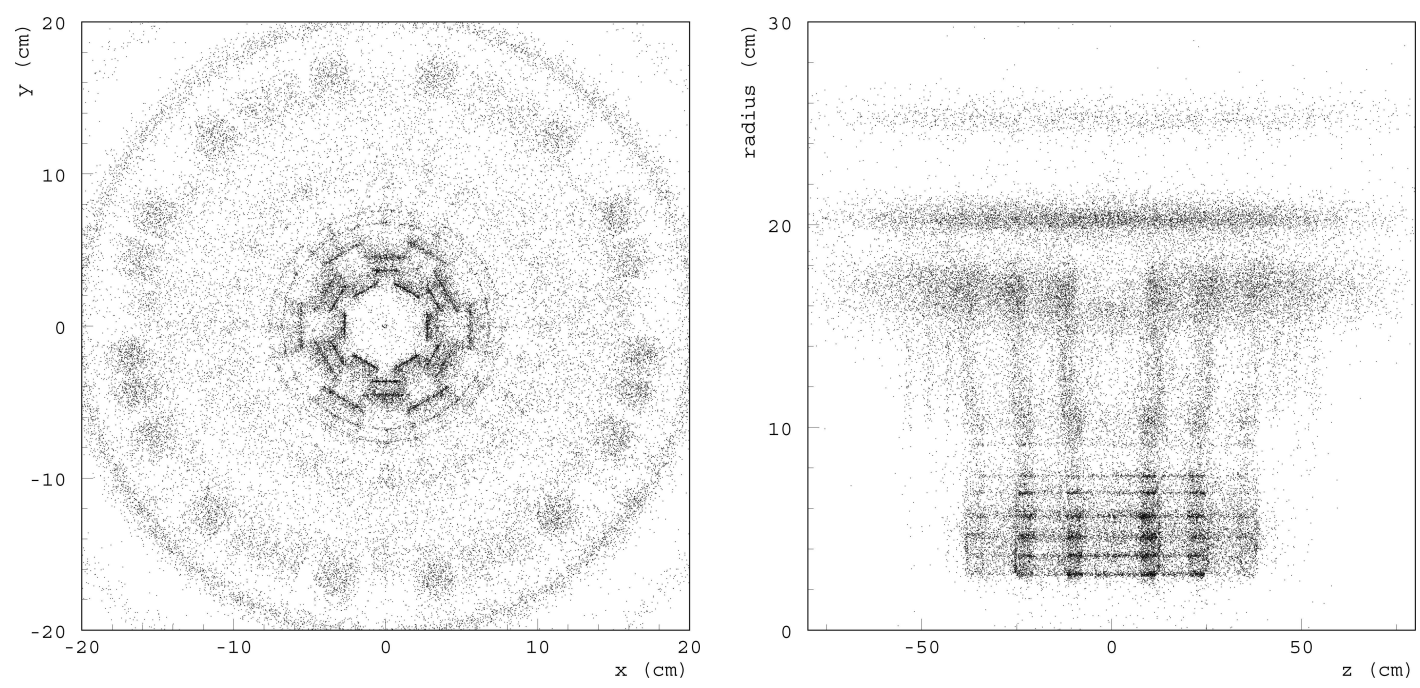

Figure 3.12: Detector imaging by reconstruction the vertex of photon conversion $\gamma \rightarrow e^{+} e^{-}$with the "AA-track" algorithm in the $x y$-plane (left) and in the $r z$-plane (right) [111]. The material of the SMT and CFT detectors can be clearly identified.

\section{The Silicon Microstrip Tracker (SMT)}

The design of the SMT was dictated by the length of the nominal interaction region, which is quite long; of the order of $50 \mathrm{~cm}$. The tracks coming from the interaction region should penetrate the sensors approximately perpendicular to allow for most precise measurements. An isometric view of the SMT is shown in Fig. 3.13. The detector consists out of 6 barrel sections with sensors parallel to the beam line and 16 disks orthogonal to the beam line. Each barrel has four layers equipped with silicon sensors. The innermost layers 1 and 2 have 12 silicon readout modules, called ladders, the two outer layers have 24 ladders, for a total of 432 ladders. Each barrel section is capped with a disk of twelve double-sided wedge detectors, called $F$-disks. In the far forward region, large-diameter $H$-disks of 24 "full" wedges, each consisting of two single-sided "half" wedges, provide tracking at high 


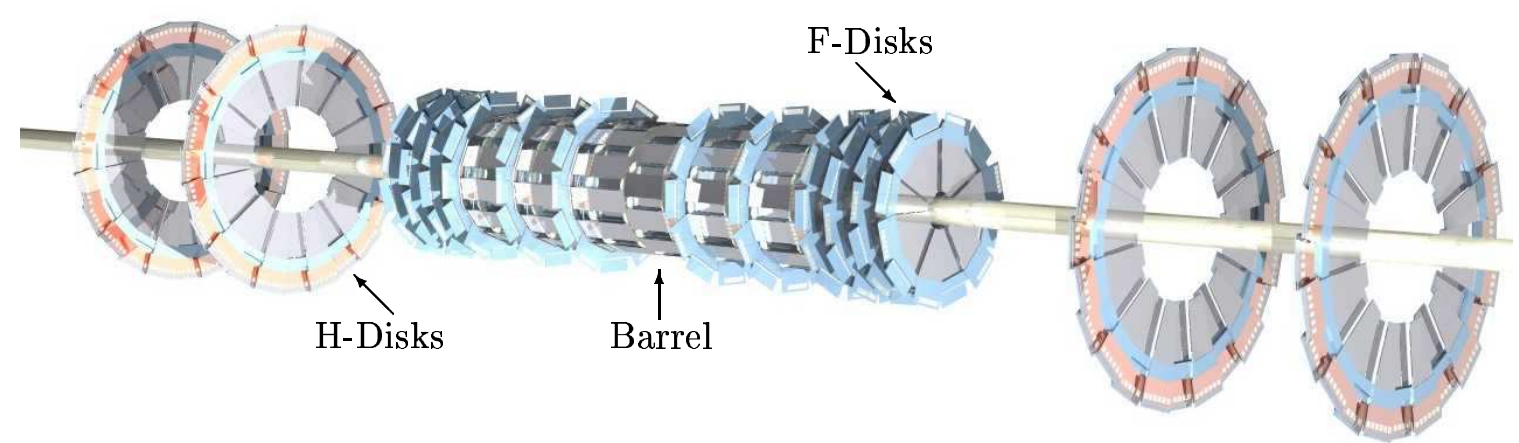

Figure 3.13: Silicon Microstrip Tracker (SMT) drawing [96].

$|\eta|$. There are $144 \mathrm{~F}$-wedges and 96 full $\mathrm{H}$-wedges. The twelve F-disks are located at $|z|=12.5,25.3,38.2,43.1,48.1$ and $53.1 \mathrm{~cm}$, the four H-disks are located at $|z|=100.4$ and $121.0 \mathrm{~cm}$.

The sensors are $300 \mu \mathrm{m}$ thick n-type silicon wafers. The ionization charge deposition of the charged particle that transits the sensor, is collected by strips of $\mathrm{p}-\mathrm{or}^{+} \mathrm{n}^{+}$-type. The pitch between two strips is between $50 \mu \mathrm{m}$ and $150 \mu \mathrm{m}$.

The barrel detectors primarily measure the $r-\phi$ coordinate, while the disk detectors measure $r-z$ as well as $\phi$. The precise knowledge of the tracks $\phi$-component is more important than the $z$-component, because the momentum reconstruction is proportional to the measurement of the track curvature, and a solenoid field affects only the $\phi$-coordinate of charged tracks.

There are three different types of sensors used for the barrel: The inner four barrel sections use double-sided sensors in all four layers which are orientated parallel to the beampipe to allow the precise measurement of the $\phi$-coordinate, and $90^{\circ}$ stereo double-sided sensors in the first and the third layer to measure the $z$-component. The $90^{\circ}$ readout was accomplished by a second metal layer insulated from the first, by a $3 \mu \mathrm{m}$ thick layer of silicon oxide. The second and the forth layers of the two outer barrels use double-sided stereo sensors; the strips of both sides are arranged under a $2^{\circ}$ angle to allow also the reconstruction of the $z$-coordinate. The first and the third layers of the outer barrel make use of single-side sensors with axial strips.

The F-disk sensors are double-sided, while two single-sided sensors are mounted back-toback to form a wedge for the H-disks. The disk-sensors have the form of a trapezoid, the strips are always parallel to one long edge of the devices. This provides an effective $30^{\circ}$ stereo angle for the 12-wedge F-disks and a $15^{\circ}$ stereo angle for the 24-wedge H-disks.

The SMT has 912 readout modules with a grand total of 792,576 channels. The cross section view of a double-sided barrel sensor and readout is shown in Fig. 3.14. The silicon sensor and the high density interconnection (HDI) to the SVXIIe readout chips are fixed to a beryllium frame which also serves as heat spreader. 
The SMT resides in the realm of high radiation. It was designed to resist a radiation dose of about $2 \mathrm{Mrad}$ or $3.5-6 \mathrm{fb}^{-1}$. It is expected that microdis-

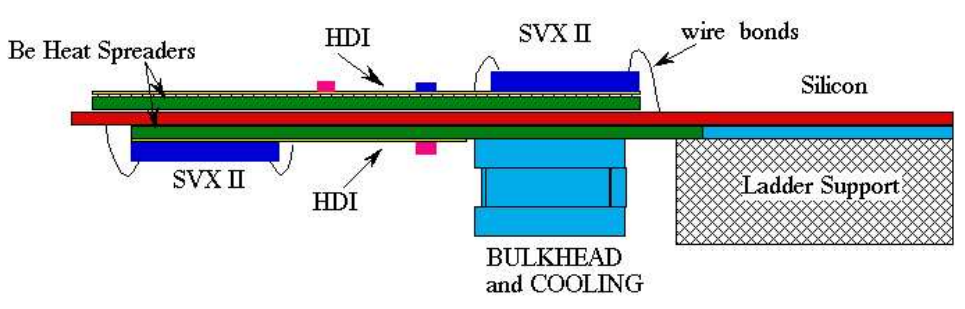
charges in the sensors will limit the lifetime of the SMT. During the long shutdown in summer 2006 the SMT was upgraded with a new barrel layer. The

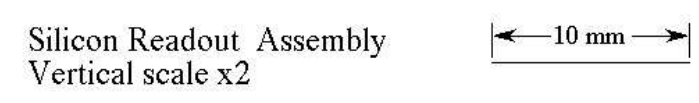

Figure 3.14: SMT Sensor and Readout [112]. Layer $\varnothing$ was inserted between the first barrel layer and a new beryllium beam pipe. Especially the secondary vertex tagging is expected to benefit from this upgrade. The Layer $\varnothing$ will ensure the $D \varnothing$ tracking capability even if parts of the present SMT die of radiation. The measurement of the time-dependent $B_{s}$ oscillation is critically depending on the tracking resolution and therefore a benchmark scenario for the improvement of the tracker: The time resolution is expected to improve from 105 fs ( $\eta$-weighted average) to $74 \mathrm{fs}$ by the addition of the Layer $\varnothing[113]$.

\section{The Central Scintillating Fiber Tracker (CFT)}

The central fiber tracker consist of scintillating fibers, $835 \mu \mathrm{m}$ in diameter, mounted on eight concentric carbon-fiber support cylinders. The two innermost cylinders are $1.66 \mathrm{~m}$ and the outer six cylinders are $2.52 \mathrm{~m}$ long; they cover a radial space from 20 to $52 \mathrm{~cm}$ from the beam pipe and a polar space of up to $|\eta|<1$.7. Each cylinder supports one doublet layer of fibers oriented along the beam axis and a second doublet layer at a stereo angle in $\phi$ of $+3^{o}$ (layer $1,3,5$ and 7 ) or $-3^{o}$ (layer 2,4,6 and 8). The two fiber layers forming a doublet are offset by half a fiber width for improved coverage, as shown in Fig. 3.15.

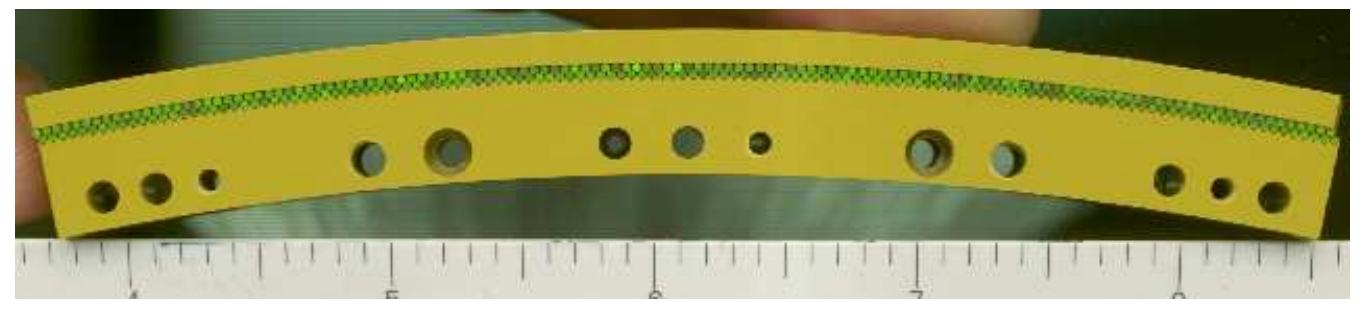

Figure 3.15: CFT fiber double layer [96]. The fibers are offset by half a fiber and coated at one end with a sputtered aluminum mirror.

While one end of the fibers is mirrored by a sputtered aluminum coating providing a reflectivity of about $90 \%$, the other end is connected to a clear waveguide fiber of $7.8 \mathrm{~m}$ to $11.9 \mathrm{~m}$ length. The waveguides end on visible light photon counters (VLPC, Fig. 3.16) housed in a liquid helium cryostat below the $\mathrm{D} \varnothing$ detector. 

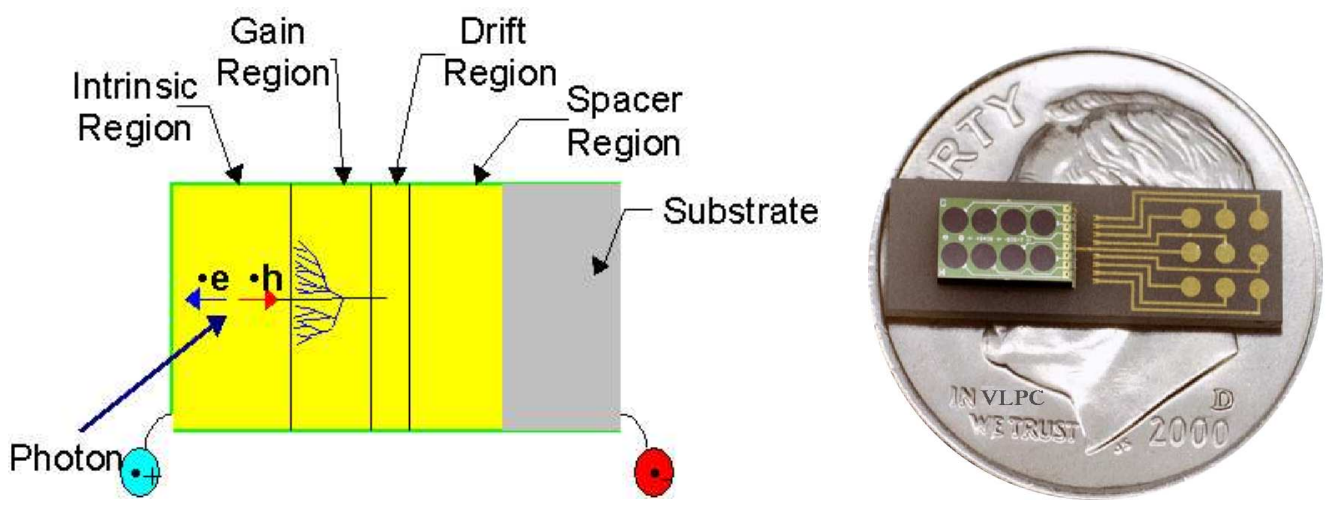

Figure 3.16: Visible Light Photon Counter (VLPC) [114].

The VLPCs are impurity-band silicon avalanche photodetectors operating at 9 Kelvin and are capable of detecting single photons with a quantum-efficiency of $85 \%$ at $530 \mathrm{~nm}$ wavelength. Only one of the two carrier types participates in carrier multiplication, in contrast to standard avalanche photo diodes. The gain dispersion is reduced considerably, since the carrier multiplication takes place across a band gap of only $50 \mathrm{meV}$. The small gap is due to the formation of an impurity band $50 \mathrm{meV}$ below the conduction band. This is accomplished by a high donor concentration (ca. $10^{17} / \mathrm{cm}^{3}$ ) and low counterdoping ca. $10^{15} / \mathrm{cm}^{3}$ ) in the p-layer of the diode [115].

The VLPC side connected to the waveguide fibers is coated with an antireflective a transparent anode metalization. An entering photon will create an electron-hole pair across the standard valence-to-conduction band gap in the intrinsic (non-doped) zone, see Fig. 3.16(a) for reference. The hole drifts through the high field depletion region ("gain layer") and into the impurity band p zone ("drift layer"). Upon collision with a neutral donor, an electron is freed, which starts the electron avalanche. Due to space charge effects (created by the slowly drifting cloud of D+'s) the avalanche process is self-limiting [115].

The fibers are fabricated out of polystyrene (PS) and doped with (1\% by weight) organic, fluorescent paraterphenyl (PT) dye and with $0.15 \%$ of 3 -hydroxyflavone (3HF) dye. If a charged particle travels through the fiber it excites the PS core material, which excites rapidly, non-radiative the $\mathrm{PT}$, which again emits very fast (order of nano-seconds) a photon with $340 \mathrm{~nm}$ wavelength. The PS is intransparent at this wavelength, but the $3 \mathrm{HF}$ acts as a wave-shifter: It absorbs the $340 \mathrm{~nm}$ photon and re-emits a photon at $530 \mathrm{~nm}$ which is well transmitted in PS.

The schematic design of the central fiber tracker is shown in Fig. 3.17. The CFT has about 76800 readout channels, the central and forward pre-shower detectors which have a similar design have additional 22564 channels. 


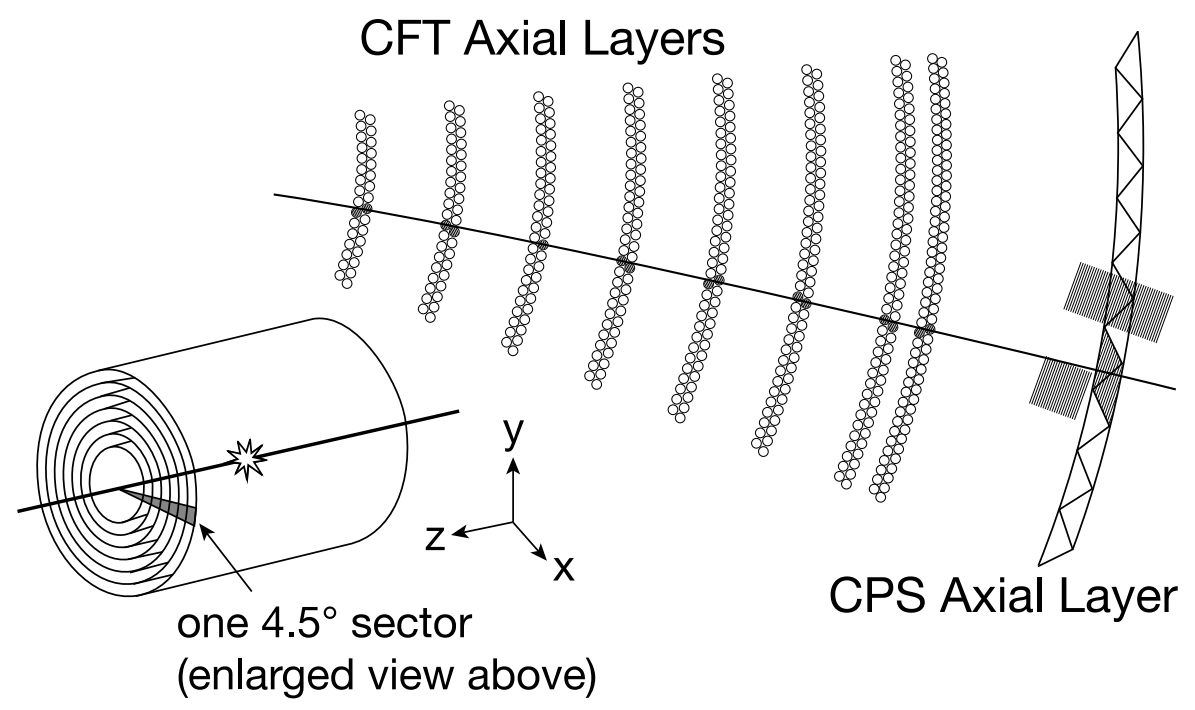

Figure 3.17: Sketch of the central scintillating fiber tracker [96]. The drawing is not to scale.

\subsubsection{The Calorimeter System}

The $\mathrm{D} \varnothing$ Calorimeter was designed to provide energy measurements for electrons, photons, and for jets and to assist in the identification of electrons, photons, jets and muons. It was inherited from $\mathrm{D} \varnothing$ Run I and the calorimeter itself is unchanged. However, more material - the tracking system and the solenoid magnet - equal to 2 to 4 radiation length ${ }^{1}$ $X_{0}$, were inserted. The readout electronics were replaced to cope with the increased readout frequency of $2.5 \mathrm{MHz}$. The calorimeter detector is divided into four parts; into the three liquid-argon sampling calorimeters, each housed in its own cryostat, and an intercryostat detector. The central calorimeter (CC) covers $|\eta| \lesssim 1$ and the two endcap calorimeters ECN (north) and ECS (south) extend the coverage up to $|\eta| \lesssim 4$. A sketch of the calorimeter system is shown in Fig. 3.18.

Together with the tracking system the identification of electrons $(E / p)$, photons (electromagnetic shower profile and absence of track) and b-jets resulting out of b-quarks is possible. Muons are minimal ionizing particles (MIP) and can be identified by the typical MIP signal, an energy deposition of $2-3 \mathrm{GeV}$ along their track. Since the transversal energy of the initially colliding partons is approximately zero, the transverse energy of not interacting particles, like neutrinos, can be reconstructed from the energy balance of the hermetically closed calorimeter. This is not possible for the total energy, since the longitudinal momentum fraction of the initial partons is unknown at hadron colliders.

\section{The Liquid Argon Calorimeter}

The DØ Liquid Argon Calorimeter is segmented in 18 layers, while each layer has 74 segments in $\eta$ and 64 segments in $\phi$, for a grand total of 85248 cells. The calorimeter cells

\footnotetext{
${ }^{1}$ One radiation length $X_{0}$ is the distance an electron can travel in material, until its energy $E$ drops to $1 / e \cdot E$ or by $63.2 \%$.
} 


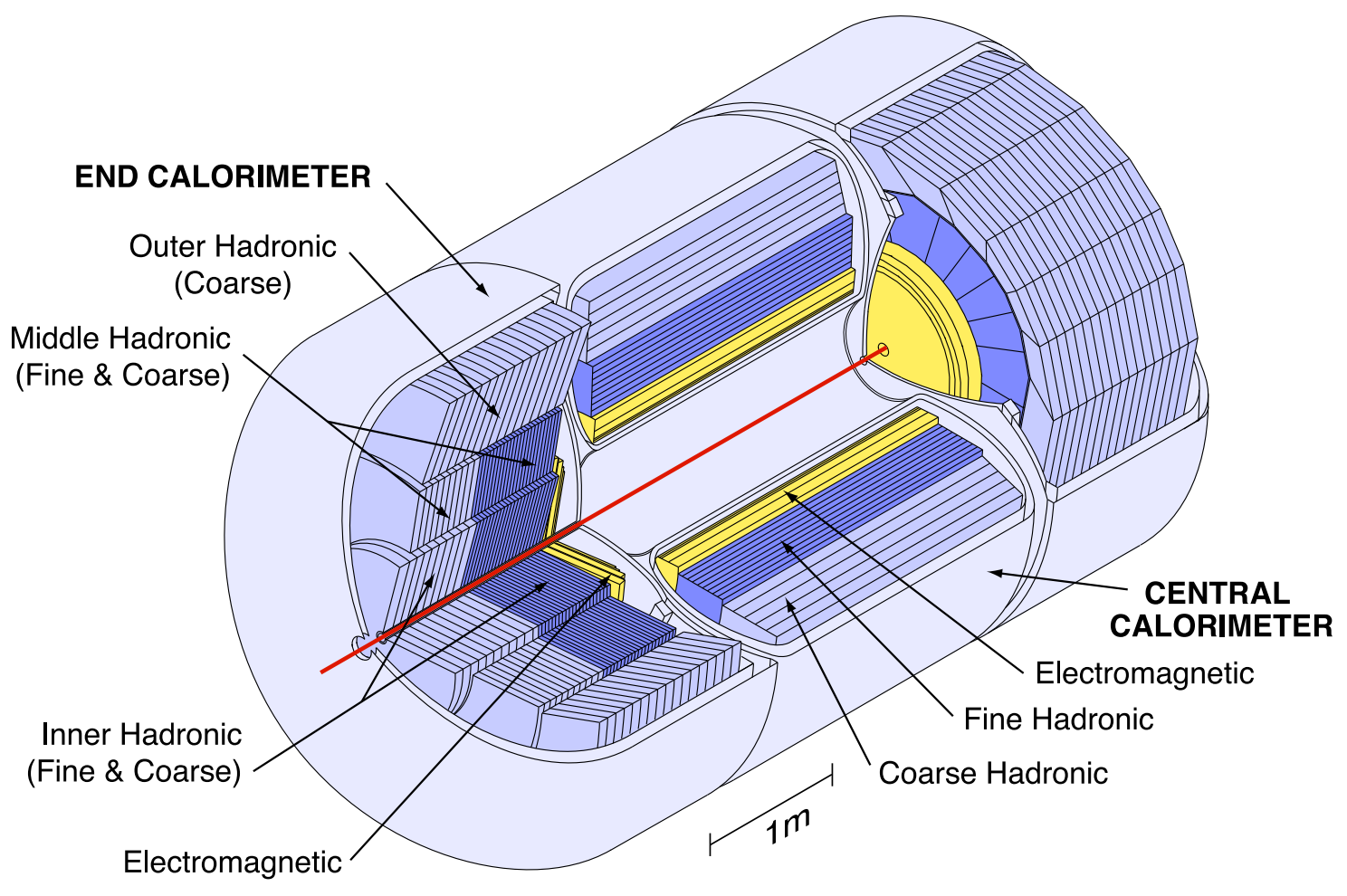

Figure 3.18: Isometric view of the central and the two endcap calorimeters [96].

are arranged in pseudo-projective towers, as shown in Fig. 3.19. The center of the cells lie on rays projecting from the center of the interaction region, but the cell boundaries are aligned perpendicular to the absorber plates, thus pseudo-projective. Different absorber plates are used in different locations, in order to achieve a similar energy response of the (compensating) calorimeter for electro-magnetic and hadronic particles. The inner electromagnetic part of the calorimeter, layer $1-4$, uses $3 \mathrm{~mm}(4 \mathrm{~mm})$ thin plates in the CC (EC) made from pure, depleted uranium. All 4 layers together have about $20 X_{0}$ interaction lengths. The absorber plates in the fine hadronic sections are made from $6 \mathrm{~mm}$ uraniumniobium alloy. The coarse hadronic modules contain $46.5 \mathrm{~mm}$ thick plates of copper in the CC and stainless steel in the EC.

A typical calorimeter cell is shown in Fig. 3.20. It consists of the absorber plate, the liquid argon active medium and a signal board. The signal boards are made from two $0.5 \mathrm{~mm}$ thick G-10 sheets. The outer surfaces facing the liquid argon gap are coated with carbon-loaded epoxy with high resistivity. The electric field is established between the grounded absorber plate and the high voltage (typically $+2.0 \mathrm{kV}$ ) epoxy electrode. The inner surface of one G-10 sheet is uncoated while the other is coated with copper. The copper pad is divided into a pattern, as necessary for the segmented readout. Charged particles (Hadrons from hadronic jets or electrons from electromagnetic jets) traversing the liquid argon gap ionize the Argon atoms. The electric field forces the drift electrons onto the charge collecting signal boards. The maximal electron drift time across the $2.3 \mathrm{~mm}$ liquid argon gap is $450 \mathrm{~ns}$, which provides a challenge for the signal charge integration, as the beam crossing frequency in the Run II is every $396 \mathrm{~ns}$. 


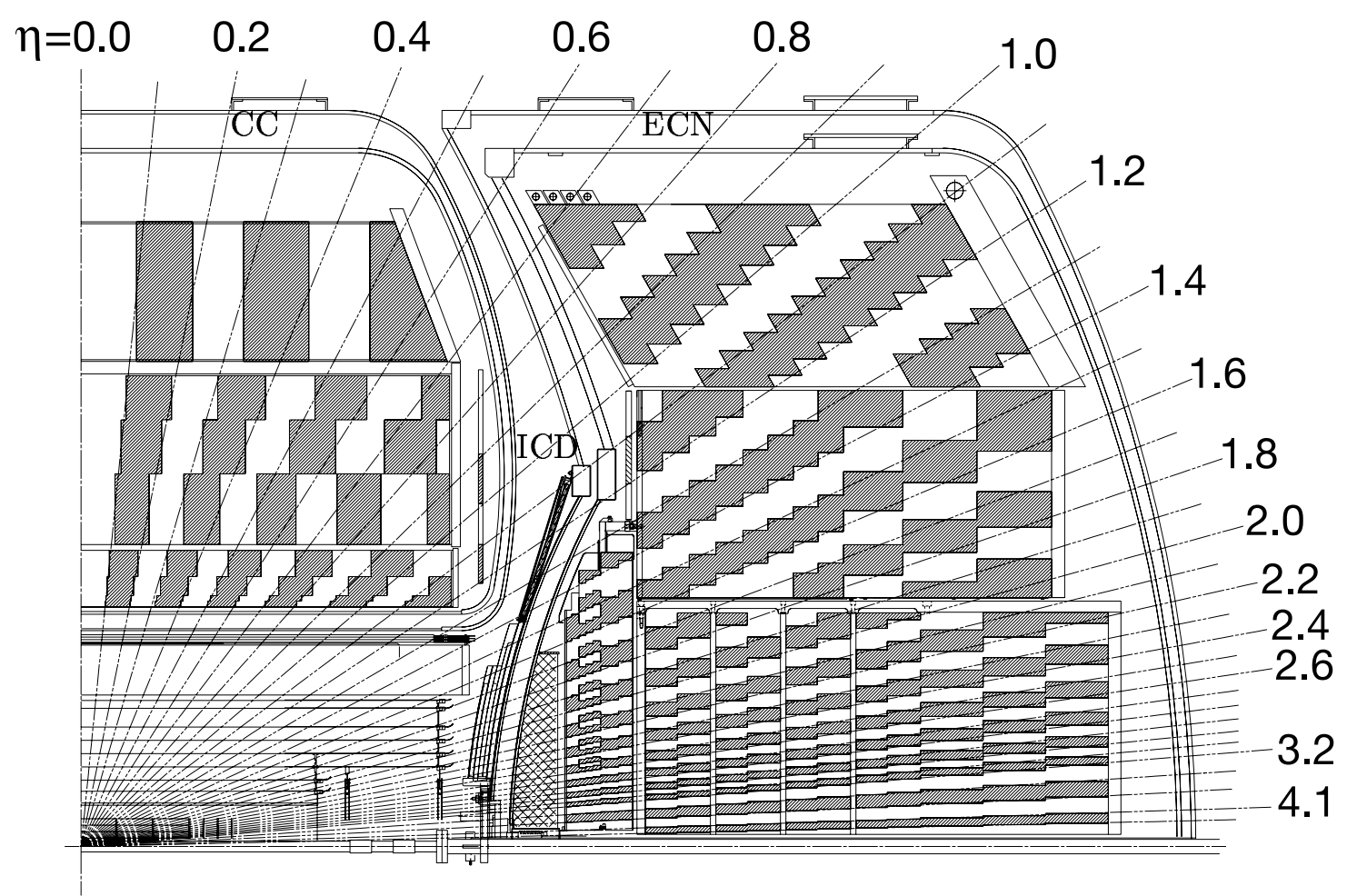

Figure 3.19: Schematic view of the $D \emptyset$ calorimeter showing the segmentation pattern. The shading indicates groups of cells ganged together for the signal readout. The intercryostat detector (ICD) is located between the central and the endcap calorimeter [96].

The inner first four layers of the calorimeter are called the electro-magnetic section, because of the electro-magnetic shower range from electrons or photons. The longitudinal shower of a hadronic jet reaches its maximum far behind the electro-magnetic layers of the calorimeter in the hadronic section due to the larger nuclear interaction length $X_{\text {nuclear }} \gg X_{0}$, while the shower from electro-magnetic objects reaches its maximum in the third layer. The size of most readout cells is $\Delta \eta \times \Delta \phi=0.1 \times 0.1$ and is therefore of the order of the transverse shower radius of hadronic jets $\mathcal{O}(10 \mathrm{~cm})$ and electro-magnetic objects $\mathcal{O}(2 \mathrm{~cm})$. To improve the spatial resolution of electrons and photons the third layer, where the EM showers are maximal, has a finer segmentation $\Delta \eta \times \Delta \phi=0.05 \times 0.05$.

\section{The Intercryostat Detector}

Since the Liquid Argon Calorimeter is divided into three cryostats, it has incomplete coverage in the region $0.8 \leq|\eta| \leq 1.4$. To account for this problem an intercryostat detector (ICD) is installed, covering $1.1 \leq|\eta| \leq 1.4$. A twice as large $|\eta|$ region was covered by the ICD in Run I, but the space is now needed for the cabling of the tracking system. The ICD is a ring of 16 trapezoid shaped, $1.27 \mathrm{~cm}$ thick scintillating tiles enclosed in light-tight aluminum boxes. Each tile covers an area of $\Delta \phi \times \Delta \eta=0.4 \times 0.3$ and is divided into 12 cells which cover $\Delta \phi \times \Delta \eta=0.1 \times 0.1$ each. The scintillator tiles are 


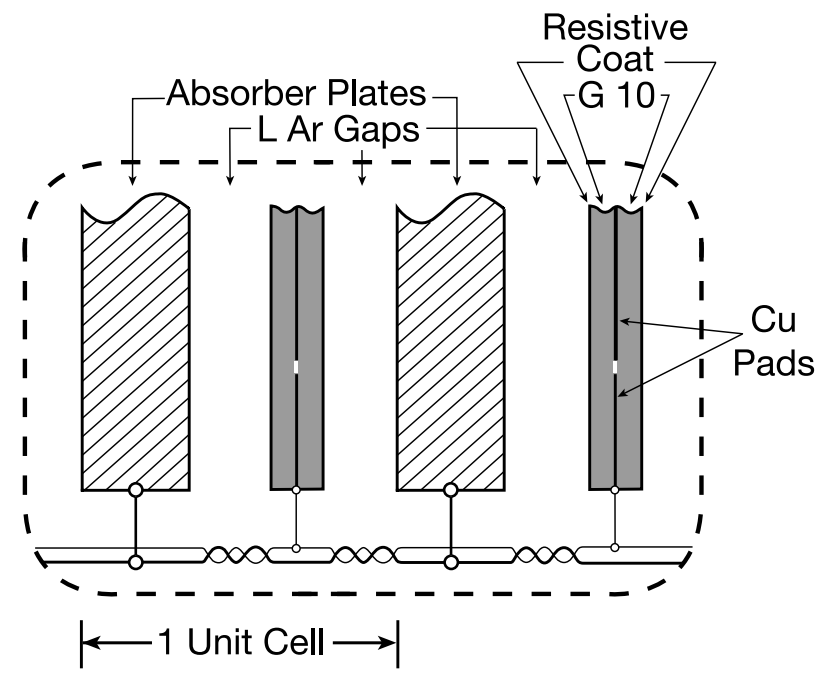

Figure 3.20: Schematic view of a calorimeter readout cell [96].

connected by optical fibers to Photomultiplier tubes contained in a drawer system in a low magnetic-field region. See Fig. 3.11 and Fig. 3.19 for the location of this detector.

\section{Calorimeter Performance}

The energy resolution of the $\mathrm{D} \varnothing$ calorimeter was studied in a test beam with pions, electrons and muons with energies between 10 and $150 \mathrm{GeV}$ [116]. The energy resolution is parametrized by

$$
\delta(E)=\frac{\sigma(E)}{E}=\frac{N}{E} \oplus \frac{S}{\sqrt{E}} \oplus C
$$

where $N$ is related to noise from the electronics or from uranium decays, the term $S$ is given by statistical sampling fluctuations and is dominating for the relevant particle energy range, and the constant term $C$ represents systematical effects like calibration errors. The three terms are added quadratically.

\begin{tabular}{lcrr}
\hline \hline type & $\mathrm{N}$ & $\mathrm{S}$ & $\mathrm{C}$ \\
\hline electro-magnetic (from $e$ ) & $0.43 \mathrm{GeV}$ & $0.135 \sqrt{\mathrm{GeV}}$ & 0.0115 \\
hadronic (from $\pi$ ) & $0.975 \mathrm{GeV}$ & $0.41 \sqrt{\mathrm{GeV}}$ & 0.032 \\
\hline \hline
\end{tabular}

Table 3.3: Calorimeter energy resolution parameters $[116,117]$.

The parameters as given in table 3.3 were determined by comparing the known beam energy $E_{\text {beam }}$ of the particle $(e / \pi)$ to the measured energy $E_{c a l}$ and minimizing the $\chi^{2}$

$$
\chi^{2}=\sum_{\text {events }} \frac{\left(E_{b e a m}-E_{c a l}\right)^{2}}{\sigma\left(E_{c a l}\right)^{2}}
$$


where $\sigma(E)$ is the resolution as given by Eq. (3.10). The jet energy resolution obtained in Run II differs from the pre-Run I testbeam pion resolution. Beside physical arguments for different calorimeter responses for hadronic jets depending on the neutral pion content $\left(\pi^{0} \rightarrow \gamma \gamma\right)$ and therefore the electromagnetic energy fraction, Run II upgrades are responsible for a degrading energy resolution:

- The 5 times higher beam crossing frequency leads to a shorter time, over which the accumulated signal charge can be integrated. This results in larger fluctuations, leading to larger $S$.

- The additional radiation length from the solenoid and the tracking system affect the $S$ term.

- The Run II calorimeter signal amplifiers were found to have a worse bearing on noise which increases $N$.

More detailed information, estimation of the DØ Run II jet energy resolution, the Jet Energy Scale (JES) calibration and jet reconstruction efficiencies will be discussed in Sec. 5.2.

\subsubsection{The Muon System}

Muons can be considered as stable particles, due to their long lifetime of $2.2 \mu \mathrm{s}$. The muon system [118] is therefore the outmost sub-system of the $\mathrm{D} \varnothing$ detector and includes a large toroid magnet. It ensures the identification of muons and provides trigger signals for muonic events. The spatial and the momentum resolution of the muon system allow for a matching with central tracks with an efficiency close to 1 , see section 5.1 for details. The muon system can be divided into the central part $|\eta| \leq 1.0$ and the two forward sections which extent over $1.0 \leq|\eta| \leq 2.1$. A detailed drawing of the complete system can be seen in Fig. 3.21.

\section{The Toroid Magnet}

The toroid was the single magnet of $\mathrm{D} \varnothing$ Run I and essential for the muon momentum measurement. With the Run II two Tesla superconducting solenoid magnet the muon momentum is now dominated by the SMT and CFT tracker. The toroid magnetic field is still useful, since it enables a low $p_{T}$ cutoff in the Level 1 muon trigger, it allows for a cleaner matching with central detector tracks, it shields the outer central muon chambers from hadronic punch-throughs and it improves the momentum resolution for high momentum muons. To save a significant amount of operation costs the current was lowered from $2500 \mathrm{~A}$ in Run I to $1500 \mathrm{~A}$ now, equivalent to a magnetic field of about 1.8 Tesla which is about $6 \%$ lower compared to Run I. The field polarization of solenoid and toroid were changed during the data taking period independently every two weeks.

The central toroid is a $109 \mathrm{~cm}$ thick square annulus, the shortest distance of its inner surface to the beamline is $318 \mathrm{~cm}$. It covers $|\eta| \lesssim 1$ and is divided into three sections to 


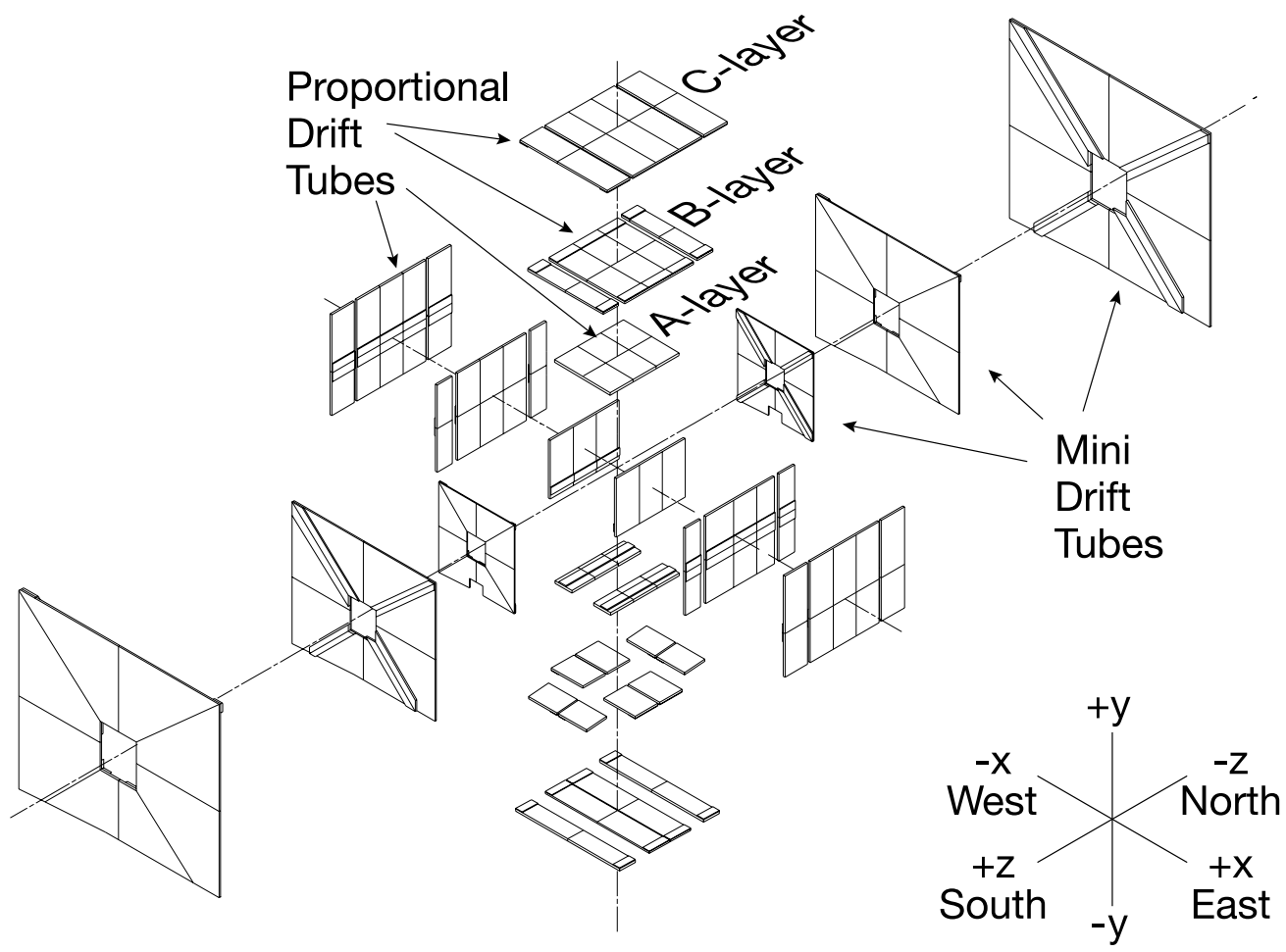

(a) Drift chambers

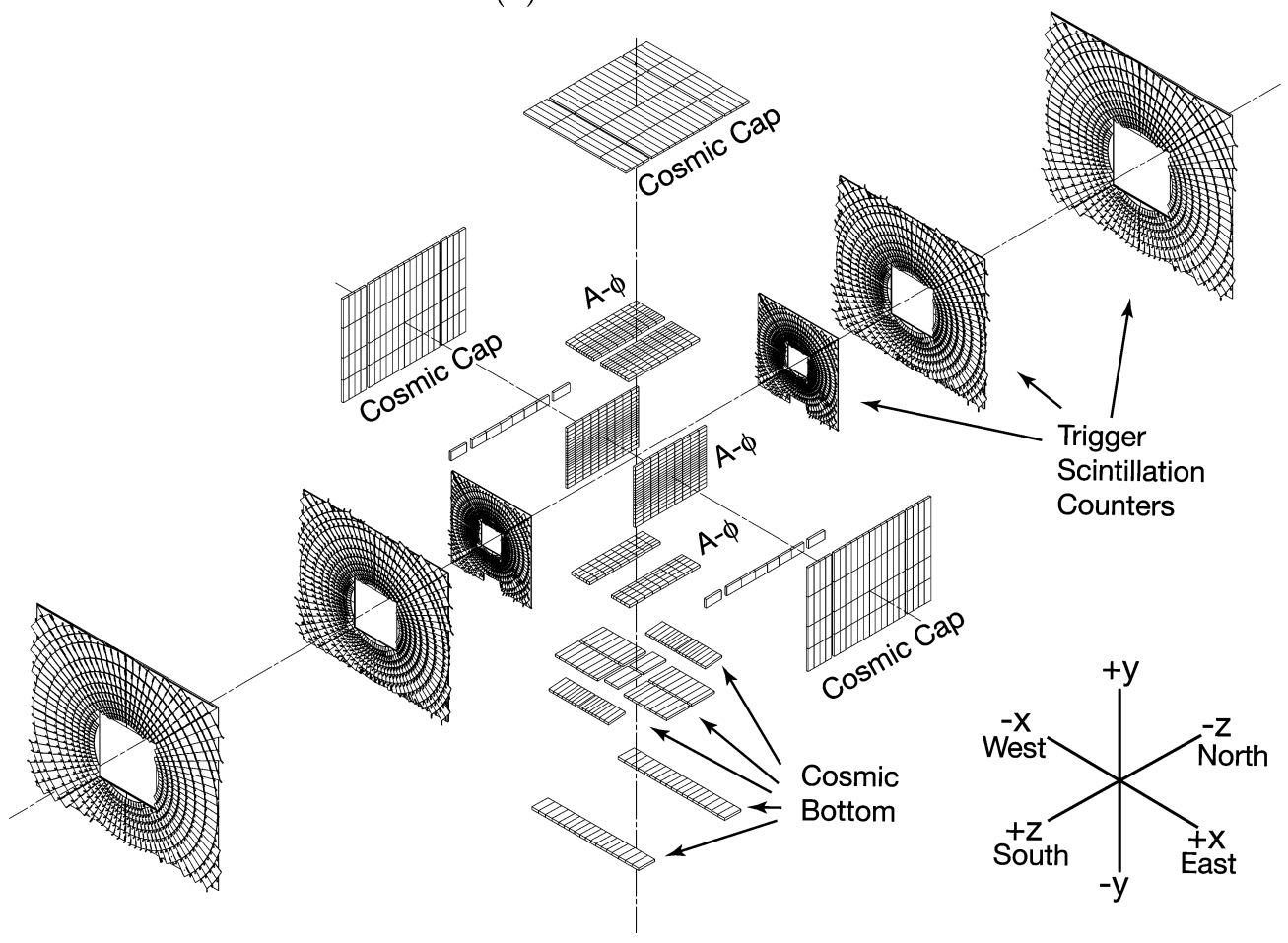

(b) Scintillator counters

Figure 3.21: Exploded drawing of the $\mathrm{D} \emptyset$ muon system [96], the proportional drift tubes as used in the barrel region and the mini drift tubes as used in the forward region (a) and the scintillator counters for cosmic veto and trigger purpose (b) are shown separately. 
allow access to the inner part of the detector. Its magnet is wound using 20 coils of 10 turns each. Two end toroids are located at $454 \leq|z| \leq 610 \mathrm{~cm}$. In the center of each end toroid is a square hole, which surface is $183 \mathrm{~cm}$ to the beam pipe. The outer surface of both end and the central toroid is $427 \mathrm{~cm}$ away from the beam pipe. The end toroid windings are 8 coils with 8 turns each.

\section{Shielding}

There are two major non-muon background sources [119] for hits in the forward muon system: Particles coming from the accelerator tunnel that travel parallel to the beam (beam halo) and p $\bar{p}$-collision remnants emitted under small angles. This background can be reduced significantly by placing shielding around the beampipe, see Fig. 3.6 for reference. The shields extent over $6 \mathrm{~m}$ from the outer surface of the forward calorimeter cryostat to the end of the $\mathrm{D} \varnothing$ detector. The low- $\beta$ focussing magnets are embedded in the shielding. The shield consist of three layers of different material:

- Steel $(50 \mathrm{~cm})$ to absorb hadronic and electro-magnetic particles like protons, pions, kaons, electrons and gammas;

- Polyethylene $(12 \mathrm{~cm})$ to absorb neutrons;

- Lead $(5 \mathrm{~cm})$ to absorb remaining gamma rays.

In the shielded detector the total particle flux is reduced by a factor $50-100$, which is in agreement within 50\% with expectations from Monte Carlo simulations [118].

\section{The Central Muon Detector}

The 94 wide angle muon system (WAMUS) drift chambers of the central muon detector are proportional drift tubes (PDT) made of rectangular extruded aluminum. The muon chambers are arranged in three layers, namely the innermost A-layer which is located between the calorimeter and the toroid magnet, the middle B-layer which is mounted on the outside of the toroid coil and the outmost layer C. The PDT chambers have 3 decks each, except for the top and side A-layer chambers, which have 4 decks. A typical chamber is 24 cells wide and covers $2.8 \times 5.6 \mathrm{~m}^{2}$. The chambers are placed such, that the signal wires are parallel to the magnetic field lines, so that the track's curvature and therefore the momentum is measured with best precision.

The rectangular PDT cells are $10.1 \times 5.5 \mathrm{~cm}^{2}$ in cross section and filled with a nonflammable mixture of $84 \%$ Argon, $8 \%$ Methane $\left(\mathrm{CH}_{4}\right)$ and $8 \%$ Tetrafluoromethane $\left(\mathrm{CF}_{4}\right)$. This fast gas is choosen to achieve a high electron drift velocity $\left(v_{\text {drift }}=10 \frac{\mathrm{cm}}{\mu \mathrm{s}}\right)$ and therefore a lower occupancy. The maximal drift time is about $500 \mathrm{~ns}$, while the time between two beam crossings is $396 \mathrm{~ns}$. The operating high voltage for the pads is $2.3 \mathrm{kV}$ and $4.7 \mathrm{kV}$ for the wires. 
The layout of the PDT cells is shown in Fig. 3.22(a). The anode signal wires of two neighboring cells are ganged together; the difference in the signal arrival time $\Delta T$ between a hit cell and the connected cell provides the distance along the wire with a resolution of $10-50 \mathrm{~cm}$, depending on the precise coordinate. If the cell is hit near the readout end, then the signal has to travel two wire lengths to the neighboring readout and disperses on the way. The pads are structured, as shown in Fig. 3.22(b), so that the longitudinal coordinate parallel to the wires can be calculated with good precision $\delta=5 \mathrm{~mm}$ from the charge deposition on the vernier pads. The pad readout is instrumented for all A-layer and $10 \%$ of all B- and C-layer PDT chambers. The resolution in drift direction is better then $500 \mu \mathrm{m}$. More details can be found in [120].

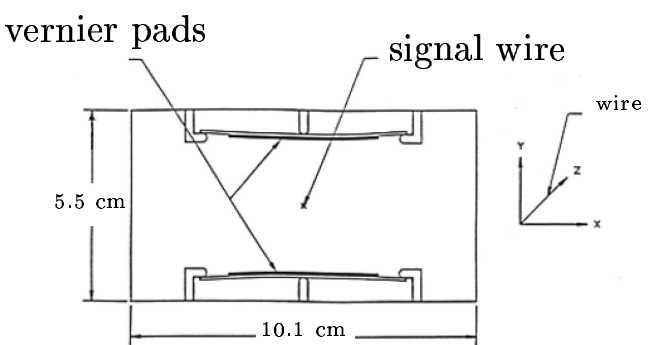

(a)

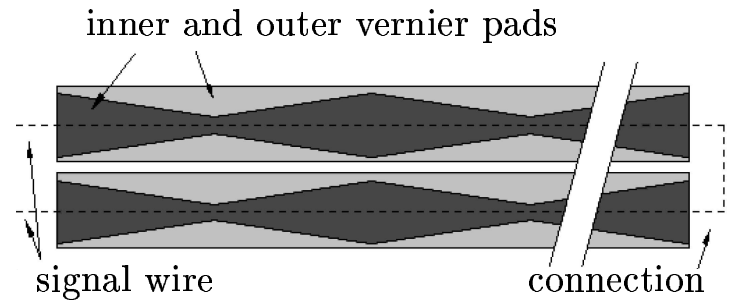

(b)

Figure 3.22: Cross section of a PDT cell (a) and top view on a vernier pad (b) [120]. The vernier pad pattern repeats every $71.0 \mathrm{~cm}$.

The set of the 240 muon scintillation counters in the barrel region is a fast detector for triggering, identifying muons and for out-of-time rejection of background events. The scintillation counters are fast enough, to allow the association of muons in the drift chambers to the correct bunch crossing and to reject cosmic muons. They are installed on top of the detector, on the two (barrel) sides and the bottom on the outer layer of the central drift chambers. An additional $A-\phi$ layer of scintillators has been installed on the inside of the first drift chamber layer between the calorimeter and the toroid magnet, see Fig. 3.6 for reference.

\section{The Forward Muon Detector}

The forward muon system (FAMUS) consists of 3 layers of mini drift tubes (MDT) and 3 layers of scintillation counters. The inner MDT A-layer chambers contain 4 cell-planes, the outer B- and C- layer chambers are built with 3-cell planes each. One MDT chamber covers one octant, the signal wires are oriented in $\phi$ direction. The base unit of a MDT chamber is a eight cell tube as displayed in Fig. 3.23. The tubes are made from $0.6 \mathrm{~mm}$ thick aluminum combs with a $0.15 \mathrm{~mm}$ thin stainless steel cover foil and are inserted into PVC sleeves with variables lengths of up to $583 \mathrm{~cm}$ and thickness $1 \mathrm{~mm}$. 


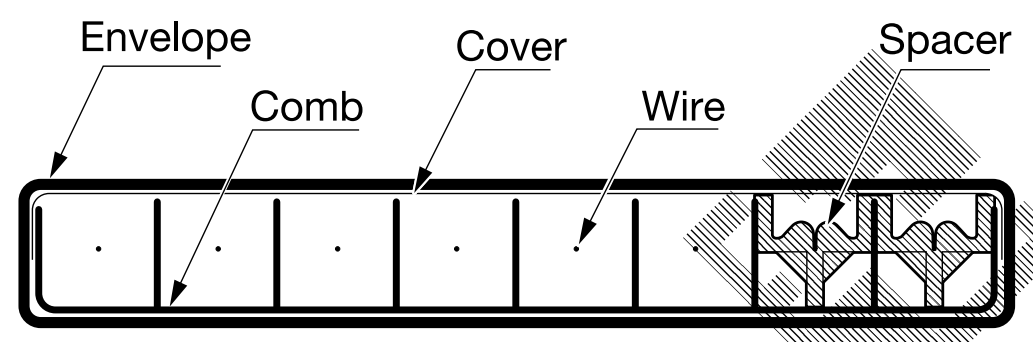

Figure 3.23: Cross section of a mini drift tube [96].

The anode wire is grounded and $-3.2 \mathrm{kV}$ is applied to the cathode. The MDT cells are $9.4 \times 9.4 \mathrm{~mm}^{2}$ in cross section and filled with a non-flammable gas mixture of $90 \% \mathrm{CF}_{4}$ and $10 \% \mathrm{CH}_{4}$. The maximal drift time is $60 \mathrm{~ns}$ and the resolution is better then $1 \mathrm{~mm}$ per cell. The drift chambers are radiation hard and have a low occupancy due to the high segmentation. The efficiency per cell is greater than $99 \%$.

The scintillation counters as well as the MDTs are used for fast trigger information. To associate the MDT hits to the correct bunch crossing, no additional scintillation counter information are necessary, because of the short drift time. More details on the forward muon system can be found in [121] and on the forward trigger scintillation counters in [122].

\section{Performance}

The overall muon momentum resolution is defined for muons with momentum up to approximately $100 \mathrm{GeV}$. The forward muon system is able to improve the resolution for higher momentum muons, in particular in the high $\eta$ region $1.6 \lesssim \eta \lesssim 2.0$, where the muon track does not traverse all layers of the CFT. The stand-alone muon system ("local muon") resolution is determined [123] by studying the variable

$$
\delta\left(p_{T}^{\mu}\right)=\frac{\sigma(q / p)}{q / p}=\frac{q / p(\text { local })-q / p(\text { central })}{q / p(\text { central })}
$$

where $q$ is the electrical charge of the muon and $p$ the muon momentum as measured by the stand-alone muon system (local) or by the central tracker (central).

The functional form of the muon momentum resolution was found to be:

$$
\delta\left(p_{T}^{\mu}\right)=\frac{\alpha \cdot(p-\beta)}{p} \oplus \gamma \cdot p
$$

The resolution is limited by multiple scattering in the calorimeter and the toroid, represented in Eq. (3.13) by $\alpha$. For muon momenta below $40 \mathrm{GeV}$ the standalone resolution is typically $\alpha=20 \%$. The term $\beta$ characterizes the minimal necessary muon momentum for reaching the muon system and is equivalent to the muon's energy loss in the calorimeter and toroid, and is $\beta=2 \mathrm{GeV}$. The last term $\gamma$ is the contribution due to finite position 
resolution in the chambers and was determined to be approximately $0.008 / \mathrm{GeV}$ for local muons without vertex-constrained fit in Run I.

The performance of the muon system in combination with the central tracker is demonstrated by Fig. 3.24, in which the possible reconstruction of several meson resonances, by calculating the invariant di-muon mass, is shown.

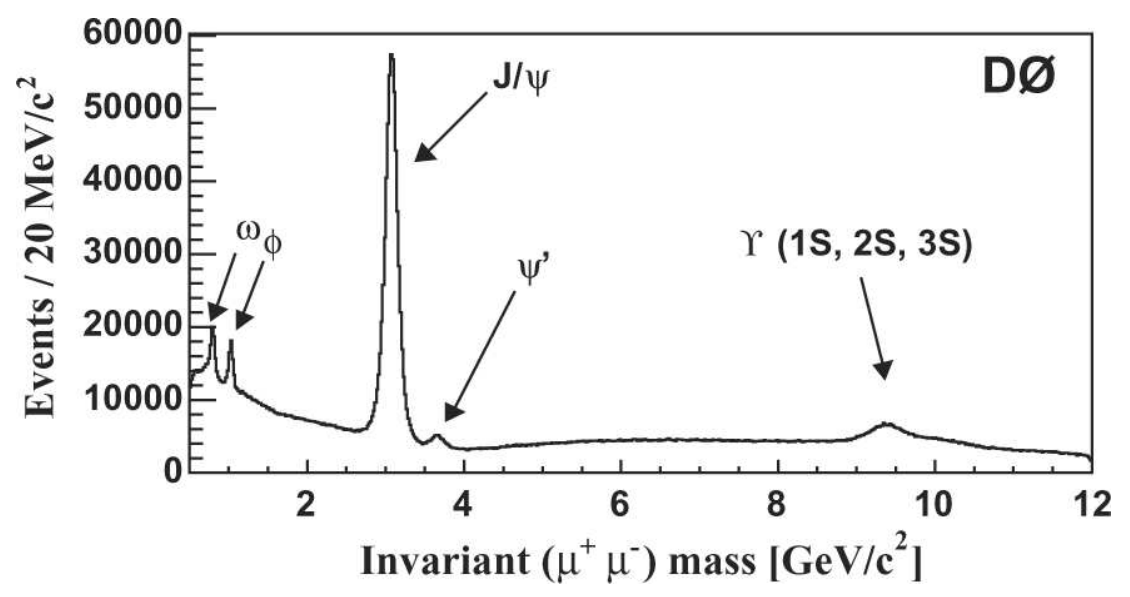

Figure 3.24: Invariant mass of $\mu^{+} \mu^{-}$pairs corresponding to $200 \mathrm{pb}^{-1}$ [96]. The peaks can be identified with mesons decaying to opposite sign di-muons: $m_{\omega}=783 \mathrm{MeV}, m_{\phi}=1020 \mathrm{MeV}$, $m_{J / \Psi}=3097 \mathrm{MeV}, m_{\Psi^{\prime}}=3686 \mathrm{MeV}$ and $m_{\Upsilon(1 S, 2 S, 3 S)}=9.5-10.4 \mathrm{GeV}$ (masses from [17]). 


\subsection{Trigger, Data Acquisition and Processing}

The interaction frequency in the $\mathrm{D} \varnothing$ detector is as large as $2.5 \mathrm{MHz}$, or a bunch crossing every 396 ns. $^{2}$ An acceptable output rate, delimited by storage, processing and transportation bandwith capabilities, is about 4 to 5 orders of magnitude lower, i.e. about $50 \mathrm{~Hz}$. The interaction event rate have to be reduced by a factor of $5 \cdot 10^{4}$. Since the cross section (and therefore the propability of occurrence) of new physics, like resonant slepton or Higgs production is much smaller in comparison to other, well known Standard Model processes like QCD or vector boson production (see Fig. 3.25), the large event rate cannot be broken down by simple randomized rejection. This would mean to loose signal at the same rate as the overwhelmingly large background. Only interesting signal events should be selected and saved. By triggering the data readout the amount of interesting events or signal events can be enriched compared to the background. The trigger decision wether the event is interesting or not is based on inputs from all subdetectors and must be made within a short time which is given by the size of the readout buffers.

The $\mathrm{D} \varnothing$ trigger is divided into three tiers; the first level of the trigger (level 1) is used to limit the event rate to $\approx 2 \mathrm{kHz}$ based on partial information from the tracking, calorimetry, and muon systems. At the next trigger stage (level 2), the rate is reduced further to $\approx$ $1 \mathrm{kHz}$. These first two levels of triggering rely mainly on hardware and firmware. The final level of the trigger, level 3 , with access to the full event information, uses software algorithms to reduce the output rate to $\approx 50 \mathrm{~Hz}$. All information about an event rejected at any stage in the trigger is lost for ever. Trigger inefficiencies arise from rejected events which are mistaken for less interesting or from dead time because the trigger systems are busy processing a previous collision. The trigger capacity must increase over time with the increasing maximal instantaneous luminosity in order to minimize the inefficiencies. Therefore especially the software trigger tools at level 3 underly continuous improvements and updates, the computing capacities at level 3 are continuously extended by adding more nodes, and also level 1 and level 2 algorithms are improved and updated. In the following a short overview about the three trigger tiers and the data acquisition (DAQ), schematical shown in Fig. 3.26, shall be given.

\subsubsection{Level 1}

The trigger level 1 decision are made by specialized programmable hardware based on input from the individual subdetectors, like the luminosity system, the central fiber tracker, the calorimeter and the muon chambers. The luminosity detectors provide a very fast measurement of the $z$-position of the interaction vertex, as discussed in section 3.3.2 and allow for fast veto decisions at L1. The level 1 central track trigger (L1CTT) reconstructs the trajectories of charged particles using fast information provided by the scintillatorbased detectors, as the central fiber tracker and the preshower detector. L1 track triggers

\footnotetext{
${ }^{2}$ Since the proton and anti-proton bunches are arranged in three trains with a $7 \mu$ s separation, as discussed in chapter 3.2, the interaction frequency averaged over time is $1.7 \mathrm{MHz}$.
} 


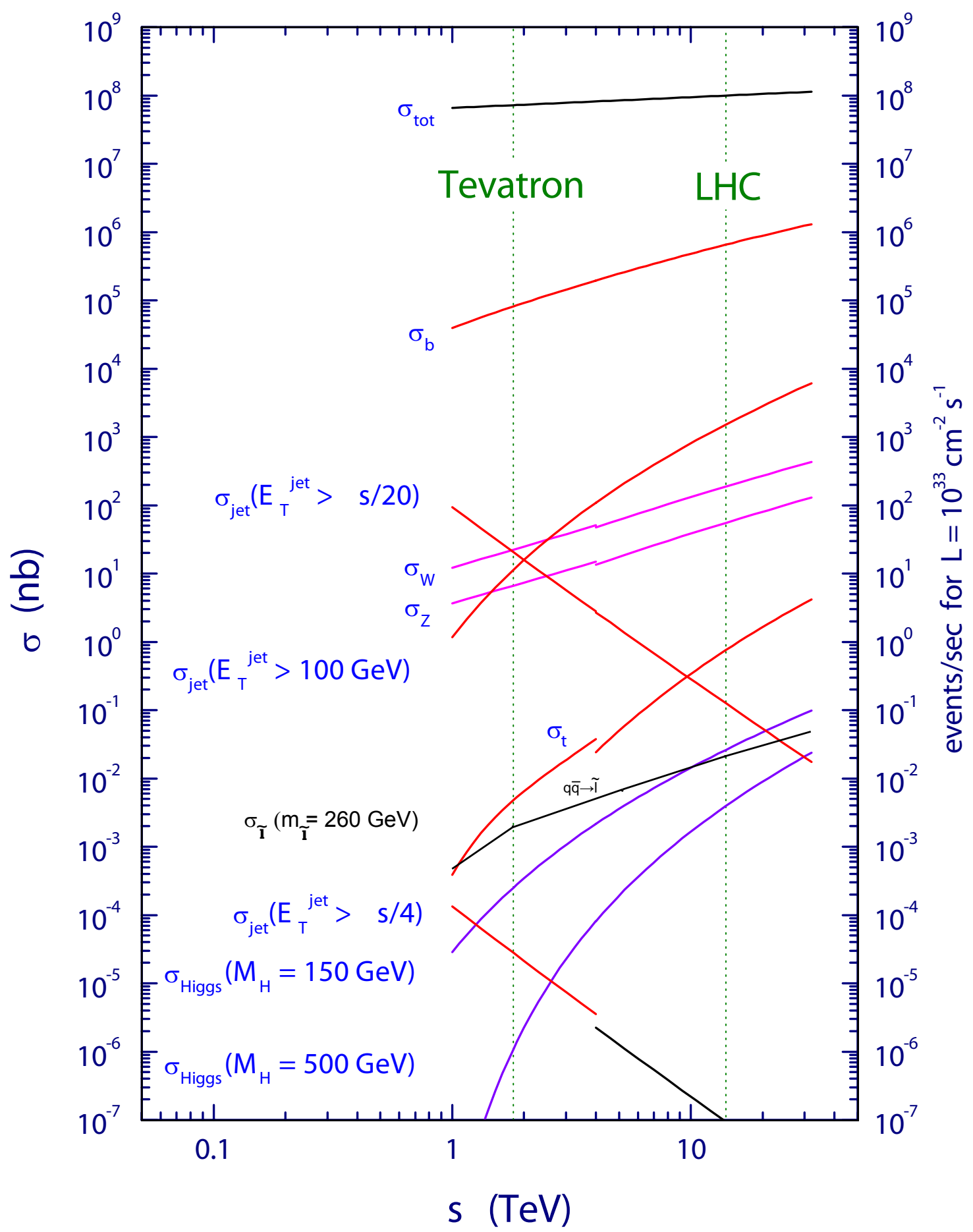

Figure 3.25: The cross section for various processes at proton-proton and proton-antiproton colliders in dependence of the center-of-mass energies [124]. The black line labeled $q \bar{q} \rightarrow \tilde{l}$ refers to resonant slepton production for $\mathrm{m}(\tilde{l})=260 \mathrm{GeV}$ and a R-parity violating coupling of size $\lambda_{211}^{\prime}=$ 0.07 . 


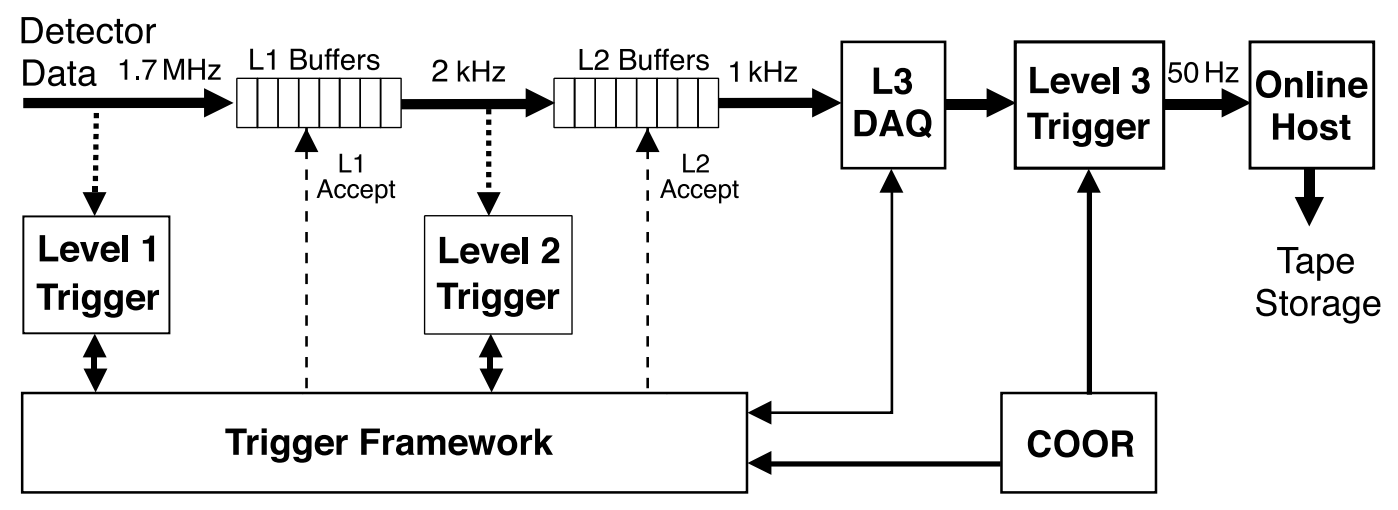

Figure 3.26: Schematic block diagram giving an overview of the $D \emptyset$ trigger and data acquisition system [96].

require tracks with transverse momenta above certain thresholds. The list of up to 480 level 1 tracks per bunch crossing are stored for later L2/L3 readout and are used as seeds for other trigger systems. The calorimeter has a separate fast level 1 readout providing the sum of energy of all electromagnetic and hadronic layers, except for the two coarse hadronic layers, in towers of size $\Delta \phi \times \Delta \eta=0.2 \times 0.2$. The level 1 calorimeter trigger require a certain amount of transverse energy in a calorimeter tower. Because of the separate level 1 calorimeter readout, the energy measurement at L1 is also used offline to identify and remove electronic noise in the more precise but slower calorimeter readout systems used for the final reconstruction. The level 1 muon triggers search for patterns consistent with muons originating from the vertex or from the L1CTT objects, in hits of the muon wire chambers and muon scintillation counters. Field programmable gate arrays (FPGAs) are used to perform the combinatorial logic. L1 muon triggers used in this analysis require hits in the muon scintillators.

The trigger framework (TFW) gathers the information of individual L1 triggers and decides on the further examination of a particular event. The TFW coordinates trigger vetoes to inhibit other triggers and manages the prescaling. Only level 1 triggers can be prescaled. A prescale $n$ on a specific L1 trigger results in a rate reduction by a factor $n$ of this trigger by "dropping" randomly events, that would have passed the trigger otherwise. However, the "dropped" events of a specific trigger can be selected by an other trigger of the same trigger level. All triggers, thresholds, prescales, etc. are programmed from COOR, see also Fig. 3.26 for reference. The level 1 trigger decision is performed after $4 \mu \mathrm{s}$ or less, leading to an accept (output) rate of approximately $2 \mathrm{kHz}$. The L1 deadtime is negligible. More information can be found in [96].

\subsubsection{Level 2}

The L2 trigger makes use of sub-detector specific hardware engines which are combined at global stage (L2Global). L2Global is the first level of trigger to examine correlations between all detectors. The subsystem preprocessors work parallel and the trigger decisions 


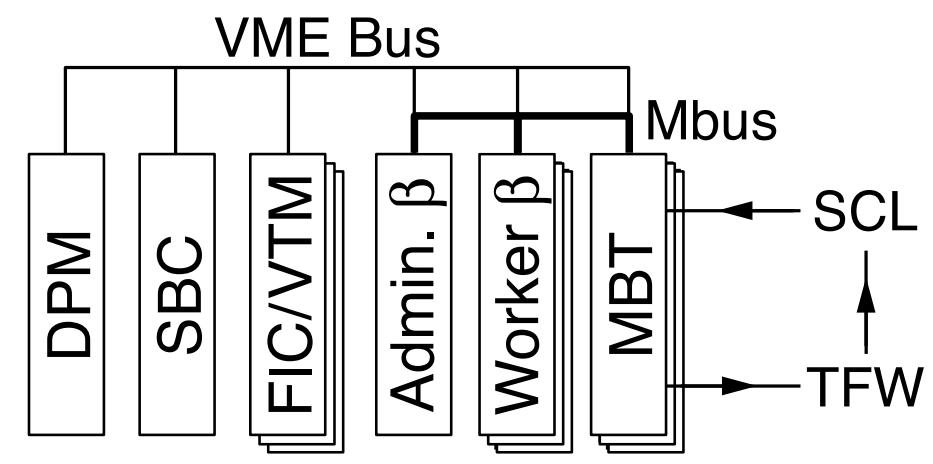

Figure 3.27: Typical layout of cards in an L2 preprocessor/global VME crate. The VME controller and dual-port-memory (DPM) card is used for downloading runtime parameters and for monitoring. The single-board-computer (SBC) send data to the level 3. The Mbus transceiver (MBT) and the fiber converter and VME transition (FIC/VTM) cards provide serial command links (SCL) to other crates. The actual work is done by the L2-beta processor cards. The L2-betas run a Linux system and all L2 programs are written in $\mathrm{C}++$. The reader is referred to a more detailed specification in [96].

are made in the L2Global based on the reconstructed L2 physics objects. The organization of a typical L2 preprocessor and the L2Global crate is given in Fig. 3.27, there are $63 \mathrm{VME}$ crates in total.

A level 2 silicon track trigger using SMT readout information and L1CTT as well as a L2 central track trigger (L2CTT) is in commissioning. The trigger level 2 was designed for an input rate of up to $10 \mathrm{kHz}$ and a maximum accept rate of $1 \mathrm{kHz}$. The L2 triggers are entirely configurable by COOR using the triggerlist which can be changed in every run (see Fig. 3.9).

\subsubsection{Level 3}

The level 3 data acquisition system (L3DAQ), depicted in Fig. 3.28, receives fully digitized data from up to 63 single board computers (SBCs), housed in the VME crates as shown in Fig. 3.27, which are connected to the various subdetectors. The Routing Master (RM) specifies routing intructions, which direct the SBCs whether and to which L3 trigger farm node the data, typically 1 to $20 \mathrm{kB}$, is to be send. The RM chooses a farm node based on the level 2 trigger decisions and the number of available buffers in each farm node. The event fragments are built into complete events on the L3 farm nodes. Events that pass the L3 trigger criteria are sent via a separate network to tape storage devices. The typical size of unreconstructed "raw" events is $250 \mathrm{kB}$. The L2 accept rate of $1 \mathrm{kHz}$ is reduced to $50 \mathrm{~Hz}$, which is today ${ }^{3}$ mainly limited by tape storage.

The L3 trigger is a high level, software-only trigger. It performs limited object reconstruction and decides on complete physics objects and relationships between such objects

\footnotetext{
${ }^{3}$ The number of L3 farm nodes was upgraded from $\sim 100$ dual-processor nodes to $\sim 200$. Other limitations were the transport and offline reconstruction capabilities. These have been improved by the development of the Grid-tool SAMGrid [125], allowing for decentral re-reconstruction of data.
} 


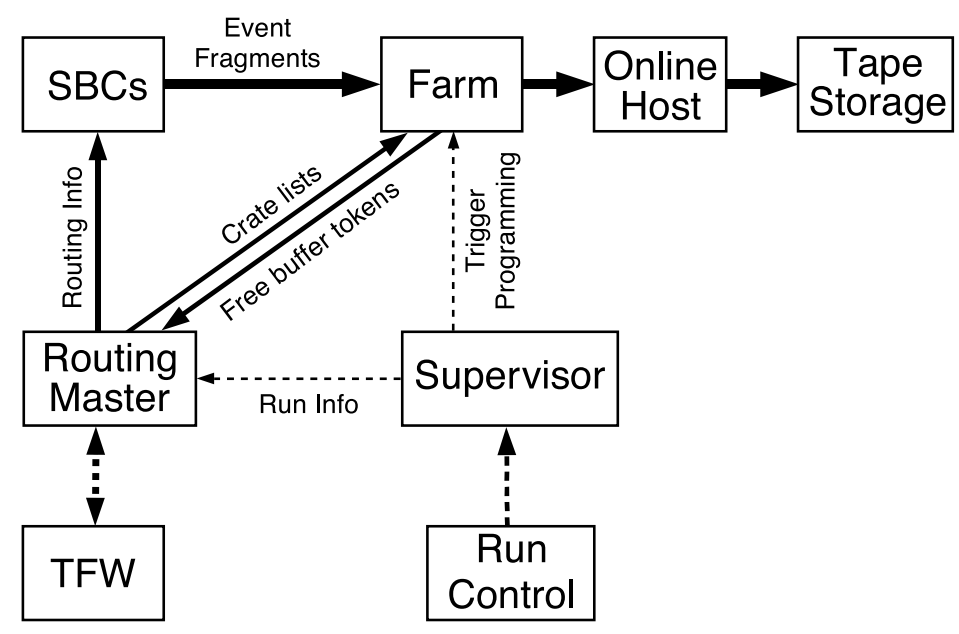

Figure 3.28: Schematic diagram of the level 3 data flow [96]. The SBCs (see Fig. 3.27) are physically located in the VME crates in the movable counting house, while most other components are located in the computer room above the control room. The tape robots are hold in the Feynman Computing Center.

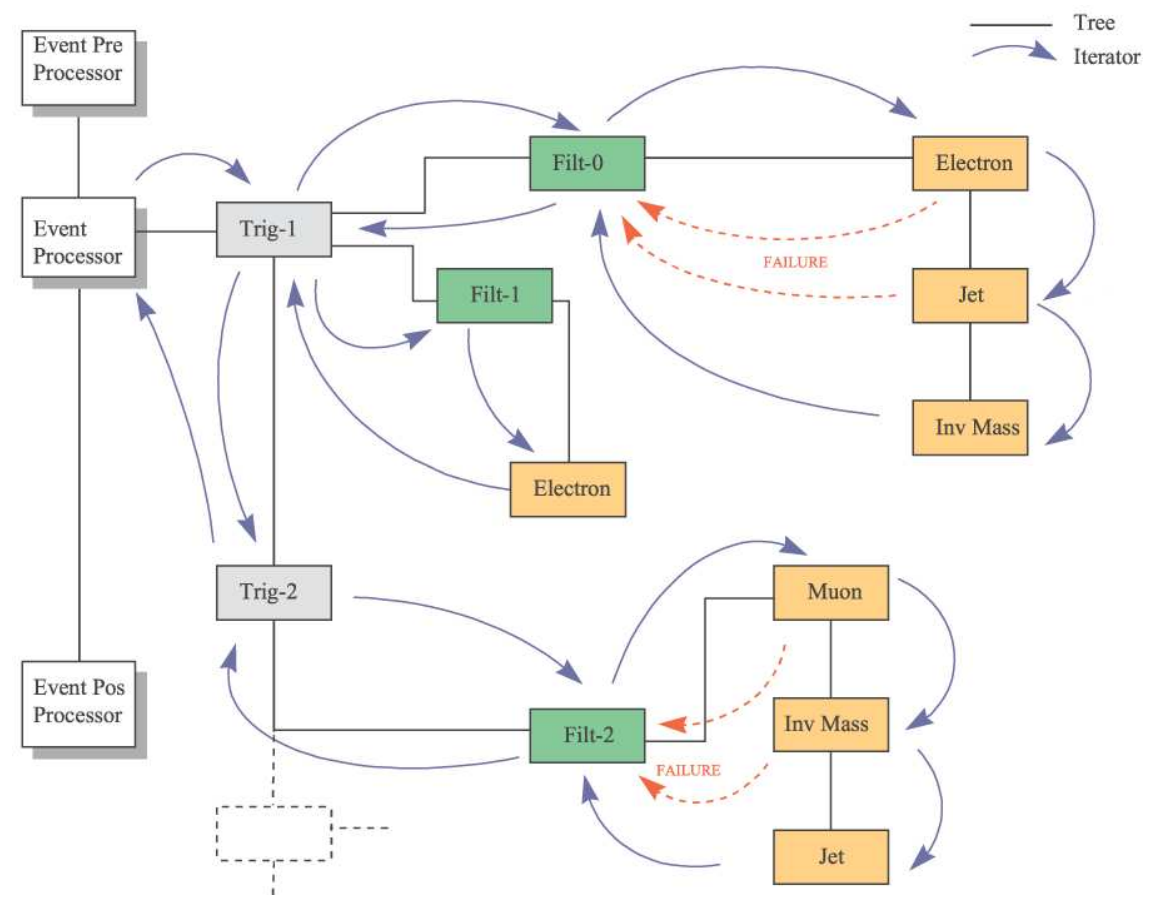

Figure 3.29: Schematic event procession at the level 3 trigger tier. The L3 trigger tools (orange) and filters (green) are called by the scriptrunner, based on the specifications of the triggerlist for a specific trigger (gray). 
(e.g. Jets, angles between objects, or their invariant masses, etc.). L3 software algorithms (tools and filters) running on the L3 farm nodes reconstruct the physics objects, calculate relations between them and perform trigger decisions, specified by the triggerlist. The triggerlist can be changed in every run by the DAQ shifter to account for changes in the instantaneous luminosity. It contains thresholds and parameters, such as jet cone radius, and trigger prescales and is downloaded to each farm node via the L3 supervisor node.

The L3 tools are called by the scriptrunner, based on the input requirements of the L3 filters or other tools. Ultimately, the set of filters and tools is specified by the individual triggers as defined in the triggerlist. A schematic event procession is shown in Fig. 3.29.

The continuous improvement of triggers with respect to event rejection is as necessary as challenging, because of the steadily increasing instantaneous luminosity and the fixed data taking rate of approximately $50 \mathrm{~Hz}$. An important level 3 algorithm to increase the event rejection without the loss of trigger efficiency is the L3 isolation tool [126]. It is used since triggerlist version v13 or June 2004 in many level 3 triggers. The isolation tool provides the possibility to calculate the isolation of a generic object and to remove those objects, which do not fulfill the requirements defined, along with other parameters, for each trigger in the triggerlist. The generic objects for which the isolation is to be calculated can be any physical object or a list of objects, i.e. any output of another tool like tracks, muons, electrons, etc; and is defined by the triggerlist, too. The quality of isolation can be calculated with respect to the number and momenta of tracks in a certain distance to the initial object (Eq. 3.14 and 3.15) or with respect to (transverse) energy depositions in the calorimeter (Eq. 3.15) inside a hollow cone as defined in Fig. 3.30. Only calorimeter cells with energy of at least $100 \mathrm{MeV}$ and all but the two outer course hadronic layers are utilized, to lower noise effects.

$$
\begin{aligned}
\text { number of tracks } & \sum_{\varangle} i \leq N_{t} \\
\text { track momenta } & \sum_{\varangle}\left(p_{T}\right)_{i}^{\text {track }} \leq P_{\text {max }}^{T} \\
\text { calorimeter activity } & \sum_{\varangle}\left(E_{T}\right)_{i}^{\text {Cal. }} \leq E_{\text {max }}^{T}
\end{aligned}
$$

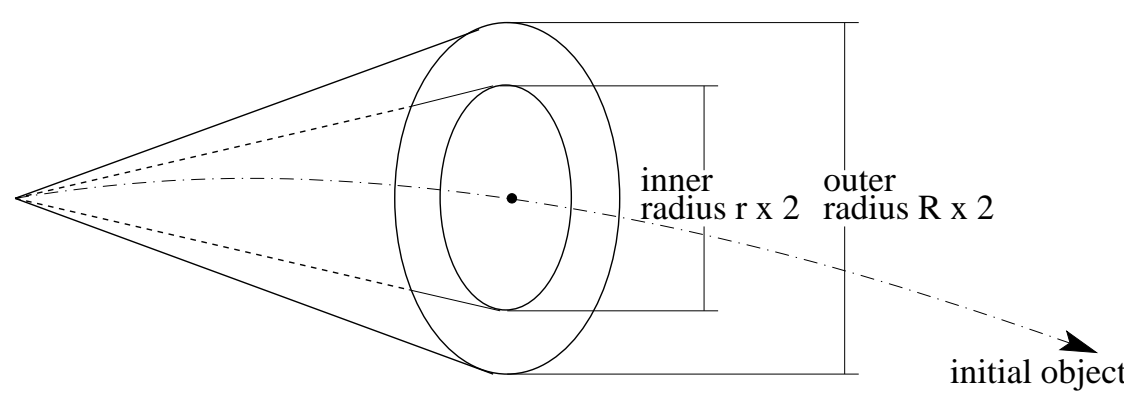

Figure 3.30: Definition of the cone shell, within which the transverse energy contents of all calorimeter cells are summed. The cone orientation is defined by the direction of the initial object at its vertex; the radii are defined by the triggerlist. 
A typical triggerlist definition for a tool producing a list of isolated objects is given in Fig. 3.31. The parameters defining the type of the object for which isolation is to be calculated, the type of isolation (track or calorimeter based) and the associated cone radii, as well as the isolation thresholds are:

\section{srctrackrefset and swarmtrackrefset}

define the tool types of the initial object and the swarm tracks, respectively. The track-isolation is calculated against the swarm tracks, which are therefore usually all tracks found in the tracking system above a certain $p_{T}$ threshold, defined by the swarm tool.

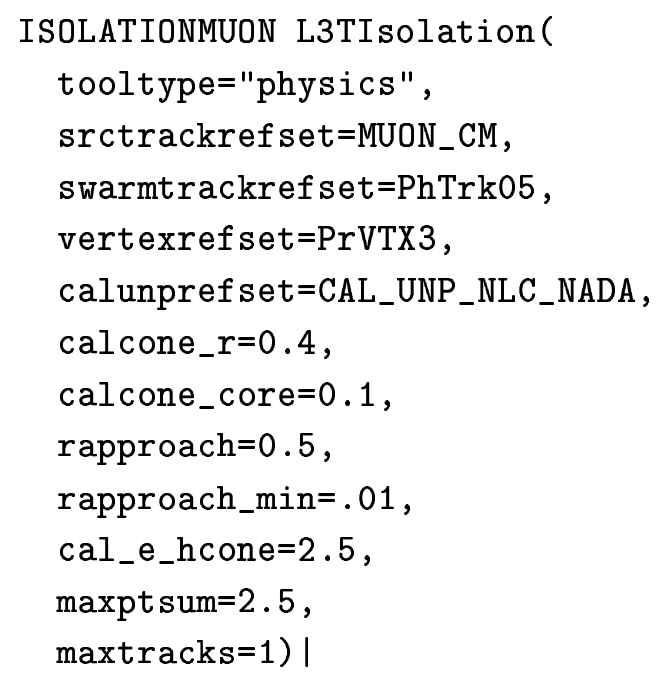

Figure 3.31: Triggerlist excerpt, defining a tool with isolated, central-matched muons as output.

vertexrefset and calunprefset are vertex and calorimeter-unpacking tools, respectively. The primary vertex is used as the origin of the source track, if this information is not provided by the source track itself, like for local muons.

calcone_r and calcone_core are the outer and inner radii of the hollow cone in which the transverse energy of the calorimeter cells is summed up. Calorimeter cells with less than $100 \mathrm{MeV}$ are not considered (in order to reject noise).

rapproach and rapproach_min are the outer and inner cone radii for track isolation. All tracks in the cone core with the radius rapproach_min are not considered, to exclude the original source track from the set of swarm tracks. The transverse momentum of all swarm tracks in the hollow cone, formed by rapproach and rapproach_min, is summed up.

cal_e_hcone $\left(E_{T}\right)_{i}^{\text {Cal. }}$, maxptsum $\left(p_{T}\right)_{i}^{\text {track }}$, and maxtracks $N_{t}$ are the maximally allowed isolation energy inside the calcone_r-calcone_core hollow cone and the maximum transverse $p_{T}$ sum and number of swarm tracks inside the hollow cone formed by rapproach and rapproach_min, respectively.

The isolation tool removes objects that are not isolated, in that sense it can also be considered as a filter. For any affiliated tool or filter the output of the isolation tool is indistinguishable from the type of the original object, which the isolation tool used as input (srctrackrefset). The isolation tool can therefore be inserted between any tool producing physics objects and the following tool or filter.

The calorimeter and the track based isolation is consistent with the isolation measured offline as shown in Fig. 3.32. Studies have been performed to find the best triggerlist- 
parameter combination with respect to efficiency, rejection and time consumption for electrons [127] and muons [128].

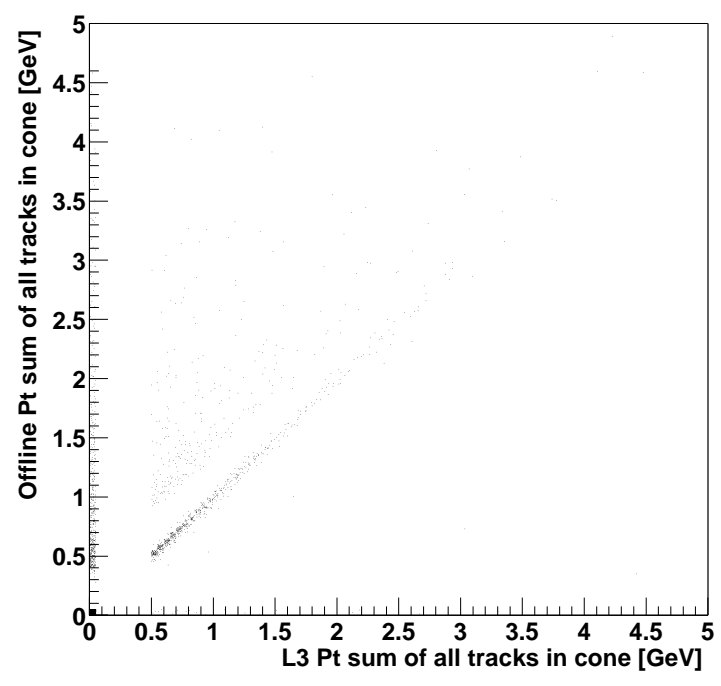

(a)

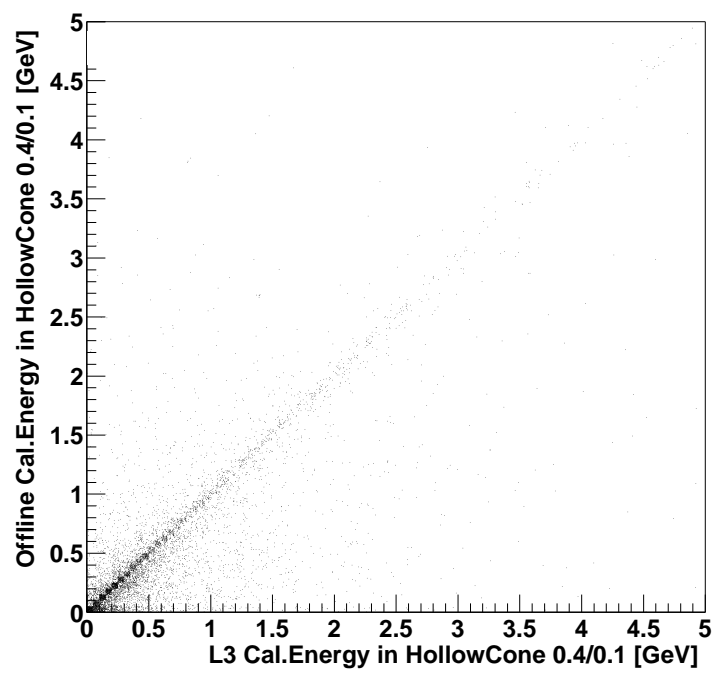

(b)

Figure 3.32: Comparison of track isolation (a) and calorimeter isolation (b) measured online at L3 (abscissa) and offline (ordinate) for the same, spatial matched object. The algorithms used online and offline for track reconstruction (affecting the momentum calculation) and calorimeter noise rejection (affecting the calorimeter isolation energy) differ significantly because of different demands with respect to the computing time. Nevertheless, a strong correlation between the isolation measured online and offline can be observed. The $0.5 \mathrm{GeV}$ thick band structure in (a) is due to a online minimum track momentum requirement of at least $0.5 \mathrm{GeV}$.

\subsubsection{Computing and Software}

The immense amount of data (a few billion events $\left(10^{9}\right)$ or the size of several Peta-bytes $\left(10^{15}\right.$ bytes $)$ ) is handled by the SAM system (sequential access via meta data) [129]. For each data file - containing raw-data from the detector, simulated data from the Monte Carlo farms or any other reconstructed data - metadata information in file format is created, for storage in a database to enhance the offline access of data. All data is stored on tapes, managed by Fermilab's Enstore system [130]; frequently used data is also cached on disk servers. SAM negotiates the data access with ENSTORE and manages the data transport. The user applications requesting stored data or creating new (Monte Carlo) data are located everywhere in the world and communicate with SAM via the meta database commands. With respect to the data distribution, SAM can be considered as the ancestor of the LHC's computing grid (LCG) [131], however all SAM jobs are constrained to computing clusters assigned by the user.

All DØ software is written in $\mathrm{C}++$ and share a persistent format which is handled by the DØ object model (DØom) [132], shown schematically in Fig. 3.33. DØom maintains a dictionary describing the layout of the used $\mathrm{C}++$ classes. The dictionary is created by a preprocessor, based on the CINT $\mathrm{C} / \mathrm{C}++$ interpreter, and can be queried at run time. 
DØом handles the adding and deleting of data members, without explicit action on part of the programmer and allows therefore intercommunication between different objects. All event data are stored in this structure and DØом provides input- and output packages, so that e.g. the reconstruction code is completely independent of the data format.

The $\mathrm{D} \varnothing$ event data model (EDM) is a library of classes and templates supporting the implementation of reconstruction and analysis software. It features the "event" class, a container class for all data associated with a single bunch crossing. This is the raw output of the detector as well as the results of the reconstruction algorithms and metadata describing the configuration of the algorithms. This allows for the multiple usage of single algorithms with different configuration parameters, e.g. a cone-jet algorithm using different cone radii. All different data formats used at $D \varnothing$ fulfill the EDM requirements:

- Raw data: All data events recorded by the $\mathrm{D} \varnothing$ detector are held on tape in the Feynman Computing Center in a raw, i.e. un-reconstructed and un-calibrated format, to allow for the reconstruction of all events by the same version of the continuously changing (improving!) D $\varnothing$ reconstruction software.

- DST (Data Summary Tape): For a long time reconstructed data and Monte Carlo were stored in the DST format, containing partial raw data information and all information about the reconstructed physical objects. Fast access to the uncompressed data was possible for the disadvantage of a large event size $(250 \mathrm{kB})$. Due to the number of expected events, $\mathcal{O}\left(10^{9}\right)$, determined from the trigger accept rate and the overall data taking efficiency, the DST was relinquished in favor of the TMB format.

- TMB (Thumbnail): Reconstructed data (from either the detector or Monte Carlo) is stored in TMB format, containing no Raw data information but only higher level Physics objects, e.g. tracks instead of single hits. The data is compressed, afflicted with loss of information, which is of the same order of magnitude as the resolution or precision of the specific object. The average event size is only $25 \mathrm{kB}$. To save processing time due to unnecessary unpacking, the events contain "tags" (e.g. «di-

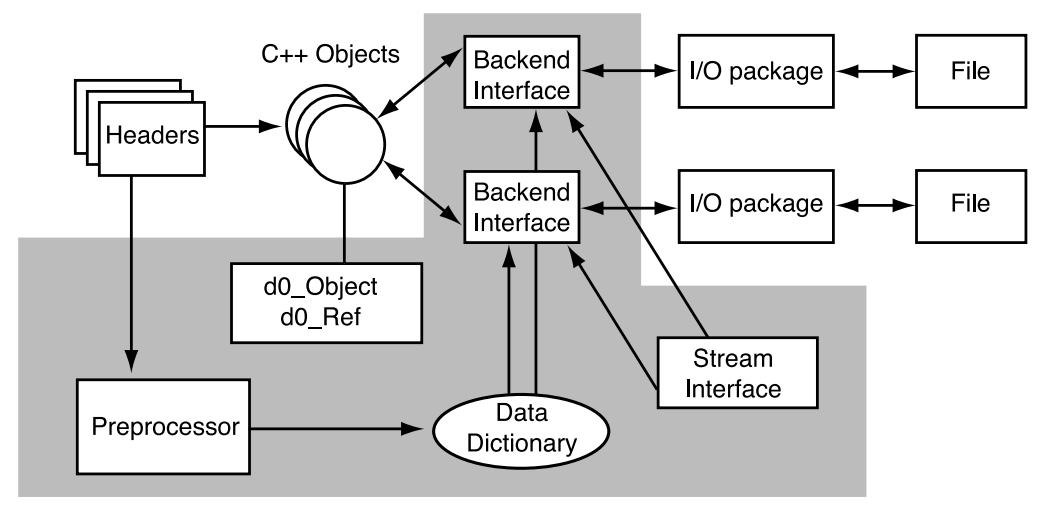

Figure 3.33: The doomed concept of the $D \varnothing$ software structure [96]. More information on the $\mathrm{D} \emptyset$ object model (DØOM) which is shaded in the figure can be found in [132]. The various DØ software packages, labeled "Headers" and "C++ Objects", have to be written in a common standard, which is discussed in [133]. 
muon event $\gg$ ) and uncompressed trigger information (not available for Monte Carlo). Every TMB event (and all descending events) is associated to a specific version of the reconstruction software (release) and is becoming obsolete.

- Root tuple: Though an analysis can already be performed on TMBs, usually a root [134] based event format is used for convenience. Recently a object orientated, common analysis format (CAF) has been developed. CAF is not used for the present analysis, but a custom root-tree format.

Events for the present analysis are simulated by Monte Carlo generators like PyTHIA [135] or Susygen [48]. For studies also the Alpgen generator was used. Almost all present generators are Fortran programs. D $\varnothing$ uses FermilaB's StdHEP code to store the generator output in a standard common block format, which is then converted by a $\mathrm{C}++$ wrapper to satisfy the EDM requirements.

The generated particles are traced through the $\mathrm{D} \varnothing$ detector and their energy depositions and secondary interactions are simulated by the Fortran program GEANT v3.21 [136]. The $\mathrm{D} \varnothing \mathrm{C}++$ wrapper, written according to the DØom conventions, is DØGstar. The simulated events are passed to DØSIM to account for all detector related effects. While the

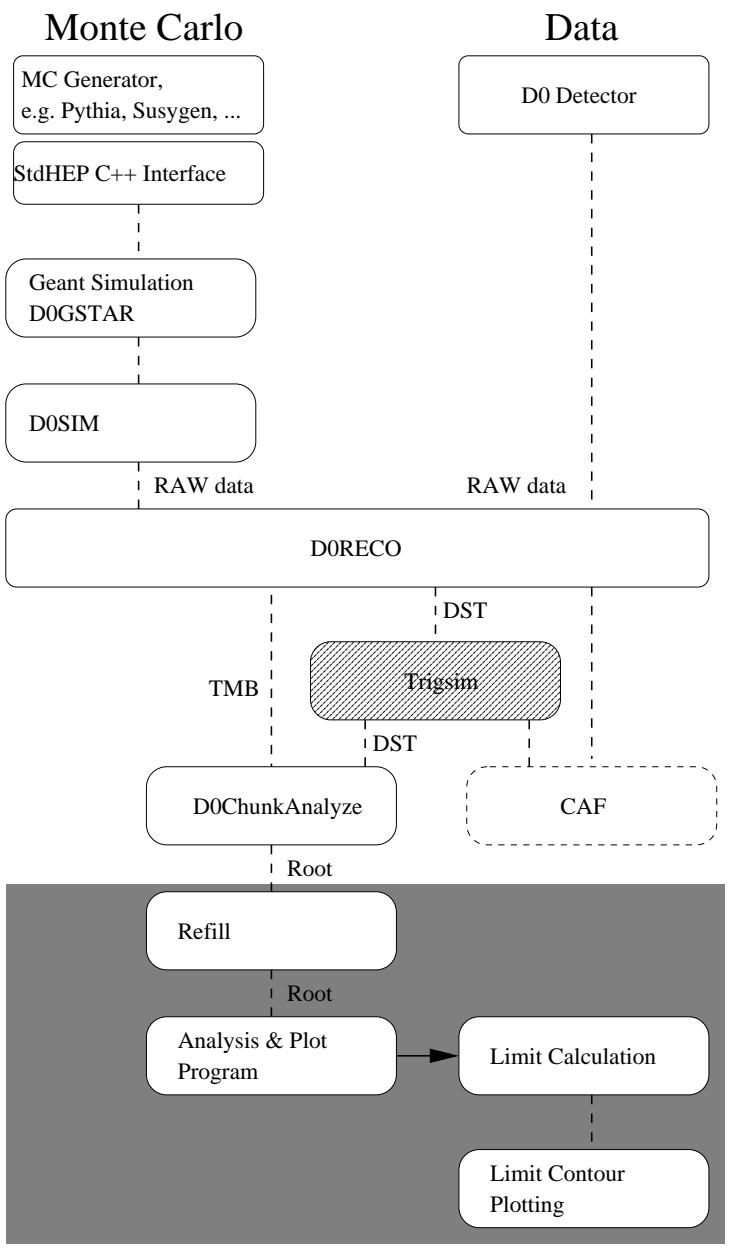

Figure 3.34: The used software and data types. events put into DØSim characterize the particles, their shower building and the material interaction; the DØSim output simulates the electrical output, which the $\mathrm{D} \varnothing$ detector would have delivered, if the interaction would have taken place in the real detector. Detector inefficiencies and noise are considered, as well as effects from multiple interactions per bunch crossing by adding minimum bias events. Minimum bias events are simulated events of elastic and inelastic $\mathrm{p} \overline{\mathrm{p}}-$ scattering, that would be recorded by the $\mathrm{D} \varnothing$ detector with a trigger requiring coinciding signals from both luminosity detectors (minimum bias). Except for additional Monte Carlo information, the MC and detector raw data files are similar. For recent simulations $(\geq \mathrm{p} 17)$ real recorded minimum data events are used.

The raw data files are reconstructed with DØRECO, a package of reconstruction algorithms from $b$-tagging to $z$-vertex position reconstruction [137]. Like most software, especially D $\varnothing \mathrm{RECO}$ is subject to a permanent improvement 
and development. All raw data is re-reconstructed with each major software upgrade (production release), typically every $1-2$ years. All data instrumentalized in the present analysis has been reconstructed with the release version p14. Reconstructed data is stored in TMB format due to the smaller event size $(\times 10)$ compared to DST format.

The tool DØ Trigsim, which is shown hatched in the schematical drawing of the software structure in Fig. 3.34, is not used directly for analyses, but for trigger studies, e.g. needed for the development of new trigger tools. Trigsim simulates only the level 1 trigger tier and provides an interface to run exactly the same L2 and L3 software as the online data acquisition system. Trigsim needs raw data information, e.g. energy information of single calorimeter cells, so that it runs only on raw data or DST, not on TMB. The impact of trigger inefficiencies on Monte Carlo events are not simulated with Trigsim in the analysis, but estimated from data and parametrized, as will be discussed in Sec. 5.1.1.

The reconstructed p14 thumbnails are translated to root format by a DØChunkAnalyze [133] derivative named TopANALYZE [138]. This program includes necessary correction algorithms to the data, which were not already comprised in the used reconstruction release, like advanced noise rejection algorithms, advanced b-tagging, jet reconstruction efficiency and resolution corrections. The used TOPANALYZE is no official D $\varnothing$ software, but a privately modified version, originally developed by the top-group. Recently a Common Analysis Format (CAF) has been introduced, intended to replace the many different privately written programs. CAF is not used for this analysis.

For the subsequent analysis of the TOPANALYzE root tuples, new software has been developed, shown shaded in Fig. 3.34. The REFILL program obeys the D $\emptyset_{\text {om }}$ coding conventions, in order to allow for an easy access to the TOPTRIGGER package [139] and with it access to the trigger parametrization. The program removes bad events due to noise or unfulfilled trigger requirements and performs a basic analysis, like the reconstruction of invariant masses and angular separation. Consisting with the EDM concept this is done event by event and sample by sample. The REFILL output is a dataset with defined object quantities, i.e. luminosity normalizable di-muon events, in root-tree format, stripped down to $\sim 100$ high level physics variables per event, used in the following more detailed analysis.

The last program in "contact" with the data events is the main analysis tool, used to select and to study the data samples. The events of all used samples, i.e. data, signaland background-MC, are filled into Standard Template Library (STL) containers and are analyzed simultaneously, allowing for convenient data studies and development of advanced selection algorithms. The concept of parallel event processing enables to study (e.g. plot) the complete data sample after each stage in the selection. The output of this program are number of found data, signal- and background- Monte Carlo events and the total uncertainty of these numbers.

The found numbers of events for multiple channels and for a specific point in the SUSY parameterspace are translated to model independent 95\% Confidence Level (CL) limits (expected and observed). The resonant slepton results are combined within mSUGRA to 
one limit on the coupling strength. The program is based on RooT's TLimit algorithm [140] which calculates $95 \%$ confidence level limits using a modified Frequentist approach, details will be discussed in Sec. 7.1. The program considers correlations between the systematic uncertainties and handles asymmetric errors [141]. The resulting limits per channel and for each parameter-space-point are written to file. These result files are interpreted within a RоOт based program, into cross-section or coupling limit contours in dependence of mass or mSUGRA parameters. 



\section{Chapter 4}

\section{Data Samples}

In this chapter the details of the used data samples shall be discussed. In Sec. 4.1 the luminosity of the recorded data of good quality is given and the utilized triggers will be discussed. The multijet QCD Standard Model background is not simulated by Monte Carlo generators but is extracted from the data, as described in Sec. 4.2. In the last section of this chapter information on the Monte Carlo simulation of Standard Model background processes (Sec. 4.3.1) and of the signal samples (Sec. 4.3.2) are given. Necessary higher order corrections due to initial and final state radiation are discussed in Sec. 4.3.3, details on how underlying events are considered in the simulation are given in Sec. 4.3.4, and the important parton distribution functions and their impact as systematic uncertainty to this analyses are discussed in Sec. 4.3.5.

\subsection{Recorded Data}

All data utilized in this analysis have been reconstructed with the $\mathrm{D} \emptyset$ software release version p14. The p14-data epoch comprises data recorded until the long shutdown that began in August 2004, as visualized in Fig. 3.10, in order to upgrade the accelerator, e.g. a scheme was introduced to cool the anti-proton beam with a second electron beam ("electron cooling") in order to increase the anti-proton density. The luminosity that was delivered in the period April 2002 until August 2004 corresponds to approximately $450 \mathrm{pb}^{-1}$. The associated recorded luminosity depends on the details of the used triggers, since only selected, i.e. triggered events are written to tape. To optimize the amount of data that is available for the analysis, several triggers were combined. However, a combination of multiple triggers which do not share the same Level 1 trigger criteria is very difficult, since different prescales and efficiencies have to be taken into account. All triggers utilized for this analysis share the same L1 trigger condition mu2ptxatxx, which requires scintillator hits at Level 1 stage from at least two muons of any momentum in any detector region. 
As discussed in chapter 3 the instantaneous luminosity is averaged over one minute of data taking, referred to as "luminosity block". One luminosity block is the smallest unit of data with a defined luminosity. Contrariwise, in case of a momentary problem, e.g. if some sub-detector component looses the synchronization, the complete luminosity block has to be rejected, since any fraction of a luminosity block would not be normalizable.

Bad events, bad luminosity blocks, and bad runs ${ }^{1}$ are not considered for the analysis and the luminosity calculation. The present analysis utilizes all major subdetectors like the muon system, the calorimeter and the tracker. Any problem with these systems, either power outage, malfunction, or unusual noise in at least one component implicate rejecting the specific LBN, run, or even store. The calorimeter is occasionally subject to noise of known and unknown origin. The affected events are flagged and removed.

The reconstructed luminosity for events of good quality, that were triggered by a di-muon trigger, is given in table 4.1 for the different triggerlist epochs. The total luminosity of the used data sample corresponds to $376.5 \pm 24.5 \mathrm{pb}^{-1}$.

\begin{tabular}{lllllll}
\hline Triggerlist version & $\mathrm{v} 8.2$ & $\mathrm{v} 9$ & $\mathrm{v} 10$ & $\mathrm{v} 11$ & $\mathrm{v} 12$ & $\mathrm{v} 13$ \\
Reconstructed luminosity $\left[\mathrm{pb}^{-1}\right]$ & 21.6 & 21.1 & 9.0 & 58.0 & 212.7 & 54.1 \\
\hline
\end{tabular}

Table 4.1: The reconstructed luminosity for each triggerlist period. The triggerlist version is increasing with the time, over the data taking period from April 2002 until August 2004. The data sample of the present analysis corresponds to an integrated luminosity of $376.5 \pm 24.5 \mathrm{pb}^{-1}$.

The luminosity as given in Tab. 4.1 is valid with respect to the di-muon triggers, listed below for each triggerlist epoch. Each data event is required to be triggered by any of these triggers, depending on the triggerlist version, defined by the run number.

- 2MU_A_L2MO (for trigger list version v10 and below)

- 2MU_A_L2MO_TRK10 or 2MU_A_L2MO_L3L15 (for trigger list version v11)

- 2MU_A_L2MO_TRK5 or 2MU_A_L2MO_L3L6 (for trigger list version v12)

- DMU1_TK5 or DMU1_LM6 (for trigger list version v13)

The abbreviations in the trigger names describe the trigger criteria. "2MU" or "DMU" stand for two muons which must be detected by scintillators on Level 1, "A" for all-muonregion without geometrical limitations. "L2M0" in a trigger name requires a medium muon on Level 2. On Level 3 "TRK10 (TK5)" requires a global track with at least 10 (5) GeV and "L3L15" a muon of loose quality with at least $15 \mathrm{GeV}$ of $p_{T}$.

Data and Monte Carlo samples are reconstructed by the software chain shown in Fig. 3.34, where the program versions are given by the $\mathrm{D} \emptyset$ release version $\mathrm{p} 14$. To reduce the number of events that have to be processed by each individual analysis, the reconstructed events are divided into different common samples, i.e. "skims", defined by the event's particle

\footnotetext{
${ }^{1} \mathrm{~A}$ run is typically a 4 hour long period of continuous datataking using the same list of triggers and prescales (triggerlist) controlled by the DAQ shifter in the D $\varnothing$ controlroom.
} 
content. For this analysis the two-muon skim "2MU" is preferred to the single-muonsingle-jet skim "MUJET", in order to use the reconstructed $Z$-boson mass peak with as much statistics as possible as control sample. The two-muon sample is valuable to check the luminosity, the muon reconstruction efficiencies and the agreement of data and Standard Model expectation with only negligible signal contamination.

In total 971187179 events were recorded between April 2002 and August 2004. The twomuon skim reduces this to $5.7 \%$ or to 55196992 events that contain at least two muons of any quality and trigger. The "preselection sample" which requires two high quality muons reduces the amount of data events to 23206 . The details of the preselection will be discussed in detail in Sec. 6.1.

\subsection{Multijet QCD Extraction from Data}

Several methods have been studied to describe the multijet or "QCD" background in the data. It is difficult to model this background with Monte Carlo generators, since the number of contributing Quantum Chromodynamic (QCD) processes and the production cross section is very large. Even an adapted $b \bar{b} \rightarrow \mu \mu+X \mathrm{MC}$ with cuts on generator level (DØmess) on the transverse momenta of both muons at $3 \mathrm{GeV}$ and $8 \mathrm{GeV}$ does not provide sufficient statistics. Of $10 \mathrm{k}$ generated events, less than 5 made it into a loose two-muon preselection. Therefore this background has to be estimated from the data, two method shall be discussed in the following:

A Selection with muon isolation criteria only: QCD events in the present analysis are dominated by $b \bar{b}$ production. The $b$-jet often contains a muon, so that these events can be enriched by asking for a muon which is not as tightly isolated as in the standard selection. One problem is the inherent cut on the event kinematics, since muons with low momenta have a higher probability to be isolated than higher energetic muons. Thus the cut on the muon isolation cannot be softened too much. The property "muon isolation" has been defined in Sec. 3.4.3.

B With additional $b$-tag: Another method to extract a di-muon QCD sample from the data is to tag $b \bar{b}$ events by a reconstructed secondary vertex. The contribution of other processes involving $b$-jets is negligible due to the large $b \bar{b}$-cross section, see Fig. 3.25. The QCD sample is dominated by heavy quarks that decay semileptonically. The contribution from light flavors is small, because significantly softer transverse momentum spectrum.

Since the QCD events are real data events, it must be ensured, that no event can be in the data sample as well as in the QCD sample. If this would be the case, a potential signal in the data sample could be masked by the QCD sample, which might contain also signal events. Therefore a variable, which ideally is capable to distinguish between signal-like and QCD events, must be used to clearly separate the data and the extracted QCD sample. The muon isolation cut in the QCD samples is applied in a different way, orthogonal to the signal and data selection: One of the two muons in these QCD events is required to be 
not as tightly isolated - in relation to the energy in a hollow cone around the muon track or the amount of other tracks around the muon - than the muons in the signal samples.

Both methods A and B shall be compared in the following and the reason to use the QCD sample with b-tag for the resonant slepton analysis shall be motivated.

For the pair and associated gaugino production analysis another approach is chosen. Here, the QCD contribution to the data is removed almost completely by cutting very hard and muon momentum depending on the muon isolation, for the disadvantage of a lower selection efficiency of events containing jets. The details shall be discuss in Sec. 4.2.3.

\subsubsection{QCD Sample without requiring a $b$-tag}

The first QCD sample is obtained from di-muon data events. The invariant di-muon mass must be lower than $65 \mathrm{GeV}$ to remove unwanted $\mathrm{Z}$ events from this QCD sample. Each muon has to be loosely isolated $<10 \mathrm{GeV}$ with respect to the transverse calorimeter energy in a hollow cone $(R=0.4, r=0.1)$ and with respect to the transverse momentum sum of tracks in a cone $(r=0.5)$ around the muons track. In order to separate the QCD sample from data and therefore from potential signal events as discussed above, one of both muons in this sample must not be tightly isolated with respect to either isolation criteria $(X>2.5 \mathrm{GeV})$, while both muons in the signal and in the data are required to be tightly isolated in both respects $(<2.5 \mathrm{GeV})$. The standard muon isolation cuts are summarized in Tab. 4.2 and the cuts of this QCD sample are given in Tab. 4.3.

\begin{tabular}{lll}
\hline \hline & $E_{T} \varangle_{0.1}^{0.4}$ & $p_{T} \varangle_{0.5}$ \\
\hline $1^{\text {st }}$ muon & $<2.5 \mathrm{GeV}$ & $<2.5 \mathrm{GeV}$ \\
$2^{\text {nd }}$ muon & $<2.5 \mathrm{GeV}$ & $<2.5 \mathrm{GeV}$ \\
& & \\
\hline \hline
\end{tabular}

Table 4.2: Standard isolation cuts.

\begin{tabular}{lll}
\hline \hline & $E_{T} \varangle_{0.1}^{0.4}$ & $p_{T} \varangle_{0.5}$ \\
\hline $1^{\text {st }}$ muon & $<10 \mathrm{GeV} \quad<10 \mathrm{GeV}$ \\
$2^{\text {nd }}$ muon & $<10 \mathrm{GeV} \quad<10 \mathrm{GeV}$ \\
\hline additional criteria: any above \\
condition $>2.5 \mathrm{GeV}$ \\
\hline \hline
\end{tabular}

Table 4.3: Selection cuts for the QCD sample without b-tag.

\subsubsection{QCD Sample with $b$-tag}

The second QCD sample is obtained from di-muon data events with a loose $b$-tag. The used secondary vertex b-tagging algorithm identifies jets arising from $b$-quark hadronization by reconstructing the decay vertex of long-lived $B$ hadrons within jets. The decay of a long-lived hadron produces several charged particles emanating from a secondary vertex, displaced from the primary $p \bar{p}$ interaction point. Details and the $b$-tag certification for the used p14-Pass 2 data set can be found in [142]. The $b$-tag should increase the amount of $b \bar{b}$ events in the QCD sample, since this is the dominating QCD process in events with $2 \mu+2$ jets final states. As before, one out of four isolation cuts for the QCD events is required to be not tight $(>2.5 \mathrm{GeV})$, to be orthogonal to the signal and data standard isolation cuts. The distance in $\Delta R=\sqrt{\Delta \phi+\Delta \eta}$ of both muons to a jet may be less than 
0.5 to increase the statistics. No $b$-tag or $b$-tag veto is applied on data or signal events, since the QCD sample and the data is already separated by the orthogonal muon isolation cuts. Thus, no additional systematic uncertainty related to the $b$-tagging efficiency has to be taken into account. For clarity, the isolation criteria are again summarized in table form:

\begin{tabular}{|c|c|c|}
\hline & $E_{T} \varangle_{0.1}^{0.4}$ & $p_{T} \varangle_{0.5}$ \\
\hline $1^{\text {st }}$ muon & - & - \\
\hline $2^{\text {nd }}$ muon & - & - \\
\hline \multicolumn{3}{|c|}{$\begin{array}{l}\text { additional criteria: } \\
\text { - any above condition }>2.5 \mathrm{GeV} \\
\text { - } b \text {-tag } \\
\text { - no cut on } \Delta \mathrm{R} \text { (muon, jet) }\end{array}$} \\
\hline
\end{tabular}

Table 4.4: Selection cuts for the QCD sample with b-tag.

Even though the loose $b$-tag requirement removes about $50 \%$ possible QCD statistics due to the $b$-tagging inefficiency, it extracts still more events than the method discussed above in Sec. $4.2 .1 ; 5927$ to 3419 events. However, a disadvantage of the b-tag method is the inherent cut on the jet multiplicity. The sample is therefore re-weighted, according to the jet multiplicity of the reference QCD sample without b-tag as shown in Fig.4.1(a). The weights for each jet multiplicity and the jet definition can be found in Tab. 4.5.

\begin{tabular}{lrrrrrr}
\hline \hline & 0 jet & 1 jet & 2 jets & 3 jets & 4 jets & $\geq 5$ jets \\
\hline events in b-tag QCD & 1775 & 2423 & 1222 & 391 & 92 & 18 \\
events in ref. QCD & 2110 & 999 & 258 & 46 & 6 & $<1$ \\
resulting weight & 1.189 & 0.412 & 0.211 & 0.118 & 0.065 & 0.0 \\
\hline \hline
\end{tabular}

Table 4.5: Weights applied on the b-tagged QCD sample to account for the inherent jet cut. A "jet" is defined as discussed in Sec. 5.2 and it's momentum is required to exceed $15 \mathrm{GeV}$.

In Fig. 4.1 both QCD samples are shown for comparison for distributions that show the largest disagreement (b)-(d) and against the jet momenta (e)-(f). Both QCD samples were added separately to the remaining Standard Model contributions simulated by the Monte Carlo generator Pythia (Sec. 4.3) and compared to the Data, in a selection where the signal contribution is still negligible. Good agreement can be observed for both QCD extraction methods, however the QCD method with $b$-tagged events tends to describe the data better, especially in events with jets, which are important for this analysis. Since this sample has also much higher statistic, particularly in events containing jets, this QCD sample with b-tag is the one used in the following resonant slepton analysis.

From the di-muon preselection sample with negligible signal contribution the QCD scale factor and a conservative uncertainty estimation is derived, as will be discussed in Sec. 6.1. In the same section control plots in different stages of the event selection are shown proving a good agreement. 


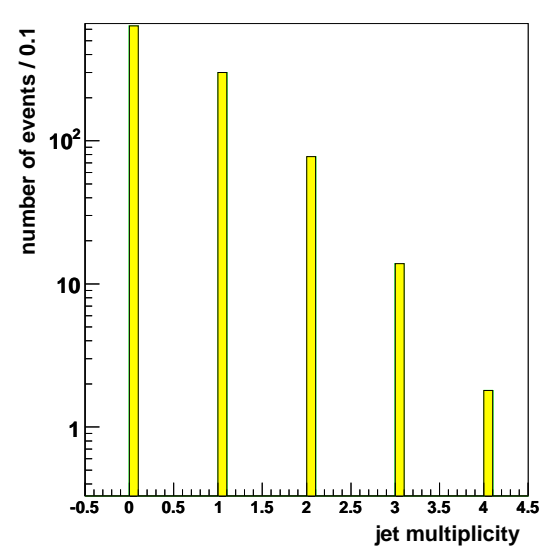

(a)

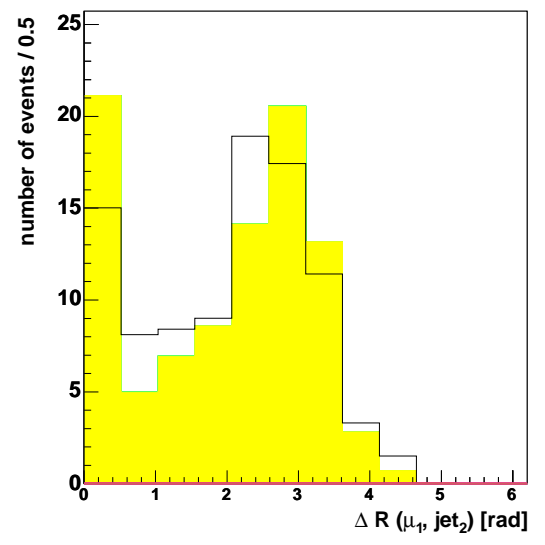

(c)

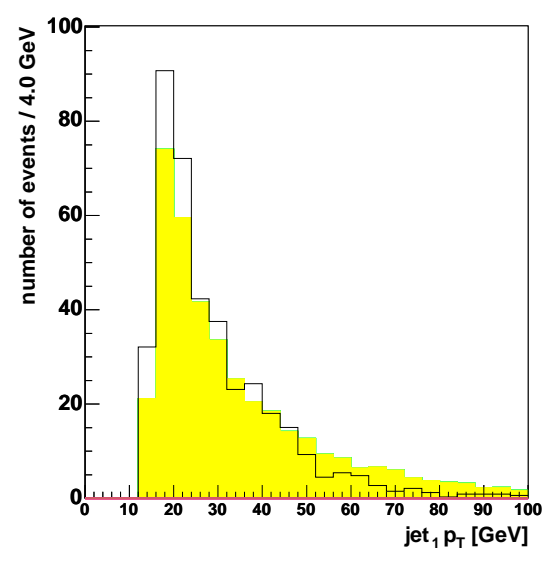

(e)

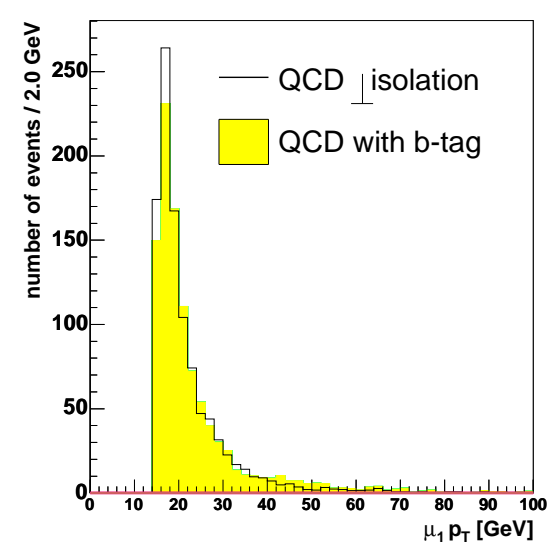

(b)

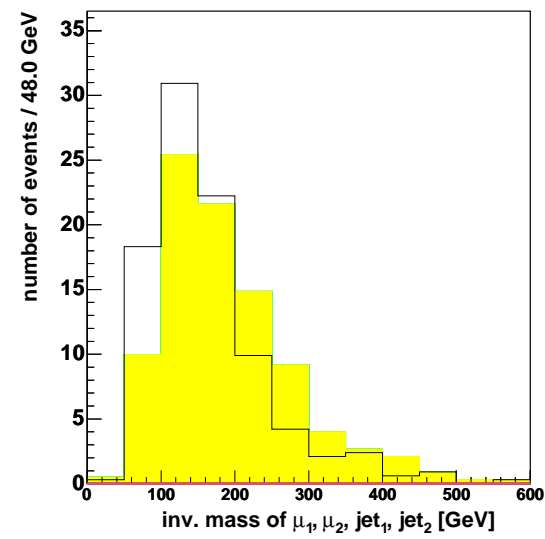

(d)

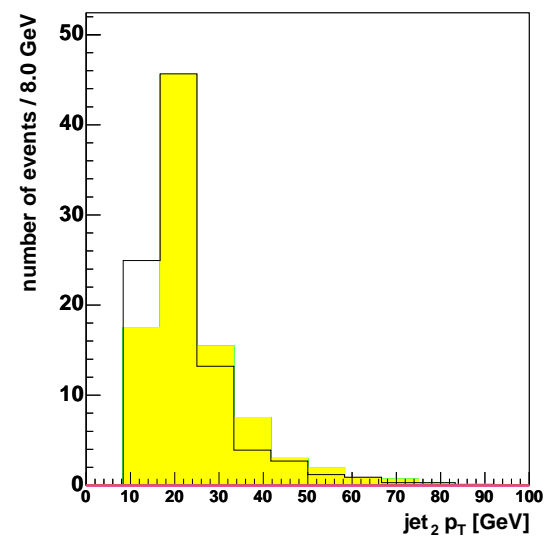

(f)

Figure 4.1: Comparison of QCD samples, extracted with and without b-tag from the data. The b-tagged sample was re-weighted to match the jet multiplicity of the sample without b-tag (a). The total entries in both samples are therefore normalized to eachother. The $1^{\text {st }}$ muon transverse momentum distribution is shown in (b), the following distributions show the largest disagreement between both samples; the distance in $\Delta R$ between the leading muon and the next-to leading jet (c), the invariant 4-body mass of both muons and both jets (d), and the momenta of both jets (e) and (f). 


\subsubsection{QCD Removal Using a Momentum Dependent Muon Isolation}

In principle it is also possible to remove nearly the complete QCD background [143, 144], by cutting harder on the muon isolation, proportional to $p_{T}^{-1}(\mu)$.

$$
\begin{aligned}
& \sum p_{T \text { cone } 0.5}^{\text {trk }} \cdot p_{T}^{-1}(\mu)<0.06 \\
& \sum E_{T h \text { hol.cone }(0.4 / 0.1)}^{\text {cal }} \cdot p_{T}^{-1}(\mu)<0.08
\end{aligned}
$$

These muon momentum dependent isolation criteria remove disproportionately many events containing jets, which are important for the analysis. Therefore these cuts are not used in the resonant slepton analysis, but the remaining multijet background in the data is modeled using the above described $b$-tag method, in order to keep a higher signal efficiency. Most QCD events can also be removed by the final selection cuts, see Sec. 6.2.2.

However, for the pair and associated production of gauginos no resonance exists and the final selection depends on the requirement of like-sign muons as discussed in Sec. 6.3. A like-sign muon QCD sample cannot be extracted from the data using the method discussed in Sec. 4.2.2, because of to low statistics. Moreover, the like-sign QCD sample cannot be normalized, since the signal contribution to any like-sign selection is not negligible. For the gaugino analysis the QCD contribution to the data is therefore removed using hard muon isolation cuts and lost of selection efficiency is accepted. Conservatively, the remaining fraction of QCD events in the data is estimated to be zero. This is a good approximation, as proved by the control plots shown in Sec. 6.3.

\subsection{Monte Carlo Simulation}

Monte Carlo generator are used to simulate the various processes that contribute to the recorded data sample. The improving understanding of physics processes is used to calibrating the simulation. Likewise, a better simulation allows for more precise measurements and searches. To search for new physics the study of the simulated signal is inevitable. The details of the used Standard Model background samples as well as the utilized signal samples shall be discussed in the following.

\subsubsection{Standard Model Background}

The dominant background in a inclusive di-muon event sample is $\mathrm{Z} / \gamma^{*} \rightarrow \mu \mu$ inclusive production, where "inclusive" refers to the production of additional jets by e.g. initial or final state radiation, see Sec. 4.3.3 for details.

The Monte Carlo generator Pythia [135] tends to underestimate jet multiplicities $\geq 2$. The $\mathrm{Z}$ inclusive Pythia sample has been compared to $\mathrm{Z}, \mathrm{Z}+\mathrm{j}, \mathrm{Z}+\mathrm{jj}, \mathrm{Z}+\mathrm{jjj}$ samples generated with the matrix element generator Alpgen [145], the samples have been normalized at a jet multiplicity of 0. A "jet" is required to pass the jet quality criteria discussed in Sec. 5.2.2 
and the transverse momentum must exceed $15 \mathrm{GeV}$. The jet multiplicity distributions (Fig. 4.2) of both samples were fitted using an exponential function and a correction factor of $1.17 \pm 0.03$ per jet for the Pythia sample was obtained. The Alpgen sample and the Pythia sample before and after the correction are shown in Fig. 4.2. The correction factor is in agreement with [146], which gives a Pythia correction factor of $1.12 \pm 0.03$ per jet for jet transverse momenta larger than $25 \mathrm{GeV}$ and Alpgen samples up to two jets.

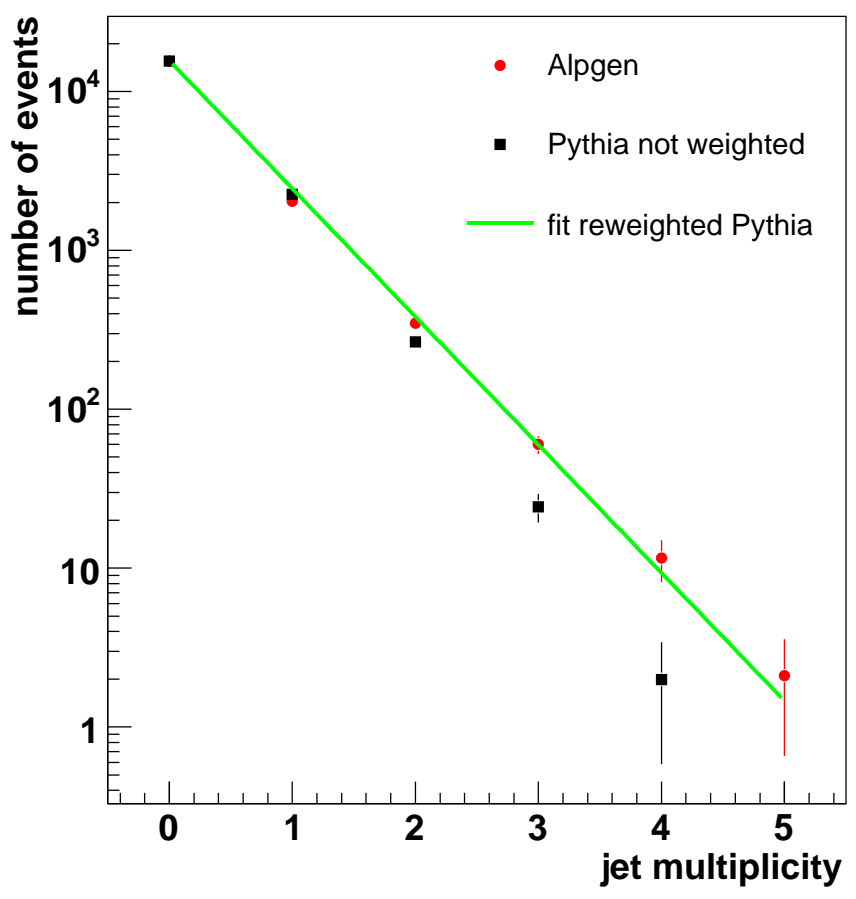

Figure 4.2: Pythia's jet multiplicity has been re-weighted using Alpgen samples as a reference. A factor of $1.17 \pm 0.03$ per jet has been obtained.

Other Standard Model processes have also been considered. Events from the inclusive $Z \rightarrow \tau \tau$ sample can pass the two-muon selection when both $\tau$ 's decay into muons, see also Sec. 5.4 for reference. Similarly, Upsilon $\Upsilon(1 s, 2 s)$ and top-pair $(t \bar{t})$ production can result in di-muon final states. Other vector boson production processes like $\mathrm{Z} \rightarrow b \bar{b}$; W, $\mathrm{WZ}, \mathrm{WW}$, and ZZ production were considered as well, but their contribution to a di-muon preselection sample was found to be negligible.

All vector boson processes have been simulated with Pythia 6.2 [135], with a scale factor [147] applied to take NLO (in the case of Z-production even NNLO) effects on the cross section [147] into account. The Upsilon sample was simulated with Pythia and the cross section is from [148]. The $t \bar{t}$ have been simulated with Alpgen. The total $t \bar{t}$ cross section is measured to be $6.77 \pm 0.42 \mathrm{pb}$ [149]. For the di-leptonic decay channel a branching fraction of $1 / 9$ is applied, and similarly, the branching fraction of the lepton-jet channel is 4/9. The used top cross sections uncertainty as given in Tab. 4.6, includes the uncertainty due to the top-quark mass. 
A summary of all used background samples is given in Tab. 4.6. All Monte Carlo samples are available from the $\mathrm{D} \emptyset$ Monte Carlo production web page [150].

\begin{tabular}{|c|c|c|c|c|}
\hline Standard Model Backgd. & $\begin{array}{l}\text { generated } \\
\text { events }\end{array}$ & $\begin{array}{l}\sigma_{\mathrm{LO}} \times \mathrm{BR}[\mathrm{pb}] \\
\pm \text { total uncert. }\end{array}$ & & $\begin{array}{l}\text { sel. events } \\
\text { preselection }\end{array}$ \\
\hline QCD from Data & $(5927)$ & scaled $\pm 20 \%$ & & 1369.4 \\
\hline$t \bar{t} \rightarrow l l$ & 47000 & $0.75 \pm 0.1$ & & 7.9 \\
\hline$t \bar{t} \rightarrow \mathrm{l}+\mathrm{jet}$ & 48500 & $3.0 \pm 0.4$ & & 0.1 \\
\hline$\Upsilon(1 s)$ & 34000 & $35.0 \pm 7.0$ & & 78.2 \\
\hline$\Upsilon(2 s)$ & 30000 & $35.0 \pm 7.0$ & & 80.8 \\
\hline$W W \rightarrow e \nu \mu \nu$ & 39500 & $0.26 \pm 0.0$ & & 0.0 \\
\hline$W W \rightarrow \mu \nu \mu \nu$ & 20750 & $0.13 \pm 0.0$ & & 11.4 \\
\hline Standard Model Backgd. & $\begin{array}{l}\text { generated } \\
\text { events }\end{array}$ & $\sigma_{\mathrm{LO}} \times \mathrm{BR}[\mathrm{pb}]$ & $\begin{array}{l}\mathrm{k} \text {-factor } \\
\pm \text { total uncert. }\end{array}$ & $\begin{array}{l}\text { sel. events } \\
\text { preselection }\end{array}$ \\
\hline$Z / \gamma^{*} \rightarrow \mu \mu[5-15 \mathrm{GeV}]$ & 219250 & 3558 & $1.25 \pm 0.07$ & 83.6 \\
\hline$Z / \gamma^{*} \rightarrow \mu \mu[15-60 \mathrm{GeV}]$ & 366500 & 330 & $1.25-1.32 \pm 0.05$ & 2446.6 \\
\hline$Z / \gamma^{*} \rightarrow \mu \mu[60-130 \mathrm{GeV}]$ & 432000 & 185 & $1.32-1.39 \pm 0.05$ & 18245.9 \\
\hline$Z / \gamma^{*} \rightarrow \mu \mu[130-250 \mathrm{GeV}]$ & 10000 & 1.4 & $1.39-1.41 \pm 0.04$ & 169.2 \\
\hline$Z / \gamma^{*} \rightarrow \mu \mu[250-500 \mathrm{GeV}]$ & 18500 & 0.1 & $1.41-1.39 \pm 0.05$ & 14.5 \\
\hline$Z / \gamma^{*} \rightarrow \mu \mu[>500 \mathrm{GeV}]$ & 9500 & $5 \cdot 10^{-3}$ & $1.39-1.37 \pm 0.08$ & 0.5 \\
\hline$Z / \gamma^{*} \rightarrow \tau \tau[5-15 \mathrm{GeV}]$ & 151250 & 3558 & $1.25 \pm 0.07$ & 0.0 \\
\hline$Z / \gamma^{*} \rightarrow \tau \tau[15-60 \mathrm{GeV}]$ & 544563 & 327 & $1.25-1.32 \pm 0.05$ & 3.5 \\
\hline$Z / \gamma^{*} \rightarrow \tau \tau[60-130 \mathrm{GeV}]$ & 655000 & 185 & $1.32-1.39 \pm 0.05$ & 165.6 \\
\hline$Z / \gamma^{*} \rightarrow \tau \tau[130-250 \mathrm{GeV}]$ & 104000 & 1.4 & $1.39-1.41 \pm 0.04$ & 2.5 \\
\hline$Z / \gamma^{*} \rightarrow \tau \tau[250-500 \mathrm{GeV}]$ & 11000 & 0.1 & $1.41-1.39 \pm 0.05$ & 0.3 \\
\hline$Z / \gamma^{*} \rightarrow \tau \tau[>500 \mathrm{GeV}]$ & 9750 & $5 \cdot 10^{-3}$ & $1.39-1.37 \pm 0.08$ & 0.02 \\
\hline$W \rightarrow \mu \nu$ & 1625050 & 2720 & $1.25-1.41 \pm 0.05$ & 0.0 \\
\hline WZ incl. & 53000 & 2.5 & $1.5 \pm 0.07$ & 11.1 \\
\hline ZZ incl. & 53500 & 1.1 & $1.3 \pm 0.05$ & 8.3 \\
\hline Total $\sum$ & $4.6 \cdot 10^{6}$ & & & 22699.3 \\
\hline
\end{tabular}

Table 4.6: All considered Standard Model backgrounds with their total statistics and cross sections. The last column gives the amount of selected events in a sample with two isolated muons with at least 8 and $15 \mathrm{GeV}$ (the "preselection" sample, Sec. 6.1), weighted according to the cross section, and for trigger and object-ID efficiencies. All samples have been generated with a Pythia Monte Carlo generator, except for the $t \bar{t}$ samples, which are Alpgen, and the QCD events which have been extracted from the data (see Sec. 4.2)

The $Z \rightarrow \mu \mu+X$ and $Z \rightarrow \tau \tau+X$ samples are separated into six mass regions of the $Z / \gamma^{*}$-boson mass on generator level, to improve the statistics in the high mass tail. The cross section is partitioned accordingly.

The 4.6 million generated SM-background events, which are held in TMB file format by SAM, have been passed through the complete detector simulation, see Sec. 3.4.4 for details. Two muons are required and bad events are removed by the REFILLER program, so that only 133405 events enter the main analysis program. This number corresponds, appropriately weighted, to 22699.3 expected Standard Model events in the preselection sample. 


\subsubsection{Signal Monte Carlo}

Two different kind of signal Monte Carlo samples have been generated for the resonant production of sleptons and for the pair and associated production of gauginos. For all samples the Monte Carlo generator Susygen v3.00-43 [48] has been utilized. The events have been reconstructed mostly on the $\mathrm{D} \varnothing$ worker-node cluster Clued0 and at GridKa [151].

The SUSY parameter space has been scanned for a fixed ratio of the vacuum expectation values of the Higgs doublets at the EW scale $\tan \beta=5$, a negative sign of the Higgs mass mixing parameter $\mu<0$, and a vanishing tri-linear coupling term $A_{0}=0$. The remaining mSUGRA parameters, the universal scalar mass $m_{0}$ and the universal gaugino mass $m_{1 / 2}$ at the GUT scale, were scanned in a typical pattern size of $20 \mathrm{GeV}$. One signal sample, or SUSY parameter "point", is defined by an unique combination of the parameters $m_{0}$ and $m_{1 / 2}$ (the other mSUGRA parameters are fixed for all studied points). In total 118 resonant-slepton-points with $1.4 \cdot 10^{6}$ events and 24 gaugino-pair/associated-points with $4 \cdot 10^{5}$ events have been generated and passed through the full detector simulation, as described in Sec. 3.4.4. The total number of studied gaugino pair/associated production points is smaller compared to the number of resonant-slepton production points, since the gaugino-analysis sensitivity depends only weakly on $m_{0}$, so that the search pattern could be chosen more coarsely. The resonant-slepton-events have all been reconstructed with the $\mathrm{D} \varnothing$ software release version p14.05.02, the pair/associated-gaugino-events have been reconstructed with p14.06.01. All events have been combined with Poisson distributed minimum bias events, 0.8 on average, to simulate the effect of multiple interactions, as discussed in Sec. 4.3.4.

Z-bosons with negative MonteCarlo generator code (Particle Data Group identification number, PDG ID $=-23$ ) from cascade decays $\tilde{\chi}_{2}^{0} \rightarrow \bar{Z} \tilde{\chi}_{1}^{0}$ are not recognized correctly by the PYEXEC interface in the used SUSYGEN version v3.00-43. The result is, that these particles are interpreted by GEANT as stable particles and do not decay. To correct for this, all events containing Z's with negative ID are removed and the remaining $\tilde{\chi}_{2}^{0} \rightarrow Z \tilde{\chi}_{1}^{0}$ events are weighted accordingly. A modification to the SUSYGEN source code has been introduced which enforces the absolute ID value of Majorana particles, see details in App. B. Studies reveal (see Sec. 6.2.1, Fig. 6.4), that samples generated with the modified SUSYGEN version differ not significantly from samples, where the affected events were removed.

All resonant-slepton-samples were fixed after the generation process as described above, while all pair- and associated-gaugino-samples were generated with the corrected SUSYGEN version.

\subsubsection{Higher Order Corrections}

At hadron colliders the initial partons can emit photons or gluons, this is called initial state radiation (ISR). Radiation emitted from the final state particles is called final state radiation (FSR). Both, ISR and FSR, can lead to additional photons or jets found in the detector. The initial partons can "adjust" their kinetic energy by ISR, so that the cross 
section is generally enhanced, if a particle is produced on shell, i. e. the particle is real. In Fig. $4.3 p \bar{p} \rightarrow \gamma$ production is shown (a) as well as ISR (b-c) for this process. The cross section in leading order for this process is given by the Feynman digram (a). However, the cross section of any process can be expanded in orders of $\alpha_{s}$, since more diagrams contribute. For the process $p \bar{p} \rightarrow \gamma$ theses next-to leading order terms (NLO) are shown in Fig. 4.3(b)-(f). Higher corrections proportional to $\alpha_{s}^{2}$ are called next-to-next-to leading order (NNLO). The ratio between a cross section in higher order and the leading order cross section is referred to as "K-factor":

$$
K_{N L O}=\frac{\sigma_{N L O}}{\sigma_{L O}}
$$

K-factors are used to correct LO cross sections from Monte Carlo generators with more recent higher order calculations.

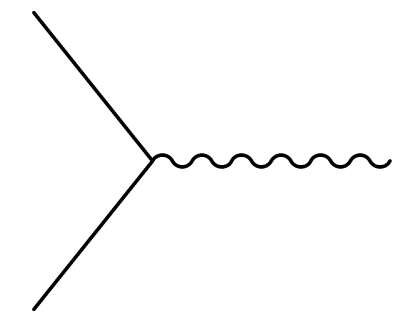

(a)

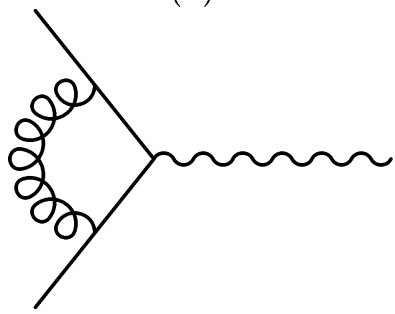

(d)

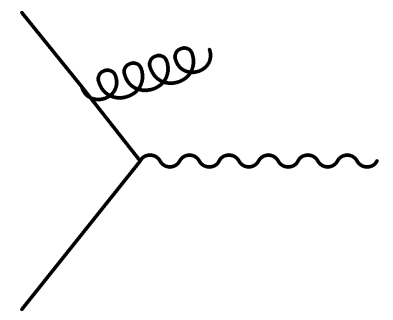

(b)

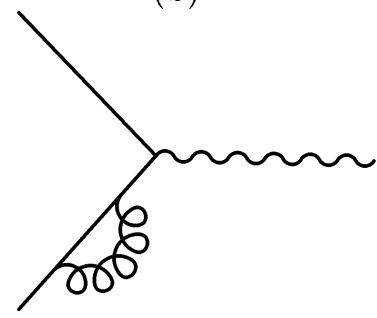

(e)

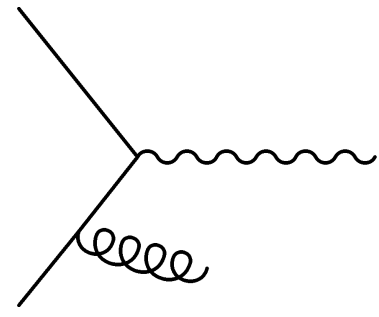

(c)

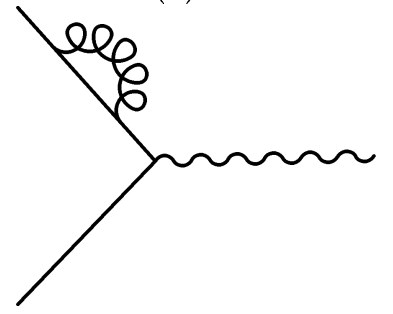

(f)

Figure 4.3: Schematic Feynman diagram in leading order for the process $q \bar{q} \rightarrow \gamma$ (a). The diagrams (b) and (c) show real corrections to the process of the order of $\alpha_{s}$. An additional correction of the same order provide the diagrams (d)-(f), which are interfering with (a).

Corrections to the $Z / \gamma^{*}$ production cross section have been calculated in NNLO [147, 152]. The $k$-factor, as shown in Fig. 4.4 , is the ratio of the NNLO $Z / \gamma^{*}$ cross section calculated with the parton distribution functions (PDF, see also Sec. 4.3.5) CTEQ6M and the LO cross section calculated with the PDF CTEQ6L. The leading order cross section of the used $Z / \gamma^{*}$-samples as calculated by PYтнIA are corrected with this mass dependend k-factor, as given in Tab. 4.6. 


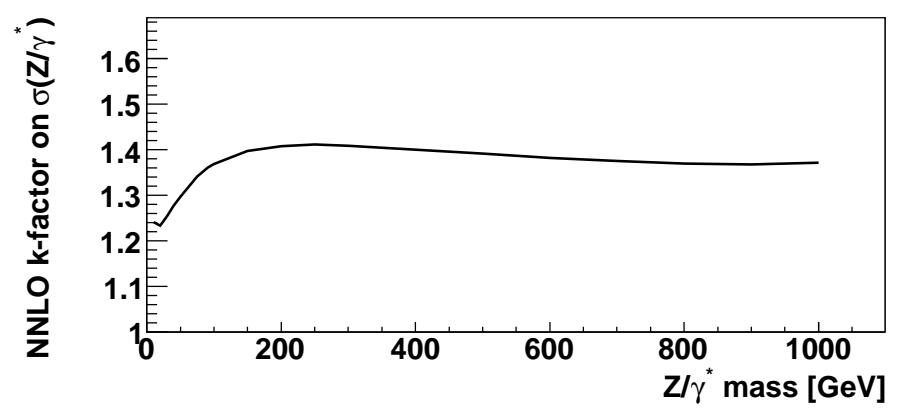

Figure 4.4: NNLO $k$-factor for the $Z / \gamma^{*}$ production cross section $[147,152]$.

\subsubsection{Multiple Interactions}

At a single bunch crossing more then one interaction may occur. The probability of $n$ minimum bias events ${ }^{2}$ at one bunch crossing is given by the Poisson probability

$$
P(n)=\frac{\mu^{n}}{n !} e^{-\mu}
$$

with $\mu$ being the expectation value. This number depends on the instantaneous luminosity, but for Monte Carlo simulation a single value for $\mu$ can be extracted from averaging over the complete data sample. In Fig. 4.5(a) the number of interactions per bunch crossing for the considered runtime is shown. The average instantaneous luminosity is $25 \cdot 10^{30} \mathrm{~cm}^{-2} \mathrm{~s}^{-1}$ and the average number of minimum bias interactions is equal to 0.75 .

For all Monte Carlo samples utilized in this analysis additional minimum bias events according to the poisson probability with an average of $\mu=0.8$, see Fig. $4.5(\mathrm{~b})$, were added to account for the additional interactions in the data. The probability of multiple interactions is used for the luminosity measurement, as was mentioned in Sec. 3.3.2.
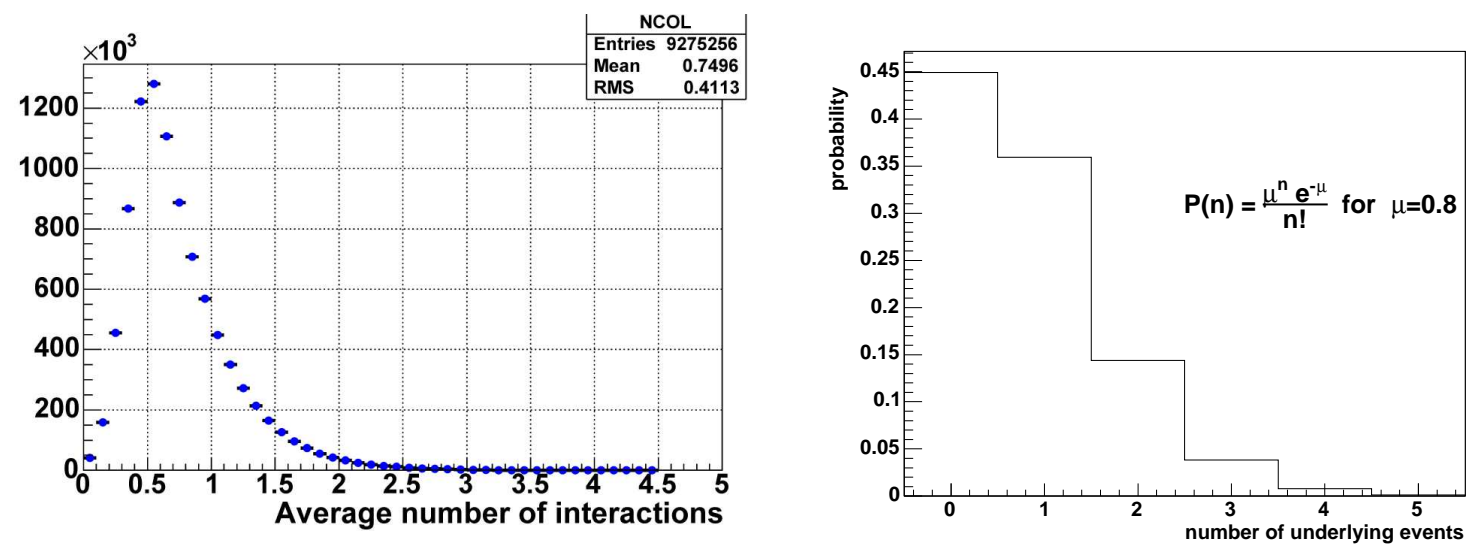

Figure 4.5: (a) Average number of interactions per bunch crossing (from [153]) and (b) the Poisson probability of underlying events for an average $\mu=0.8$.

\footnotetext{
${ }^{2} \mathrm{~A}$ minimum bias event is some particle interaction in one bunch crossing, that is (or would have been in case of Monte Carlo simulations) triggered by the luminosity detectors.
} 
Event pile-up e.g. from previous bunch crossings can lead to a different calorimeter response or to wrong information from the muon system, because the maximal drift-times are of the same order as the bunch crossing frequency. To minimize this effect fast driftgas is used in the muon system and calorimeter cells with energy less then $100 \mathrm{MeV}$ are zero suppressed. At the future LHC collider event pile-up will increase, however the finer segmentation of detectors allows to retain small occupancies.

\subsubsection{Parton Distribution Functions}

The parton distribution functions $(\mathrm{PDF}) f_{a \mid A}(x)$ were introduced in Sec. 2.3.1 as the probability to find a parton of type $a$ in a hadron of type $A$ with a certain momentum fraction $x$ ("Bjørken $x$ ") of the hadron's momentum. In general the cross section for lepton-hadron or hadron-hadron collisions can be factorized [154], as given below for a generic process $p \bar{p} \rightarrow X$ at the Tevatron:

$$
\sigma_{p \bar{p} \rightarrow X}=\sum_{i, j} \int d x_{1} f_{i \mid p}\left(x_{1}, \mu_{f}\right) \cdot d x_{2} f_{j \mid \bar{p}}\left(x_{2}, \mu_{f}\right) \cdot \hat{\sigma}_{i j \rightarrow X}\left(\hat{s}, \mu_{f}\right),
$$

where $\hat{\sigma}_{i j \rightarrow X}$ is the partonic cross section which can be calculated in perturbative QCD. The PDFs and $\hat{\sigma}$ depend on the factorization scale $\mu_{f}$. The effective center of mass energy $\hat{s}$, depends on the total center of mass energy $\sqrt{s}$, as well as on the momentum fractions $x_{1}$ and $x_{2}$ of both interacting partons:

$$
\hat{s}=x_{1} \cdot x_{2} \cdot s
$$

In order to model such an interaction, the parton distribution functions $f_{a \mid A}\left(x, \mu_{f}\right)$ have to be known. The factorization as given in Eq. (4.5) is proven to hold in all types of interactions, like $e p, p \bar{p}$, or $p p$ scattering [154]. This allows to measure the $\operatorname{PDFs} f_{a \mid A}\left(x, Q^{2}\right)$ with great precision in $e^{+} p$ and $e^{-} p$ collisions, for example at the lepton-hadron collider HERA or at the fixed target experiment BCDMS ( $\mu$ scattering), where the parton momentum fraction $x$ and the four-momentum transfer squared $Q^{2}$ can be reconstructed from the final state. The measured PDFs can then be applied for simulations of hadron-hadron interactions.

In Fig. 4.6(a) the kinematic plane $x-Q^{2}$ explored by the various experiments is shown. In Fig. 4.6(b) $x \cdot f_{a \mid A}(x)$ for $a=u, d, S$ ( $u, d$ valence quark, $S$ sea quark) and $a=g$ (gluon) as determined by the HERA experiments are compared to the PDF fits by the CTEQ collaboration $[49,50]$.

The parton content of a proton fluctuates, since virtual quarks are created in the sea, as well as gluon radiation $q \rightarrow q g$ or gluon conversion $g \rightarrow q \bar{q}$ and $g \rightarrow g g$. The evolution 


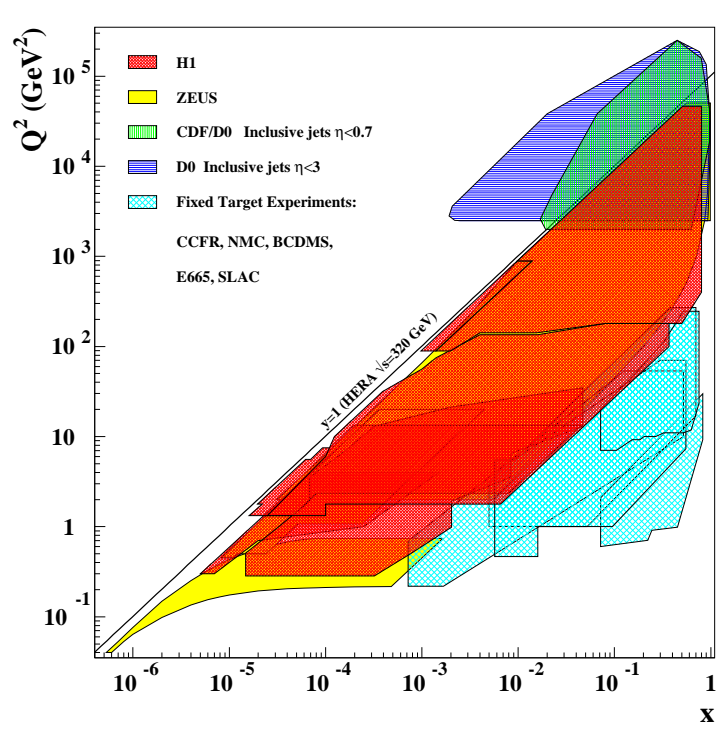

(a)

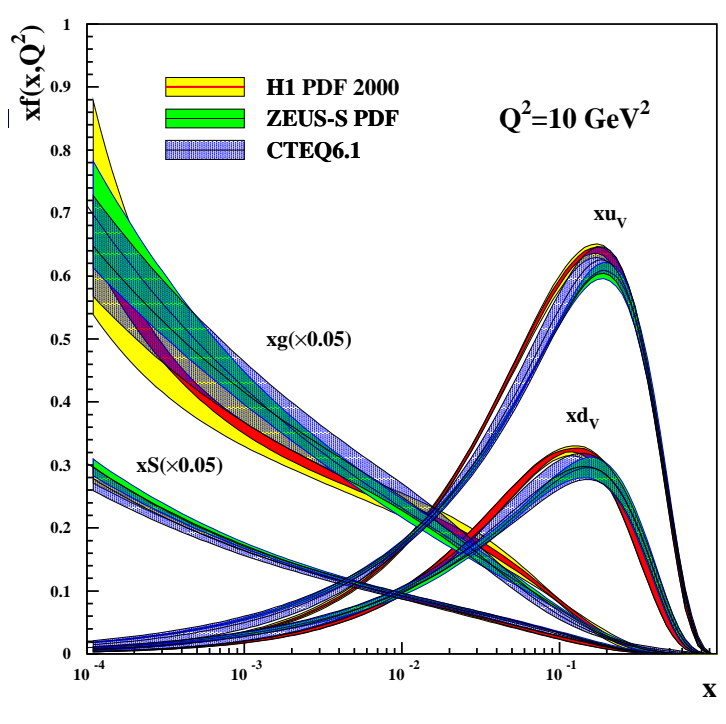

(b)

Figure 4.6: The kinematic plane in $x-Q^{2}$ covered by various experiments (a) and the parton and gluon distribution functions as measured by the HERA experiments (b) $[155,156]$.

of quark and gluon densities is given by differential equations. The DGLAP (Dokshitzer, Gribov, Lipatov, Altarelli, Parisi) $[157,158,159]$ equations describe the evolution in $Q^{2}$ :

$$
\frac{d}{d \ln Q^{2}}\left(\begin{array}{c}
f_{i}\left(x, Q^{2}\right) \\
f_{g}\left(x, Q^{2}\right)
\end{array}\right)=\frac{\alpha_{s}\left(Q^{2}\right)}{2 \pi} \sum_{j} \int_{x}^{1} \frac{d y}{y}\left(\begin{array}{cc}
P_{f_{i} f_{j}}\left(\frac{x}{y}\right) & P_{f_{i} f_{g}}\left(\frac{x}{y}\right) \\
P_{f_{g} f_{j}}\left(\frac{x}{y}\right) & P_{f_{g} f_{g}}\left(\frac{x}{y}\right)
\end{array}\right)\left(\begin{array}{c}
f_{j}\left(y, Q^{2}\right) \\
f_{g}\left(y, Q^{2}\right)
\end{array}\right)
$$

where the $f_{i}\left(x, Q^{2}\right)$ and $f_{g}\left(x, Q^{2}\right)$ are the parton density functions for quarks and gluons and the terms $P_{a b}$ give the probability to observe a parton with a momentum fraction $x$ that originates from another parton $b$ with momentum fraction $y$.

For the simulation of signal and background Monte Carlo events in this analysis, the PDFfits provided by the CTEQ collaboration $[49,50]$ were used. The systematic uncertainties to the analysis due to the PDF fits were calculated using $1 \sigma$ variations of the CTEQ6 parton distribution functions [147], as will be discussed in detail in Sec. 6.2.3. 


\section{Chapter 5}

\section{Object Identification}

Simulated data must be in agreement with the recorded data, to prove good understanding of the involved Standard Model processes and of the detector. The detector understanding is growing with time, but it is very difficult to keep all Monte Carlo samples on the same level. Hence, the determination of key efficiencies such as the trigger or the muon reconstruction efficiency, for both simulated and recorded data, is essential.

The lower the difference between data efficiency and Monte Carlo efficiency the better is the data simulation. Higher efficiencies themselves result in a better discovery potential or a stronger signal limit. In fact, all selection or acceptance efficiencies enter the signal efficiency linearly. For the best limit, they are therefore as important as for example the luminosity.

\section{$5.1 \quad$ Muons}

A widely used method to estimate muon related efficiencies is the "tag and probe" method. Objects are tagged by very tight selection cuts under the constraint that all cuts are "orthogonal" to the cut for which the efficiency is determined. The tightly selected or tagged sample is then probed for the attribute in question. The corresponding efficiency is the percentage of successfully probed events to all tagged events. Well known resonances, like the Z-boson, are used for this kind of studies, due to their clear signature. Contamination with background processes can be controlled and a possible signal contamination is negligible. All efficiencies are determined separately for data and Monte Carlo.

The Monte Carlo correction factor is the ratio of Data and Monte Carlo efficiency, and may depend on $\eta, \phi, p_{T}$ or other variables like the vertex or the instantaneous luminosity. The error of the efficiency ratio does not only have to include the (statistical, binomial) errors of both efficiencies, but also the systematic uncertainties. The uncertainties are here defined as the deviation in some interval $\left(\delta \eta, \delta \phi, \delta p_{T}\right)$ from a fit to the data sample using an appropriate function $f\left(\eta, \phi, p_{T}\right)$. The estimation of these uncertainties is quite difficult, since they depend on the bin-width. However, the maximal deviation between 
the fit and any bin (under consideration of the statistical errors) of the ratio, is a good approximation of the systematic uncertainty. Another method is to use the difference of the fit and a correction factor of 1 (perfect agreement of Data and MC expectation) as the total error of the ratio. Both methods result in very similar uncertainties, but the last method is more reliable and is used in the following for simplicity.

\subsubsection{Di-Muon Trigger Efficiency}

In most $\mathrm{D} \varnothing$ analyses the event trigger efficiencies are not simulated but parametrized. The parametrization functions are obtained from the data, using independent triggers. For the determination of the di-muon trigger efficiency, e.g. the data events were required to be triggered by EM or jet triggers. If offline reconstructed muons were found in these events, then the trigger requirements for each trigger level can be probed.

For the di-muon trigger efficiency parametrization, the D $\varnothing$ package TRIGGEREFFICIENCY [139], is utilized. All Monte Carlo events, background and signal, are weighted according to the event properties like muon energy, angular distributions, $\mathbb{F}_{T}$, and jets, in order to model the di-muon trigger efficiency in the data. These Monte Carlo event weights correspond to the di-muon trigger efficiency in data.

The used di-muon triggers as listed in Tab. 5.1 require conditions on all three trigger levels to be fulfilled. The trigger efficiency is parametrized on each trigger level L1, L2, and L3, separately.

The Level 1 turn-on function for MU1PTXATXX (Level 1, all muon region, single muon scintillator trigger without $p_{T}$ requirement.) and for $\operatorname{MUON}(1$, med) (One muon on Level 2, with medium quality) was found to be parametrized best by the following function [139]:

$$
f(\eta)=A_{3}+A_{0} \cdot e^{-A_{1}\left(\eta^{2}-A_{2}\right)} \sin \left(\eta^{2}-A_{2}\right)
$$

\begin{tabular}{lcllll}
\hline \hline Trigger & triggerlist & Level 1 & Level 2 & Level 3 & \\
\hline DMU1_TK5 & v13 & MU2PTXATXX & medium $\mu$ & 1 track, & $p_{T}>5 \mathrm{GeV}$ \\
DMU1_LM6 & v13 & MU2PTXATXX & medium $\mu$ & 1 loose $\mu$, & $p_{T}>6 \mathrm{GeV}$ \\
2MU_A_L2M0_TRK5 & v12 & MU2PTXATXX & medium $\mu$ & 1 track, & $p_{T}>10 \mathrm{GeV}$ \\
2MU_A_L2M0_L3L6 & v12 & MU2PTXATXX & medium $\mu$ & 1 loose $\mu$, & $p_{T}>6 \mathrm{GeV}$ \\
2MU_A_L2M0_TRK10 & v11 & MU2PTXATXX & medium $\mu$ & 1 track, & $p_{T}>10 \mathrm{GeV}$ \\
2MU_A_L2M0_L3L15 & v11 & MU2PTXATXX & medium $\mu$ & 1 loose $\mu$, & $p_{T}>15 \mathrm{GeV}$ \\
2MU_A_L2M0 & v9,v10 & MU2PTXATXX & medium $\mu$ & & - \\
\hline \hline
\end{tabular}

Table 5.1: The di-muon trigger requirements at the three different trigger tiers. The triggerlist version increases with time. Triggers within the same triggerlist epoch are OR-ed. The L1 requirement MU2PTXATXX asks for two muons (mu2) of any momenta (ptx) in any detector region (atxx) detected by scintillators. All di-muon triggers require at least one muon of medium quality at Level 2 in any detector region. The triggers differ with respect to their Level 3 requirement and their Level 1 pre-scale factors. 


\begin{tabular}{lcccc} 
& $A_{0}$ & $A_{1}$ & $A_{2}$ & $A_{3}$ \\
\hline Level 1: mu1ptxatxx & $-0.8 \pm 0.2$ & $2.8 \pm 0.5$ & $0.1 \pm 0.1$ & $0.99 \pm 0.01$ \\
Level 2: MUON(1,med) & 0 & $8.7 \pm 7.0$ & $1.779 \pm 0.006$ & $0.981 \pm 0.007$
\end{tabular}

Table 5.2: Parameterization for the Level 1 and Level 2 di-muon trigger turn-on function $f(\eta)$ as given in equation (5.1) [139].

Where the parameters $A_{0}$ through $A_{3}$ are defined in Tab. 5.2. The L1 turn-on functions versus $p_{T}$ for the trigger list epochs $\mathrm{v} 9{ }_{-\mathrm{v} 11}$ and $\mathrm{v} 12$ are shown in Fig. 5.1. The $\eta$ depending parametrization for L1 and L2 is shown in Fig. 5.2.

The total trigger efficiency $P(L 1, L 2, L 3)$ is the probability that the trigger conditions at all three trigger levels are satisfied and is given by:

$$
P(L 1, L 2, L 3)=P(L 1) \cdot P(L 2 \mid L 1) \cdot P(L 3 \mid L 1, L 2) .
$$

Where $P(L 2 \mid L 1)$ and $P(L 3 \mid L 1, L 2)$ represent the conditional probability for an event to satisfy a set of criteria, given it has already passed the requirements imposed at the previous triggering level(s).

Under the condition that the probability for a single object to satisfy a certain trigger condition is independent of the presence of other objects in the event, the total probability can be factored:

$$
P(O 1, O 2)=P(O 1) \cdot P(O 2)
$$

If the probability for a single object to pass a specific condition is given by $P$, then the total probability for any object out of a set of $N$ similar objects to pass the condition is given by:

$$
P=1-\prod_{i=1}^{N}\left(1-P_{i}\right)
$$

All utilized triggers (Tab. 5.1) require the trigger condition MU2PTXATXX, or two muons to be found at Level 1. The Level 1 parameterization as given by Eq. (5.1) describes only one muon. Using Eq. (5.3) the probability $P(L 1)$ can be expressed as $P(L 1)=f_{L 1}^{2}$. The Level 2 condition requires only one muon of medium quality, however at least two muons at L1 have been found. The probability $P(L 2 \mid L 1)=\left(1-\left(1-f_{L 2}\right)^{2}\right)$ can be calculated using Eq. (5.4). All Level 3 requirements were found to be $100 \%$ efficient: $P_{L 3 \mid L 1, L 2}=1$ [139]. The total trigger efficiency parametrization function is then given by:

$$
\begin{aligned}
P(L 1, L 2, L 3) & =P(L 1) \cdot P(L 2 \mid L 1) \cdot P(L 3 \mid L 1, L 2) \\
& =f_{L 1}^{2} \cdot\left(1-\left(1-f_{L 2}\right)^{2}\right) \cdot 1
\end{aligned}
$$

Where the $\eta$-depending functions $f_{L 1}$ and $f_{L 2}$ are given by equation (5.1) using the parameters as defined in Tab. 5.2. 

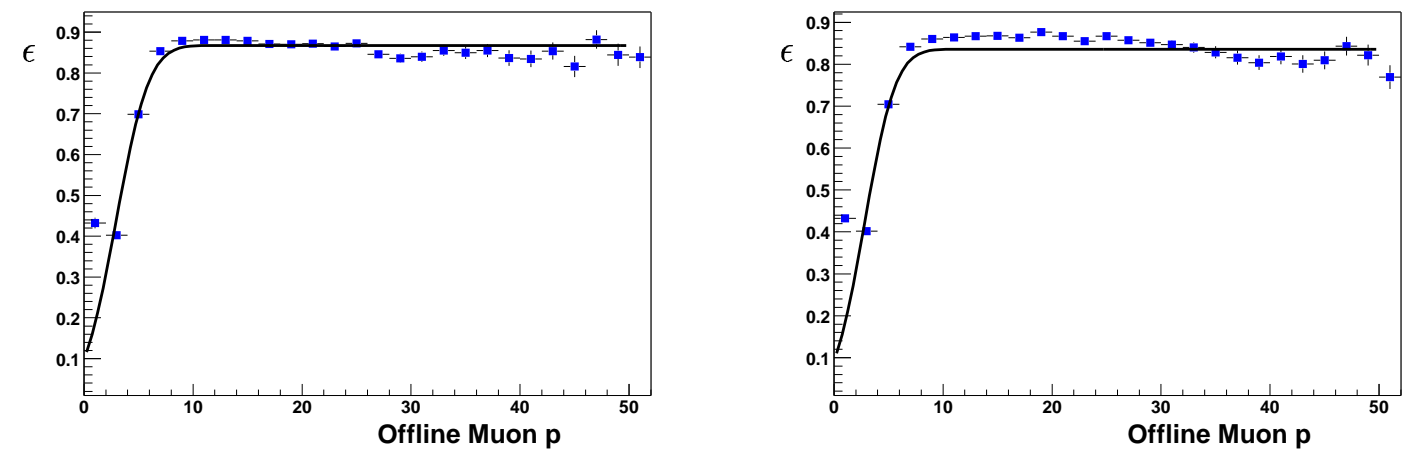

Figure 5.1: Level 1 muon trigger efficiency versus muon $p_{T}$ for trigger list version $v 9-v 11$ (left) and v12 (right) [139].
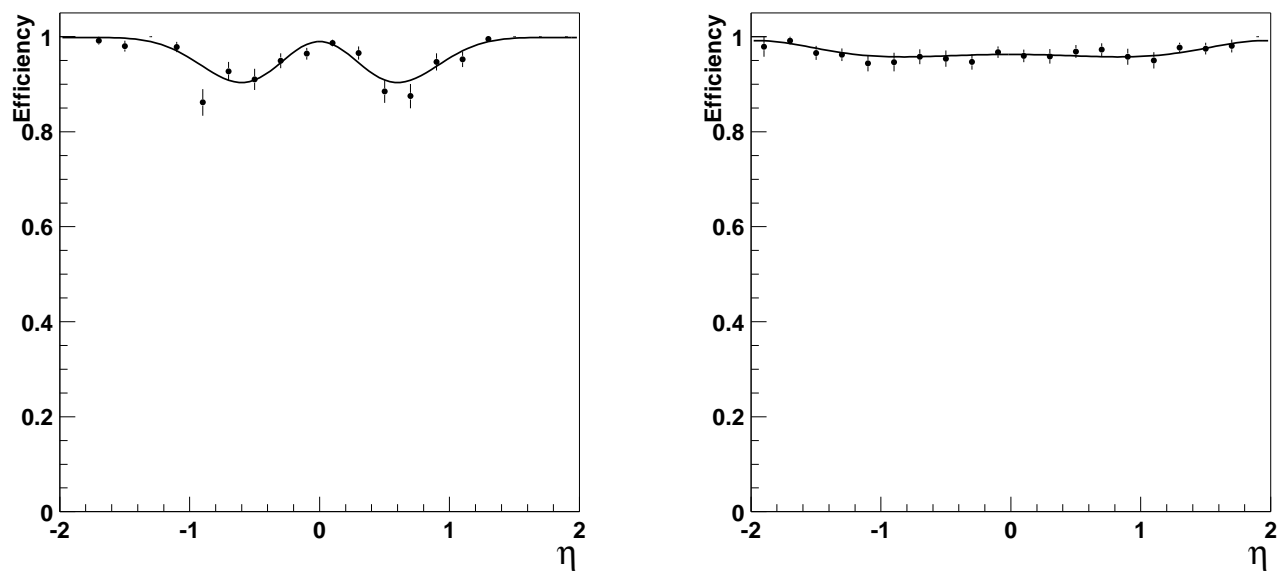

Figure 5.2: Muon Level 1 (left) and Level 2 (right) trigger efficiency parametrization versus the muon's pseudo-rapidity $\eta[139]$.
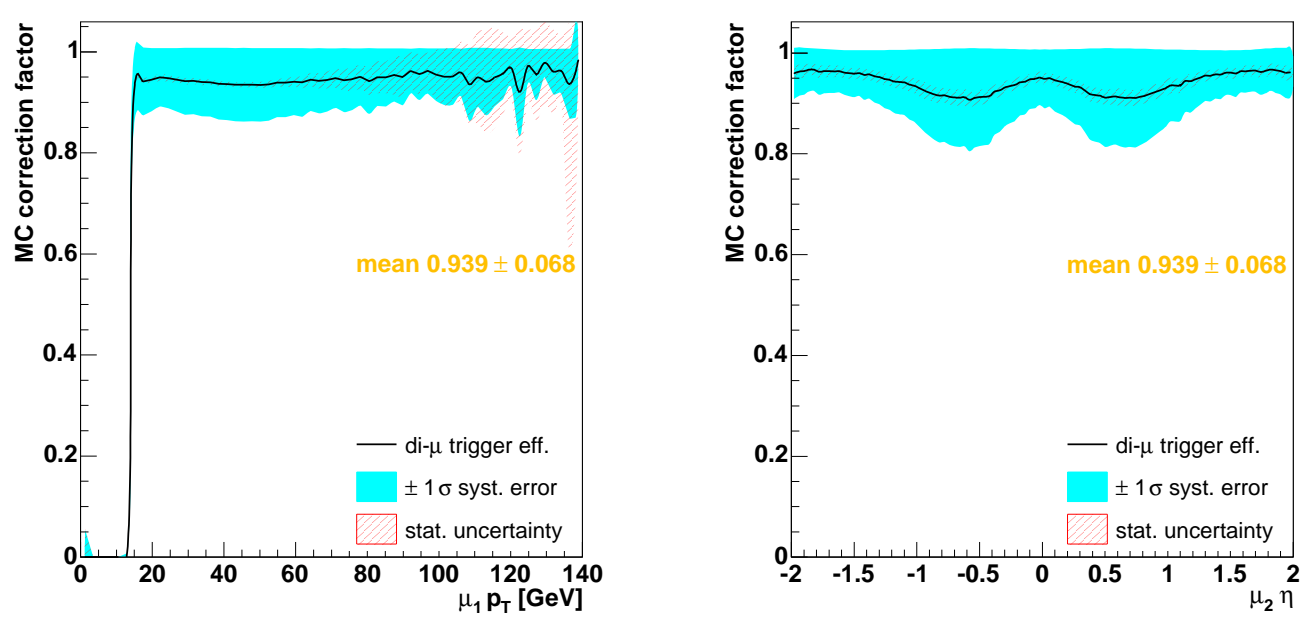

Figure 5.3: Di-muon trigger efficiency weight as applied on all Monte Carlo Samples, as a function of the offline reconstructed transverse momentum $\left(p_{T}\right)$ of the leading muon (left) and $\eta$ of the next to leading muon (right). 
The total di-muon trigger efficiency, or the weight as applied on all Monte Carlo events, $0.939 \pm 0.068$ on average over the total Standard Model background data set, is shown in Fig. 5.3 as a function of the offline muon momentum $p_{T}$ and as a function of the pseudo-rapidity $\eta$.

The total uncertainty of $6.8 \%$ reflects the uncertainty of the fit parameters as given in Tab. 5.2 and is overestimating the true di-muon trigger uncertainty by far, see the blue band in Fig. 5.3. Nonetheless, the trigger uncertainty contribution to the total systematic error will be negligible in the end, see Sec. ?? for reference. Another shortcomming of the di-muon TRIggerEfFiciency class is the L2 parametrization, which is defined only for muons exceeding $15 \mathrm{GeV}$. Since this analysis requires at least one muon with $p_{T}>15 \mathrm{GeV}$ this is no issue here. However, the TRIGGEREFFICIENCY package is rewritten for the new data epoch p17 in order to handle muons with lower transverse momenta and to minimize the systematic trigger parametrization uncertainty.

\subsubsection{Muon Reconstruction Efficiency}

The muon reconstruction efficiency is the probability that a muon is detected and reconstructed by the DØ muon system with at least "loose" quality. A loose muon must meet at least two of the following three tests:

- at least one A layer scintillator hit and at least two A layer wire chamber hits

- at least one BC layer scintillator hit

- at least two BC layer wire chamber hits

A muon which passes all three of the above tests is called "medium". The muon reconstruction efficiency is calculated on the Z-peak.

The $\mathrm{Z}$ sample is selected by requiring a well measured medium, isolated and track matched muon with at least $15 \mathrm{GeV} p_{T}$. The event must not contain more than one jet and anticosmic cuts are applied. Secondly a well measured track in the inner detector (SMT, $\mathrm{CFT}$ ) with at least $20 \mathrm{GeV} p_{T}$ must exist opposite in $\phi$ from the first muon. The fiducial region corresponding to the bottom hole in the muon system is removed: the track must not point toward the sixth octant in $\phi$ for $|\eta|<1.3$. The muon and the track must be oppositely charged, and their invariant mass must match the Z-mass within $20 \mathrm{GeV}$.

To minimize trigger bias, all events must be triggered by a non-muon trigger, i.e. electron or jet triggers, or by any single-muon trigger, where the tagged muon has pulled the trigger. No event is recorded based alone on di-muon triggers, which would have influenced the measurement of the muon reconstruction efficiency in data.

The tagged events are probed for a second muon in two different ways: 
A Any loose muon matches the tagged track within $\Delta R=\sqrt{\Delta \phi^{2}+\Delta \eta^{2}} \leq 0.5$. The cone size within the track and the muon is matched is large enough, so that the matching is fully efficient.

B Any loose muon is matched to the tagged track by the standard D $\varnothing$ algorithm implemented in the $\mathrm{D} \varnothing$ reconstruction software [137]. Here, the product of muon reconstruction and track matching efficiency is measured.

Due to the large cone radius of method $A$, the measured efficiency is the pure muon reconstruction probability. The result of method $B$ is the product of muon reconstruction and track matching efficiency.

The Pass 2 data sample used was the top-group "MURECOEFF" skim, which is based on the common sample skim "1MULOosE" and converted to root format by TopANALYZE version Ipanema. The skim contains events with at least one loose, isolated muon with more that $15 \mathrm{GeV} p_{T}$ and at least two tracks with at least $15 \mathrm{GeV} p_{T}$ each. The Pass 2 Monte Carlo sample contains $\mathrm{Z} / \gamma^{*}$ events in the mass region of $60-130 \mathrm{GeV}$. CTEQ5L parton distribution functions and the TopAnalyze version "Ipanema" [138] was used, as for the data.

\begin{tabular}{lll} 
Method & $\epsilon$ Data & $\epsilon$ Monte Carlo \\
\hline A $\quad$ reco. efficiency & $0.9151 \pm 0.0021$ & $0.9376 \pm 0.0012$ \\
B $\quad$ reco. * track match. efficiency & $0.9148 \pm 0.0021$ & $0.9378 \pm 0.0012$
\end{tabular}

Table 5.3: Muon reconstruction efficiency in data and in the Monte Carlo simulation.

As given in Tab. 5.1.2, the muon reconstruction efficiency for data is $91.5 \pm 0.2 \%$, for Monte Carlo $93.8 \pm 0.1 \%$. The efficiencies of both methods A \& B agree, for a given track matching efficiency of $99.8 \%$, which is consistent with the track finding times track matching efficiency in section 5.1.3. The reconstruction efficiency in data and Monte Carlo versus muon $p_{T}, \phi$, and $\eta$ is shown in Fig. 5.4.

The corresponding correction, i.e. the ratio of data and Monte Carlo efficiency, is shown in Fig. 5.5 for $\phi, \eta, p_{T}$ and also $M_{\mu \mu}$, for reference; the muon reconstruction efficiency should not depend on $M_{\mu \mu}$. The ratio is flat in $\phi, p_{T}$ and $M_{\mu \mu}$, however a slight $\eta$ dependence can
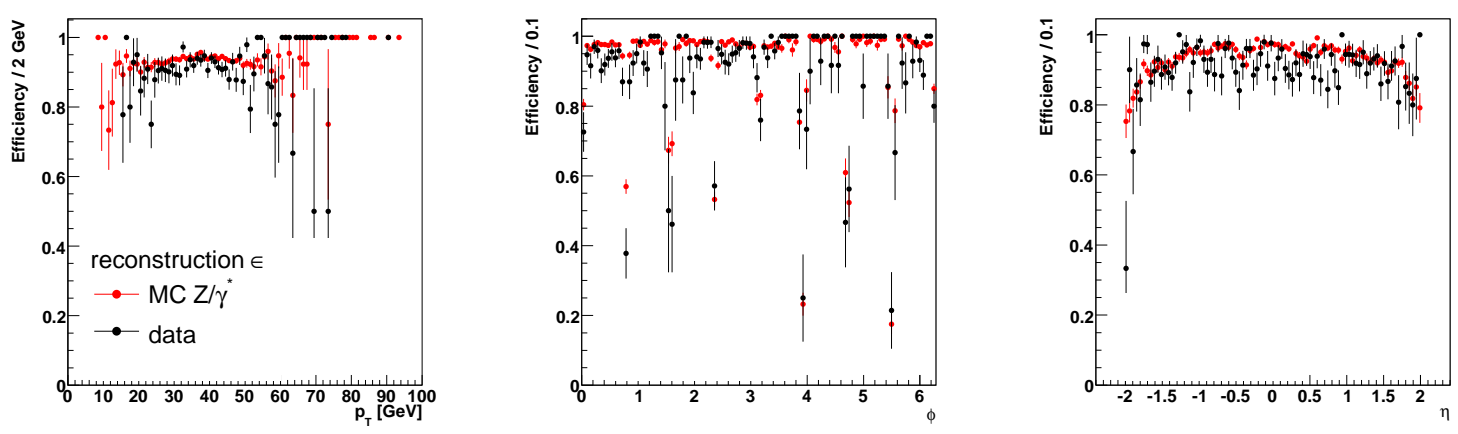

Figure 5.4: Muon reconstruction efficiency as a function of muon $p_{T}, \phi$, and $\eta$ from left to right. 
Data (MURECOEFF skim)
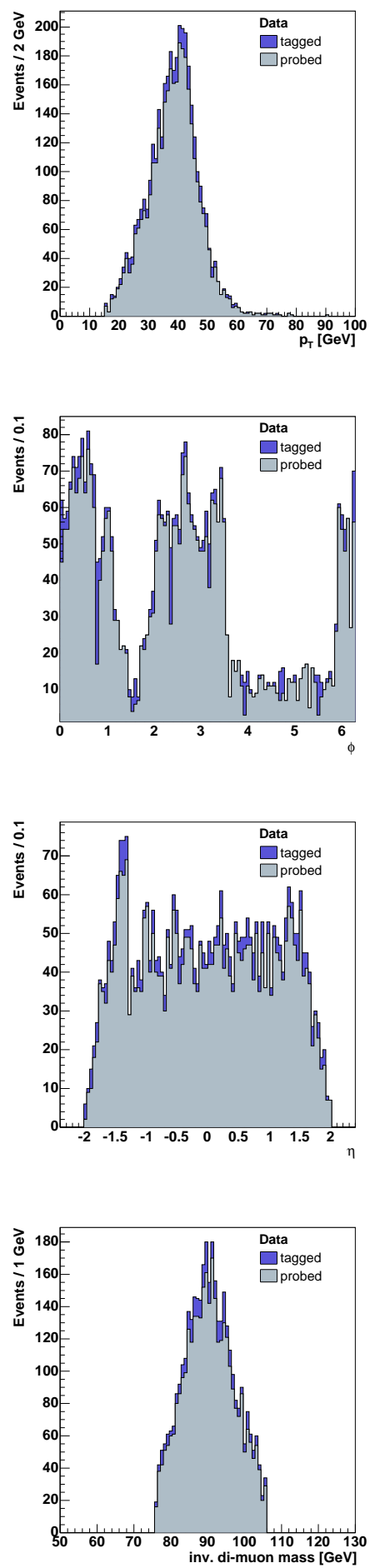

$Z / \gamma^{*}$-Monte Carlo
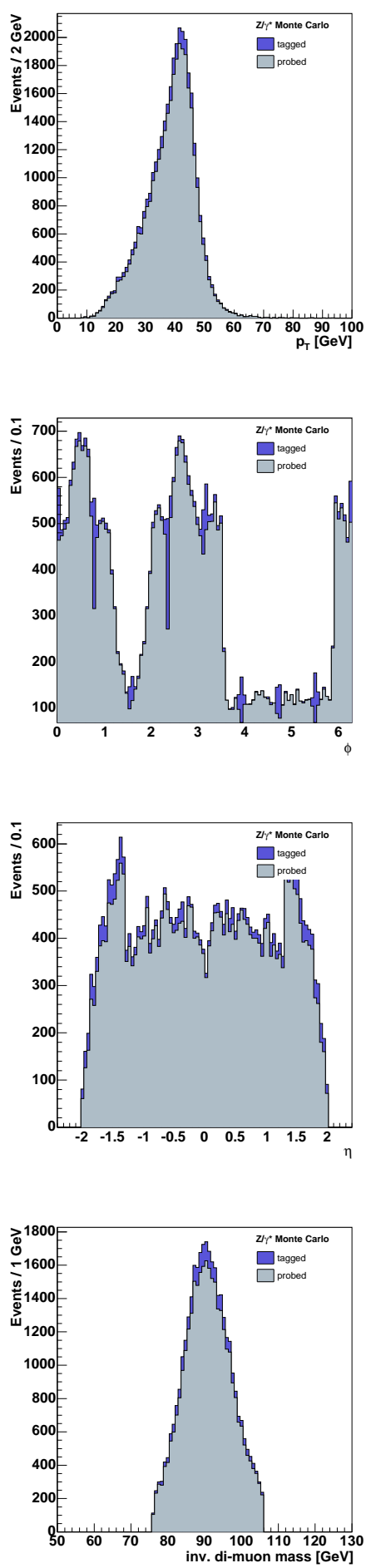

correction $\epsilon_{d a t a} / \epsilon_{M C}$
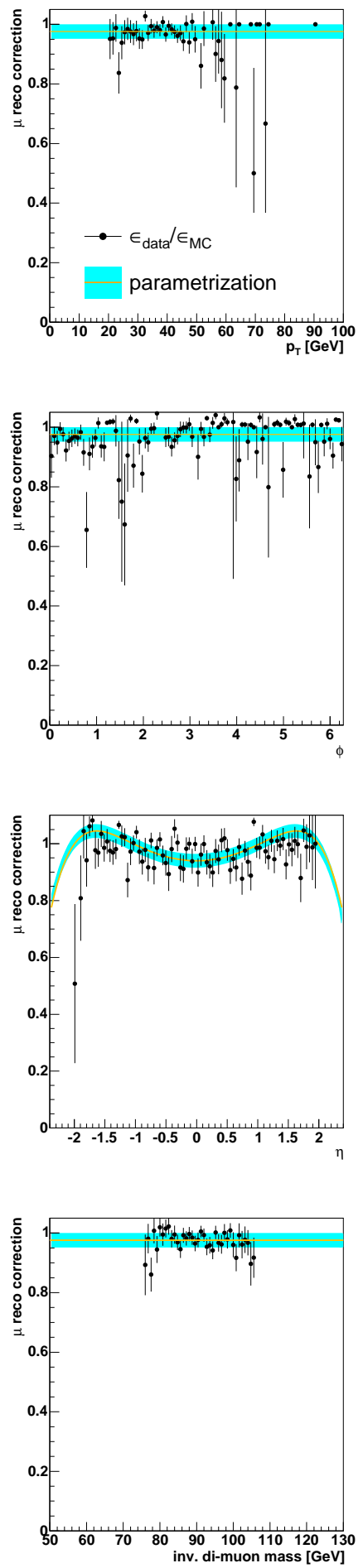

Figure 5.5: Muon reconstruction correction factor: Tagged and probed muons in the MURECOEFF data skim (left column), in the $Z / \gamma^{*}$-Monte Carlo (middle column) and the resulting correction for the Monte Carlo samples $\epsilon_{\text {data }} / \epsilon_{M C}$ (right column) as a function of $p_{T}, \phi, \eta$ and the invariant mass of the tag-muon and the track of the muon candidate, from the top to the bottom row. 
be observed. All simulated events are therefore re-weighted, according to the fit-function given by:

$$
f(\eta)=0.94+0.06 \eta^{2}-0.003 \eta^{6}
$$

The resulting muon reconstruction correction averaged over $\eta$ is equal to a factor of $0.976 \pm$ 0.024 per muon.

\subsubsection{Muon Track Finding and Matching Efficiency}

The muon momentum resolution is poor if measured only in the local muon system. Hence, only muons which are matched to a track measured by the central tracking detectors, the SMT or the CFT, are used for most analyses. The efficiency of finding a central track and matching it to the muon is measured by requiring one well measured, medium, isolated and central track-matched muon with at least $30 \mathrm{GeV} p_{T}$, and a second medium muon without requiring a central track. The "probe" muon must have at least $20 \mathrm{GeV} p_{T}$ measured by the local muon system, a separation in $\phi$ from the first muon of at least 2.8, and form together with the first muon an invariant mass in the range of $30 \mathrm{GeV}$ around the nominal Z-mass. The track finding times matching efficiency is the percentage of tagged events for which the second muon is matched to a central track.

Trigger bias is avoided, by asking for the events to be triggered by any trigger, but a di-muon trigger with track requirement. If the event is selected by a single muon trigger with track requirement, then the trigger object must be matched to the "tagged" muon. No event is exclusively selected by a trigger with track requirement, that is fired by the "probe" muon.

The used data samples are the common sample group's Pass 2 " $2 M U$ " skim with two loose muons and a CTEQ5L Z $/ \gamma^{*}$ Monte Carlo sample in a Z-mass region of $60-130 \mathrm{GeV}$. Both samples are processed with TopAnalyze version "Ipanema" [138].
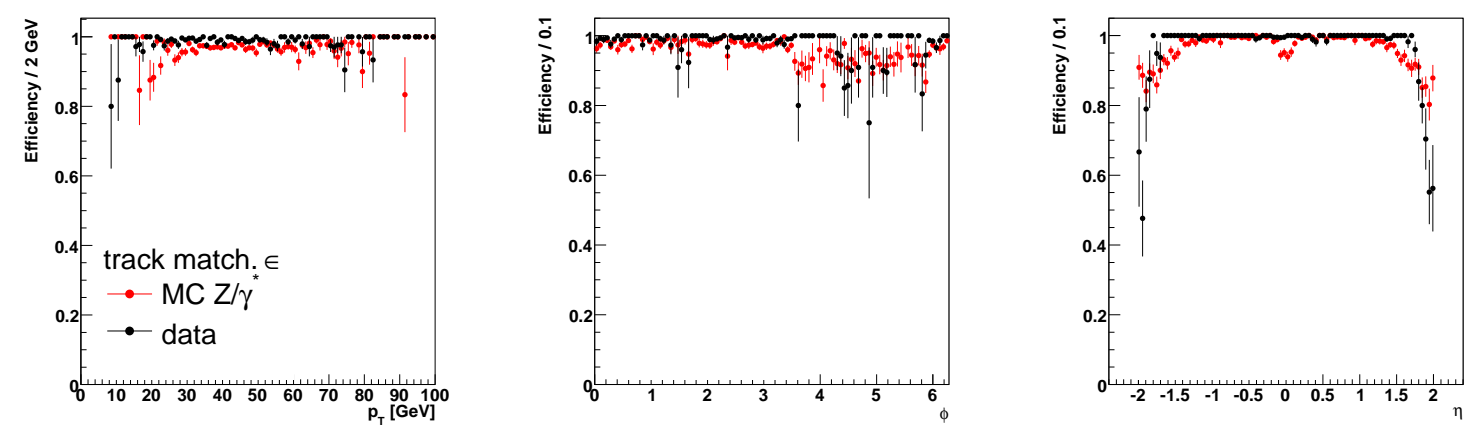

Figure 5.6: Track finding and matching efficiency as a function of muon $p_{T}, \phi$, and $\eta$ from left to right. 
Data (2MU skim)
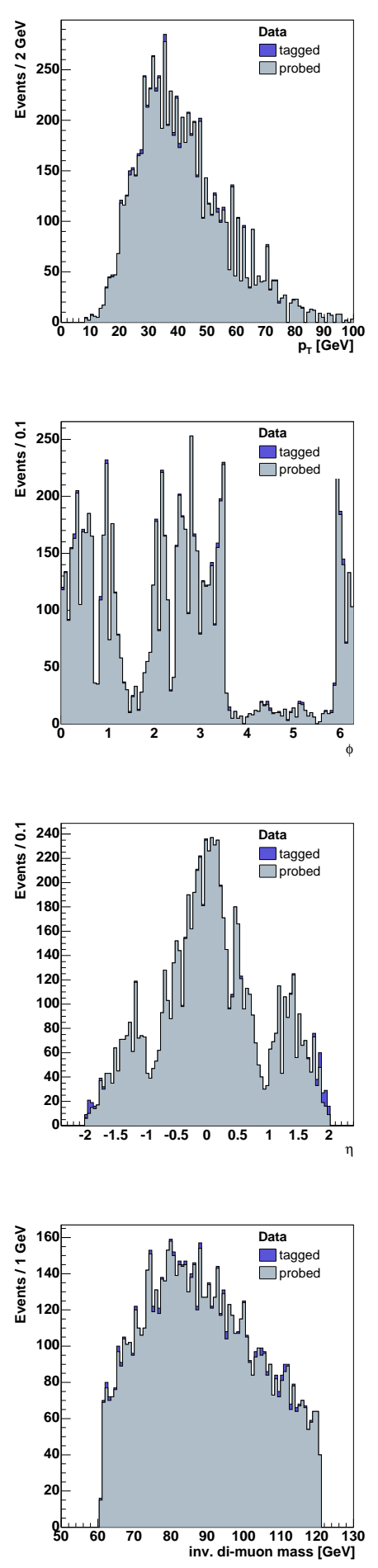

$Z / \gamma^{*}$-Monte Carlo
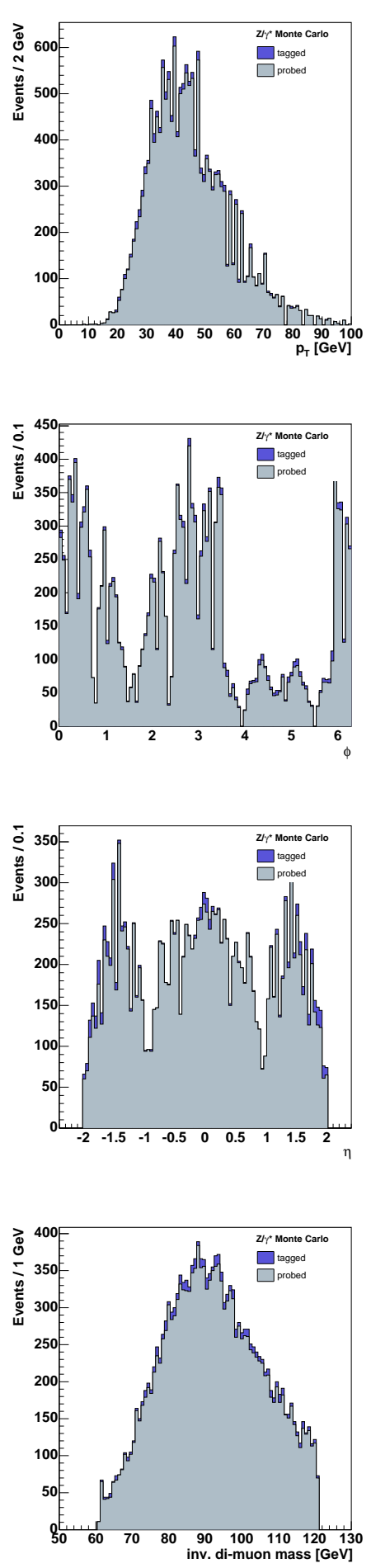

correction $\epsilon_{d a t a} / \epsilon_{M C}$
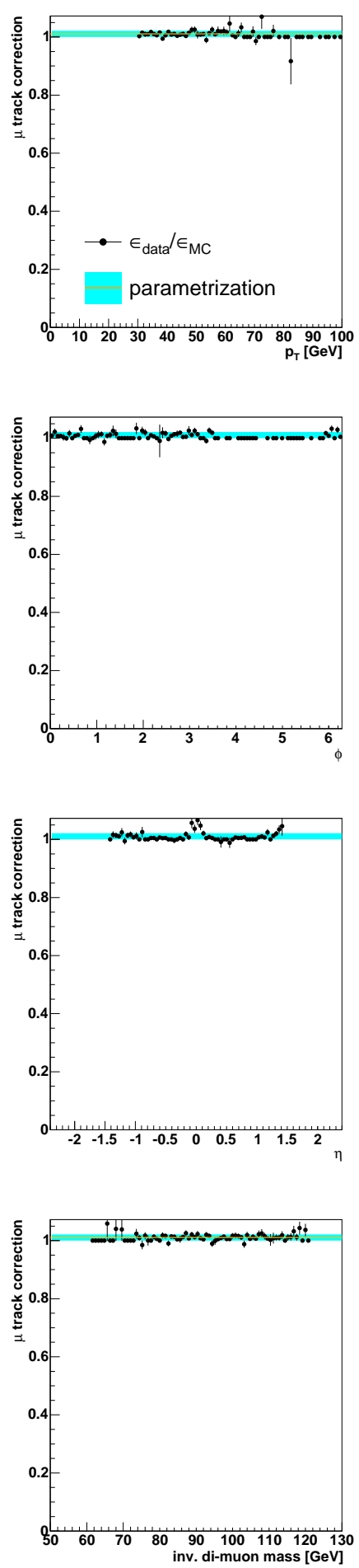

Figure 5.7: Muon track finding \& matching correction factor: Tagged and probed muons in the $2 \mathrm{MU}$ data skim (left column), in the $Z / \gamma^{*}$-Monte Carlo (middle column) and the resulting correction for the Monte Carlo samples $\epsilon_{d a t a} / \epsilon_{M C}$ (right column) as a function of $p_{T}, \phi, \eta$ and the invariant di-muon mass, from the top to the bottom row. 


\begin{tabular}{lll} 
& $\epsilon$ Data & $\epsilon$ Monte Carlo \\
\hline track efficiency & $0.998 \pm 0.001$ & $0.988 \pm 0.001$
\end{tabular}

Table 5.4: Track finding times matching efficiency in data and in the Monte Carlo simulation.

The track matching and finding efficiencies in data and Monte Carlo have a similar shape (Fig. 5.6), therefore the ratio is flat, as shown in Fig. 5.7 for $\phi, \eta, p_{T}$ and the invariant di-muon mass. A constant correction factor of $1.010 \pm 0.01$ is applied to all Monte Carlo events per muon.

\subsubsection{Muon Isolation Efficiency}

The muon isolation efficiency is measured in a similar way as the muon track matching efficiency. As before, the used Pass 2 samples are the common sample group's $2 M U$ skim and the $\mathrm{Z} / \gamma^{*}$ Monte Carlo in the mass region of $60-130 \mathrm{GeV}$. The muon isolation efficiency versus a variable $x$ is the probability that a muon inside the range $\delta x$ is isolated. A muon is isolated when the amount of unclustered calorimeter energy around the muon track, in a hollow cone with inner radius $r=0.1,\left(r=\sqrt{\Delta \eta^{2}+\Delta \phi^{2}}\right)$ and outer radius $R=0.4$ does not exceed $2.5 \mathrm{GeV}$ and when the sum of $p_{T}$ of tracks around the muon inside a cone with radius $R=0.5$ is smaller than $2.5 \mathrm{GeV}$. The isolation efficiency is calculated for events with one muon with isolation and track match (the tagged muon) and a second muon with track match (the probe muon). The invariant mass of both muons has to be $\left|M_{\mu \mu}-91 \mathrm{GeV}\right|<30 \mathrm{GeV}$, the $p_{T}$ of the tag-muon has to be larger than $8 \mathrm{GeV}$ and $p_{T}>15 \mathrm{GeV}$ for the probe-muon.

The muon isolation efficiency is not biased by any trigger, since the complete data set has been taken with triggers regardless of isolation. This will change from triggerlist version v14, however.

The influence of the instantaneous luminosity was studied by [160]. The isolation efficiency in the data changes roughly by $\Delta \epsilon^{i s o}=5 \%$ when the instantaneous luminosity goes from 0 to $50 \cdot 10^{30} \mathrm{~cm}^{-2} \mathrm{~s}^{-1}$. In this analysis and in the sample used to estimate the isolation efficiency, the luminosity profile is identical. No other selections in the analysis are correlated to the instantaneous luminosity. If therefore the isolation efficiency in the data is shifted because of the instantaneous luminosity, then this effect would be included in the calculated correction factor. A systematic uncertainty would arise, if the luminosity profile in the analysis data and in the data used for the efficiency would be different, which is not the case.

\begin{tabular}{lll} 
& $\epsilon$ Data & $\epsilon$ Monte Carlo \\
\hline muon isolation efficiency & $0.944 \pm 0.002$ & $0.956 \pm 0.001$
\end{tabular}

Table 5.5: Muon isolation efficiency in data and in the Monte Carlo simulation. The simulated events contain minimum bias events to simulate the pile-up, as discussed in Sec. 4.3.4. 
Data (2MU skim)
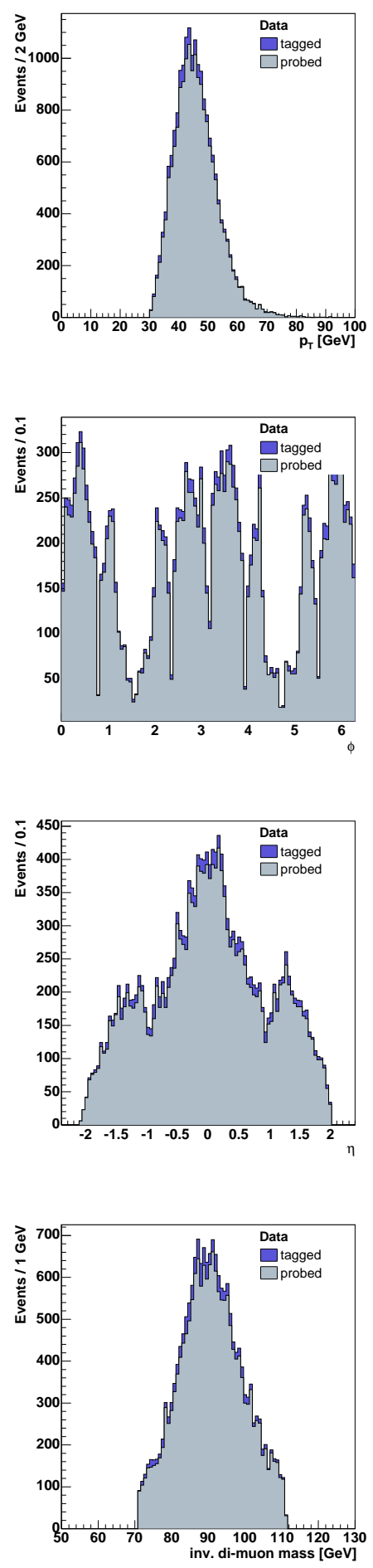

$Z / \gamma^{*}$-Monte Carlo
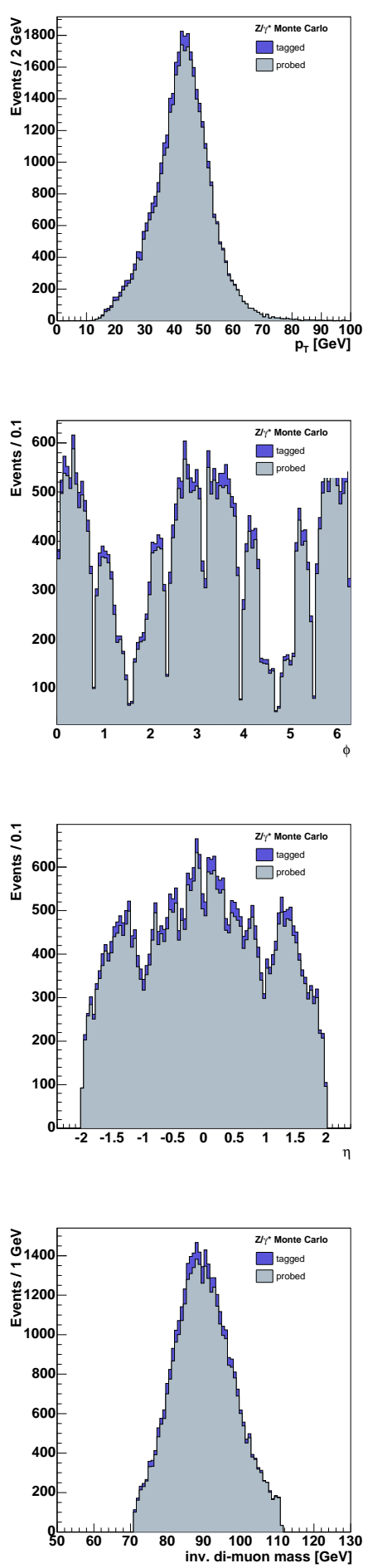

correction $\epsilon_{d a t a} / \epsilon_{M C}$
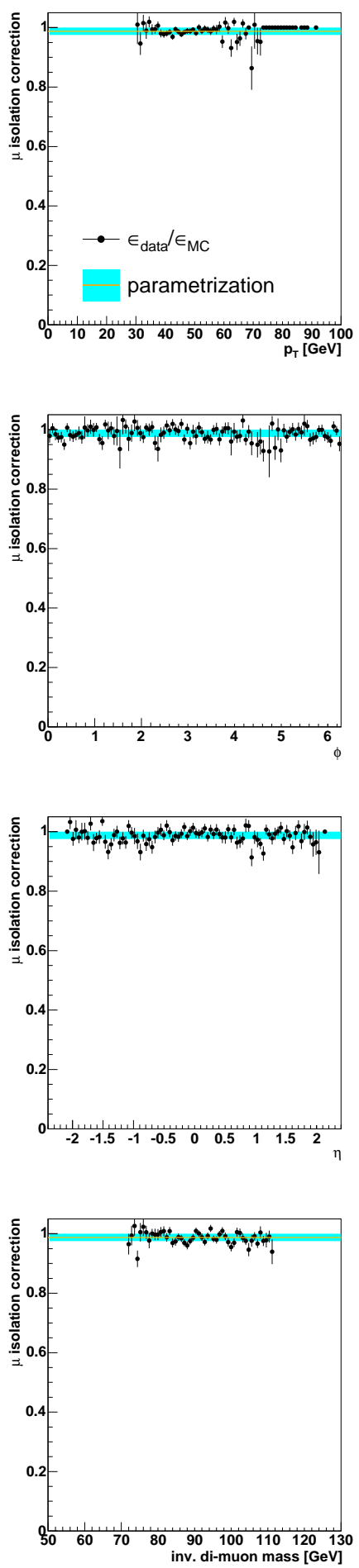

Figure 5.8: Muon isolation correction factor: Tagged and probed muons in the 2MU data skim (left column), in the $Z / \gamma^{*}$-Monte Carlo (middle column) and the resulting correction for the Monte Carlo samples $\epsilon_{d a t a} / \epsilon_{M C}$ (right column) as a function of $p_{T}, \phi, \eta$ and the invariant di-muon mass, from the top to the bottom row. 


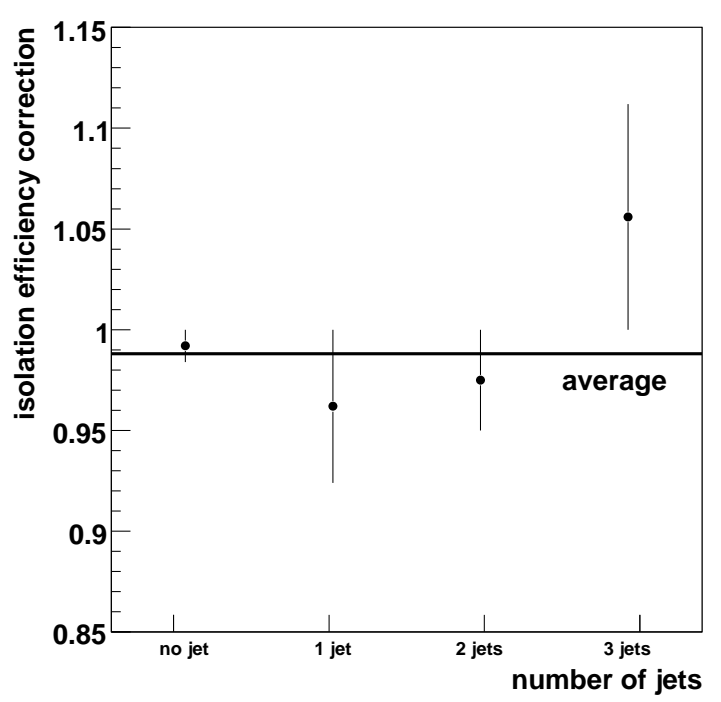

Figure 5.9: Muon isolation correction factor dependence on the number of jets in the event.
The dependence of the correction factor on the jet multiplicity has been studied as shown in Fig. 5.9 and found to be negligible. A constant correction independent of jet activity is used.

The muon isolation efficiency is approximately flat for $\phi, \eta, p_{T}$ and the invariant di-muon mass as shown in Fig. 5.10 and summarized in Tab. 5.5. Therefore a constant correction factor of $0.988 \pm$ 0.012 per muon (Fig. 5.8) is applied to all used Monte Carlo samples. The uncertainty of this factor as all other efficiency correction uncertainties are considered for the systematic errors of all simulated processes (Section ??).
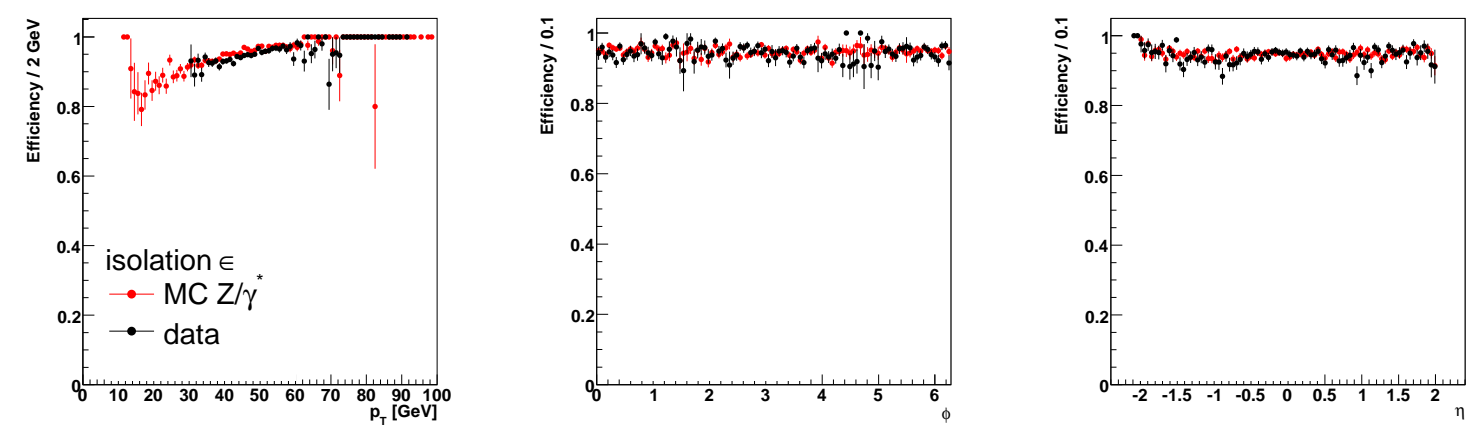

Figure 5.10: Isolation efficiency as a function of muon $p_{T}, \phi$, and $\eta$ from left to right.

\subsection{5 "Medium" Muon Efficiency}

The "medium" efficiency is calculated by tagging loose muons and probing them for medium quality, as defined in section 5.1.2 and explained in more detail in [161]. The data samples used are the common sample group $2 M U$ skim with two loose muons or the MURECOEFF skim with one loose muon and a $\mathrm{Z} / \gamma^{*}$ Monte Carlo sample in the mass range of $60-130 \mathrm{GeV}$. The muon-system bottom hole is removed. Again, both Pass 2 data and Monte Carlo samples are transfered to root-format by the TopAnalyze version "Ipanema" [138].

Two types of events are used to calculate the medium efficiency in data and Monte Carlo. 
1. The Z-resonance is selected by asking for two well measured, isolated and trackmatched muons with an invariant mass of the Z-boson $\pm 15 \mathrm{GeV}$ and a distance in $\phi$ of at least 2.8 ;

2. Only a single loose muon is required and is probed for medium quality. The distributions for this method are shown in Fig. 5.12.

The data events must be selected by triggers that do not require medium muons, that are either calorimeter based triggers or single muon triggers, where the "tag" muon is matched to the L1/L2/L3 trigger object.

\begin{tabular}{lll} 
& $\epsilon$ Data & $\epsilon$ Monte Carlo \\
\hline medium efficiency Z-peak & $0.982 \pm 0.001$ & $0.956 \pm 0.001$ \\
medium efficiency single- $\mu$ & $0.950 \pm 0.001$ & $0.961 \pm 0.0002$
\end{tabular}

Table 5.6: Medium muon efficiency in data and in Monte Carlo simulation.
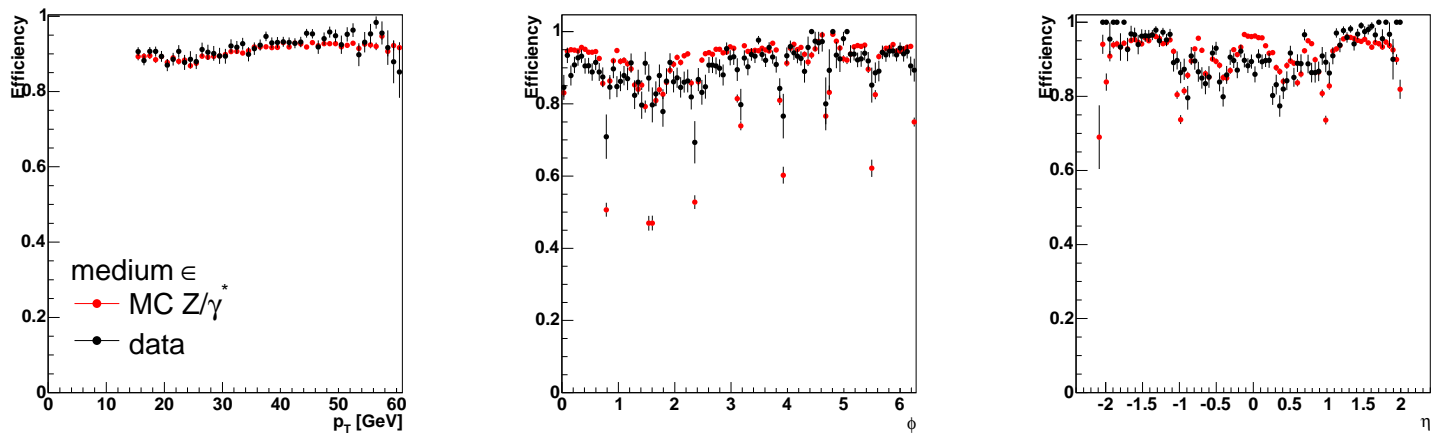

Figure 5.11: Medium efficiency as a function of muon $p_{T}, \phi$, and $\eta$ from left to right.

The ratio of the data and Monte Carlo medium muon efficiency is flat in $\phi, \eta$ and $p_{T}$ as shown in Fig. 5.11. However, different methods and different quality criteria on the events give different correction factors that are compatible with $1.000 \pm 0.027$, see Fig. 5.12. For the medium efficiency the Monte Carlo is taken as truth (the correction factor is equal to 1 ), with an uncertainty of $2.7 \%$. 
Data (MURECOEFF skim)
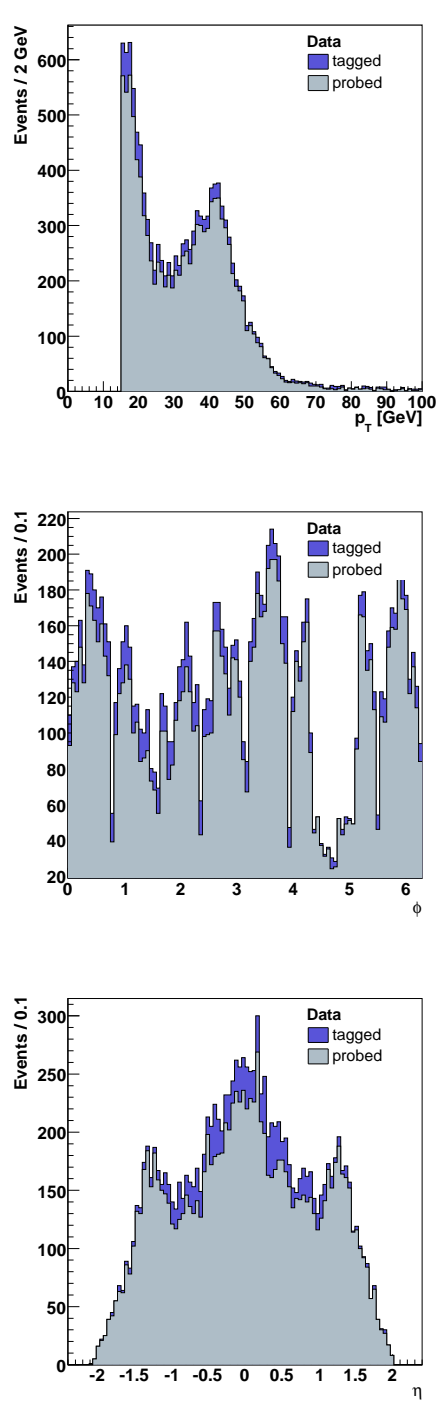

$Z / \gamma^{*}$-Monte Carlo
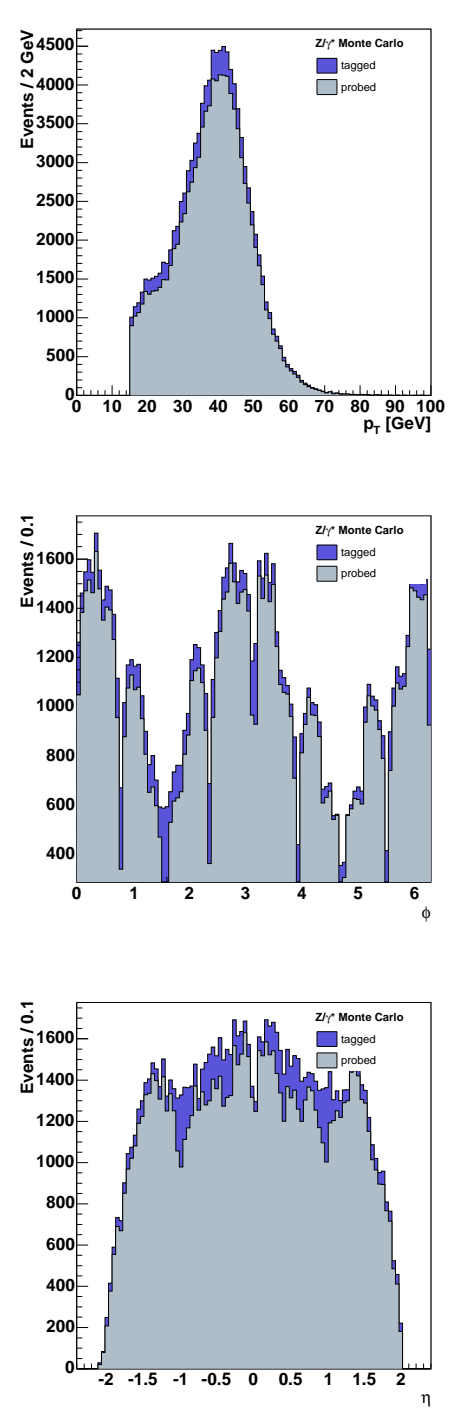

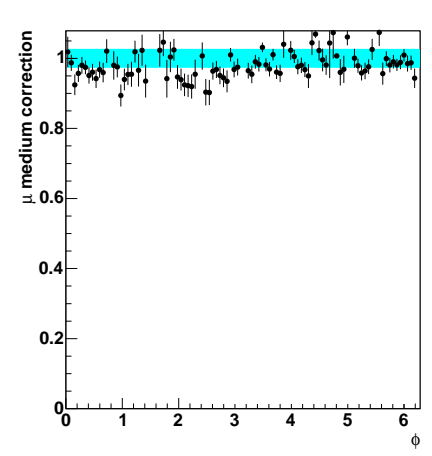

correction $\epsilon_{\text {data }} / \epsilon_{M C}$
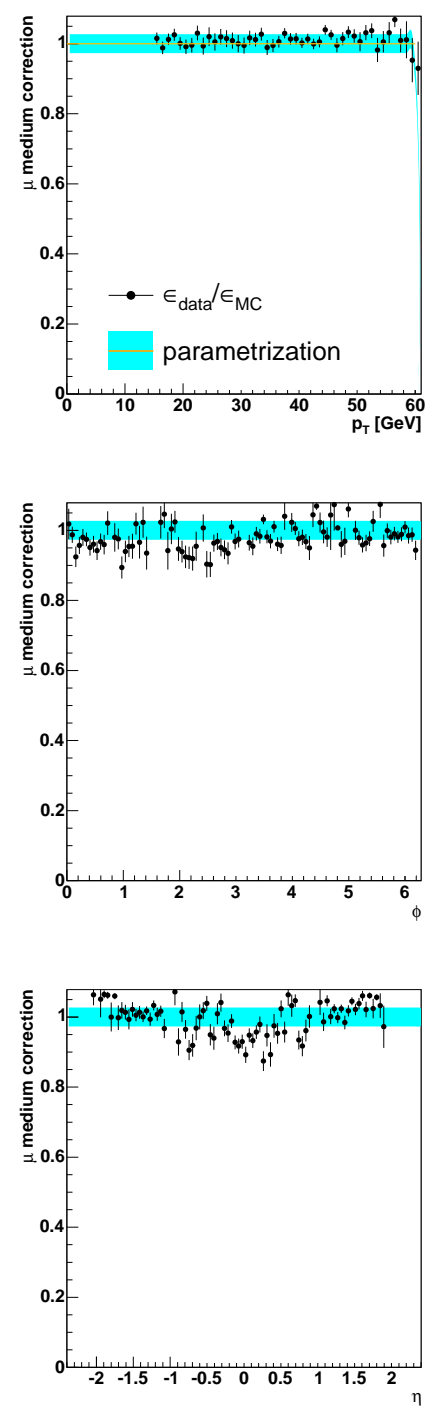

Figure 5.12: Medium muon correction factor: Tagged and probed muons in the MURECOEFF data skim (left column), in the $Z / \gamma^{*}$-Monte Carlo (middle column) and the resulting correction for the Monte Carlo samples $\epsilon_{d a t a} / \epsilon_{M C}$ (right column) as a function of $p_{T}, \phi$ and $\eta$, from the top to the bottom row. 


\subsubsection{Muon Momentum Smearing}

The muon momentum resolution is simulated too optimistically by the detector simulation, therefore the muon $p_{T}$ is smeared to match the $\mathrm{D} \varnothing$ detector resolution as observed in the data.

$$
\frac{1}{p_{T}}=\frac{1}{p_{T}^{\prime} \cdot S}+\operatorname{Gauss}(0, \sigma)
$$

Where $S$ is a scale factor to the unsmeared muon momentum $p_{T}^{\prime}$ and $\operatorname{Gauss}(0, \sigma)$ is a Gaussian distributed random number with mean 0 and a width of $\sigma$. The size of $S$ and $\sigma$ are given in Tab. 5.7 for the central and the forward muon system. For more details see Ref. [161].

\begin{tabular}{lll}
\hline \hline & central $|\eta| \leq 1.6$ & forward $|\eta|>1.6$ \\
\hline scale $S$ & $0.9910_{-0.0026}^{+0.0026}$ & $0.9962_{-0.0077}^{+0.0051}$ \\
width $\sigma$ & $0.0025_{-0.0002}^{+0.0001}$ & $0.0043_{-0.0006}^{+0.0001}$ \\
\hline \hline
\end{tabular}

Table 5.7: Muon momentum smearing coefficients [161].

The $1 \sigma$ uncertainties of $S$ and $\sigma$ as given in Tab. 5.7 are considered as systematic error source.

\subsubsection{Summary Muon Efficiencies}

In Tab. 5.8 the various muon efficiencies and the resulting correction factors are summarized and compared to the correction factors obtained by [144] on a "Pass 1" data sample. The "Pass 2" data utilized for this analysis have been re-reconstructed, i.e. corrected, with the more recent "DØcorrect" version p14.fixtmb2.02.

\begin{tabular}{lccc|c}
\hline \hline Requirement & $\epsilon_{\text {Data }}$ & $\epsilon_{\mathrm{MC}}$ & $\begin{array}{c}\text { correction } \\
(\text { Pass 2) }\end{array}$ & $\begin{array}{c}\text { correction } \\
(\text { Pass 1) }\end{array}$ \\
\hline di-muon trigger $_{\text {loose muon reconstruct. }}{ }^{1}$ & $0.939 \pm 0.068$ & - & $0.939 \pm 0.068$ & $0.939 \pm 0.068$ \\
medium quality & $0.950 \pm 0.001$ & $0.961 \pm 0.001$ & $1.000 \pm 0.027$ & $0.999 \pm 0.021$ \\
track finding \& match. & $0.998 \pm 0.001$ & $0.988 \pm 0.001$ & $1.010 \pm 0.010$ & $1.020 \pm 0.013$ \\
isolation & $0.944 \pm 0.002$ & $0.956 \pm 0.001$ & $0.988 \pm 0.012$ & $0.986 \pm 0.016$ \\
\hline total per muon & $0.794 \pm 0.048$ & - & $0.974 \pm 0.039$ & $0.996 \pm 0.029$ \\
\hline \hline
\end{tabular}

Table 5.8: Summary of the muon efficiencies in data and Monte Carlo and the resulting MC corrections. Similar corrections as obtained for "Pass 1" data are shown for reference [144]. The total efficiency per muon is correct for muons that exceed $p_{T} \gtrsim 10 \mathrm{GeV}$ and includes the di-muon trigger efficiency broken down for one muon. The total correction factor per muon does not include the di-muon trigger efficiency.

\footnotetext{
${ }^{1}$ The muon reconstruction efficiency correction factor is $\eta$ dependend. The given number in Tab. 5.8 is the average over the complete Standard Model background.
} 


\subsection{Jets}

High energetic quarks or gluons from Standard Model processes like $Z \rightarrow b \bar{b}$ or $W \rightarrow q^{\prime} \bar{q}$ or from other, supersymmetric decays like $\tilde{\chi}_{2}^{0} \rightarrow \tilde{\chi}_{1}^{0} q \bar{q}$ or $\tilde{\chi}_{1}^{0} \rightarrow \mu q^{\prime} \bar{q}$ cannot be observed as free particles, as discussed in Sec. 3.1.2, but hadronize into a jet of particles comprised out of hadrons and leptons. This particle jet creates some amount of hits in the tracking systems and is stopped finally in the calorimeter. While the reconstruction from tracks is possible, the best momentum resolution and reliability is obtained from the calorimeter based jet reconstruction. Additional tracking information is used to tag jets originating from secondary vertices, i.e. from $B$-meson decays.

In Sec. 5.2.1 the requirements on jet finding algorithms and the cone algorithm used at DØ Run II shall be discussed. The jet identification and the rejection of jets that do not originate from hadronic energy but noise or detector effects will be discussed in Sec. 5.2.2. Finally the jet reconstruction differences in the data and Monte Carlo are discussed in Sec. 5.2.3, the jet energy calibration is described in Sec. 5.2.4, and the jet energy resolution for the utilized version of the DØ Run II jet algorithm is given in Sec. 5.2.5.

\subsubsection{Jet Algorithm}

Traditionally, cone algorithms have been the jet-algorithm of choice for hadron-hadron experiments [162]. The particle jet has an opening angle and can be enclosed by a cone with radius $R$ in the $\eta \times \phi$ plane, which is invariant under boosts along the $z$-axis. The cone axis coincides with the jet direction as defined by the transverse Energy $E_{T}$ weighted centroid of the particles within the cone. Ideally, all particle trajectories lie in the area defined by the cone size and the reconstructed jet energy corresponds to the pristine quark's energy from which the particle jet originates. However, in practice many problems have to be solved by the jet algorithm. In principle the algorithms should start with a "seed" corresponding to the most energetic particle in the event. In detector terms however, usually a calorimeter tower exceeding a threshold energy of typically a few hundred $\mathrm{MeV}$ is used. A calorimeter tower consists out of calorimeter cells with the same $\eta$ and $\phi$ and covers at $\mathrm{D} \emptyset$ the space $\Delta \eta \times \Delta \phi=0.1 \times 0.1$. The $E_{T}$-weighted centroids are calculated for the particles in each seed cone and then the centroids are used as centers for new cones in $\eta \times \phi$ space. This is iterated until the geometrical cone axis matches the $E_{T}$-weighted centroids of the comprised calorimeter towers. The contents of the stable cone is called a proto-jet. Unfortunately, a single particle can belong to more than one cone, so that a rule to split or to merge overlapping proto-jets needs to be specified.

The cone algorithm needs to be collinear safe. One example is shown in Fig. 5.13; if the energy of a particle is split among several detector towers, then it might fail to produce a seed, while the energy would suffice if it were distributed more narrowly. Using seed thresholds of $1 \mathrm{GeV}$, the seed-based jet algorithm used at $\mathrm{D} \varnothing$ was found to be fully collinear safe for sufficient large $E_{T}>20 \mathrm{GeV}$. Another collinear problem can arise, if the jet algorithm is sensitive to the $E_{T}$ ordering of particles, as shown in Fig. 5.14. If the seeds 


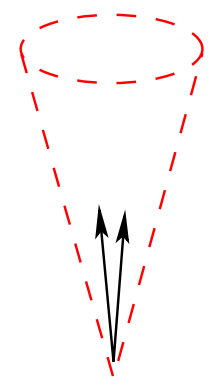

Figure 5.13: Seed collinear sensitivity

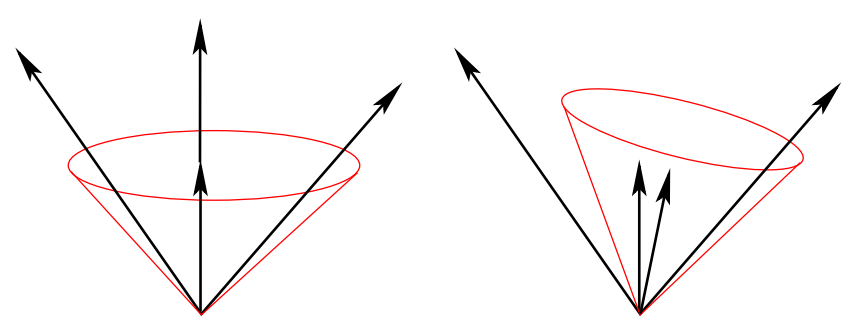

Figure 5.14: $E_{T}$ ordering sensitivity

are treated in order of decreasing energy, then a different cone might be reconstructed, if the hardest particle splits into two collinear towers and a different particle serves as new seed.

Another important requirement is the infrared safety of the algorithm. As illustrated in Fig. 5.15 two clearly separated particles can be merged into one cone if one particle radiates a soft gluon, which serves as a seed. Jet algorithms that only look for seed towers exceeding a minimum amount of energy are not infrared safe.

The jet algorithm needs to find the same solutions independent of boosts in the longitudinal directions, particularly important at hadron colliders. There should not be any dependence
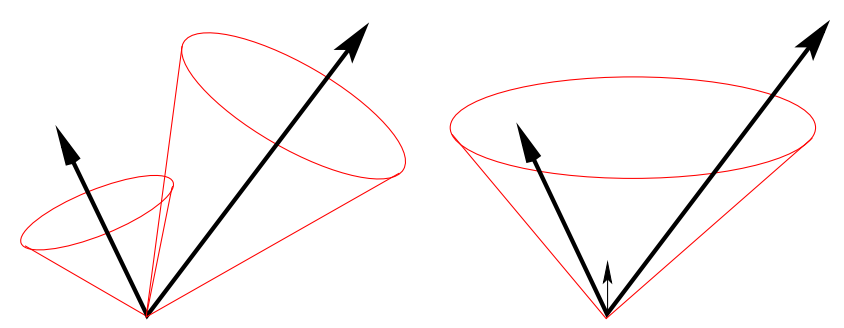

Figure 5.15: Infrared sensitivity on the detector performance, detector region, cell type, or cell size. The influence of the instantaneous luminosity should be under control and finally a reliable calibration of the kinematic properties of the jet must be found.

The problems described above arise from seed based algorithms. Seedless clustering is infrared safe and is insensitive to collinear radiation. To accomplish an unbiased set of seeds, each calorimeter tower should be considered to be a seed. This translates for the $\mathrm{D} \varnothing$ detector with a fiducial volume of $-2.4 \leq \eta \leq 2.4$ and $0 \leq \phi \leq 2 \pi$, and a segmentation of $0.1 \times 0.1$, to the order of $3 \cdot 10^{3}$ seed towers. The efficiency to detect physical meaningful jets is maximal for seedless algorithms, but the necessary computing effort is too large.

Seed-based cone algorithms are comparatively efficient in CPU time by considering only towers that pass a seed cut

$$
E_{T}^{\text {tower }}>E_{T}^{\text {seed }}
$$

as starting points for the initial jet cones. The seedless algorithm can be approximated by the addition of "midpoints". The sensitivity to soft radiation as shown in Fig. 5.15 
is essentially removed by adding a starting point for clustering at the position given by $\sum_{i} p_{i}$. Widely separated seeds cannot be clustered to a proto-jet, so that it is sufficient to consider all seeds $p_{i}$ that lie within a distance of

$$
\Delta R<2.0 \cdot R_{\text {cone }}
$$

This midpoint algorithm is also called Improved Legacy Cone Algorithm (ILCA). The last step in the jet reconstruction ILCA is the recombination or splitting of the proto-jets. Two independent proto-jets can share one or more calorimeter towers (particles) and need to be split, or two proto-jets belong to the same source and need to be recombined. The proto-jets, in descending order of transverse energy $E_{T}$, are probed for calorimeter towers, that belong also to other proto-jets. If this is not the case, then the proto-jet is removed from the list and is considered as final. Otherwise, both proto-jets are merged if the shared transverse energy is larger than the fraction $f$ of the total transverse energy of the neighbor. If the shared transverse energy is smaller, then the shared towers are assigned to the nearest cone. In both cases the cones need to be recalculated as described above, and will be added to the list of proto-jets. This procedure is iterated until no proto-jets are left.

In Tab. 5.9 the cone algorithm specifications as used for this analysis are listed.

\begin{tabular}{ll}
\hline cone size $R=\sqrt{\Delta \eta+\Delta \phi}$ & 0.5 \\
seed threshold $p_{T}$ & $1.0 \mathrm{GeV}$ \\
Split/Merge fraction $f$ & 0.5 \\
jet threshold $E_{T}$ & $8 \mathrm{GeV}$ \\
\hline
\end{tabular}

Table 5.9: DØ Run II cone jet specifications

\subsubsection{Jet Identification}

Further quality criteria are posed onto the found jets, to remove fake-jets, which were not created by hadronic particles, but by calorimeter noise or by electromagnetic particles created in the collision like electrons, photons or taus:

- The number of calorimeter towers containing $90 \%$ of a jet's energy ( $n 90)$ has to be larger than one, to reduce noise jets.

- Jets clustered from hot cells are removed by requiring the ratio of the highest to next-to-highest transverse energy cell to be $\mathrm{HotF}<10$.

- To reduce jets originating from calorimeter noise, the T42 algorithm (T-four-two = threshold $4-2 \sigma$, pronounced tea-for-two) is applied. Calorimeter cells with less than $4 \sigma$ energy above threshold, or with less than $2 \sigma$, if an adjacent cell has at least $4 \sigma$ energy above threshold, are rejected [163]. Between $30 \%$ and $60 \%$ cells are rejected by the T42 algorithm, compared to the number of cells in the event. In the main part of the calorimeter $|\eta|<3.2$ the number of rejected cells corresponds 
to the number of expected noise cells, while in the very forward calorimeter region more cells from pile-up effects, which accumulate close to the beampipe, are rejected $[164]$.

- To reject electromagnetic and noise like jets, the electromagnetic fraction $(E M F)$ of the energy deposition in the calorimeter is required to be $5 \%<E M F<95 \%$.

- The coarse hadronic fraction $(C H F)$ of the energy deposition in the coarse hadronic layers compared to all layers of the calorimeter is required to be less than $40 \%$, due to the higher noise level in the coarse hadronic part of the calorimeter.

- To reduce the noise influence of the calorimeter readout, a Level 1 trigger confirmation is required, since the L1 trigger is using a different readout chain. Jets have to fulfill the cut given in Tab. 5.10 for the different calorimeter $\eta$-regions. Where $L 1 S E T$ is the scalar sum of the transverse energy of all trigger towers within the jet's cone.

\begin{tabular}{lrrr} 
& CC & ICD & EC \\
\hline$\frac{L 1 S E T}{E_{T}^{r e c o} \cdot(1-C H F)}>$ & 0.4 & 0.2 & 0.4
\end{tabular}

Table 5.10: Cut values on the L1 confirmation energy for different calorimeter regions.

Fig. 5.16 shows the behavior of good and bad jets in the jet quality variables CHF, EMF, HotF and n90 [143].

\subsubsection{Jet Reconstruction Efficiency}

A correction that accounts for different jet reconstruction efficiencies in data and Monte Carlo is applied to all Monte Carlo events per jet by TopAnalyze [138] using JETCoRR version 5.3 [165]. For the specific parametrization of the correction, as given by Eq. (5.11), the error function was used. The error function is known e.g. from trigger turn-on curves, where the trigger threshold is given by a Heavyside step function depending on some object's transverse momentum $p_{T}$ and the measured $p_{T}$ of the object is Gaussian distributed around the true value.

The data efficiencies have been studied using $\gamma+$ jet and $\mathrm{Z}+$ jet events. The obtained fit values for Eq. (5.11) can be found in Tab. 5.11.

$$
f\left(p_{T}\right)=A 2 \cdot\left(\frac{1}{2}+\frac{1}{\sqrt{\pi}} \int_{0}^{\frac{p_{T}-A 0}{\sqrt{p_{T}} \cdot A 1}} e^{-x^{2}} d x\right)
$$

In Fig. 5.17 the jet reconstruction efficiency correction functions $f\left(p_{T}\right)$ are shown for the CC, ICD and EC regions. 

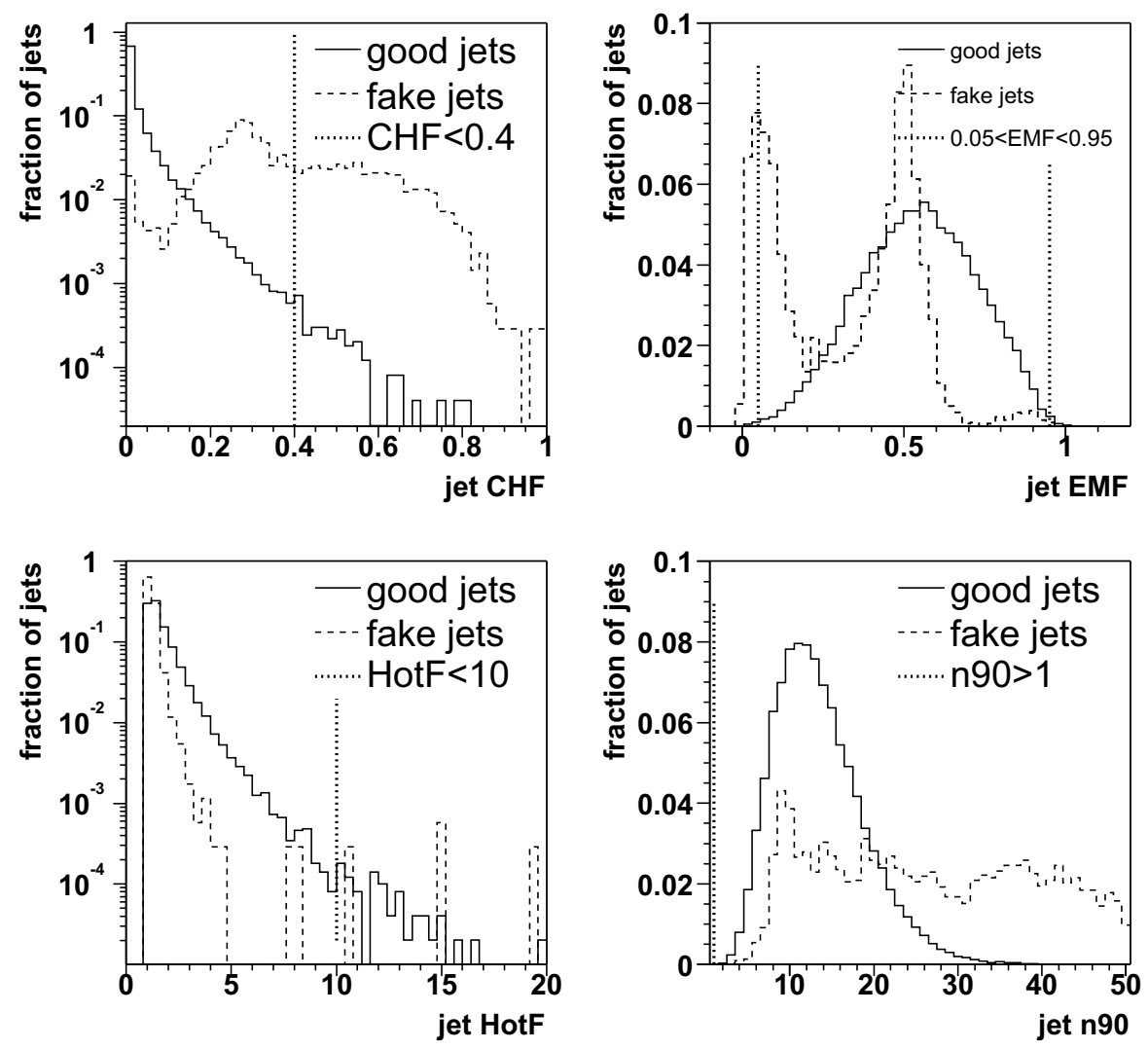

Figure 5.16: Behavior of good and bad jets in the quality variables [143]. Good jets are assumed to be found in events containing one good jet or one electromagnetic object and a second jet, which is probed for the attribute in question. Bad jets events are selected by either requiring two balanced, good jets to tag the event and a third jet which would violate momentum conservation or in 6-jet events, where the probe bad jet must not be matched to tracks exceeding $p_{T}>0.5 \mathrm{GeV}$.

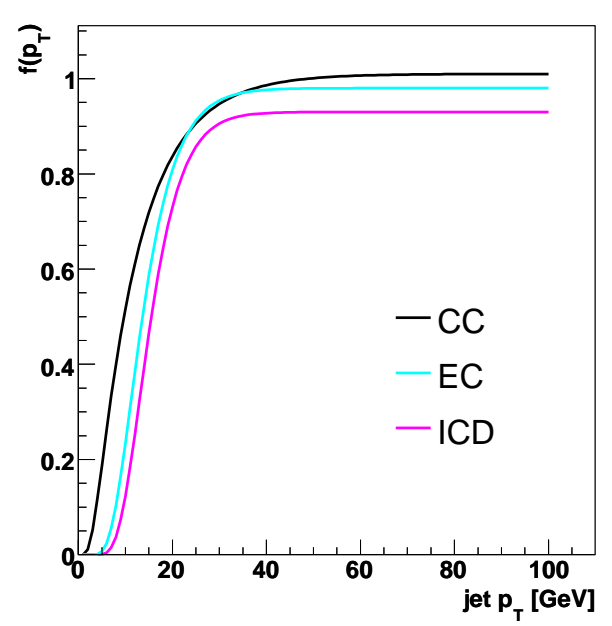

Figure 5.17: Jet reconstruction efficiency correction functions for the three different calorimeter regions (CC, ICD, EC) using the parametrization given by Eq. (5.11). 


\begin{tabular}{lrrr}
\hline \hline Calorimeter region & $\mathrm{A} 0[\mathrm{GeV}]$ & $\mathrm{A} 1[\sqrt{\mathrm{GeV}}]$ & $\mathrm{A} 2$ \\
\hline $\mathrm{CC}$ & $9.8 \pm 2.7$ & $3.4 \pm 0.7$ & $1.01 \pm 0.01$ \\
$\mathrm{ICD}$ & $15.0 \pm 2.3$ & $2.0 \pm 0.7$ & $0.93 \pm 0.02$ \\
$\mathrm{EC}$ & $13.5 \pm 3.3$ & $2.2 \pm 1.0$ & $0.98 \pm 0.02$ \\
\hline \hline
\end{tabular}

Table 5.11: Fit values for the jet reconstruction efficiency correction, parametrized by Eq. (5.11) taken from [138].

\subsubsection{Jet Energy Scale}

In the previous sections the ILCA cone algorithm has been discussed, which finds jets in the calorimeter. The measured jet energy $E_{\text {meas }}$ is the sum of the energy contents of all calorimeter cells within a cone of size $\Delta R=0.5$ around the jet's axis, above a certain threshold. $E_{\text {meas }}$ deviates from the true energy of the initial parton that created the jet and needs therefore calibration. This is provided by the Jet Energy Scale (JES). For a nice introduction to this topic see Ref. [166]. The jet energy scale corrections for reconstructed jet energies $E_{\text {meas }}$ back to the original parton energy $E_{\text {corr }}$ is parametrized by the following function:

$$
E_{\text {corr }}=\frac{E_{\text {meas }}-O}{R \times S}
$$

Where the relevant variables that characterize the deviation of original parton and measured jet energy are:

Calorimeter Response $\mathbf{R}$ can be distorted for different partons, different calorimeter regions, i.e. a strong $\eta$-dependence, inhomogeneous instrumentation, dead material and a non-linear response to the particle energies. $R$ is determined by the examination of QCD Compton events, i.e. $q g \rightarrow q \gamma$. The purely electromagnetic energy of the photon is know with high precision, due to the accurate EM energy scale calibration, for example on the $Z \rightarrow e e$ peak; and can be used as an estimator for the parton energy, if $q$ and $\gamma$ are back to back. This is the largest relative correction and can be as large as $30 \%$, see Fig. 5.20(a).

Energy Offset $\mathbf{O}$ corresponds to additional energy in the calorimeter cells within the jet cone, due to underlying events (beam remnants and multiple parton interactions), energy pile-up or noise from the electronics or the uranium absorber material. This has to be subtracted from the reconstructed energy. $O$ is determined in events without a hard interaction, the minimum bias events, which are triggered by the luminosity detectors. The energy offset depends on $\eta$ as well as on the number of primary vertices and is typically of the size of a few GeV, see Fig. 5.20(b).

Showering Corrections S: Some amount of the original energy might escape the cone; for example low energetic, charged hadrons can curl out of the jet cone due to the magnetic field. $S$ is measured from jet energy profiles and is typically smaller than $4 \%$ for jet momenta larger than $20 \mathrm{GeV}$, see Fig. 5.20(c). 

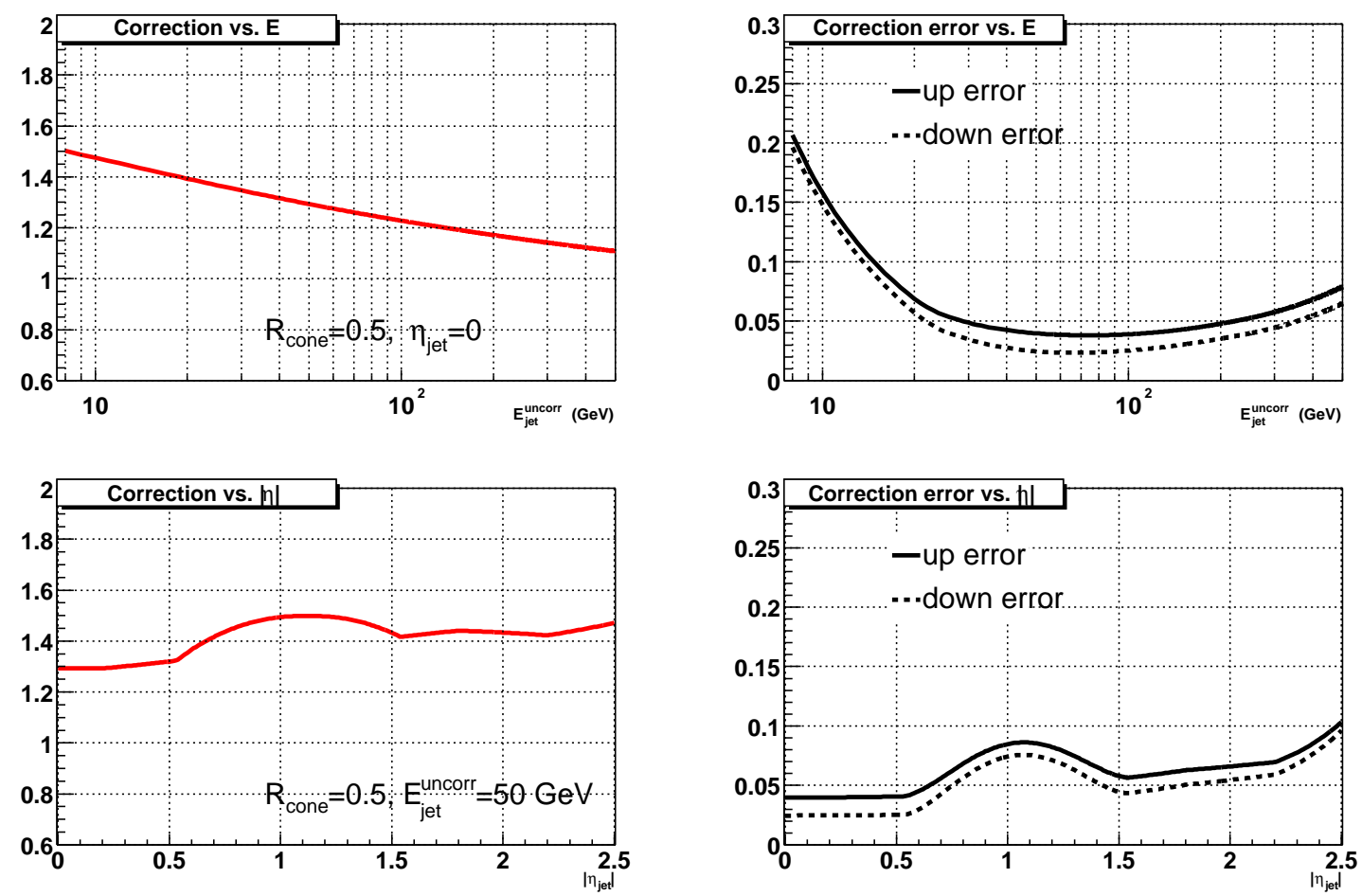

Figure 5.18: Jet energy scale correction for data events versus $E_{T}$ of the unreconstructed jet (top) and $|\eta|$ (bottom) [165]. The associated uncertainties are shown on the right hand side.
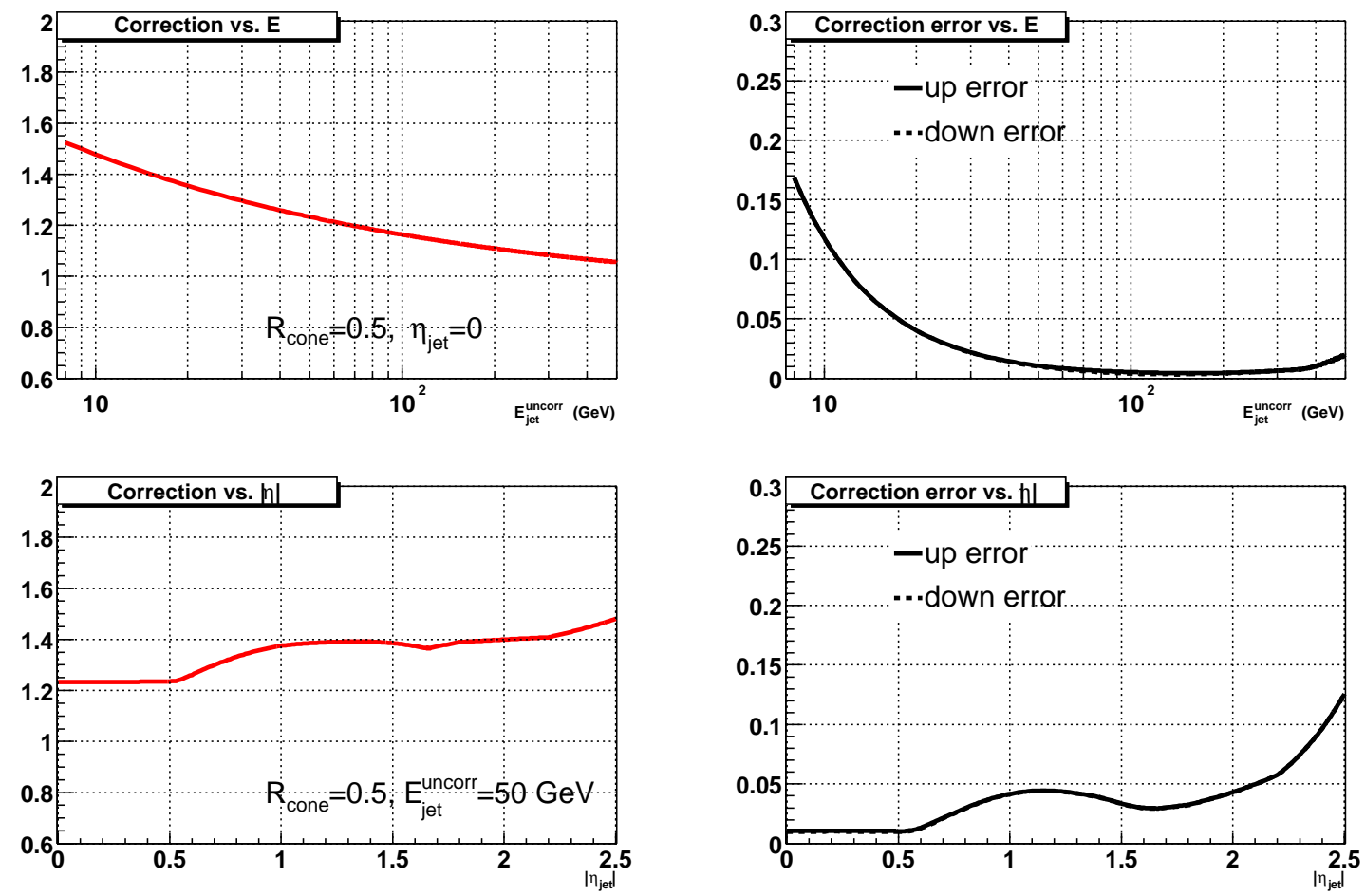

Figure 5.19: Jet energy scale correction for Monte Carlo events [165]. 


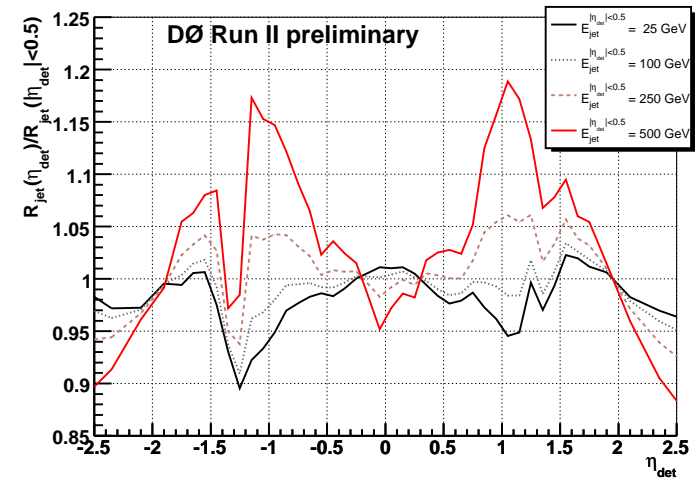

(a)

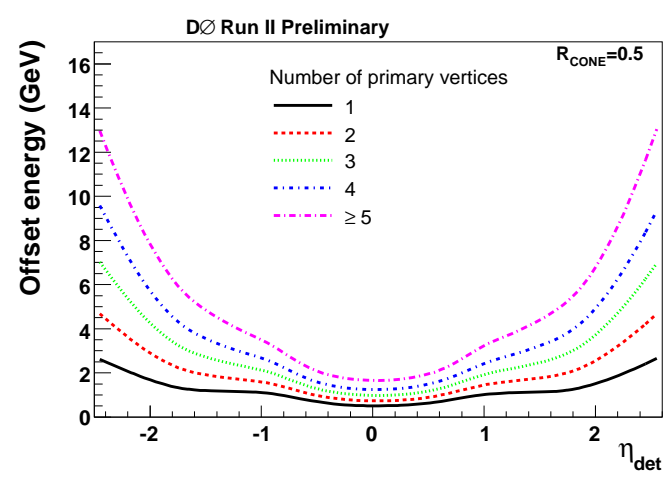

(b)

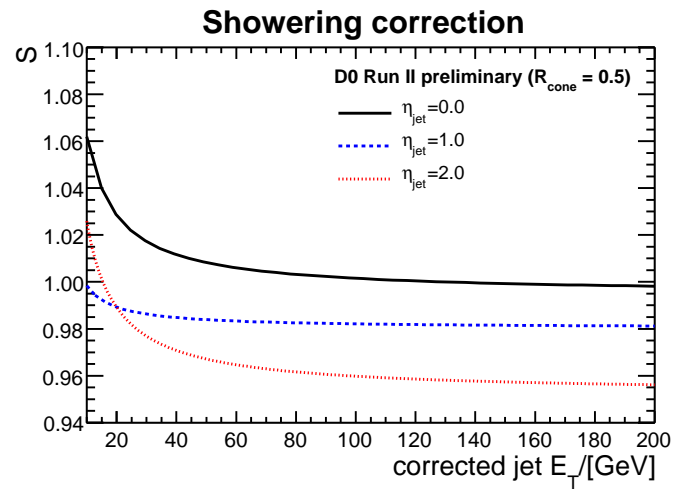

(c)

Figure 5.20: (a) Relative response correction in data. (b) Offset energy for different primary vertex multiplicities, as a function of jet $\eta$. (c) Showering correction for jets in data as a function of corrected jet transverse energy. All plots are taken from [166].

The JES correction and it's uncertainty depends on the initial parton's $p_{T}$ as well as on $\eta$. Since the jet energies in the data are not correctly described by the Monte Carlo, a different correction is applied on simulated events. The corrections and their uncertainties are given in Fig. 5.18 for the data and in Fig. 5.19 for simulated events versus $E_{T}$ and $\eta$ of the uncorrected jet.

The total systematic uncertainty assigned to the JES correction arises from the statistical and the systematic error of both data and Monte Carlo measurement and is added in quadrature:

$$
\sigma_{\mathrm{JES}}=\sqrt{\sigma_{\mathrm{stat}, \mathrm{data}}^{2}+\sigma_{\mathrm{syst}, \mathrm{data}}^{2}+\sigma_{\text {stat }, M C}^{2}+\sigma_{\text {syst }, M C}^{2}}
$$

The JES correction depends on the flavor of the initial parton. In particular $b$-quark jets that decay semileptonically and that are tagged by a muon within the jet cone, are corrected with twice the muon energy (Where the neutrino energy is approximated to be equal to the muon's). Since in the signal studied in this analysis no $b$-quarks are created, with the exception of possible cascade decays, effectively the standard jet energy calibration is utilized. 


\subsubsection{Jet Energy Resolution}

The jet energy resolution was studied in a di-jet event sample, taken with the jet triggers JT_25TT_NG, JT_45TT, JT_65TT, JT_95 TT with the average prescale factors $150,4.7,1.3$, and 1 , respectively. The luminosity of this sample corresponds to $143 \mathrm{pb}^{-1}$ and both jets were required to pass the standard jet ID cuts, as discussed previously in Sec. 5.2.2. Both jets are required to be back-to-back; $|\Delta \phi-\pi| \leq 5$ degrees. The details of this study can be found in [167].

The sample is split into bins of $20 \mathrm{GeV}$ width, in the variable of average momentum of the two jet system $\left\langle p_{T}\right\rangle=\frac{1}{2}\left(p_{T}^{\text {jet } 1}+p_{T}^{\text {jet } 2}\right)$. Then the momentum asymmetry $A$ is calculated:

$$
A=\frac{\left|p_{T}^{j e t 1}-p_{T}^{j e t 2}\right|}{p_{T}^{j e t 1}+p_{T}^{j e t 2}}
$$

The jet $p_{T}$ resolution is directly related to the asymmetry resolution $\sigma_{A}$, which is obtained by a Gaussian fit of the $A$ distribution, with a mean value set to zero.

$$
\frac{\sigma_{p_{T}}}{p_{T}}=\sqrt{2} \cdot \sigma_{A}
$$

The resolutions are fit using the following formula, which is already known from the calorimeter performance (Sec. 3.3.4):

$$
\frac{\sigma_{p_{T}}}{p_{T}}=\sqrt{\left(\frac{N}{p_{T}}\right)^{2}+\left(\frac{S}{\sqrt{p_{T}}}\right)^{2}+C^{2}}
$$

The terms $N$ (noise), $S$ (statistical sampling fluctuations), and $C$ (calibration errors) were already defined in the context of Eq. (3.10).

The jet energy resolution versus the transverse jet momentum $p_{T}$ as well as the obtained $\chi^{2}$-fit results for the parameters $N, S$, and $C$ are shown in Fig. 5.21 for different bins in $\eta$ of width 0.4 .

\subsection{Missing $E_{T}$}

Information about particles that do not interact with the detector material, like neutrinos, can be found indirectly, by calculating the missing transverse energy $\mathbb{E}_{T}$. The initial longitudinal energy along the beam axis of both interacting partons is unknown at hadronhadron colliders, so that the $z$-component of the missing energy cannot be reconstructed. The vectorial $\mathbb{E}_{T}$ is a vector in the $\phi$-plane and is calculated by the negative sum of the transverse energy contents of all calorimeter cells, with an energy content of at least $100 \mathrm{MeV}$ above threshold. The transverse energy component of each cell is calculated for the true, reconstructed vertex $z$-component. If muons were reconstructed in the muon system, then the reconstructed muon momentum is added to the visible calorimeter energy. 

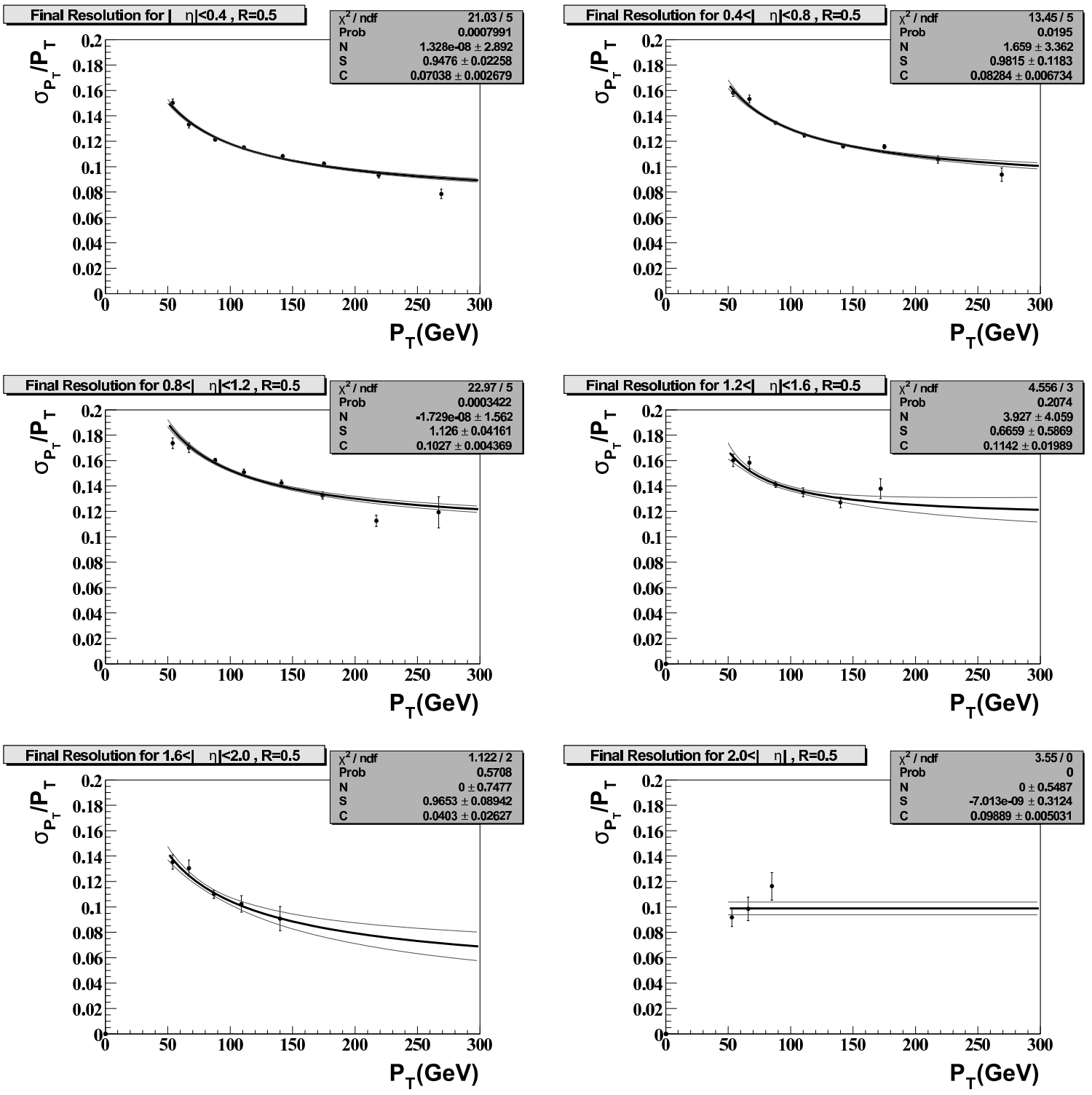

Figure 5.21: Jet resolution for JETCORR v5.3 with T42 in different rapidity bins after soft radiation corrections and particle imbalance corrections. Bands of $\pm 1 \sigma$ statistical error are shown as well [167]. 
Muons are minimal ionizing particles and leave only little energy, typical $2-3 \mathrm{GeV}$, in the calorimeter.

Corrections to the jet energy scale, muon momentum smearing, or noise suppression by the T42 algorithm are passed through to the $\mathscr{E}_{T}$ calculation. The systematic uncertainties of JES and muon $p_{T}$ smearing have a significant influence on the missing $E_{T}$ and are considered in this analysis.

The signal channels in this analysis have only little true physical missing $E_{T}$, coming from cascade decays of gauginos to the lightest neutralino, e.g. $\tilde{\chi}_{1}^{ \pm} \rightarrow \tilde{\chi}_{1}^{0} l \nu$ or $\tilde{\chi}_{2}^{0} \rightarrow \tilde{\chi}_{1}^{0} \nu \nu$, so that the $\not_{T}$ distribution is an excellent control distribution, see Sec. 6.1 for details. The amount of reconstructed missing $E_{T}$ in the signal channel $\tilde{\mu} \rightarrow \mu \tilde{\chi}_{1}^{0} \rightarrow \mu \mu q q$ or the dominant background channel $Z q q \rightarrow \mu \mu q q$ is an indication of the muon and jet momentum resolution.

\subsection{Taus}

Tau leptons $\tau$ are unimportant for the present analysis, so that the reader is referred to [168] which describes the $\tau$ identification in detail. However, $\tau$ leptons decay to muons $\tau \rightarrow \mu \nu_{\tau} \nu_{\mu}$, so that the di-muon final state receives contributions from Standard Model processes involving $\tau$ 's. The branching fraction of tau decay to muon and two neutrinos is about $17 \%$ [17]; considering the lower muon momentum compared to the tau's, this translates to a probability of about $10 \%$ for a tau from a $Z \rightarrow \tau \tau$ decay to "fake" (create) a muon, which passes the selection criteria as defined in Sec 6.1, i.e. a transverse momentum of at least $15 \mathrm{GeV}$. This probability can be extracted from Tab. 4.6. 


\section{Chapter 6}

\section{Data Analysis}

In order to discover a signal in the data, the underlying processes need to be under control. In Sec. 6.1 the preselection sample is presented to compare the recorded data to the Standard Model expectation in a domain where the signal is still negligible. In the following sections advanced selection criteria are discussed, to separate SM background from the signal, to discover either "Resonant slepton production" (Sec. 6.2) or "Pair and associated gaugino production" (Sec. 6.3). These sections include detailed studies, control plots and a detailed discussion of systematic uncertainties.

\subsection{The Common Event Preselection}

In Sec. 2.3.1 a preselection sample with two muons has been motivated. To control the background and for the ability to use the standard candle $Z / \gamma^{*}$ production for studies and control plots, two muons are selected. This sample is called "Preselection" sample.

Two "medium" quality (for details of the muon reconstruction see Sec. 5.1.2 and Sec. 5.1.5 for medium quality) muons with a transverse momentum of at least $15 \mathrm{GeV}$ and $8 \mathrm{GeV}$ are required per event. Both muons have to be isolated, so that the sum of the transverse energy in calorimeter cells in a hollow cone $(R=0.4, r=0.1)$ along the muon track does not exceed $2.5 \mathrm{GeV}$, and the sum of the transverse momenta of tracks in a cone with radius $\sqrt{\phi^{2}+\eta^{2}}=0.5$ is smaller than $2.5 \mathrm{GeV}$ (details can be found in Sec. 5.1.4). Both muons are also required to match a central track found by the central tracking systems (Sec. 5.1.3). The muon $z$-vertex has to be within $60 \mathrm{~cm}$ of the nominal interaction point and cosmic muons are rejected by requiring the muon system scintillator to be hit in the A-layer (BC-layer) within $10 \mathrm{~ns}$ (15 ns) of the nominal interaction. These criteria are summarized in Tab. 6.1.

In $376.5 \mathrm{pb}^{-1}$ of data, corresponding to di-muon trigger selected events (Sec. 5.1.1), 23206 data events and $22700 \pm 70$ (stat) \pm 2900 (syst) Standard Model background events match the preselection criteria. Each simulated event has been weighted according to its specific event kinematics, to account for the di-muon trigger efficiency, as well as to correct 


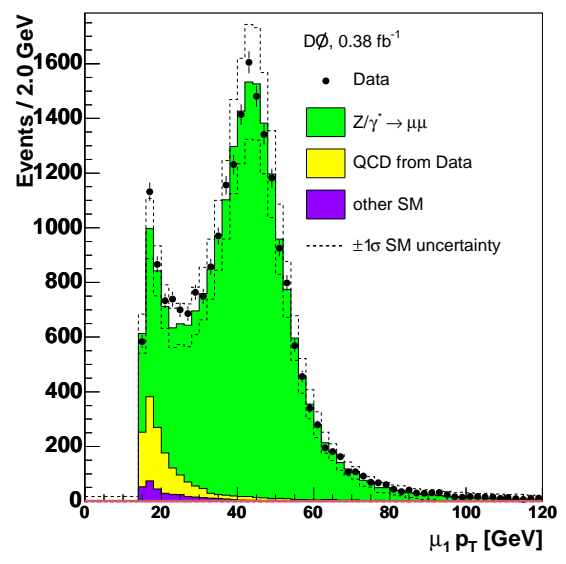

(a)

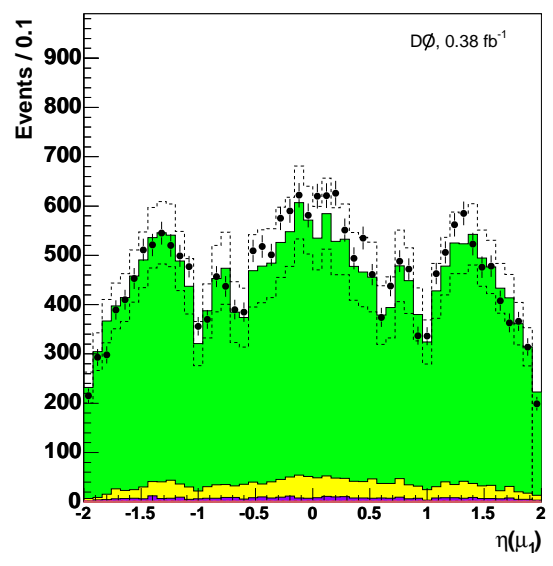

(c)

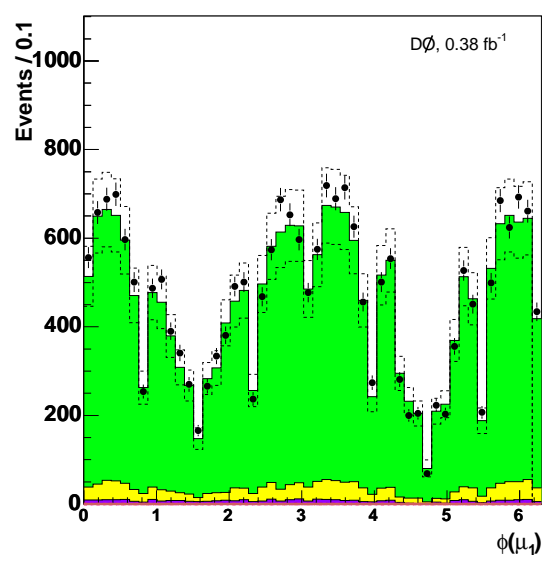

(e)

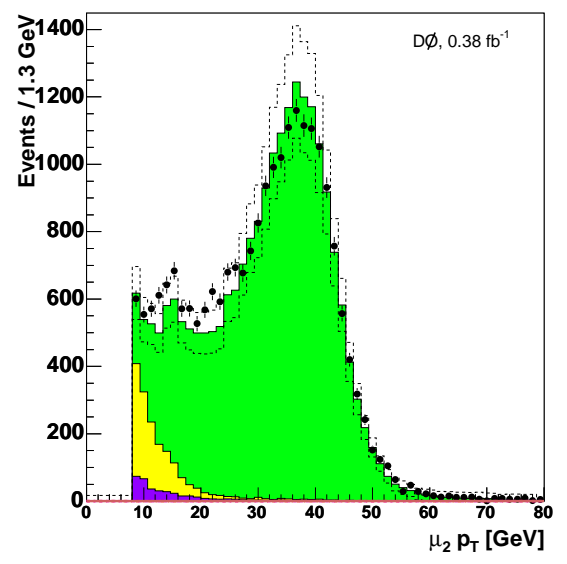

(b)

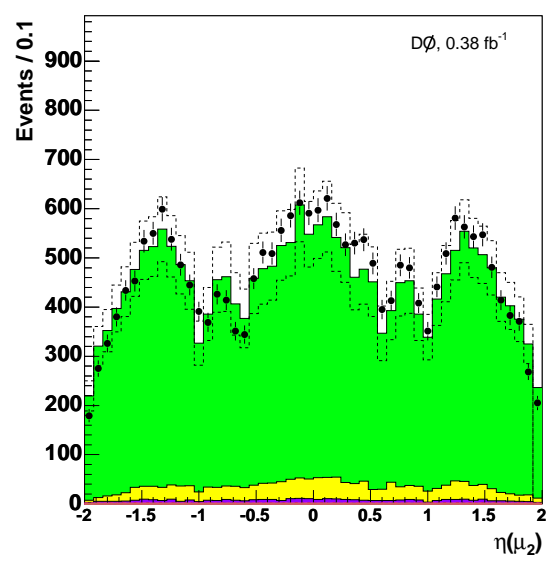

(d)

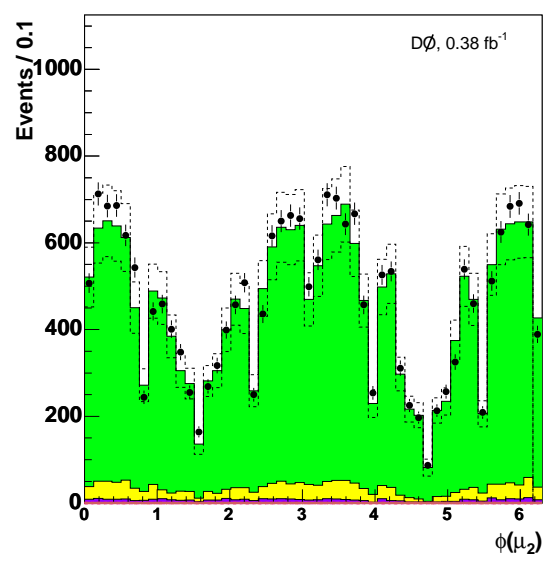

(f)

Figure 6.1: Preselection sample ( $2 \mu$-selection). The plotted variables are the transverse momentum $p_{T}$ (top), the pseudo-rapidity $\eta$ (middle) and the angular angle $\Phi$ (bottom) of the two leading muons. On the left hand side the $1^{\text {st }}$ muon is shown and on the right the $2^{\text {nd }}$ muon. The various Standard Model processes have been added and the dashed lines correspond to the total systematic uncertainty of the background. 


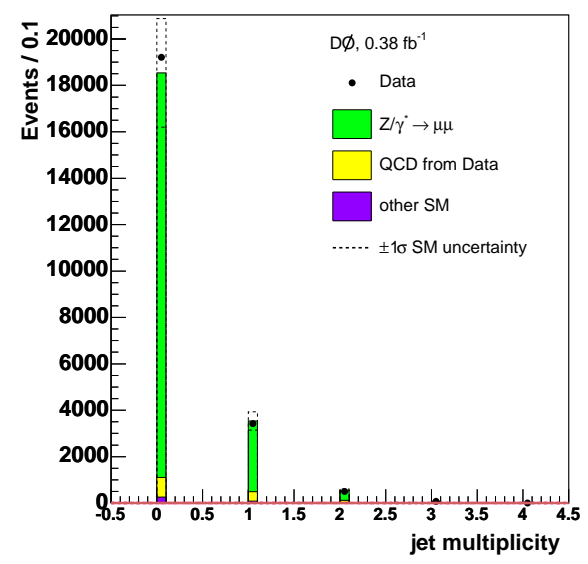

(a)

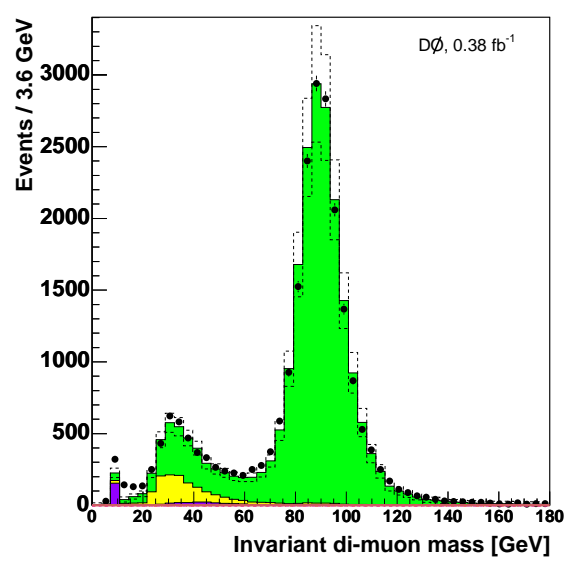

(c)

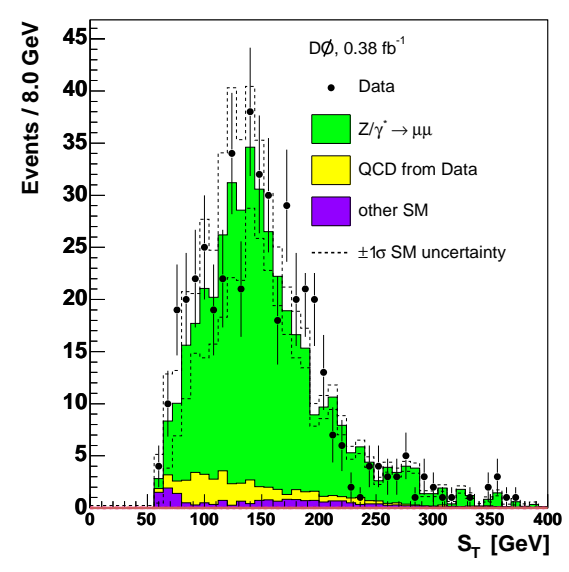

(e)

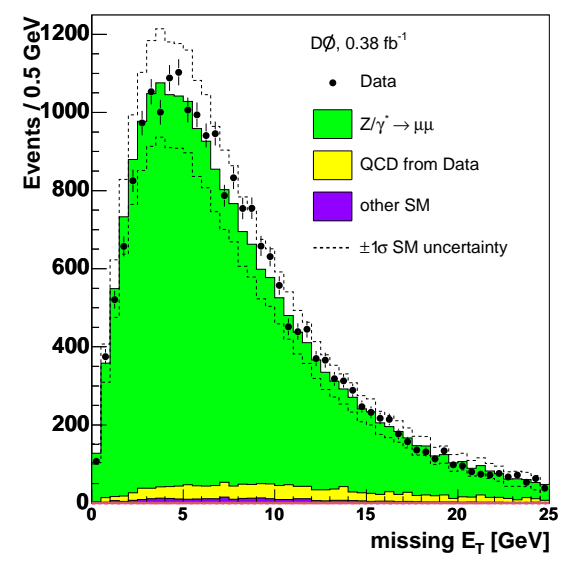

(b)

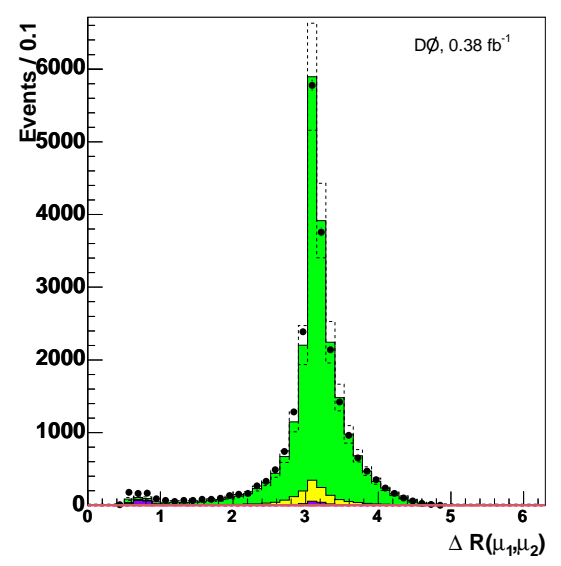

(d)

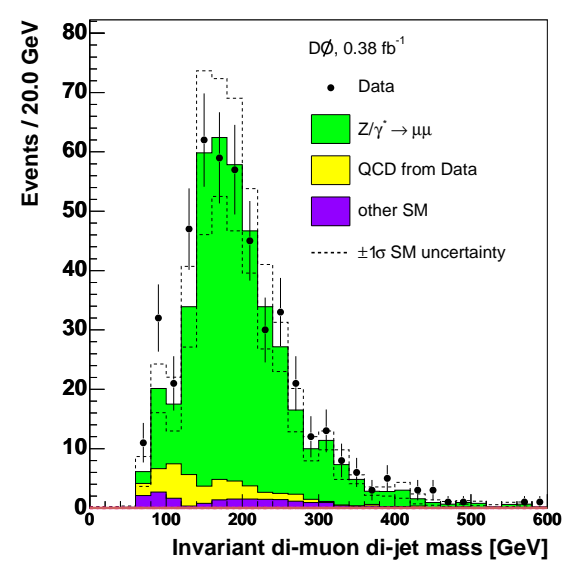

(f)

Figure 6.2: (a)-(d) Preselection sample ( $2 \mu$-selection), plots (e)-(f) show a $2 \mu$-2jet-selection; the shown variables are the jet multiplicity, the missing transverse momentum $\mathbb{E}_{T}$, the invariant dimuon mass $M_{\mu_{1} \mu_{2}}$, the opening angle between both muons $\Delta R\left(\mu_{1}, \mu_{2}\right)$, the scalar sum of both muon- and both jet-transverse momenta $S_{T}$, and the invariant di-muon-di-jet mass $M_{\mu_{1} \mu_{2} j e t_{1} j_{2} t_{2}}$. 


\begin{tabular}{lll}
\hline \hline Selection criterion & $1^{\text {st }}$ muon $\mu_{1}$ & $2^{\text {nd }}$ muon $\mu_{2}$ \\
\hline quality & $\geq$ medium & $\geq$ medium \\
transverse momentum $p_{T}$ & $\geq 15 \mathrm{GeV}$ & $\geq 8 \mathrm{GeV}$ \\
calorimeter isolation $E_{T} \varangle_{0.1}^{0.4}$ & $\leq 2.5 \mathrm{GeV}$ & $\leq 2.5 \mathrm{GeV}$ \\
tracker isolation $p_{T} \varangle_{0.5}$ & $\leq 2.5 \mathrm{GeV}$ & $\leq 2.5 \mathrm{GeV}$ \\
central track in & $\mathrm{CFT}$ or SMT & $\mathrm{CFT}$ or SMT \\
muon $z$-vertex & $\leq 60 \mathrm{~cm}$ & $\leq 60 \mathrm{~cm}$ \\
cosmic veto: scintillator time & $\left|t_{A-\text { layer }}\right|<10 \mathrm{~ns}$ & $\left|t_{A-\text { layer }}\right|<10 \mathrm{~ns}$ \\
& $\left|t_{B C-\text { layer }}\right|<15 \mathrm{~ns}$ & $\left|t_{B C-\text { layer }}\right|<15 \mathrm{~ns}$ \\
\hline \hline
\end{tabular}

Table 6.1: The di-muon preselection criteria.

for different muon identification efficiencies in data and in Monte Carlo, as discussed in Sec. 5.1.

In Fig. 6.1-6.2 characterizing variables of the di-muon preselection sample are plotted. The total systematic uncertainty of the background expectation, details will be discussed in Sec. 6.2.3, is shown as dashed lines. In the following sections more control plots will be shown for variables that are used for the signal selection, like jet momenta.

The above preselection sample was utilized to scale the chosen QCD sample (Sec. 4.2). For a scale factor of 0.4 the di-muon preselection data as shown in Fig. 6.1-6.2 is best described in the invariant muon mass and the muon transverse momentum contributions. The signal contribution to the di-muon selection is negligible, so that the background processes, i.e. the normalization of the QCD contribution, can be studied in this sample. After requiring one or two jets of at least $15 \mathrm{GeV}$ transverse momenta, the data is still in good agreement with the sum of all Standard Model processes. The QCD sample is compared to the difference of data and Standard Model Monte Carlo simulations in Fig. 6.3 for the di-muon preselection and a di-muon di-jet selection. (The same information can be extracted from the pre-selection control plots). From the fluctuation of data and the sum of all background processes, the QCD sample and other Standard Model Monte Carlo simulations, in an arbitrary bin range a conservative relative scale error of $20 \%$ has been estimated.

Overall, an agreement of the data with the sum of all Standard Model background processes in all distributions within the systematical and statistical uncertainties can be observed. 


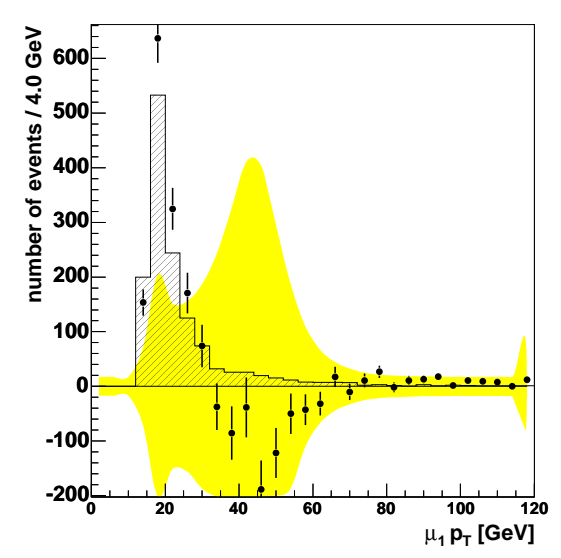

(a)

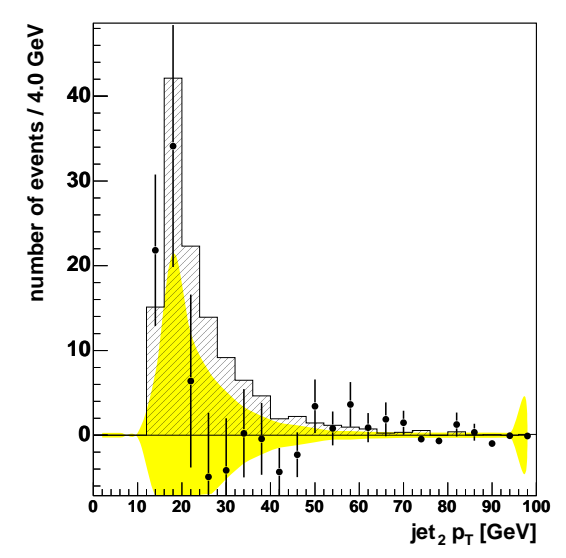

(c)

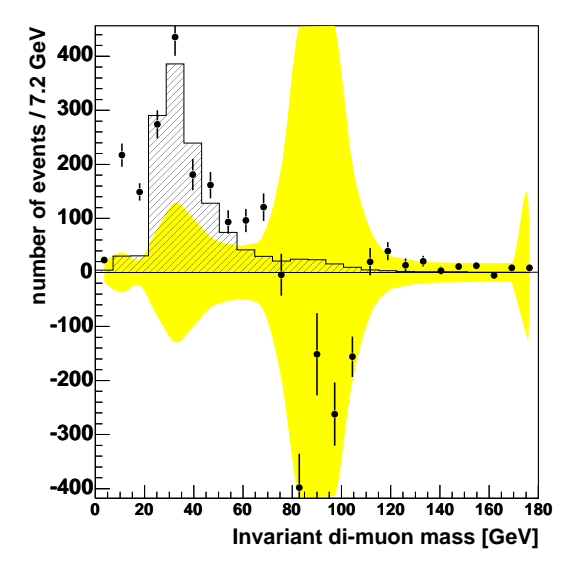

(e)

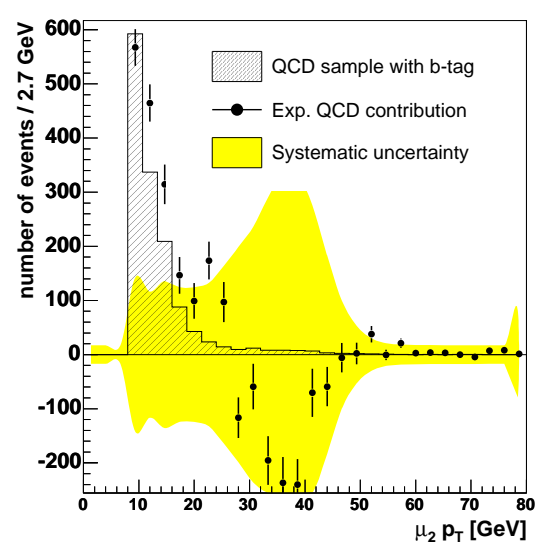

(b)

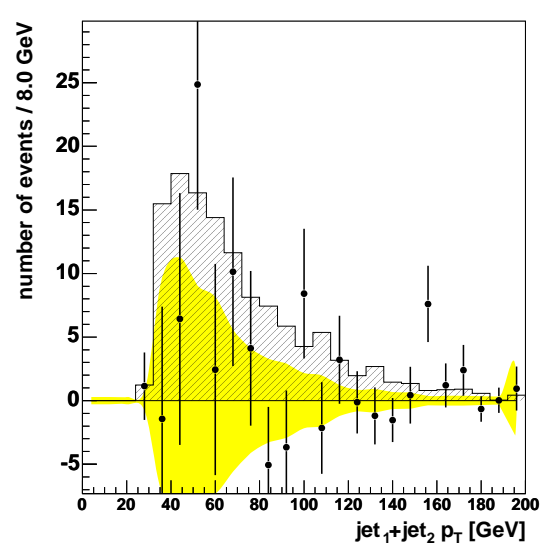

(d)

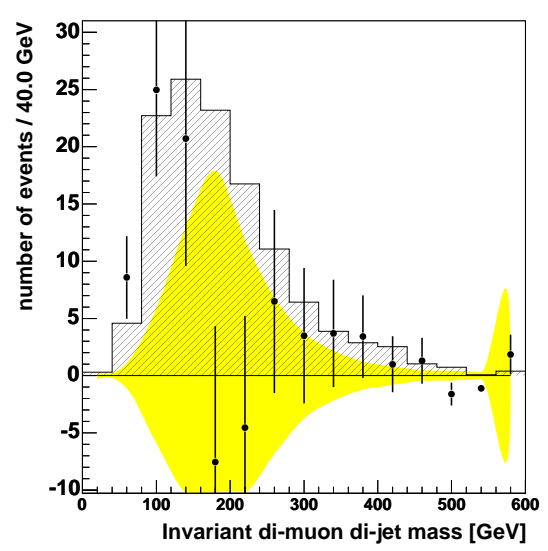

(f)

Figure 6.3: Comparison of the extracted QCD sample with $b$-tag (black hatched) to the difference of data and Standard Model Monte Carlo (black dots) in the di-muon preselection sample (a)-(b) and in the di-muon and di-jet sample (c)-(f). The QCD sample has to fit into the gap of the selected data and the Standard Model Monte Carlo simulations without multijet QCD processes. The error bars represent the data statistical uncertainties. The simulation's systematical uncertainties are denoted by the solid yellow contour, which is drawn symmetric to the $x$-axis. The QCD sample agrees well with the difference between data and SM Monte Carlo simulation, especially in regions where the QCD dominates, i.e. the remaining Standard Model expectation and it's uncertainty is small. 


\subsection{Search for Resonant Slepton Production}

The theoretical foundations of resonant slepton production, as well as the slepton branching ratios and the different contributing signal channels have been discussed in Sec. 2.3.1. In this section, the analysis strategy, how to separate resonant-slepton-signal-events from the background shall be described. Detailed signal studies are given in Sec. 6.2.1, in Sec. 6.2.2 the signal selection and in Sec. 6.2.3 the systematic uncertainties are discussed, and finally the resulting final resonant slepton sample is presented in Sec. 6.2.4.

\subsubsection{Signal Monte Carlo Studies}

In Sec. 4.3.2 the SUSYGEN problem has been mentioned, that prevents the decay of Zbosons with negative generator ID. The affected events have been removed from all generated resonant slepton signal samples and the remaining events of the affected process $\tilde{\chi}_{2}^{0} \rightarrow Z \tilde{\chi}_{1}^{0}$ were reweighted accordingly. In Fig. 6.4, a corrected sample is compared to a sample which was generated with the corrected version of SUSYGEN, see App. B for details. No significant deviation of both samples can be observed. The influence of different parton distribution functions (PDFs) and $\mathrm{D} \varnothing$ reconstruction release versions on the signal kinematics have also been studied and were found to be negligible, as shown in Fig. 6.4.

For all points, $\lambda_{211}^{\prime}$ has been fixed to 0.07 which is the best limit given by previous D $\varnothing$ analyses or theoretical predictions $[92,169,170]$ for any parameter point. The cross section depends on the coupling strength as given by Eq. (2.121). In this analysis different hypotheses with respect to the size of $\lambda_{211}^{\prime}$ have been tested, and the cross section has been re-calculated using:

$$
\sigma\left(\lambda_{211}^{\prime}\right)=\frac{\sigma(0.07)}{0.07^{2}} \cdot\left(\lambda_{211}^{\prime}\right)^{2}
$$

To test the quadratic dependence of the resonant slepton signal cross section $\sigma\left(\lambda_{211}^{\prime}\right)$ as generated with SUSYGEN to the coupling strength-squared $\left(\lambda_{211}^{\prime}\right)^{2}, \sigma$ has been fitted with a quadratical function as shown in Fig. 6.5 for different slepton masses. The used parametrization as given by Eq. (6.1) is legitimate for the studied masses and cross sections.

The leading-order SusYgen signal cross sections have been corrected with a slepton mass dependent k-factor, see Fig. 6.6, to account for higher order QCD-corrections ([171], [172]). A non-vanishing tri-linear SUSY breaking coupling $A_{0}$ can change the LO cross section prediction by $\sim 10 \%$ [172]. For non-zero $A_{0}$ additional SUSY-QCD contributions arise at NLO, the size of which depends sensitively on the choice of $A_{0}$ and the squark and gluino masses. The cross section can be changed by approximately $\pm 30 \%$ for $|A|<1 \mathrm{TeV}$ and squark and gluino masses beyond $200 \mathrm{GeV}$ [172]. For this analysis the tri-linear coupling has been fixed to zero $A_{0}=0$ and no higher order SUSY-QCD corrections have been taken into account.

The slepton $p_{T}$ distribution has been calculated in next-to-leading order [51] and is compared to the Susygen and Herwig [173] Monte Carlo generators as shown in Fig. 6.7. 


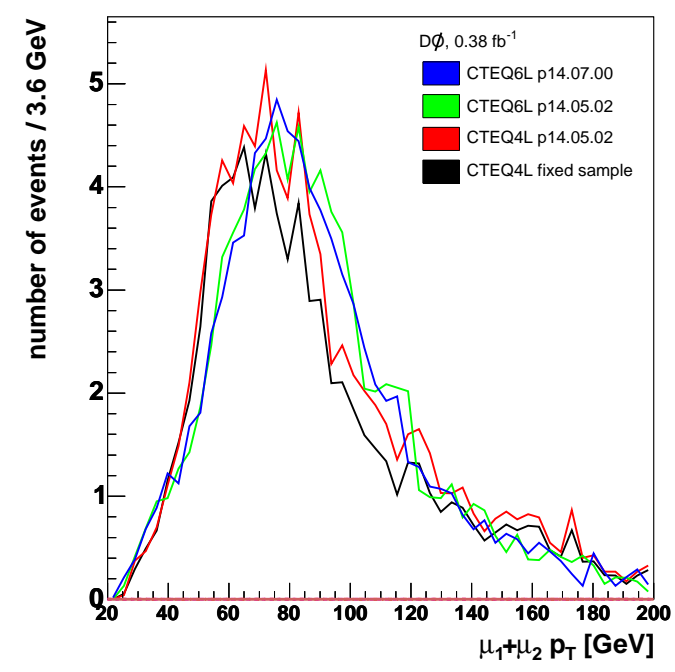

(a)

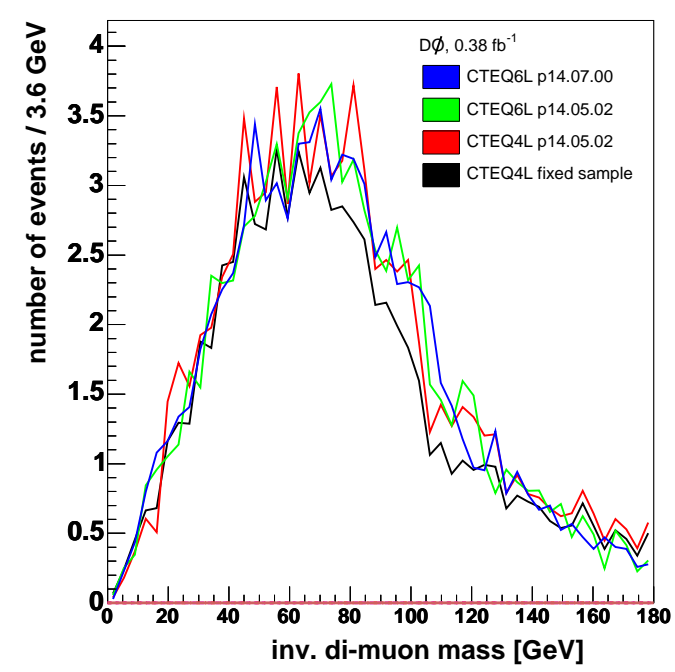

(c)

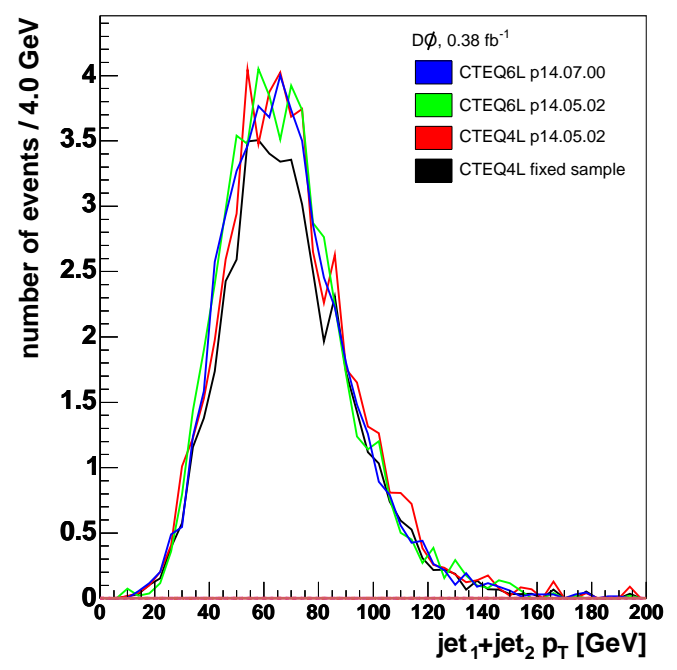

(b)

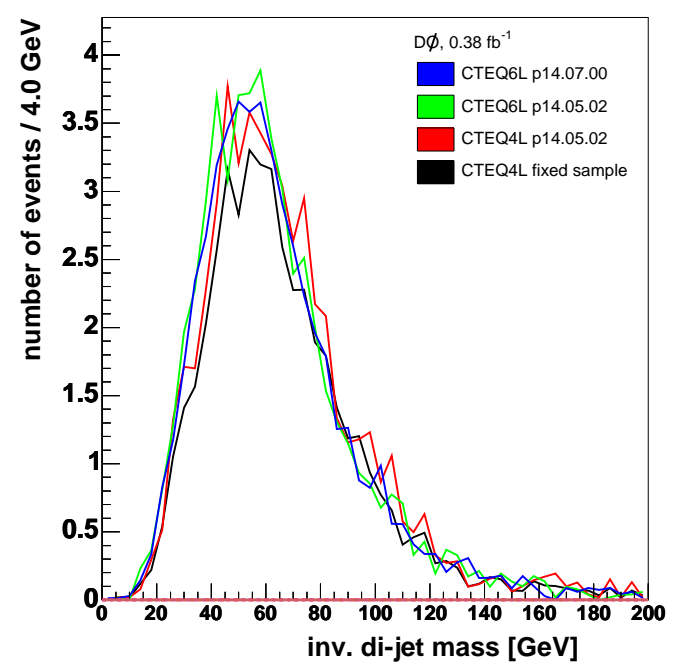

(d)

Figure 6.4: A SUSYGEn sample with ID problem and that was corrected after the generation (black) is compared to samples generated with a corrected SUSYGEN version. The influence of different parton distribution functions and $D \emptyset$ reconstruction release versions on the signal kinematics is shown as well. In (a) and (b) the sum of the muon and jet momenta is shown, in (c) and (d) the invariant di-muon and di-jet mass is depicted. The black line refers to a signal generated by the unfixed SUSYGEN version with CTEQ4L PDFs and reconstructed with DØRECo version p14.05.02, the red curve was generated by a corrected version of SuSYGEN. The blue and the green curve were generated using CTEQ6L PDFs with a Les Houches Accord (LHA) event interface. The blue curve was reconstructed with the more recent DØRECO version p14.07.00. No significant deviations can be observed. 


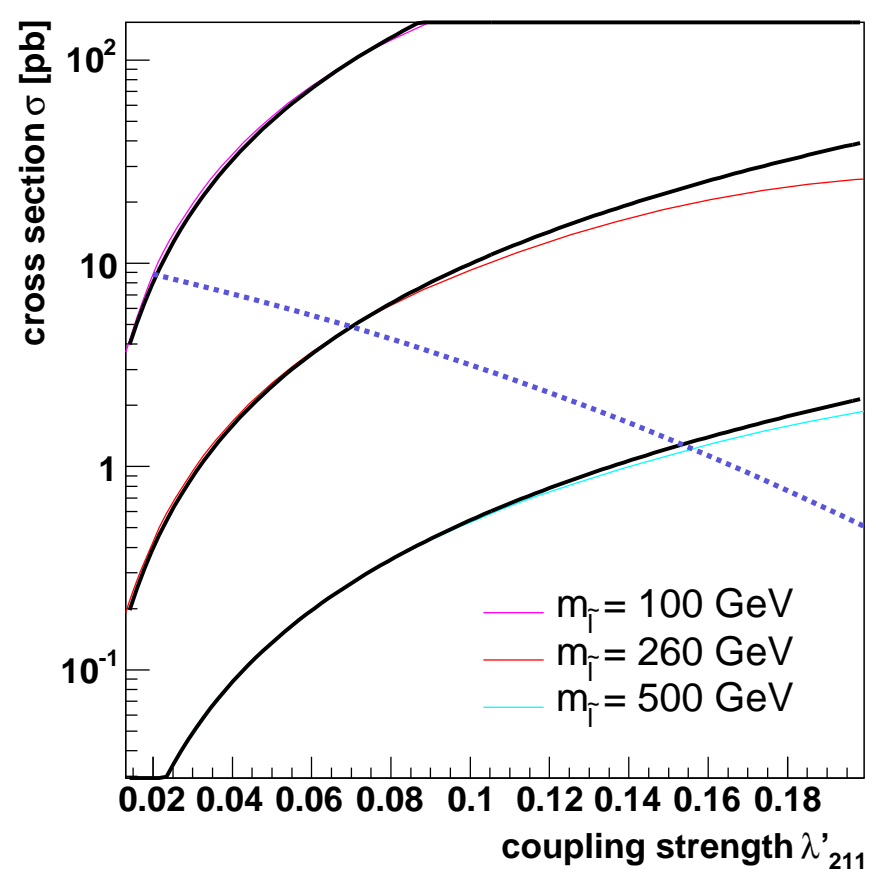

Figure 6.5: Signal cross section as predicted by SUSYGEN for different slepton masses in dependence of the coupling strength $\lambda_{211}^{\prime}$. The black lines represent the $\sigma$-parametrization as used by the present analysis assuming $\sigma \simeq\left(\lambda_{211}^{\prime}\right)^{2}$. The reach of this analysis is indicated by the dashed blue line; the analysis is sensitive to all cross sections and coupling strengths down to this line, for most studied mSUGRA parameters. The reach does also depend on the neutralino mass, if the mass is smaller than $\approx 50 \mathrm{GeV}$.

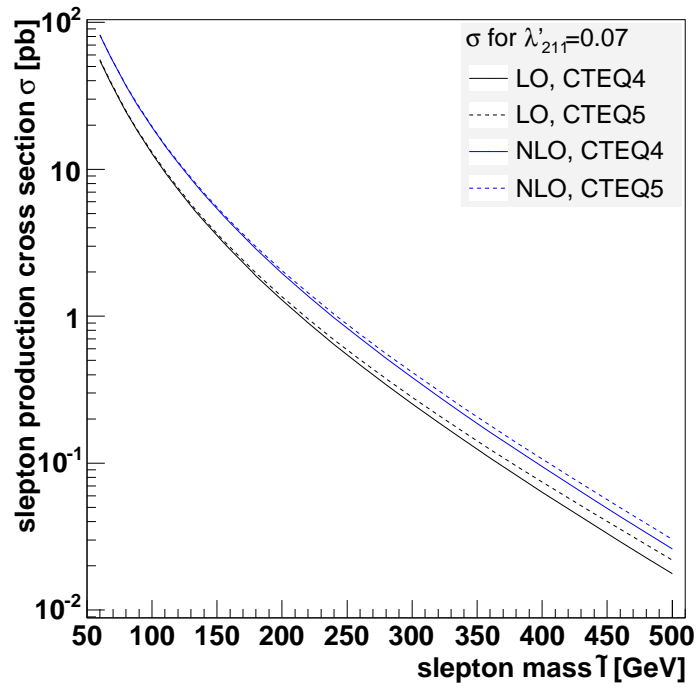

(a)

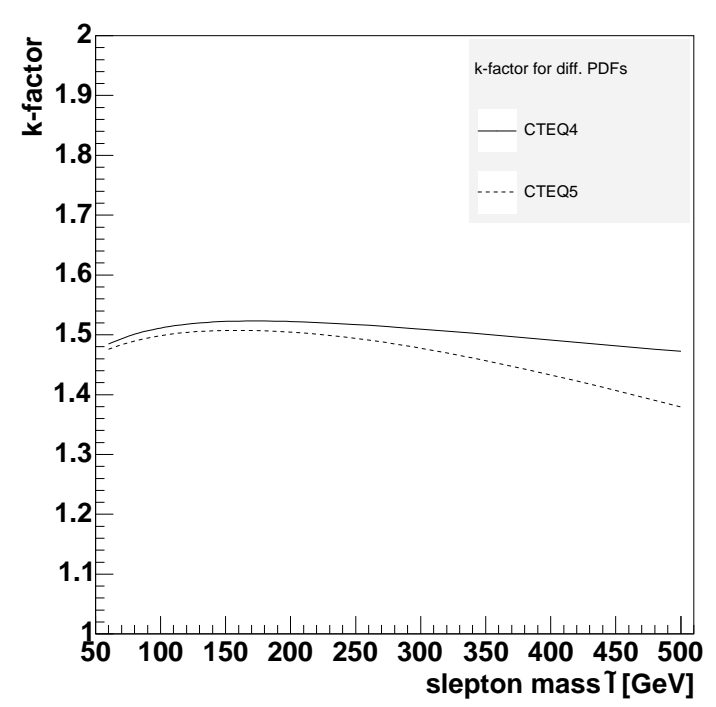

(b)

Figure 6.6: Leading order and next-to leading order calculation [171] of the slepton production cross section (a) for $\lambda_{211}^{\prime}=0.07$ and the resulting k-factor (b) for two PDFs. 
The slepton $p_{T}$ spectrum from SUSYGEN is softer then the spectrum predicted by HERWIG, however the NLO calculation agrees quite well at Tevatron energies with the SUSYgEN prediction. The slepton $p_{T}$ as predicted by SuSYgEN is used for this analysis.

In Sec. 2.3.1 the three different signal channels, which are defined by the slepton decay mode, (i) $\tilde{\mu} \rightarrow \tilde{\chi}_{1}^{0} \mu$, (ii) $\tilde{\mu} \rightarrow \tilde{\chi}_{2,(3,4)}^{0} \mu$, and (iii) $\tilde{\nu}_{\mu} \rightarrow \tilde{\chi}_{1,(2)}^{ \pm} \mu$ were given. For a specific point in the supersymmetric parameter space the kinematics of the latter two channels do not differ significantly since $\mathrm{m}\left(\tilde{\chi}_{1}^{ \pm}\right) \simeq \mathrm{m}\left(\tilde{\chi}_{2}^{0}\right)$ and $\mathrm{m}\left(\tilde{\chi}_{2}^{ \pm}\right) \simeq \mathrm{m}\left(\tilde{\chi}_{3}^{0}\right) \simeq \mathrm{m}\left(\tilde{\chi}_{4}^{0}\right)$. However, because of the different lightest neutralino mass, i.e. $2 \cdot \mathrm{m}\left(\tilde{\chi}_{1}^{0}\right) \simeq \mathrm{m}\left(\tilde{\chi}_{2}^{0}\right) \simeq \mathrm{m}\left(\tilde{\chi}_{1}^{ \pm}\right)$in mSUGRA, there are differences to channel (i). In Fig. 6.8 the transverse momenta of the leading (the $1^{\text {st }}$ ) and the next-to-leading (the $2^{\text {nd }}$ ) muon and jet are shown for the different signal channels. The particles from channels (ii) and (iii) are softer compared to the particles from channel (i). This observation is especially true for the leading muon. The leading muon originates with high probability from the slepton decay $\tilde{l} \rightarrow \mu \tilde{\chi}$ as proven by Fig. 6.9 and Fig. 6.10. Since the gauginos $\tilde{\chi}_{2}^{0}$ and $\tilde{\chi}_{1}^{ \pm}$of channels (ii) and (iii) are heavier then the $\tilde{\chi}_{1}^{0}$ of channel (i) accordingly less energy remains for the $1^{\text {st }}$ muon created in the $\tilde{l} \rightarrow \mu \tilde{\chi}$ decay.

The point which is used throughout the complete analysis as reference point is created with very high statistics (42k events) for an exemplary parameter set as listed in Tab. 6.2. The same point was right on the exclusion contour obtained by the DØ-Run I analysis [82] for a coupling strength of $\lambda_{211}^{\prime}=0.09$, as marked in Fig. 2.27.

\begin{tabular}{lc|lc}
\hline \hline mSUGRA & & masses & \\
\hline$m_{0}$ & $200 \mathrm{GeV}$ & $\mathrm{m}(\tilde{\mu})$ & $264.0 \mathrm{GeV}$ \\
$m_{1 / 2}$ & $243 \mathrm{GeV}$ & $\mathrm{m}\left(\tilde{\nu}_{\mu}\right)$ & $252.6 \mathrm{GeV}$ \\
$\tan (\beta)$ & 5 & $\mathrm{~m}\left(\tilde{\chi}_{1}^{0}\right)$ & $99.8 \mathrm{GeV}$ \\
$\operatorname{sign}(\mu)$ & -1 & $\mathrm{~m}\left(\tilde{\chi}_{2}^{0}\right)$ & $192.4 \mathrm{GeV}$ \\
$A_{0}$ & $0 \mathrm{GeV}$ & $\mathrm{m}\left(\tilde{\chi}_{1}^{ \pm}\right)$ & $192.8 \mathrm{GeV}$ \\
\hline$\lambda_{211}^{\prime}$ & 0.07 & $\sigma_{\mathrm{NLO}}$ & $6.06 \mathrm{pb}$ \\
\hline \hline
\end{tabular}

Table 6.2: The signal reference point. The cross section corresponds to the coupling strength $\lambda_{211}^{\prime}=0.07$. 


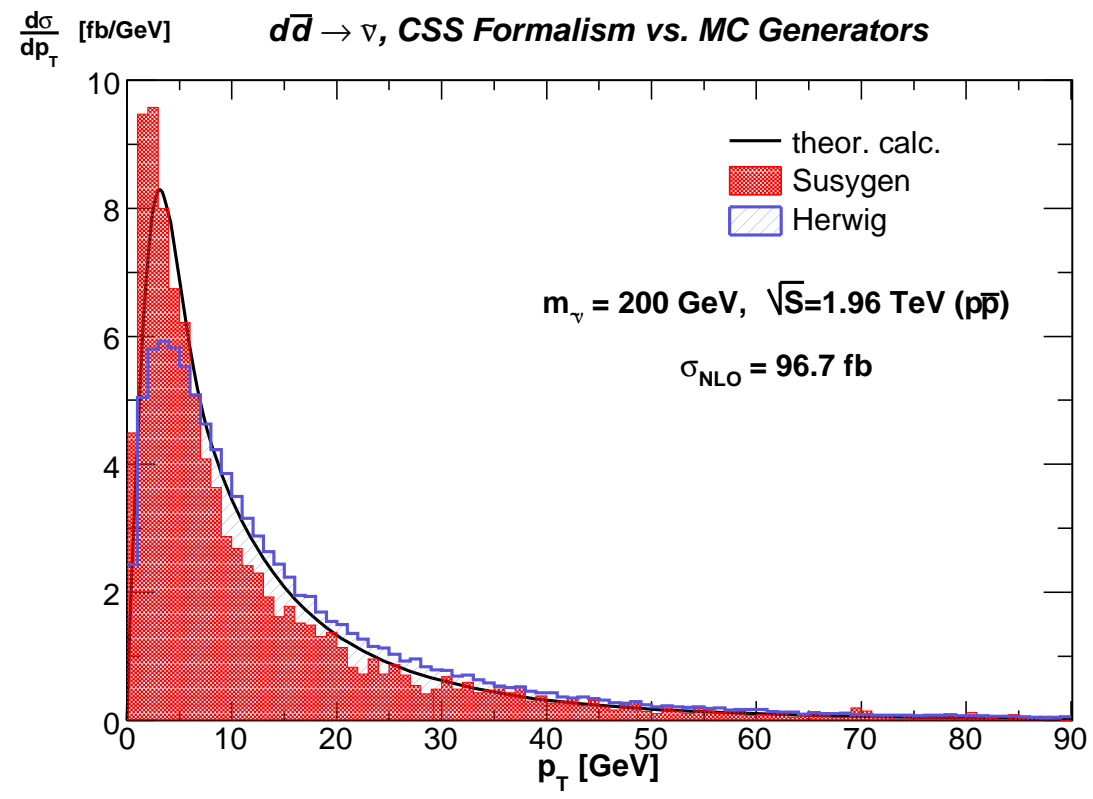

Figure 6.7: Slepton $p_{T}$ in NLO calculation and simulated by PYTHIA and HERWIG for a coupling strength $\lambda_{211}^{\prime}=0.01$ for $p \bar{p}$-collisions at $\sqrt{s}=1.96 \mathrm{TeV}[51]$.

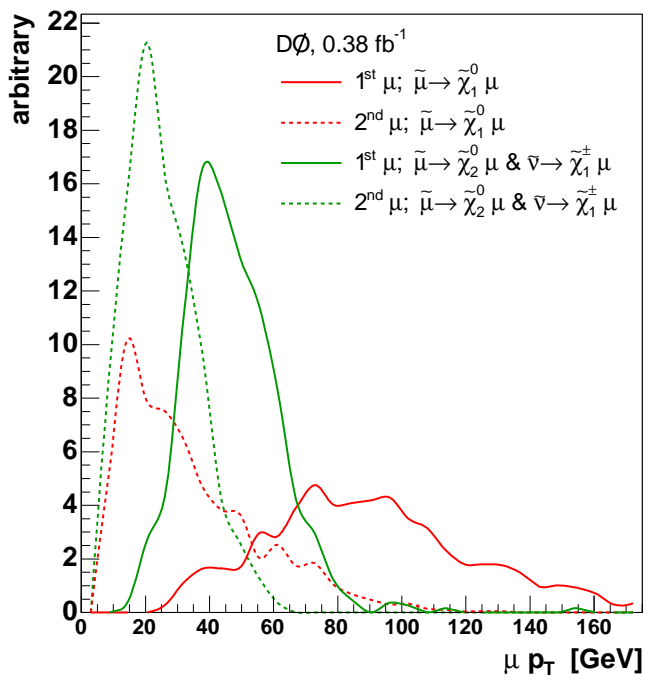

(a)

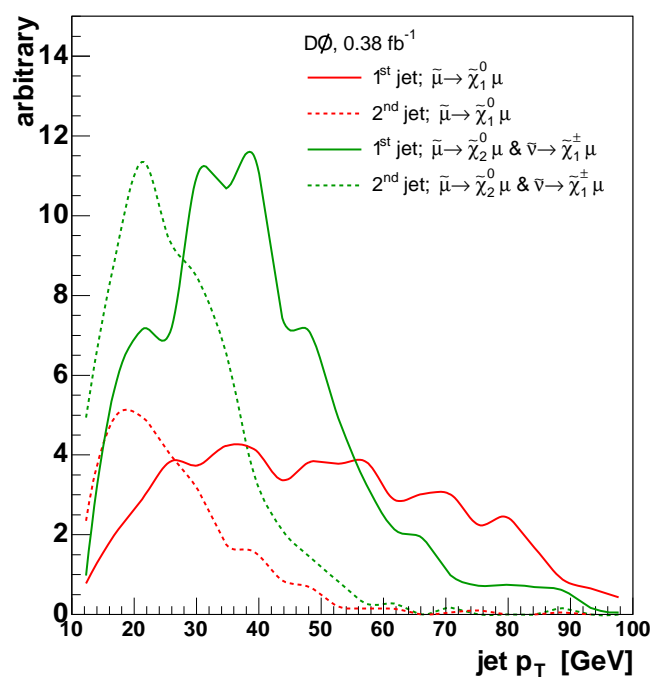

(b)

Figure 6.8: Muon (a) and jet (b) transverse momenta of the signal with $\mathrm{m}(\tilde{\mu})=260 \mathrm{GeV}$ and $\mathrm{m}\left(\tilde{\chi}_{1}^{0}\right)=100 \mathrm{GeV}$. The leading particle (solid) as well the next-to-leading particle (dashed) are shown for the channels $\tilde{\mu} \rightarrow \tilde{\chi}_{1}^{0} \mu, \tilde{\mu} \rightarrow \tilde{\chi}_{2}^{0} \mu$ and $\tilde{\nu}_{\mu} \rightarrow \tilde{\chi}_{1}^{ \pm} \mu$. The latter two channels are added, since the event kinematics are very similar. 


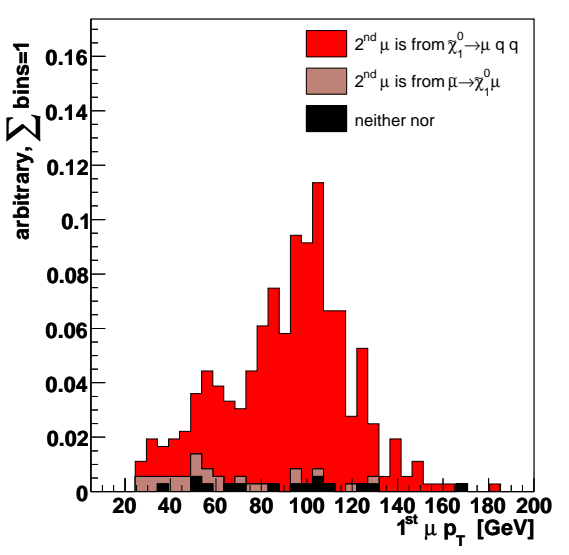

(a) $1^{\text {st }}$ muon momentum

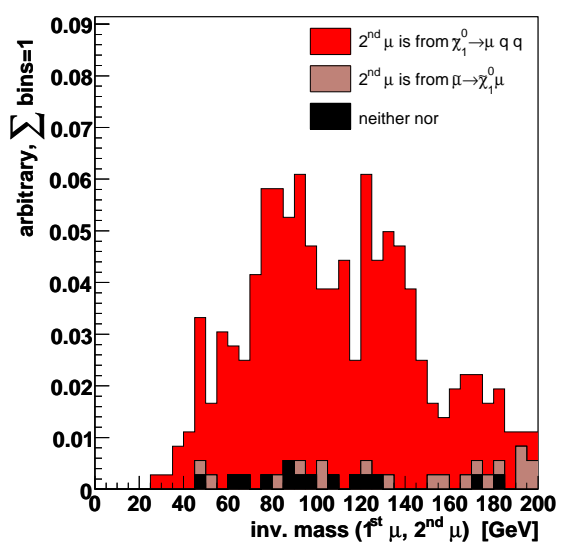

(c) Invariant di-muon mass

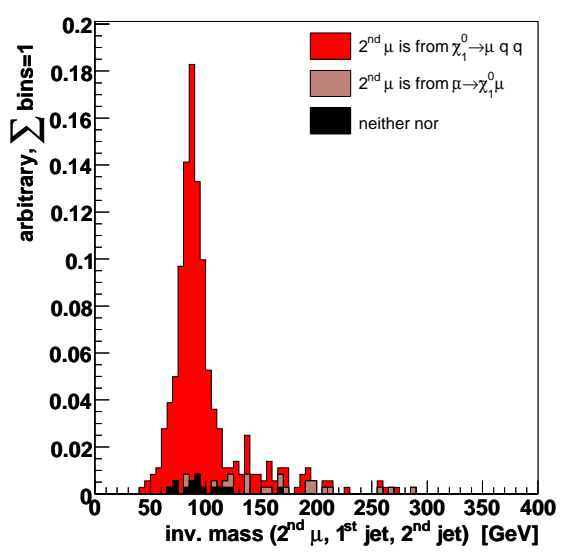

(e) Reconstructed $\tilde{\chi}_{1}^{0}$-mass with $2^{\text {nd }}$ muon

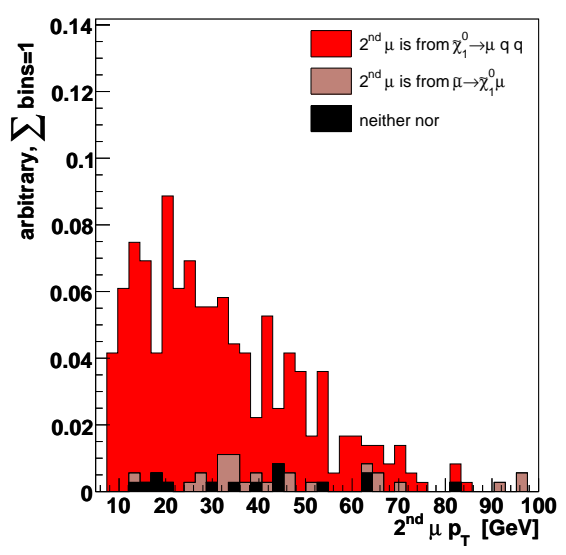

(b) $2^{\text {nd }}$ muon momentum

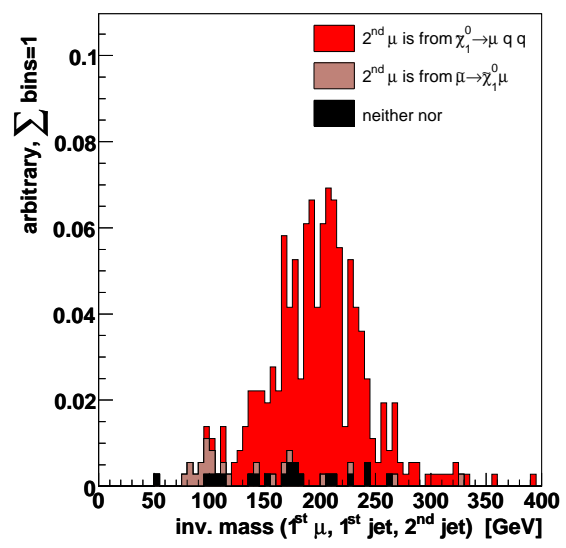

(d) Reconstructed $\tilde{\chi}_{1}^{0}$-mass with $1^{\text {st }}$ muon

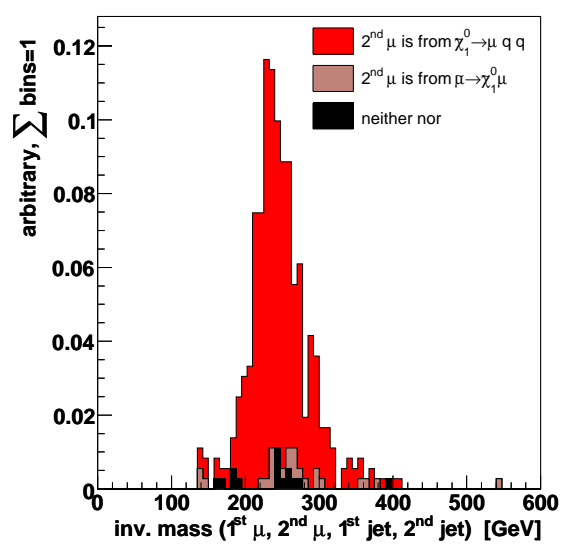

(f) Reconstructed $\tilde{\mu}$-mass

Figure 6.9: Signal studies for $\tilde{\mu} \rightarrow \tilde{\chi}_{1}^{0} \mu$. The events were generated by SUSYGEN with $\mathrm{m}(\tilde{\mu})=$ $260 \mathrm{GeV}$ and $\mathrm{m}\left(\tilde{\chi}_{1}^{0}\right)=100 \mathrm{GeV}$ and have passed the $\mathrm{D} \emptyset$ detector simulation. All shown events contain at least two muons and two jets (selection as discussed in this Sec.). The color refers to the origin of the muons and is obtained from generator information. In most events the $1^{\text {st }}$ muon with the highest transverse momentum originates from the $\tilde{\mu}$-decay, so that the $2^{\text {nd }}$ muon is from $\tilde{\chi}_{1}^{0} \rightarrow \mu q q$ and can be used to reconstruct the neutralino mass, as shown in (e). 


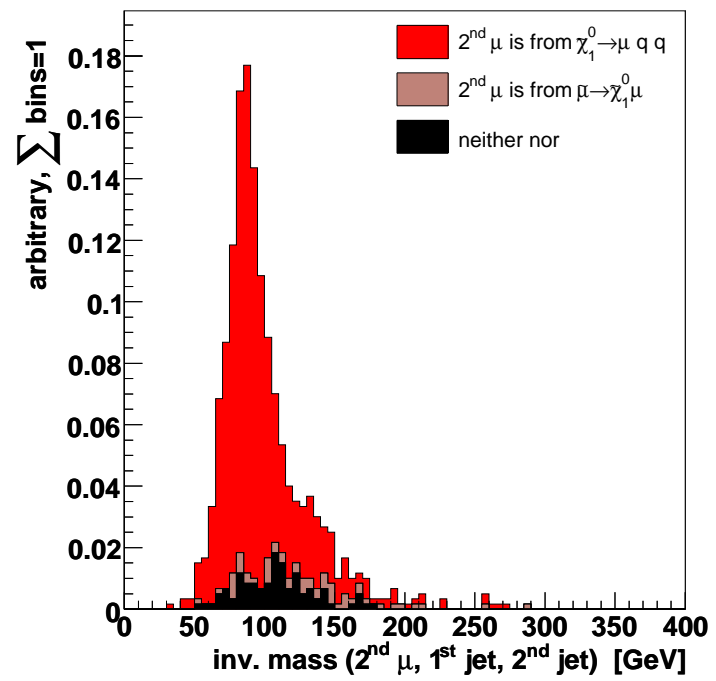

(a) Reconstructed $\tilde{\chi}_{1}^{0}$-mass with $2^{\text {nd }}$ muon

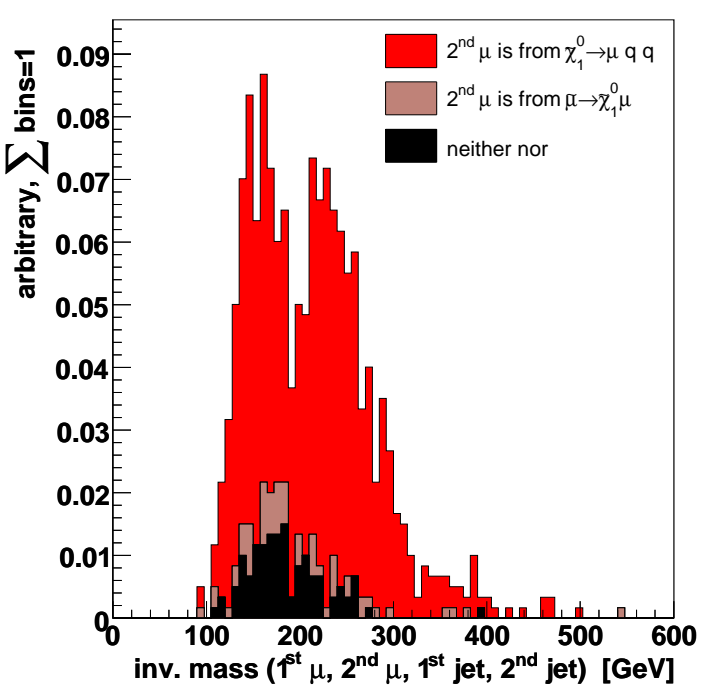

(b) Reconstructed $\tilde{l}$-mass

Figure 6.10: Signal studies for $\tilde{\mu} \rightarrow \tilde{\chi}_{2}^{0} \mu$ and $\tilde{\nu}_{\mu} \rightarrow \tilde{\chi}_{1}^{ \pm} \mu$. As in Fig. 6.9 the color refers to the origin of the muons and the sparticle masses are $\mathrm{m}(\tilde{\mu}) \simeq \mathrm{m}\left(\tilde{\nu}_{\mu}\right)=260 \mathrm{GeV}$ and $2 \cdot \mathrm{m}\left(\tilde{\chi}_{1}^{0}\right) \simeq$ $\mathrm{m}\left(\tilde{\chi}_{2}^{0}\right)=\mathrm{m}\left(\tilde{\chi}_{1}^{ \pm}\right)=190 \mathrm{GeV}$. Here, however, additional muons from the cascade decay of the heavier gauginos $\tilde{\chi}_{2}^{0}$ and $\tilde{\chi}_{1}^{ \pm}$to the lightest neutralino $\tilde{\chi}_{1}^{0}$ can be present (black). In these channels the reconstructed neutralino and slepton masses are more washed-out and the slepton mass is smaller, since energy is lost to particles created in the cascade decay.

In Tab. 6.3 the signal cutflow from the signal generator level down to a preselection with two muons and two jets is given. The cut efficiency is given for the reference point of Tab. 6.2 sequentially, if the cut would have been applied as first cut, and if the cut would have been applied as last cut. The selection efficiency depends on the signal point under study. For the $2^{\text {nd }}$ muon and the $2^{\text {nd }}$ jet, both originating from the neutralino decay $\tilde{\chi}_{1}^{0} \rightarrow \mu q q$, the selection efficiencies are shown in Fig. 6.11 in dependence of the neutralino mass $\mathrm{m}\left(\tilde{\chi}_{1}^{0}\right)$. 


\begin{tabular}{lccc}
\hline \hline Cut & sequentially & if applied as $1^{\text {st }}$ cut & if applied as last cut \\
\hline$p_{T}\left(\mu_{1}\right)>15 \mathrm{GeV}$ & $90.3 \%$ & $90.3 \%$ & $99.9 \%$ \\
isolation $\mu_{1}$ & $90.2 \%$ & $96.0 \%$ & $100.0 \%$ \\
central track $\mu_{1}$ & $77.3 \%$ & $79.9 \%$ & $84.8 \%$ \\
\hline$p_{T}\left(\mu_{2}\right)>8 \mathrm{GeV}$ & $38.3 \%$ & $36.8 \%$ & $92.3 \%$ \\
isolation $\mu_{2}$ & $27.5 \%$ & $38.4 \%$ & $75.0 \%$ \\
central track $\mu_{2}$ & $22.6 \%$ & $38.3 \%$ & $81.5 \%$ \\
\hline$p_{T}\left(\right.$ jet $\left._{1}\right)>15 \mathrm{GeV}$ & $20.7 \%$ & $91.5 \%$ & $99.9 \%$ \\
$p_{T}\left(\right.$ jet $\left._{2}\right)>15 \mathrm{GeV}$ & $10.7 \%$ & $49.9 \%$ & $84.5 \%$ \\
\hline$\varangle\left(\right.$ jet $_{1}$, jet $\left._{2}\right)>0.5$ & $10.7 \%$ & $91.5 \%$ & $100.0 \%$ \\
$\mid \eta\left(\right.$ jet $\left._{1}\right) \mid<2.1$ & $10.4 \%$ & $53.4 \%$ & $98.0 \%$ \\
$\mid \eta\left(\right.$ jet $\left._{2}\right) \mid<2.1$ & $10.2 \%$ & $55.6 \%$ & $97.2 \%$ \\
\hline \hline
\end{tabular}

Table 6.3: Signal cut flow for $\tilde{\mu} \rightarrow \tilde{\chi}_{1}^{0} \mu$ with $\mathrm{m}(\tilde{\mu})=260 \mathrm{GeV}$ and $\mathrm{m}\left(\tilde{\chi}_{1}^{0}\right)=100 \mathrm{GeV}$ from generator level to preselection. The given numbers refer to the fraction of events left, after a specific cut has been applied. The first column gives the cut flow in top-down order. The second column is the survival rate if the cut is applied as first cut, and likewise the third column gives the relative cut efficiency if the cut is applied last. In total 41750 events were generated for this mass-combination, 18685 events correspond to the process $\tilde{\mu} \rightarrow \tilde{\chi}_{1}^{0} \mu$. This table corresponds to that fraction of events (9576) where the neutralino decays to a muon and two jets $\tilde{\chi}_{1}^{0} \rightarrow \mu q q$, so that the final state should consist out of at least two muons and two jets. Note, that i.e. a cut on $p_{T}\left(\mu_{2}\right)$ implies, that a muon $\mu_{1}$ with at least this energy exists, and similarly for jets.

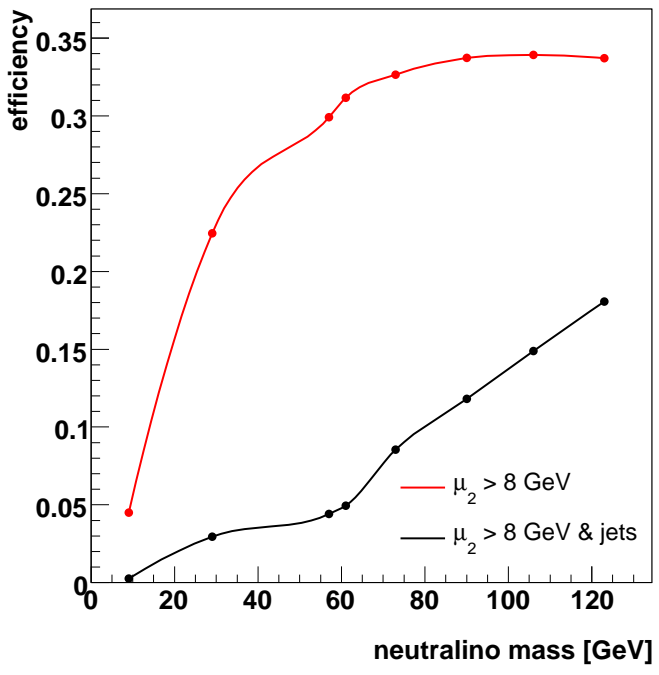

(a)

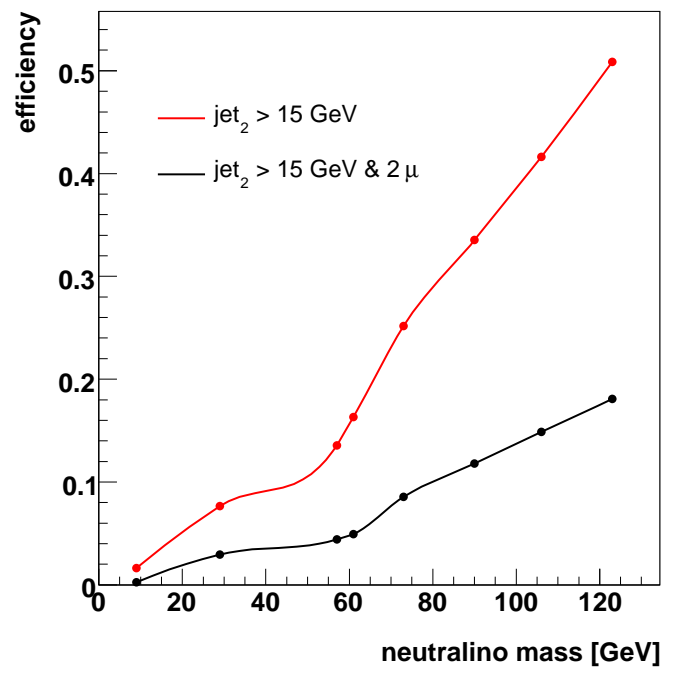

(b)

Figure 6.11: Efficiency to find the $2^{\text {nd }}$ muon of at least $8 \mathrm{GeV}$ (a) and the $2^{\text {nd }}$ jet with at least $15 \mathrm{GeV}$ (b) of signal in dependence of the neutralino mass $\tilde{\chi}_{1}^{0}$. The mSUGRA parameter $m_{0}$ is fixed to $m_{0}=100 \mathrm{GeV}$ for all shown points. The black line refers to the probability to find a $2^{\text {nd }}$ muon and the $2^{\text {nd }}$ jet and is therefore identical in both plots. The transverse momentum of the $1^{\text {st }}$ muon (jet) is always larger than $p_{T}\left(\mu_{2}\right)$, or $p_{T}\left(\right.$ jet $\left._{2}\right)$ respectively, by definition. 


\subsubsection{Event Selection}

All signal events contain at least two jets from the neutralino decay $\tilde{\chi}_{1}^{0} \rightarrow \mu q q$, therefore two jets with minimum transverse momentum $p_{T} \geq 15 \mathrm{GeV}$ are required ${ }^{1}$, reconstructed with the DØ Run II ILCA cone algorithm $(R=0.5)$ which was discussed in Sec. 5.2. Each jet is required to have a distance from any muon in $\Delta R$ greater than 0.5 . The distance of both jets has to be in the range of $\Delta R_{\text {jet } 1, j e t 2}<3.2$. The upper limit removes some background events, since all signal jets originate directly from gaugino decays, which are recoiling against the muon from the slepton decay. All jets are required to be $|\eta|<2.0$.

In Fig. 6.12 the preselection sample with two additional required jets (the " $2 \mu$-2jetsample") is shown. The displayed variables are the leading and the next-to-leading jet transverse momentum $p_{T}\left(\right.$ jet $\left._{1}\right)$ and $p_{T}\left(\right.$ jet $\left._{2}\right)$, the invariant di-jet-mass, the sum of both jet momenta, the 3 -body mass $M_{\mu_{2}, \text { jet }_{1}, j e t_{2}}$ which in the signal is equivalent to the reconstructed $\tilde{\chi}_{1}^{0}$ mass, and the 4-body mass $M_{\mu_{1}, \mu_{2}, j e t_{1}, j e t_{2}}$ which in the signal corresponds to the reconstructed $\tilde{l}$ mass. The signal is scaled up by factor 100 in (a) and by a factor of 10 in (b)-(f). Overall a good agreement of data and Standard Model prediction within the statistical and systematical uncertainties can be observed.

The cut flow is summarized in Tab. 6.4. The cuts are applied in the same way for all SUSY parameter points. At all steps in the cut flow, the number of data events is in agreement with the Standard Model expectation, within its uncertainties.

\begin{tabular}{|c|c|c|c|c|c|c|}
\hline Cut & Data & SM Exp. \pm & stat. \pm & syst. & Signal Eff. \pm & $\delta \epsilon$ \\
\hline $2 \mu$ preselection & 23206 & $22700 \pm$ & $70 \quad \pm$ & 2900 & $5.5 \% \pm$ & $0.7 \%$ \\
\hline$p_{T}$ jet $_{1}>15 \mathrm{GeV}$ & 3852 & $3760 \pm$ & 40 & 560 & $4.8 \% \pm$ & $0.6 \%$ \\
\hline$p_{T}$ jet $_{2}>15 \mathrm{GeV}$ & 538 & $500 \pm$ & 10 & 100 & $2.5 \% \pm$ & $0.3 \%$ \\
\hline$\Delta R_{\text {jet } 1, j e t 2}<3.2$ & 475 & $430 \pm$ & 10 & 80 & $2.4 \% \pm$ & $0.3 \%$ \\
\hline
\end{tabular}

Table 6.4: Cut flow toward a clean di-muon, di-jet selection. The signal efficiency is given for the reference point with $\mathrm{m}(\tilde{l})=260 \mathrm{GeV}$ and $\mathrm{m}\left(\tilde{\chi}_{1}^{0}\right)=100 \mathrm{GeV}$ in relation to the total slepton production cross section, which includes also inaccessible channels, e.g. $\tilde{\nu}_{\mu} \rightarrow \nu_{\mu} \tilde{\chi}_{1}^{0}$, and all channels with $\tilde{\chi}_{1}^{0} \rightarrow \nu_{\mu} q q$.

From this point on, the analysis differs for the three signal channels (i) $\tilde{\mu} \rightarrow \tilde{\chi}_{1}^{0} \mu$, (ii) $\tilde{\mu} \rightarrow \tilde{\chi}_{2,(3,4)}^{0} \mu$, (iii) $\tilde{\nu}_{\mu} \rightarrow \tilde{\chi}_{1,(2)}^{ \pm} \mu$ and for all studied SUSY parameter points.

Several methods have been studied to separate the signal from the Standard Model background, from a simple cut approach, a like-sign di-muon selection, to more complex algorithms like neural nets. The best separation has been accomplished with 2-dimensional cuts. Since the scanned SUSY variables cover a large range of slepton and neutralino masses (from a few $\mathrm{GeV}$ to several hundred $\mathrm{GeV}$ ), the final selection cuts have to be very flexible and have to be tuned in a consistent way. Because of the large number of examined SUSY parameter combinations (117 points with $\tan \beta=5, \mu<0, A_{0}=0$ ) it is not feasible to inspect all individual distributions visually. An algorithm was developed,

\footnotetext{
${ }^{1}$ The possibility of lowering the threshold for the cut on the $2^{\text {nd }}$ jet $p_{T}$ has been discarded due to the badly known jet energy scale in the low energy region.
} 


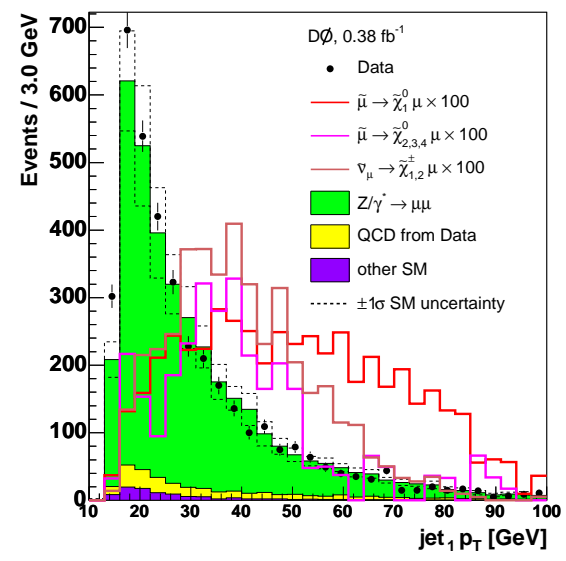

(a)

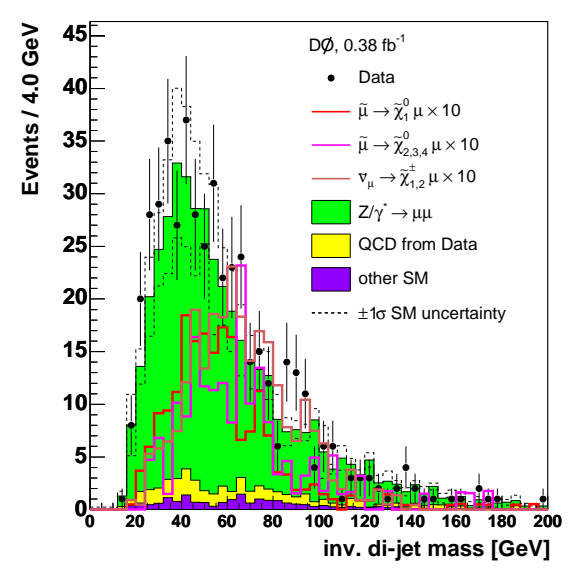

(c)

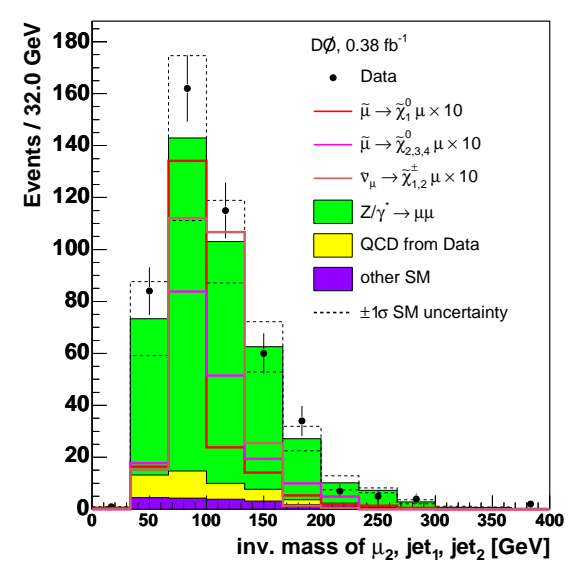

(e)

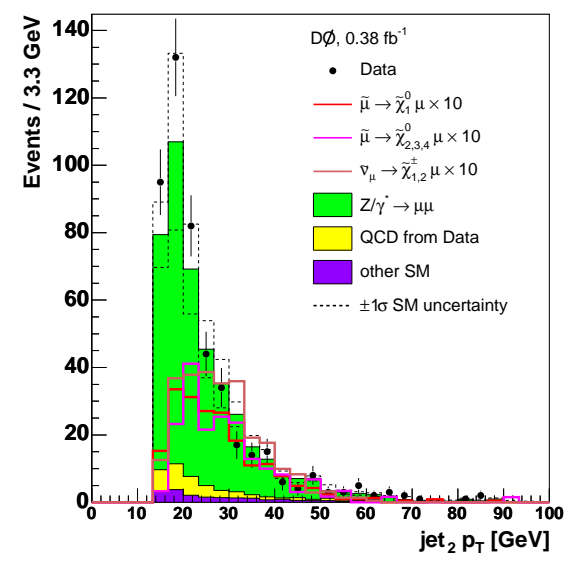

(b)

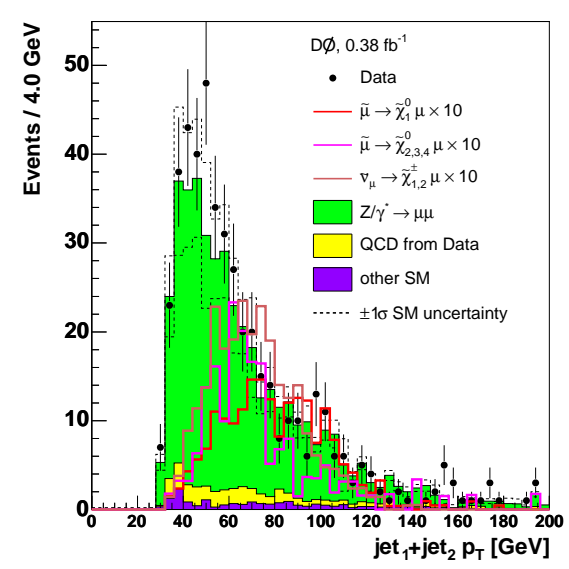

(d)

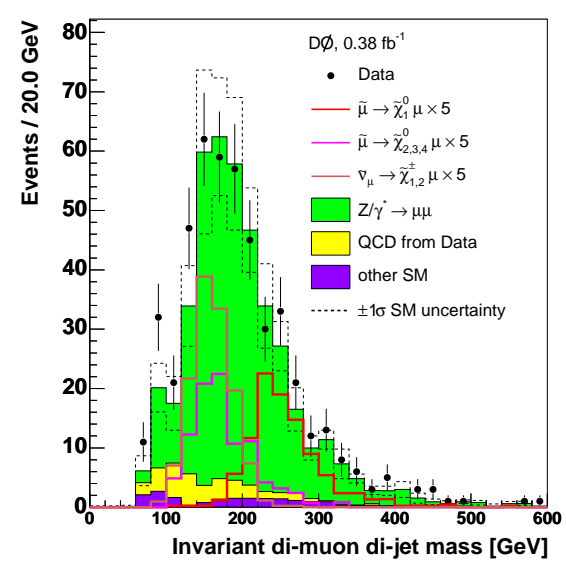

(f)

Figure 6.12: The " $2 \mu$-2jet-sample". The reference point signal with $\mathrm{m}(\tilde{\mu})=260 \mathrm{GeV}$ and $\mathrm{m}\left(\tilde{\chi}_{1}^{0}\right)=$ $100 \mathrm{GeV}$ is scaled ub in (a) by a factor of 100 and in (c)-(f) by a factor of 10 . The total systematic uncertainty of the SM background is shown as dashed black lines. 
which allows to optimize the final selection for each SUSY parameter point, depending only on the slepton mass $\mathrm{m}(\tilde{l})$ and the neutralino mass $\mathrm{m}\left(\tilde{\chi}_{1}^{0}\right)$, which define the point in the SUSY parameter space under study, equivalent to the mSUGRA parameter pair $m_{0}$ and $m_{1 / 2}$ as described in Sec. 4.3.2.

The three signal channels (i)-(iii), are analyzed separately. The final selection procedure, which shall be discussed in the following, is repeated for each channel, as well as for each SUSY parameter point.

The final selection cuts are applied in six different, 2-dimensional planes spanned by seven different variables; the reconstructed slepton mass, the reconstructed neutralino mass, the invariant di-muon mass, the invariant di-jet mass, the sum of the muon transverse momenta, the sum of the jet transverse momenta and the angle between both jets. The choice of these variables is inspired by the characteristics of the resonant slepton production. The slepton as well as the neutralino mass can be reconstructed for signal events with high efficiency and precision, as discussed in Sec. 6.2.1. The signal is therefore clustered in a small region in a plane spanned by the reconstructed slepton mass and the reconstructed neutralino mass. An ideal cut in this plane has therefore the form of an ellipse. However, as discussed previously, the spacial orientation of the ellipse, as well as the ellipse radii, depend on the slepton and neutralino masses and their widths so that the cut details depend on the SUSY parameter point and also on the signal channel.

For each of the six cut planes one cut template in the form of an analytical function $f(x, y)$ is defined, e.g. an elliptical function for the $\mathrm{m}(\tilde{l})-\mathrm{m}\left(\tilde{\chi}_{1}^{0}\right)$-plane. Also the slepton mass $\mathrm{m}(\tilde{l})$ and the neutralino mass $\mathrm{m}\left(\tilde{\chi}_{1}^{0}\right)$, which are defined by the current SUSY point under study, are used as parameters. Additional parameters $p_{i}$, which are constrained to the interval $p_{i}=[0 . .1]$, are used, to introduce some degree of freedom to the cut function's parametrization. This freedom is necessary to optimize each cut over a wide range of signal points with very different kinematics. The details of the six cut planes and the associated cut parametrization will be discussed below.

The algorithm which tunes the free parameters for each final selection cut is depicted schematically in Fig. 6.13. In the first step, all Monte Carlo samples (signal as well as background) are split into a training sample (1/3) and a test sample (2/3). The training sample is used to find the optimal cut combination for a point, which is then applied on the re-weighted (factor 3/2) test sample and the data. The cuts are tuned on a different sample (the training sample) to avoid any bias due to over-training, which might happen in regions of large fluctuations of signal and background. It is unlikely that the test and the training sample are subject to random fluctuations in the same phase space region.

The cut optimization for one cut in any 2D-plane, for each of the three signal channels, and all 117 SUSY points is repeated $10^{4}-10^{5}$ times, depending of the number of free parameters for the specific cut. In each optimization step, first a new set of parameters is randomly chosen. The cut parametrization using these parameters is then applied on 


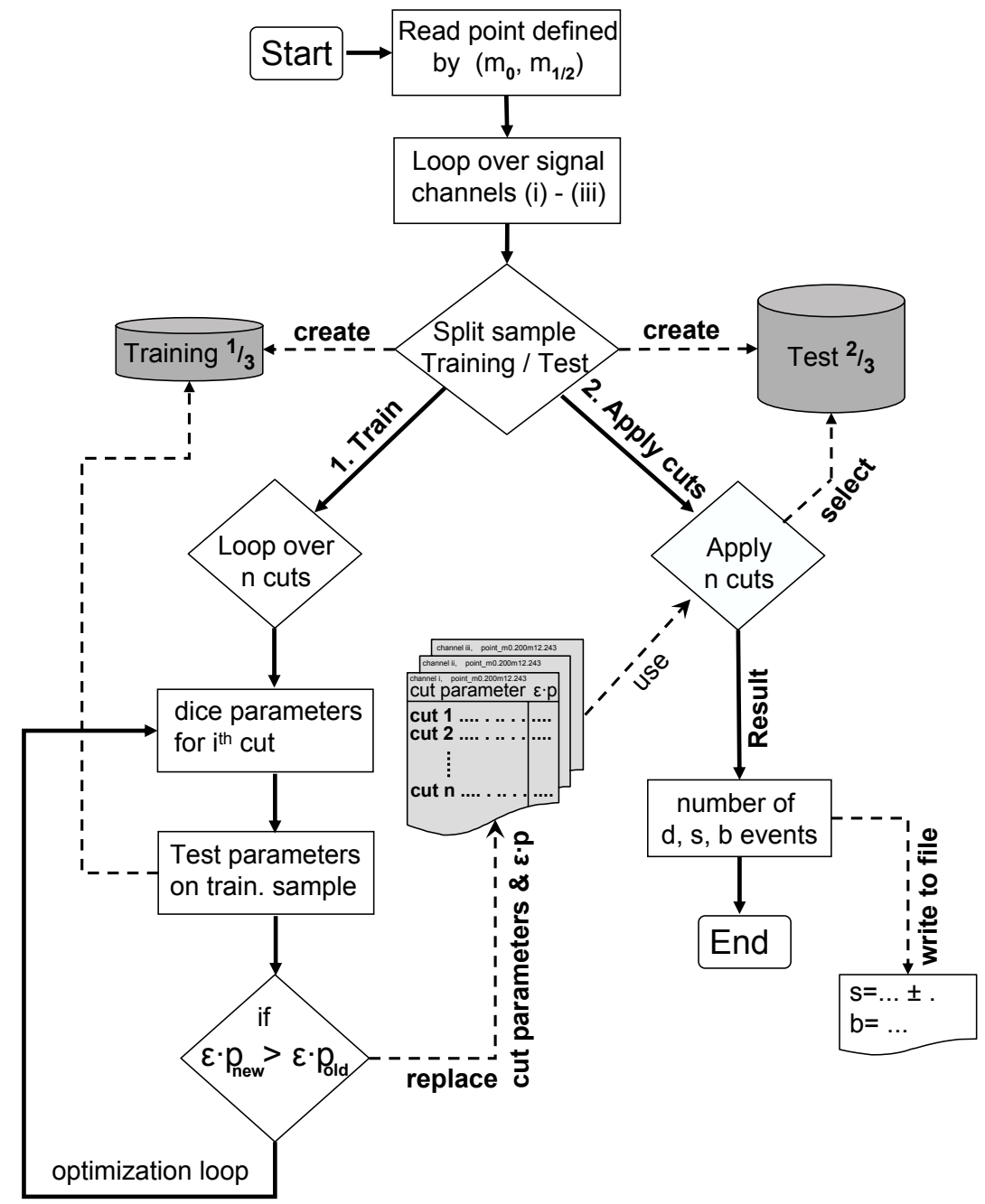

Figure 6.13: Flowchart of the final 2D-cut optimization algorithm. The algorithm is repeated for all three signal channels (i)-(iii) and for each of the 117 SUSY parameter points. The cutoptimization procedure ("1. Train") can be skipped if an optimized set of parameters for the specific cut, signal channel, and SUSY point already exists. This set of parameters is then loaded from a file. The result of the shown algorithm is the finally selected sample of data, signal and background events.

the training sample. The signal efficiency (without acceptance, i.e. trigger efficiency corrections) is given by:

$$
\epsilon_{\text {sig }}=\frac{n_{\text {selected signal }}}{n_{\text {generated signal }}}
$$


The signal purity is defined as:

$$
p_{\text {sig }}=\frac{n_{\text {signal }}}{n_{\text {signal }}+n_{\text {background }}} .
$$

The product $\epsilon_{\mathrm{sig}} \cdot p_{\mathrm{sig}}$ is used to score the cuts. This is equivalent to the optimization of $n_{\text {signal }} / \sqrt{{ }^{n} \text { background }}$. To keep the numerical values of $\epsilon_{\text {sig }}$ handy, the signal efficiency is redefined to a relative signal efficiency with respect to the last selection, i.e. $n_{\text {generated signal }}$ in Eq. (6.2) is replaced with the number of signal events that were present in the sample just before the cut was applied. If the new score $\epsilon_{\text {sig }} \cdot p_{\text {sig }}$ is larger than an old score for this cut, then the new set of optimized parameters $p_{i}$ is saved. The optimized cuts using the parameters which led to the largest scores are then applied on the test sample, if the score was larger than 0.2 , or the signal efficiency larger than 0.5 . This barrier prevents the selection of small fluctuations, e.g. a cut which selects only very few signal events in the training sample will result in $p=1$ and a non vanishing signal efficiency, however $\epsilon_{\text {sig }} \cdot p_{\text {sig }}$ in the test sample would be much worse.

\section{Neutralino-mass reconstruction}

The slepton and neutralino masses are reconstructed in different ways for the three signal channels to account for the different signal channel decay topologies.

Channel (i) $\tilde{\mu} \rightarrow \tilde{\chi}_{1}^{0} \mu$ : The slepton mass is reconstructed with the two leading jets and both muons. The neutralino mass is reconstructed with both jets and the next-toleading muon. The leading muon originates in this channel with high probability from the smuon decay vertex;

Channel (ii) $\tilde{\mu} \rightarrow \tilde{\chi}_{2,(3,4)}^{0} \mu$ and channel (iii) $\tilde{\nu}_{\mu} \rightarrow \tilde{\chi}_{1,(2)}^{ \pm} \mu:$ If only two jets are found, then the above method is used to reconstruct the slepton and neutralino mass. However, more jets from hadronic decays of vector bosons from the gaugino cascade to $\tilde{\chi}_{1}^{0}$ can be present. A simple likelihood is calculated for each combination to reconstruct a vector-boson and the neutralino mass of the point under study. The neutralino mass of the combination with the smallest deviation from the nominal mass is used. The slepton mass is reconstructed from all found jets and the two leading muons.

\section{Cut Parameterization}

In the following the functions used to parametrize the $2 \mathrm{D}$ cuts in the six different planes are given. The free parameters $p_{i}$ are scanned and the set of $p_{i}$ giving the best signal efficiency $\times$ purity ("score") $\epsilon_{\text {sig }} \cdot p_{\text {sig }}$ for a specific cut is used when applying the selection on the data. 
1. Cut in the slepton mass - neutralino mass plane

The details of the slepton mass $M_{\mu_{1}, \mu_{2}, \text { jet }_{1}, \text { jet }_{2}}$ and neutralino mass $M_{\mu_{2}, \text { jet }_{1}, \text { jet }_{2}}$ reconstruction depend on the studied signal channel as described above.

The cut is an elliptical function, all events in the outside of the ellipse are rejected. To parametrize the function, $\mathrm{m}(\tilde{l})$ and $\mathrm{m}\left(\tilde{\chi}_{1}^{0}\right)$ which are given by the signal sample or SUSY point under study, and four additional free parameters $p_{1}, p_{2}, p_{3}, p_{4}$ are used. The free parameters are constraint to the interval $p_{i}=[0 . .1]$ for all cuts.

$$
\begin{aligned}
\text { plane : } \quad x=M_{\mu_{1}, \mu_{2}, \text { jet }_{1}, \text { jet }_{2}} / \mathrm{GeV} \\
y=M_{\mu_{2}, \text { jet }_{1}, \text { jet }_{2}} / \mathrm{GeV} \\
\text { cut param. : } \quad 1=\left(\frac{x-2 \cdot p_{1} \cdot m_{\tilde{l}} / \mathrm{GeV}}{0.2 \cdot p_{3} \cdot m_{\tilde{l}} / \mathrm{GeV}}\right)^{2}+\left(\frac{y-2 \cdot p_{2} \cdot m_{\tilde{\chi}_{1}^{0}} / \mathrm{GeV}}{20 \cdot p_{4}}\right)^{2}
\end{aligned}
$$

\begin{tabular}{ll|llll|lll}
\hline \hline \multicolumn{2}{c|}{ signal channel } & $p_{1}$ & $p_{2}$ & $p_{3}$ & $p_{4}$ & $\epsilon_{\text {sig }}$ & $p_{\text {sig }}$ & score \\
\hline (i) & $\tilde{\mu} \rightarrow \tilde{\chi}_{1}^{0} \mu$ & 0.47 & 0.40 & 0.81 & 0.93 & 0.53 & 0.58 & 0.31 \\
(ii) & $\tilde{\mu} \rightarrow \tilde{\chi}_{2,(3,4)}^{0} \mu$ & 0.90 & 0.79 & 0.67 & 0.35 & 0.83 & 0.08 & 0.07 \\
$(\mathrm{iii})$ & $\tilde{\nu}_{\mu} \rightarrow \tilde{\chi}_{1,(2)}^{ \pm} \mu$ & 0.72 & 0.53 & 0.45 & 0.24 & 0.72 & 0.18 & 0.13 \\
\hline \hline
\end{tabular}

Table 6.5: Parameters and score for cut 1 for the reference signal point.

2. Cut on the scalar sum of the transverse di-muon momenta

This cut is one-dimensional but it is optimized in the same way as all other final cuts using one free parameter $p_{1}$.

$$
\begin{aligned}
\text { "plane" : } & x=\left(p_{T}\left(\mu_{1}\right)+p_{T}\left(\mu_{2}\right)\right) / \mathrm{GeV} \\
\text { cut parametrization : } & x \geq p_{1} \cdot 100
\end{aligned}
$$

\begin{tabular}{|c|c|c|c|c|}
\hline signal channel & $p_{1}$ & $\epsilon_{\mathrm{sig}}$ & $p_{\text {sig }}$ & score \\
\hline (i) $\quad \tilde{\mu} \rightarrow \tilde{\chi}_{1}^{0} \mu$ & 0.90 & 0.91 & 0.64 & 0.59 \\
\hline$\tilde{\mu} \rightarrow \tilde{\chi}_{2,(3,4)}^{0} \mu$ & 0.47 & 0.97 & 0.09 & 0.09 \\
\hline$\tilde{\nu}_{\mu} \rightarrow \tilde{\chi}_{1,(2)}^{ \pm} \mu$ & 0.47 & 0.94 & 0.23 & 0.21 \\
\hline
\end{tabular}

Table 6.6: Parameters and score for cut 2 for the reference signal point.

3. Cut in the invariant di-muon mass - -muon transverse momentum sum plane

This cut makes use of two free parameters $p_{1}$ and $p_{2}$ and is only applied for the two signal channels (ii)-(iii) with higher gaugino masses. The intention of this cut is to 
remove the QCD background which accumulates in the low sum of muon $p_{T}$ and the low invariant di-muon mass region.

$$
\text { plane : } \quad \begin{aligned}
x & =M_{\mu_{1}, \mu_{2}} / \mathrm{GeV} \\
y & =\left(p_{T}\left(\mu_{1}\right)+p_{T}\left(\mu_{2}\right)\right) / \mathrm{GeV}
\end{aligned}
$$

cut parametrization: $\quad f(x)=200 \cdot p_{1}-8 \cdot p_{2} \cdot x$

\begin{tabular}{ll|ll|lll}
\hline \hline \multicolumn{2}{c|}{ signal channel } & $p_{1}$ & $p_{2}$ & $\epsilon_{\text {sig }}$ & $p_{\text {sig }}$ & score \\
\hline (ii) & $\tilde{\mu} \rightarrow \tilde{\chi}_{2,(3,4)}^{0} \mu$ & 0.34 & 0.03 & 0.98 & 0.09 & 0.09 \\
(iii) & $\tilde{\nu}_{\mu} \rightarrow \tilde{\chi}_{1,(2)}^{ \pm} \mu$ & 0.24 & 0.02 & 0.94 & 0.23 & 0.21 \\
\hline \hline
\end{tabular}

Table 6.7: Parameters and score for cut 3 for the reference signal point.

4. Cut in the invariant di-jet mass - jet transverse momentum sum plane

This cut which makes use of two free parameters $p_{1}$ and $p_{2}$ is able to separate Z/DrellYan events from signal events with high neutralino masses, since the jets stemming from the neutralino decay tend to have harder transverse momentum spectra and invariant di-jet masses in comparison to the initial or final state parton showers from $\mathrm{Z} / \gamma^{*}$.

$$
\begin{aligned}
\text { plane : } \quad x & =M_{\text {jet }_{1}, \text { jet }_{2}} / \mathrm{GeV}, \\
y & =\left(p_{T}\left(\text { jet }_{1}\right)+p_{T}\left(\text { jet }_{2}\right)\right) / \mathrm{GeV} \\
\text { cut parametrization : } \quad f(x) & =240 \cdot p_{1}-\frac{4}{3} \cdot p_{2} \cdot x
\end{aligned}
$$

\begin{tabular}{lc|ll|lll}
\hline \hline \multicolumn{2}{c|}{ signal channel } & $p_{1}$ & $p_{2}$ & $\epsilon_{\text {sig }}$ & $p_{\text {sig }}$ & score \\
\hline (ii) & $\tilde{\mu} \rightarrow \tilde{\chi}_{2,(3,4)}^{0} \mu$ & 0.30 & 0.20 & 0.90 & 0.13 & 0.12 \\
(iii) & $\tilde{\nu}_{\mu} \rightarrow \tilde{\chi}_{1,(2)}^{ \pm} \mu$ & 0.41 & 0.80 & 0.93 & 0.31 & 0.29 \\
\hline \hline
\end{tabular}

Table 6.8: Parameters and score for cut 4 for the reference signal point. 
5. Cut in the invariant di-muon mass - jet transverse momentum sum plane

This cut which uses four free parameters $p_{1}$ through $p_{4}$ is intended to remove $Z$ events in the peak region around $91 \mathrm{GeV}$ with low jet momenta.

$$
\begin{aligned}
\text { plane : } \quad x & =M_{\mu_{1}, \mu_{2}} / \mathrm{GeV}, \\
y & =\left(p_{T}\left(\text { jet }_{1}\right)+p_{T}\left(\text { jet }_{2}\right)\right) / \mathrm{GeV} \\
\text { cut parametrization : } \quad & \\
f_{1}(x) & =200 \cdot p_{1}-2 \cdot p_{2} \cdot x \\
f_{2}(x) & =160 \cdot p_{3}-0.18 \cdot p_{4} \cdot(x-91)^{2} \\
f(x) & =\operatorname{maximum}\left(f_{1}(x), f_{2}(x)\right)
\end{aligned}
$$

\begin{tabular}{lc|llll|lll}
\hline \hline \multicolumn{2}{c|}{ signal channel } & $p_{1}$ & $p_{2}$ & $p_{3}$ & $p_{4}$ & $\epsilon_{\text {sig }}$ & $p_{\text {sig }}$ & score \\
\hline (ii) & $\tilde{\mu} \rightarrow \tilde{\chi}_{2,(3,4)}^{0} \mu$ & 0.98 & 0.51 & 0.55 & 0.47 & 0.51 & 0.38 & 0.18 \\
(iii) & $\tilde{\nu}_{\mu} \rightarrow \tilde{\chi}_{1,(2)}^{ \pm} \mu$ & 0.56 & 0.56 & 0.54 & 0.49 & 0.65 & 0.66 & 0.43 \\
\hline \hline
\end{tabular}

Table 6.9: Parameters and score for cut 5 for the reference signal point.

6. Cut in the di-muon mass - di-jet opening angle plane

The last cut using two free parameters $p_{1}, p_{2}$ is intended to remove $Z$-events in the peak region around $91 \mathrm{GeV}$ with large opening angles between both jets.

$$
\begin{aligned}
& \text { plane : } \quad x=M_{\mu_{1}, \mu_{2}} / \mathrm{GeV} \text {, } \\
& y=\Delta R\left(\text { jet }_{1}, \text { jet }_{2}\right)
\end{aligned}
$$

\begin{tabular}{ll|ll|lll}
\hline \hline \multicolumn{2}{c|}{ signal channel } & $p_{1}$ & $p_{2}$ & $\epsilon_{\text {sig }}$ & $p_{\text {sig }}$ & score \\
\hline (ii) & $\tilde{\mu} \rightarrow \tilde{\chi}_{2,(3,4)}^{0} \mu$ & 0.14 & 0.99 & 0.87 & 0.16 & 0.14 \\
(iii) & $\tilde{\nu}_{\mu} \rightarrow \tilde{\chi}_{1,(2)}^{ \pm} \mu$ & 0.01 & 0.79 & 0.77 & 0.34 & 0.26 \\
\hline \hline
\end{tabular}

Table 6.10: Parameters and score for cut 6 for the reference signal point.

In case of channel $\tilde{\mu} \rightarrow \tilde{\chi}_{1}^{0} \mu$ only up to two cuts are applied, namely the $1^{\text {st }}$ cut on the neutralino mass-slepton mass-plane and the $2^{\text {nd }}$ cut on the sum of muon $p_{T}$. These two cuts are sufficient, since the relevant gaugino mass $\left(\tilde{\chi}_{1}^{0}\right)$ in this channel is significantly lighter than in the other two channels $\tilde{\mu} \rightarrow \tilde{\chi}_{2,(3,4)}^{0} \mu$, and $\tilde{\nu}_{\mu} \rightarrow \tilde{\chi}_{1,(2)}^{ \pm} \mu$. For the same slepton mass, the kinetic energy of both muons is therefore in this channel much higher (see Fig. 6.8). The energy in the cascade decays of a heavy gaugino to the lightest neutralino $\left(\tilde{\chi}_{1}^{0}\right)$ does not contribute to the muon energy, since the selected muons in this analysis 
originate from either the slepton decay vertex or from the decay of the lightest neutralino $\left(\tilde{\chi}_{1}^{0}\right)$.

The cut in the slepton mass - neutralino mass plane is optimized and applied on the $2 \mu$ 2jet sample. All other final cuts are then optimized on the remaining sample and applied successively. For this reason the data distributions in Fig. 6.14-6.16 are shown for two selections; the selection where the cut parameters have been optimized (left column), and the selection on which the plot is applied (right column). These two samples are obviously identical for cut 1 as well as for cut 2 . The cuts show the signal sample generated at the reference point with $\mathrm{m}(\tilde{l})=260 \mathrm{GeV}, \mathrm{m}\left(\tilde{\chi}_{1}^{0}\right)=100 \mathrm{GeV}$ and $\mathrm{m}\left(\tilde{\chi}_{2}^{0}\right) \approx \mathrm{m}\left(\tilde{\chi}_{1}^{ \pm}\right)=193 \mathrm{GeV}$.

Plotted in Fig. 6.14-6.16 is the test sample, while all cuts have been optimized using the training sample. The Standard Model Monte Carlo samples in these 2D-plots have been normalized to their effective cross section. For a better legibility the signal Monte Carlo sample is not normalized. The data is not shown in the plots. The various cuts might overlap and are sometimes redundant and not independent. This flexibility is necessary due to the wide mass spectrum of the signal.

Typically, the $\epsilon \cdot p \geq 0.2$ or $\epsilon \geq 0.5$ barrier is met by all cuts, so that all cuts are applied, except for SUSY parameter points with neutralino masses smaller than $\sim 50 \mathrm{GeV}$, where the signal efficiency is too small to find a more stringent selection.

Channel (i) $\tilde{\mu} \rightarrow \tilde{\chi}_{1}^{0}$

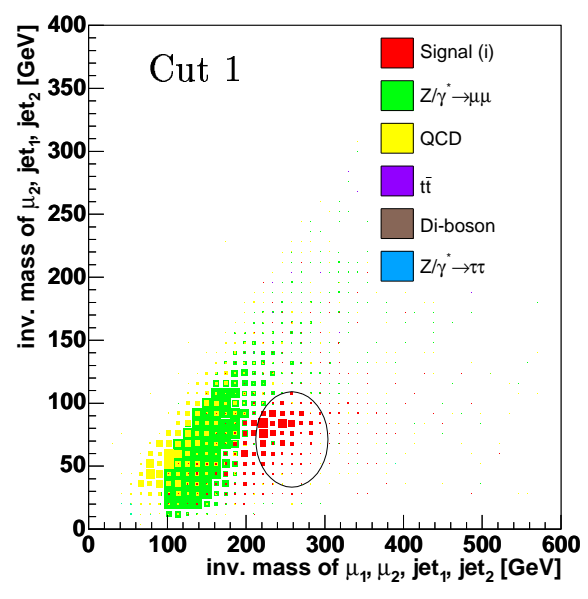

(a)

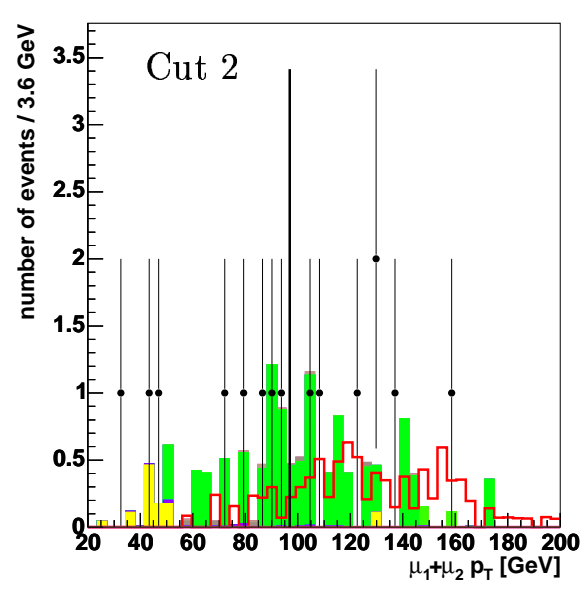

(b)

Figure 6.14: Final selection cuts for channel $\tilde{\mu} \rightarrow \tilde{\chi}_{1}^{0} \mu$, optimized for the reference point with $\mathrm{m}(\tilde{\mu})=260 \mathrm{GeV}$ and $\mathrm{m}\left(\tilde{\chi}_{1}^{0}\right)=100 \mathrm{GeV}$. The plot (a) shows the 2-dimensional cut parametrized by an ellipse in the smuon mass - neutralino mass plane. (b) The $2^{\text {nd }}$ cut on the sum of the muon transverse momenta was optimized after the $1^{\text {st }}$ cut (a) was applied. 
Channel (ii) $\tilde{\mu} \rightarrow \tilde{\chi}_{2,(3,4)}^{0}$

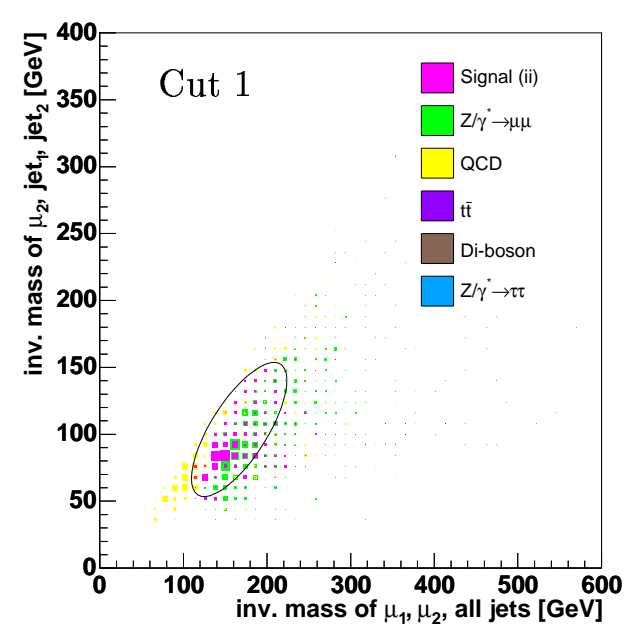

(a) $2 \mu$-2jet sample

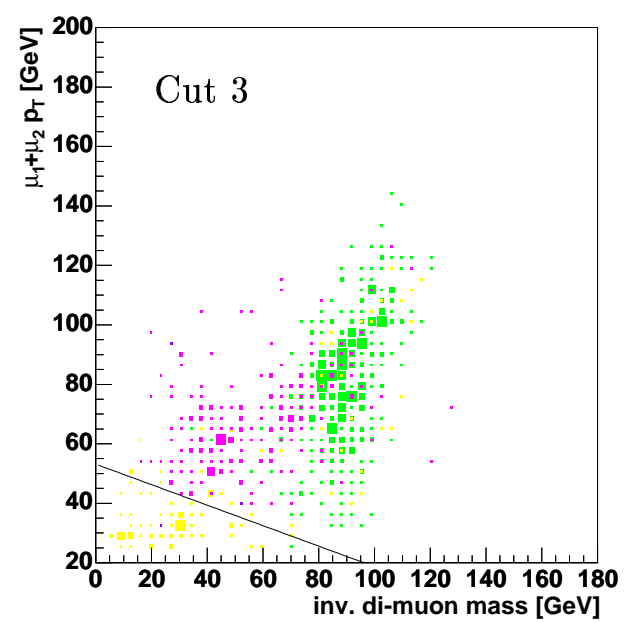

(c) sample after $1^{\text {st }}$ cut (plot a)

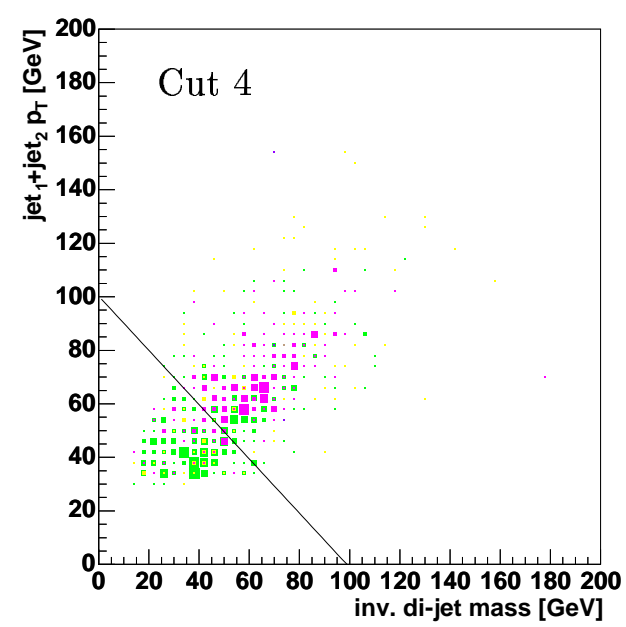

(e) sample after $1^{\text {st }}$ cut (plot a)

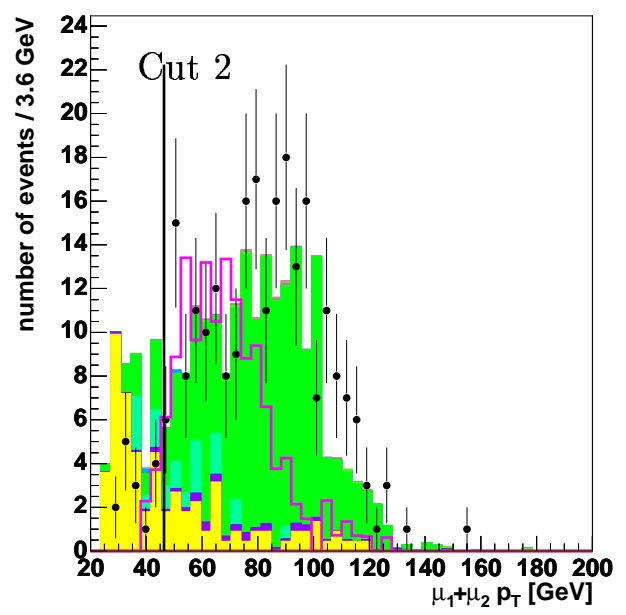

(b) sample after $1^{\text {st }}$ cut (plot a)

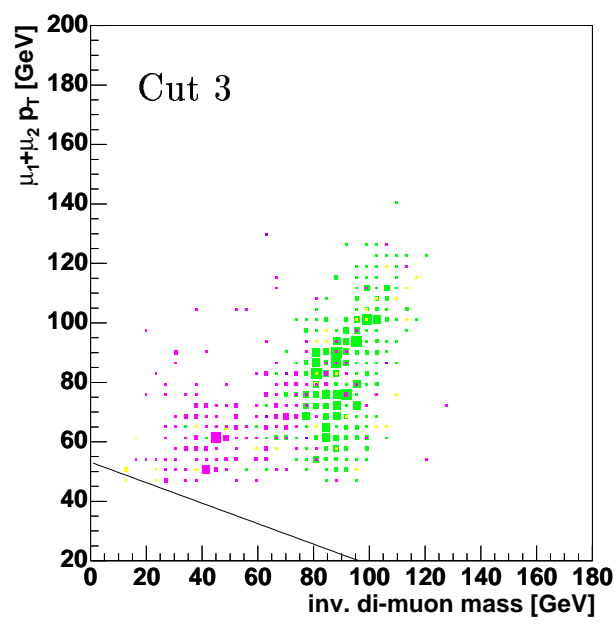

(d) sample after $2^{\text {nd }}$ cut (plot b)

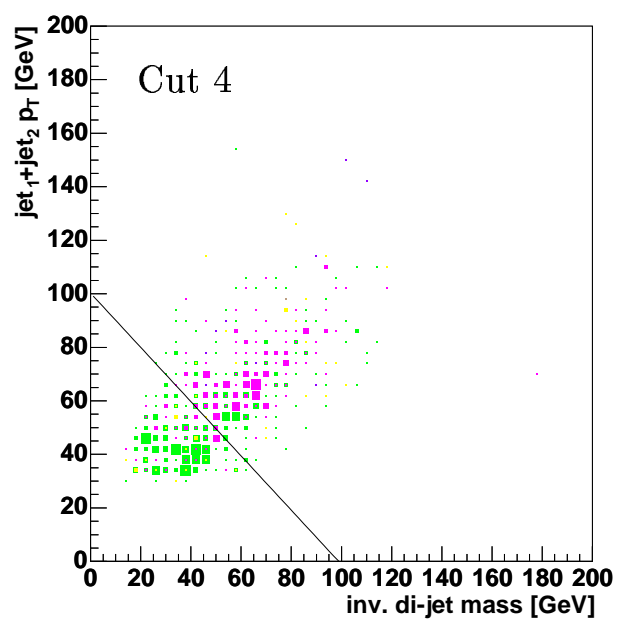

(f) sample after $3^{\text {rd }}$ cut (plot c, d) 


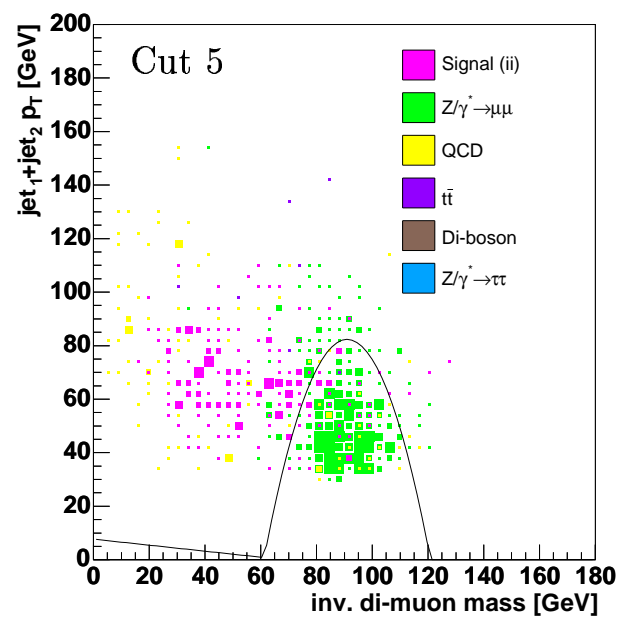

(g) sample after $1^{\text {st }}$ cut (plot a)

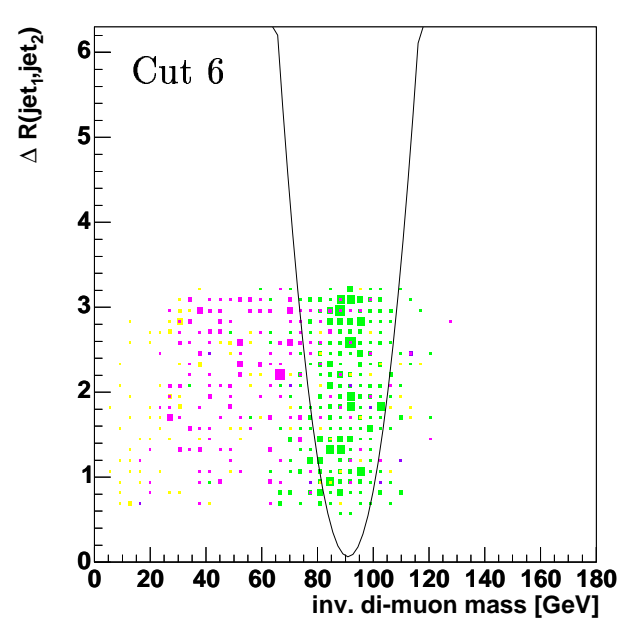

(i) sample after $1^{\text {st }}$ cut (plot a)

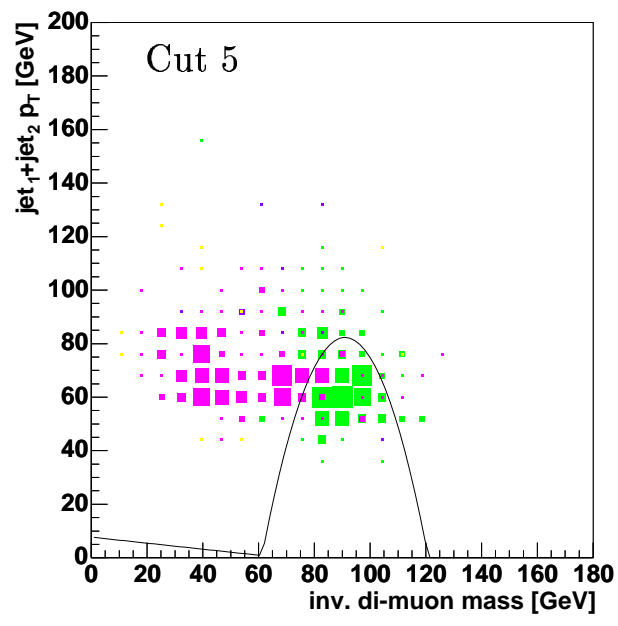

(h) sample after $4^{\text {th }}$ cut (plot e, f)

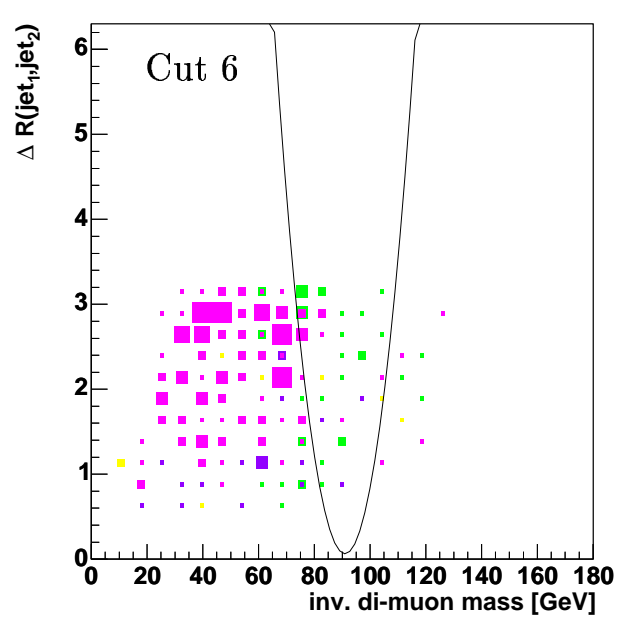

(j) sample after $5^{\text {th }}$ cut (plot g, h)

Figure 6.15: Final selection cuts for channel $\tilde{\mu} \rightarrow \tilde{\chi}_{2,(3,4)}^{0} \mu$, optimized for the reference point with $\mathrm{m}(\tilde{\mu})=260 \mathrm{GeV}$ and $\mathrm{m}\left(\tilde{\chi}_{2}^{0}\right)=192 \mathrm{GeV}$. The plot (a) shows the 2-dimensional cut parametrized by an ellipse in the smuon-neutralino ${ }_{1}$-plane. First, this cut 1 is applied, then the other cuts 2-6 are optimized on the remaining sample. The cuts are shown in the sample where they have been optimized (left) and directly before they are applied (right). For cut 1, and cut 2 these samples are identical. The cut-flow is top down. 
Channel (iii) $\tilde{\nu}_{\mu} \rightarrow \tilde{\chi}_{1,(2)}^{ \pm}$

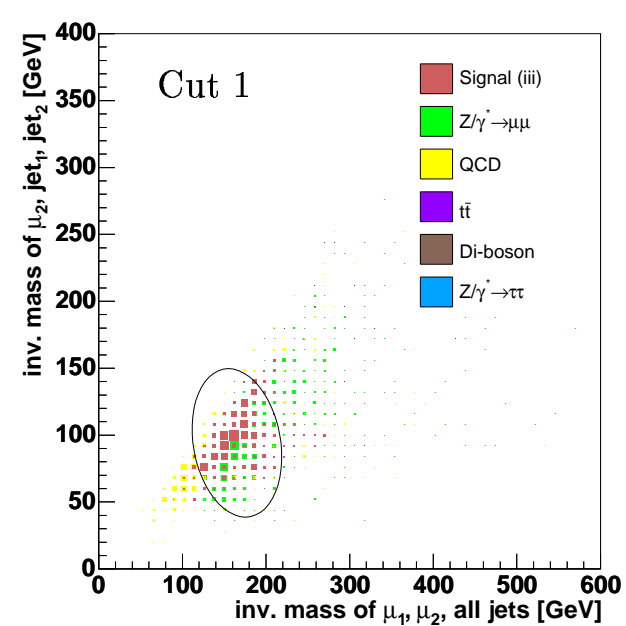

(a) $2 \mu$-2jet sample

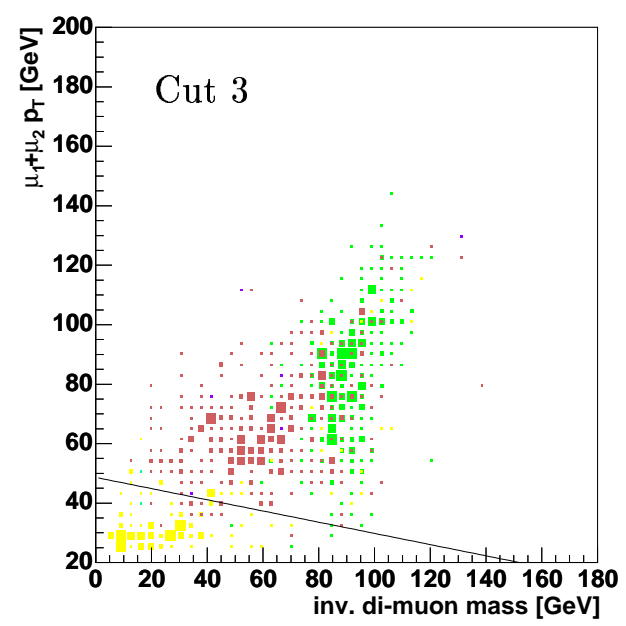

(c) sample after $1^{\text {st }}$ cut (plot a)

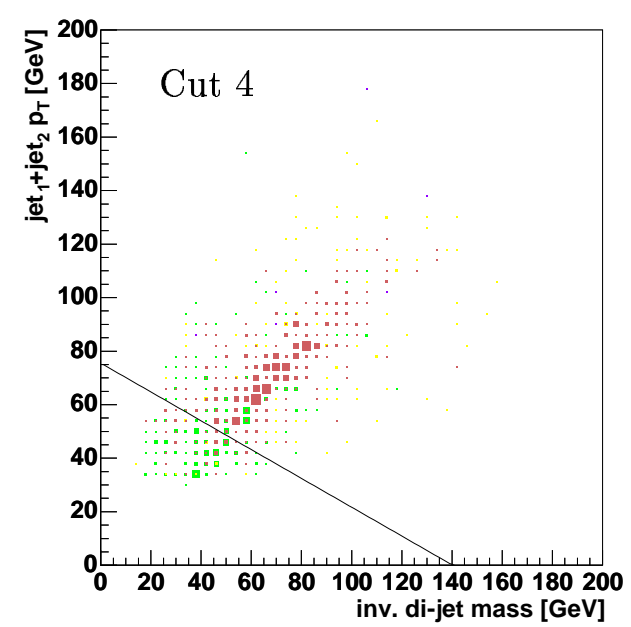

(e) sample after $1^{\text {st }}$ cut (plot a)

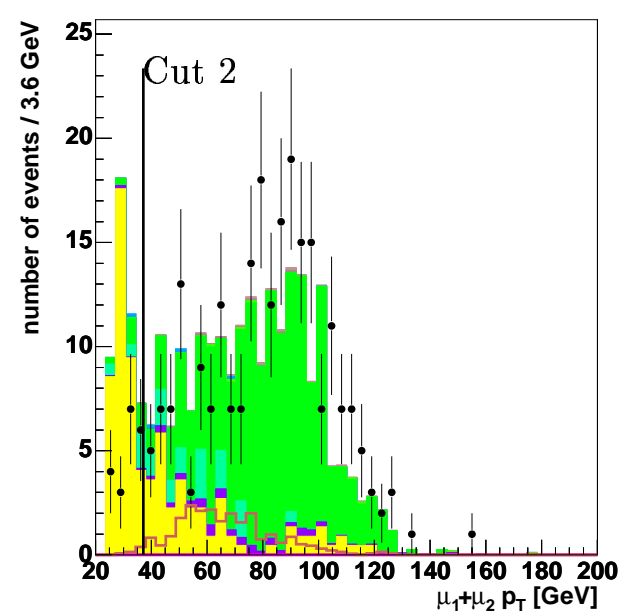

(b) sample after $1^{\text {st }}$ cut (plot a)

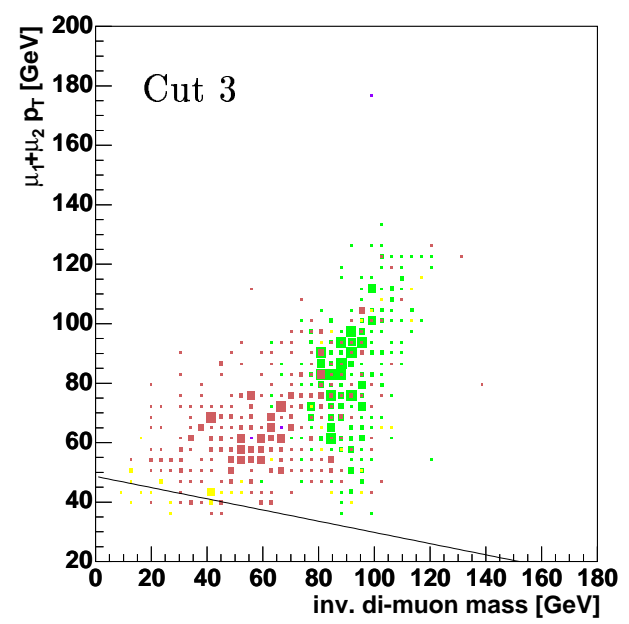

(d) sample after $2^{\text {nd }}$ cut (plot b)

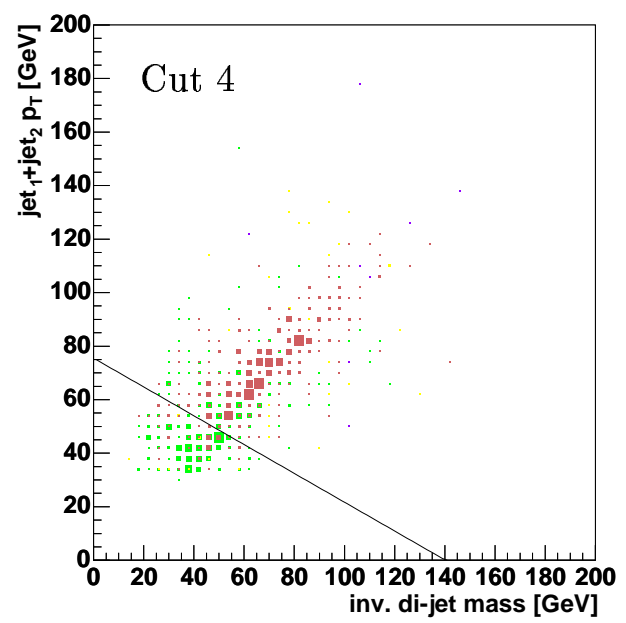

(f) sample after $3^{\text {rd }}$ cut (plot c,d) 


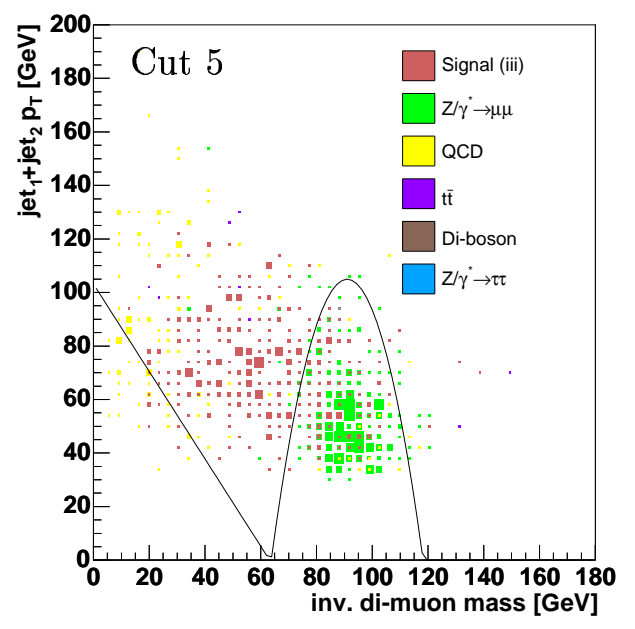

(g) sample after $1^{\text {st }}$ cut (plot a)

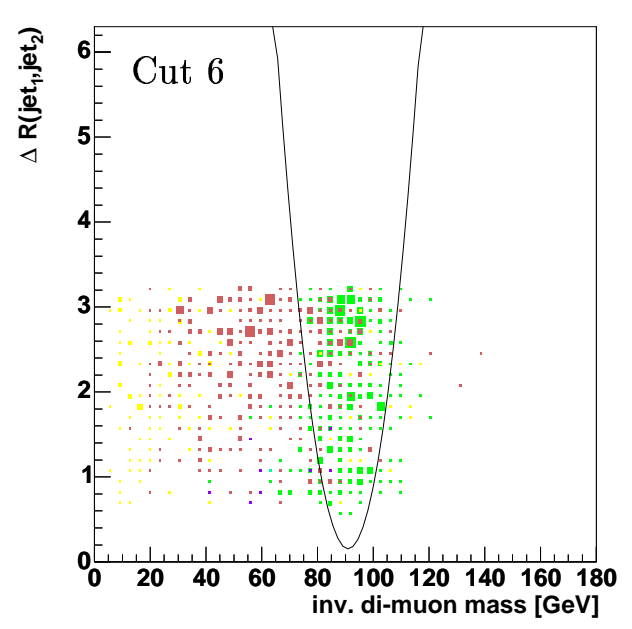

(i) sample after $1^{\text {st }}$ cut (plot a)

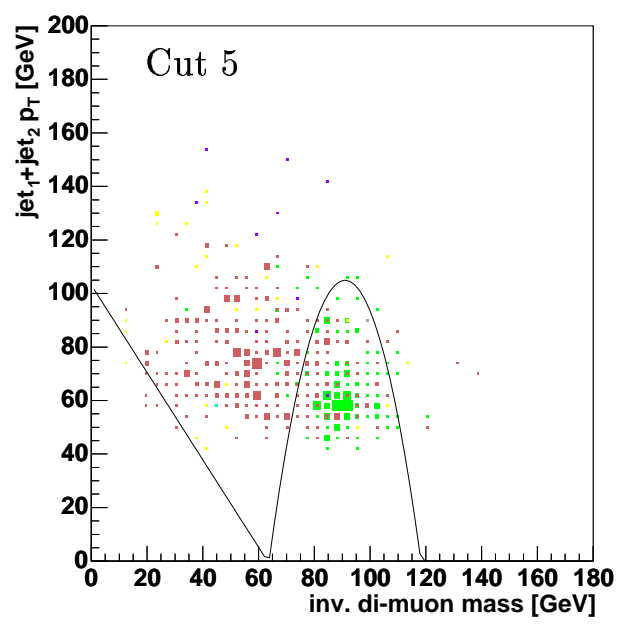

(h) sample after $4^{\text {th }}$ cut (plot e, f)

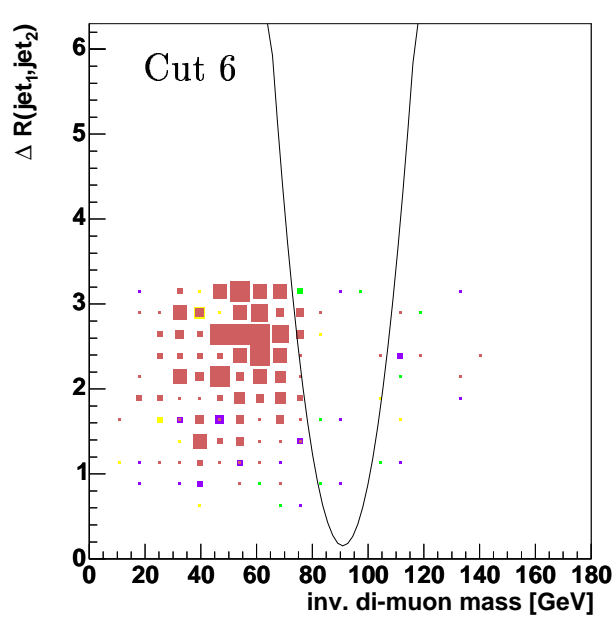

(j) sample after $5^{\text {th }}$ cut (plot g, h)

Figure 6.16: Final selection cuts for channel $\tilde{\nu}_{\mu} \rightarrow \tilde{\chi}_{1}^{ \pm} \mu$, optimized for the reference point with $\mathrm{m}\left(\tilde{\nu_{\mu}}\right)=260 \mathrm{GeV}$ and $\mathrm{m}\left(\tilde{\chi}_{1}^{ \pm}\right)=193 \mathrm{GeV}$. The plot (a) shows the 2-dimensional cut parametrized by an ellipse in the smuon-neutralino 1 -plane. First, this cut 1 is applied, then the other cuts 2-6 are optimized on the remaining sample. The cuts are shown in the sample where they have been optimized (left) and directly before they are applied (right). For cut 1, and cut 2 these samples are identical. The cut-flow is top down. 


\subsubsection{Systematic Uncertainties}

The systematic errors on the Standard Model background and on the signal prediction arise from different error sources, which are listed in the following in the order of the typical significance:

- The largest single systematic error source is due to the jet energy scale uncertainty and is derived by varying the jet $E_{T}$ correction together for the data and the Monte Carlo samples by $1 \sigma$ (one standard deviation), see also Sec. 5.2.4. The variations are propagated correctly to other variables $\left(\not_{T}\right)$. The complete analysis is then redone using the modified jet energies;

- All muon related efficiencies have been discussed in detail in Sec. 5.1. The ratio of the muon reconstruction, track finding and matching, isolation and medium-quality efficiency in data and in the simulation have been used to calculate correction factors, which depend in the case of the muon reconstruction on the muon pseudo-rapidity $\eta$. The corrections are calculated per muon. Since di-muon events are selected, each muon uncertainty is multiplied by a factor of 2 and summed quadratically. This conservatively takes full correlation between both muons into account;

- The relative luminosity uncertainty for the utilized datataking period is equal to $6.5 \%$ [174]. The luminosity uncertainty does not apply to the QCD sample. Since the QCD contribution to the $2 \mu$-2jet sample is approximately $15 \%$, the resulting total luminosity error on the total background in the $2 \mu$-2jet sample is $85 \% * 6.5 \%=5.5 \%$;

- The systematic error of the di-muon trigger parameterization is obtained by varying the parameters given in Sec. 5.1.1, Tab. 5.2 within their errors;

- The uncertainty on the production cross section times k-factor, arising from the parton density function uncertainties, is taken into account by varying the (N)NLO cross section correction by one standard deviation, as given in [147]. The massdependent corrections were calculated using the CTEQ6.1M parton density error functions and are shown in Fig. 6.17(a) for the dominant Standard Model background process $(Z / \gamma)^{*}$-production and in Fig. 6.17(b) for the resonant slepton production.

- The choice of the factorization scale $\mu_{f}=m(\tilde{l})$ has influence on the resonant slepton production cross section. In Fig. 6.18 the change in the cross section is shown, when varying the factorization scale in the range $m(\tilde{l}) / 2 \leq \mu_{f} \leq 2 \cdot m(\tilde{l})$. The renormalization scale $\mu_{r}$ which is important at higher orders is usually chosen to be equal to $\mu_{f}$. The total systematic uncertainty to the resonant slepton cross section due to the factorization and renormalization scale uncertainties is conservatively estimated to be less than $5 \%$;

- The QCD sample cross section uncertainty has been estimated and is less than 20\%; 


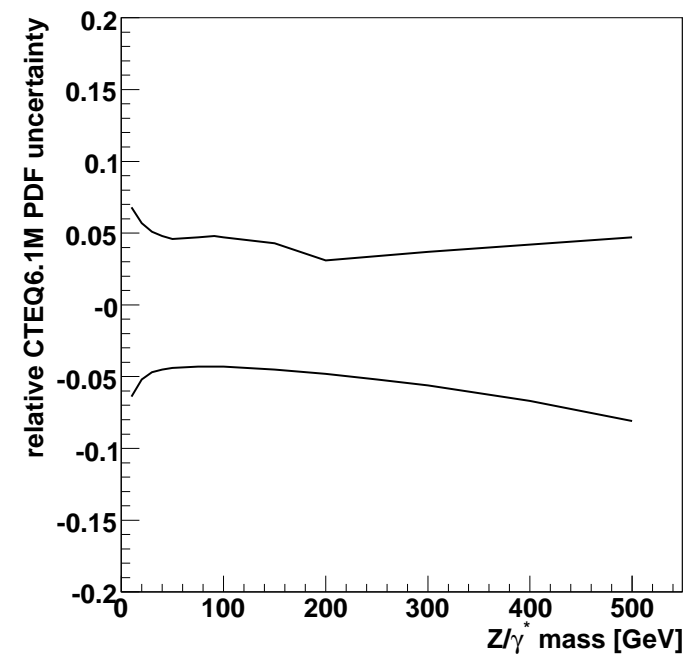

(a)

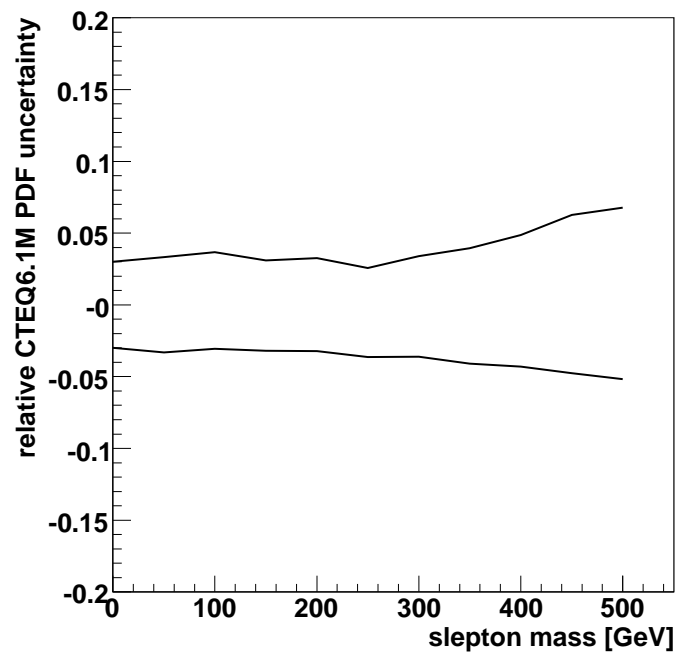

(b)

Figure 6.17: Uncertainty on the (N)NLO production cross section arising from the parton density function uncertainties for (a) the $Z / \gamma^{*}$ production in dependence of $\mathrm{m}\left(Z / \gamma^{*}\right)$ and (b) the resonant slepton production in dependence of the slepton mass $\mathrm{m}(\tilde{l})$. The plot data is from [147].

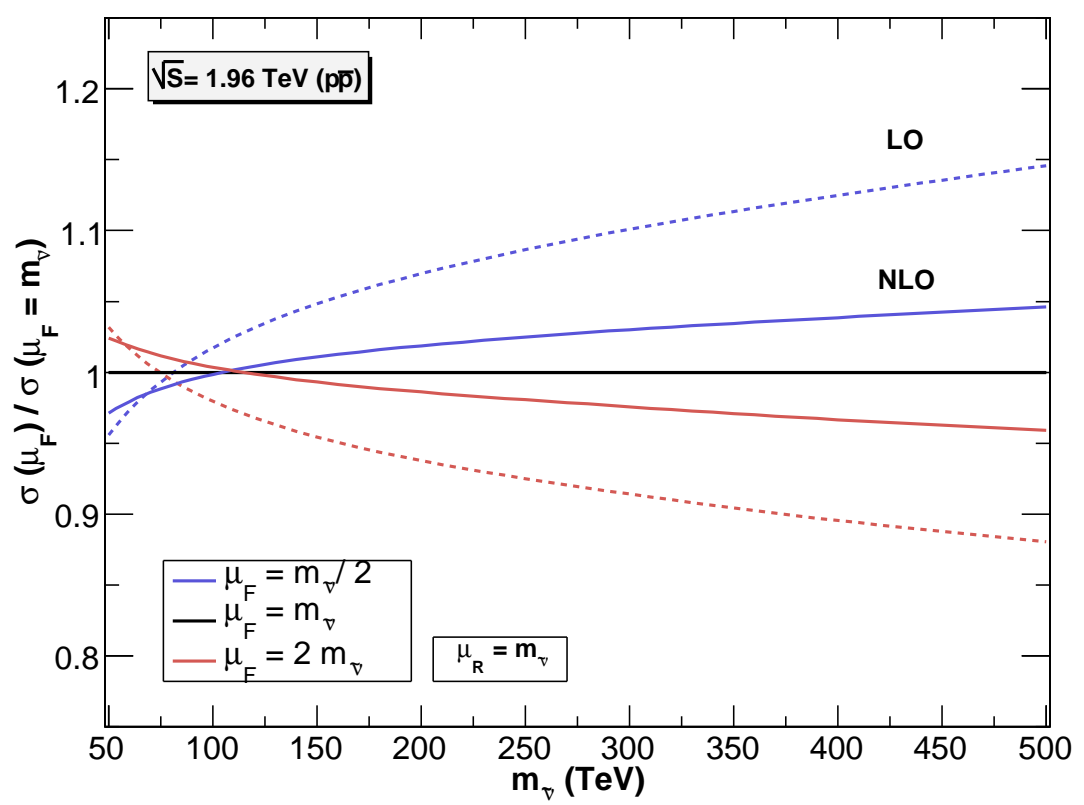

Figure 6.18: The total systematic uncertainty to the NLO resonant slepton cross section due to the factorization and renormalization scale uncertainties is less than $5 \%$ in the complete range of studied slepton masses [51]. 
- The statistical error $\delta w_{i}^{\text {stat }}$ of a specific Monte Carlo sample " $i$ " with an average event weight $w_{i}=\sigma_{i} \cdot \mathcal{L} / N_{g e n}^{i}$ is calculated with the unweighted number of selected events:

$$
\delta w_{i}^{\text {stat }}=\sqrt{s_{\text {selected }}} \cdot w_{i} .
$$

The statistical errors of all background samples are added in quadrature, as they are independent;

- The error of the muon momentum smearing, see Sec. 5.1.6 for details, is handled in a similar way as the JES uncertainty.

A summary of the systematic errors can be found in Tab. 6.11 with their contribution to the total Standard Model background and to the resonant slepton signal in the $2 \mu$-2jet sample.

\begin{tabular}{lcc}
\hline \hline uncertainty & tot. syst. error backgd. & tot. syst. error signal \\
\hline jet energy scale & $13.7 \%$ & $1.0-26 \%$ \\
Muon (reco,iso,track,med.) & $7.8 \%$ & $7.8 \%$ \\
Luminosity & $5.5 \%$ & $6.5 \%$ \\
$2 \mu$ trigger & $5.2 \%$ & $4.2-9.1 \%$ \\
MC cross-section \& k-factor & $3.7 \%$ & $5 \%$ \\
QCD & $3.1 \%$ & - \\
MC statistics & $2.2 \%$ & $2.7-33 \%$ \\
muon $p_{T}$ smearing & $0.1 \%$ & $0.0-14 \%$ \\
\hline \hline
\end{tabular}

Table 6.11: Effect of the systematic uncertainties relative to the $2 \mu-2$ jet sample on background and signal. The systematic errors on the signal depend heavily on the neutralino mass and therefore on the SUSY parameter point under study, so a typical error range is given.

The systematic uncertainties on the background and on the signal are handled in different ways: All background error sources are uncorrelated and are summed quadratically, except for the muon ID efficiencies, which are correlated between both muons.

The systematic error sources which influence the signal cross section, i.e. the PDF and the scale uncertainties, are separated from the other signal uncertainty contributions, and are not considered for the total signal uncertainty. Instead, the signal cross section is diminished by the total quadratical sum of the cross section affecting systematical uncertainties. This conservative procedure is the recommended approach by the $\mathrm{D} \varnothing$ adhoc committee on limit-setting procedures [175]. In this context it is clear, that the signal PDF and scale errors are not considered for the calculation of cross section limits, as the cross section limit does not depend on the signal's cross section. 


\subsubsection{The Final Sample}

The event selection cuts vary with the signal point under study and so does the cut flow. A detailed list with the final results for all points and all three signal channels is given in App. C.1-C.3. For the reference point with $\mathrm{m}(\tilde{l})=260 \mathrm{GeV}$ and $\mathrm{m}\left(\tilde{\chi}_{1}^{0}\right)=100 \mathrm{GeV}$ the final resulting numbers of events in the three signal channels are given in Tab. 6.12.

\begin{tabular}{llrrr|cc}
\hline \hline & channel & $n_{\text {data }}$ & \multicolumn{1}{c}{$\mathrm{b}$} & signal $\epsilon$ & $\sigma[\mathrm{pb}]$ & 95\% CL limit [pb] \\
\hline (i) & $\tilde{\mu} \rightarrow \tilde{\chi}_{1}^{0} \mu$ & 14 & $10.0 \pm 1.9_{-1.6}^{+1.2}$ & $3.0 \pm 0.4 \%$ & 1.10 & 1.53 \\
(ii) & $\tilde{\mu} \rightarrow \tilde{\chi}_{2,(3,4)}^{0} \mu$ & 28 & $24.6 \pm 3.2_{-4.0}^{+7.2}$ & $2.3 \pm 0.4 \%$ & 0.98 & 3.06 \\
(iii) & $\tilde{\nu}_{\mu} \rightarrow \tilde{\chi}_{1,(2)}^{ \pm} \mu$ & 8 & $8.3 \pm 1.9_{-2.4}^{+1.0}$ & $2.6 \pm 0.3 \%$ & 1.74 & 2.24 \\
\hline \hline
\end{tabular}

Table 6.12: Selected number of data events $n_{\text {data }}$ and $b$ expected Standard Model events. The signal efficiency is given for the reference point with $\mathrm{m}(\tilde{l})=260 \mathrm{GeV}$ and $\mathrm{m}\left(\tilde{\chi}_{1}^{0}\right)=100 \mathrm{GeV}$ with respect to the specific decay channel. The cross section has been calculated within mSUGRA using a coupling strength of $\lambda_{211}^{\prime}=0.07$. The cross section limit has been calculated using the $\mathrm{CL}_{s}$ method, which is discussed in more detail in Sec. 7.1.

These number of selected events for the reference point are typical for all studied SUSY points. The final sample for all three signal channels for this point is shown in Fig. 6.19.

The deviation $\sigma$ of data and Standard Model expectation after the final selection is calculated for all SUSY points (Fig. 6.20) and for all three signal channels:

$$
\sigma=\frac{n_{d a t a}-b}{\sqrt{\sigma_{b}^{2}+b}} .
$$

Where $n_{d a t a}$ is the number of data events and $\sigma_{b}$ the total error of the Standard-Model expectation $b$. The deviation $\sigma$ is not completely independent with respect to different points in the studied SUSY parameter space, since the underlying data and background event samples are the same for each point, only the final selection cuts differ.

The mean values of Fig. 6.20 are indicative for the general agreement between data and Standard Model expectation on the $2 \mu-2$ jet level, before a specific final selection is applied. The width of the distributions is the result of 117 more or less independent selections. The deviations between data and background are in good agreement with the statistical expectations.

Overall, no significant excess in the data has been observed, the selected data events agree within uncertainties with the Standard Model prediction. Since no sign for resonant slepton production was found limits on this process were calculated and will be discussed in Sec. 7.2. 


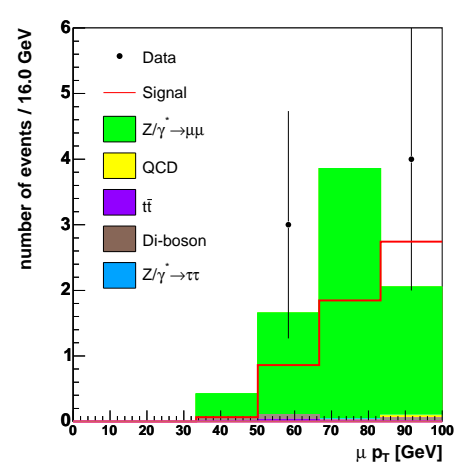

(a)

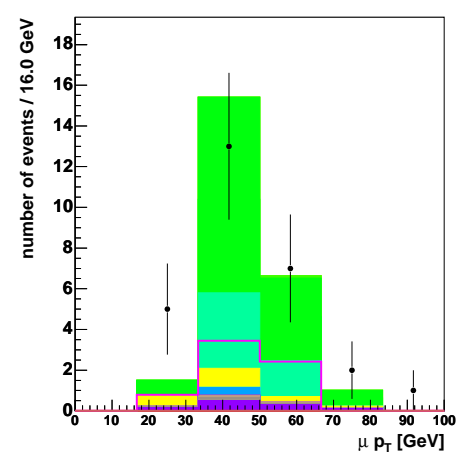

(d)

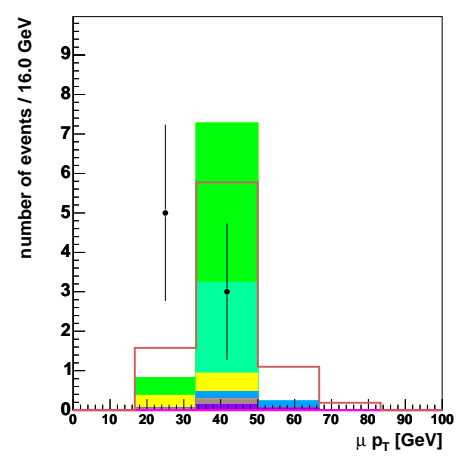

(g)

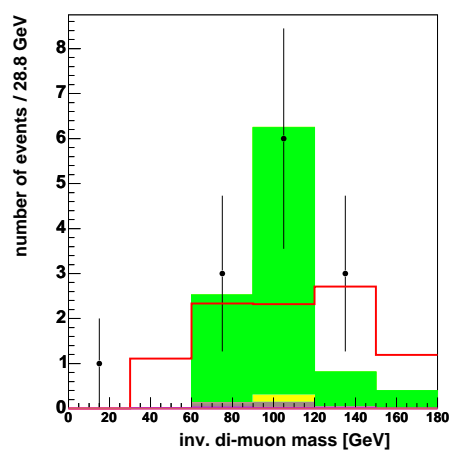

(b)

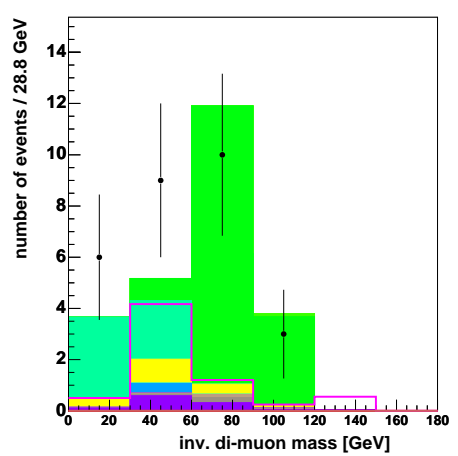

(e)

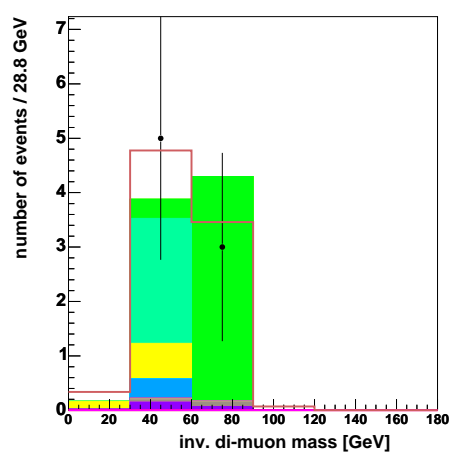

(h)

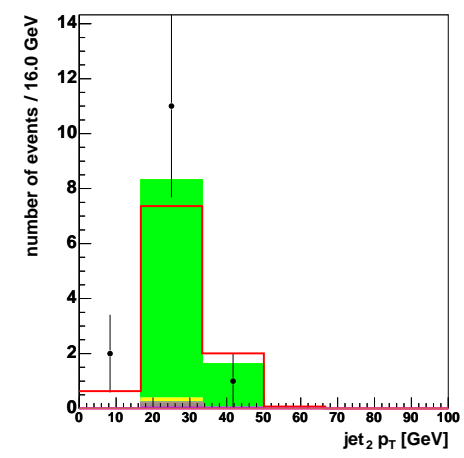

(c)

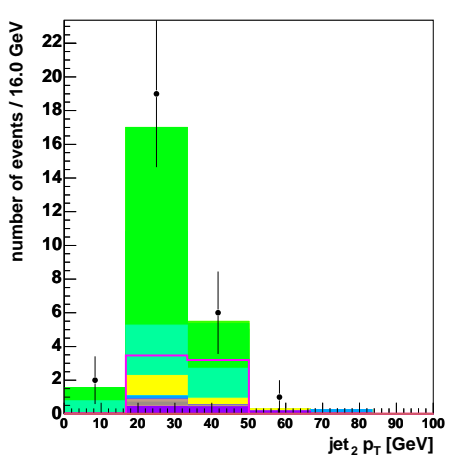

(f)

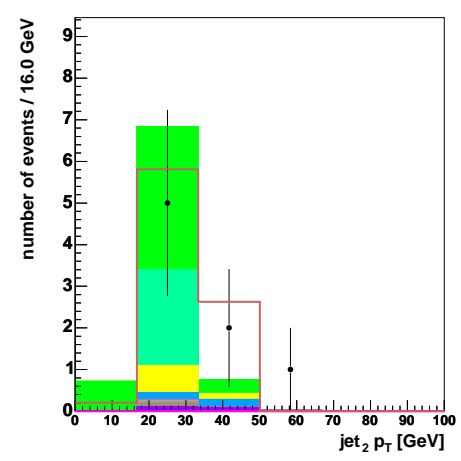

(i)

Figure 6.19: Final selected sample found for the point with $\mathrm{m}(\tilde{\mu})=260 \mathrm{GeV}$ and $\mathrm{m}\left(\tilde{\chi}_{1}^{0}\right)=$ $100 \mathrm{GeV}$ for channel $\tilde{\mu} \rightarrow \tilde{\chi}_{1}^{0} \mu$ (a)-(c), $\tilde{\mu} \rightarrow \tilde{\chi}_{2,(3,4)}^{0} \mu(\mathrm{d})$-(f) and $\tilde{\nu}_{\mu} \rightarrow \tilde{\chi}_{1,(2)}^{ \pm} \mu(\mathrm{g})$-(i) in the variables $p_{T}\left(\mu_{1}\right)$ (left), di- $\mu$ mass (middle) and $p_{T}\left(\right.$ jet $\left.t_{2}\right)$ (right). 


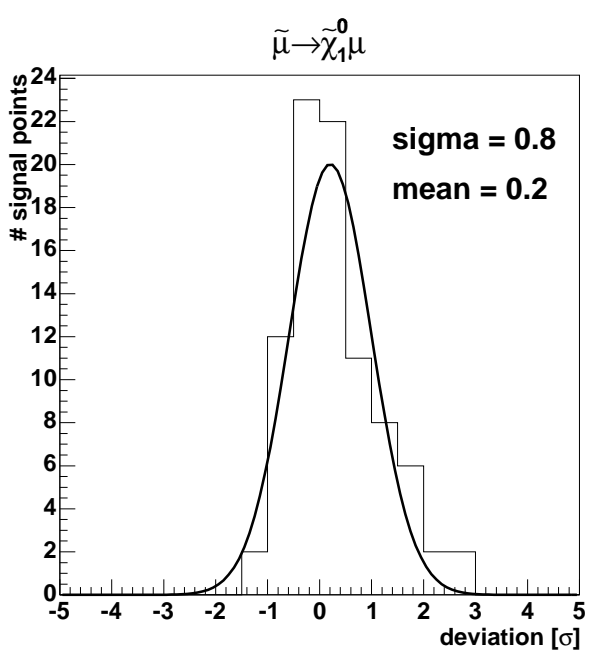

(a)

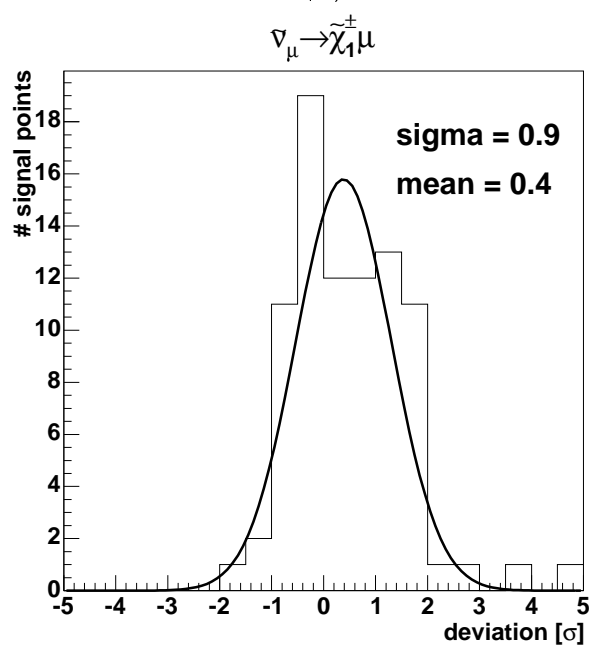

(c)

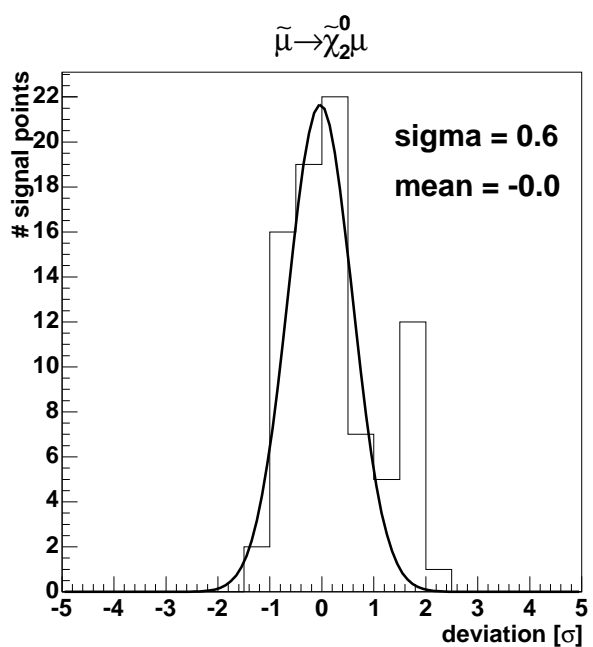

(b)

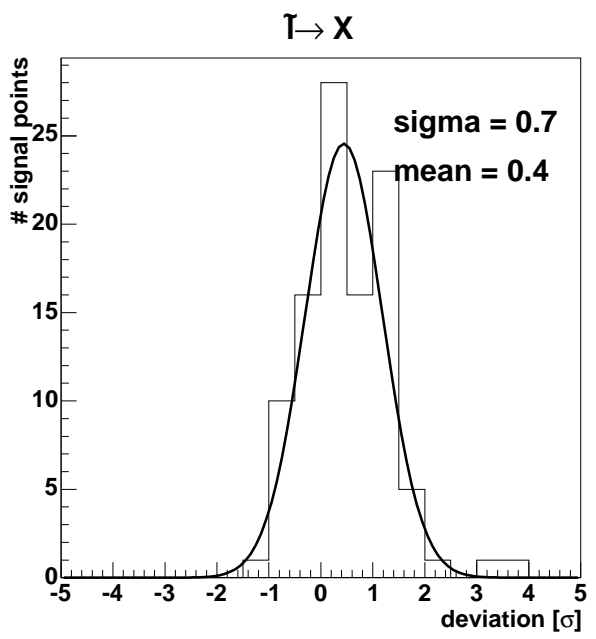

(d)

Figure 6.20: Deviation of data and Standard Model expectation after the final selection for all studied SUSY points for (a) channel $\tilde{\mu} \rightarrow \tilde{\chi}_{1}^{0} \mu$, (b) channel $\tilde{\mu} \rightarrow \tilde{\chi}_{2,(3,4)}^{0} \mu,(\mathrm{c})$ channel $\tilde{\nu}_{\mu} \rightarrow \tilde{\chi}_{1,(2)}^{ \pm} \mu$ and $(\mathrm{d})$ the combination of all three.

The points with a deviation larger than 3.0 in (c) are \#0 and \#12 and in (d) \#94 and \#109, see App. C for details.

\subsubsection{Candidate events}

In Fig. 6.21 and in Fig. 6.22 two data events are displayed. These events were found in the data by the analysis optimized for channel $\tilde{\mu} \rightarrow \tilde{\chi}_{1}^{0} \mu$ with $m(\tilde{l})=260 \mathrm{GeV}$ and $m\left(\tilde{\chi}_{1}^{0}\right)=100 \mathrm{GeV}$. The events are compatible with the resonant slepton production signal process but also with the main background process, Drell-Yan production with additional partons from initial or final state radiation $Z / \gamma^{*} q q \rightarrow \mu^{+} \mu^{-} q q$. 


\begin{tabular}{|c|c|c|}
\hline \multirow{2}{*}{\multicolumn{2}{|c|}{$\begin{array}{l}\text { event number } \\
\text { run number }\end{array}$}} & 6545223 \\
\hline & & 179895 \\
\hline \multirow[t]{3}{*}{$1^{\text {st }}$ muon } & $\overline{p_{T}}$ & $=113 \mathrm{GeV}$ \\
\hline & $\phi$ & $=5.7 \mathrm{rad}$ \\
\hline & $\eta$ & $=\quad-0.3$ \\
\hline \multirow[t]{3}{*}{$2^{\text {nd }}$ muon } & $p_{T}$ & $=18 \mathrm{GeV}$ \\
\hline & $\phi$ & $=1.8 \mathrm{rad}$ \\
\hline & $\eta$ & $=\quad 0.0$ \\
\hline \multirow[t]{3}{*}{$1^{s t}$ jet } & $p_{T}$ & $=78 \mathrm{GeV}$ \\
\hline & $\phi$ & $=2.7 \mathrm{rad}$ \\
\hline & $\eta$ & -1.0 \\
\hline \multirow[t]{3}{*}{$2^{n d}$ jet } & $p_{T}$ & $=35 \mathrm{GeV}$ \\
\hline & $\phi$ & $=2.7 \mathrm{rad}$ \\
\hline & $\eta$ & $=\quad 0.0$ \\
\hline \multicolumn{2}{|c|}{ missing $E_{T}$} & $13 \mathrm{GeV}$ \\
\hline \multicolumn{2}{|c|}{ di-muon mass } & $84 \mathrm{GeV}$ \\
\hline \multicolumn{2}{|c|}{ di-jet mass } & $57 \mathrm{GeV}$ \\
\hline \multicolumn{2}{|c|}{$\mathrm{m}\left(\mu_{2}\right.$, jet $_{1}$, jet $\left._{2}\right)$} & $75 \mathrm{GeV}$ \\
\hline \multicolumn{2}{|c|}{$\mathrm{m}\left(\mu_{1}, \mu_{2}\right.$, jet $_{1}$, jet $\left._{2}\right)$} & ) $262 \mathrm{GeV}$ \\
\hline
\end{tabular}
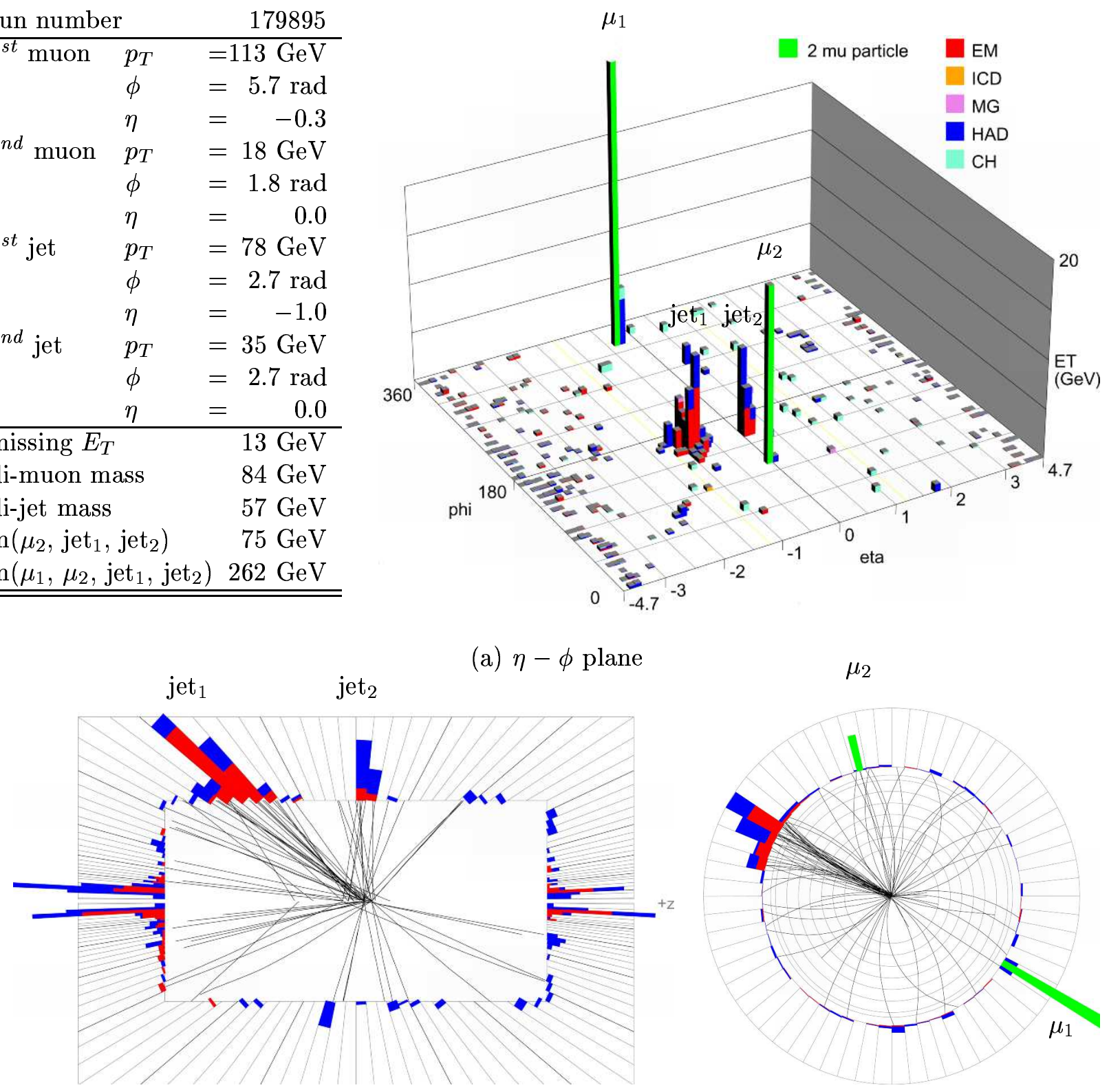

(b) $\eta-r$ cross section

(c) $\phi-r$ cross section

Figure 6.21: Event 6545223 is a candidate event found in the data by the analysis optimized for channel $\tilde{\mu} \rightarrow \tilde{\chi}_{1}^{0} \mu$ with $m(\tilde{l})=260 \mathrm{GeV}$ and $m\left(\tilde{\chi}_{1}^{0}\right)=100 \mathrm{GeV}$. The reconstructed 3-body mass of $2^{n d}$ muon and both jets (4-body mass of both muons and both jets) matches the neutralino (slepton) mass, as required from the analysis. However, the event's invariant di-muon mass is also compliant with the major background process $Z q q \rightarrow \mu \mu q q$. 


\begin{tabular}{|c|c|c|}
\hline \multirow{2}{*}{\multicolumn{2}{|c|}{$\begin{array}{l}\text { event number } \\
\text { run number }\end{array}$}} & \multirow{2}{*}{$\begin{array}{r}30837455 \\
191329\end{array}$} \\
\hline & & \\
\hline \multirow[t]{3}{*}{$1^{\text {st }}$ muon } & $p_{T}$ & $=88 \mathrm{GeV}$ \\
\hline & $\phi$ & $=5.7 \mathrm{rad}$ \\
\hline & $\eta$ & 0.2 \\
\hline \multirow[t]{3}{*}{$2^{\text {nd }}$ muon } & $p_{T}$ & $=34 \mathrm{GeV}$ \\
\hline & $\phi$ & $1.2 \mathrm{rad}$ \\
\hline & $\eta$ & -0.6 \\
\hline \multirow[t]{3}{*}{$1^{s t}$ jet } & $p_{T}$ & $=43 \mathrm{GeV}$ \\
\hline & $\phi$ & $3.3 \mathrm{rad}$ \\
\hline & $\eta$ & -0.8 \\
\hline \multirow[t]{3}{*}{$2^{\text {nd }}$ jet } & $p_{T}$ & $21 \mathrm{GeV}$ \\
\hline & $\phi$ & $2.8 \mathrm{rad}$ \\
\hline & $\eta$ & -1.7 \\
\hline \multicolumn{2}{|c|}{ missing $E_{T}$} & $23 \mathrm{GeV}$ \\
\hline \multicolumn{2}{|c|}{ di-muon mass } & $96 \mathrm{GeV}$ \\
\hline \multicolumn{2}{|c|}{ di-jet mass } & $33 \mathrm{GeV}$ \\
\hline \multicolumn{2}{|c|}{$\mathrm{m}\left(\mu_{2}\right.$, jet $_{1}$, jet $\left._{2}\right)$} & $86 \mathrm{GeV}$ \\
\hline \multicolumn{2}{|c|}{$\mathrm{m}\left(\mu_{1}, \mu_{2}\right.$, jet $_{1}$, jet $\left._{2}\right)$} & $225 \mathrm{GeV}$ \\
\hline
\end{tabular}

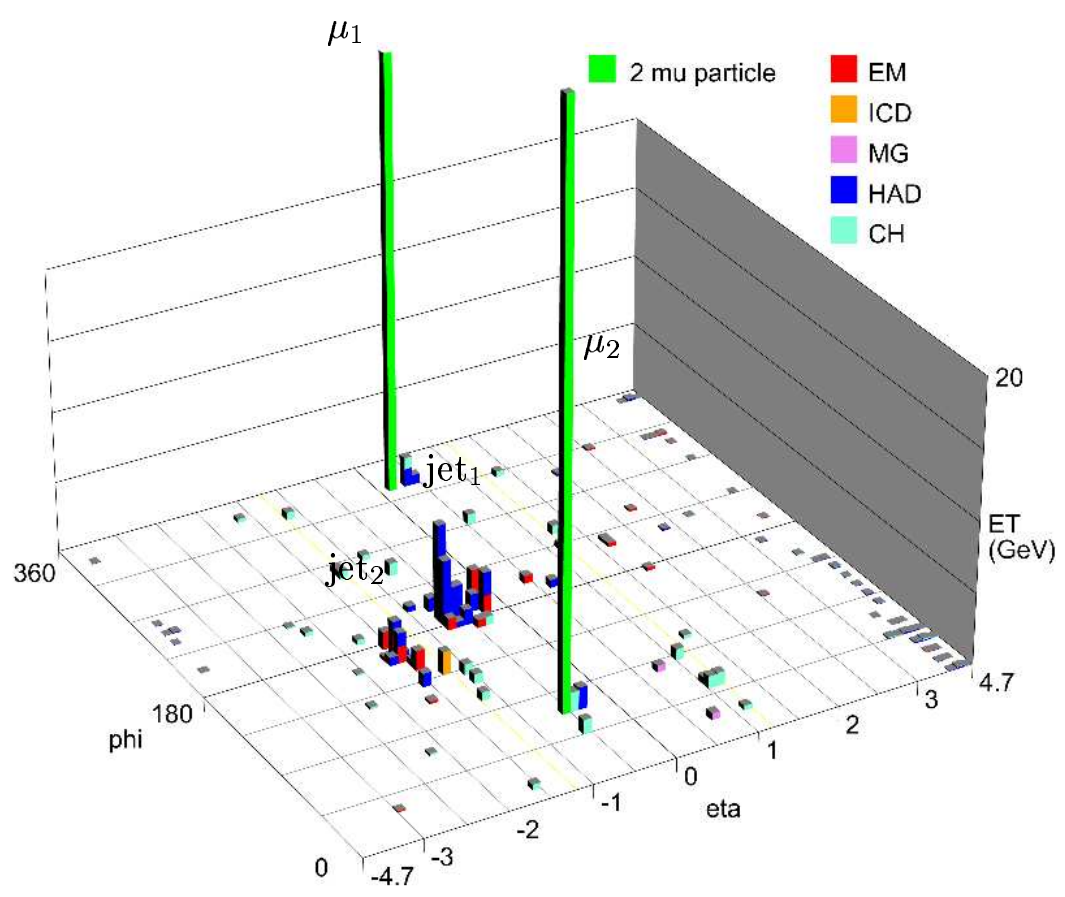

(a) $\eta-\phi$ plane

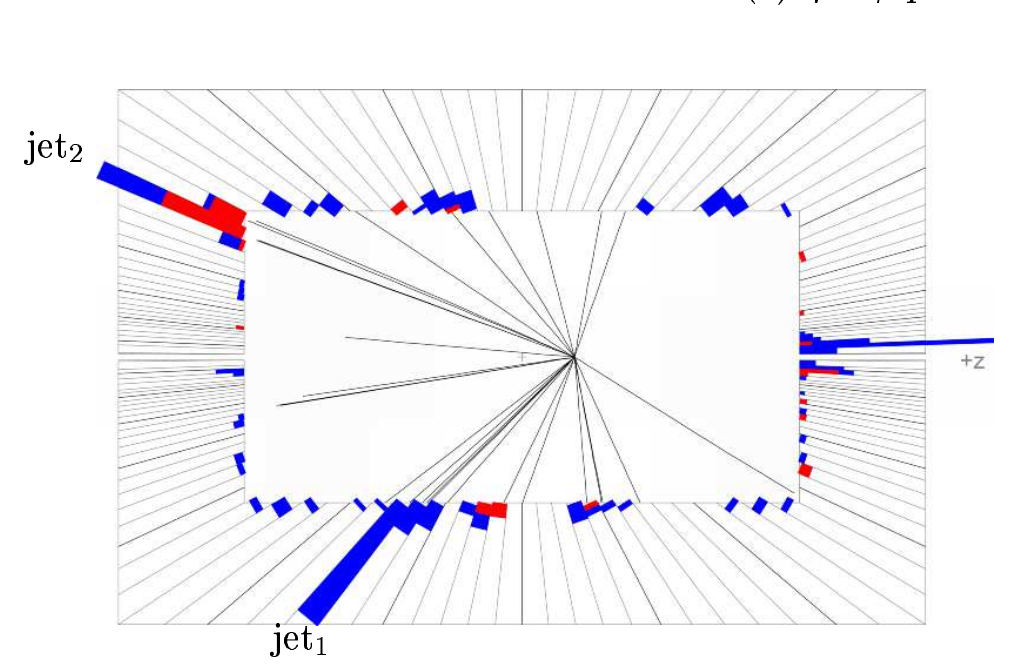

(b) $\eta-r$ cross section

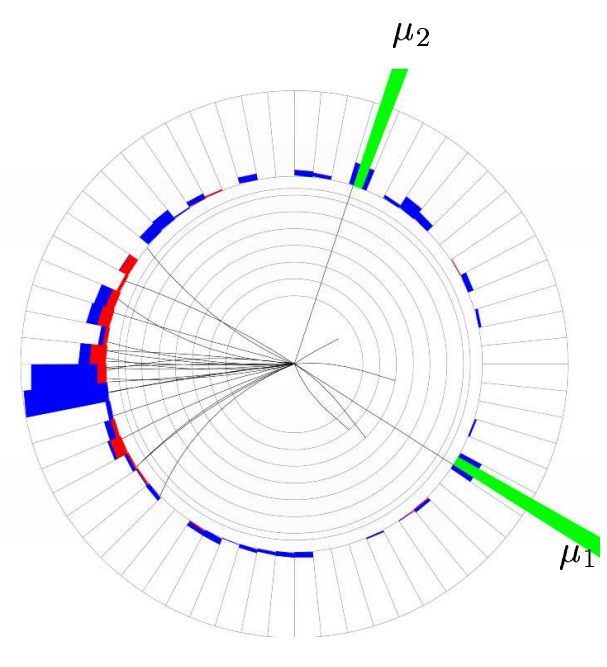

(c) $\phi-r$ cross section

Figure 6.22: Event 30837455 is another candidate event found in the data by the analysis optimized for channel $\tilde{\mu} \rightarrow \tilde{\chi}_{1}^{0} \mu$ with $m(\tilde{l})=260 \mathrm{GeV}$ and $m\left(\tilde{\chi}_{1}^{0}\right)=100 \mathrm{GeV}$. The reconstructed 3 -body mass of $2^{\text {nd }}$ muon and both jets (4-body mass of both muons and both jets) matches the neutralino (slepton) mass, as required from the analysis. However, the event's invariant di-muon mass is also compliant with the major background process $Z q q \rightarrow \mu \mu q q$. 


\subsection{Search for Gaugino Pair and Associated Production}

Using the same $2 \mu$ preselection sample as for the search for the resonant production of sleptons, it is possible to search for the $R$-parity conserving pair and associated production of supersymmetric particles, that decay finally via some non-zero R-parity violating coupling into final states with two muons. Recently, a search for this topology via a $L L \bar{e}$ coupling $\lambda_{122}$ or $\lambda_{121}$ in final states with two muons and a third electron or muon has been published [91]. This analysis concentrates therefore on $L Q \bar{d}$ couplings $\lambda_{2 j k}^{\prime} \geq 0.01$, with $j=1,2$ and $k=1,2,3$, resulting in $2 \mu$ final states with jets.

\subsubsection{Signal Monte Carlo Studies}

In the parameter region to which this analysis is sensitive to $\left(m_{1 / 2} \lesssim 150 \mathrm{GeV}\right)$, the event kinematics are determined solely by the gaugino masses. Production and decays involving squarks, gluinos, or sleptons are negligible due to their large masses compared to all gaugino masses, except for very low values of $m_{0}$ and $m_{1 / 2}$ (Fig. 2.7). Contrary to resonant slepton production, here both muons originate from neutralino $\tilde{\chi}_{1}^{0}$ decays; the muon momenta and therefore their reconstruction efficiency depends on $\mathrm{m}\left(\tilde{\chi}_{1}^{0}\right)$. Detailed studies concerning the pair and associated production of gauginos can be found in [176].

The obtained results are valid for all $\lambda_{2 j k}^{\prime} \geq 0.01$, with $j=1,2$ and $k=1,2,3$, since the event kinematics, as well as the sensitivity of the analysis do not depend on the quark flavors. The size of the $\mathbb{R}_{p}$ coupling $\lambda_{2 j k}^{\prime}$ determines in this case of R-parity conserving gaugino pair and associated production the neutralino lifetime only. The neutralino decay length is negligible for all couplings larger than 0.01 .

The point which is used throughout this thesis as a reference point for pair and associated gaugino production is created with high statistics (24.5k events) for an exemplary parameter set as listed in Tab. 6.2. This point (number 11) is close to the expected limit on the neutralino $\tilde{\chi}_{1}^{0}$ mass for an intermediate value of $m_{0}$.

\begin{tabular}{lr|lr}
\hline \hline mSUGRA & & masses & \\
\hline$m_{0}$ & $200 \mathrm{GeV}$ & $\mathrm{m}(\tilde{\mu})$ & $217.0 \mathrm{GeV}$ \\
$m_{1 / 2}$ & $100 \mathrm{GeV}$ & $\mathrm{m}\left(\tilde{d}_{R}\right)$ & $252.6 \mathrm{GeV}$ \\
$\tan (\beta)$ & 5 & $\mathrm{~m}\left(\tilde{\chi}_{1}^{0}\right)$ & $39.6 \mathrm{GeV}$ \\
$\operatorname{sign}(\mu)$ & -1 & $\mathrm{~m}\left(\tilde{\chi}_{2}^{0}\right)$ & $74.2 \mathrm{GeV}$ \\
$A_{0}$ & $0 \mathrm{GeV}$ & $\mathrm{m}\left(\tilde{\chi}_{1}^{ \pm}\right)$ & $75.8 \mathrm{GeV}$ \\
\hline$\lambda_{222}^{\prime}$ & 0.01 & $\sigma_{\mathrm{LO}}$ & $6.86 \mathrm{pb}$ \\
\hline \hline
\end{tabular}

Table 6.13: The signal reference point for gaugino pair and associated production.

In Tab. 6.14 the cutflow from the signal generator level down to the final selection (which will be discussed in detail in Sec. 6.3.2) is given. The cut efficiency is given for the reference point of Tab. 6.13 sequentially, if the cut would have been applied as first cut, and if the cut would have been applied as last cut. The signal selection efficiency depends 
on the parameter $m_{1 / 2}$ which defines the neutralino mass and does not include detector acceptance corrections (i.e. the trigger efficiency parameterization).

\begin{tabular}{|c|c|c|c|}
\hline Cut & sequentially & if applied as $1^{\text {st }}$ cut & if applied as last cut \\
\hline medium quality $\mu_{1}$ & $44.1 \%$ & $44.1 \%$ & $80.2 \%$ \\
\hline$p_{T}\left(\mu_{1}\right)>15 \mathrm{GeV}$ & $24.6 \%$ & $24.6 \%$ & $68.6 \%$ \\
\hline isolation $\mu_{1}$ & $24.6 \%$ & $44.1 \%$ & $100.0 \%$ \\
\hline central track $\mu_{1}$ & $8.9 \%$ & $15.9 \%$ & $88.0 \%$ \\
\hline medium quality $\mu_{2}$ & $1.9 \%$ & $7.1 \%$ & $84.4 \%$ \\
\hline$p_{T}\left(\mu_{2}\right)>8 \mathrm{GeV}$ & $1.9 \%$ & $7.1 \%$ & $71.7 \%$ \\
\hline isolation $\mu_{2}$ & $1.9 \%$ & $7.1 \%$ & $46.8 \%$ \\
\hline central track $\mu_{2}$ & $0.8 \%$ & $2.1 \%$ & $95.3 \%$ \\
\hline$\Delta \phi\left(\mu_{1}, \mu_{2}\right)<2.9$ & $0.7 \%$ & $95.5 \%$ & $84.4 \%$ \\
\hline $\operatorname{sign}\left(\mu_{1}\right)=\operatorname{sign}\left(\mu_{2}\right)$ & $0.3 \%$ & $58.9 \%$ & $42.0 \%$ \\
\hline$p_{T}\left(\right.$ jet $\left._{1}\right)>8 \mathrm{GeV}$ & $0.3 \%$ & $94.5 \%$ & $96.4 \%$ \\
\hline sphericity $S<0.98$ & $0.3 \%$ & $6.1 \%$ & $100.0 \%$ \\
\hline aplanarity $A>0.02$ & $0.2 \%$ & $5.8 \%$ & $45.5 \%$ \\
\hline
\end{tabular}

Table 6.14: Signal cut flow for point 11 with $\mathrm{m}\left(\tilde{\chi}_{1}^{0}\right)=39.6 \mathrm{GeV}$ from generator level to the final sample. The given numbers refer to the fraction of events left, after a specific cut has been applied. The first column gives the cut flow in top-down order. The second column is the survival rate if the cut is applied as first cut, and likewise the third column gives the relative cut efficiency if the cut is applied last. In total 24500 events were generated for this mSUGRA parameter combination, in $74.4 \%$ of all events at least one neutralino decays into a muon $\tilde{\chi}_{1}^{0} \rightarrow \mu q \bar{q}^{\prime}$ and in 5867 events or $24.2 \%$ both neutralinos decay to muons and quarks. The percentage of events given in this table correspond to the total number of events (24500), since also muons from the cascade to $\tilde{\chi}_{1}^{0}$ contribute. The trigger efficiency parametrization, which affects the signal acceptance is not applied here. Note, that e.g. a cut on $p_{T}\left(\mu_{2}\right)$ implies, that a muon $\mu_{1}$ with at least this energy exists.

\subsubsection{Event Selection}

The pair and associated production of gauginos, followed by a cascade decay into two neutralinos and their decay via any $L Q \bar{d}$ coupling $\lambda_{2 j k}^{\prime}$ with $j=1,2$ and $k=1,2,3$ can result in a final state including two muons, as discussed in Sec. 2.3.2. It is assumed, that only one $\lambda_{2 j k}^{\prime}$ coupling is non-zero, however, the specific kind of coupling is unimportant for the analysis, as the coupling determines only the flavor of the quarks from the R-parity violating neutralino decay. All flavors, except for top which would be $j=3$, are possible. The analysis is insensitive to this quark or jet flavor. The event kinematics are dominated by the involved particle masses, i.e. the neutralino mass, as long as the $L Q \bar{d}$ couplings are not too small $\lambda_{2 i j}^{\prime} \gtrsim 0.01$.

This analysis differs from the previous search, as here no resonance exists to separate signal and background by cutting on reconstructed masses. To obtain a reasonable signal over square-root of background value (this is equivalent to a good signal efficiency times signal purity) a like-sign muon selection is chosen. This has implications on the multijet QCD sample estimation, as the QCD sample used previously was extracted from the data under 


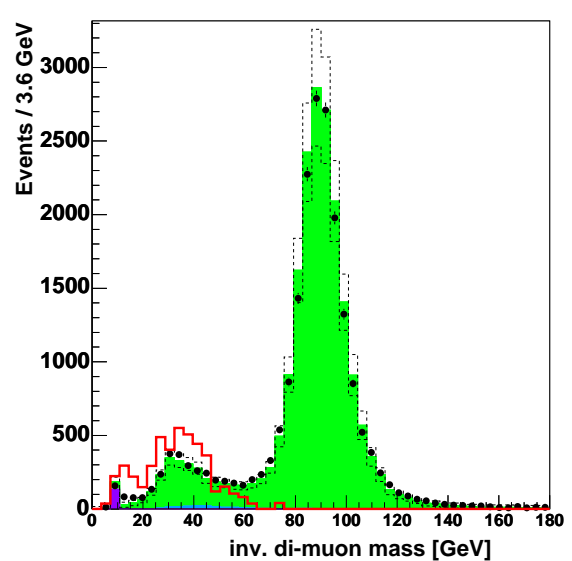

(a)

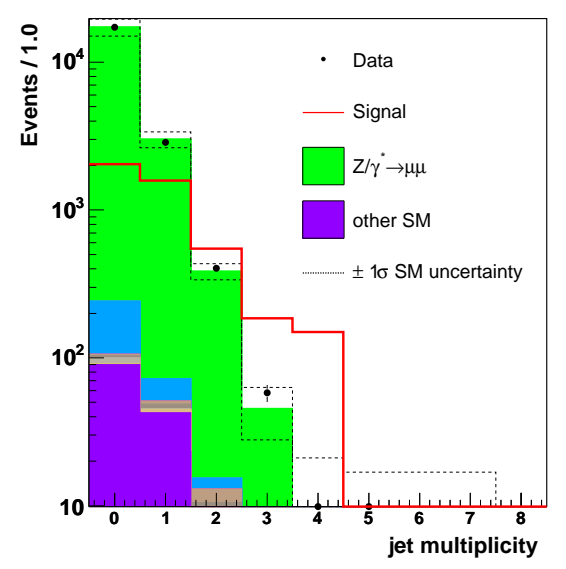

(c)

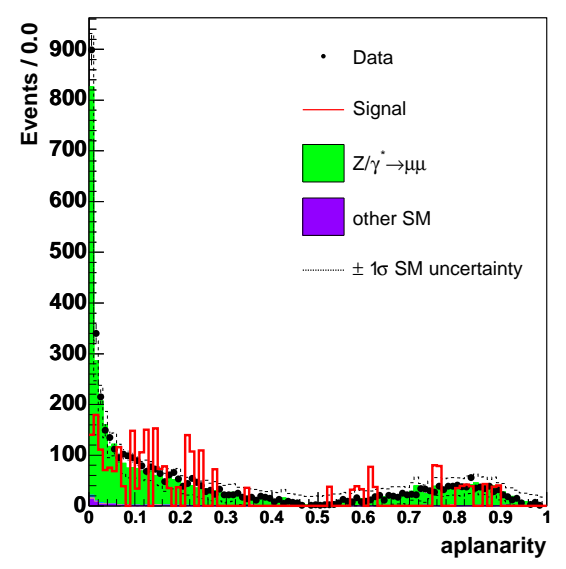

(e)

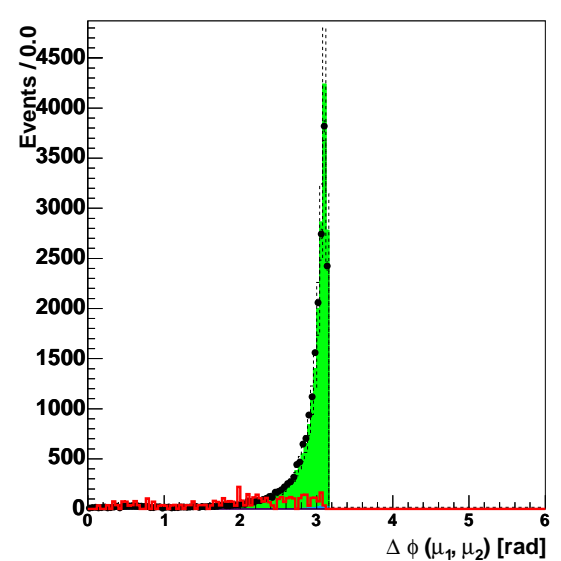

(b)

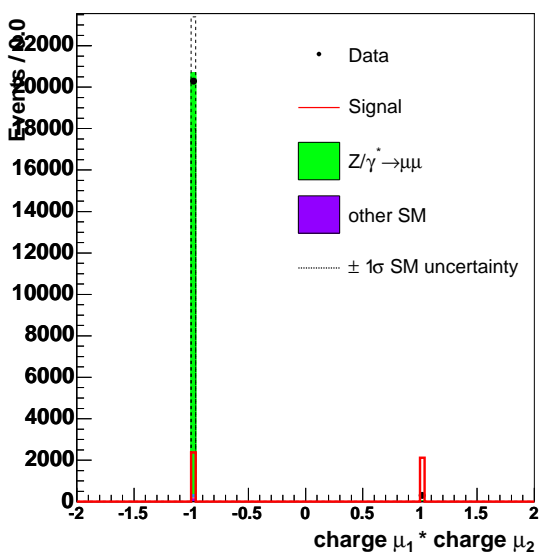

(d)

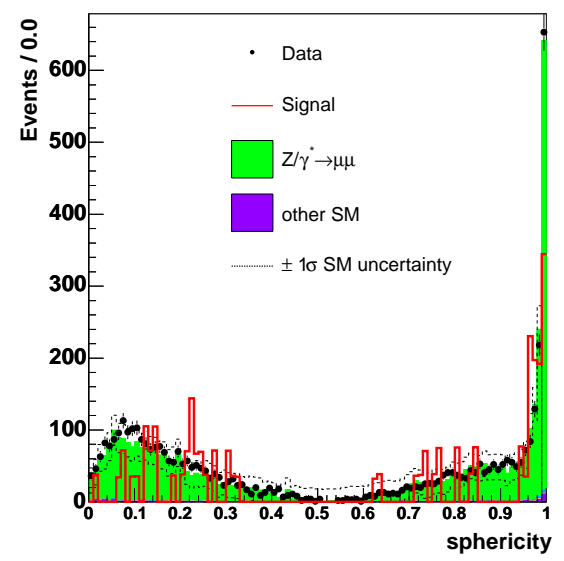

(f)

Figure 6.23: Tight isolation di- $\mu$-selection. The signal (Gaugino pair and associated production) with $\mathrm{m}\left(\tilde{\chi}_{1}^{0}\right)=40 \mathrm{GeV}$ is scaled by a factor of 100 . The total Standard Model background uncertainty is shown as dashed black lines. 
the assumption of dominant $b \bar{b}$-contribution, see Sec. 4.2 for details. This assumption is no longer valid. A like-sign muon QCD sample extraction from the data is prevented by the small statistics of any like-sign sample. Consequently, hard muon $p_{T}$ dependent isolation cuts as discussed in Sec. 4.2.3 were applied to remove the QCD like events from the data. Conservatively, the QCD expectation is neglected in the following. This selection with tightend muon isolation criteria and without a QCD background sample is shown in Fig. 6.23. A good agreement in all distributions can be observed. In total 20591 tight isolated $2 \mu$ data events have been selected, while the Standard Model expectation yields $20720 \pm 70 \pm 2760$ events.

The dominant background process is $Z / \gamma^{*} \rightarrow \mu^{+} \mu^{-}$production including additional jets. Because of the large $Z$-mass the two muons tend to be back-to-back in the $x-y$ plane. The muon's $\phi$ coordinate is measured with high precision independent of the transverse momentum, so that the cut $\Delta \phi<2.9$ removes 7 out of 10 Drell-Yan events. The signal distribution in $\Delta \phi$ is flat, as shown in Fig. 6.23(b).

The sphericity $S$ of an event is essentially a measure of the summed $p_{T}^{2}$ with respect to the event axis [135]. In a two-jet event the event axis is defined by both jets and no energy is perpendicular to this axis, so the sphericity is 0 . Contrariwise, $S=1$ for an isotropic event follows. The aplanarity measures the transverse momentum component out of the event plane. A planar event has $A=0$ and an isotropic event has $A=1$. The sphericity tensor $S^{\alpha \beta}$ is defined [177] as:

$$
S^{\alpha \beta}=\frac{\sum_{i} p_{i}^{\alpha} p_{i}^{\beta}}{\sum_{i}\left|p_{i}\right|^{2}}
$$

The sums extent over the two muons with largest transverse momentum and all, but at least one, jet(s) in the event. The $\alpha, \beta=1,2,3$ correspond to the $x, y, z$ components of the particle momenta. By standard diagonalization of $S^{\alpha \beta}$ one finds three eigenvalues $\lambda_{1} \leq \lambda_{2} \leq \lambda_{3}$, with $\lambda_{1}+\lambda_{2}+\lambda_{3}=1$. The sphericity $S$ and the aplanarity $A$ of the event is then defined as

$$
\begin{aligned}
S & =\frac{3}{2} \cdot\left(\lambda_{1}+\lambda_{2}\right), \\
A & =3 \cdot \lambda_{3},
\end{aligned}
$$

so that $0 \leq S \leq 1$ and $0 \leq A \leq 1$. In this analysis at least two muons and at least one jet are required to calculate $S^{\alpha \beta}$, so that implicitly one jet with at transverse momentum of at least $8 \mathrm{GeV}$ is required. The sphericity is required to be $S<0.98$ and the aplanarity has to be $A>0.02$ to remove isotropic background events, see Fig. 6.23(e),(f) for reference.

The cutflow of this analysis is identical for all studied points in the SUSY parameter space and summarized in Tab. 6.15. 


\begin{tabular}{lrrrr}
\hline \hline Cut & Data & SM Exp. \pm stat. & syst. & Signal Eff. $\delta \epsilon$ \\
\hline 2MU Preselection & 23206 & $22700 \pm 70$ & \pm 2900 & $1.021 \% \pm 0.138 \%$ \\
$p_{T}(\mu)$ dependent isolation & 20591 & $20720 \pm 70$ & \pm 2760 & $0.731 \% \pm 0.100 \%$ \\
$\Delta \phi\left(\mu_{1}, \mu_{2}\right)<2.9$ & 6163 & $5750 \pm 40$ & \pm 760 & $0.621 \% \pm 0.088 \%$ \\
like sign $\left(\mu_{1}, \mu_{2}\right)$ & 5 & $1.9 \pm 0.5$ & \pm 0.2 & $0.285 \% \pm 0.048 \%$ \\
jet $1>8 \mathrm{GeV}$ & 2 & $0.9 \pm 0.4$ & \pm 0.1 & $0.267 \% \pm 0.046 \%$ \\
Sphericity $S<0.98$ & 2 & $0.7 \pm 0.3$ & \pm 0.1 & $0.253 \% \pm 0.044 \%$ \\
Aplanarity $A>0.02$ & 1 & $0.2 \pm 0.2$ & \pm 0.005 & $0.220 \% \pm 0.040 \%$ \\
\hline \hline
\end{tabular}

Table 6.15: Cut flow of the gaugino pair and associated production analysis. The signal efficiency is given for the reference point with $\mathrm{m}\left(\tilde{\chi}_{1}^{0}\right)=39.6 \mathrm{GeV}$. In this analysis all selection cuts are independent of the SUSY parameter point.

\subsubsection{Systematic Uncertainties}

The systematic uncertainties, that were listed in detail in Sec. 6.2.3, are calculated in a similar way for this analysis, except for the QCD sample uncertainty which does not apply here. For the signal, a constant uncertainty of $5 \%$ is used, to account for PDF uncertainties affecting the cross section. This uncertainty is not considered for the calculation of cross section limits. The systematic uncertainties of signal and background are conservatively assumed to be fully correlated.

\subsubsection{The Final Sample}

After all cuts one candidate event was found in the data while $0.238 \pm 0.164$ (stat) \pm 0.032 (sys) Standard Model background events were expected. The Poisson probability that an expected measurement of 0.238 fluctuates to 1 is $19 \%$, without considering the uncertainty on this background expectation. This is no significant excess, so that the result can be interpreted as a limit with respect to the gaugino pair and associated production, as will be discussed in Sec. 7.3.

In total 24 points were simulated, the final results for each point are shown in Tab. 6.16. The background expectation and the candidate event found in the data are shown in Fig. 6.24 together with the signal expectation for the point 11 with $\mathrm{m}\left(\tilde{\chi}_{1}^{0}\right)=39.6 \mathrm{GeV}$.

\subsubsection{Candidate event}

The event shown in Fig. 6.25 is the only candidate event found by the analysis in the data for the signal process of pair and associated gaugino production. The event consists of two positive charged muons with not too high momenta and some hadronic activity, so that a mismeasured $Z / \gamma^{*} q \rightarrow \mu^{+} \mu^{-} q$ event is very unlikely. The event is compliant with the signal process, but it is also possible, that the event originates from di-boson production or from an QCD process. 


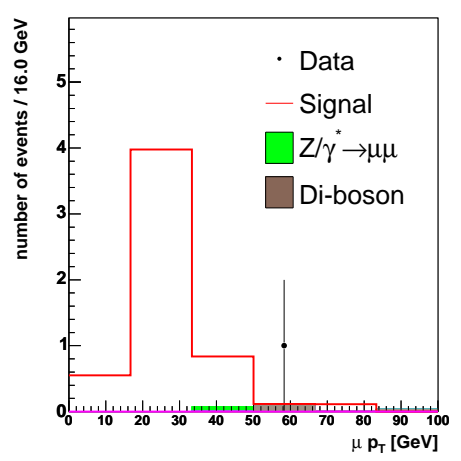

(a)

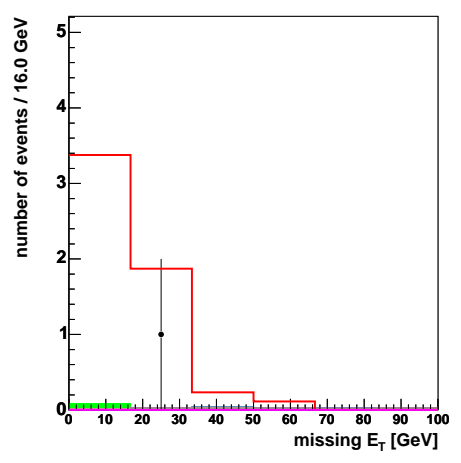

(d)

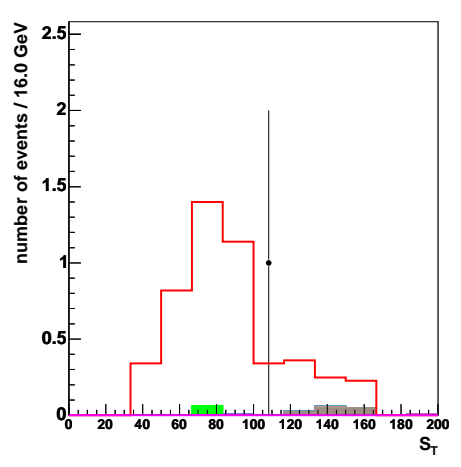

(g)

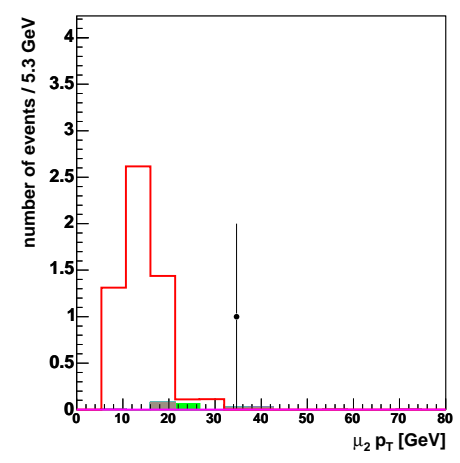

(b)

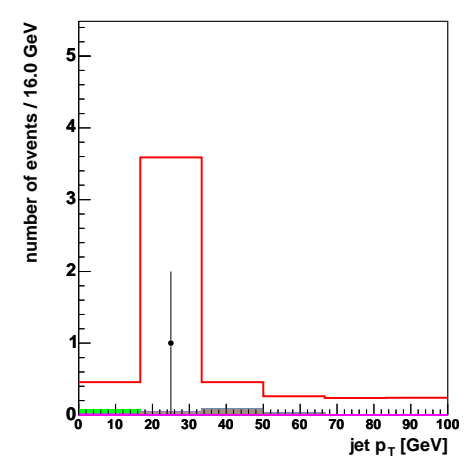

(e)

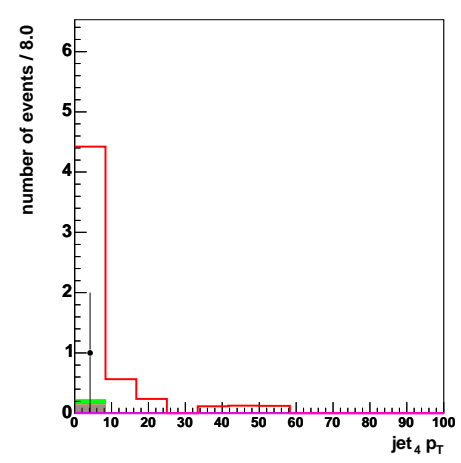

(h)

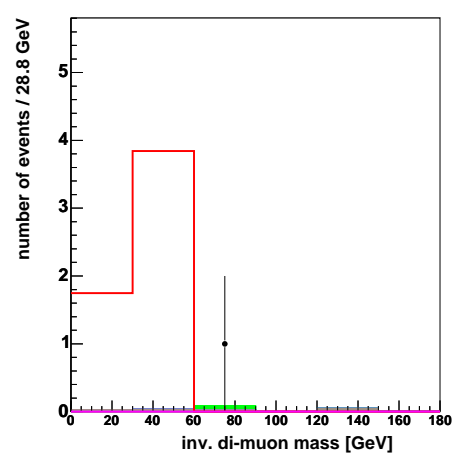

(c)

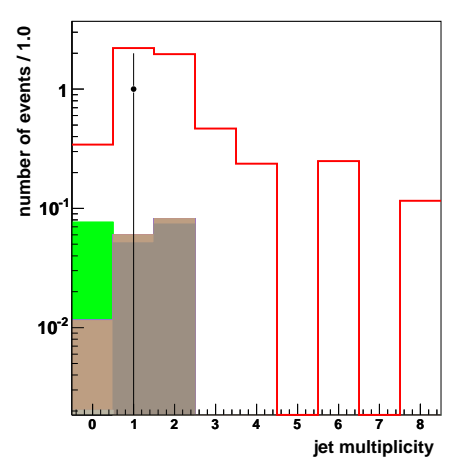

(f)

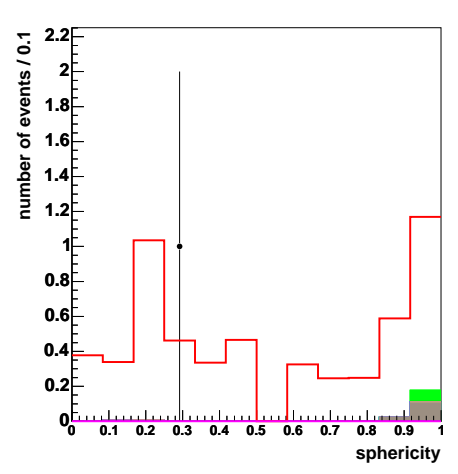

(i)

Figure 6.24: Final selected sample found by the gaugino pair and associated production analysis. One candidate event was found in the data while $0.238 \pm 0.164$ (stat) \pm 0.032 (sys) Standard Model background events where expected. The shown signal is the generated point number 11 with $\mathrm{m}\left(\tilde{\chi}_{1}^{0}\right)=39.6 \mathrm{GeV}$. 


\begin{tabular}{|c|c|c|c|c|c|c|c|}
\hline \# & $\begin{array}{r}m_{0} \\
{[\mathrm{GeV}]}\end{array}$ & $\begin{array}{r}m_{1 / 2} \\
{[\mathrm{GeV}]}\end{array}$ & $\begin{array}{l}\mathrm{m}\left(\tilde{\chi}_{1}^{0}\right) \\
{[\mathrm{GeV}]}\end{array}$ & $\begin{array}{r}\mathrm{m}\left(\tilde{d}_{R}\right) \\
{[\mathrm{GeV}]}\end{array}$ & $\begin{array}{c}\epsilon_{\text {signal }} \\
{[\%]}\end{array}$ & $\begin{array}{l}\sigma_{L O} \\
{[\mathrm{pb}]}\end{array}$ & $\begin{array}{r}\text { 95\% CL limit } \\
{[\mathrm{pb}]}\end{array}$ \\
\hline 1 & 1 & 150 & 58 & 353 & $0.57 \pm 0.10$ & 1.1 & 2.3 \\
\hline 2 & 40 & 120 & 46 & 292 & $0.26 \pm 0.06$ & 3.4 & 5.1 \\
\hline 3 & 75 & 75 & 29 & 205 & $0.05 \pm 0.03$ & 47.4 & 41.3 \\
\hline 4 & 100 & 100 & 39 & 266 & $0.25 \pm 0.07$ & 8.3 & 5.7 \\
\hline 5 & 125 & 125 & 48 & 325 & $0.38 \pm 0.06$ & 2.5 & 3.4 \\
\hline 6 & 150 & 150 & 58 & 383 & $0.63 \pm 0.12$ & 1.0 & 2.0 \\
\hline 7 & 175 & 175 & 68 & 440 & $0.60 \pm 0.13$ & 0.5 & 2.2 \\
\hline 8 & 200 & 40 & 17 & 228 & $0.002 \pm 0.002$ & 2621.7 & 2130.4 \\
\hline 9 & 200 & 60 & 24 & 253 & $0.02 \pm 0.01$ & 133.0 & 88.6 \\
\hline 10 & 200 & 80 & 32 & 283 & $0.11 \pm 0.03$ & 20.5 & 13.2 \\
\hline 11 & 200 & 100 & 40 & 315 & $0.22 \pm 0.04$ & 6.9 & 6.0 \\
\hline 12 & 200 & 120 & 47 & 350 & $0.31 \pm 0.07$ & 2.9 & 4.2 \\
\hline 13 & 200 & 140 & 55 & 387 & $0.34 \pm 0.05$ & 1.4 & 3.7 \\
\hline 14 & 300 & 20 & 9 & 309 & - & 15423.5 & - \\
\hline 15 & 300 & 40 & 17 & 320 & $0.002 \pm 0.002$ & 2549.3 & 2184.0 \\
\hline 16 & 300 & 60 & 24 & 337 & $0.03 \pm 0.03$ & 96.6 & 89.5 \\
\hline 17 & 300 & 80 & 32 & 360 & $0.13 \pm 0.04$ & 17.5 & 11.3 \\
\hline 18 & 300 & 100 & 40 & 386 & $0.23 \pm 0.06$ & 6.2 & 6.1 \\
\hline 19 & 300 & 120 & 47 & 414 & $0.38 \pm 0.08$ & 2.7 & 3.4 \\
\hline 20 & 300 & 140 & 55 & 445 & $0.50 \pm 0.12$ & 1.4 & 2.7 \\
\hline 21 & 400 & 100 & 40 & 467 & $0.33 \pm 0.06$ & 6.1 & 3.9 \\
\hline 22 & 400 & 120 & 48 & 491 & $0.47 \pm 0.07$ & 2.7 & 2.7 \\
\hline 23 & 400 & 140 & 55 & 516 & $0.61 \pm 0.08$ & 1.4 & 2.0 \\
\hline 24 & 500 & 120 & 48 & 575 & $0.49 \pm 0.07$ & 2.7 & 2.6 \\
\hline
\end{tabular}

Table 6.16: After all selection cuts, 1 data event and $0.238 \pm 0.164$ (stat) \pm 0.032 (sys) expected Standard Model background events are left in the final sample. The final amount of expected signal events for all studied mSUGRA parameter combinations is listed in this table. The cross section limit has been calculated using the $\mathrm{CL}_{s}$ method as will be discussed in detail in Sec. 7.1. For point number 14 no limit can be given, since no signal event has been selected. However, this does not mean that this point cannot be excluded; it is simply not possible to generate enough statistic. For the points 8 and 15 e.g. 60000 events were generated in order to select a single event. The signal efficiency $\epsilon_{\text {signal }}$ does not depend on the signal cross section $\sigma$. However, both $\epsilon_{\text {signal }}$ and $\sigma$ depend on the neutralino mass and therefore on the mSUGRA parameter $m_{1 / 2}$ of the point under study, as was discussed in Sec. 2.3.2. 


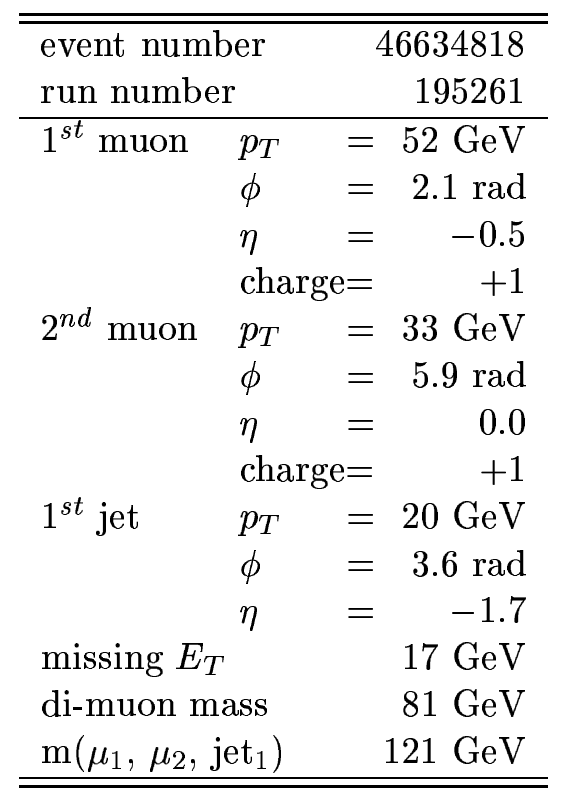

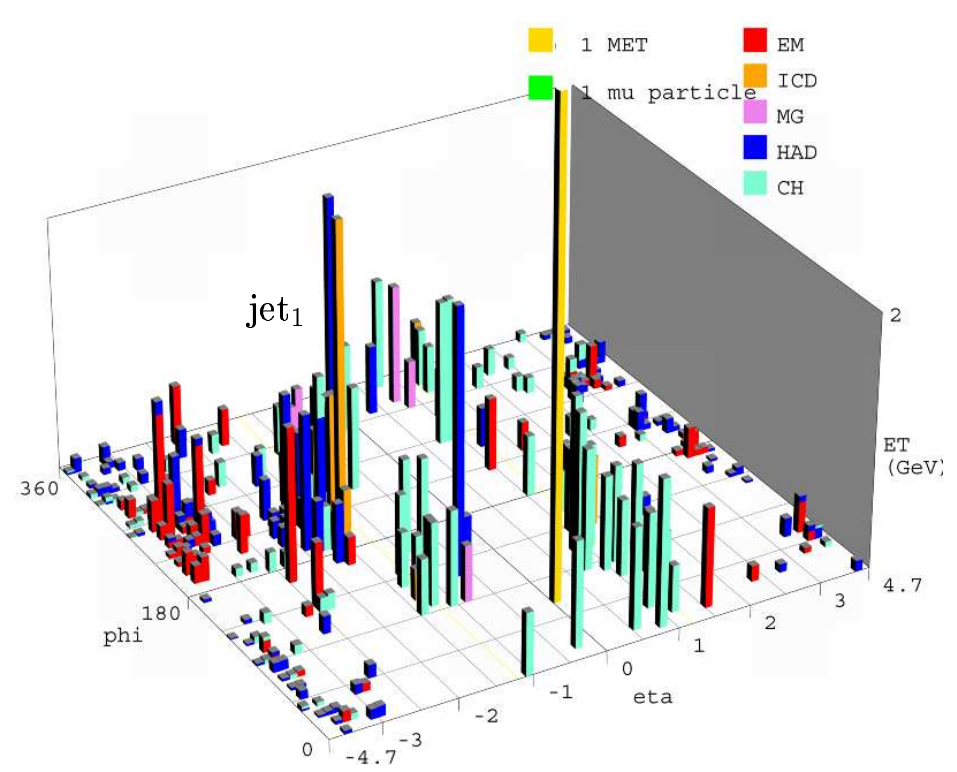

(a) $\eta-\phi$ plane

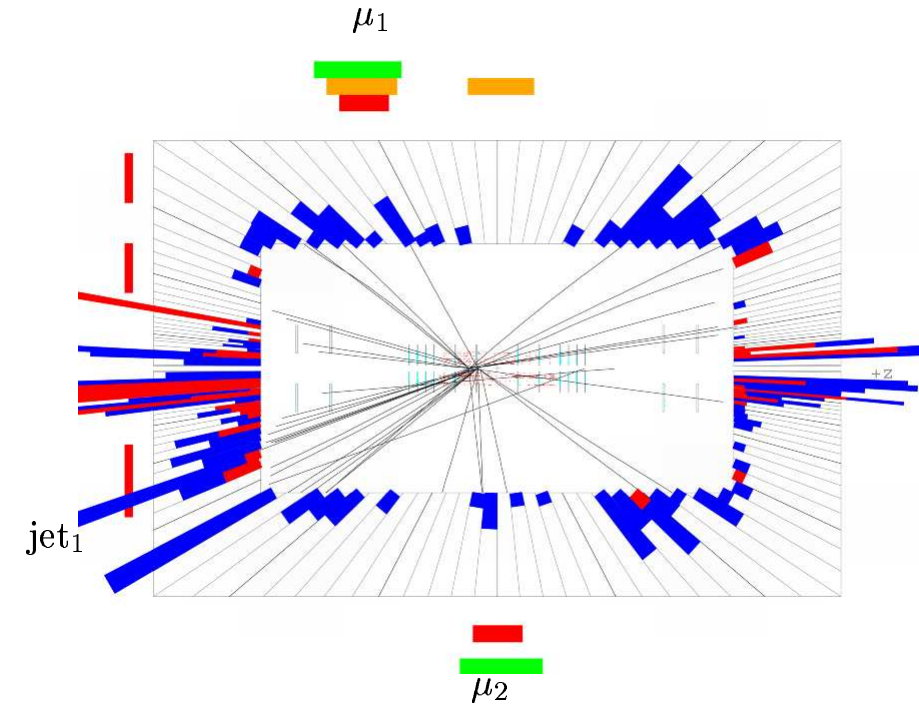

(b) $\eta-r$ cross section

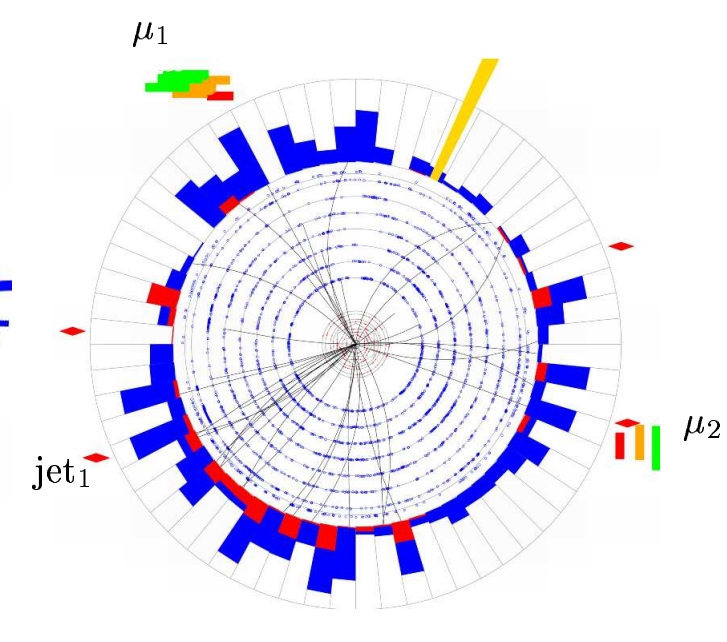

(c) $\phi-r$ cross section

Figure 6.25: Event 46634818 is the candidate event found in the data. Both muons are positively charged and their momenta are not too high, so that a mismeasured $Z \rightarrow \mu^{+} \mu^{-}$background event is unlikely. 


\section{Chapter 7}

\section{Results}

Since no excess was found in the data, limits on the $R$-parity violating supersymmetry have been calculated. In Sec. 7.1 the $\mathrm{CL}_{s}$ limit calculation method will be discussed. In Sec. 7.2 and in Sec. 7.3 the resulting limits on resonant slepton production and on the pair and associated production of gauginos will be given, respectively.

\subsection{The $\mathrm{CL}_{s}$ Limit Calculation Method}

Various methods have been developed to calculate upper limits on a signal cross section, if no signal is seen, or to give confidence levels (CL) for a seen signal. While in general the methods are consistent, no mathematical proof for this statement exists and the differences rise when large negative background fluctuations are found or if the uncertainties are large compared to the background expectation and the acceptance [175]. The Particle Data Group [17] suggests the Bayesian and the Frequentist approach as the most robust methods. Though the Bayesian method was used by the Run I analysis [92], now a modified Frequentist approach is chosen. The $\mathrm{CL}_{s}$ method [178] was used by LEP Higgs searches [179] and many D $\varnothing$ Run II searches, e.g. [180]. The advantage of the $\mathrm{CL}_{s}$ method is the possibility of considering correlated errors in signal and background expectation and the easy combination of multiple channels (denoted in the following with subscript $i$ ), where different channels can mean different signal processes or, for example, different bins in one distribution as a function of some variable. The $\mathrm{CL}_{s}$ algorithm is implemented in the class TLimit [140] in root [134] and shall be briefly described in the following.

A test statistic $X_{i}$ is defined as the likelihood ratio of two Poisson probabilities. The test statistic separates experiments where the measured data $d_{i}$ is either background like, or where the data is in agreement with a signal+background hypothesis. 


$$
\begin{aligned}
X & =\prod_{i=1}^{N} X_{i} \\
X_{i} & =\frac{\left(s_{i}+b_{i}\right)^{d_{i}} \cdot e^{-\left(s_{i}+b_{i}\right)}}{d_{i} !} / \frac{\left(b_{i}\right)^{d_{i}} \cdot e^{-b_{i}}}{d_{i} !}
\end{aligned}
$$

Here $s_{i}$ and $b_{i}$ denote the expected signal and background events of one channel $i . N$ is the total number of channels. An important feature of the above test statistic definition is the possibility of combining multiple channels, since likelihoods are multiplicative.

The confidence level $\mathrm{CL}_{s+b}$ for a measured number of data events $d=\left\{d_{i}\right\}$ not being compatible with the assumption that both signal and background events are present (the $\mathrm{s}+\mathrm{b}$ hypothesis), is given by the fraction of Monte Carlo experiments in a large ensemble of signal+background experiments, which would result in a less signal-like test statistic than the observed data:

$$
\mathrm{CL}_{s+b}(d)=P_{s+b}\left(X \leq X_{o b s}\right)
$$

This probability is the sum of Poisson probabilities over all possible outcomes $d^{\prime}=\left\{d_{i}^{\prime}\right\}$, which have a statistic $X\left(d^{\prime}\right)$ less than or equal to the observed $X_{o b s}(d)$ :

$$
\mathrm{CL}_{s+b}(d)=\sum_{X\left(d_{i}^{\prime}\right) \leq X^{o b s}\left(d_{i}\right)} \prod_{i=1}^{N} \frac{\left(s_{i}+b_{i}\right) d^{\prime} \cdot e^{-\left(s_{i}+b_{i}\right)}}{d_{i}^{\prime !} !}
$$

The confidence level that the measured data events $d$ are compatible with the $\mathrm{s}+\mathrm{b}$ hypothesis is $1-\mathrm{CL}_{s+b}(d)$. The $\mathrm{CL}_{b}$ probability is obtained in a similar way.

The presence of a signal in the data is excluded with $95 \%$ confidence level, if $1-\mathrm{CL}_{s+b} \geq$ 0.95 , by definition. However, the $\mathrm{CL}_{s+b}$ probability is sensitive to fluctuations in the background. Negative statistical fluctuations can lead to larger $1-\mathrm{CL}_{s+b}$, so that the signal+background hypothesis is excluded even if the experimental sensitivity is low. Therefore the confidence level $\mathrm{CL}_{s}$ is defined:

$$
\mathrm{CL}_{s}=\frac{\mathrm{CL}_{s+b}}{\mathrm{CL}_{b}}
$$

A limit on a signal cross section is set with $95 \% \mathrm{CL}$ if $\mathrm{CL}_{s}<0.05$. The usage of $\mathrm{CL}_{s}$ reduces the sensitivity but the limit is never more restrictive than the one obtained by using $\mathrm{CL}_{s+b}$. This is sometimes referred to as the "Modified Frequentist Approach".

The statistical and systematical errors on the signal and background expectations are taken into account by replacing the $s_{i}$ and $b_{i}$ by appropriate probability distribution functions. By default the uncertainties are assumed to be Gaussian. For this analysis a modified TLimit version is used, that can handle asymmetric errors [141]. 


\subsection{Limits on Resonant Slepton Production}

\subsubsection{Model Independent Limits}

In the absence of an excess in the data, cross section limits on the resonant production were set. To be as model independent as possible, $95 \%$ confidence level limits $\mathrm{CL}_{s}$ with respect to the slepton production cross section times branching ratio to gaugino plus muon in the slepton mass - gaugino mass plane is given. The systematic errors of the simulation are expected to be fully correlated, this is considered in the limit calculation. These are the first published cross section limits on resonant slepton production in the plane of the relevant gaugino and slepton masses [181].

The $95 \%$ C.L. cross section limit for the three independent analyzed channels (i) $\tilde{\mu} \rightarrow \tilde{\chi}_{1}^{0} \mu$, (ii) $\tilde{\mu} \rightarrow \tilde{\chi}_{2,(3,4)}^{0} \mu$, and (iii) $\tilde{\nu}_{\mu} \rightarrow \tilde{\chi}_{1,(2)}^{ \pm} \mu$ are given in Fig. 7.1a-c. The combination of the two $\tilde{\mu} \rightarrow \tilde{\chi}_{i}^{0} \mu$ channels smuon production (i) and (ii) is shown in Fig. $7.1 \mathrm{~d}$; this holds under the constraint that $\mathrm{m}\left(\tilde{\chi}_{2}^{0}\right) \approx 2 \cdot \mathrm{m}\left(\tilde{\chi}_{1}^{0}\right)$ and that the relative branching fractions of both channels are similar to the ratio predicted by mSUGRA.

The combined LEP limits [42] for R-Parity violating Supersymmetry via $L Q \bar{d}$ couplings, $\tilde{\chi}_{1}^{0} \geq 39 \mathrm{GeV}, \tilde{\chi}_{1}^{ \pm} \geq 103 \mathrm{GeV}, \tilde{\nu}_{\mu} \geq 78 \mathrm{GeV}$ and $\tilde{\mu} \geq 90 \mathrm{GeV}$ are included in all limit figures. The LEP limit from $\tilde{\chi}_{1}^{0}$ and $\tilde{\chi}_{1}^{ \pm}$is drawn left-hatched, while the exclusion region originating from the slepton limits are drawn right-hatched.

\subsubsection{Combined Limits within mSUGRA}

Assuming relations between the smuon, muon sneutrino and gaugino masses, in other words, introducing a model, the channels can be combined. In this analysis mSUGRA with its 5 parameters $m_{0}, m_{1 / 2}, A_{0}, \tan (\beta)$ and $\operatorname{sign}(\mu)$ is used. The trilinear coupling parameter $A_{0}$ is set to zero, since the influence is very small. Setting the parameter $A_{0}$ to $\pm 1 \mathrm{TeV}$ would vary the total slepton production cross section by $\pm 10 \%$ due to next-to leading order SUSY-QCD corrections to the $q \bar{q}^{\prime} \rightarrow \tilde{l}$ process [172], see also Sec. 6.2.1. For the signal generation the sign of $\mu$ was chosen negative and $\tan (\beta)$ was set to 5 . With these 3 parameters set, $m_{0}$ and $m_{1 / 2}$ are given by the slepton and the gaugino mass of a certain SUSY parameter point. The obtained results are interpreted within this exemplary mSUGRA parameter set, just like previous searches, e.g. ??.

At first the two channels $\tilde{\mu} \rightarrow \tilde{\chi}_{1}^{0} \mu$ and $\tilde{\mu} \rightarrow \tilde{\chi}_{2,(3,4)}^{0} \mu$ are combined to a "smuon production" channel, then the muon sneutrino channel $\tilde{\nu}_{\mu} \rightarrow \tilde{\chi}_{1,(2)}^{ \pm} \mu$ is added. While the signal has no overlap in different channels, due to the different processes, the background expectation and the data might have. In that case, those events are removed from that channel with the worse signal-over-background ratio. A combined cross section limit is calculated, considering again correlations of the systematic uncertainties.

The systematic errors on the signal cross section, arising from the signal production cross section, PDF and factorization scale uncertainties, is separated from the total systematic 
error on the number of selected signal events, following [175]. The signal cross section is then decreased by this value, to calculate a conservative limit on $\lambda_{211}^{\prime}$.

As an example the combination of the three signal channels is shown for the reference point with $\mathrm{m}(\tilde{\mu})=260 \mathrm{GeV}$ and $\mathrm{m}\left(\tilde{\chi}_{1}^{0}\right)=100 \mathrm{GeV}$ in Tab. 7.1.

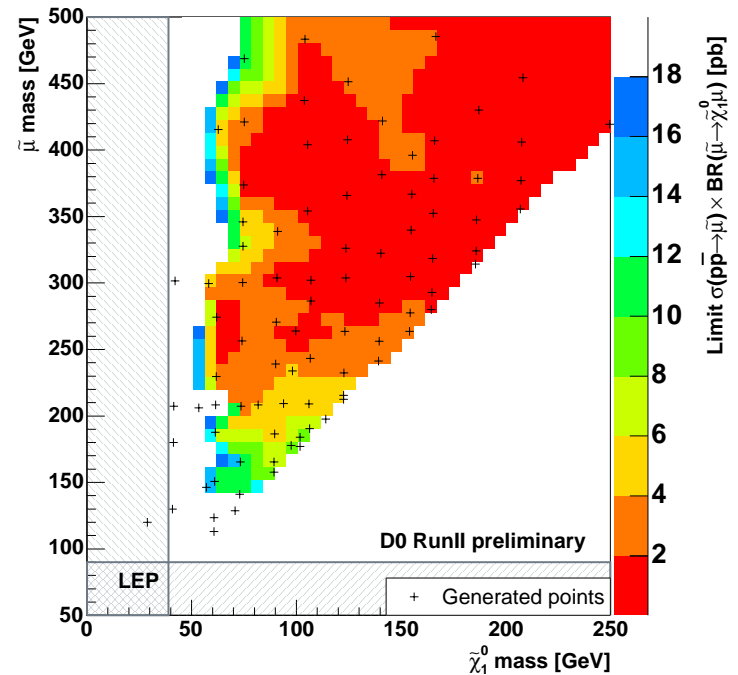

(a)

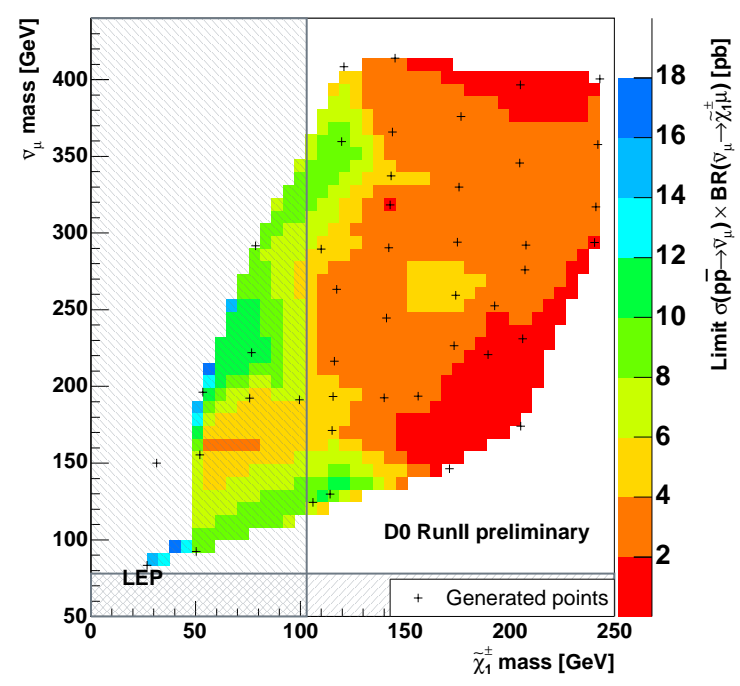

(c)

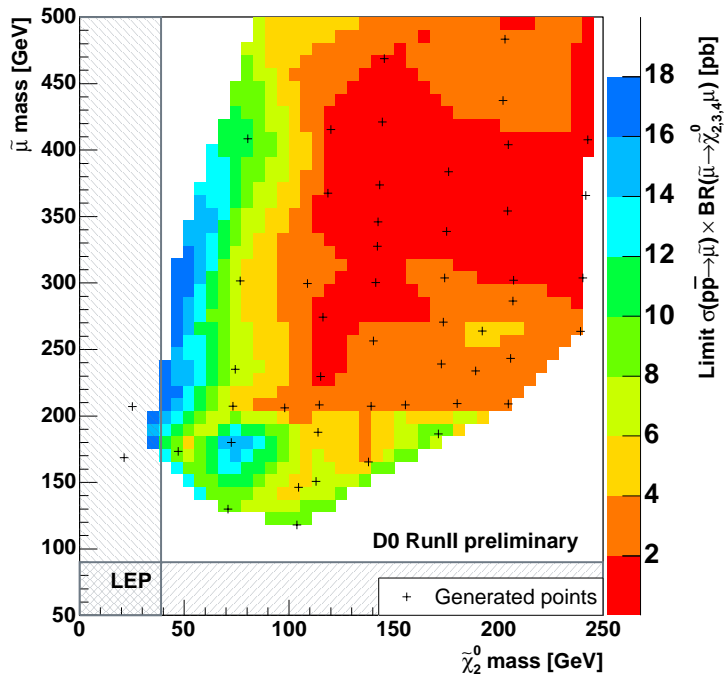

(b)

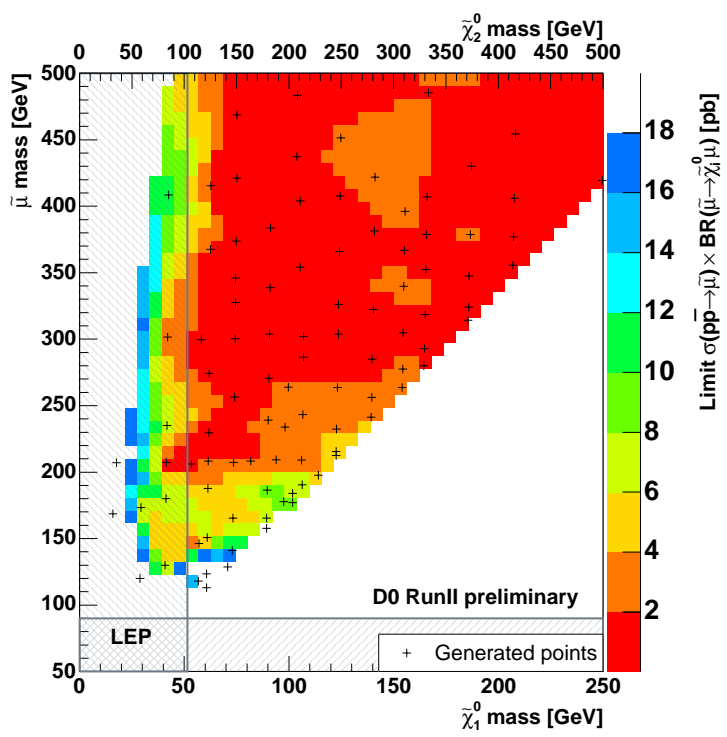

(d)

Figure 7.1: Model independent 95\% CL Cross section $\times$ Branching ratio limits for (a) channel $\tilde{\mu} \rightarrow \tilde{\chi}_{1}^{0} \mu$, (b) channel $\tilde{\mu} \rightarrow \tilde{\chi}_{2,(3,4)}^{0} \mu$, (c) channel $\tilde{\nu}_{\mu} \rightarrow \tilde{\chi}_{1,(2)}^{ \pm} \mu$ and (d) the combination of the two $\tilde{\mu} \rightarrow \tilde{\chi}_{i}^{0} \mu$ smuon channels (i) and (ii) under the constraint $\mathrm{m}\left(\tilde{\chi}_{2}^{0}\right) \approx 2 \cdot \mathrm{m}\left(\tilde{\chi}_{1}^{0}\right)$ and assuming the relative branching fraction is like in mSUGRA. The LEP gaugino-limits are drawn left-hatched, while the LEP limits originating from slepton constraints are shown right-hatched. 


\begin{tabular}{|c|c|c|c|c|c|}
\hline channel & $\begin{array}{c}\text { data } \\
\text { [events] }\end{array}$ & $\begin{array}{l}\text { SM exp. } \\
\text { [events] }\end{array}$ & $\begin{array}{l}\text { signal exp. } \\
\text { [events] }\end{array}$ & $\begin{array}{c}\sigma_{\mathrm{NLO}} \\
{[\mathrm{pb}]}\end{array}$ & $\begin{array}{c}95 \% \text { CL limit } \\
{[\mathrm{pb}]}\end{array}$ \\
\hline $1: \tilde{\mu} \rightarrow \tilde{\chi}_{1}^{0} \mu$ & 14 & $11.9 \pm 2.1_{-1.6}^{+1.5}$ & $9.9 \pm 0.8_{-1.2}^{+1.2}$ & 1.10 & 1.51 \\
\hline $2: \tilde{\mu} \rightarrow \tilde{\chi}_{2,(3,4)}^{0} \mu$ & 28 & $25.4 \pm 3.2_{-4.2}^{+\overline{6} .7}$ & $7.3 \pm 1.3_{-1.1}^{+1.6}$ & 0.98 & 4.49 \\
\hline $3: \tilde{\nu}_{\mu} \rightarrow \tilde{\chi}_{1,(2)}^{ \pm} \mu$ & 8 & $6.5 \pm 1.6_{-1.2}^{+2.0}$ & $8.0 \pm 0.7_{-0.9}^{+1.2}$ & 1.74 & 2.33 \\
\hline combination & \multicolumn{3}{|c|}{ overlapping events } & $\sigma_{\mathrm{NLO}}[\mathrm{pb}]$ & limit [pb] \\
\hline 4: 1. and 2. & 0 & 0.0 & - & 3.15 & 3.33 \\
\hline 5: 3. and 4. & 4 & 3.8 & - & 6.06 & 1.17 \\
\hline
\end{tabular}

Table 7.1: Combination of the three channels for the reference point $\mathrm{m}(\tilde{\mu})=260 \mathrm{GeV}$ and $\mathrm{m}\left(\tilde{\chi}_{1}^{0}\right)=100 \mathrm{GeV}$. The limit refers to the total cross section times branching ratio of a specific channel. The cross section is given for $\lambda_{211}^{\prime}=0.07$. Note, that the data and SM events given for the combined channels are duplicate events, that are present in more than one channel. Inaccessible channels like $\tilde{\nu}_{\mu} \rightarrow \tilde{\chi}_{1}^{0} \nu_{\mu}$ are not listed, but they contribute to the cross section of the total smuon $\tilde{\mu} \rightarrow X$ or total second generation slepton $\tilde{l} \rightarrow X$ production.

Since the cross section is proportional to $\left(\lambda_{211}^{\prime}\right)^{2}$, limits with respect to the coupling strength $\lambda_{211}^{\prime}$ can be given. Using Eq. (6.1) the 95\% C.L. cross section limits can be translated into limits on the coupling strength $\lambda_{211}^{\prime}$. For the reference point listed in Tab. 7.1 the total slepton production cross section limit of $\sigma \leq 1.17 \mathrm{pb}$ translates therefore to $\lambda_{211}^{\prime} \leq 0.03$.

In Fig. 7.2 exclusion contours are given in the $m_{0}-m_{1 / 2}$ plane. The black colored region at low $m_{0}$ and $m_{1 / 2}$ has no mSUGRA solution. The presented analysis is insensitive in regions of high $m_{1 / 2}$ (high neutralino masses) and low $m_{0}$ because here the neutralino is no longer the lightest supersymmetric particle (LSP), but the stau ( $\tilde{\tau})$. Thus the decay channels to final states with muons are suppressed. In Fig. 7.2 this region is filled light-gray. However, the analysis does not require that $\tilde{\chi}_{1}^{0}$ is the LSP! In regions of low $m_{1 / 2}$ or low neutralino masses $\mathrm{m}\left(\tilde{\chi}_{1}^{0}\right)<50 \mathrm{GeV}$ the analysis is constricted by the minimal transverse momentum requirements for muons and jets - the decay products of the neutralino. In regions of high $m_{0}$ or high $m_{1 / 2}$, the cross section runs off, while the limit is constant or improving slightly, resulting in a slowly degrading limit on $\lambda_{211}^{\prime}$.

In Fig. 7.3 the limit on $\lambda_{211}^{\prime}$ is given in the $\mathrm{m}\left(\tilde{\chi}_{1}^{0}\right)-\mathrm{m}(\tilde{l})$-plane and in the $\mathrm{m}\left(\tilde{d}_{R}\right)-\mathrm{m}(\tilde{l})-$ plane for $\tan \beta=5$ and $\mu<0$. The hatched areas represent the limits given by LEP,

\begin{tabular}{lcl}
\hline \hline & excluded mass range & coupling strength \\
\hline second generation slepton & $\mathrm{m}(\tilde{l}) \leq 210 \mathrm{GeV}$ & for $\lambda_{211}^{\prime} \geq 0.04$ \\
& $\mathrm{~m}(\tilde{l}) \leq 330 \mathrm{GeV}$ & for $\lambda_{211}^{\prime} \geq 0.06$ \\
& $\mathrm{~m}(\tilde{l}) \leq 358 \mathrm{GeV}$ & for $\lambda_{211}^{\prime} \geq 0.10$ \\
\hline right handed down squark & $\mathrm{m}\left(\tilde{d}_{R}\right) \leq 408 \mathrm{GeV}$ & for $\lambda_{211}^{\prime} \geq 0.04$ \\
& $\mathrm{~m}\left(\tilde{d}_{R}\right) \leq 430 \mathrm{GeV}$ & for $\lambda_{211}^{\prime} \geq 0.08$ \\
& $\mathrm{~m}\left(\tilde{d}_{R}\right) \leq 443 \mathrm{GeV}$ & for $\lambda_{211}^{\prime} \geq 0.12$ \\
\hline \hline
\end{tabular}

Table 7.2: Limits on the slepton mass $\tilde{l}$ and the right-handed down-squark mass $\tilde{d}_{R}$, obtained from Fig. 7.3. 
the gray shaded areas have no mSUGRA solutions. These regions are calculated with [43] and [48] and cross-checked with [182]. From Fig. 7.3 absolute limits on the slepton mass $\tilde{l}$ and the right-handed down-squark mass $\tilde{d}_{R}$ can be obtained. Some exemplary limits are marked by arrows and are summarized in Tab. 7.2. The exclusion contour in the slepton mass - coupling strength $\lambda_{211}^{\prime}$ plane is shown in Fig. 7.4.

In Fig. 7.5 (a) - (b) the calculated limit as a function of $\mathrm{m}\left(\tilde{\chi}_{1}^{0}\right)$ for two different values of $\mathrm{m}(\tilde{\mu})$ and in Fig. 7.5 (c) - (d) as a function of $\mathrm{m}(\tilde{\mu})$ for two different values of $\mathrm{m}\left(\tilde{\chi}_{1}^{0}\right)$ are shown. The solid gray area, calculated with [43], is kinematically not allowed in mSUGRA; for a certain slepton mass there is a maximal possible neutralino mass and vice versa. For example, for a neutralino mass of $\mathrm{m}\left(\tilde{\chi}_{1}^{0}\right)=100 \mathrm{GeV}$, the slepton mass has to be larger than $\mathrm{m}(\tilde{l}) \approx 180 \mathrm{GeV}$, as one can see in Fig. 7.2. The limit obtained from the lepton universality in the pion decay $[54,57,183]$ is not plotted since it is too weak to be of interest here.

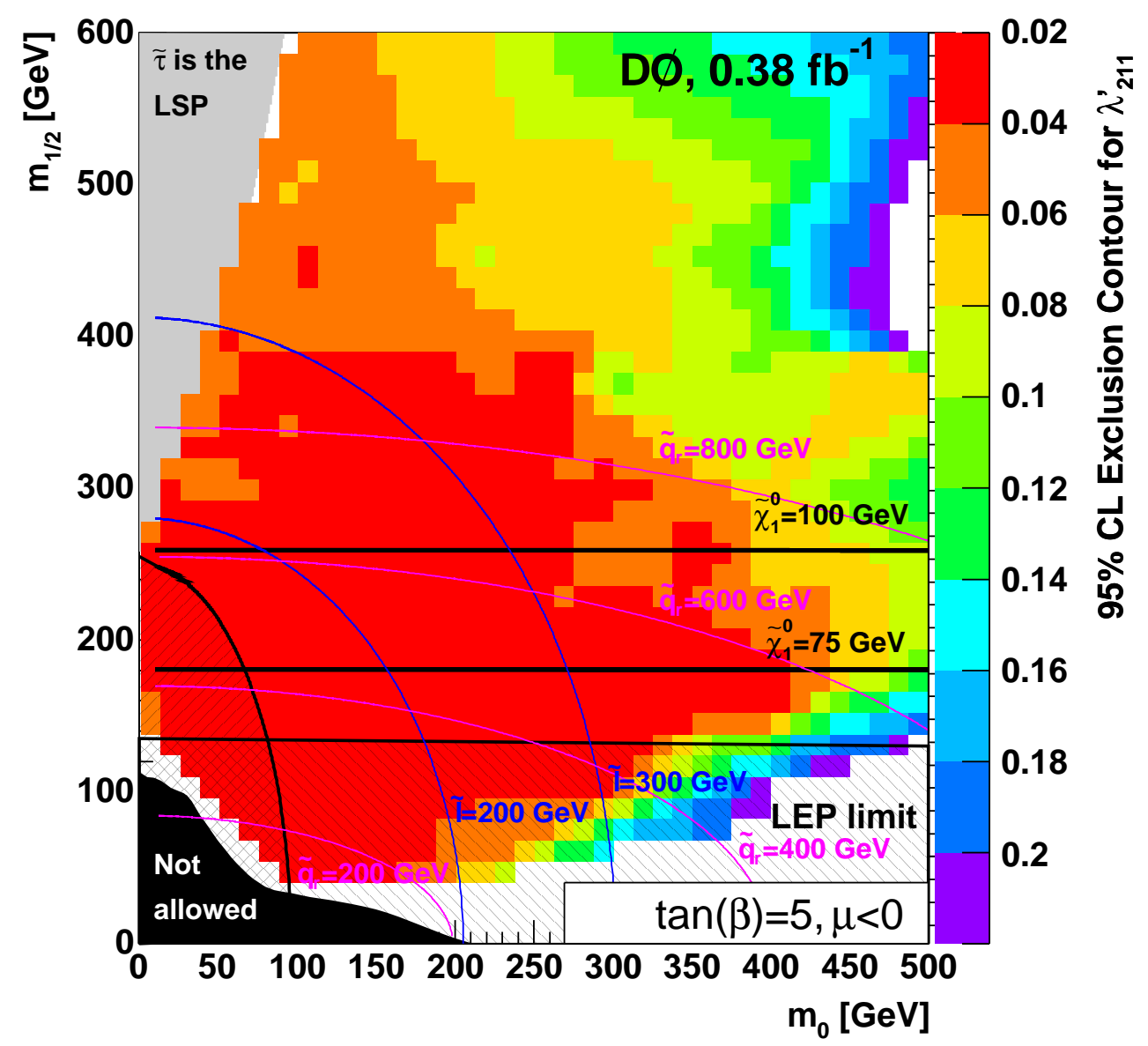

Figure 7.2: $95 \%$ C.L. exclusion contours for different coupling strengths $\lambda_{211}^{\prime}$ in the mSUGRA $m_{0}-m_{1 / 2}$ plane for $\tan \beta=5, \mu<0$, and $A_{0}=0$. In the black shaded region no electroweak symmetry breaking occurs. The gray shaded region is not accessable by this analysis, since the stau $(\tilde{\tau})$ is the lightest supersymmetric particle and the $\not R_{p}$ decays of the neutralino are suppressed, in favor of the R-parity conserving decay $\tilde{\chi}_{1}^{0} \rightarrow \tilde{\tau}^{ \pm} \tau^{\mp}$. The LEP limit on the slepton mass is shown right-hatched, the LEP gaugino limits are shown left-hatched. For better orientation, lines of constant neutralino mass (black), slepton mass (blue) and squark mass (magenta) are shown. 

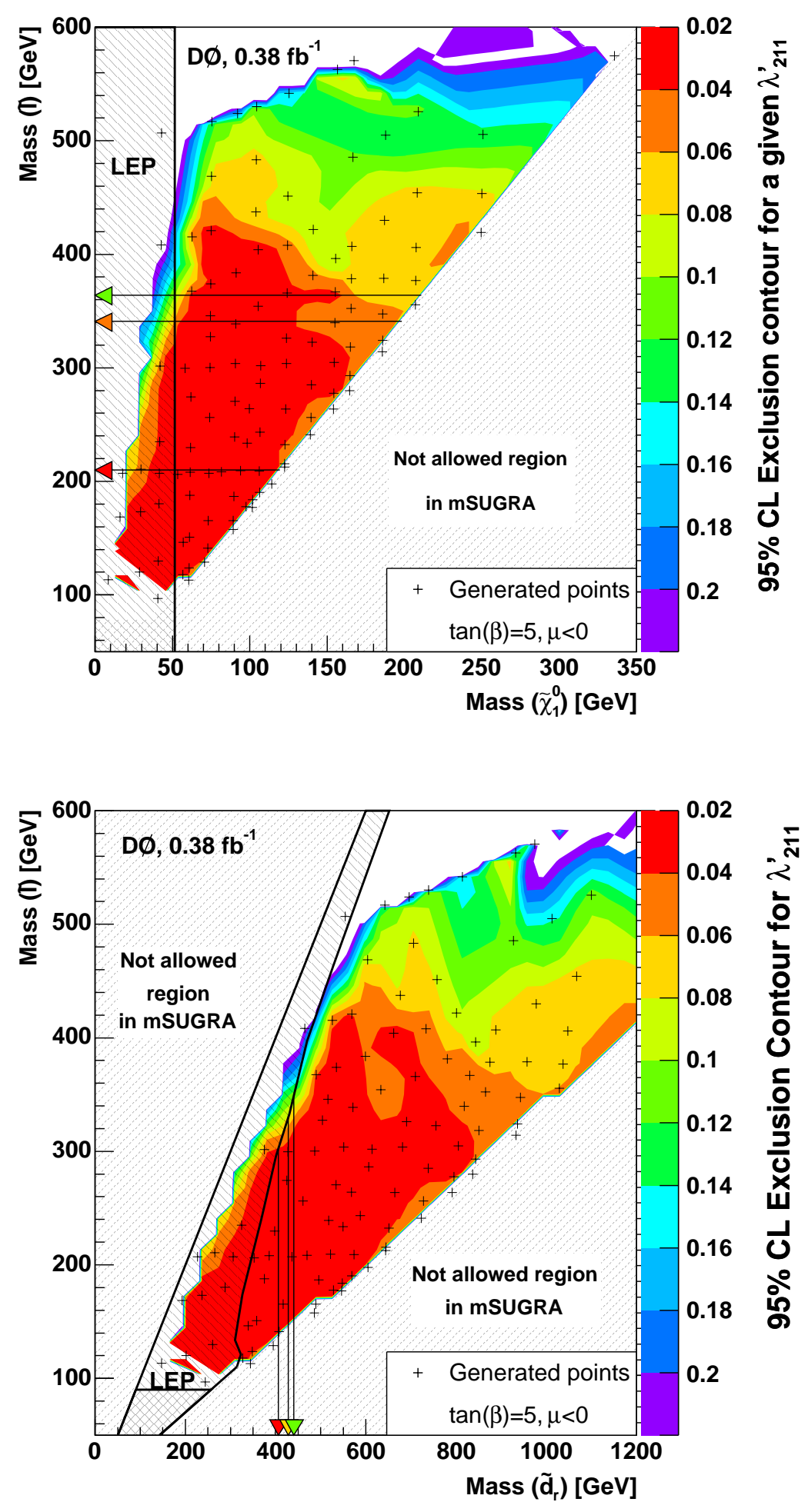

Figure 7.3: $95 \%$ CL exclusion contours for different coupling strengths $\lambda_{211}^{\prime}$ interpreted (top) in the neutralino mass $\mathrm{m}\left(\tilde{\chi}_{1}^{0}\right)$ - slepton mass $\mathrm{m}(\tilde{l})$ plane and (bottom) in the right-handed down squark $\mathrm{m}\left(\tilde{d}_{R}\right)$ - slepton mass $\mathrm{m}(\tilde{l})$ plane for $\tan \beta=5, \mu<0$ and $A_{0}=0$. The cross marks indicate where points have been generated. The LEP limits on the gaugino mass and on the slepton mass are shown left-hatched and right-hatched, respectively. The gray shaded region is not allowed in mSUGRA. Absolute limits on the slepton and the down squark mass can be derived from these plots as indicated by the arrows. See also Fig. 7.4 for more details. 


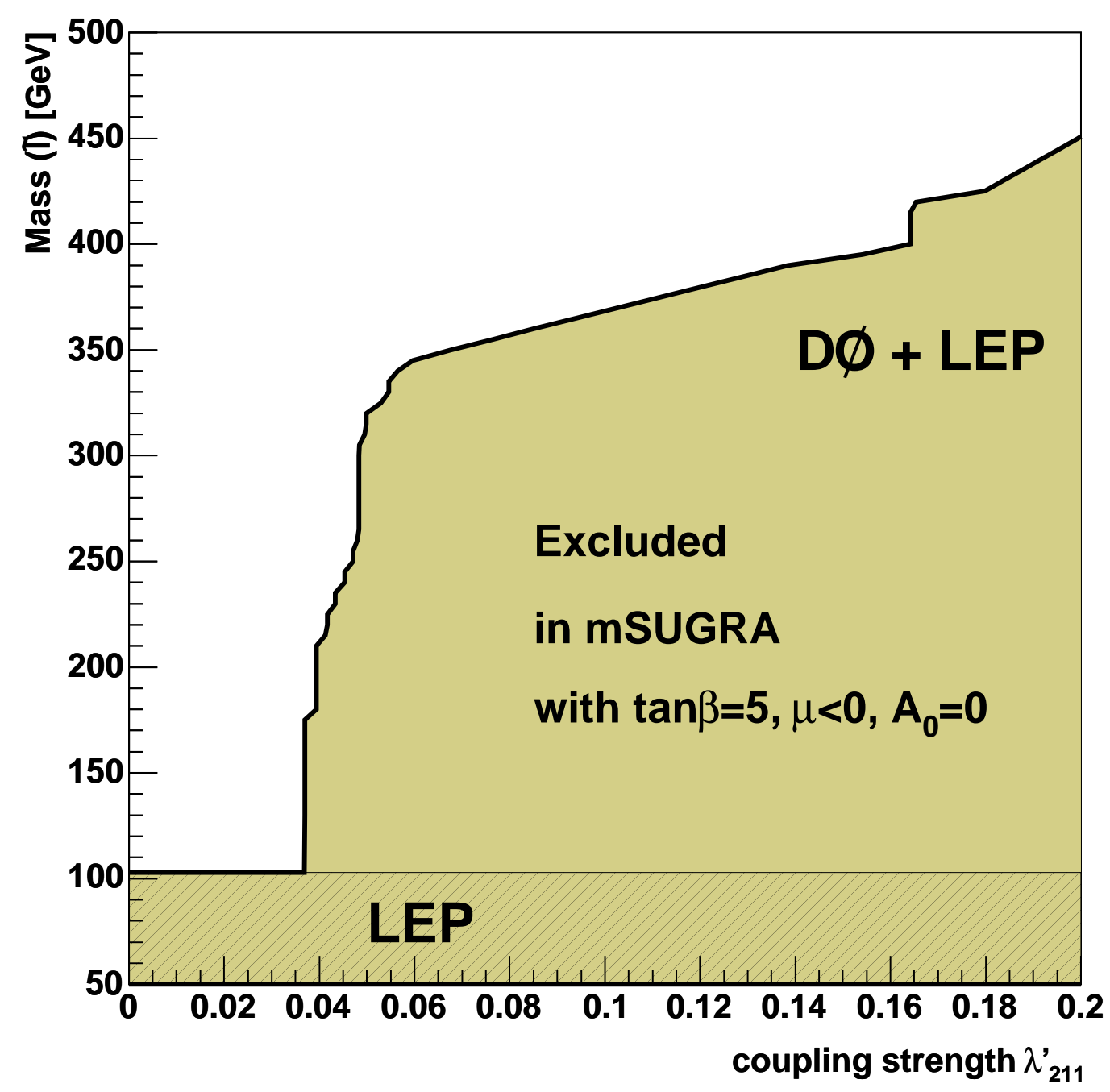

Figure 7.4: Interpretation in the slepton mass - coupling strength $\lambda_{211}^{\prime}$ plane within mSUGRA with $\tan \beta=5, \mu<0$ and $A_{0}=0$. The indicated region can be excluded using the limits obtained by this analysis, as well as the LEP limits on the neutralino and chargino mass. The shown exclusion contour can be derived from Fig. 7.3a.

The form of the plot contour is determined by the sensitivity of the analysis to muons from the neutralino decay. As can be seen in Fig. 7.3a, this sensitivity does also depend on $\mathrm{m}(\tilde{l})$, since at higher slepton masses, slepton decays to heavier gauginos become possible, so that less kinematic energy remains for the lightest neutralino. Thus, particles from neutralino decays in parameter space regions of heavier slepton masses tend to have less energy. LEP excludes charginos $\mathrm{m}\left(\tilde{\chi}_{1}^{ \pm}\right)<103 \mathrm{GeV}$, which translates to $\mathrm{m}\left(\tilde{\chi}_{1}^{0}\right)<51.5 \mathrm{GeV}$ in the studied mSUGRA parameter space [182]. For slepton masses larger than $\approx 320 \mathrm{GeV}$ particles from neutralino decays with $\mathrm{m}\left(\tilde{\chi}_{1}^{0}\right)=51.5 \mathrm{GeV}$ are no longer reconstructed efficiently, which is resulting in a turning-point in the slepton mass - coupling strength $\lambda_{211}^{\prime}$ curve. A larger coupling strength or a larger signal cross section makes up for a lower reconstruction efficiency. 


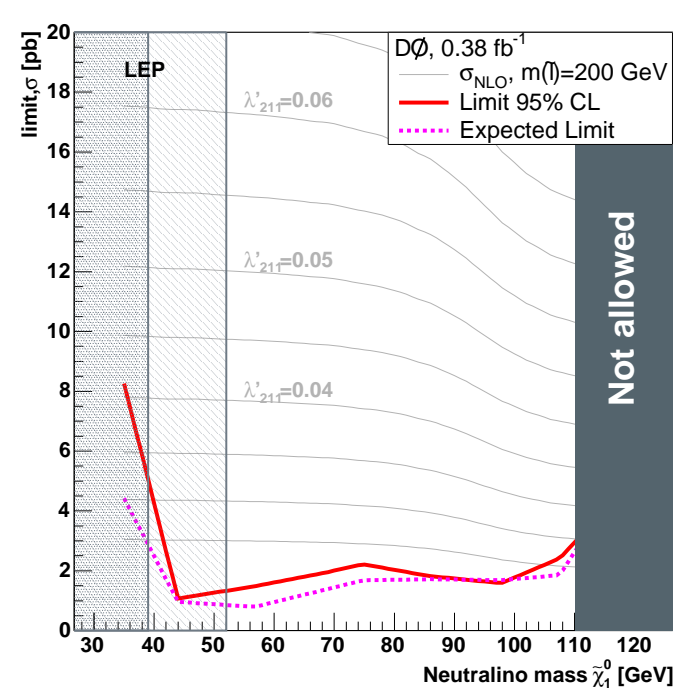

(a)

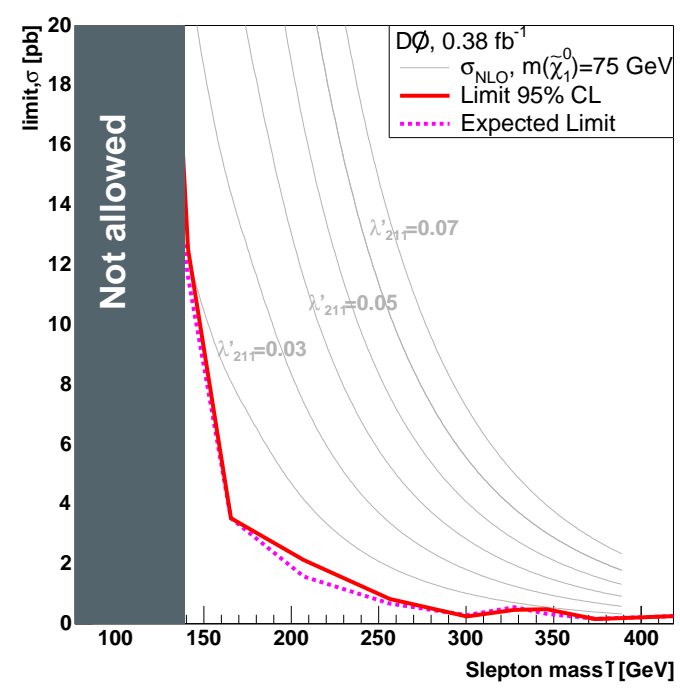

(c)

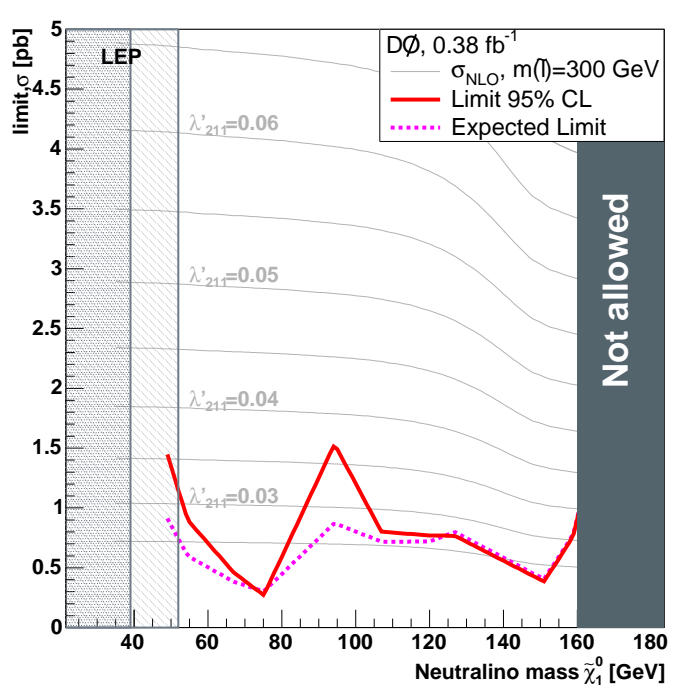

(b)

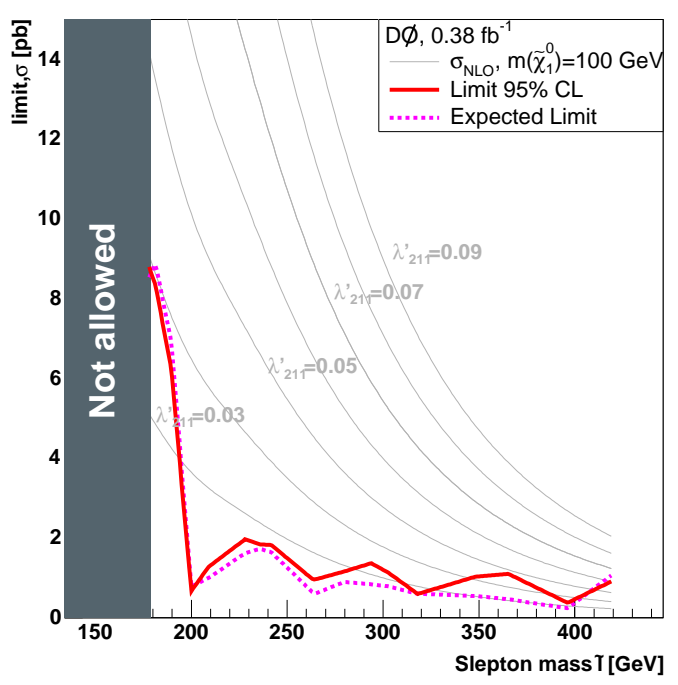

(d)

Figure 7.5: Cross section and 95\% CL limits for $\tan \beta=5, \mu<0, A_{0}=0$ and fixed slepton masses $200 \mathrm{GeV}$ (a) and $300 \mathrm{GeV}$ (b) and for fixed neutralino masses $75 \mathrm{GeV}$ (c) and $100 \mathrm{GeV}$ (d). The solid gray area in all graphs is in mSUGRA kinematically not allowed. The indirect limit from the pion decay is too weak to appear in the plots. The LEP limit $\tilde{\chi}_{1}^{0} \geq 39 \mathrm{GeV}$ and $\tilde{\chi}_{1}^{ \pm} \geq 103 \mathrm{GeV}$ is shown left-hatched in plot (a) and (b). The limit is generally constant in $\mathrm{m}\left(\tilde{\chi}_{1}^{0}\right)$ and $\mathrm{m}(\tilde{l})$; the slight fluctuation is due to the individual final selection optimization for each point. The limit gets worse for low neutralino masses $\mathrm{m}(\tilde{l}) \lesssim 50 \mathrm{GeV}$, since here the muon and jets from the neutralino decay have no longer enough energy to fulfill the selection criteria, see Fig. 6.11 for details. At low slepton masses $\mathrm{m}(\tilde{l}) \lesssim 2 \cdot \mathrm{m}\left(\tilde{\chi}_{1}^{0}\right)$ the limits worsen, since the slepton decay channel to $\tilde{\chi}_{2}^{0}$ or to $\tilde{\chi}_{1}^{ \pm}$is closing. 


\subsection{Limits on Gaugino Pair and Associated Production}

In the absence of an excess in the data (Sec. 6.3) limits on the gaugino pair and associated production cross sections are obtained. In Sec. 7.3.1 general cross section limits and signal efficiencies are given, while in Sec. 7.3.2 interpretations within mSUGRA are shown.

\subsubsection{Cross Section Limits}

In Fig. 7.6 the signal selection efficiency and in Fig. 7.7 the limit on the gaugino pair and associated production cross section (a) as well as a mSUGRA cross section prediction (b) are shown. The signal production cross section has been calculated for the parameters $\tan \beta=5, \mu<0$ and $A_{0}=0$. The points, where signal Monte Carlo has been generated, have been marked in the figures. The signal selection efficiency, and therefore the cross section limit as well, mainly depend on the size of the neutralino mass $\tilde{\chi}_{1}^{0}$ or on the mSUGRA parameter $m_{1 / 2}$, which controls the gaugino masses. In the region of low values of $m_{0}$ and $m_{1 / 2}$, near the dark shaded area, the squark and gluino masses become light, and the signal production is enhanced by squark-squark, squark-gluino, and gluino-gluino pair and associated production, as observable in Fig. 7.7(b).

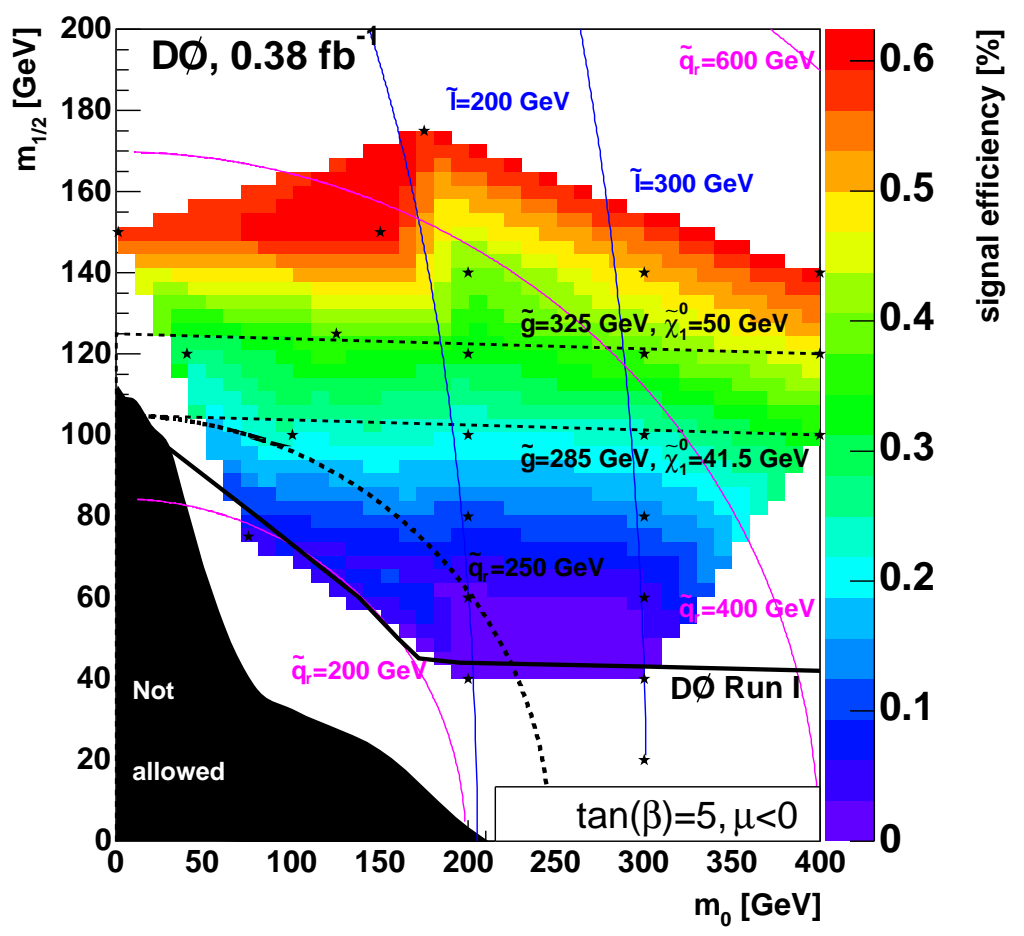

Figure 7.6: Gaugino pair and associated production signal selection efficiency. The cross marks reffer to points where signal Monte Carlo has been generated. The signal selection efficiency effectively only depends on the neutralino mass $\mathrm{m}\left(\tilde{\chi}_{1}^{0}\right)$ and therefore on $m_{1 / 2}$. 


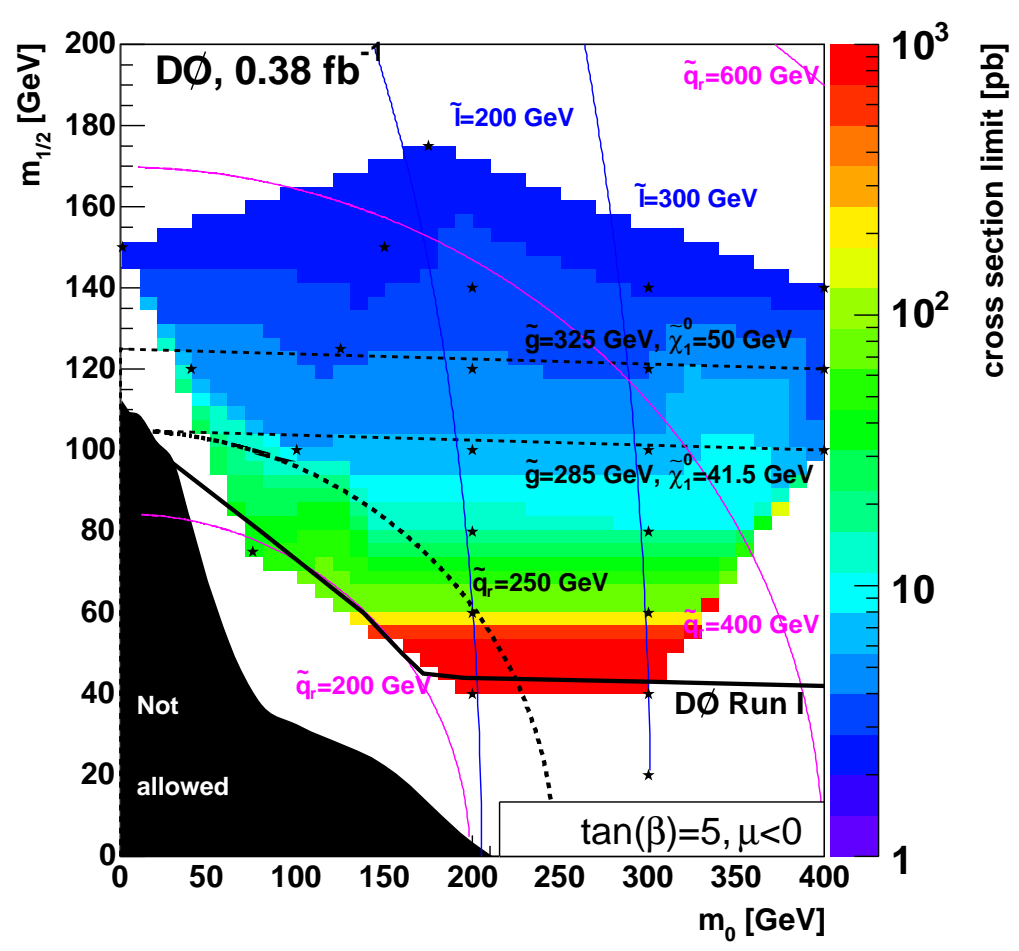

(a) cross section limit $95 \%$ CL $[\mathrm{pb}]$

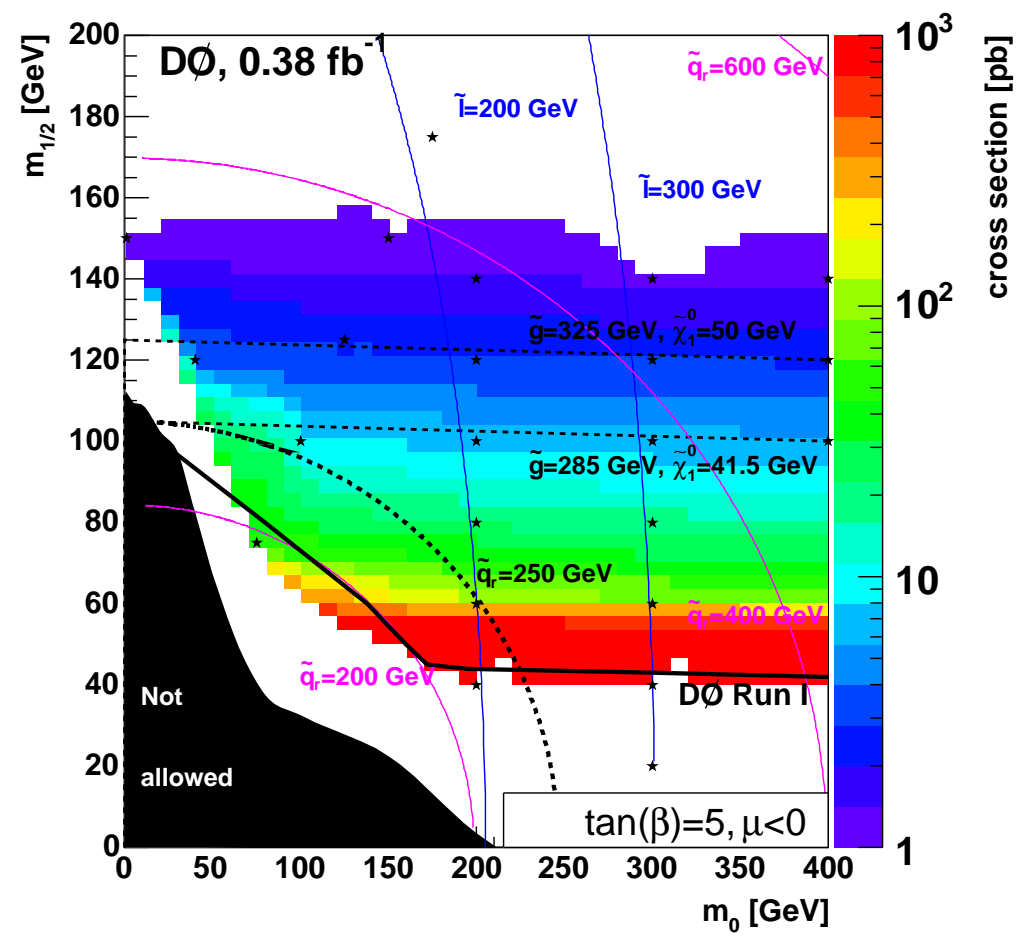

(b) cross section $\sigma[\mathrm{pb}]$

Figure 7.7: Gaugino pair and associated production cross section limit (a) and mSUGRA cross section (b). The shown cross section has been obtained with the parameters $\tan \beta=5, \mu<0$ and $A_{0}=0$. The cross section limit is independent of these mSUGRA parameters. The D $\varnothing$ Run I limit [82] excludes the parameter space below the solid black line for $\tan \beta=6, \mu<0$ and $A_{0}=0$. Note, that the color-coded $z$-axis is logarithmic. 


\subsubsection{Interpretation within mSUGRA}

To obtain an exclusion contour in the $m_{0}-m_{1 / 2}$ space and mass limits on the involved particles, the cross section limit contour (Fig.7.7a) is compared to the production cross section (Fig.7.7b) calculated for the mSUGRA parameters $\tan \beta=5, \mu<0$ and $A_{0}=0$. The intersection line of both planes outlines the excluded space, as shown in Fig. 7.8. The limit is approximately constant in $m_{1 / 2}$ since the signal selection efficiency as well as the signal cross section in this region depends mostly on $\mathrm{m}\left(\tilde{\chi}_{1}^{0}\right)$ and therefore $m_{1 / 2}$. The complete space between this limit contour and the previous D $\varnothing$ Run I limit can be excluded.

In Fig. 7.9 projections onto lines in the $m_{0}-m_{1 / 2}$ space are shown. The intersection points between the signal cross section and the cross section limit indicates the current limit. The $\mathrm{D} \varnothing$ Run I limit is indicated by the arrows. For low neutralino (or low squark) masses the cross section is large, but the signal selection efficiency is low, as discussed in Sec. 2.3.2 to be one of the challenges of this analysis. The cross section limit is dominated in this region by the signal statistics. The points with $m_{0}=200 \mathrm{GeV}$ (Fig. 7.9a) have been generated with high statistics, while the statistics for the points with $m_{0}=300 \mathrm{GeV}$ (Fig. 7.9b) is just enough, see also Tab. 6.16 for reference.

Neutralino masses below $41.5 \mathrm{GeV}$ and gluino masses below $285 \mathrm{GeV}$ are excluded, if any $L Q \bar{d}$ coupling $\lambda_{2 i j}^{\prime}$ with $j=1,2$ and $k=1,2,3$ is larger than 0.01 . This result is a significant extension of the Run I analysis [82] and is comparable to the LEP results, which limit the neutralino mass to $\mathrm{m}\left(\tilde{\chi}_{1}^{0}\right)>39 \mathrm{GeV}$ and the chargino mass to $\mathrm{m}\left(\tilde{\chi}_{1}^{ \pm}\right)$ $>103 \mathrm{GeV}$, corresponding to $\mathrm{m}\left(\tilde{\chi}_{1}^{0}\right) \gtrsim 50 \mathrm{GeV}$ within mSUGRA with $\tan \beta=5, \mu<0$ and $A_{0}=0$, see Sec. 2.4 for details. 


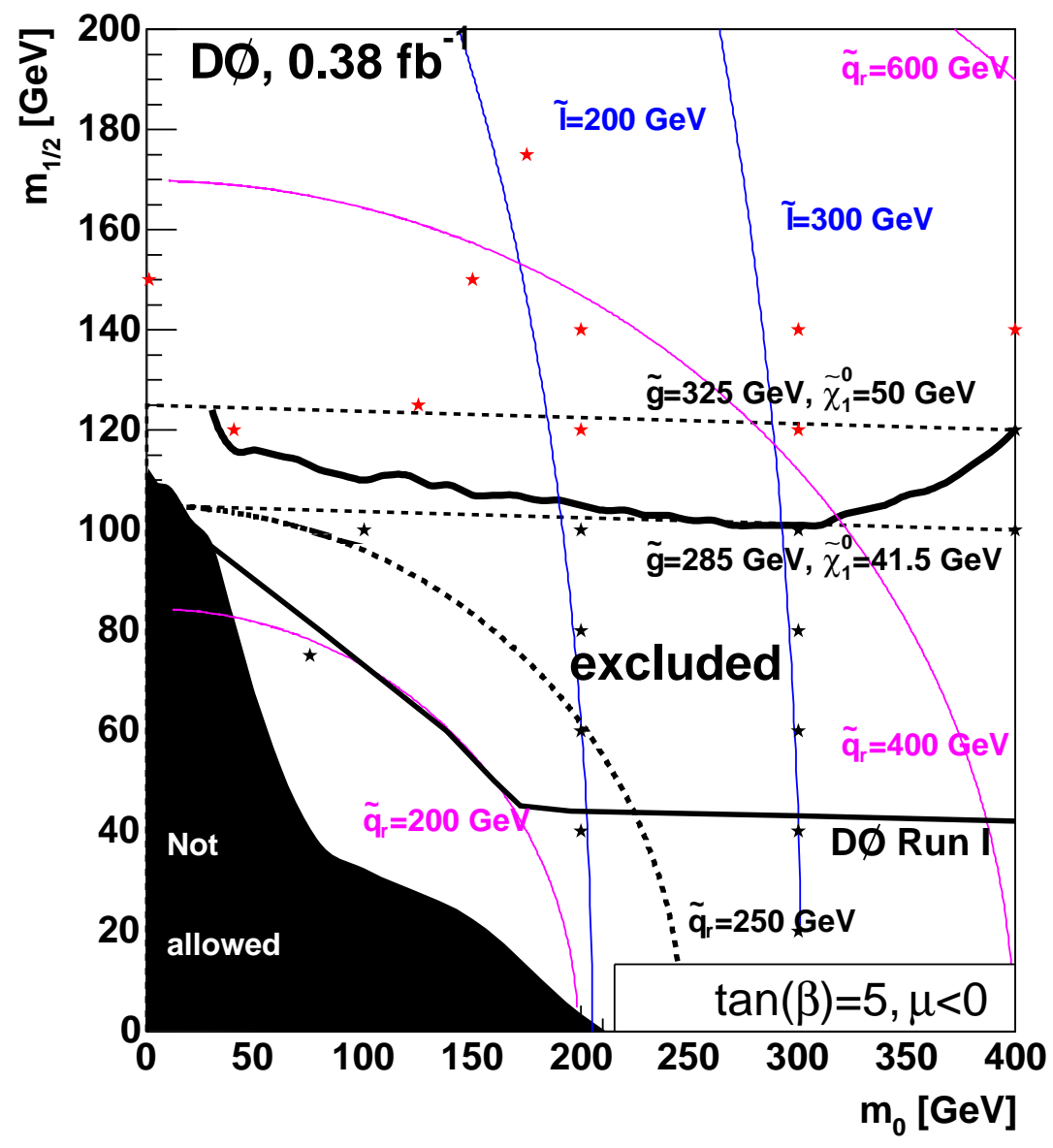

Figure 7.8: Exclusion contour in the mSUGRA $m_{0}-m_{1 / 2}$ plane with $\tan \beta=5, \mu<0$ and $A_{0}=0$ for all $\lambda_{2 i j}^{\prime}>0.01$ with $j=1,2$ and $k=1,2,3$. The D $\emptyset$ Run I limit calculated for $\tan \beta=6, \mu<0$ and $A_{0}=0$ is from [82]. The space below the solid black line is excluded by this analysis. The markers refer to the points where signal events have been generated; the marker color indicates wether a point can be excluded (black) or not (red). The various iso-mass lines have been calculated with [43] and cross checked with [182]. For small values of $m_{0} \lesssim 40 \mathrm{GeV}$ no statement can be made; the parameter space is limited here only by D $\varnothing$ Run I. In the black indicated region the $\tilde{\tau}$ can be the LSP and no electroweak symmetry breaking occurs. 


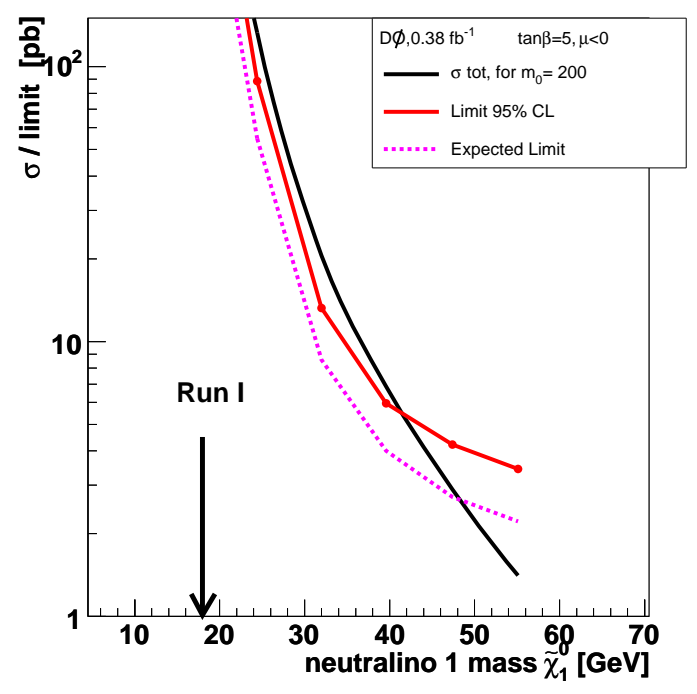

(a)

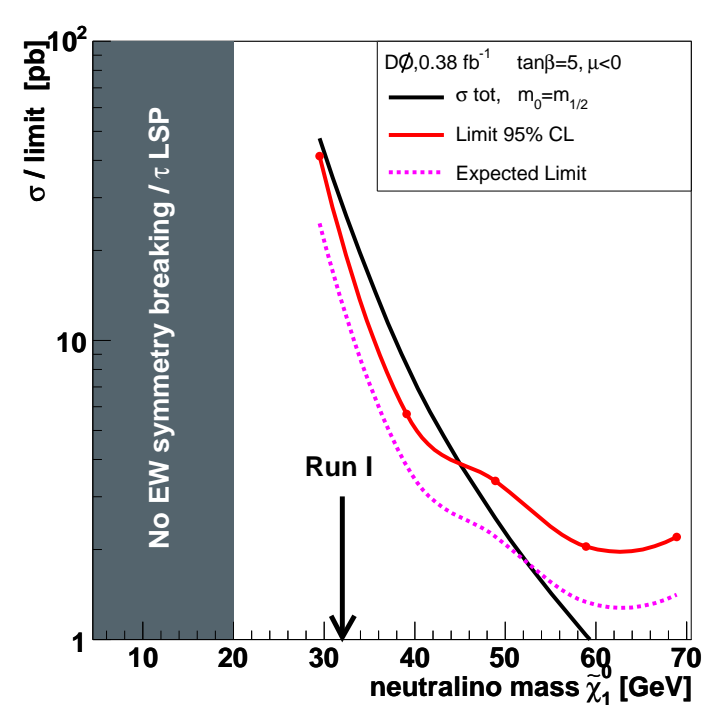

(c)

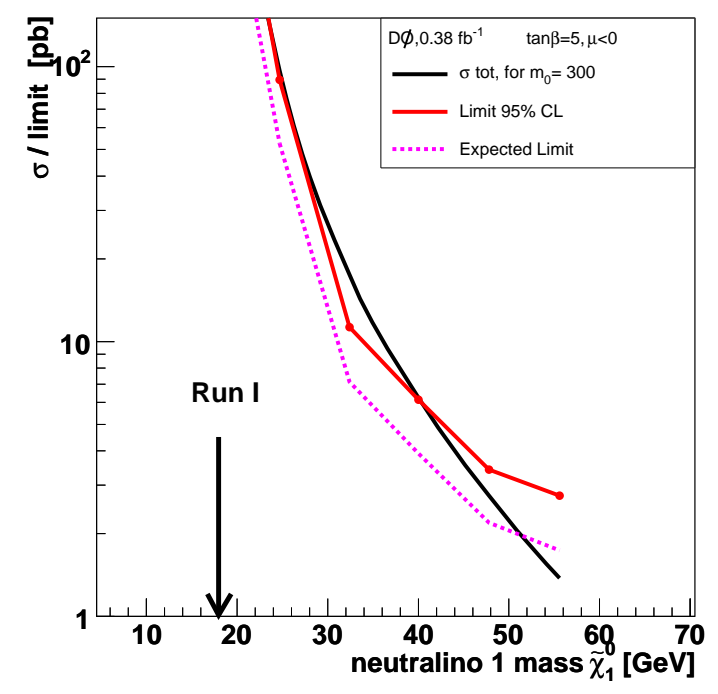

(b)

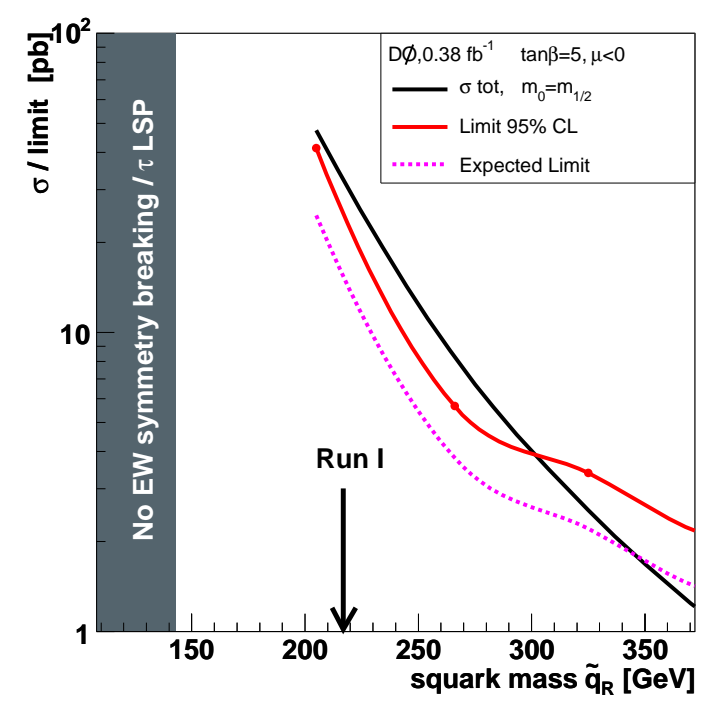

(d)

Figure 7.9: Mass limits on the lightest neutralino $\tilde{\chi}_{1}^{0}$ for $m_{0}=200 \mathrm{GeV}$ (a), for $m_{0}=300 \mathrm{GeV}$ (b) and for $m_{0}=m_{1 / 2}(\mathrm{c})$, and the mass limit on the right handed down squark $\tilde{d}_{R}$ mass for $m_{0}=m_{1 / 2}(\mathrm{~d})$. Masses and cross sections have been calculated within mSUGRA with $\tan \beta=5$, $\mu<0$ and $A_{0}=0$. The given limits are valid for all $\lambda_{2 i j}^{\prime}>0.01$ with $j=1,2$ and $k=1,2,3$. For low neutralino masses the cross section limits are dominated by the signal statistics. The signal points with $m_{0}=200 \mathrm{GeV}\left(m_{0}=300 \mathrm{GeV}\right)$ have been generated with high (just enough) statistics. 


\section{Chapter 8}

\section{Conclusions}

In this thesis different searches for R-parity violating supersymmetry in the DØ Run II data using $375 \mathrm{pb}^{-1}$ of integrated luminosity were presented:

1. For resonant production of $2^{\text {nd }}$ generation sleptons via the $L Q \bar{d}$ coupling $\lambda_{211}^{\prime}$, in final states with at least two jets and two muons, agreement between the data and the Standard Model expectation is observed, and model-independent limits as a function of gaugino and slepton masses have been set. The typical limit for each signal channel $\tilde{\mu} \rightarrow \tilde{\chi}_{1}^{0} \mu$, $\tilde{\mu} \rightarrow \tilde{\chi}_{2,(3,4)}^{0} \mu$, and $\tilde{\nu}_{\mu} \rightarrow \tilde{\chi}_{1,(2)}^{ \pm} \mu$ on the signal cross section is lower than $4 \mathrm{pb}$ if the lightest neutralino mass exceeds $80 \mathrm{GeV}$.

Within mSUGRA with $\tan \beta=5$ and $\mu<0$ the three separately analyzed signal channels $\tilde{\mu} \rightarrow \tilde{\chi}_{1}^{0} \mu, \tilde{\mu} \rightarrow \tilde{\chi}_{2,(3,4)}^{0} \mu$, and $\tilde{\nu}_{\mu} \rightarrow \tilde{\chi}_{1,(2)}^{ \pm} \mu$ have been combined and the best constraints to date on the coupling strength $\lambda_{211}^{\prime}$ have been derived. D $\varnothing$ Run I excluded slepton masses up to $280 \mathrm{GeV}$ for $\lambda_{211}^{\prime}=0.09$ and $m\left(\tilde{\chi}_{1}^{0}\right)=200 \mathrm{GeV}$. Now, for example slepton masses up to $358 \mathrm{GeV}$ can be excluded, for $\lambda_{211}^{\prime}=0.09$, independent of other sparticle masses. The point with $\mathrm{m}(\tilde{l})=260 \mathrm{GeV}$ and $\mathrm{m}\left(\tilde{\chi}_{1}^{0}\right)=100 \mathrm{GeV}$ or $m_{0}=200 \mathrm{GeV}, m_{1 / 2}=243 \mathrm{GeV}$, $\tan \beta=5$ and $\mu<0$ was used in the Run I analysis [89, 92] and in this analysis as a reference point. For this specific point, the Run I analysis was able to exclude $\lambda_{211}^{\prime}$ coupling parameters larger than 0.09 , and now a limit of $\lambda_{211}^{\prime} \leq 0.03$ can be achieved. This translates into an improvement of $1: 10$ with respect to a cross section limit, since $\sigma \propto \lambda_{211}^{\prime}{ }^{2}$.

2. The search for the $R$-parity conserving pair and associated production of gauginos and their decay via any $R$-parity violating $L Q \bar{d}$ coupling $\lambda_{2 j k}^{\prime}$ with $j=1,2$ and $k=1,2,3$ into a final state consisting of two (like-sign) muons and jets has been performed on the same $375 \mathrm{pb}^{-1}$ data sample as was used in (1). Since no significant excess is observed in the data, neutralino masses below $41.5 \mathrm{GeV}$ and gluino masses below $285 \mathrm{GeV}$ can be excluded within mSUGRA with $\tan \beta=5$ and $\mu<0$, if any $L Q \bar{d}$ coupling $\lambda_{2 j k}^{\prime}$ with $j=1,2$ and $k=1,2,3$ is larger than 0.01 . This result is a significant improvement over the Run I analysis [82] and is comparable to the LEP limits. 
For the $L Q \bar{d}$ coupling $\lambda_{211}^{\prime}$ larger than 0.01 , the results of both analysis strategies can be compared: The second analysis of the pair and the associated production of gauginos (2) is sensitive to smaller neutralino masses compared to (1), since fewer particles are required in the final state. The analysis (2) is insensitive to the coupling strength (as long as it is larger than 0.01 ) because the production is $R$-parity conserving, allowing to exclude the parameter space $\mathrm{m}\left(\tilde{\chi}_{1}^{0}\right) \leq 41.5 \mathrm{GeV}$ for all couplings strengths $\lambda_{2 j k}^{\prime}>0.01$.

The resonant slepton analysis (1) on the other hand is covering a much larger SUSY parameter space. The cross section is proportional to $\left(\lambda_{211}^{\prime}\right)^{2}$, so that also parameter sets with large slepton masses could be probed and excluded depending on the size of the coupling strength. The obtained combined cross section limit is of the order of $1-2 \mathrm{pb}$ if the neutralino mass exceeds $50 \mathrm{GeV}$ and if the decay channels $\tilde{\mu} \rightarrow \tilde{\chi}_{2,(3,4)}^{0} \mu$, and $\tilde{\nu}_{\mu} \rightarrow \tilde{\chi}_{1,(2)}^{ \pm} \mu$ are kinematically allowed; $\mathrm{m}(\tilde{l})>2 \cdot \mathrm{m}\left(\tilde{\chi}_{1}^{0}\right)$. The obtained cross section limit from the pair and associated gaugino production analysis, of the order of $2 \mathrm{pb}$, is comparable to the resonant slepton analysis, if the neutralino mass is larger than $60 \mathrm{GeV}$. However, the gaugino production cross section is steeply falling with $\mathrm{m}\left(\tilde{\chi}_{1}^{0}\right)$ and is negligible compared to the resonant slepton production cross section, for all coupling strengths $\lambda_{211}^{\prime}$, to which the resonant slepton analysis is sensitive to.

To conclude, both analyses cover complementary regions in the SUSY parameter space. In the resonant slepton analysis model independent cross section limits for any slepton mass - neutralino mass combinations were derived, for coupling strengths $\lambda_{211}^{\prime}>0.01$, and interpreted within mSUGRA to give the tightest constraints to date with respect to $\lambda_{211}^{\prime}$. The resonant slepton analysis is insensitive to the SUSY parameter space $\mathrm{m}\left(\tilde{\chi}_{1}^{0}\right)<50 \mathrm{GeV}$. This parameter region can be excluded by the search for pair and associated production of gauginos for all $L Q \bar{d}$ couplings $\lambda_{2 j k}^{\prime}>0.01$ with $j=1,2$ and $k=1,2,3$.

Until 2009 the Tevatron is expected to deliver up to $8 \mathrm{fb}^{-1}$ integrated luminosity, which would multiply the utilized luminosity for this analysis by a factor of 20 . This will improve the sensitivity to smaller coupling strengths and could enable the discovery of $\not_{p}$ supersymmetry, or at least improve the limits. At the Large Hadron Collider (LHC), which is expected to see first collisions in 2007, the studied parameter space can be expanded to SUSY masses at the TeV scale. The seven times larger center of mass energy of $\sqrt{s}=14 \mathrm{TeV}$ at the LHC compared to the Tevatron, and an expected integrated luminosity of several $100 \mathrm{fb}^{-1}$ in the first years, will enable the discovery of supersymmetry, if this is realized in nature. The large LHC luminosity will allow to probe very small $R$ parity violating couplings, but it will be difficult to improve the Tevatron results for rather light slepton masses $\mathrm{m}(\tilde{l}) \lesssim 300 \mathrm{GeV}$, since a large number of multiple interactions and trigger limitations have to be expected. Moreover, the slepton production cross section at the $p p$-collider LHC, compared to the Tevatron $p \bar{p} \rightarrow \tilde{l}$ cross section, does not benefit equally for all $\mathrm{m}(\tilde{l})$ from the larger center of mass energy. For $\mathrm{m}(\tilde{l}) \lesssim 300 \mathrm{GeV}$ the ratio $\sigma_{\mathrm{LHC}} / \sigma_{\text {Tevatron }}$ is only $\approx 10$, but increasing for larger slepton masses, as shown in Fig. 8.1.

The search for $R_{p}$ supersymmetry will remain an exciting topic of research at the Tevatron and at future colliders. 


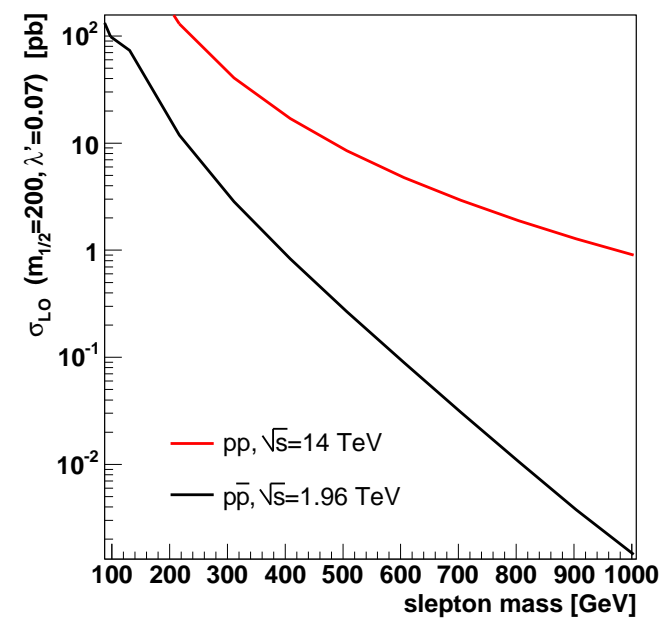

(a)

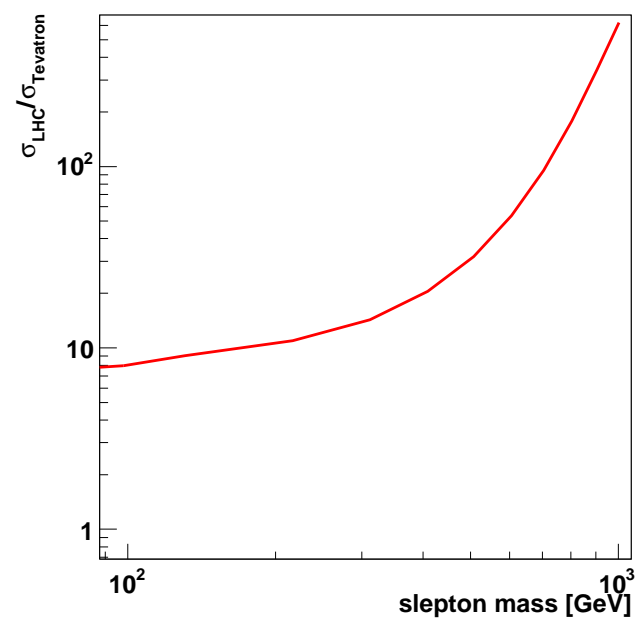

(b)

Figure 8.1: Resonant slepton cross section $\sigma_{L O}$ at the Tevatron and the LHC for a coupling strength $\lambda_{211}^{\prime}=0.07$ (a) and the ratio $\sigma_{\mathrm{LHC}} / \sigma_{\text {Tevatron }}(\mathrm{b})$. 



\section{Appendix A}

\section{Feynman Diagrams of the Signal}

\section{A.1 Neutralino Decay Channels}

In Fig. A.1 all possible decay modes of the Neutralino via the R-Parity violating coupling $\lambda_{211}^{\prime}$ are given.

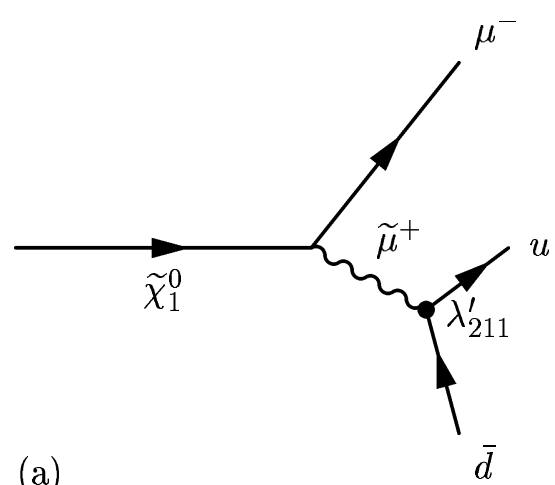

(a)

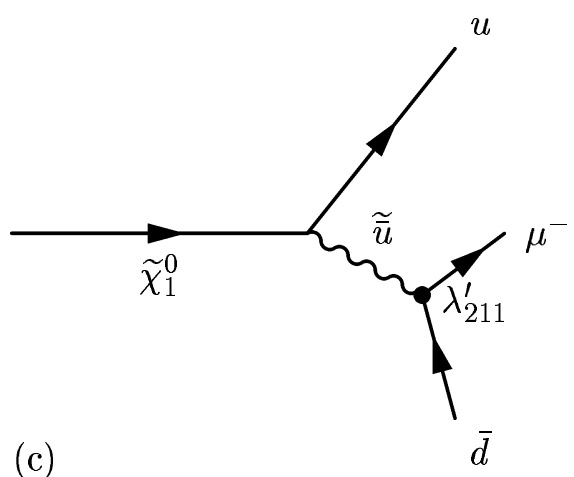

(b)

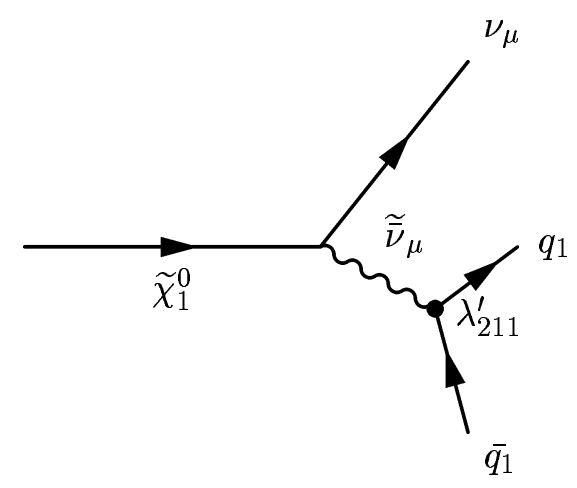

(d)

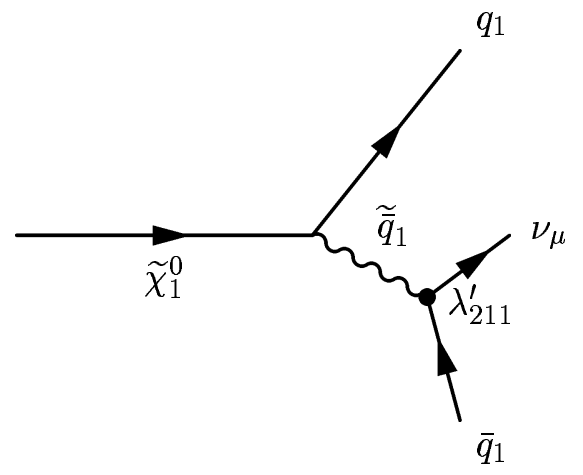




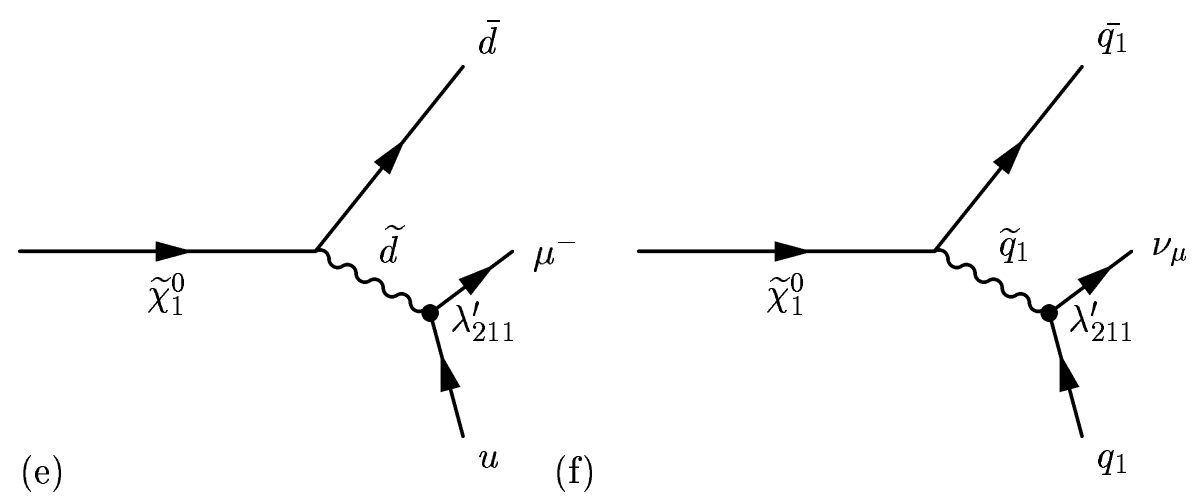

Figure A.1: Possible neutralino decay channels into one muon and two quarks (left) and into one neutrino and two quarks (right). The three decay channels (a),(c),(e) or (b),(d),(f) with similar final states are indistinguishable. 


\section{A.2 Chargino Decay Channels}

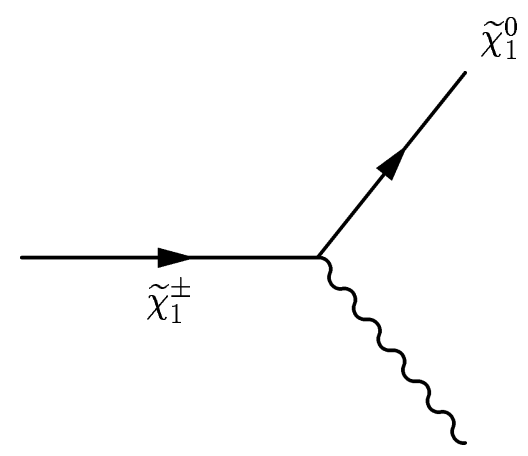

(a)

$$
W^{ \pm}
$$

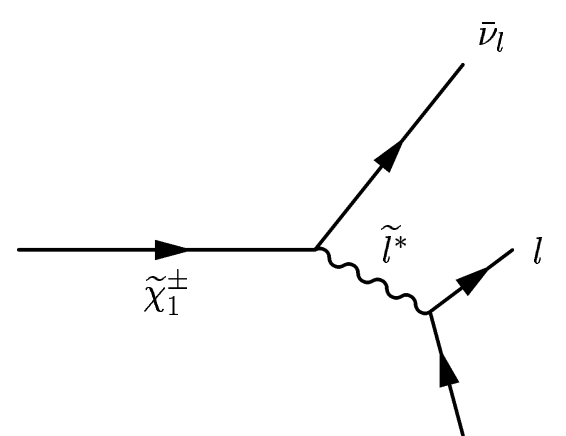

(b)

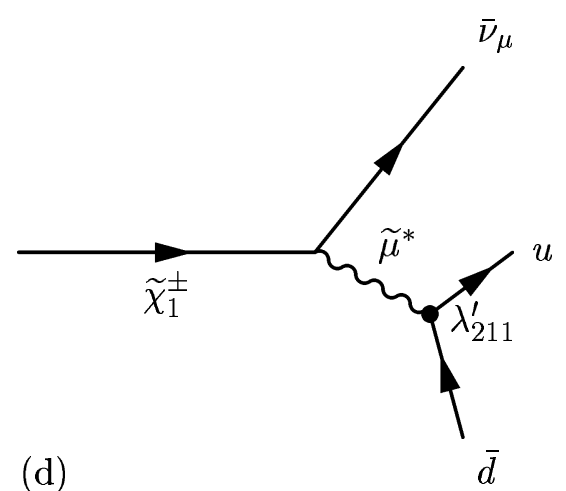

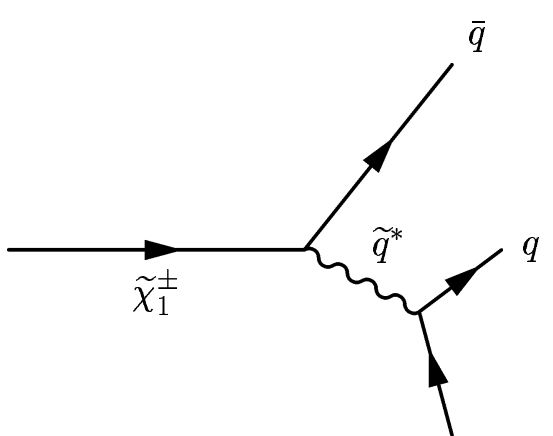

(c)
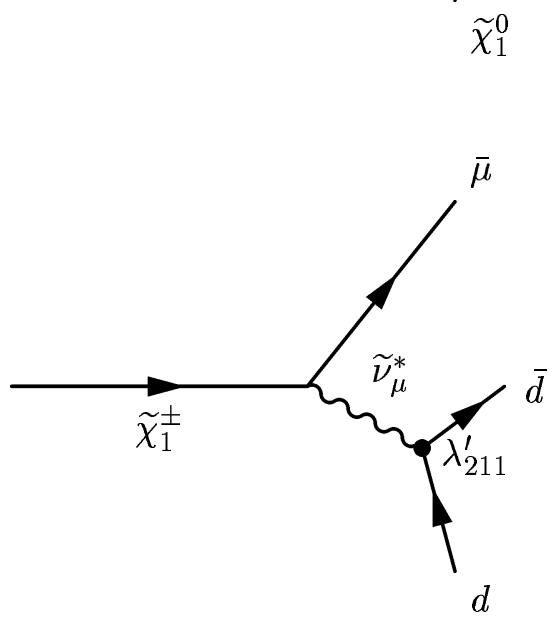

(e)

Figure A.2: Possible chargino decay channels, (a) is the dominant channel in large regions of the SUSY parameter space, while (b) and (c) are suppressed by the large slepton and squark mass and similarly the R-Parity violating decays via $\lambda_{211}^{\prime},(\mathrm{d})$ and (e). 


\section{A.3 Neutralino 2 Decay Channels}
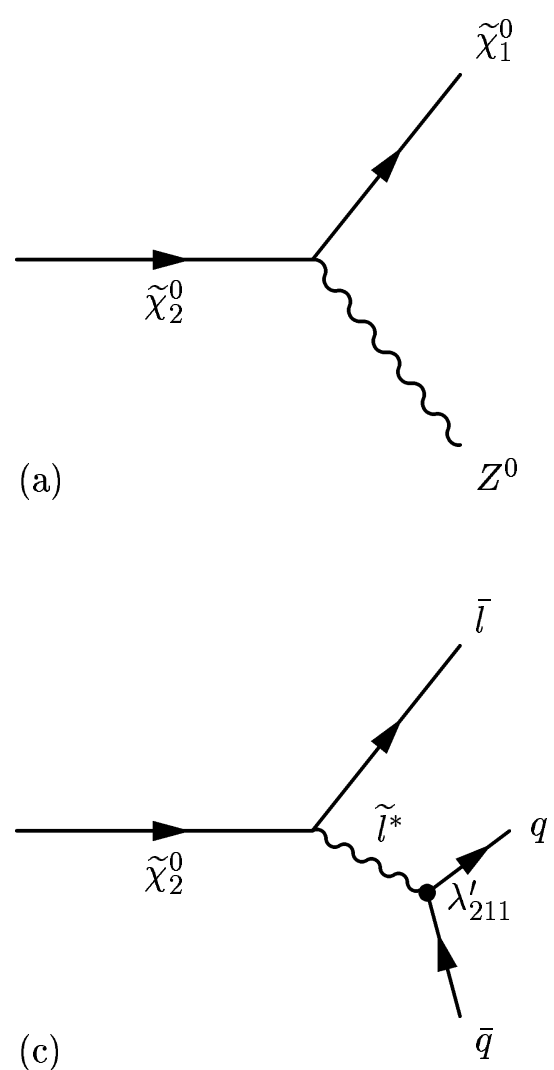

(b)

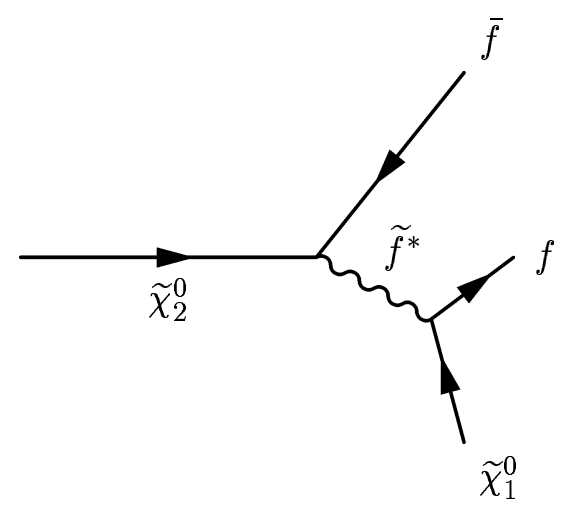

(d)

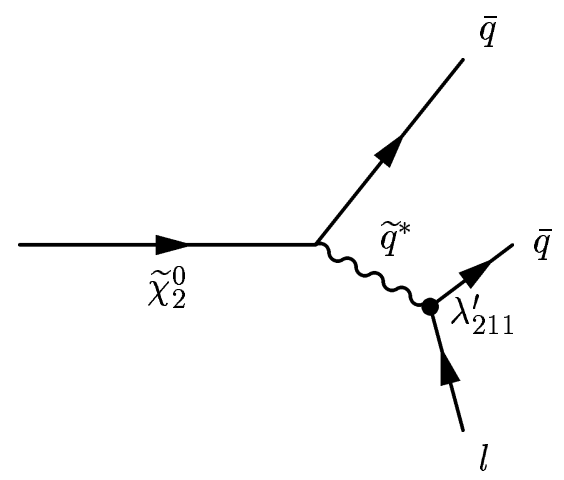

Figure A.3: Possible neutralino 2 decays channels, (a) is the dominant channel, while (b) is suppressed by the large slepton or squark mass and similarly the R-Parity violating decays via $\lambda_{211}^{\prime},(\mathrm{c})$ and $(\mathrm{d})$. 


\section{Appendix B}

\section{Susygen Modification}

The Susygen source code was changed, to enforce an absolute ID value for all Majorana particles [184]. This is important to ensure the decay of the $Z$-bosons that originate from $\tilde{\chi}_{2}^{0}$ cascade decays. The particle ID of the $Z$ may come out negative $(-23)$, if the $\tilde{\chi}_{2}^{0}$ was a (Majorana) anti-particle. An ID -23 particle is not recognized by the succeeding simulation programs Pythia and Geant as $Z$-boson and remains stable. This is fixed by the following Fortran code change to the SusYgen source code (susygen.f):

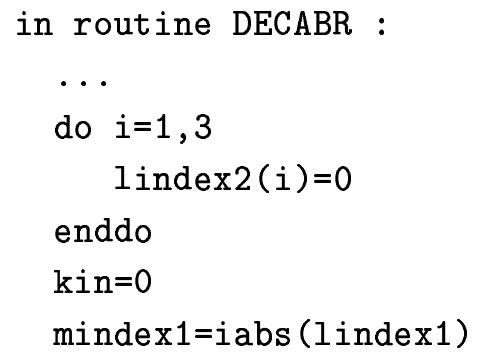





\section{Appendix $\mathrm{C}$}

\section{Final Results in Detail}

In this chapter the final results of the search for resonant slepton production are given in detail. The number of selected data events $d$ and the number of expected Standard Model and signal events are given for the three studied signal channels (i) $\tilde{\mu} \rightarrow \tilde{\chi}_{1}^{0} \mu$ in Sec. C.1, (ii) $\tilde{\mu} \rightarrow \tilde{\chi}_{2,(3,4)}^{0} \mu$ in Sec. C.2, and (iii) $\tilde{\nu}_{\mu} \rightarrow \tilde{\chi}_{1,(2)}^{ \pm} \mu$ in Sec. C.3. The number of selected signal events and the given next-to-leading order cross section correspond to a coupling strength $\lambda_{211}^{\prime}=0.07$. The $95 \%$ C.L. cross section limits have been calculated using the $\mathrm{CL}_{s}$ method discussed in Sec. 7.1. A modified version of TLimit was utilized [141].

\section{C.1 Channel $\tilde{\mu} \rightarrow \tilde{\chi}_{1}^{0} \mu$ with $\tan \beta=5$ and $\operatorname{sign}(\mu)=-1$}

\begin{tabular}{|c|c|c|c|c|c|c|c|}
\hline \# & $\begin{array}{r}\mathrm{m}(\tilde{\mu}) \\
{[\mathrm{GeV}]}\end{array}$ & $\begin{array}{l}\mathrm{m}\left(\tilde{\chi}_{1}^{0}\right) \\
{[\mathrm{GeV}]}\end{array}$ & $\begin{array}{c}\text { data } \\
\text { [evts] }\end{array}$ & $\begin{array}{l}\text { SM exp. } \\
\text { [events] }\end{array}$ & $\begin{array}{c}\text { signal exp. } \\
\text { [events] }\end{array}$ & $\begin{array}{r}\sigma_{N L O} \\
{[\mathrm{pb}]}\end{array}$ & $\begin{array}{r}\sigma_{95} \\
{[\mathrm{pb}]}\end{array}$ \\
\hline 0. & 113 & 60 & 0 & $1.7 \pm 0.8_{-0.6}^{+0.3}$ & $5.3 \pm 5.3_{-0.6}^{+0.5}$ & 68.9 & 59.4 \\
\hline 1. & 128 & 70 & 26 & $22.3 \pm 3.2_{-4.9}^{+4.7}$ & $59.3 \pm 14.0_{-16.7}^{+9.5}$ & 45.2 & 25.9 \\
\hline 2. & 157 & 89 & 57 & $50.4 \pm 4.4_{-12.3}^{+10.5}$ & $81.0 \pm 10.8_{-10.5}^{+9.7}$ & 23.2 & 7.7 \\
\hline 3. & 177 & 101 & 87 & $76.9 \pm 5.5_{-12.5}^{+24.2}$ & $75.9 \pm 8.2_{-11.8}^{+7.8}$ & 15.7 & 8.8 \\
\hline 4. & 197 & 114 & 46 & $45.0 \pm 4.1_{-11.5}^{+9.2}$ & $48.8 \pm 5.7_{-5.6}^{+5.1}$ & 10.8 & 5.1 \\
\hline 5. & 212 & 122 & 33 & $32.4 \pm 3.6_{-3.9}^{+15.4}$ & $30.3 \pm 3.8_{-5.1}^{+4.8}$ & 8.4 & 4.8 \\
\hline 6. & 190 & 106 & 66 & $64.9 \pm 4.9_{-14.7}^{+13.3}$ & $62.2 \pm 4.4_{-14.1}^{+7.9}$ & 12.5 & 8.2 \\
\hline 10. & 123 & 60 & 4 & $2.9 \pm 1.3_{-0.5}^{+0.5}$ & $16.3 \pm 8.1_{-8.8}^{+2.3}$ & 43.7 & 36.4 \\
\hline 11. & 141 & 72 & 40 & $31.9 \pm 3.6_{-8.1}^{+5.1}$ & $60.3 \pm 12.1_{-9.5}^{+12.4}$ & 34.2 & 12.5 \\
\hline 12. & 165 & 89 & 63 & $67.5 \pm 5.1_{-21.8}^{+8.9}$ & $82.7 \pm 10.3_{-10.2}^{+9.3}$ & 20.4 & 6.9 \\
\hline 13. & 177 & 97 & 78 & $77.7 \pm 5.5_{-20.4}^{+14.5}$ & $63.9 \pm 8.0_{-7.7}^{+6.9}$ & 15.9 & 9.1 \\
\hline 14. & 183 & 101 & 38 & $38.2 \pm 3.9_{-11.8}^{+10.2}$ & $53.1 \pm 7.0_{-14.3}^{+6.0}$ & 14.1 & 7.8 \\
\hline 15 . & 215 & 122 & 62 & $56.5 \pm 4.7_{-8.5}^{+10.6}$ & $48.1 \pm 4.7_{-9.1}^{+5.0}$ & 8.1 & 5.1 \\
\hline 16 . & 241 & 139 & 44 & $44.7 \pm 4.1_{-6.7}^{+9.1}$ & $35.7 \pm 3.3_{-3.9}^{+3.6}$ & 5.3 & 2.7 \\
\hline 17. & 263 & 154 & 54 & $45.7 \pm 4.1_{-9.2}^{+4.8}$ & $29.8 \pm 2.5_{-3.4}^{+3.0}$ & 3.8 & 3.2 \\
\hline 18. & 279 & 164 & 37 & $38.3 \pm 3.8_{-6.3}^{+6.1}$ & $28.0 \pm 2.2_{-3.5}^{+3.3}$ & 3.0 & 1.8 \\
\hline
\end{tabular}




\begin{tabular}{|c|c|c|c|c|c|c|c|}
\hline$\#$ & $\begin{array}{r}\tilde{\mu} \\
{[\mathrm{GeV}]} \\
\end{array}$ & $\begin{array}{r}\tilde{\chi}_{1}^{0} \\
{[\mathrm{GeV}]} \\
\end{array}$ & $\begin{array}{l}\text { data } \\
{[\text { evts }]}\end{array}$ & $\begin{array}{c}\text { SM exp. } \\
\text { [events] }\end{array}$ & $\begin{array}{c}\text { signal exp. } \\
\text { [events] }\end{array}$ & $\begin{array}{r}\sigma[p b] \\
{[\mathrm{pb}]} \\
\end{array}$ & $\begin{array}{r}\sigma_{95} \\
{[\mathrm{pb}]} \\
\end{array}$ \\
\hline 19. & 314 & 185 & 15 & $10.1 \pm 1.9_{-2.9}^{+2.2}$ & $13.2 \pm 1.2_{-1.5}^{+1.3}$ & 1.9 & 1.6 \\
\hline 22 . & 120 & 28 & 1 & $0.8 \pm 0.5_{-0.3}^{+0.3}$ & $6.7 \pm 6.7_{-2.5}^{+2.4}$ & 18.7 & 21.5 \\
\hline 23. & 129 & 40 & 3 & $3.5 \pm 1.1_{-0.7}^{+0.7}$ & $5.0 \pm 5.0_{-0.5}^{+0.5}$ & 14.6 & 30.6 \\
\hline 24. & 146 & 57 & 8 & $6.1 \pm 1.5_{-1.4}^{+0.9}$ & $10.6 \pm 5.3_{-1.6}^{+1.5}$ & 12.6 & 18.2 \\
\hline 25. & 150 & 61 & 14 & $10.4 \pm 1.9_{-1.4}^{+2.7}$ & $21.1 \pm 7.0_{-3.6}^{+3.3}$ & 12.4 & 8.7 \\
\hline 26. & 165 & 73 & 44 & $40.6 \pm 3.9_{-6.7}^{+8.7}$ & $33.9 \pm 5.0_{-10.5}^{+3.8}$ & 12.2 & 11.5 \\
\hline 27. & 186 & 89 & 37 & $31.4 \pm 3.4_{-5.1}^{+5.3}$ & $51.3 \pm 6.9_{-7.2}^{+6.7}$ & 11.6 & 5.1 \\
\hline 28. & 208 & 106 & 58 & $54.3 \pm 4.4_{-10.7}^{+9.3}$ & $48.6 \pm 3.4_{-5.7}^{+5.1}$ & 9.4 & 4.7 \\
\hline 29. & 232 & 122 & 49 & $51.7 \pm 4.5_{-9.5}^{+10.7}$ & $42.4 \pm 3.9_{-6.1}^{+4.6}$ & 6.4 & 3.3 \\
\hline 30. & 256 & 139 & 43 & $38.9 \pm 3.7_{-5.2}^{+5.5}$ & $35.2 \pm 3.0_{-6.9}^{+3.5}$ & 4.4 & 2.5 \\
\hline 31. & 277 & 154 & 20 & $13.0 \pm 2.2_{-1.8}^{+1.7}$ & $18.4 \pm 1.8_{-2.2}^{+2.5}$ & 3.2 & 2.6 \\
\hline 32. & 293 & 164 & 27 & $30.3 \pm 3.4_{-7.8}^{+3.1}$ & $22.4 \pm 1.7_{-2.6}^{+2.4}$ & 2.6 & 1.7 \\
\hline 33. & 324 & 185 & 12 & $16.3 \pm 2.5_{-2.3}^{+2.2}$ & $15.4 \pm 1.3_{-1.7}^{+1.6}$ & 1.7 & 0.8 \\
\hline 34. & 355 & 207 & 17 & $7.7 \pm 1.6_{-1.0}^{+1.6}$ & $9.9 \pm 0.8_{-1.1}^{+1.2}$ & 1.1 & 1.5 \\
\hline 35. & 419 & 249 & 1 & $1.9 \pm 0.9_{-1.3}^{+1.1}$ & $3.9 \pm 0.3_{-0.5}^{+0.4}$ & 0.5 & 0.4 \\
\hline 36. & 209 & 93 & 39 & $39.0 \pm 3.8_{-9.0}^{+5.2}$ & $24.9 \pm 2.6_{-3.2}^{+4.3}$ & 6.1 & 4.6 \\
\hline 37. & 187 & 61 & 2 & $0.7 \pm 0.5_{-0.3}^{+0.3}$ & $4.3 \pm 2.2_{-1.0}^{+0.9}$ & 4.1 & 7.3 \\
\hline 38. & 208 & 81 & 28 & $20.6 \pm 2.7_{-3.6}^{+3.5}$ & $16.3 \pm 2.2_{-3.1}^{+1.9}$ & 3.9 & 4.1 \\
\hline 41. & 180 & 41 & 3 & $0.9 \pm 0.6_{-0.2}^{+0.3}$ & $1.5 \pm 1.5_{-1.5}^{+0.5}$ & 4.1 & 43.4 \\
\hline 42. & 207 & 73 & 12 & $11.5 \pm 2.1_{-1.4}^{+3.5}$ & $8.6 \pm 1.7_{-1.2}^{+1.3}$ & 3.2 & 3.6 \\
\hline 43. & 233 & 98 & 16 & $13.7 \pm 2.2_{-3.3}^{+3.2}$ & $10.9 \pm 1.5_{-1.7}^{+1.5}$ & 3.2 & 4.1 \\
\hline 44. & 243 & 106 & 17 & $15.0 \pm 2.3_{-2.9}^{+3.3}$ & $18.9 \pm 2.7_{-3.4}^{+3.7}$ & 3.3 & 2.2 \\
\hline 45. & 263 & 123 & 12 & $7.6 \pm 1.6_{-1.0}^{+0.9}$ & $15.5 \pm 2.0_{-2.7}^{+2.3}$ & 3.2 & 2.2 \\
\hline 46. & 285 & 139 & 11 & $10.0 \pm 1.8_{-1.4}^{+1.1}$ & $16.7 \pm 1.7_{-1.7}^{+2.5}$ & 2.9 & 1.4 \\
\hline 47. & 318 & 165 & 17 & $19.0 \pm 2.7_{-4.1}^{+1.9}$ & $18.2 \pm 1.4_{-2.1}^{+2.0}$ & 1.9 & 1.2 \\
\hline 48. & 347 & 186 & 10 & $7.8 \pm 1.7_{-2.0}^{+2.1}$ & $12.3 \pm 1.0_{-1.4}^{+1.3}$ & 1.3 & 0.9 \\
\hline 49. & 376 & 207 & 8 & $3.6 \pm 1.1_{-0.7}^{+0.7}$ & $8.7 \pm 0.7_{-1.0}^{+0.9}$ & 0.9 & 0.9 \\
\hline 50. & 304 & 154 & 9 & $10.9 \pm 2.1_{-1.7}^{+1.7}$ & $14.7 \pm 0.9_{-1.9}^{+2.0}$ & 2.3 & 1.1 \\
\hline 51. & 208 & 61 & 5 & $1.5 \pm 0.8_{-0.4}^{+0.4}$ & $0.9 \pm 0.5_{-0.4}^{+0.3}$ & 2.5 & 51.2 \\
\hline 52. & 206 & 53 & 1 & $0.9 \pm 0.6_{-0.3}^{+0.3}$ & $0.8 \pm 0.6_{-0.6}^{+0.3}$ & 2.5 & 25.4 \\
\hline 53. & 239 & 90 & 17 & $11.7 \pm 2.0_{-1.8}^{+3.3}$ & $11.7 \pm 2.3_{-1.8}^{+1.7}$ & 2.2 & 2.7 \\
\hline 54. & 207 & 41 & 0 & $1.5 \pm 0.8_{-0.8}^{+0.8}$ & $0.3 \pm 0.3{ }_{-0.1}^{+0.0}$ & 2.4 & 33.5 \\
\hline 57. & 229 & 61 & 0 & $0.5 \pm 0.4_{-0.1}^{+0.1}$ & $3.2 \pm 1.3_{-1.6}^{+0.7}$ & 1.6 & 2.1 \\
\hline 58. & 263 & 99 & 14 & $11.9 \pm 2.1_{-1.6}^{+1.5}$ & $9.9 \pm 0.8_{-1.2}^{+1.2}$ & 1.5 & 1.5 \\
\hline 59. & 453 & 250 & 2 & $0.6 \pm 0.4_{-0.6}^{+1.1}$ & $2.5 \pm 0.2_{-0.4}^{+0.3}$ & 0.4 & 0.6 \\
\hline 60. & 574 & 335 & 1 & $0.5 \pm 0.4_{-0.2}^{+0.5}$ & $0.3 \pm 0.0_{-0.0}^{+0.0}$ & 0.1 & 0.9 \\
\hline 62. & 256 & 74 & 4 & $3.0 \pm 1.0_{-0.6}^{+0.6}$ & $3.3 \pm 0.7_{-0.4}^{+0.4}$ & 1.1 & 2.0 \\
\hline 63. & 270 & 90 & 5 & $2.2 \pm 0.9_{-0.5}^{+0.2}$ & $3.2 \pm 1.3_{-0.7}^{+0.8}$ & 1.1 & 3.6 \\
\hline 64. & 286 & 106 & 7 & $3.1 \pm 1.0_{-0.4}^{+0.3}$ & $5.3 \pm 0.7_{-0.9}^{+0.5}$ & 1.1 & 1.9 \\
\hline 65. & 303 & 123 & 9 & $5.3 \pm 1.3_{-0.7}^{+0.5}$ & $5.2 \pm 0.6_{-0.6}^{+0.5}$ & 1.1 & 1.7 \\
\hline 66. & 322 & 140 & 6 & $6.9 \pm 1.5_{-0.8}^{+0.8}$ & $6.5 \pm 0.9_{-0.7}^{+1.7}$ & 1.1 & 0.9 \\
\hline
\end{tabular}




\begin{tabular}{|c|c|c|c|c|c|c|c|}
\hline$\#$ & $\begin{array}{r}\tilde{\mu} \\
{[\mathrm{GeV}]}\end{array}$ & $\begin{array}{r}\tilde{\chi}_{1}^{0} \\
{[\mathrm{GeV}]}\end{array}$ & $\begin{array}{l}\text { data } \\
{[\text { evts }]}\end{array}$ & $\begin{array}{l}\text { SM exp. } \\
\text { [events] }\end{array}$ & $\begin{array}{l}\text { signal exp. } \\
\text { [events] }\end{array}$ & $\begin{array}{r}\sigma[p b] \\
{[\mathrm{pb}]}\end{array}$ & $\begin{array}{r}\sigma_{95} \\
{[\mathrm{pb}]} \\
\end{array}$ \\
\hline 67. & 339 & 154 & 5 & $6.7 \pm 1.6_{-0.9}^{+0.9}$ & $5.1 \pm 0.7_{-0.7}^{+0.6}$ & 1.1 & 1.1 \\
\hline 68. & 352 & 165 & 6 & $5.8 \pm 1.5_{-0.7}^{+0.7}$ & $6.0 \pm 0.7_{-0.7}^{+0.6}$ & 1.0 & 1.0 \\
\hline 69. & 378 & 186 & 7 & $2.9 \pm 1.0_{-0.6}^{+0.6}$ & $3.9 \pm 0.6_{-0.4}^{+0.4}$ & 0.9 & 2.1 \\
\hline 70. & 405 & 207 & 5 & $3.5 \pm 1.1_{-0.6}^{+0.5}$ & $4.9 \pm 0.4_{-0.6}^{+0.7}$ & 0.7 & 0.8 \\
\hline 71. & 302 & 107 & 7 & $6.1 \pm 1.5_{-1.2}^{+1.2}$ & $4.3 \pm 0.6{ }_{-0.5}^{+0.5}$ & 0.8 & 1.2 \\
\hline 72 . & 274 & 62 & 0 & $0.4 \pm 0.4_{-0.4}^{+0.4}$ & $2.6 \pm 0.9_{-0.5}^{+0.5}$ & 0.7 & 0.8 \\
\hline 73. & 326 & 123 & 2 & $2.8 \pm 1.0_{-1.7}^{+0.4}$ & $4.1 \pm 0.7_{-1.1}^{+1.0}$ & 0.7 & 0.7 \\
\hline 74. & 303 & 90 & 2 & $0.0 \pm 0.0_{-0.0}^{+0.3}$ & $1.7 \pm 0.3_{-0.3}^{+0.2}$ & 0.6 & 1.5 \\
\hline 75. & 366 & 155 & 3 & $3.6 \pm 1.2_{-0.5}^{+1.4}$ & $3.0 \pm 0.5_{-0.3}^{+0.3}$ & 0.6 & 0.9 \\
\hline 76. & 378 & 165 & 8 & $4.7 \pm 1.3{ }_{-0.9}^{+0.9}$ & $4.3 \pm 0.5_{-0.4}^{+0.4}$ & 0.6 & 1.4 \\
\hline 77. & 300 & 74 & 0 & $0.5 \pm 0.5_{-0.2}^{+0.1}$ & $0.6 \pm 0.2_{-0.1}^{+0.1}$ & 0.5 & 2.9 \\
\hline 78. & 299 & 58 & 0 & $0.0 \pm 0.0_{-0.0}^{+0.1}$ & $0.3 \pm 0.2_{-0.1}^{+0.1}$ & 0.5 & 3.5 \\
\hline 79 . & 301 & 42 & 1 & $0.3 \pm 0.3{ }_{-0.3}^{+0.3}$ & $0.1 \pm 0.1_{-0.0}^{+0.0}$ & 0.5 & 51.7 \\
\hline 81. & 327 & 74 & 0 & $0.0 \pm 0.0_{-0.0}^{+0.1}$ & $0.1 \pm 0.1_{-0.1}^{+0.1}$ & 0.3 & 6.4 \\
\hline 82. & 338 & 91 & 0 & $0.4 \pm 0.4_{-0.1}^{+0.1}$ & $0.3 \pm 0.2_{-0.1}^{+0.1}$ & 0.3 & 3.9 \\
\hline 83. & 365 & 124 & 0 & $1.5 \pm 0.8_{-0.3}^{+0.3}$ & $1.1 \pm 0.3_{-0.1}^{+0.2}$ & 0.3 & 0.7 \\
\hline 84. & 381 & 140 & 0 & $0.5 \pm 0.5_{-0.1}^{+0.2}$ & $0.7 \pm 0.2_{-0.1}^{+0.1}$ & 0.3 & 1.3 \\
\hline 85. & 396 & 155 & 0 & $0.0 \pm 0.0_{-0.0}^{+0.1}$ & $0.3 \pm 0.1_{-0.2}^{+0.0}$ & 0.3 & 3.8 \\
\hline 86. & 407 & 166 & 2 & $1.4 \pm 0.8_{-0.2}^{+0.2}$ & $0.9 \pm 0.2_{-0.1}^{+0.1}$ & 0.3 & 1.5 \\
\hline 87. & 430 & 187 & 0 & $0.0 \pm 0.0_{-0.0}^{+0.3}$ & $0.9 \pm 0.2_{-0.2}^{+0.1}$ & 0.3 & 0.7 \\
\hline 88. & 454 & 208 & 2 & $2.7 \pm 1.0_{-0.4}^{+0.4}$ & $1.7 \pm 0.2_{-0.2}^{+0.2}$ & 0.3 & 0.6 \\
\hline 89. & 505 & 250 & 1 & $0.5 \pm 0.5_{-0.5}^{+0.8}$ & $0.7 \pm 0.1_{-0.1}^{+0.1}$ & 0.2 & 1.0 \\
\hline 90. & 354 & 105 & 6 & $0.4 \pm 0.1_{-0.4}^{+0.5}$ & $1.7 \pm 0.3_{-0.2}^{+0.2}$ & 0.3 & 1.2 \\
\hline 91. & 345 & 74 & 0 & $0.0 \pm 0.0_{-0.0}^{+0.0}$ & $0.1 \pm 0.1_{-0.1}^{+0.0}$ & 0.3 & 5.3 \\
\hline 93. & 373 & 74 & 0 & $0.5 \pm 0.5_{-0.1}^{+0.1}$ & $0.4 \pm 0.1_{-0.1}^{+0.1}$ & 0.2 & 1.4 \\
\hline 95. & 407 & 124 & 0 & $0.6 \pm 0.5_{-0.2}^{+0.2}$ & $0.7 \pm 0.2_{-0.1}^{+0.1}$ & 0.2 & 0.5 \\
\hline 96. & 421 & 141 & 2 & $0.5 \pm 0.3_{-0.1}^{+0.5}$ & $0.5 \pm 0.1_{-0.2}^{+0.1}$ & 0.2 & 2.2 \\
\hline 97. & 403 & 105 & 0 & $0.1 \pm 0.0_{-0.0}^{+0.0}$ & $0.2 \pm 0.1_{-0.0}^{+0.1}$ & 0.1 & 1.5 \\
\hline 99. & 415 & 62 & 0 & $0.0 \pm 0.0_{-0.0}^{+0.0}$ & $0.1 \pm 0.1_{-0.0}^{+0.0}$ & 0.1 & 4.9 \\
\hline 100. & 421 & 75 & 0 & $0.0 \pm 0.0_{-0.0}^{+0.0}$ & $0.1 \pm 0.1_{-0.0}^{+0.0}$ & 0.1 & 2.3 \\
\hline 101. & 437 & 103 & 0 & $0.8 \pm 0.6_{-0.1}^{+0.1}$ & $0.4 \pm 0.1_{-0.1}^{+0.1}$ & 0.1 & 0.7 \\
\hline 102. & 451 & 124 & 1 & $0.4 \pm 0.3_{-0.4}^{+0.5}$ & $0.2 \pm 0.1_{-0.1}^{+0.1}$ & 0.1 & 2.2 \\
\hline 103. & 485 & 166 & 0 & $0.0 \pm 0.0_{-0.0}^{+0.2}$ & $0.1 \pm 0.0_{-0.0}^{+0.0}$ & 0.1 & 2.0 \\
\hline 104. & 504 & 187 & 0 & $0.0 \pm 0.0_{-0.0}^{+0.0}$ & $0.2 \pm 0.1_{-0.1}^{+0.0}$ & 0.1 & 1.6 \\
\hline 105. & 525 & 209 & 0 & $0.5 \pm 0.5_{-0.5}^{+0.1}$ & $0.3 \pm 0.1_{-0.1}^{+0.0}$ & 0.1 & 0.7 \\
\hline 106. & 570 & 251 & 2 & $0.2 \pm 0.1_{-0.2}^{+1.2}$ & $0.3 \pm 0.0_{-0.0}^{+0.0}$ & 0.1 & 0.9 \\
\hline 107. & 468 & 75 & 0 & $0.0 \pm 0.0_{-0.0}^{+0.0}$ & $0.0 \pm 0.0_{-0.0}^{+0.0}$ & 0.1 & 11.9 \\
\hline 108. & 483 & 104 & 0 & $0.0 \pm 0.0_{-0.0}^{+0.0}$ & $0.1 \pm 0.0_{-0.0}^{+0.0}$ & 0.0 & 1.9 \\
\hline 109. & 506 & 42 & 7 & $2.2 \pm 0.9_{-0.4}^{+0.4}$ & $0.0 \pm 0.0_{-0.0}^{+0.0}$ & 0.0 & 68.2 \\
\hline 110. & 516 & 75 & 0 & $0.0 \pm 0.0_{-0.0}^{+0.0}$ & $0.0 \pm 0.0_{-0.0}^{+0.0}$ & 0.0 & 10.2 \\
\hline 111. & 524 & 92 & 0 & $0.0 \pm 0.0_{-0.0}^{+0.0}$ & $0.0 \pm 0.0_{-0.0}^{+0.0}$ & 0.0 & 4.5 \\
\hline
\end{tabular}




\begin{tabular}{|c|c|c|c|c|c|c|c|}
\hline \# & $\begin{array}{r}\tilde{\mu} \\
{[\mathrm{GeV}]}\end{array}$ & $\begin{array}{r}\tilde{\chi}_{1}^{0} \\
{[\mathrm{GeV}]}\end{array}$ & $\begin{array}{l}\text { data } \\
{[\text { evts] }}\end{array}$ & $\begin{array}{l}\text { SM exp. } \\
\text { [events] }\end{array}$ & $\begin{array}{c}\text { signal exp. } \\
\text { [events] }\end{array}$ & $\begin{array}{r}\sigma[p b] \\
{[\mathrm{pb}]} \\
\end{array}$ & $\begin{array}{r}\sigma_{95} \\
{[\mathrm{pb}]} \\
\end{array}$ \\
\hline 112. & 530 & 104 & 1 & $0.0 \pm 0.0_{-0.0}^{+0.2}$ & $0.0 \pm 0.0_{-0.0}^{+0.0}$ & 0.0 & 3.1 \\
\hline 113. & 541 & 125 & 1 & $0.0 \pm 0.0_{-0.0}^{+0.3}$ & $0.0 \pm 0.0_{-0.0}^{+0.0}$ & 0.0 & 2.9 \\
\hline 114. & 562 & 156 & 0 & $0.0 \pm 0.0_{-0.0}^{+0.0}$ & $0.1 \pm 0.0_{-0.0}^{+0.0}$ & 0.0 & 0.7 \\
\hline 115. & 570 & 167 & 0 & $0.0 \pm 0.0_{-0.0}^{+0.0}$ & $0.1 \pm 0.0_{-0.0}^{+0.0}$ & 0.0 & 1.2 \\
\hline 116. & 605 & 209 & 0 & $0.0 \pm 0.0_{-0.0}^{+0.2}$ & $0.0 \pm 0.0_{-0.0}^{+0.0}$ & 0.0 & 1.6 \\
\hline 117. & 644 & 252 & 0 & $0.1 \pm 0.0_{-0.1}^{+0.0}$ & $0.1 \pm 0.0_{-0.0}^{+0.0}$ & 0.0 & 0.6 \\
\hline
\end{tabular}

Table C.1: Final Results for channel $\tilde{\mu} \rightarrow \tilde{\chi}_{1}^{0} \mu$ with $\tan \beta=5$ and $\operatorname{sign}(\mu)=-1$. The slepton and neutralino mass ( $2^{\text {nd }}$ and $3^{\text {rd }}$ column) are given in $\mathrm{GeV}$, the next-to-leading order cross section $\sigma=\sigma_{q q \rightarrow \tilde{\mu}}^{N L O} \cdot B R\left(\tilde{\mu} \rightarrow \mu \tilde{\chi}_{1}^{0}\right)$ as well as the $95 \%$ C.L. limit are given in pb (last two columns). If 0.049 or less events are expected, then 0.0 is stated, due to the finite column width. 


\section{C.2 Channel $\tilde{\mu} \rightarrow \tilde{\chi}_{2,(3,4)}^{0} \mu$ with $\tan \beta=5$ and $\operatorname{sign}(\mu)=-1$}

\begin{tabular}{|c|c|c|c|c|c|c|c|c|}
\hline$\#$ & $\begin{array}{r}\mathrm{m}(\tilde{\mu}) \\
{[\mathrm{GeV}]}\end{array}$ & $\begin{array}{l}\mathrm{m}\left(\tilde{\chi}_{2}^{0}\right) \\
{[\mathrm{GeV}]}\end{array}$ & $\begin{array}{l}\text { data } \\
{[\text { evts] }}\end{array}$ & $\begin{array}{l}\text { SM exp. } \\
\text { [events] }\end{array}$ & $\begin{array}{r}\text { signal ex] } \\
\text { [events] }\end{array}$ & & $\begin{array}{r}\sigma_{N L O} \\
{[\mathrm{pb}]}\end{array}$ & $\begin{array}{r}\sigma_{95} \\
{[\mathrm{pb}]} \\
\end{array}$ \\
\hline 0. & 113 & 111 & 1 & $1.4 \pm 0.6_{-0.9}^{+1.1}$ & & - & - & - \\
\hline 1. & 128 & 132 & 84 & $57.3 \pm 5.2_{-14.2}^{+12.9}$ & & - & - & - \\
\hline 2. & 157 & 170 & 6 & $5.6 \pm 1.5_{-1.8}^{+1.0}$ & & - & - & - \\
\hline 3. & 177 & 195 & 82 & $68.9 \pm 5.2_{-9.5}^{+22.2}$ & & - & - & - \\
\hline 4. & 197 & 220 & 9 & $4.1 \pm 1.3_{-0.8}^{+0.8}$ & & - & - & - \\
\hline 5. & 212 & 237 & 4 & $6.1 \pm 1.8_{-0.7}^{+0.7}$ & & - & - & 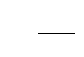 \\
\hline 6. & 190 & 204 & 0 & $0.0 \pm 0.0_{-0.0}^{+0.0}$ & & - & - & - \\
\hline 10. & 123 & 112 & 90 & $59.6 \pm 5.2_{-15.9}^{+12.1}$ & & - & - & - \\
\hline 11. & 141 & 137 & 41 & $26.1 \pm 3.9_{-5.2}^{+5.1}$ & & - & - & - \\
\hline 12. & 165 & 170 & 3 & $3.7 \pm 1.2_{-0.7}^{+1.1}$ & & - & - & 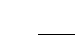 \\
\hline 13. & 177 & 187 & 12 & $7.2 \pm 2.1_{-1.6}^{+1.5}$ & & - & - & - \\
\hline 14. & 183 & 195 & 111 & $74.3 \pm 5.8_{-20.0}^{+13.9}$ & & - & 一 & 一 \\
\hline 15. & 215 & 237 & 47 & $45.6 \pm 3.9_{-8.4}^{+5.6}$ & & - & - & - \\
\hline 16. & 241 & 271 & 84 & $89.3 \pm 5.8_{-14.4}^{+16.9}$ & & - & - & - \\
\hline 17. & 263 & 300 & 47 & $44.7 \pm 4.1_{-10.5}^{+6.5}$ & & - & - & - \\
\hline 18. & 279 & 321 & 109 & $109.1 \pm 6.4_{-22.6}^{+17.6}$ & & - & - & - \\
\hline 19. & 314 & 363 & 8 & $12.0 \pm 2.3_{-2.3}^{+3.1}$ & & - & 一 & - \\
\hline 22 . & 120 & 44 & 0 & $0.1 \pm 0.0_{-0.0}^{+0.0}$ & & - & - & - \\
\hline 23. & 129 & 70 & 1 & $2.8 \pm 1.1_{-0.9}^{+0.4}$ & $14.3 \pm 8.3$ & $\begin{array}{l}+2.8 \\
-2.9\end{array}$ & 20.4 & 8.4 \\
\hline 24. & 146 & 104 & 2 & $0.9 \pm 0.7_{-0.9}^{+0.9}$ & $16.0 \pm 6.5$ & $\begin{array}{l}+4.6 \\
-4.7\end{array}$ & 11.1 & 4.3 \\
\hline 25. & 150 & 113 & 7 & $1.6 \pm 0.7_{-0.4}^{+3.6}$ & $14.7 \pm 6.0$ & $\begin{array}{l}+4.8 \\
-1.7\end{array}$ & 9.3 & 8.0 \\
\hline 26. & 165 & 138 & 27 & $16.7 \pm 2.8_{-2.8}^{+5.1}$ & $27.0 \pm 4.4$ & $\begin{array}{l}+5.1 \\
-5.3\end{array}$ & 5.0 & 3.8 \\
\hline 27. & 186 & 171 & 1 & $0.2 \pm 0.2_{-0.2}^{+0.3}$ & $1.1 \pm 1.1$ & $\begin{array}{l}+0.1 \\
{ }_{-0.1}\end{array}$ & 1.4 & 10.3 \\
\hline 28. & 208 & 204 & 4 & $1.3 \pm 0.7_{-0.1}^{+0.1}$ & $0.5 \pm 0.3$ & $\begin{array}{l}+0.2 \\
-0.2\end{array}$ & 0.1 & 3.4 \\
\hline 29. & 232 & 238 & 96 & $59.4 \pm 5.1_{-14.3}^{+17.2}$ & & - & - & - \\
\hline 30. & 256 & 271 & 10 & $5.5 \pm 1.7_{-1.4}^{+1.6}$ & & - & - & - \\
\hline 31. & 277 & 300 & 3 & $2.0 \pm 0.8_{-0.4}^{+0.4}$ & & - & 一 & - \\
\hline 32. & 293 & 321 & 57 & $40.8 \pm 3.9_{-5.5}^{+12.1}$ & & - & - & - \\
\hline 33. & 324 & 363 & 41 & $28.8 \pm 3.3_{-3.7}^{+8.1}$ & & - & 一 & - \\
\hline 34. & 355 & 405 & 2 & $1.8 \pm 0.6_{-0.8}^{+0.4}$ & & - & - & - \\
\hline 35. & 419 & 489 & 1 & $3.7 \pm 1.4_{-0.5}^{+0.5}$ & & - & - & - \\
\hline 36. & 209 & 180 & 2 & $1.7 \pm 0.3{ }_{-0.3}^{+0.3}$ & $4.7 \pm 1.1$ & $\begin{array}{l}+0.5 \\
-1.5\end{array}$ & 1.9 & 2.5 \\
\hline 37. & 187 & 113 & 2 & $0.0 \pm 0.0_{-0.0}^{+0.4}$ & $6.4 \pm 2.6$ & $\begin{array}{l}+0.7 \\
-1.5\end{array}$ & 5.5 & 5.0 \\
\hline 38. & 208 & 155 & 32 & $33.3 \pm 4.1_{-6.2}^{+6.1}$ & $25.0 \pm 2.8$ & $\begin{array}{l}+3.4 \\
-3.5\end{array}$ & 3.1 & 2.1 \\
\hline 41. & 180 & 72 & 0 & $0.7 \pm 0.5_{-0.3}^{+0.7}$ & $2.6 \pm 1.9$ & $\begin{array}{l}+0.9 \\
-1.6\end{array}$ & 7.4 & 16.6 \\
\hline 42. & 207 & 139 & 20 & $11.5 \pm 2.4_{-1.6}^{+2.7}$ & $13.7 \pm 2.1$ & $\begin{array}{l}+2.9 \\
-1.9\end{array}$ & 3.6 & 3.8 \\
\hline 43. & 233 & 189 & 6 & $4.5 \pm 1.6_{-1.0}^{+1.0}$ & $4.8 \pm 1.0$ & $\begin{array}{l}+1.1 \\
{ }_{-0.6}\end{array}$ & 1.7 & 2.8 \\
\hline 44. & 243 & 205 & 0 & $0.6 \pm 0.4_{-0.2}^{+0.2}$ & $1.9 \pm 1.1$ & $\begin{array}{l}+0.5 \\
{ }_{-0.5}\end{array}$ & 1.2 & 2.6 \\
\hline 45. & 263 & 239 & 0 & $0.0 \pm 0.0_{-0.0}^{+0.2}$ & $0.7 \pm 0.5$ & $\begin{array}{l}+0.2 \\
-0.2\end{array}$ & 0.5 & 2.8 \\
\hline
\end{tabular}




\begin{tabular}{|c|c|c|c|c|c|c|c|}
\hline$\#$ & $\begin{array}{r}\mathrm{m}(\tilde{\mu}) \\
{[\mathrm{GeV}]} \\
\end{array}$ & $\begin{array}{l}\mathrm{m}\left(\tilde{\chi}_{2}^{0}\right) \\
{[\mathrm{GeV}]} \\
\end{array}$ & $\begin{array}{l}\text { data } \\
{[\text { evts] }}\end{array}$ & $\begin{array}{l}\text { SM exp. } \\
\text { [events] }\end{array}$ & $\begin{array}{l}\text { signal exp. } \\
\text { [events] }\end{array}$ & $\begin{array}{r}\sigma_{N L O} \\
{[\mathrm{pb}]} \\
\end{array}$ & $\begin{array}{r}\sigma_{95} \\
{[\mathrm{pb}]} \\
\end{array}$ \\
\hline 46. & 285 & 272 & 0 & $0.0 \pm 0.0_{-0.0}^{+0.3}$ & $0.2 \pm 0.2_{-0.0}^{+0.0}$ & 0.1 & 2.2 \\
\hline 47. & 318 & 322 & 3 & $1.1 \pm 0.0_{-0.3}^{+0.3}$ & - & - & - \\
\hline 48. & 347 & 364 & 63 & $51.8 \pm 4.5_{-9.0}^{+10.7}$ & - & - & - \\
\hline 49 . & 376 & 406 & 43 & $40.9 \pm 3.8_{-6.1}^{+6.0}$ & - & - & - \\
\hline 50. & 304 & 301 & 0 & $0.1 \pm 0.0_{-0.1}^{+0.5}$ & $0.1 \pm 0.1_{-0.0}^{+0.0}$ & 0.0 & 0.4 \\
\hline 51. & 208 & 114 & 25 & $22.1 \pm 2.9_{-3.1}^{+4.9}$ & $16.7 \pm 2.3_{-2.0}^{+3.1}$ & 4.0 & 3.4 \\
\hline 52. & 206 & 98 & 10 & $8.8 \pm 1.8_{-1.5}^{+1.6}$ & $15.8 \pm 2.4_{-2.9}^{+2.7}$ & 4.4 & 2.5 \\
\hline 53. & 239 & 172 & 11 & $9.1 \pm 2.0_{-2.6}^{+1.5}$ & $7.5 \pm 1.9_{-0.9}^{+2.8}$ & 2.0 & 2.7 \\
\hline 54. & 207 & 73 & 7 & $7.9 \pm 1.7_{-1.5}^{+0.9}$ & $8.1 \pm 1.7_{-2.1}^{+1.6}$ & 4.6 & 4.9 \\
\hline 57. & 229 & 115 & 6 & $10.0 \pm 1.9_{-2.0}^{+1.9}$ & $13.0 \pm 2.6_{-2.1}^{+2.0}$ & 2.9 & 1.5 \\
\hline 58. & 263 & 192 & 28 & $25.4 \pm 3.2_{-4.2}^{+6.7}$ & $7.3 \pm 1.3_{-1.1}^{+1.6}$ & 1.4 & 4.5 \\
\hline 59 . & 453 & 490 & 59 & $72.3 \pm 5.1_{-15.7}^{+8.0}$ & - & - & - \\
\hline 60. & 574 & 658 & 13 & $8.3 \pm 1.6_{-1.1}^{+2.5}$ & - & - & - \\
\hline 62. & 256 & 140 & 21 & $22.9 \pm 2.9_{-5.3}^{+4.7}$ & $11.0 \pm 1.3_{-1.7}^{+1.6}$ & 1.9 & 2.4 \\
\hline 63. & 270 & 173 & 3 & $5.3 \pm 1.4_{-1.1}^{+1.1}$ & $4.7 \pm 1.6_{-1.1}^{+1.1}$ & 1.4 & 2.1 \\
\hline 64. & 286 & 207 & 30 & $26.5 \pm 3.4_{-3.9}^{+7.6}$ & $6.4 \pm 1.1_{-0.8}^{+0.7}$ & 1.0 & 2.8 \\
\hline 65. & 303 & 240 & 11 & $20.1 \pm 3.0_{-3.6}^{+3.6}$ & $4.6 \pm 0.9{ }_{-0.6}^{+0.5}$ & 0.7 & 1.2 \\
\hline 66. & 322 & 273 & 15 & $13.5 \pm 2.1_{-2.7}^{+2.7}$ & $3.0 \pm 0.8_{-0.4}^{+0.3}$ & 0.4 & 1.7 \\
\hline 67. & 339 & 303 & 0 & $0.0 \pm 0.0_{-0.0}^{+0.0}$ & $0.1 \pm 0.1_{-0.0}^{+0.0}$ & 0.2 & 7.4 \\
\hline 68. & 352 & 323 & 0 & $0.0 \pm 0.0_{-0.0}^{+0.0}$ & - & - & - \\
\hline 69 . & 378 & 365 & 0 & $0.0 \pm 0.0_{-0.0}^{+0.0}$ & - & - & - \\
\hline 70. & 405 & 407 & 56 & $49.8 \pm 4.3_{-8.3}^{+11.2}$ & - & - & - \\
\hline 71. & 302 & 207 & 12 & $14.4 \pm 2.4_{-2.8}^{+2.9}$ & $5.8 \pm 1.1_{-0.8}^{+0.8}$ & 0.9 & 1.4 \\
\hline 72. & 274 & 116 & 6 & $5.4 \pm 1.3_{-0.8}^{+0.8}$ & $7.7 \pm 1.5_{-1.1}^{+1.1}$ & 1.5 & 1.3 \\
\hline 73. & 326 & 240 & 1 & $0.1 \pm 0.0_{-0.1}^{+0.3}$ & - & - & - \\
\hline 74. & 303 & 174 & 1 & $0.2 \pm 0.1_{-0.1}^{+0.5}$ & $1.2 \pm 0.3_{-0.2}^{+0.4}$ & 0.9 & 2.6 \\
\hline 75 . & 366 & 303 & 20 & $17.6 \pm 2.4_{-2.9}^{+7.0}$ & $3.7 \pm 1.0_{-0.4}^{+0.3}$ & 0.3 & 0.9 \\
\hline 76 . & 378 & 324 & 0 & $0.1 \pm 0.0_{-0.1}^{+0.2}$ & - & - & - \\
\hline 77. & 300 & 141 & 9 & $10.0 \pm 1.9_{-1.2}^{+3.0}$ & $5.9 \pm 0.7_{-0.9}^{+0.9}$ & 1.1 & 1.3 \\
\hline 78. & 299 & 108 & 5 & $1.9 \pm 0.8_{-0.4}^{+0.5}$ & $3.0 \pm 0.5_{-0.5}^{+0.4}$ & 1.1 & 2.6 \\
\hline 79. & 301 & 76 & 2 & $0.6 \pm 0.4_{-0.2}^{+0.2}$ & $1.0 \pm 0.3_{-0.2}^{+0.2}$ & 1.1 & 5.5 \\
\hline 81. & 327 & 142 & 0 & $0.1 \pm 0.0_{-0.1}^{+0.2}$ & $1.1 \pm 0.4_{-0.3}^{+0.3}$ & 0.7 & 1.6 \\
\hline 82. & 338 & 175 & 13 & $14.3 \pm 2.3_{-2.1}^{+2.0}$ & $5.2 \pm 0.8_{-0.7}^{+0.6}$ & 0.6 & 1.1 \\
\hline 83. & 365 & 241 & 4 & $2.8 \pm 0.9_{-1.1}^{+2.7}$ & $2.2 \pm 0.6_{-0.3}^{+0.3}$ & 0.4 & 1.3 \\
\hline 84. & 381 & 275 & 0 & $0.3 \pm 0.1_{-0.2}^{+0.2}$ & - & - & - \\
\hline 85. & 396 & 304 & 0 & $0.4 \pm 0.4_{-0.4}^{+0.2}$ & - & - & - \\
\hline 86. & 407 & 325 & 0 & $0.0 \pm 0.0_{-0.0}^{+0.0}$ & - & - & - \\
\hline 87. & 430 & 367 & 0 & $0.0 \pm 0.0_{-0.0}^{+0.0}$ & - & - & - \\
\hline 88. & 454 & 408 & 0 & $0.1 \pm 0.0_{-0.1}^{+0.2}$ & - & - & - \\
\hline 89. & 505 & 492 & 0 & $0.3 \pm 0.2_{-0.3}^{+0.3}$ & - & - & - \\
\hline 90. & 354 & 204 & 3 & $2.6 \pm 1.0_{-0.8}^{+0.8}$ & $1.9 \pm 0.4_{-0.2}^{+0.2}$ & 0.5 & 1.3 \\
\hline
\end{tabular}




\begin{tabular}{|c|c|c|c|c|c|c|c|}
\hline \# & $\begin{array}{r}\mathrm{m}(\tilde{\mu}) \\
{[\mathrm{GeV}]}\end{array}$ & $\begin{array}{l}\mathrm{m}\left(\tilde{\chi}_{2}^{0}\right) \\
{[\mathrm{GeV}]}\end{array}$ & $\begin{array}{l}\text { data } \\
{[\text { evts }]}\end{array}$ & $\begin{array}{l}\text { SM exp. } \\
\text { [events] }\end{array}$ & $\begin{array}{l}\text { signal exp. } \\
\text { [events] }\end{array}$ & $\begin{array}{r}\sigma_{N L O} \\
{[\mathrm{pb}]}\end{array}$ & $\begin{array}{r}\sigma_{95} \\
{[\mathrm{pb}]}\end{array}$ \\
\hline 91. & 345 & 142 & 1 & $1.0 \pm 0.6_{-0.1}^{+0.1}$ & $1.3 \pm 0.2_{-0.2}^{+0.1}$ & 0.6 & 1.5 \\
\hline 93. & 373 & 143 & 3 & $4.5 \pm 1.2_{-1.5}^{+0.6}$ & $2.8 \pm 0.3{ }_{-0.4}^{+0.3}$ & 0.4 & 0.7 \\
\hline 95. & 407 & 242 & 0 & $1.1 \pm 0.6_{-0.2}^{+0.1}$ & $0.7 \pm 0.3_{-0.3}^{+0.1}$ & 0.3 & 1.6 \\
\hline 96. & 421 & 276 & 0 & $1.1 \pm 0.6_{-0.1}^{+0.1}$ & $0.1 \pm 0.1_{-0.1}^{+0.1}$ & 0.2 & 8.0 \\
\hline 97. & 403 & 204 & 4 & $2.3 \pm 0.8_{-0.6}^{+0.6}$ & $1.3 \pm 0.2_{-0.2}^{+0.2}$ & 0.3 & 1.3 \\
\hline 99 . & 415 & 120 & 0 & $0.5 \pm 0.5_{-0.2}^{+0.1}$ & $0.6 \pm 0.2_{-0.1}^{+0.1}$ & 0.3 & 1.2 \\
\hline 100. & 421 & 144 & 0 & $0.7 \pm 0.4_{-0.3}^{+0.3}$ & $0.8 \pm 0.2_{-0.2}^{+0.2}$ & 0.2 & 0.8 \\
\hline 101. & 437 & 202 & 0 & $0.6 \pm 0.4_{-0.2}^{+0.2}$ & $0.3 \pm 0.1_{-0.1}^{+0.1}$ & 0.2 & 3.1 \\
\hline 102. & 451 & 243 & 0 & $0.0 \pm 0.0_{-0.0}^{+0.1}$ & - & - & 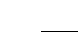 \\
\hline 103. & 485 & 327 & 0 & $0.0 \pm 0.0_{-0.0}^{+0.2}$ & $0.1 \pm 0.1_{-0.0}^{+0.1}$ & 0.1 & 6.6 \\
\hline 104. & 504 & 369 & 0 & $0.5 \pm 0.5_{-0.1}^{+0.1}$ & - & - & - \\
\hline 105. & 525 & 410 & 0 & $0.0 \pm 0.0_{-0.0}^{+0.3}$ & - & - & - \\
\hline 106. & 570 & 494 & 1 & $1.8 \pm 0.9_{-0.7}^{+0.7}$ & - & - & 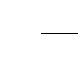 \\
\hline 107. & 468 & 145 & 0 & $0.0 \pm 0.0_{-0.0}^{+0.1}$ & $0.2 \pm 0.1_{-0.0}^{+0.0}$ & 0.1 & 1.8 \\
\hline 108. & 483 & 203 & 1 & $0.6 \pm 0.5_{-0.2}^{+0.2}$ & $0.2 \pm 0.1_{-0.0}^{+0.1}$ & 0.1 & 3.3 \\
\hline 109. & 506 & 83 & 2 & $0.0 \pm 0.0_{-0.0}^{+0.0}$ & $0.1 \pm 0.0_{-0.0}^{+0.0}$ & 0.1 & 6.6 \\
\hline 110. & 516 & 146 & 0 & $0.0 \pm 0.0_{-0.0}^{+0.1}$ & $0.1 \pm 0.0_{-0.0}^{+0.0}$ & 0.1 & 3.7 \\
\hline 111. & 524 & 179 & 0 & $0.0 \pm 0.0_{-0.0}^{+0.4}$ & $0.1 \pm 0.0_{-0.0}^{+0.0}$ & 0.1 & 2.1 \\
\hline 112. & 530 & 204 & 0 & $0.0 \pm 0.0_{-0.0}^{+0.1}$ & $0.1 \pm 0.1_{-0.0}^{+0.0}$ & 0.1 & 1.3 \\
\hline 113. & 541 & 245 & 0 & $0.2 \pm 0.1_{-0.2}^{+0.1}$ & $0.1 \pm 0.0_{-0.0}^{+0.0}$ & 0.1 & 1.8 \\
\hline 114. & 562 & 308 & 2 & $1.4 \pm 0.5_{-0.2}^{+0.2}$ & $0.5 \pm 0.2_{-0.1}^{+0.1}$ & 0.0 & 0.4 \\
\hline 115. & 570 & 329 & 1 & $0.6 \pm 0.4_{-0.2}^{+0.3}$ & $0.0 \pm 0.0_{-0.0}^{+0.0}$ & 0.0 & 18.0 \\
\hline 116. & 605 & 412 & 1 & $0.6 \pm 0.4_{-0.1}^{+0.1}$ & - & - & - \\
\hline 117. & 644 & 496 & 0 & $0.0 \pm 0.0_{-0.0}^{+0.1}$ & $0.0 \pm 0.0_{-0.0}^{+0.0}$ & 0.0 & 3.5 \\
\hline
\end{tabular}

Table C.2: Final Results for channel $\tilde{\mu} \rightarrow \tilde{\chi}_{2,(3,4)}^{0} \mu$ with $\tan \beta=5$ and $\operatorname{sign}(\mu)=-1$. The slepton and neutralino $\mathrm{O}_{2}$ mass $\left(2^{\text {nd }}\right.$ and $3^{\text {rd }}$ column) are given in $\mathrm{GeV}$, the next-to-leading order cross section $\sigma=\sigma_{q q \rightarrow \tilde{\mu}}^{N L O} \cdot B R\left(\tilde{\mu} \rightarrow \mu \tilde{\chi}_{2}^{0}\right)$ as well as the $95 \%$ C.L. limit are given in pb (last two columns). If 0.049 or less events are expected, then 0.0 is stated, due to the finite column width. For some points the signal generation for this channel was not possible, because $\mathrm{m}\left(\tilde{\chi}_{2}^{0}\right)>\mathrm{m}(\tilde{\mu})$, here the signal expectation and cross section is marked "-". 


\section{C.3 Channel $\tilde{\nu}_{\mu} \rightarrow \tilde{\chi}_{1,(2)}^{ \pm} \mu$ with $\tan \beta=5$ and $\operatorname{sign}(\mu)=-1$}

\begin{tabular}{|c|c|c|c|c|c|c|c|}
\hline$\#$ & $\begin{array}{l}\mathrm{m}\left(\tilde{\nu}_{\mu}\right) \\
{[\mathrm{GeV}]}\end{array}$ & $\begin{array}{r}\mathrm{m}\left(\tilde{\chi}_{1}^{ \pm}\right) \\
{[\mathrm{GeV}]}\end{array}$ & $\begin{array}{l}\text { data } \\
{[\text { evts }]}\end{array}$ & $\begin{array}{l}\text { SM exp. } \\
\text { [events] }\end{array}$ & $\begin{array}{l}\text { signal exp. } \\
\text { [events] }\end{array}$ & $\begin{array}{r}\sigma_{N L O} \\
{[\mathrm{pb}]}\end{array}$ & $\begin{array}{r}\sigma_{95} \\
{[\mathrm{pb}]}\end{array}$ \\
\hline 0. & 82 & 112 & 18 & $6.1 \pm 1.8_{-1.1}^{+1.0}$ & - & - & - \\
\hline 1. & 103 & 133 & 26 & $15.7 \pm 2.9_{-6.6}^{+3.9}$ & - & - & - \\
\hline 2. & 137 & 170 & 47 & $27.9 \pm 3.9{ }_{-10.8}^{+4.8}$ & - & - & - \\
\hline 3. & 159 & 195 & 119 & $77.1 \pm 5.7_{-17.6}^{+22.7}$ & - & - & - \\
\hline 4. & 182 & 220 & 56 & $36.3 \pm 4.2_{-12.1}^{+6.3}$ & - & - & - \\
\hline 5. & 198 & 237 & 7 & $10.2 \pm 2.1_{-1.9}^{+1.8}$ & - & - & - \\
\hline 6. & 174 & 205 & 0 & $0.0 \pm 0.0_{-0.0}^{+0.2}$ & $0.3 \pm 0.3{ }_{-0.0}^{+0.0}$ & 0.0 & 0.1 \\
\hline 10. & 96 & 113 & 55 & $39.2 \pm 4.4_{-14.8}^{+4.2}$ & - & - & - \\
\hline 11. & 118 & 138 & 3 & $3.1 \pm 0.9_{-1.4}^{+1.7}$ & - & - & - \\
\hline 12. & 146 & 171 & 2 & $0.1 \pm 0.0_{-0.1}^{+0.1}$ & $1.5 \pm 1.5_{-0.5}^{+0.5}$ & 0.0 & 0.1 \\
\hline 13. & 160 & 187 & 2 & $1.3 \pm 0.7_{-0.6}^{+0.6}$ & - & - & - \\
\hline 14. & 167 & 196 & 2 & $0.5 \pm 0.0{ }_{-0.1}^{+0.9}$ & - & - & 一 \\
\hline 15. & 201 & 237 & 8 & $5.5 \pm 1.4_{-2.2}^{+2.1}$ & - & - & - \\
\hline 16. & 228 & 271 & 155 & $138.0 \pm 7.4_{-27.7}^{+32.8}$ & - & - & - \\
\hline 17. & 252 & 300 & 4 & $5.3 \pm 1.4_{-0.7}^{+1.0}$ & - & - & - \\
\hline 18. & 269 & 321 & 232 & $189.4 \pm 8.6_{-40.7}^{+40.7}$ & - & - & 一 \\
\hline 19. & 304 & 363 & 22 & $15.7 \pm 2.2_{-2.6}^{+2.6}$ & - & - & - \\
\hline 22. & 92 & 50 & 2 & $3.1 \pm 1.1_{-0.6}^{+0.6}$ & $20.8 \pm 12.0_{-4.4}^{+4.2}$ & 26.5 & 9.4 \\
\hline 23. & 104 & 73 & 11 & $6.3 \pm 2.0_{-1.6}^{+1.5}$ & - & - & - \\
\hline 24. & 124 & 106 & 14 & $7.0 \pm 1.9_{-0.9}^{+3.4}$ & $33.3 \pm 9.2_{-10.8}^{+4.5}$ & 16.4 & 10.0 \\
\hline 25. & 129 & 114 & 3 & $1.7 \pm 0.9_{-0.3}^{+0.4}$ & $9.4 \pm 4.7_{-0.9}^{+0.8}$ & 14.1 & 12.8 \\
\hline 26. & 146 & 138 & 0 & $0.0 \pm 0.0_{-0.0}^{+0.0}$ & - & - & - \\
\hline 27. & 170 & 171 & 159 & $123.9 \pm 7.0_{-22.1}^{+30.5}$ & - & - & 一 \\
\hline 28. & 194 & 205 & 0 & $0.0 \pm 0.0_{-0.0}^{+0.0}$ & - & - & 一 \\
\hline 29. & 219 & 238 & 0 & $0.0 \pm 0.0_{-0.0}^{+0.0}$ & - & - & 一 \\
\hline 30. & 244 & 271 & 0 & $0.0 \pm 0.0_{-0.0}^{+0.0}$ & - & - & - \\
\hline 31. & 266 & 301 & 0 & $0.0 \pm 0.0_{-0.0}^{+0.0}$ & - & - & - \\
\hline 32. & 282 & 321 & 1 & $0.6 \pm 0.0_{-0.1}^{+0.1}$ & - & - & - \\
\hline 33. & 314 & 363 & 14 & $15.0 \pm 2.6_{-2.4}^{+2.3}$ & - & - & — \\
\hline 34. & 347 & 405 & 12 & $10.5 \pm 1.8_{-2.0}^{+2.5}$ & - & - & 一 \\
\hline 35. & 412 & 489 & 5 & $4.6 \pm 1.4_{-0.9}^{+1.0}$ & - & - & 一 \\
\hline 36. & 194 & 180 & 0 & $0.0 \pm 0.0_{-0.0}^{+0.0}$ & - & - & - \\
\hline 37. & 171 & 115 & 6 & $8.7 \pm 2.2_{-3.4}^{+1.5}$ & $17.6 \pm 4.4_{-3.3}^{+3.2}$ & 8.7 & 4.5 \\
\hline 38. & 193 & 156 & 40 & $28.8 \pm 3.7_{-9.1}^{+5.8}$ & $43.2 \pm 3.7_{-5.8}^{+5.4}$ & 5.3 & 2.4 \\
\hline 41. & 162 & 75 & 0 & $0.0 \pm 0.0_{-0.0}^{+0.3}$ & - & - & - \\
\hline 42. & 192 & 139 & 22 & $13.1 \pm 2.7_{-3.3}^{+3.4}$ & $35.5 \pm 3.4_{-4.7}^{+4.3}$ & 6.0 & 2.7 \\
\hline 43. & 220 & 189 & 15 & $9.8 \pm 2.0_{-1.4}^{+4.8}$ & $26.0 \pm 2.2_{-2.8}^{+2.6}$ & 3.0 & 1.3 \\
\hline 44. & 230 & 206 & 1 & $1.6 \pm 0.0_{-0.5}^{+0.5}$ & $4.9 \pm 1.4_{-0.7}^{+0.7}$ & 2.2 & 1.7 \\
\hline 45. & 252 & 239 & 0 & $0.0 \pm 0.0_{-0.0}^{+0.0}$ & - & - & - \\
\hline
\end{tabular}




\begin{tabular}{|c|c|c|c|c|c|c|c|c|}
\hline$\#$ & $\begin{array}{l}\mathrm{m}\left(\tilde{\nu}_{\mu}\right) \\
{[\mathrm{GeV}]} \\
\end{array}$ & $\begin{array}{r}\mathrm{m}\left(\tilde{\chi}_{1}^{ \pm}\right) \\
{[\mathrm{GeV}]} \\
\end{array}$ & $\begin{array}{l}\text { data } \\
{[\text { evts] }}\end{array}$ & $\begin{array}{c}\text { SM exp. } \\
\text { [events] }\end{array}$ & $\begin{array}{r}\text { signal exp } \\
\text { [events] }\end{array}$ & & $\begin{array}{r}\sigma_{N L O} \\
{[\mathrm{pb}]} \\
\end{array}$ & $\begin{array}{r}\sigma_{95} \\
{[\mathrm{pb}]} \\
\end{array}$ \\
\hline 46. & 274 & 272 & 80 & $81.9 \pm 5.7_{-21.4}^{+13.4}$ & & - & - & - \\
\hline 47. & 309 & 322 & 0 & $1.3 \pm 0.4_{-0.3}^{+0.2}$ & & - & - & - \\
\hline 48. & 338 & 364 & 27 & $13.9 \pm 2.7_{-1.9}^{+1.8}$ & & - & - & - \\
\hline 49 . & 369 & 406 & 283 & $269.6 \pm 10.0_{-49.7}^{+53.0}$ & & - & - & - \\
\hline 50. & 295 & 302 & 0 & $0.0 \pm 0.0_{-0.0}^{+0.0}$ & $0.1 \pm 0.1$ & $\begin{array}{l}+0.0 \\
{ }_{-0.0}\end{array}$ & 0.0 & 0.9 \\
\hline 51. & 193 & 115 & 17 & $8.9 \pm 2.1_{-2.8}^{+1.5}$ & $15.4 \pm 2.2$ & $\begin{array}{l}+4.3 \\
{ }_{-2.3}\end{array}$ & 6.4 & 5.7 \\
\hline 52. & 191 & 99 & 16 & $9.1 \pm 1.8_{-1.6}^{+1.3}$ & $13.7 \pm 2.3$ & $\begin{array}{l}+2.7 \\
-1.6\end{array}$ & 6.6 & 6.1 \\
\hline 53. & 226 & 173 & 28 & $20.0 \pm 3.1_{-5.0}^{+3.7}$ & $22.4 \pm 3.2$ & $\begin{array}{l}+3.3 \\
-3.5\end{array}$ & 3.5 & 2.8 \\
\hline 54. & 192 & 75 & 4 & $6.9 \pm 1.6_{-1.2}^{+1.2}$ & $9.0 \pm 1.8$ & $\begin{array}{l}+1.1 \\
-2.4\end{array}$ & 6.3 & 5.0 \\
\hline 57. & 216 & 116 & 6 & $2.3 \pm 1.1_{-0.7}^{+0.7}$ & $12.9 \pm 2.6$ & $\begin{array}{l}+1.5 \\
+1.6\end{array}$ & 4.7 & 2.6 \\
\hline 58. & 252 & 192 & 8 & $6.5 \pm 1.6_{-1.2}^{+2.0}$ & $8.0 \pm 0.7$ & $\begin{array}{l}+1.2 \\
{ }_{-0.9}\end{array}$ & 2.4 & 2.3 \\
\hline 59 . & 447 & 490 & 29 & $16.9 \pm 2.4_{-1.7}^{+5.8}$ & & - & - & - \\
\hline 60. & 569 & 658 & 3 & $0.1 \pm 0.0_{-0.1}^{+0.5}$ & & - & - & - \\
\hline 62. & 244 & 141 & 18 & $17.3 \pm 2.6_{-3.6}^{+4.4}$ & $10.3 \pm 1.2$ & $\begin{array}{l}+1.7 \\
{ }_{-1.3}\end{array}$ & 3.2 & 3.6 \\
\hline 63. & 259 & 174 & 1 & $1.3 \pm 0.9_{-0.2}^{+0.2}$ & $2.6 \pm 1.2$ & $\begin{array}{l}{ }_{-0.7}^{+1.4} \\
{ }_{0}\end{array}$ & 2.5 & 4.9 \\
\hline 64. & 275 & 207 & 42 & $38.0 \pm 4.0_{-6.5}^{+5.2}$ & $12.0 \pm 1.0$ & $\begin{array}{l}+1.8 \\
-1.5\end{array}$ & 1.8 & 3.1 \\
\hline 65. & 293 & 240 & 16 & $19.4 \pm 2.7_{-3.2}^{+7.1}$ & $6.9 \pm 0.7$ & $\begin{array}{l}+0.8 \\
{ }_{-1.1}\end{array}$ & 1.2 & 1.9 \\
\hline 66. & 313 & 273 & 6 & $2.1 \pm 0.8_{-0.5}^{+0.6}$ & $2.6 \pm 0.6$ & $\begin{array}{l}+0.4 \\
{ }_{-0.4}\end{array}$ & 0.8 & 2.4 \\
\hline 67. & 330 & 303 & 0 & $0.0 \pm 0.0_{-0.0}^{+0.0}$ & $0.1 \pm 0.1$ & $\begin{array}{l}{ }_{-0.0}^{+0.0} \\
-0.0\end{array}$ & 0.4 & 15.0 \\
\hline 68. & 343 & 323 & 0 & $0.0 \pm 0.0_{-0.0}^{+0.0}$ & & - & - & - \\
\hline 69 . & 370 & 365 & 0 & $0.0 \pm 0.0_{-0.0}^{+0.3}$ & & - & - & - \\
\hline 70. & 398 & 407 & 217 & $217.3 \pm 9.1_{-42.8}^{+41.8}$ & & - & - & - \\
\hline 71. & 292 & 207 & 24 & $20.1 \pm 2.5_{-3.0}^{+6.4}$ & $9.4 \pm 0.8$ & $\begin{array}{l}+1.1 \\
{ }_{-1.2}\end{array}$ & 1.6 & 2.4 \\
\hline 72. & 263 & 117 & 4 & $8.0 \pm 1.7_{-1.3}^{+1.3}$ & $5.8 \pm 1.3$ & $\begin{array}{l}+0.9 \\
-1.0\end{array}$ & 2.5 & 2.4 \\
\hline 73. & 316 & 241 & 24 & $20.1 \pm 2.8_{-2.8}^{+2.8}$ & $5.9 \pm 0.9$ & $\begin{array}{l}+0.8 \\
-0.9\end{array}$ & 1.1 & 2.9 \\
\hline 74. & 294 & 174 & 25 & $21.2 \pm 2.8_{-4.5}^{+5.6}$ & $7.2 \pm 0.7$ & $\begin{array}{l}{ }_{-0.9}^{+0.8} \\
-0.9\end{array}$ & 1.7 & 3.5 \\
\hline 75 . & 358 & 303 & 7 & $16.7 \pm 2.5_{-4.9}^{+1.7}$ & $1.3 \pm 0.3$ & $\begin{array}{l}+0.3 \\
{ }_{-0.3}\end{array}$ & 0.5 & 5.6 \\
\hline 76 . & 370 & 324 & 0 & $0.1 \pm 0.0_{-0.1}^{+0.0}$ & & - & - & - \\
\hline 77. & 290 & 142 & 5 & $2.6 \pm 0.9_{-0.4}^{+0.3}$ & $3.4 \pm 0.5$ & $\begin{array}{l}+0.4 \\
{ }_{-0.4}\end{array}$ & 1.8 & 3.6 \\
\hline 78 . & 289 & 110 & 21 & $13.7 \pm 2.2_{-2.0}^{+2.3}$ & $5.9 \pm 0.7$ & $\begin{array}{l}+0.6 \\
{ }_{-1.1}\end{array}$ & 1.8 & 5.3 \\
\hline 79 . & 291 & 78 & 0 & $0.4 \pm 0.4_{-0.4}^{+0.2}$ & $0.6 \pm 0.2$ & $\begin{array}{l}{ }_{-0.1}^{+0.1} \\
-1\end{array}$ & 1.8 & 8.7 \\
\hline 81. & 318 & 142 & 1 & $2.9 \pm 1.0_{-0.3}^{+0.3}$ & $2.5 \pm 0.6$ & $\begin{array}{l}+0.5 \\
{ }_{-0.4}\end{array}$ & 1.3 & 1.8 \\
\hline 82. & 329 & 175 & 18 & $17.5 \pm 2.5_{-4.1}^{+2.7}$ & $4.3 \pm 0.7$ & $\begin{array}{l}{ }_{-0.8}^{+0.9} \\
-0.8\end{array}$ & 1.1 & 3.9 \\
\hline 83. & 357 & 242 & 9 & $6.5 \pm 1.4_{-0.9}^{+0.8}$ & $2.5 \pm 0.4$ & $\begin{array}{l}+0.3 \\
{ }_{-0.3}\end{array}$ & 0.7 & 2.5 \\
\hline 84. & 373 & 275 & 6 & $5.9 \pm 1.3_{-0.8}^{+1.3}$ & $1.5 \pm 0.3$ & $\begin{array}{l}+0.2 \\
{ }_{-0.2}\end{array}$ & 0.6 & 2.7 \\
\hline 85. & 388 & 304 & 0 & $0.0 \pm 0.0_{-0.0}^{+0.0}$ & & - & - & - \\
\hline 86. & 399 & 325 & 6 & $8.3 \pm 1.8_{-1.1}^{+1.2}$ & $0.8 \pm 0.2$ & $\begin{array}{l}+0.1 \\
-0.1\end{array}$ & 0.3 & 2.8 \\
\hline 87. & 423 & 367 & 0 & $0.0 \pm 0.0_{-0.0}^{+0.1}$ & & - & - & - \\
\hline 88. & 447 & 409 & 0 & $0.0 \pm 0.0_{-0.0}^{+0.0}$ & & - & - & - \\
\hline 89. & 499 & 492 & 1 & $1.4 \pm 0.7_{-0.3}^{+0.2}$ & & - & - & - \\
\hline 90. & 345 & 204 & 9 & $4.3 \pm 1.1_{-1.7}^{+1.7}$ & $3.1 \pm 0.4$ & $\begin{array}{l}{ }_{-0.7}^{0.5} \\
\end{array}$ & 0.9 & 3.1 \\
\hline
\end{tabular}




\begin{tabular}{|c|c|c|c|c|c|c|c|}
\hline$\#$ & $\begin{array}{l}\mathrm{m}\left(\tilde{\nu}_{\mu}\right) \\
{[\mathrm{GeV}]}\end{array}$ & $\begin{array}{r}\mathrm{m}\left(\tilde{\chi}_{1}^{ \pm}\right) \\
{[\mathrm{GeV}]}\end{array}$ & $\begin{array}{c}\text { data } \\
\text { [evts] }\end{array}$ & $\begin{array}{l}\text { SM exp. } \\
\text { [events] }\end{array}$ & $\begin{array}{l}\text { signal exp. } \\
\text { [events] }\end{array}$ & $\begin{array}{r}\sigma_{N L O} \\
{[\mathrm{pb}]}\end{array}$ & $\begin{array}{r}\sigma_{95} \\
{[\mathrm{pb}]}\end{array}$ \\
\hline 91. & 337 & 143 & 2 & $0.1 \pm 0.0_{-0.1}^{+0.3}$ & $0.9 \pm 0.2_{-0.2}^{+0.3}$ & 1.0 & 4.6 \\
\hline 93. & 365 & 144 & 2 & $1.8 \pm 0.8_{-0.2}^{+0.7}$ & $1.3 \pm 0.2_{-0.2}^{+0.2}$ & 0.7 & 2.3 \\
\hline 95. & 400 & 243 & 3 & $1.9 \pm 0.7_{-0.7}^{+0.7}$ & $1.3 \pm 0.2_{-0.1}^{+0.1}$ & 0.5 & 1.8 \\
\hline 96. & 414 & 276 & 0 & $0.2 \pm 0.1_{-0.1}^{+0.3}$ & $0.0 \pm 0.0_{-0.0}^{+0.0}$ & 0.4 & 48.0 \\
\hline 97. & 396 & 205 & 0 & $1.3 \pm 0.6_{-0.4}^{+0.2}$ & $1.3 \pm 0.2_{-0.2}^{+0.1}$ & 0.5 & 0.9 \\
\hline 99 . & 408 & 120 & 0 & $0.0 \pm 0.0_{-0.0}^{+0.3}$ & $0.3 \pm 0.1_{-0.1}^{+0.0}$ & 0.5 & 4.9 \\
\hline 100. & 413 & 145 & 1 & $1.1 \pm 0.6_{-0.3}^{+0.2}$ & $0.8 \pm 0.2_{-0.1}^{+0.1}$ & 0.4 & 2.0 \\
\hline 101. & 430 & 202 & 0 & $0.0 \pm 0.0_{-0.0}^{+0.0}$ & - & - & - \\
\hline 102. & 444 & 244 & 0 & $0.2 \pm 0.1_{-0.1}^{+0.7}$ & $0.1 \pm 0.0_{-0.0}^{+0.0}$ & 0.3 & 21.0 \\
\hline 103. & 479 & 327 & 0 & $0.5 \pm 0.4_{-0.3}^{+0.1}$ & $0.0 \pm 0.0_{-0.0}^{+0.0}$ & 0.2 & 39.3 \\
\hline 104. & 498 & 369 & 0 & $0.0 \pm 0.0_{-0.0}^{+0.0}$ & - & - & - \\
\hline 105. & 519 & 410 & 1 & $0.0 \pm 0.0_{-0.0}^{+0.1}$ & - & - & - \\
\hline 106. & 565 & 494 & 1 & $0.5 \pm 0.5_{-0.1}^{+0.1}$ & $0.0 \pm 0.0_{-0.0}^{+0.0}$ & 0.0 & 46.5 \\
\hline 107. & 462 & 146 & 0 & $0.0 \pm 0.0_{-0.0}^{+0.0}$ & $0.0 \pm 0.0_{-0.0}^{+0.0}$ & 0.3 & 39.3 \\
\hline 108. & 477 & 203 & 0 & $0.0 \pm 0.0_{-0.0}^{+0.0}$ & $0.0 \pm 0.0_{-0.0}^{+0.0}$ & 0.2 & 50.4 \\
\hline 109. & 500 & 84 & 0 & $0.0 \pm 0.0_{-0.0}^{+0.1}$ & - & - & - \\
\hline 110. & 511 & 147 & 0 & $0.0 \pm 0.0_{-0.0}^{+0.0}$ & $0.1 \pm 0.0_{-0.0}^{+0.0}$ & 0.2 & 8.4 \\
\hline 111. & 518 & 179 & 0 & $0.0 \pm 0.0_{-0.0}^{+0.1}$ & $0.0 \pm 0.0_{-0.0}^{+0.0}$ & 0.1 & 11.0 \\
\hline 112. & 524 & 204 & 0 & $0.0 \pm 0.0_{-0.0}^{+0.0}$ & - & - & - \\
\hline 113. & 536 & 246 & 0 & $0.4 \pm 0.4_{-0.4}^{+0.1}$ & $0.1 \pm 0.0_{-0.0}^{+0.0}$ & 0.1 & 5.9 \\
\hline 114. & 557 & 308 & 0 & $0.0 \pm 0.0_{-0.0}^{+0.2}$ & - & - & 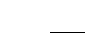 \\
\hline 115. & 565 & 329 & 1 & $0.2 \pm 0.1_{-0.1}^{+0.1}$ & $0.0 \pm 0.0_{-0.0}^{+0.0}$ & 0.1 & 18.3 \\
\hline 116. & 600 & 412 & 0 & $0.0 \pm 0.0_{-0.0}^{+0.2}$ & - & - & - \\
\hline 117. & 639 & 496 & 0 & $0.4 \pm 0.4_{-0.4}^{+0.4}$ & $0.0 \pm 0.0_{-0.0}^{+0.0}$ & 0.0 & 44.6 \\
\hline
\end{tabular}

Table C.3: Final Results for channel $\tilde{\nu}_{\mu} \rightarrow \tilde{\chi}_{1,(2)}^{ \pm} \mu$ with $\tan \beta=5$ and $\operatorname{sign}(\mu)=-1$. The slepton and chargino mass ( $2^{\text {nd }}$ and $3^{\text {rd }}$ column) are given in GeV, the next-to-leading order cross section $\sigma=\sigma_{q q \rightarrow \tilde{\nu}}^{N L O} \cdot B R\left(\tilde{\nu} \rightarrow \mu \tilde{\chi}_{1}^{ \pm}\right)$as well as the $95 \%$ C.L. limit are given in pb (last two columns). If 0.049 or less events are expected, then 0.0 is stated, due to the finite column width. For some points the signal generation for this channel was not possible, because $\mathrm{m}\left(\tilde{\chi}_{1}^{ \pm}\right)>\mathrm{m}\left(\tilde{\nu}_{\mu}\right)$, here the signal expectation and cross section is marked "-". 


\section{Bibliography}

[1] J. Lykken. Solving the hierarchy problem. 2004. Talk given at the Slac Summer Institute (SSI).

[2] X. Tata. Supersymmetry basics. 2004. Lecture given at the Slac Summer Institute (SSI).

[3] T. Ohl. Feynmandiagramme für Anfänger. 2002. Lecture given at the Maria Laach Summer School.

[4] S. P. Martin. A supersymmetry primer. 1997.

[5] E. Noether. Invariante Variationsprobleme. Nachr. d. König. Gesellsch. d. Wiss. zu Göttingen, Math.-phys. Klasse:235-257, 1918.

[6] H. A. Kastrup. Scriptum zur Vorlesung Theoretrische Mechanik. Bibliothek RWTH Aachen, 1997.

[7] C. N. Yang and R. L. Mills. Conservation of Isotopic Spin and Isotopic Gauge Invariance. Phys. Rev., 96:191-195, 1954.

[8] G. 't Hooft. Renormalization of massless Yang-Mills fields. Nucl. Phys., B 33:173$199,1971$.

[9] G. 't Hooft. Renormalizable Lagrangians for massive Yang-Mills fields. Nucl. Phys., B 35:167-188, 1971.

[10] S. Weinberg. Nonabelian gauge theories of the strong interactions. Phys. Rev. Lett., 31:494-497, 1973.

[11] T. Nakano. Experimental evidence for penta-quark system. Prepared for International Conference on Color Confinement and Hadrons in Quantum Chromodynamics - Confinement 2003, Wako, Japan, 21-24 Jul 2003.

[12] D. J. Gross and F. Wilczek. Asymptotically free gauge theories. 1. Phys. Rev., D8:3633-3652, 1973.

[13] H. D. Politzer. Reliable perturbative results for strong interactions? Phys. Rev. Lett., 30:1346-1349, 1973.

[14] S. L. Glashow. Partial symmetries of weak interactions. Nucl. Phys., 22:579-588, 1961. 
[15] A. Salam and N. Svartholm (ed.). Proceedings of the 8th nobel symposium. Almquist and Wiksells, Stockholm, page 367, 1968.

[16] S. Weinberg. A model of leptons. Phys. Rev. Lett., 19:1264-1266, 1967.

[17] S. Eidelman et al. Review of Particle Physics. Phys. Lett., B 592, 2004. URL http://pdg.lbl.gov.

[18] N. Cabibbo. Unitary symmetry and leptonic decays. Phys. Rev. Lett., 10:531-532, 1963.

[19] M. Kobayashi and T. Maskawa. Cp violation in the renormalizable theory of weak interaction. Prog. Theor. Phys., 49:652-657, 1973.

[20] P. W. Higgs. Broken symmetries and the masses of gauge bosons. Phys. Rev. Lett., 13:508-509, 1964.

[21] The LEP Collaborations. A combination of preliminary electroweak measurements and constraints on the standard model. 2005.

URL http://lepewwg. web.cern.ch/LEPEWWG/.

[22] J.F. Gunion, H.E. Haber, G. Kane, and S. Dawson. The Higgs Hunter's Guide. Addison-Wesley Publishing Company, Redwood City, CA, 1990.

[23] Fukuda, Y. et al. (The Super-Kamiokande Collaboration). Evidence for oscillation of atmospheric neutrinos. Phys. Rev. Lett., 81:1562-1567, 1998.

[24] M. C. Gonzalez-Garcia and Y. Nir. Developments in neutrino physics. Rev. Mod. Phys., 75:345-402, 2003.

[25] S. T. Petcov. Theoretical prospects of neutrinoless double beta decay. Phys. Scripta, T121:94-101, 2005.

[26] M. Gell-Mann, P. Ramond, R. Slansky, and D. Freedman (ed.). Complex spinors and unified theories. Supergravity, North Holland, pages 315-321, 1979.

[27] K. G. Wilson. Quantum field theory models in less than four-dimensions. Phys. Rev., D7:2911-2926, 1973.

[28] M.H. Shamos (ed.). Great experiments in physics. Henry Holt and Company, New York, page 75, 1959.

[29] N. Arkani-Hamed, S. Dimopoulos, and G.R. Dvali. The hierarchy problem and new dimensions at a millimeter. Phys. Lett., B429:263-272, 1998.

[30] Abazov, V. M. et al. (The DØ Collaboration). Search for Large Extra Dimensions in the Dimuon Channel with $250 \mathrm{pb}^{-1}$ of Run II Data. Phys. Rev. Lett., 95:161602, 2005 .

[31] S.J. Smullin et al. New constraints on yukawa-type deviations from newtonian gravity at 20 -microns. 2005 .

[32] K. Riesselmann. Limitations of a standard model Higgs boson. 1997. 
[33] H.-C. Cheng and I. Low. TeV symmetry and the Little Hierarchy Problem. JHEP, 09:051, 2003.

[34] L. Girardello and M. T. Grisaru. Soft breaking of supersymmetry. Nucl. Phys., B194:65, 1982.

[35] J. Wess and B. Zumino. Supergauge transformations in four-dimensions. Nucl. Phys., B70:39-50, 1974.

[36] J. Wess and B. Zumino. A lagrangian model invariant under supergauge transformations. Phys. Lett., B49:52, 1974.

[37] S. Dimopoulos and D. Sutter. The supersymmetric flavor problem. Nucl. Phys., B 452:496-512, 1995.

[38] P. Fayet and J. Iliopoulos. Spontaneously broken supergauge symmetries and goldstone spinors. Phys. Lett., B51:461-464, 1974.

[39] L. O'Raifeartaigh. Spontaneous symmetry breaking for chiral scalar superfields. Nucl. Phys., B96:331, 1975.

[40] A. H. Chamseddine, R. Arnowitt, and P. Nath. Locally supersymmetric grand unification. Phys. Rev. Lett., 49:970, 1982.

[41] S. Abel et al. Report of the sugra working group for run ii of the tevatron. 2000.

[42] R. Barbier et al. R-parity violating supersymmetry. 2004.

[43] T. Hebbeker. Calculation of SUSY masses in MSUGRA or CMSSM framework. 2005. URL http://www.physik.rwth-aachen.de/ ${ }^{2}$ hebbeker/mysusy/.

[44] A. Salam and J. A. Strathdee. Supersymmetry and fermion number conservation. Nucl. Phys., B87:85, 1975.

[45] P. Fayet. Supergauge invariant extension of the higgs mechanism and a model for the electron and its neutrino. Nucl. Phys., B90:104-124, 1975.

[46] G. R. Farrar and P. Fayet. Phenomenology of the production, decay, and detection of new hadronic states associated with supersymmetry. Phys. Lett., B76:575-579, 1978 .

[47] H. K. Dreiner and G. L. Kane (ed.). Perspectives on supersymmetry. page 479, 1998. Singapore, Singapore: World Scientific.

[48] N. Ghodbane, S. Katsanevas, P. Morawitz, and E. Perez. SUSYGEN 3. 1999.

[49] J. Pumplin et al. JHEP, 0207:012, 2002.

[50] D. Stump et al. JHEP, 0310:046, 2003.

[51] M. Trenkel. private communication.

[52] S. Wolfram. Abundances of stable particles produced in the early universe. Phys. Lett., B82:65, 1979. 
[53] C. B. Dover, T. K. Gaisser, and G. Steigman. Cosmological constraints on new stable hadrons. Phys. Rev. Lett., 42:1117, 1979.

[54] B. C. Allanach, A. Dedes, and Herbert K. Dreiner. Bounds on r-parity violating couplings at the weak scale and at the gut scale. Phys. Rev., D 60:075014, 1999.

[55] B. C. Allanach, A. Dedes, and H. K. Dreiner. The R parity violating minimal supergravity model. Phys. Rev., D69:115002, 2004.

[56] Abreu, P. et al. (The DELPHI Collaboration). Search for supersymmetry with R-parity violating decays via $\lambda$ couplings at $\sqrt{s}=183 \mathrm{GeV}$. pages 13-20, 1999 . Prepared for 34th Rencontres de Moriond: Electroweak Interactions and Unified Theories, Les Arcs, France.

[57] V. D. Barger, G. F. Giudice, and T. Han. Some new aspects of Supersymmetry R Parity Violating interactions. Phys. Rev., D40:2987, 1989.

[58] P. Langacker (ed.). Precision tests of standard model of electroweak interactions. World Scientific, Singapore, 1996.

[59] J. E. Kim, P. Ko, and D.-G. Lee. More on r-parity and lepton-family number violating couplings from muon(ium) conversion, and $\tau$ and $\pi^{0}$ decays. Phys. Rev., D 56:100, 1997.

[60] D. Choudhury and P. Roy. New constraints on lepton nonconserving r-parity violating couplings. Phys. Lett., B 378:153, 1996.

[61] Y. Grossman, Z. Ligeti, and E. Nardi. First limit on inclusive b $-i \mathrm{x} / \mathrm{s}$ neutrino antineutrino decay and constraints on new physics. Nucl. Phys., B465:369-398, 1996.

[62] M. Chaichian and K. Huitu. Constraints on $r$-parity violating interactions from $\mu \rightarrow$ eү. Phys. Lett., B384:157-160, 1996.

[63] G. Bhattacharyya and A. Raychaudhuri. New constraints on r-parity violation from k and b systems. Phys. Rev., D57:3837-3841, 1998.

[64] P. Wintz for the SINDRUM II Collaboration. Proceedings of the 14th international conference on particles and nuclei (panic96). World Scientific, 458, 1997.

[65] A. Y. Smirnov and F. Vissani. Upper bound on all products of r-parity violating couplings $\lambda^{\prime}$ and $\lambda^{\prime \prime}$ from proton decay. Phys. Lett., B380:317-323, 1996.

[66] LEPSUSYWG, ALEPH, DELPHI, L3 and OPAL experiments. R-parity violation with LLE couplings (LEPSUSYWG/02-10.1).

URL http://lepsusy . web.cern.ch/lepsusy/Welcome.html.

[67] Heister, A. et al. (The ALEPH Collaboration). Search for R-parity violating decays of supersymmetric particles in $e^{+} e^{-}$collisions at center-of-mass energies near $\sqrt{s}=$ $183 \mathrm{GeV}$. Eur. Phys. J., C 13:29, 2000. 
[68] Berggren, M. et al. (The ADLO Collaboration). Combined LEP Selectron/Smuon/Stau Results $183-208$ GeV. 2001. LEP SUSY Working Group.

[69] Heister, A. et al. (The ALEPH Collaboration). Search for supersymmetric particles with $\mathrm{R}$ parity violating decays in e+ e- collisions at $\sqrt{s}$ up to $209 \mathrm{GeV}$. Eur. Phys. J., C 31:1-16, 2003.

[70] Heister, A. et al. (The ALEPH Collaboration). Absolute mass lower limit for the lightest neutralino of the MSSM from $e^{+} e^{-}$data at $\sqrt{s}$ up to $209 \mathrm{GeV}$. Phys. Lett., B 583:247-263, 2004.

[71] Abreu, P. et al. (The DELPHI Collaboration). Search for SUSY with R-parity violating LLE couplings at $\sqrt{(s)}=189 \mathrm{GeV}$. Phys. Lett., B 487:36-52, 2000.

[72] Abreu, P. et al. (The DELPHI Collaboration). Search for R-parity violation with a UUD coupling at $\sqrt{s}=189 \mathrm{GeV}$. Phys. Lett., B 500:22-36, 2001.

[73] Abreu, P. et al. (The DELPHI Collaboration). Search for spontaneous R-parity violation at $\sqrt{s}=183 \mathrm{GeV}$ and $189 \mathrm{GeV}$. Phys. Lett., B 502:24-36, 2001.

[74] M. Acciarri et al. (The L3 Collaboration). Search for R-Parity Breaking Sneutrino Exchange at LEP. Phys. Lett., B 414:373-381, 1997.

[75] P. Achard et al. (The L3 Collaboration). Search for Colour Reconnection Effects in $e^{+} e^{-} \rightarrow W^{+} W^{-} \rightarrow$ hadrons through Particle-Flow Studies at LEP. Phys. Lett., B $561: 202-212,2003$.

[76] P. Achard et al. (The L3 Collaboration). Search for R-parity Violating Decays of Supersymmetric Particles in $e^{+} e^{-}$Collisions at LEP. Phys. Lett., B 524:65-80, 2001.

[77] M. Acciarri et al. (The L3 Collaboration). Search for R-parity Violating Decays of Supersymmetric Particles in $e^{+} e^{-}$Collisions $\sqrt{s}=189 \mathrm{GeV}$. Eur. Phys. Journal, C19:397-414, 2001.

[78] G. Abbiendi et al. (The Opal Collaboration). Search for R-parity Violating Decays of Sfermions at LEP. Eur. Phys. Journal, C33:149, 2004. Cern preprint 2003-036.

[79] Aktas, A. et al. (The H1 Collaboration). Search for squark production in R-parity violating supersymmetry at HERA. Eur. Phys. J., C36:425-440, 2004.

[80] The Zeus Collaboration. Search for Stop Production in RPV SUSY at HERA. 2006. Proceeding submitted to ICHEP 2006, Moscow, Russia, ZEUS-pub-06-007.

[81] The DØ Collaboration. Search for R-parity Violating Supersymmetry in the Dielectron Channel. Phys. Rev. Lett., 83:4476, 1999. hep-ex/9907019.

[82] The DØ Collaboration. Search for R-parity violating supersymmetry in two-muon and four-Jet Channel. Phys. Rev. Lett., 89:171801, 2002. hep-ex/0111053.

[83] The Zeus Collaboration. Search for gaugino production in R-parity violating supersymmetry at HERA. 2005. Talk given at Lepton-Photon 2005, Uppsala, Sweden, ZEUS-prel-05-003. 
[84] The Zeus Collaboration. Search for gravitino production in R-parity violating supersymmetry at HERA. 2005. Talk given at Lepton-Photon 2005, Uppsala, Sweden, ZEUS-rel-05-019.

[85] F. Abe et al. (The CDF Collaboration). Search for R-parity Violating Supersymmetry using Like-Sign Dielectrons in $p \bar{p}$ Collisions at $\sqrt{s}=1.8 \mathrm{TeV}$. Phys. Rev. Lett., 83:2133, 1999.

[86] D. Acosta et al. (The CDF Collaboration). Search for Pair Production of Scalar Top Quarks in R-parity Violating Decay Modes in $p \bar{p}$ Collisions at $\sqrt{s}=1.8 \mathrm{TeV}$. Phys. Rev. Lett., 92:051803, 2004.

[87] M. Chertok et al. (The CDF Collaboration). Search for Supersymmetric Top Quarks Decaying to a b Quark and a tau Lepton. CDF note, 7835, 2005.

[88] K. Hahn et al. (The CDF Collaboration). High Mass Snuetrino Search in the RPV Electron-Muon Channel. CDF note, 7747, 2005.

[89] Abazov, V. M. et al. (The D $\varnothing$ Collaboration). Search for the production of single sleptons through $\mathrm{R}$ parity violation in $p \bar{p}$ collisions at $\sqrt{s}=1.8 \mathrm{TeV}$. Phys. Rev. Lett., 89:261801, 2002.

[90] The D $\varnothing$ Collaboration. Search for R-parity Violation in Multilepton Final States in $p \bar{p}$ Collisions at $\sqrt{s}=1.8 \mathrm{TeV}$. Phys. Rev. D Rapid. Comm., 62:071701, 2000. hep-ex/0005034.

[91] Abazov, V. M. et al. (The DØ Collaboration). Search for R-parity violating supersymmetry via the LLE couplings $\lambda_{121}, \lambda_{122}$ or $\lambda_{133}$ in $p \bar{p}$-collisions at $\sqrt{s}=1.96 \mathrm{TeV}$. Phys. Lett., B 638:441, 2006.

[92] A. Abdesselam, P. Petroff, and C. Royon. First Search for Resonant Slepton Production through R-parity Violation at Tevatron Run I. 2001. DØ Note 3838.

[93] F. Déliot. PhD thesis: Reconstruction et identification des muons dans l'experience $\mathrm{D} \varnothing$ Étude de la production résonnante de sleptons. 2002.

URL http://www-d0.fnal.gov/results/publications-talks/thesis.

[94] The DØ Collaboration. Complete list of D $\varnothing$ Run I publications.

URL http://www-d0.fnal.gov/www_buffer/pub/Run1_publications.html.

[95] Fermilab Beams Division. The Tevatron Accelerator Complex. 2004.

URL http://www-bdnew.fnal.gov/operations/rookie_books/rbooks.html.

[96] V. M. Abazov et al. (The DØ Collaboration). The Upgraded D $\varnothing$ Detector. 2005. submitted to Elsevier Science, physics/0507191.

[97] R. Bock. The Particle Detector BriefBook. 1999.

URL: http://rd11. web.cern.ch/RD11/rkb/titleD.html.

[98] R. L. Gluckstern. Uncertainties in track momentum and direction due to multiple scattering and measurement errors. Nucl. Instrum. Meth., 24:381, 1963. 
[99] R. M. Sternheimer and R. F. Peierls. General expression for the density effect for the ionization loss of charged particles. Phys. Rev., B3:3681, 1971.

[100] C. W. Fabjan and T. Ludlam. Calorimetry in High-Energy Physics. Ann. Rev. Nucl. Part. Sci., 32, 1982. reprinted in Formulae and Methods in Experimental Data Evaluation, R.K. Bock ed., European Physical Society 1984.

[101] W. Walraff. Prospects for Large Scintillating Noble Liquid Calorimeters. World Scientific, 1991. in: Proceedings of Calorimetry in High-energy Physics, Capri 1991, A.Ereditato ed.

[102] A. Lyon. A search for squarks and gluinos using the jets and missing energy signature at DØ. 1997.

[103] F. Zimmermann. Quantum Mechanical Limits on Beam Demagnification and Luminosity. 2002. Proceedings to the talk given at the Nanobeam Conference, CERN.

[104] J. D. Jackson. Classical Electrodynamics. 1999. Hamilton Printing Company, ISBN $047130932 \mathrm{X}$.

[105] Amos, N. et al. (The E710 Collaboration). Measurement of rho, the ratio of the real to imaginary part of the $p \bar{p}$ forward elastic scattering amplitude, at $\sqrt{s}=1.8 \mathrm{TeV}$. Phys. Rev. Lett., 68:2433-2436, 1992.

[106] Avila, C. et al. (The E811 Collaboration). A measurement of the proton anti-proton total cross section at $\sqrt{s}=1.8 \mathrm{TeV}$. Phys. Lett., B 445:419-422, 1999.

[107] Abe, F. et al. (The CDF Collaboration). Measurement of the anti-proton proton total cross-section at $\sqrt{s}=546 \mathrm{GeV}$ and $1800 \mathrm{GeV}$. Phys. Rev., D 50:5550-5561, 1994.

[108] M. Begel and H. Schellman. On averaging inelastic cross sections across experiments. DØ note 4153, 2004.

[109] G. Snow. Adjustments to DØ's RunIIa measured Luminosity. 2006. Talk given at the Manchster Collaboration Meeting.

[110] DØ Run Coordinators. DØ Operations Webpage. 2006.

URL http://www-d0.fnal.gov/runcoor/.

[111] G. Borissov. Status of DØTrack Reconstruction. Feb. 2003. Plenary Talk given at the $\mathrm{D} \emptyset$ collaboration meeting at Fermilab.

[112] DØ SMT group. DØ SMT home page. 2006.

URL http://dOserver1.fnal.gov/projects/Silicon/www/silicon.html.

[113] A. Kreymadhi and R. Van Kooten. Estimated Improvement in Proper Time Resolution with the Addition of Layer 0 Silicon. 2004. Internal DØ note 4418.

[114] DØ CFT group. DØ CFT home page. 2006.

URL http://d0server1.fnal.gov/projects/SciFi/cft_home.html. 
[115] D. Adams et al. First Large Sample Study of Visible Light Photon Counters (VLPC's). 1994.

URL: http://d0server1.fnal.gov/users/stefan/www/como94/como.html.

[116] The DØ Collaboration. Beam Test of The D $\varnothing$ Uranium Liquid Argon End Calorimeters. 1992. Fermilab-Pub-92/162, D $\varnothing$ Note 1434.

[117] Abbott, B. et al. (The D $\varnothing$ Collaboration). A measurement of the $\mathrm{W}$ boson mass. Phys. Rev., D58:092003, 1998.

[118] The D0 Muon Group; V. Abazov and others. The Muon System of the Run II D0 Detector. Nucl. Inst. Meth. A, 552:372, 2005.

[119] D. Denisov. The D $\varnothing$ Muon System. 2004. Talk at the D $\varnothing / C D F$ Muon Workshop.

[120] B. Baldin and others. Technical Design of the Central Muon System. 1998. D Note 3365 .

[121] G. Alexeev and others. Technical Design Report for the DØ Forward Muon Tracking Detector based on Mini Drift Tubes. 1997. DØ Note 3366.

[122] V. Abramov and others. Technical Design Report for the D $\varnothing$ forward trigger scintillation counters. 1997. DØ Note 3237.

[123] J. M. Butler. Local Muon Momentum Resolution. 2002. DØ Note 4002.

[124] S. Catani. Aspects of QCD, from the Tevatron to the LHC. 2000.

[125] Fermilab Computing Division. The SAM Grid project. 2005. URL http://projects.fnal.gov/samgrid/atwork.html.

[126] C. Autermann. The Level 3 isolation tool. 2004. DØ Note 4436.

[127] C. Magass. Track Isolation for EM Objects at Level 3. 2004. DØ Note 4664.

[128] V. Lesne. Muon isolation (cal and track) at L3. 2006. DØ Note 4988.

[129] A. Baranovski et al. SAM: Sequential access via meta data. Fermilab-TM-2175, 2002. URL http://www-d0.fnal.gov/computing/sam/.

[130] Enstore. URL http://www-d0en.fnal.gov/enstore/.

[131] The LHC Computing Grid. URL http://lcg.web.cern.ch/LCG/.

[132] S. Snyder. The DØObject Model. presented at Computing in High Energy Physics '98, Chicago, 1998. URL http://www.hep.net/chep98/PDF/130.pdf.

[133] R. Hauser. The DØFramework Software Tutorial. presented at the DØCollaboration week, 2003. URL http://www-d0.fnal.gov/computing/algorithms/howto/ tutorial/rhauser1.pdf.

[134] R. Brun, F. Rademakers, and S. Panacek. Root, an object oriented data analysis framework. Prepared for CERN School of Computing (CSC 2000), Marathon, Greece, 17-30 Sep 2000. 
[135] T. Sjostrand, L. Lonnblad, and S. Mrenna. PYTHIA 6.2: Physics and manual. 2001.

[136] R. Brun and F. Carminati. Geant 3. CERN Program Library Long Writeup W5013, unpublished, 1993.

[137] The DØ Collaboration. The D0 Run II Reconstruction Progrom. 2006. The DØ code repository;

URL http://www-d0.fnal.gov/computing/algorithms/status/index.html.

[138] M. Klute, M. Phaf, and D. Whiteson. TopAnalyze - A Framework Analyze Package for Top Group Analyses, and the source code of the TopAnalyze package. 2003. DØ Note 4122, URL http://www-d0.fnal.gov/Run2Physics/top/d0-private/ wg/top-analyze/Ipanema/Ipanema.html.

[139] B. Vachon et al. Top Trigger Efficiency Measurements and the top_trigger package. 2004. D $\varnothing$ Note 4512 and part of the DØ code repository;

URL http://www-d0.fnal.gov/Run2Physics/top/d0-private/wg/ top-analyze/Ipanema/Ipanema.html.

[140] T. Junk. Confidence level computation for combining searches with small statistics. Nucl. Instrum. Meth., A 434:435-443, 1999.

[141] V. Büscher. A modified version of TLimit, which handles asymmetric errors. 2005. Private communication.

[142] D. Boline and L. Feligioni and M. Narain. Update on b-quark jet identification with Secondary Vertex reconstruction using DØreco version p14-Pass2 Samples. 2005. DØ note 4796.

[143] T. Golling. Measurement of the tt Production Cross-Section at $\sqrt{s}=1.96 \mathrm{TeV}$ in the Muon+Jets Final State using a Topological Method. 2005. DØ Note 4667.

[144] D. Käfer and A. Meyer. A search for RPV-SUSY via the LLE-coupling $\lambda_{122}$ in the $\mu \mu l$ channel $(\mathrm{l}=\mu, \mathrm{e}) .2005$. D $\varnothing$ Note 4841.

[145] M. L. Mangano, M. Moretti, F. Piccinini, R. Pittau, and A. Polosa. ALPGEN, a generator for hard multiparton processes in hadronic collisions. JHEP, 307:001, 2003 .

[146] T. Christiansen and R. Ströhmer. Search for Second-Generation Leptoquarks in $\mu j+\mu j$ Events at $\sqrt{s}=1.96 \mathrm{TeV}$. 2005. DØ Note 4679 .

[147] T. Nunnemann. NNLO Cross-Sections for Drell-Yan, $Z$ and $W$ Production using Modern Parton Distribution Functions. 2004. DØ Note 4476, URL http: //www-clued0.fnal.gov/ nunne/cross-sections/nnlo-xsect.html.

[148] 2005. Upsilon cross section, private communication.

[149] N. Kikonakis and R. Vogt. Next-to-leading order soft-gluon corrections in top quark hadroproduction. Phys. Rev., D 68:114014, 2003. 
[150] DØ Run II Monte Carlo Production Webpage.

URL http://www-d0.fnal.gov/computing/mcprod/mcc.html.

[151] H. Marten, K. P. Mickel, and R. Kupsch. A Grid Computing Centre at Forschungszentrum Karlsruhe. 2001.

[152] R. Hamberg, W. L. van Neerven, and T. Matsuura. A complete calculation of the order $\alpha_{s}^{2}$ correction to the drell-yan k-factor. Nucl. Phys., B 359:343, 1991. Erratumibid. B 644, 403 (2002),

URL http://www. lorentz. leidenuniv.nl/“neerven/.

[153] P. Verdier et al. Search for Squarks ans Gluinos in the Jets + MET Topology with the DØ Detector. 2005. DØ Note 5267.

[154] D. E. Soper. Parton Distribution Functions. Rev. of Mod. Phys. Proceedings of talk given at the Lattice 96 Conference, St. Louis, published in hep-lat/9609018

URL http://zebu. uoregon.edu/ ${ }^{\sim}$ parton/.

[155] The H1 Collaboration. Complete list of H1 publications.

URL http://www-h1.desy.de/h1/www/publications/H1_sci_results.shtml.

[156] The ZEUS Collaboration. Complete list of ZEUS publications.

URL http://www-zeus.desy.de/publications.php3.

[157] V. N. Gribov and L. N. Lipatov. Deep inelastic e p scattering in perturbation theory. Sov. J. Nucl. Phys., 15:438-675, 1972.

[158] G. Altarelli and G. Parisi. Asymptotic freedom in parton language. Nucl. Phys., B 126:298, 1977.

[159] Y. L. Dokshitzer. Calculation of the structure functions for deep inelastic scattering and $e^{+} e^{-}$annihilation by perturbation theory in quantum chromodynamics. Sov. Phys. JETP, 46:641-653, 1977.

[160] B. Tuchming. Luminosity and isolation. 2005. Talk given at $\mathrm{W} / \mathrm{Z}$ to muons meeting, (2005) URL http://www-d0.hef.kun.nl///fullAgenda.php?ida=a05202.

[161] DØ Muon ID Group.

URL http://www-d0.fnal.gov/phys-id/muon-id/d0-private/muon-id.html.

[162] G. Blazey et al., U. Baur (ed.). Run II Jet Physics. pages 47-77, 2000. FermilabPub-00/297.

[163] U. Bassler and G. Bernadi. Towards a Coherent Treatment of Calorimetric Energies: Missing Transverse Energy, Jets, E.M. Objects and the T42 Algorithm. 2003. D Note 4124.

[164] G. Bernardi, E. Busato, and J.-R. Vlimant. Improvements from the T42 Algorithm on Calorimeter Objects Reconstruction. 2004. DØ Note 4335.

[165] DØ Jet Energy Scale Group. Jetcorr v5.3 with t42. URL http://www-d0.fnal. gov/phys-id/jes/d0-private/certified/v5.3/links.html. 
[166] A. Juste and C. Royon. DZero Preliminary Jet Energy Scale. 2006. URL http: //www-d0.fnal.gov/phys_id/jes/public/plots_v7.1/.

[167] M. Agelou, J.-L. Agram, and C. Royon. 0.5 and 0.7 jet $p_{T}$ resolution using JES v05-03-00. 2005. DØ Note 4775.

[168] Abazov, V. M. et al. (The DØ Collaboration). Measurement of $\sigma(p \bar{p} \rightarrow Z) \cdot \operatorname{Br}(Z \rightarrow$ $\tau \tau)$ at $\sqrt{s}=1.96 \mathrm{TeV}$. Phys. Rev., D 71:072004, 2005.

[169] S. Dimopoulos and L. J. Hall. Lepton and baryon number violating collider signatures from supersymmetry. Phys. Lett., B 207:210, 1988.

[170] H. K. Dreiner and G. G. Ross. R-parity violation at hadron colliders. Nucl. Phys., B 365:597-613, 1991.

[171] D. Choudhury, S. Majhi, and V. Ravindran. QCD corrections to resonant slepton production in hadron colliders. Nucl. Phys., B660:343-361, 2003.

[172] H. K. Dreiner, S. Grab, M. Kramer, and M. K. Trenkel. Supersymmetric NLO QCD corrections to resonant slepton production and signals at the Tevatron and the LHC. 2006. hep-ph/0611195.

[173] G. Corcella, I. G. Knowles, G. Marchesini, S. Moretti, P. Richardson K. Odagiri, M.H. Seymour, and B.R. Webber. HERWIG 6.5. JHEP, 0101, 2001.

[174] T. Edwards et al. The Updated D $\varnothing$ Luminosity Determination - Short Summary. 2004. D $\varnothing$ Note 4328.

[175] V. Büscher et al. Recommendation of the Ad-Hoc Committee on Limit-Setting Procedures to be used by D $\varnothing$ in Run II. 2004. D $\varnothing$ Note 4629.

[176] D. Käfer. A search for R-Parity Violating Supersymmetry in Multilepton Final States with the D $\varnothing$ Detector. 2006. Dissertation at the RWTH Aachen.

[177] J. D. Bjorken and Stanley J. Brodsky. Statistical model for electron - positron annihilation into hadrons. Phys. Rev., D 1:1416-1420, 1970.

[178] A. L. Read. Modified Frequentist Analysis of Search Results (The $C L_{S}$ Method). Talk given at the Workshop on Confidence Limits at CERN, 2000. CERN 2000-005.

[179] R. Barate et al. Search for the standard model Higgs boson at LEP. Phys. Lett., B 565:61-75, 2003.

[180] Abazov, V. M. et al. (The D $\varnothing$ Collaboration). Search for supersymmetry via associated production of charginos and neutralinos in final states with three leptons. Phys. Rev. Lett., 95:151805, 2005.

[181] Abazov, V. M. et al. (The DØ Collaboration). Search for resonant second generation slepton production at the tevatron. Phys. Rev. Lett., 97:111801, 2006.

[182] S. Kraml. Comparison of SUSY spectrum generators. 2005. URL http://kraml. home. cern.ch/kraml/comparison/. 
[183] F. Ledroit and G. Sajot. Indirect limits on SUSY R-parity violating couplings $e^{+} e^{-} \rightarrow f \bar{f}$ and $\lambda^{\prime}$. GDR-S-008, 1998. ISN Grenoble.

[184] E. Perez. private communication. 2005. 


\section{List of Figures}

2.1 The Higgs potential . . . . . . . . . . . . . . 10

2.2 Deviations from Newtonian Gravitation . . . . . . . . . . . . 15

2.3 One loop corrections to the Higgs mass . . . . . . . . . . . . 16

2.4 The supersymmetry breaking in the "hidden sector" $\ldots \ldots \ldots \ldots$. . . . 27

2.5 Schematic evolution of the coupling constants . . . . . . . . . . 28

2.6 Mass degeneration at the GUT scale . . . . . . . . . . . . . . 29

2.7 The mSUGRA parameter space . . . . . . . . . . . . . 34

2.8 Basic $\not_{p}$ trilinear interactions $\ldots \ldots \ldots \ldots \ldots \ldots \ldots$

2.9 Relative slepton branching fraction $\tilde{l} \rightarrow q \bar{q} \ldots \ldots \ldots \ldots$

2.10 Resonant slepton production and decay $\ldots \ldots \ldots \ldots$

2.11 Leading order slepton cross section . . . . . . . . . . . . . 39

2.12 Branching ratio of the slepton decay channels . . . . . . . . . . . 41

2.13 Pair- and associated gaugino production . . . . . . . . . . . 42

2.14 Cross section and $\mathrm{m}\left(\tilde{\chi}_{1}^{0}\right)$ dependence on $m_{1 / 2} \ldots \ldots \ldots \ldots$

2.15 Pion decay $\ldots \ldots \ldots \ldots \ldots \ldots \ldots \ldots$

2.16 Neutrino scattering involving $L Q \bar{d}$ couplings . . . . . . . . . . . . 45

2.17 The proton decay . . . . . . . . . . . . . . . 46

$2.18 R_{p}$ SUSY at LEP . . . . . . . . . . . . . . . . 48

$2.19 \not R_{p}$ Aleph limits . . . . . . . . . . . . . . . . 49

2.20 Aleph limits in the $3^{r d}$ family sector . . . . . . . . . . . . . 49

$2.21 \not R_{p} \mathrm{~L} 3$ limits . . . . . . . . . . . . . . . 50

$2.22 \not R_{p}$ Opal limits . . . . . . . . . . . . . . . 50

2.23 LEP limits in the $\mu-M_{2}$ plane $\ldots \ldots \ldots \ldots \ldots \ldots \ldots \ldots$ 


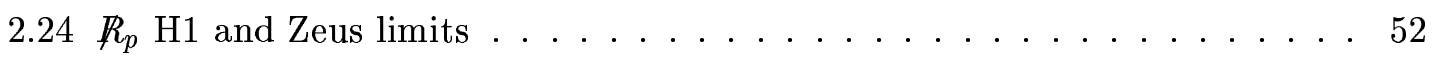

2.25 Resonant squark production at HERA . . . . . . . . . . . . . 53

2.26 Gaugino production at HERA . . . . . . . . . . . . . 53

2.27 DØ Run I resonant slepton exclusion contours within mSUGRA . . . . . . 54

$2.28 \mathrm{D} \varnothing$ Run I limit for pair and associated gaugino production $\ldots \ldots \ldots 5$

3.1 Energy loss for heavy charged particles . . . . . . . . . . . . 59

$3.2 d E / d x$ for electrons . . . . . . . . . . . . . . . . . 59

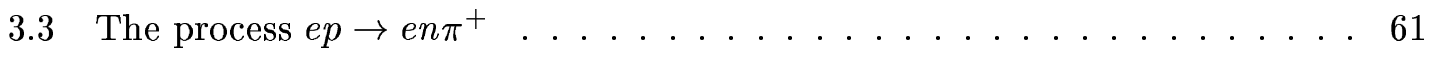

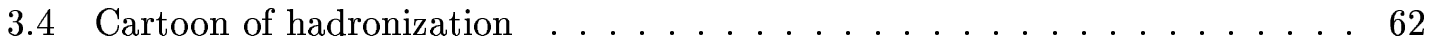

3.5 The accelerator chain of Fermilab . . . . . . . . . . 63

3.6 The $\mathrm{D} \emptyset$ detector $\ldots \ldots \ldots \ldots \ldots \ldots$

3.7 The Vertex distribution at $\mathrm{D} \emptyset \ldots \ldots \ldots \ldots \ldots$

3.8 The luminosity detector $\ldots \ldots \ldots \ldots \ldots$

3.9 A typical physics store . . . . . . . . . . . . 70

3.10 Total integrated luminosity and absolute data taking efficiency $\ldots$. . . . 70

3.11 The $\mathrm{D} \emptyset$ tracking system . . . . . . . . . . . . . . 71

3.12 Detector imaging by $\gamma \rightarrow e^{+} e^{-}$vertex reconstruction $\ldots \ldots \ldots . \ldots 72$

3.13 Silicon Microstrip Tracker . . . . . . . . . . . . . . 73

3.14 SMT Readout . . . . . . . . . . . . . . . . . 74

3.15 CFT fiber double layer . . . . . . . . . . . . . . . . . 74

3.16 Visible Light Photon Counter . . . . . . . . . . . . . . 75

3.17 The central scintillating fiber tracker . . . . . . . . . . . 76

3.18 The central and the two endcap calorimeters $\ldots \ldots \ldots \ldots$

3.19 Schematic view of the $\mathrm{D} \emptyset$ calorimeter $\ldots \ldots \ldots \ldots \ldots$

3.20 Schematic view of a calorimeter readout cell . . . . . . . . . . . 79

3.21 Exploded drawing of the $\mathrm{D} \emptyset$ muon system . . . . . . . . . . . 81

3.22 Cross section of a proportional drift tubes cell . . . . . . . . . . 83

3.23 Cross section of a mini drift tube . . . . . . . . . . . 84

3.24 Performance of the muon system . . . . . . . . . . . 85

3.25 The cross section for various processes at hadron-hadron colliders . . . . . 87 
3.26 Schematic block diagram of the trigger and DAQ . . . . . . . . . 88

3.27 Layout of an L2 preprocessor/global VME crate . . . . . . . . . . . 89

3.28 Schematic diagram of the level 3 data flow . . . . . . . . . . . . 90

3.29 Schematic event procession at the level 3 trigger tier $\ldots \ldots$. . . . . 90

3.30 Definition of the isolation cone shell f . . . . . . . . . . . . 91

3.31 Triggerlist excerpt $\ldots \ldots \ldots \ldots \ldots \ldots \ldots \ldots$

3.32 Comparison of measured online at L3 and offline . . . . . . . . . . 93

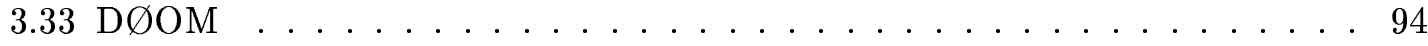

3.34 The used software and data types. . . . . . . . . . . . . 95

4.1 Comparison of QCD samples, extracted with and without b-tag . . . . . . 104

4.2 Pythia MC reweighting with respect to the jet multiplicity . . . . . . 106

4.3 Next-to leading order corrections of the process $q \bar{q} \rightarrow \gamma \ldots \ldots$. . . . 109

4.4 NNLO $k$-factor for the $Z / \gamma^{*}$ production cross section . . . . . . . . 110

4.5 Average number of interactions per bunch crossing . . . . . . . . . . 110

4.6 Kinematic plane in $x-Q^{2}$ and parton distribution functions . . . . . 112

5.1 L1 muon trigger efficiency . . . . . . . . . . . . . . 116

5.2 Muon trigger efficiency parametrization on L1 and L2 . . . . . . . 116

5.3 Di-muon trigger efficiency weight $\ldots \ldots \ldots \ldots \ldots$

5.4 Muon reconstruction efficiency $\ldots \ldots \ldots \ldots \ldots$

5.5 Muon reconstruction correction factor . . . . . . . . . . . . 119

5.6 Track finding and matching efficiency . . . . . . . . . . . 120

5.7 Muon track finding \& matching correction factor . . . . . . . . . . 121

5.8 Muon isolation correction factor . . . . . . . . . . . . . . 123

5.9 Jet dependence of the muon isolation correction . . . . . . . . . . . . . 124

5.10 Isolation efficiency . . . . . . . . . . . . . . . . . . . 124

5.11 Medium muon efficiency . . . . . . . . . . . . . . . . 125

5.12 Medium muon correction factor . . . . . . . . . . . . 126

5.13 Seed collinear sensitivity . . . . . . . . . . . . . . . 129

$5.14 E_{T}$ ordering sensitivity . . . . . . . . . . . . . . 129 
5.15 Infrared sensitivity . . . . . . . . . . . . . . . 129

5.16 Behavior of good and bad jets in the quality variables . . . . . . . . . 132

5.17 Jet reconstruction efficiency correction . . . . . . . . . . . . . 132

5.18 Jet energy scale correction for data events . . . . . . . . . . . . . 134

5.19 Jet energy scale correction for Monte Carlo events . . . . . . . . . . 134

5.20 Jet energy correction variables . . . . . . . . . . . . . 135

5.21 Jet resolution . . . . . . . . . . . . . . . . 137

6.1 Preselection sample $(2 \mu$-selection $) \ldots \ldots \ldots \ldots$. . . . . . . . . . . . . .

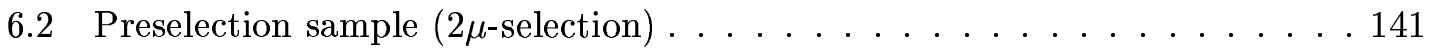

6.3 Comparison of the QCD sample to the expected QCD contribution . . . . 143

6.4 Influence of different PDFs and DØRECO versions on the signal kinematics 145

6.5 The $\left(\lambda_{211}^{\prime}\right)^{2}$ of the resonant slepton cross section . . . . . . . . . 146

6.6 Slepton production cross section in LO and NLO . . . . . . . . . . 146

6.7 Slepton $p_{T}$ in NLO calculation and MC simulation . . . . . . . . . 148

6.8 Muon and jet transverse momenta of the signal . . . . . . . . . . . . 148

6.9 Signal studies for $\tilde{\mu} \rightarrow \tilde{\chi}_{1}^{0} \mu \ldots \ldots \ldots \ldots \ldots \ldots$

6.10 Signal studies for $\tilde{\mu} \rightarrow \tilde{\chi}_{2}^{0} \mu$ and $\tilde{\nu}_{\mu} \rightarrow \tilde{\chi}_{1}^{ \pm} \mu \ldots \ldots \ldots \ldots$

6.11 Muon and jet efficiency in signal versus $\mathrm{m}\left(\tilde{\chi}_{1}^{0}\right) \ldots \ldots \ldots \ldots 151$

6.12 Preselection sample with additional jet requirement $(1) \ldots . . \ldots 153$

6.13 Flowchart final 2D-cut optimization algorithm. . . . . . . . . . 155

6.14 Final selection cuts for channel $\tilde{\mu} \rightarrow \tilde{\chi}_{1}^{0} \mu \ldots \ldots \ldots$. . . . . . . 160

6.15 Final selection cuts for channel $\tilde{\mu} \rightarrow \tilde{\chi}_{2,(3,4)}^{0} \mu \ldots \ldots \ldots 2$

6.16 Final selection cuts for channel $\tilde{\nu}_{\mu} \rightarrow \tilde{\chi}_{1}^{ \pm} \mu \ldots \ldots \ldots$. . . . . . 164

6.17 Parton density function uncertainties . . . . . . . . . . 166

6.18 Factorization and renormalization scale uncertainties . . . . . . . . 166

6.19 Final selected resonant slepton sample . . . . . . . . . . . 169

6.20 Deviation of data and Standard Model expectation . . . . . . . . . 170

6.21 Candidate event number $6545223 \ldots \ldots \ldots$. . . . . . . . . . 171

6.22 Candidate event number $30837455 \ldots \ldots$. . . . . . . . . . 172

6.23 Tight isolation di $-\mu$ selection $\ldots \ldots \ldots \ldots \ldots \ldots$ 
6.24 Final selected pair production sample . . . . . . . . . . . . 178

6.25 Candidate event number $46634818 \ldots \ldots \ldots$. . . . . . . . . 180

7.1 Model independent 95\% CL Cross section $\times$ Branching ratio limits . . . . . 184

7.2 Interpretation within the mSUGRA $m_{0}-m_{1 / 2}$-plane $\ldots \ldots \ldots 6$

7.3 Interpretation within mSUGRA mass planes . . . . . . . . . . . 187

7.4 Interpretation in the slepton mass - coupling strength plane . . . . . . . 188

7.5 Projections onto constant slepton and neutralino masses . . . . . . . . 189

7.6 Gaugino pair and associated production signal efficiency . . . . . . . 190

7.7 Gaugino pair and associated production cross section and limit . . . . . 191

7.8 Exclusion contour in the $m_{0}-m_{1 / 2}$ plane $\ldots \ldots \ldots$

7.9 Mass limits on $\mathrm{m}\left(\tilde{\chi}_{1}^{0}\right)$ and $\mathrm{m}\left(\tilde{d}_{R}\right) \ldots \ldots \ldots . \ldots \ldots 4$

8.1 Resonant slepton cross section at the Tevatron and the LHC $\ldots . . \ldots 197$

A.1 Possible neutralino decay channels . . . . . . . . . . . . . 200

A.2 Possible chargino decays channels . . . . . . . . . . . . . . 201

A.3 Possible neutralino 2 decays channels . . . . . . . . . . . . . 202 



\section{List of Tables}

2.1 The fermions of the Standard Model . . . . . . . . . . . . 7

2.2 The gauge bosons of the Standard Model $\ldots \ldots \ldots$

2.3 Chiral super-multiplets in the Minimal Supersymmetric Standard Model . . 23

2.4 Gauge or vector super-multiplets in the MSSM . . . . . . . . . . 24

2.5 Particle mass eigenstates in the minimal supersymmetric model . . . . . . 24

2.6 Limits from low-energy experiments on the product of two $\not R_{p}$-couplings $\ldots 47$

2.7 LEP limits for non-zero $L Q \bar{d}$-couplings $\lambda_{i j k}^{\prime} \ldots \ldots \ldots \ldots \ldots \ldots$

2.8 Summary of all constraints in case of a non-zero $L Q \bar{d}$-coupling $\lambda_{211}^{\prime} \ldots \ldots$

3.1 Basic characteristics of the accelerator chain and the Tevatron $\ldots \ldots$. . 64

3.2 Total and inelastic proton scattering cross section at $\sqrt{s}=1.8 \mathrm{TeV} \ldots \ldots 8$

3.3 Calorimeter energy resolution parameters $\ldots \ldots \ldots \ldots$. . . . . . 79

4.1 The reconstructed luminosity for each triggerlist period . . . . . . . 100

4.2 Standard isolation cuts . . . . . . . . . . . . . . . . . 102

4.3 Selection cuts for the QCD sample without b-tag. . . . . . . . . . . 102

4.4 Selection cuts for the QCD sample with b-tag. . . . . . . . . . 103

4.5 Weights applied on the b-tagged QCD sample . . . . . . . . . 103

4.6 All considered Standard Model backgrounds . . . . . . . . . . . . 107

5.1 The di-muon trigger requirements at L1/L2/L3 . . . . . . . . . . . 114

5.2 di-muon trigger turn-on functions . . . . . . . . . . . . 115

5.3 Muon reconstruction efficiency $\ldots \ldots \ldots \ldots \ldots$

5.4 Track finding times matching efficiency . . . . . . . . . . . . 122 
5.5 Muon isolation efficiency . . . . . . . . . . . . . . 122

5.6 Medium muon efficiency . . . . . . . . . . . . . . . . . 125

5.7 Muon momentum smearing coefficients . . . . . . . . . . . . . 127

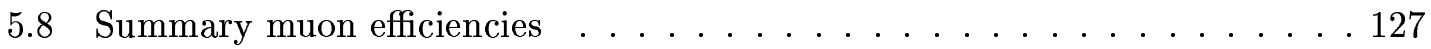

5.9 DØ Run II cone jet specifications . . . . . . . . . . . . . 130

5.10 Cut values on the $\mathrm{L} 1$ confirmation energy $\ldots \ldots \ldots \ldots$. . . . . . . 131

5.11 Fit values for the jet reconstruction efficiency correction . . . . . . . 133

6.1 The Preselection . . . . . . . . . . . . . . . . . . . 142

6.2 The signal reference point . . . . . . . . . . . . . . . . 147

6.3 Signal cut flow for $\tilde{\mu} \rightarrow \tilde{\chi}_{1}^{0} \mu$ with $\mathrm{m}(\tilde{\mu})=260 \mathrm{GeV}$ and $\mathrm{m}\left(\tilde{\chi}_{1}^{0}\right)=100 \mathrm{GeV} \ldots 151$

6.4 Cut flow toward a clean di-muon, di-jet selection . . . . . . . . . . 152

6.5 Parameters and score for cut 1 for the reference signal point. . . . . . . 157

6.6 Parameters and score for cut 2 for the reference signal point. . . . . . . 157

6.7 Parameters and score for cut 3 for the reference signal point. . . . . . . 158

6.8 Parameters and score for cut 4 for the reference signal point. . . . . . . . 158

6.9 Parameters and score for cut 5 for the reference signal point. . . . . . . 159

6.10 Parameters and score for cut 6 for the reference signal point. . . . . . . 159

6.11 Summary of the systematic uncertainties . . . . . . . . . . 167

6.12 Final result of an example point for resonant slepton production . . . . 168

6.13 The signal reference point . . . . . . . . . . . . . 173

6.14 Signal cut flow for point 11 with $\mathrm{m}\left(\tilde{\chi}_{1}^{0}\right)=39.6 \mathrm{GeV} \ldots \ldots . \ldots . . \ldots 174$

6.15 Cut flow of the gaugino pair and associated production analysis . . . . . 177

6.16 Final results for all studied mSUGRA parameter combinations . . . . . 179

7.1 Combination for $\mathrm{m}(\tilde{\mu})=260 \mathrm{GeV}$ and $\mathrm{m}\left(\tilde{\chi}_{1}^{0}\right)=100 \mathrm{GeV} \ldots \ldots \ldots$

7.2 Limits on the slepton mass and the right-handed down-squark mass . . . 185

C.1 Final Results for channel $\tilde{\mu} \rightarrow \tilde{\chi}_{1}^{0} \mu \ldots \ldots$. . . . . . . . . . . . .

C.2 Final Results for channel $\tilde{\mu} \rightarrow \tilde{\chi}_{2,(3,4)}^{0} \mu \ldots \ldots \ldots \ldots$. . . . . . . . . .

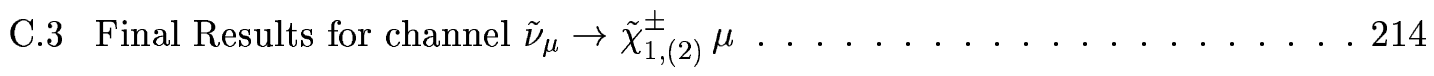




\section{Acknowledgments}

I like to thank my supervisor Prof. Dr. Thomas Hebbeker for the opportunity to work on an interesting analysis topic, for enabling my long visit at the $\mathrm{D} \varnothing$ experiment, and for the valuable advice and discussions. Many thanks to Prof. Dr. Lutz Feld for agreeing to be my second referee.

I am very thankful to Dr. Arnd Meyer for long inspiring discussions over a large variety of topics, for different perspectives, and much more; least of all for sharing the pain of lunch in the Mensa or the cafeteria.

I am deeply grateful to the collaborators who make the $\mathrm{D} \varnothing$ experiment successful. I like to thank the New Phenomena convenor Dr. Jean-Francois Grivaz and the former convenors Dr. Volker Büscher and Arnd, as well as the current and former editorial board chairs Dr. Marc Besançon and Dr. Pierre Pétroff for the help and the advice to prepare the resonant slepton analysis for publication.

Thanks also to Prof. Dr. Krämer and Maike Trenkel for the answers to many questions concerning supersymmetry, especially to Prof. Krämer for proof-reading the theory chapter. Thanks to Dr. Emmanuelle Perez for the quick support with Susygen.

Many thanks to my colleagues in Aachen; Arnd, Carsten, Daniel, Daniela, Jan, Matthias, Steffen and Volker for the nice working atmosphere and enlightening discussions during many coffee breaks. Special thanks to Dr. Daniela Käfer for the good teamwork on many $\not R_{p}$ SUSY topics.

I also thank the Deutsche Forschungsgemeinschaft, BMBF, and the HUK24 for their financial support.

Finally, I like to thank my parents and my sister for their support and for their interest in my work, not only during the last years of this dissertation. 



\section{Curriculum Vitae}

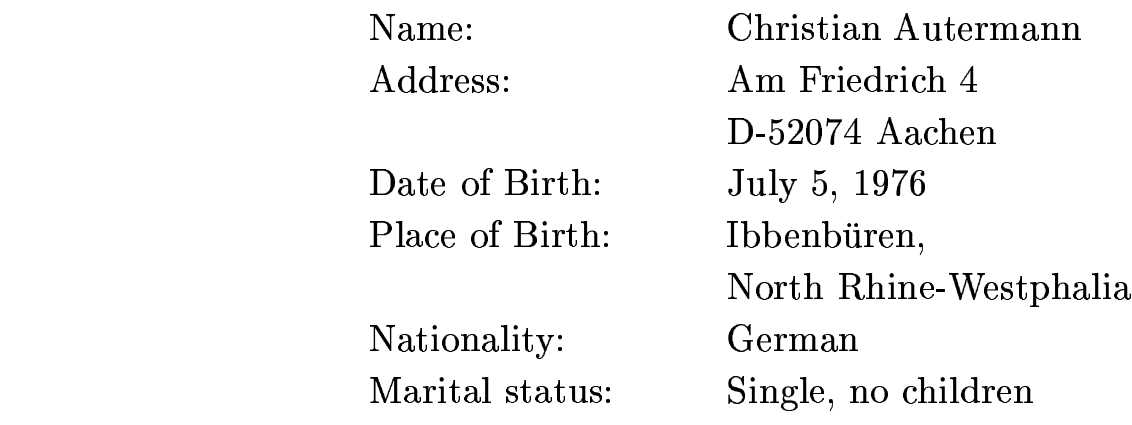

EDUCATION

School entry

June 1983

St. Ludgerus-Grundschule Hörstel, NRW, Germany

General qualification for university entrance (Abitur) June 1996 Johannes-Kepler-Gymnasium Ibbenbüren, NRW, Germany

Study of physics (Dipl.-Phys.)

April 1997 - June 2002

RWTH Aachen, NRW, Germany

Dissertation

since July 2002

III. Physikalische Institut A, RWTH Aachen, NRW, Germany

SERVICE

Compulsory military service

July 1996 - April 1997

German Bundeswehr Army, Coesfeld and Rheine

REASEARCH AND Diploma thesis

June 2001 - June 2002

EXPERIENCE

Funktionstests von Myonkammern für die Verwendung am CMS

Experiment am LHC am CERN, III. Phys. Inst. A, RWTH Aachen, Supervisor: Prof. Dr. T. Hebbeker

Physics diploma

June 2002

RWTH Aachen

Scholarship of the DFG July 2002 - June 2005

Scholarship of the Deutsche Forschungsgemeinschaft within the Graduate Seminar "Elementary Particle Physics at the TeV Scale"

Research stay

July 2003 - August 2004

Guest scientist at the Fermi National Accelerator Laboratory (FNAL), Batavia, Illinois, USA

Research Assistant

since July 2005

III. Physikalische Institut A, RWTH Aachen 

